Illinois State University

ISU ReD: Research and eData

Theses and Dissertations

3-29-2019

\title{
Synthesis And Metalation Of Internally Alkylated Porphyrinoids
}

Alissa Nicole Latham

Illinois State University, alissa.latham1@gmail.com

Follow this and additional works at: https://ir.library.illinoisstate.edu/etd

Part of the Organic Chemistry Commons

\section{Recommended Citation}

Latham, Alissa Nicole, "Synthesis And Metalation Of Internally Alkylated Porphyrinoids" (2019). Theses and Dissertations. 1085.

https://ir.library.illinoisstate.edu/etd/1085

This Thesis is brought to you for free and open access by ISU ReD: Research and eData. It has been accepted for inclusion in Theses and Dissertations by an authorized administrator of ISU ReD: Research and eData. For more information, please contact ISUReD@ilstu.edu. 


\section{SYNTHESIS AND METALATION OF INTERNALLY ALKYLATED PORPHYRINOIDS}

\section{ALISSA NICOLE LATHAM}

310 pages

The mechanism for alkyl group migration in palladium(II) benzocarbaporphyrins was investigated by synthesizing a 23-methylcarbaporphyrin and reacting it with palladium(II) acetate. It was found that palladium insertion led to methyl group migration to C21 and it is proposed that this occurs through an oxidative addition onto the palladium metal center, followed by a reductive elimination to transfer the methyl to the internal carbon. A similar rearrangement was observed when 23-methylcarbaporphyrin was reacted with $\left[\mathrm{Rh}(\mathrm{CO})_{2} \mathrm{Cl}\right]_{2}$ and this resulted in the formation of a rhodium(III) complex containing a three-membered rhodacycle. The syntheses of rhodium(I) and rhodium(III) derivatives of other carbaporphyrins, including 21- and 22-methylbenzocarbaporphyrins, were probed and four of these metalated complexes were characterized by X-ray crystallography. To further investigate the effect of internal substituents on porphyrinoid systems, a series of $N$-methylated porphyrinoids were synthesized using the versatile ' $3+1$ ' variant of the MacDonald condensation. The spectroscopic data for the internally methylated derivatives were compared to their $N$-unsubstituted counterparts. It was found that internal alkylation greatly affects the chromophore of these systems, as is evident by bathochromic shifts and peak broadening in the UV-Vis spectra. However, the aromatic character of these systems is fully retained after the introduction of an internal substituent, and proton NMR spectroscopy shows the presence of large macrocyclic ring currents. 
KEYWORDS: carbaporphyrin, rhodium, aromaticity, metalation, alkylation, rearrangement, crystallography 
ALISSA NICOLE LATHAM

A Thesis Submitted in Partial

Fulfilment of the Requirements for the Degree of

MASTER OF SCIENCE

Department of Chemistry

ILLINOIS STATE UNIVERSITY

2019 
(C) 2019 Alissa Nicole Latham 
ALISSA NICOLE LATHAM

COMMITTEE MEMBERS:

Timothy D. Lash, Chair

Gregory M. Ferrence

Shawn R. Hitchcock 


\section{ACKNOWLEDGMENTS}

The author would like to acknowledge the members of her research group and thank them for their support throughout the duration of her time at Illinois State University. The author would also like to thank Dr. Timothy D. Lash for his endless patience, guidance and encouragement throughout the time she spent working with him. The writer acknowledges financial support from the National Science Foundation under grants no. CHE-1212691 and CHE-1465049 and the Petroleum Research Fund. The author also thanks NSF grant no. CHE-1039689 for funding the Xray diffractometer.

The writer would also like to thank the members of her committee. Dr. Gregory M. Ferrence for his assistance in obtaining all the X-ray crystal structures and his guidance, and Dr. Shawn R. Hitchcock for his encouragement and support. Finally, the author thanks her parents for their unending love and encouragement and her dog Nala for always lifting her spirits.

A. N. L 


\section{CONTENTS}

Page

ACKNOWLEDGMENTS

CHARTS

FIGURES

SCHEMES $\quad \mathrm{x}$

CHAPTERS

$\begin{array}{lr}\text { I. INTRODUCTION } & 1\end{array}$

II. ALKYL GROUP MIGRATION IN INTERNALLY METHYLATED

PALLADIUM(II) BENZOCARBAPORPHYRINS 32

$\begin{array}{ll}\text { Introduction } & 32\end{array}$

Results and Discussion 36

$\begin{array}{ll}\text { Conclusion } & 51\end{array}$

$\begin{array}{ll}\text { Experimental } & 52\end{array}$

III. RHODIUM COMPLEXES OF CARBAPORPHYRINOID SYSTEMS 59

$\begin{array}{ll}\text { Introduction } & 59\end{array}$

$\begin{array}{ll}\text { Results and Discussion } & 61\end{array}$

$\begin{array}{ll}\text { Conclusion } & 94\end{array}$

$\begin{array}{ll}\text { Experimental } & 95\end{array}$

IV. SYNTHESIS AND CHARACTERIZATION OF N-METHYLATED

$\begin{array}{ll}\text { PORPHYRINOIDS } & 102\end{array}$

$\begin{array}{ll}\text { Introduction } & 102\end{array}$

$\begin{array}{ll}\text { Results and Discussion } & 102\end{array}$ 
V. CONCLUSIONS

APPENDIX B: SPECTROSCOPIC DATA FOR

23-METHYLBENZOCARBAPORPHYRIN AND PALLADIUM(II) COMPLEXES 


\section{CHARTS}

Chart

Page

1. Examples of porphyrin and porphyrin analogues 3

2. Organometallic benzocarbaporphyrins 4

3. Organometallic complexes of benziporphyrin and azuliporphyrin 4

4. Rhodium(III) and iridium(III) azuliporphyrins $\quad 5$

5. Porphyrin analogues and their metal complexes 6

6. Hetero- and diheteroporphyrinoids 9

7. Common $\pi$-electron delocalization pathways observed in porphyrinoids 12

8. Examples of methylated N-confused porphyrins 26

9. Examples of metalated azuliporphyrins 61 


\section{FIGURES}

Figure

Page

1. Shielding and deshielding regions due to porphyrin ring current 13

2. Dipolar resonance structure for azuliporphyrins 17

3. $500 \mathrm{MHz}{ }^{1} \mathrm{H}$ NMR spectrum of pyrrole ester $\mathbf{7 8}$ in $\mathrm{CDCl}_{3}$. The multiplet at $5.23 \mathrm{ppm}$ corresponds to a small amount of the related isopropyl ester pyrrole (ca. 3\%) that results from the transesterification with the solvent 2-propanol

4. $500 \mathrm{MHz}{ }^{1} \mathrm{H}$ NMR spectrum of 23-methylbenzocarbaporphyrin 76 in $\mathrm{CDCl}_{3}$

5. UV-vis spectra of 23-methylcarbaporphyrin $\mathbf{7 6}$ in $1 \%$ triethylamine-dichloromethane (free base, red line) and 1\% TFA-dichloromethane (monocation $\mathbf{7 6} \mathrm{H}^{+}$, purple line)

6. Color POV-Ray rendered ORTEP III drawing (50\% probability level, hydrogen atoms rendered arbitrarily small for clarity) of 23-methylcarbaporphyrin $\mathbf{7 6}$

7. UV-vis spectra of palladium(II) carbaporphyrin complexes 84 (23-methyl, red line) and 72 (21-methyl, blue line)

8. Downfield region for the ${ }^{1} \mathrm{H}$ NMR spectra of 23-methyl palladium complex 84 (top spectrum) and 21-methyl palladium complex 72 (bottom spectrum)

9. Color POV-Ray rendered ORTEP III drawing (50\% probability level, hydrogen atoms rendered arbitrarily for clarity) of 21-methyl palladium(II) complex 72

10. Time course experiments on the rate of alkyl group migration, showing the conversion of 84 (black line) into 72 (red line) a) Timed reflux reactions b) Reaction of 84 in $d_{3}$-acetonitrile at $70{ }^{\circ} \mathrm{C}$ in an NMR tube within a $500 \mathrm{MHz}$ NMR instrument

11. $500 \mathrm{MHz}{ }^{1} \mathrm{H}$ NMR spectrum of rhodium(I) carbaporphyrin diester 89 in $\mathrm{CDCl}_{3}$ 
13. IR spectrum of rhodium(I) carbaporphyrin diester $\mathbf{8 9}$

14. UV-Vis spectrum of rhodium(I) carbaporphyrin diester 89

15. Color POV-Ray rendered ORTEP III drawing (50\% probability level, hydrogen atoms rendered arbitrarily small for clarity) of rhodium(I) carbaporphyrin $\mathbf{8 9}$

16. $500 \mathrm{MHz}{ }^{1} \mathrm{H}$ NMR spectrum of rhodium(III) carbaporphyrin diester 90 in $d_{6}$-benzene

17. UV-Vis spectrum of rhodium(III) carbaporphyrin diester $\mathbf{9 0}$

18. Color POV-Ray rendered ORTEP III drawing (50\% probability level, hydrogen atoms rendered arbitrarily small or omitted for clarity) of rhodium(III) complex $\mathbf{9 0}$

19. $500 \mathrm{MHz}{ }^{1} \mathrm{H}$ NMR spectrum of rhodium(I) naphthocarbaporphyrin 91 in $\mathrm{CDCl}_{3}$

20. $125 \mathrm{MHz}{ }^{13} \mathrm{C}$ NMR spectrum of rhodium(I) naphthocarbaporphyrin 91 in $\mathrm{CDCl}_{3}$

21. IR spectrum of rhodium(I) naphthocarbaporphyrin 91

22. UV-Vis spectrum of rhodium(I) naphthocarbaporphyrin 91

23. Color POV-Ray rendered ORTEP III drawing (50\% probability level, hydrogen atoms rendered arbitrarily small for clarity) of rhodium(I) naphthocarbaporphyrin 91

24. $500 \mathrm{MHz}{ }^{1} \mathrm{H}$ NMR spectrum of rhodium(III) naphthocarbaporphyrin 92 in $d_{6}$-benzene

25. $125 \mathrm{MHz}{ }^{13} \mathrm{C}$ NMR spectrum of rhodium(III) naphthocarbaporphyrin 92 in $d_{6}$-benzene

26. UV-Vis spectrum of rhodium(III) naphthocarbaporphyrin 92

27. $500 \mathrm{MHz}{ }^{1} \mathrm{H}$ NMR spectrum of rhodium(I) 22-methylbenzocarbaporphyrin 93 in $\mathrm{CDCl}_{3}$

28. $125 \mathrm{MHz}{ }^{13} \mathrm{C}$ NMR spectrum of rhodium(I) 22-methylbenzocarbaporphyrin 93 in $\mathrm{CDCl}_{3} 84$

29. IR spectrum of rhodium(I) 22-methylbenzocarbaporphyrin 93

30. UV-Vis spectrum of rhodium(I) 22-methylbenzocarbaporphyrin 93

31. $500 \mathrm{MHz}{ }^{1} \mathrm{H}$ NMR spectrum of rhodium(I) 21-methylbenzocarbaporphyrin 94 in $\mathrm{CDCl}_{3}$

32. $125 \mathrm{MHz}{ }^{13} \mathrm{C}$ NMR spectrum of rhodium(I) 21-methylbenzocarbaporphyrin 94 in $\mathrm{CDCl}_{3}$ 
33. IR spectrum of rhodium(I) 21-methylbenzocarbaporphyrin 94

34. UV-Vis spectrum of rhodium(I) 21-methylbenzocarbaporphyrin 94

35. $400 \mathrm{MHz}{ }^{1} \mathrm{H}$ NMR spectrum of bridged rhodium(III) complex 95 in $\mathrm{CDCl}_{3}$

36. $100 \mathrm{MHz}{ }^{13} \mathrm{C}$ NMR spectrum of bridged rhodium(III) complex 95 in $\mathrm{CDCl}_{3}$

37. UV-Vis spectrum of bridged rhodium(III) complex 95

38. Color POV-Ray rendered ORTEP III drawing (50\% probability level, hydrogen atoms rendered arbitrarily small for clarity) of methylene-bridged rhodium(III) carbaporphyrin 95

39. $500 \mathrm{MHz}{ }^{1} \mathrm{H}$ NMR spectrum of 23-methyl-21-oxaporphyrin $96 \mathbf{b}$ in $\mathrm{CDCl}_{3}$

40. $125 \mathrm{MHz}{ }^{13} \mathrm{C}$ NMR spectrum of 23-methyl-21-oxaporphyrin 96b in $\mathrm{CDCl}_{3}$

41. UV-Vis spectra of $\mathbf{9 6 \mathbf { b }}$ in $1 \%$ triethylamine-dichloromethane (free base, pink line) and 96a in 1\% triethylamine-dichloromethane (free base, black line)

42. UV-Vis spectra of $\mathbf{9 6 a}$ in $1 \%$ triethylamine-dichloromethane (free base, dark blue line) or 1\% TFA-dichloromethane (dication, light blue line), and $\mathbf{9 6 b}$ in $1 \%$ triethylamine-dichloromethane (free base, red line) or 1\% TFA-dichloromethane (dication, pink line)

43. $500 \mathrm{MHz}{ }^{1} \mathrm{H}$ NMR spectrum of $N$-methyl thiaporphyrin $97 \mathbf{b}$ in $\mathrm{CDCl}_{3}$

44. $125 \mathrm{MHz}{ }^{13} \mathrm{C}$ NMR spectrum of $\mathrm{N}$-methyl thiaporphyrin $\mathbf{9 7 \mathbf { b }}$ in $\mathrm{CDCl}_{3}$

45. UV-Vis spectra of $\mathbf{9 7 \mathbf { b }}$ in $1 \%$ triethylamine-dichloromethane (free base, purple line) and 97a in 1\% triethylamine-dichloromethane (free base, black line) 
46. UV-Vis spectra of $\mathbf{9 7 \mathbf { b }}$ in $1 \%$ triethylamine-dichloromethane (free base, blue line) and 1\% TFA-dichloromethane (dication, green line), and 97a in 1\% triethylaminedichloromethane (free base, purple line) and 1\% TFA-dichloromethane (dication, red line)

47. $500 \mathrm{MHz}{ }^{1} \mathrm{H}$ NMR spectrum of $N$-methyl porphyrin $99 \mathbf{b}$ in $\mathrm{CDCl}_{3}$

48. $125 \mathrm{MHz}{ }^{13} \mathrm{C}$ NMR spectrum of $N$-methyl porphyrin 99b in $\mathrm{CDCl}_{3}$

49. UV-Vis spectra of $N$-methyl porphyrin 99b (red line) and $N$-unsubstituted porphyrin 99a (black line)

50. UV-Vis spectra of $\mathbf{9 9 b}$ in $1 \%$ triethylamine-dichloromethane (free base, black line) and 1\% TFA-dichloromethane (dication, purple line)

51. $500 \mathrm{MHz}{ }^{1} \mathrm{H}$ NMR spectrum of $N$-methyloxybenziporphyrin 100a in $\mathrm{CDCl}_{3}$

52. $125 \mathrm{MHz}{ }^{13} \mathrm{C}$ NMR spectrum of $N$-methyloxybenziporphyrin 100a in $\mathrm{CDCl}_{3}$

53. UV-Vis spectra of $N$-methyloxybenziporphyrin 100a (blue line) and $N$-unsubstituted oxybenziporphyrin 7a (black line)

54. UV-Vis spectra of 100a in 1\% triethylamine-dichloromethane (free base, black line) and 1\% TFA-dichloromethane (dication, blue line)

55. IR spectrum of $N$-methyloxybenziporphyrin 100a

56. $500 \mathrm{MHz}{ }^{1} \mathrm{H}$ NMR spectrum of palladium(II) oxybenziporphyrin complex 102 in $\mathrm{CDCl}_{3}$

57. $125 \mathrm{MHz}{ }^{13} \mathrm{C}$ NMR spectrum of palladium(II) oxybenziporphyrin complex 102 in $\mathrm{CDCl}_{3}$

58. IR spectrum of palladium(II) oxybenziporphyrin complex 102

59. $500 \mathrm{MHz}{ }^{1} \mathrm{H}$ NMR spectrum of $N$-methyloxypyriporphyrin 100b in $\mathrm{CDCl}_{3}$ 
60. $125 \mathrm{MHz}{ }^{13} \mathrm{C}$ NMR spectrum of $N$-methyloxypyriporphyrin $\mathbf{1 0 0 b}$ in $\mathrm{CDCl}_{3}$

61. IR spectrum of $N$-methyloxypyriporphyrin 100b

62. UV-Vis spectra of $N$-methyloxypyriporphyrin $\mathbf{1 0 0 b}$ (green line) and the $N$-unsubstituted oxypyriporphyrin $\mathbf{7 b}$ (black line)

63. UV-Vis spectra of $\mathbf{1 0 0 b}$ in $1 \%$ triethylamine-dichloromethane (free base, black line) and 1\% TFA-dichloromethane (monocation, green line)

64. $500 \mathrm{MHz}{ }^{1} \mathrm{H}$ NMR spectrum of tert-butyl azuliporphyrin $\mathbf{1 0 3 H}_{2}{ }^{2+}$ in TFA- $d_{6}$-DMSO

65. $125 \mathrm{MHz}{ }^{13} \mathrm{C}$ NMR spectrum of tert-butyl azuliporphyrin $\mathbf{1 0 3 H}_{2}{ }^{2+}$ in TFA- $d_{6}$-DMSO

66. UV-Vis spectra of 103a in 1\% triethylamine-dichloromethane (free base, black line) and $\mathbf{1 0 3 b}$ in 1\% triethylamine-dichloromethane (free base, purple line)

67. UV-Vis spectra of $\mathbf{1 0 3 b}$ in $1 \%$ triethylamine-dichloromethane (free base, black line) and in 1\% TFA-dichloromethane (dication, red line) 


\section{SCHEMES}

Scheme

Page

1. Formation of palladium(II) complexes of 23-oxa-, 23-thia- and 23-selena-21benzocarbaporphyrins

2. Formation of nickel(II) complexes 24a,b and oxyheterocarbaporphyrin

3. C-H activation of aryl units by Rh(III) porphyrins

4. Ring contraction of Rh(III) benziporphyrins into Rh(III) carbaporphyrins containing a three-membered rhodacycle

5. Mono- and diprotonation of benzocarbaporphyrin 3

6. Protonation of tropiporphyrin 9

7. Protonation of benziporphyrin $\mathbf{5}$

8. Protonation of dimethoxybenziporphyrin $\mathbf{8}$

9. Tetramerization of monopyrroles to produce porphyrins

10. Etioporphyrin type-isomers from the acid-catalyzed tetramerization of a pyrrole

11. '2+2' MacDonald condensation reaction

12. '3+1'variant of the MacDonald Condensation

13. Synthesis of a tripyrrane

14. Synthesis of heteroporphyrins through the ' $3+1$ ' variant of the MacDonald reaction

15. Methylation of NCP to give a $N, N$-dimethyl NCP

16. Alkylation of an oxidized benziporphyrin derivative

17. Alkylation of palladium(II) oxybenziporphyrin

18. Base-promoted rearrangement of palladium(II) p-benziporphyrin to palladium(II) carbaporphyrin 
19. Alkyl group migration in a palladium(II) benzocarbaporphyrin complex 30

20. Preparation of benzocarbaporphyrin via the ' $3+1$ ’ MacDonald condensation 33

21. Methylation of benzocarbaporphyrin 73

22. Proposed [1,5] sigmatropic rearrangement for alkyl group migration 35

23. Synthesis and protonation of 23-methylbenzocarbaporphyrin $\mathbf{7 6} 36$

24. Barton-Zard synthesis and methylation of a pyrrole precursor 37

25. Synthesis of acetoxymethylpyrrole $\mathbf{8 0} 39$

26. Synthesis of $N$-methyl tripyrrane 54d $\quad 40$

27. Metalation of 23-methylbenzocarbaporphyrin to give 23- and 21-methyl

$\begin{array}{ll}\text { palladium(II) complexes } & 45\end{array}$

28. Proposed mechanism for migration of an alkyl group from the 23- to 21-position

$\begin{array}{ll}\text { of benzocarbaporphyrins } & 50\end{array}$

29. Metalation of benzocarbaporphyrin 3 to give rhodium(I) and rhodium(III) derivatives 60

30. Rhodium(I) and rhodium(III) complexes of carbaporphyrin diester $\mathbf{8 8}$

31. Rhodium(I) and rhodium(III) complexes of naphthocarbaporphyrin 87

32. Formation of 21- and 22-methyl rhodium(I) derivatives $\mathbf{9 4}$ and $\mathbf{9 3} 81$

33. Formation of rhodium derivative $\mathbf{9 5}$ of 23-methylcarbaporphyrin $\mathbf{7 6} 89$

34. '3+1’ Synthesis of 23-methyl-21-oxaporphyrin 96

35. '3+1' Synthesis of 23-methyl-21-thiaporphyrin $97 \quad 108$

36. '3+1' Synthesis of 23-methylporphyrin 99

37. ' $3+1$ ' Synthesis of $N$-methylated and $N$-unsubstituted oxybenzi- and $\begin{array}{ll}\text { oxypyriporphyrins } & 117\end{array}$

38. Metalation of $N$-methyloxybenziporphyrin 100a with palladium(II) acetate 122 
39. Synthesis of $\mathrm{N}$-methylazuliporphyrin $\mathbf{1 0 3 b \mathbf { b } _ { 2 }}{ }^{2+}$ 


\section{CHAPTER I: INTRODUCTION}

Porphyrins 1 (Chart 1) and metalloporphyrins have been widely investigated, due not only to their biological importance, but also to their numerous applications. Metalloporphyrins provide important functions in nature, including oxygen transportation by hemoglobin and photosynthesis with chlorophyll, oxidation reactions with the cytochromes, and many redox functions as well. ${ }^{1,2-}$

${ }^{5}$ Porphyrins and closely related phthalocyanines are two classes of conjugated systems that are used in areas such as dyes and inks, as well as in the development of optical materials with potential use in optical recording technology. ${ }^{1,6}$ Metalloporphyrins, incorporating numerous different metals, have been investigated in the development of artificial molecular recognition receptors, enzyme mimics, DNA cleaving agents, chiral catalysts for asymmetric synthesis, materials, and in the discovery of new reactions. ${ }^{2,5,7-11}$ Certain porphyrins are also known for their applications as photosensitizers within the medical field, because of their high affinity for tumor cells and ability to produce singlet oxygen in Photodynamic Therapy (PDT). ${ }^{12}$

To act as a photosensitizer, the compound needs to absorb energy from light, which it then uses to produce the singlet oxygen. Unfortunately, light is strongly absorbed by bodily tissue over much of the visible region, which places limitations on clinical applications. ${ }^{6}$ Light has the greatest penetration at higher wavelengths, specifically between $650-800 \mathrm{~nm}$, which is the reason there has been extensive research directed towards developing photosensitizers that absorb in this range of wavelengths. Therefore, modifying porphyrin and porphyrin analogue structures has been explored in an attempt to produce molecules that absorb within the needed wavelength range. Investigations into modifying the porphyrin chromophore also gives insights into the coordination 
chemistry of porphyrin-like systems, as well as the nature of conjugation and aromaticity seen in these macrocyclic molecules. ${ }^{6}$

Porphyrin is one of nature's most versatile ligands, forming coordination complexes with virtually every known metal. ${ }^{13}$ The majority of elements in the periodic table can form complexes with porphyrins, and many of them can also take on additional axial ligands. ${ }^{14}$ These macrocyclic compounds possess an internal cavity that contains four nitrogen atoms and this allows for the flexibility in coordination. However, the internal atoms of this cavity can be altered, which gives rise to a number of different porphyrin derivatives. Carbaporphyrins ${ }^{15-18}$ are porphyrin analogues in which one or more of the internal nitrogen atoms are replaced with a carbon atom, and include true carbaporphyrins such as 2 and $3,{ }^{16,18}$ azuliporphyrins $\mathbf{4},{ }^{19}$ benziporphyrins $5,{ }^{20} \mathrm{~N}$-confused porphyrins $\mathbf{6},{ }^{21}$ oxybenziporphyrins 7a, ${ }^{22,23}$ dimethoxybenziporphyrins $\mathbf{8}^{24}$ and tropiporphyrins $\mathbf{9}^{25}$ (Chart 1). 


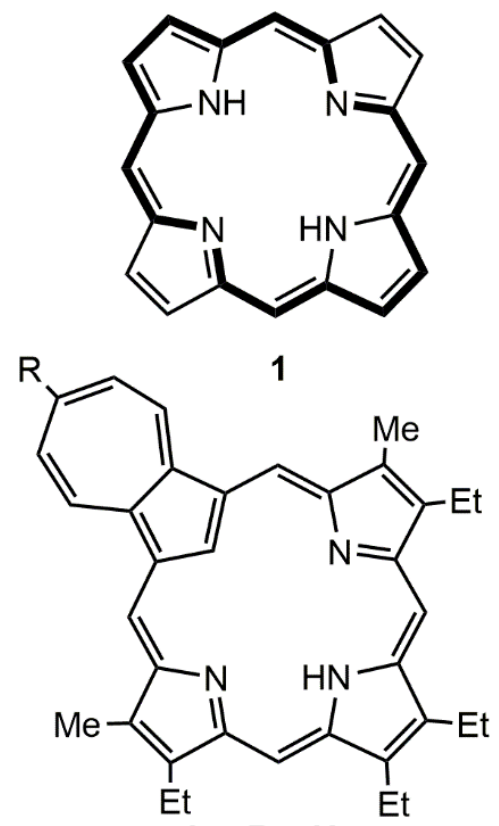

4 a. $R=H$

b. $R=t$-butyl

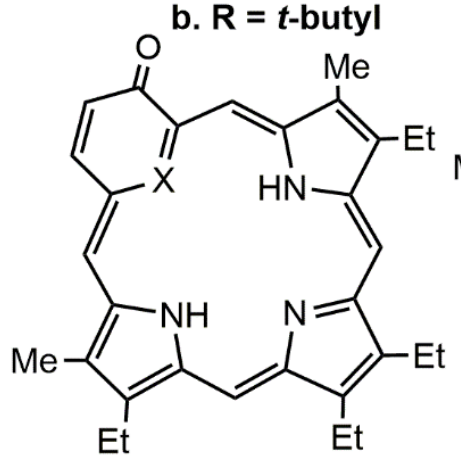

7 a. $X=C H$

b. $X=N$

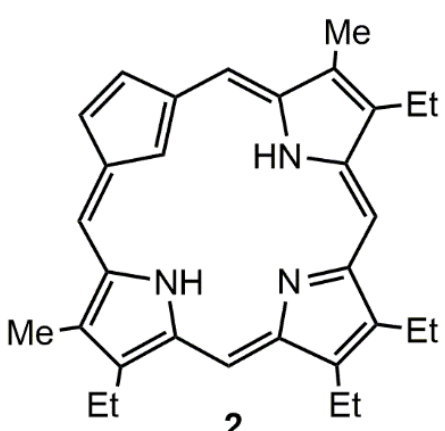<smiles>CCC1=C(C)/C2=C/c3cccc(c3)/C=C3\N=C(/C=c4/[nH]/c(c(CC)c4CC)=C\C1=N2)C(C)=C3CC</smiles>

5<smiles>CCC1=C(C)C2=NC1=Cc1cc(c(OC)cc1OC)C=C1N=C(C=c3[nH]c(c(CC)c3CC)=C2)C(C)=C1C</smiles>

8<smiles>CCC1=C(CC)c2cc3[nH]c(cc4cc(cc5[nH]c(cc1n2)c(C)c5C)-c1ccccc1-4)c(C)c3CC</smiles><smiles>[R]C1=Nc2cc1cc1[nH]c(cc3[nH]c(cc4nc(c2)C(CC)=C4CC)c(C)c3CC)c(CC)c1C</smiles>

6 a. $\mathbf{R}=\mathbf{H}$

b. $\mathbf{R}=\mathbf{M e}$<smiles>CCC1=C(C)C2=NC1=CC1C=CC=CC(=C1)C=C1N=C(C=c3[nH]c(c(CC)c3CC)=C2)C(C)=C1C</smiles>

9

Chart 1. Examples of porphyrin and porphyrin analogues

Even with the modified core, these carbaporphyrinoids still undergo metal coordination, albeit with a smaller number of mainly late transition metals. ${ }^{26}$ True carbaporphyrins, such as 2 and 3, normally act as trianionic ligands. Benzocarbaporphyrin $\mathbf{3}$ has been shown to form complexes with metals such as $\mathrm{Ag}(\mathrm{III}),{ }^{27-29} \mathrm{Au}(\mathrm{III}),{ }^{28} \mathrm{Rh}(\mathrm{III}){ }^{30}$ and $\mathrm{Ir}(\mathrm{III})^{30}$ to give organometallic derivatives such as 10a,b and 11a,b (Chart 2). On the other hand, benziporphyrins ${ }^{31}$ act as dianionic ligands affording metal complexes such as 12a-c, and azuliporphyrins similarly react to 
afford $\mathrm{Ni}(\mathrm{II}),{ }^{32} \mathrm{Pd}(\mathrm{II})^{32}$ and $\mathrm{Pt}(\mathrm{II})^{32}$ complexes 13a-c, as well as Ru(II) derivative $\mathbf{1 4}^{33}$ (Chart 3). Azuliporphyrins can also form Rh(III) complexes 15a-c and 16, and $\operatorname{Ir}(\mathrm{III})$ derivatives 17a-d (Chart 4), but these metal centers have an additional axial ligand. ${ }^{34,35}$

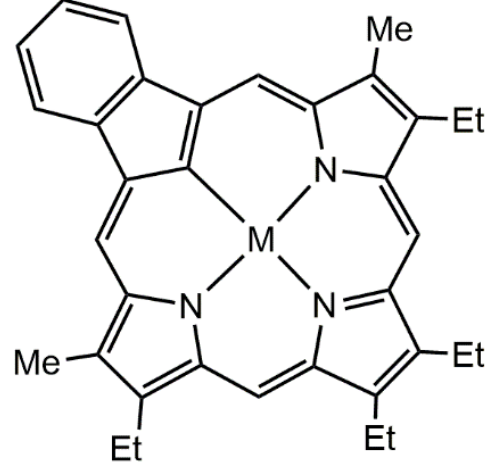

10 a. $M=A g(I I I)$

b. $M=A u(I I I)$

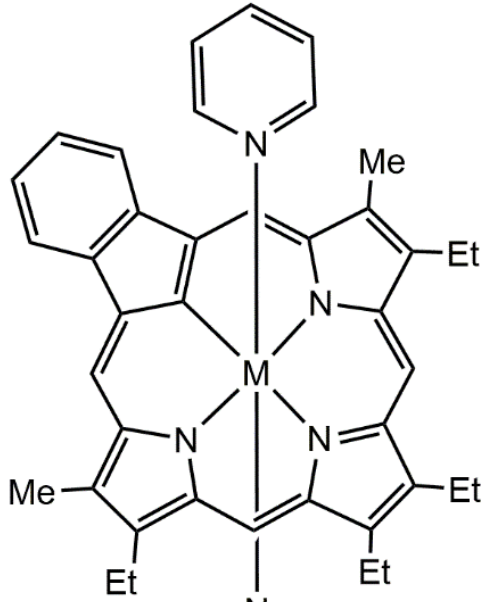

11 a. $M=\operatorname{Rh}(I I I)$

b. $M=\operatorname{Ir}(I I I)$

Chart 2. Organometallic benzocarbaporphyrins

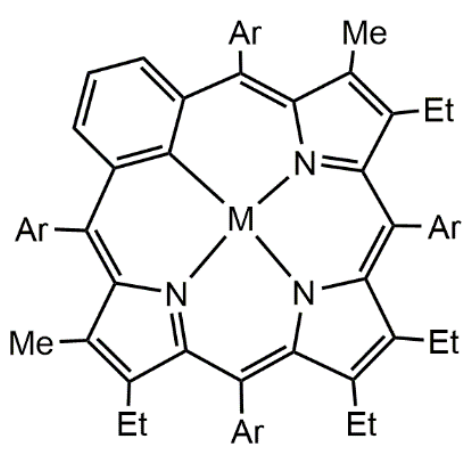

12 a. $\mathrm{M}=\mathrm{Ni}(\mathrm{II})$

b. $M=P d(I I)$

c. $M=P t(I I)$

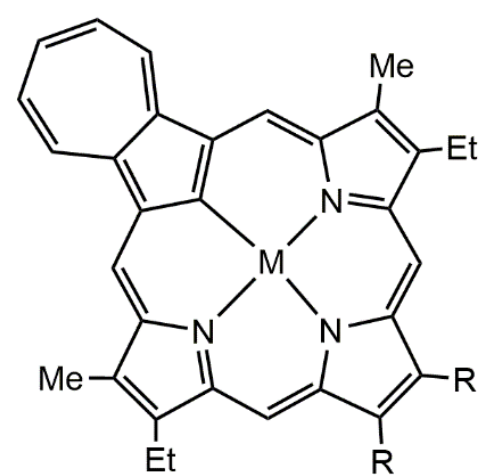

13 a. $M=N i(I I)$

b. $M=P d(I I)$

c. $M=P t(I I)$

$\mathrm{R}=\mathrm{Et}$ or $\mathrm{Ph}$

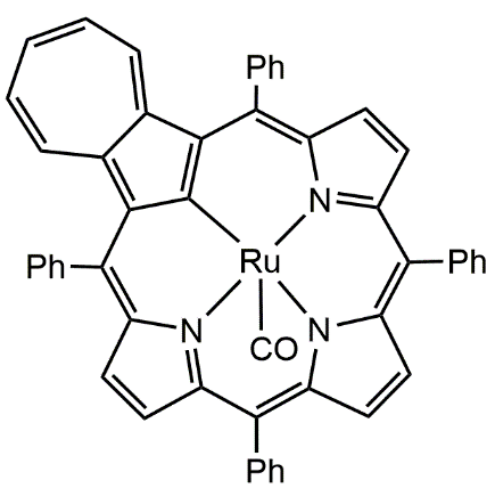

14

Chart 3. Organometallic complexes of benziporphyrin and azuliporphyrin 


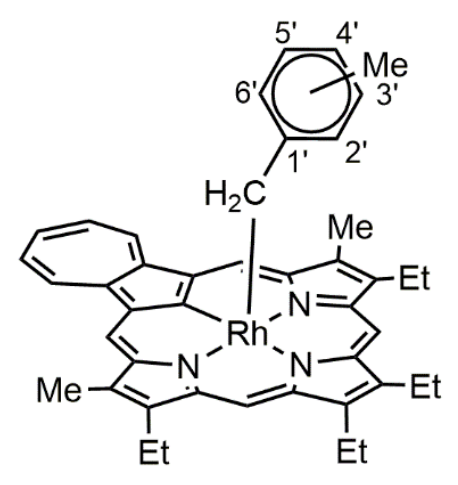

15

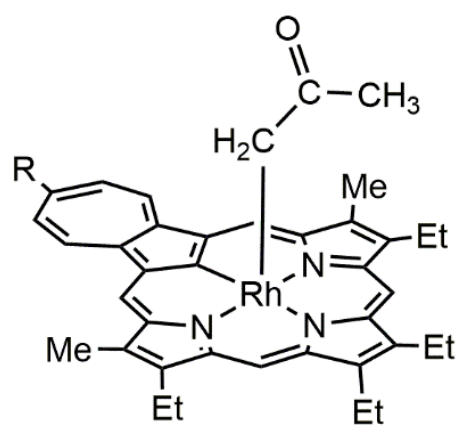

16

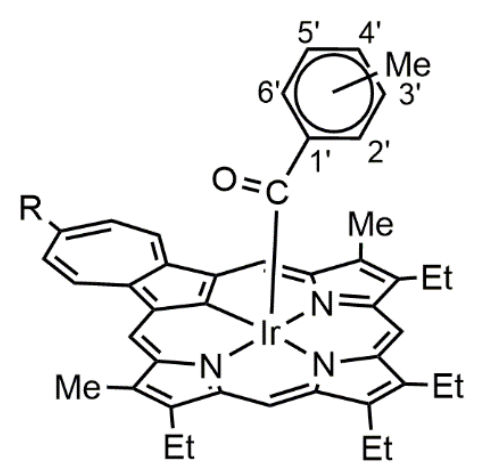

17

a. 2'-Me; b. 3'-Me; c. 4'-Me

Chart 4. Rhodium(III) and iridium(III) azuliporphyrins

$\mathrm{N}$-confused porphyrins (NCPs) ${ }^{36}$ typically act as both dianionic and trianionic ligands. Reaction of $N$-confused porphyrin with nickel(II) chloride in refluxing chloroform/methanol gave a nickel(II) complex where one of the NH protons had moved to the external nitrogen. ${ }^{37} \mathrm{An}$ example of $\mathrm{N}$-confused porphyrin acting as a trianionic ligand can be seen in the reaction of NCP with silver(I) trifluoracetate, which affords the Ag(III) complex. ${ }^{38}$ Oxybenziporphyrin also gave Ag(III) complex 18a (Chart 5) when treated with silver(I) acetate, ${ }^{39}$ but also reacted with palladium(II) chloride to form an anionic Pd(II) complex 19a. ${ }^{40}$ Oxybenziporphyrin also formed a related anionic platinum(II) complex 19b when reacted with platinum(II) chloride. ${ }^{41}$ Tetraaryloxybenziporphyrins not only formed Ag(III) derivatives 18b, but also gave $\mathrm{Au}(\mathrm{III})$ complexes 18c ${ }^{42}$ (Chart 5). Dimethoxy benziporphyrins 20a,b and 21a,b formed nickel(II) complexes 22a-d when reacted with nickel(II) acetate. ${ }^{43,44}$ They also reacted with palladium(II) acetate to afford the related palladium(II) complexes 23a-d ${ }^{44}$ (Chart 5). Similarly, dimethoxybenziporphyrins with two meso-substituents were prepared and reacted with palladium(II) acetate to give the corresponding palladium(II) complexes. ${ }^{45}$ When tropiporphyrins 
9 were reacted with silver(I) acetate at room temperature, only trace amounts of the corresponding silver complexes were formed. However, when they were refluxed with silver(I) acetate and DBU in pyridine, the corresponding silver(III) complexes were obtained in good yields. ${ }^{46}$

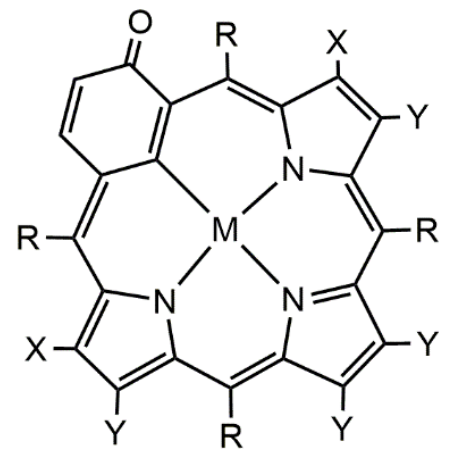

18

a. $M=A g(I I I), R=H, X=M e, Y=E t$

b. $M=A g(I I I), R=A r, X$ and $Y=H$

c. $M=A u(I I I), R=A r, X$ and $Y=H$<smiles>CCCCCO</smiles>

19 a. $M=P d(I I)$

b. $M=P t(I I)$

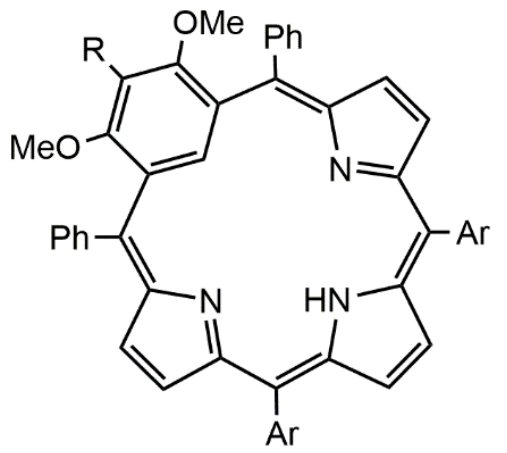

$20 \mathrm{R}=\mathrm{H} \quad 21 \mathrm{R}=\mathrm{Me}$

a. $\mathrm{Ar}=\mathrm{Ph} ;$ b. $\mathrm{Ar}=p-t-\mathrm{Bu}$

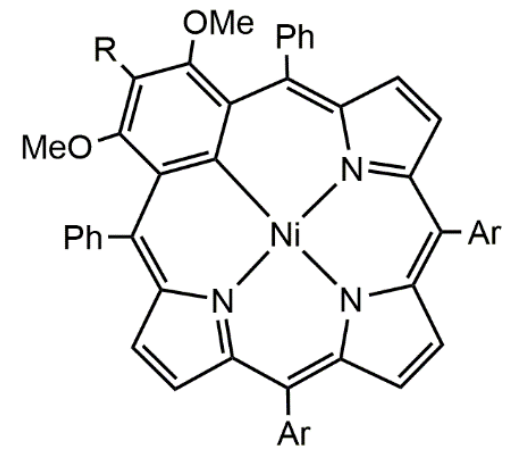

22

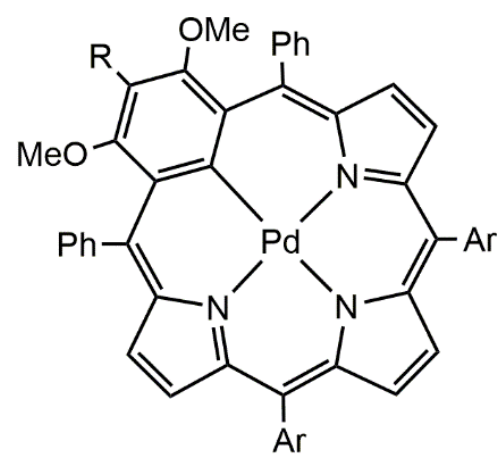

23
a. $\mathrm{R}=\mathrm{H} ; \mathrm{Ar}=\mathrm{Ph}$;
b. $\mathrm{R}=\mathrm{H} ; \mathrm{Ar}=p-t-\mathrm{Bu}$
c. $\mathrm{R}=\mathrm{Me} ; \mathrm{Ar}=\mathrm{Ph}$
d. $\mathrm{R}=\mathrm{Me} ; \mathrm{Ar}=p-t-\mathrm{Bu}$

Chart 5. Porphyrin analogues and their metal complexes

Further modifications to the reactivity of carbaporphyrins have been observed in heterocarbaporphyrins, where the internal cavity of 21-benzocarbaporphyrins 3 not only contains nitrogen and carbon atoms, but also oxygen, sulfur or selenium atoms $24 \mathbf{a}-\mathbf{c}^{46}$ at the 23-position 
(Scheme 1). Normally, benzocarbaporphyrins are trianionic, possessing three internal hydrogens that can be given up in favor of coordinating with metal centers. 23-Oxa-, 23-thia- and 23-selena21-benzocarbaporphyrins 24a-c were reacted with palladium(II) acetate to afford the corresponding palladium(II) complexes 25a-c (Scheme 1), where the porphyrin was shown to act as a dianionic ligand instead. ${ }^{46}$ This is due to there being one fewer internal hydrogens when one of the remaining nitrogen atoms is replaced by the oxygen, sulfur or selenium. The same dianionic character was seen when the same benzocarbaporphyrins were reacted with nickel(II) acetate in DMF, affording the corresponding nickel(II) complexes 26a,b. However, in the presence of air oxidation to oxyheterocarbaporphyrins $\mathbf{2 7}$ was observed (Scheme 2). ${ }^{46}$ Heteroporphyrins with an internal core of XNNN 28a-d (Chart 6), in general, have much lower reactivity towards metalation, which is due in part to the reduced planarity of the macrocycle. ${ }^{47}$ Furthermore, incorporation of larger heteroatoms (S, Se and Te) result in a change in the cavity size, which plays a role in reducing metal-binding in heteroporphyrins. ${ }^{48}$ However, despite this weaker metal-binding behavior, examples of metalated heteroporphyrins do exist. For example, Latos-Grazynski and coworkers reported the first synthesis of $\mathrm{Cu}(\mathrm{II}), \mathrm{Fe}(\mathrm{II})$ and Ni(II) complexes 29a-c of tetraphenyl21-thiaporphyrin $\mathbf{3 0}$ by treating the free-base with copper, iron and nickel chloride salts under reflux conditions (Chart 6). ${ }^{49}$ 


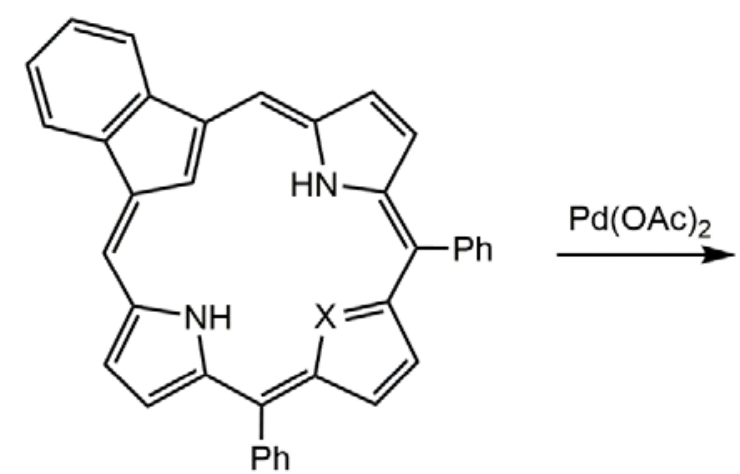

24 a. $X=0$

b. $X=S$

c. $X=S e$

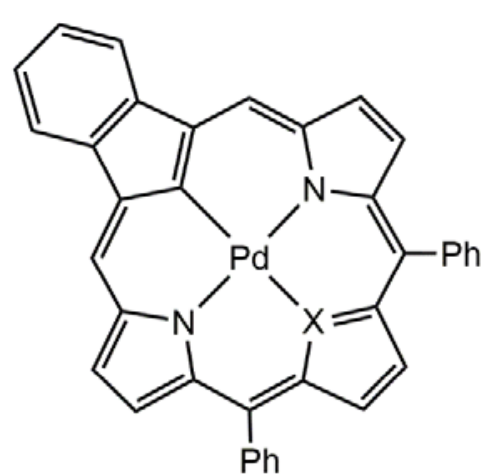

25 a. $X=0$

b. $X=S$

c. $X=S e$

Scheme 1. Formation of palladium(II) complexes of 23-oxa-, 23-thia- and 23-selena-21benzocarbaporphyrins

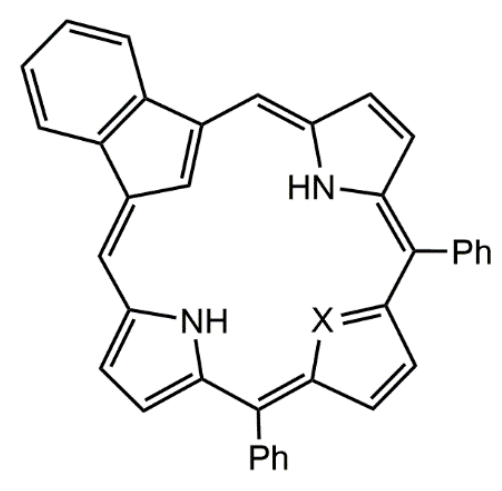

24 a. $X=0$

b. $X=S$

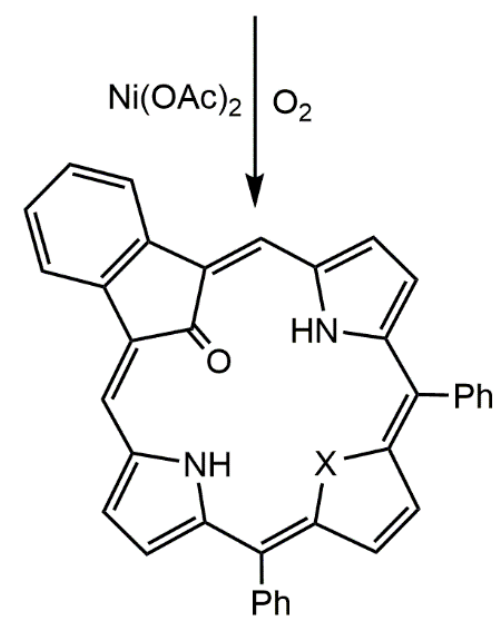

27
26 a. $X=0$

b. $X=S$

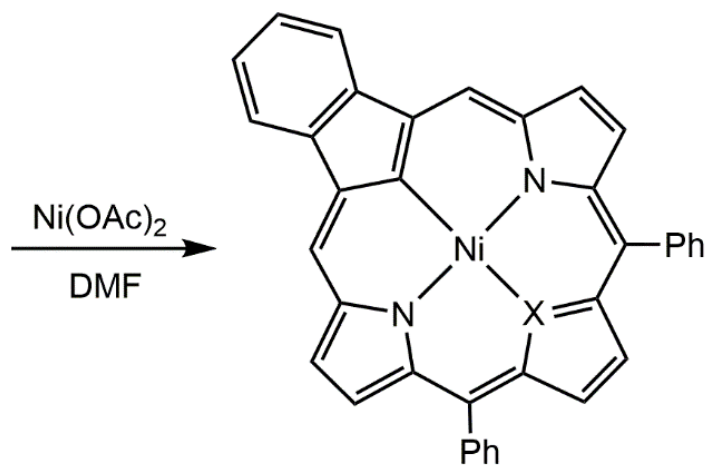

Scheme 2. Formation of nickel(II) complexes 24a,b and oxyheterocarbaporphyrin 


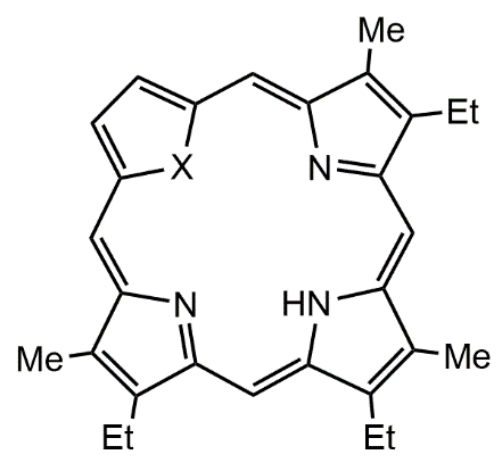

28 a. $X=0$

b. $X=S$

c. $X=\mathrm{Se}$

d. $X=\mathrm{Te}$

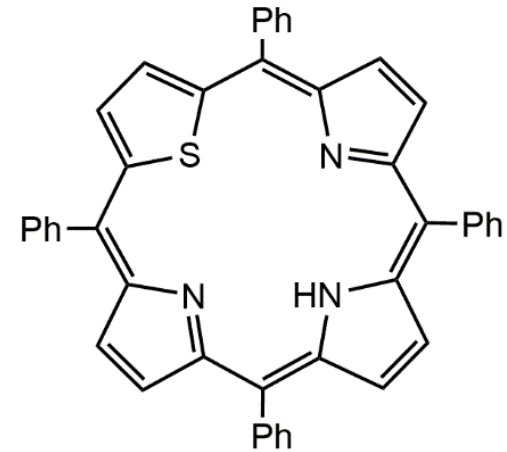

30

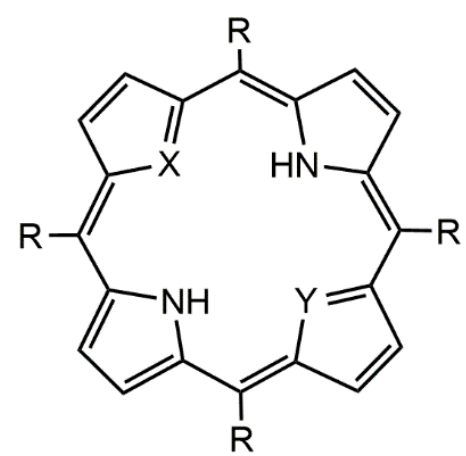

$37 \mathrm{R}=\mathrm{H} \quad 38 \mathrm{R}=\mathrm{Ph}$

a. $X=N ; Y=N$

b. $X=O ; Y=O$

c. $X=S ; Y=S$

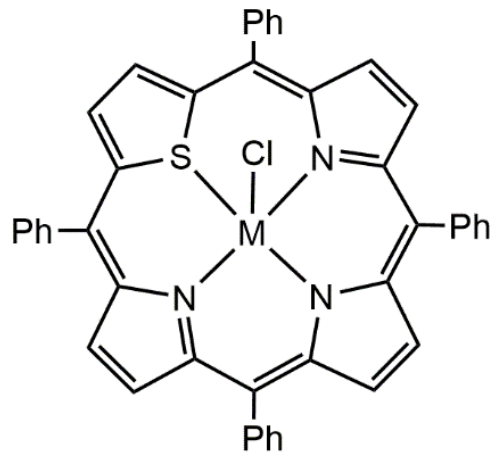

29 a. $\mathrm{M}=\mathrm{Cu}(\mathrm{II})$

b. $M=F e(I I)$

c. $\mathrm{M}=\mathrm{Ni}(\mathrm{II})$

Chart 6. Hetero- and diheteroporphyrinoids

All three of these metal complexes incorporated chloride as an axial ligand and were stable in the solid state. However, the Fe(II) complex demetalated in solution upon standing. ${ }^{47}$ Similarly, the palladium(II) complex was obtained by refluxing 21-thiaporphyrin with palladium(II) chloride. $\mathrm{Rh}(\mathrm{III})^{50}$ and $\mathrm{Ru}(\mathrm{III})^{51}$ complexes were also reported for the same heteroporphyrin, along with many other metals. ${ }^{52-54}$ Likewise, 21-oxaporphyrin has been shown to form $\mathrm{Ni}(\mathrm{II}),{ }^{55} \mathrm{Fe}(\mathrm{III}),{ }^{56}$ $\mathrm{Zn}(\mathrm{II}),{ }^{58}, \mathrm{Re}(\mathrm{I}){ }^{54} \mathrm{Mn}(\mathrm{II}),{ }^{59} \mathrm{Co}(\mathrm{II})^{59}$ and $\mathrm{Cu}(\mathrm{II})^{59}$ complexes.

Rhodium complexes of porphyrins have attracted much interest and have been studied for the past 50 years, leading to important discoveries such as catalytic enantioselective 
cyclopropanations, as well as more recently, selective C-H and C-C activation transformations. ${ }^{1}$ Due to its small size in all of its oxidation states, rhodium can fit neatly into the nearly planar porphyrin ligand to form rhodium(I), rhodium(II) and rhodium(III) complexes with varying geometries. The rhodium(I) complexes possess square planar geometries, while rhodium(II) porphyrins exhibit square planar monomeric and square pyramidal dimeric geometries, and rhodium(III) porphyrins are commonly associated with square pyramidal or octahedral geometries. ${ }^{1}$ The square planar and square pyramidal geometries of some of these rhodium complexes allow axial ligands to easily coordinate to the metal center. Rhodium coordination has been investigated thoroughly and examples of five- and six-coordinate complexes have been seen with nitrogen, sulfur, phosphorus, oxygen and carbon-based ligands. ${ }^{1,60-62}$ The vast number of rhodium complexes is due to their high association constants, ranging from $10^{5}$ for nitrogen-based ligands and $10^{8}$ for phosphorus-based ligands. ${ }^{1}$ Rhodium(III) porphyrins have been shown to facilitate carbon-hydrogen bond activation, ${ }^{63-65}$ specifically, $\mathrm{Rh}(\mathrm{OEP}) \mathrm{Cl}$ complexes 31 (OEP = 2,3,7,8,12,13,17,18-octaethylporphyrin) were found to be prone to electrophilic cleavage of aryl C-H bonds to form aryl rhodium(III) porphyrins 32. ${ }^{66,67}$ This occurred through 31 being activated by either $\mathrm{AgClO}_{4}$ or $\mathrm{AgBF}_{4}$ to generate the electrophilic metalloporphyrin (Scheme 3).
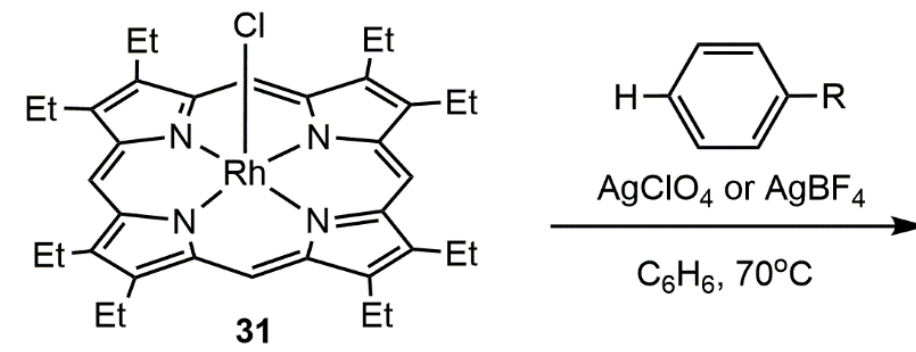

$$
\mathrm{R}=\mathrm{H}, \mathrm{OCH}_{3}, \mathrm{CH}_{3} \text { or } \mathrm{Cl}
$$

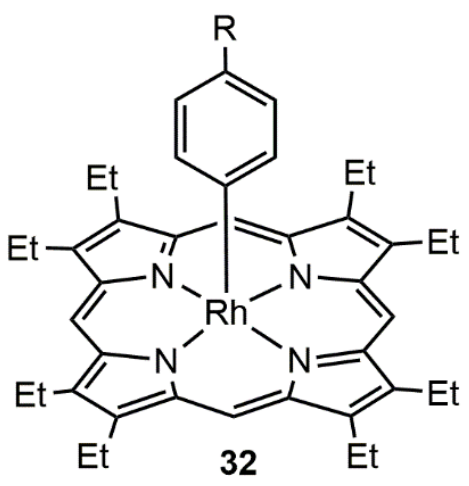

Scheme 3. C-H activation of aryl units by Rh(III) porphyrins 
The resulting cationic species can then activate the $\mathrm{C}-\mathrm{H}$ bonds in benzene, anisole, toluene and chlorobenzene to form the corresponding aryl species with selective metalation at the para positions. ${ }^{66,67}$ Rhodium(III) porphyrinoid complexes have also been shown to undergo ring contractions. Specifically, $p$ - and $m$ - benziporphyrins ring contracted into true carbaporphyrins while also generating a three-membered ring rhodacycle 33 (Scheme 4). ${ }^{68}$

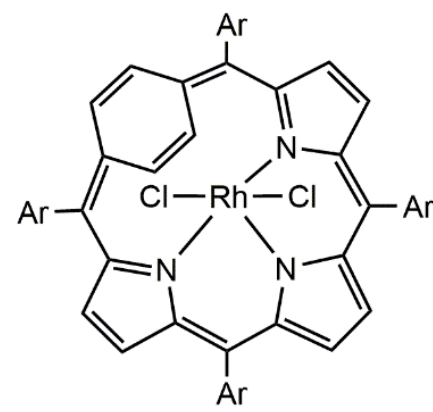

$$
\text { p-xylene }
$$

$\Delta$

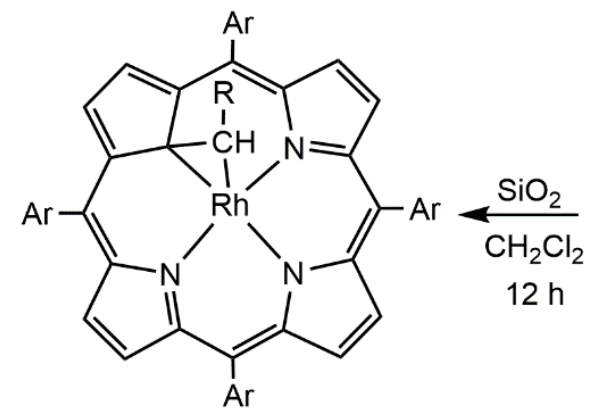

33 a. $\mathrm{R}=\mathrm{H}$

b. $\mathrm{R}=\mathrm{CHO}$

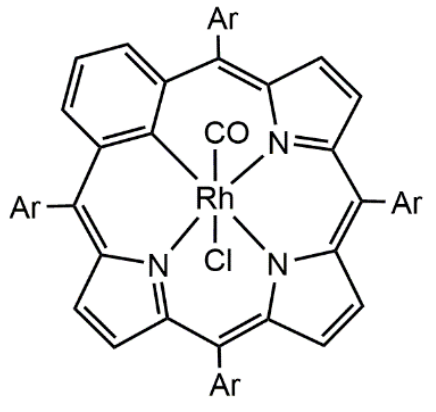

Scheme 4. Ring contraction of Rh(III) benziporphyrins into Rh(III) carbaporphyrins containing a three-membered rhodacycle

Alongside the interest that arises from studying these stable organometallic complexes, porphyrin derivatives are widely investigated for their aromatic properties. This aromatic character is evident through X-ray crystallography, where the absence of bond length alternation implies the presence of a conjugated pathway around the macrocycle, as well as from characteristic chemical shifts in proton NMR spectroscopy, and the ability of the porphyrin nucleus to maintain its integrity when analyzed by electron impact mass spectrometry. ${ }^{3}$ However, there has been much dispute over the factors that are responsible for porphyrinoid aromaticity. The porphyrin system contains $26 \pi$ electrons, which includes two "lone pairs" found on the internal nitrogens. However, it was proposed by Sondheimer, ${ }^{69}$ and later Emmanuel Vogel $^{70,71}$ during a study on bridged annulenes, that an $18 \pi$-electron pathway (shown in bold for structure $\mathbf{1}$, Chart 1 ) found within the 
system resembles [18]annulene $\mathbf{3 4}$ (Chart 7) and is a major contributor to the aromaticity observed in porphyrinoids.

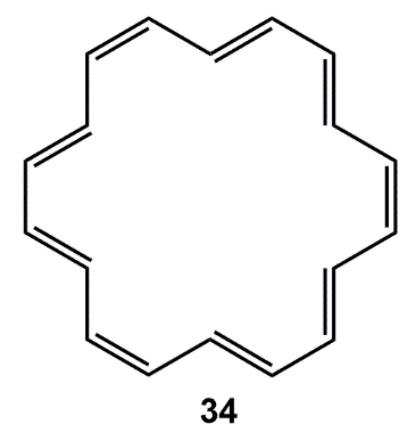

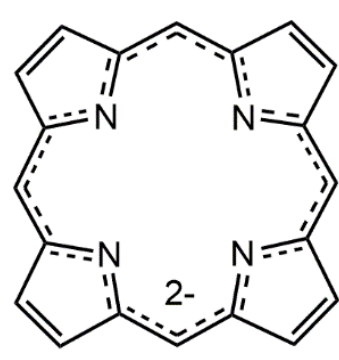

35

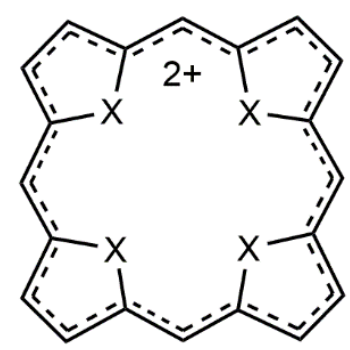

36 a. $X=S$

b. $\mathrm{X}=\mathrm{NH}$

Chart 7. Common $\pi$-electron delocalization pathways observed in porphyrinoids

Schleyer ${ }^{72}$ suggested that these macrocycles are better considered to be $22 \pi$-electron systems, due to the "lone pair" electrons located on two of the pyrrole nitrogens. These lone pair electrons may give rise to multiple different $\pi$-electron pathways that could contribute to the overall aromaticity of a porphyrin. Other models have been proposed for porphyrin aromaticity, although these speculations tend to be a better fit for metalloporphyrins than free-base porphyrins. ${ }^{73}$ A $16 \pi$ electron-pathway 35 (Chart 7) related to the dianion of [16]annulene has been proposed for the porphyrin ligands, ${ }^{74}$ and a 20 -atom delocalized pathway around the outside of the system which resembles a dication of [20]annulene may play a role in dirprotonated porphyrins and related heteroporphyrins. The latter pathway was initially proposed for the tetrathiaporphyrin dication 36a ${ }^{75}$ which resembles porphyrin dications $36 \mathbf{b}$ (Chart 7). The delocalized pathways in 35 and 36 both consist of $18 \pi$-electrons and therefore follow Huckel's Rule, which states that a molecule possessing [ $4 \mathrm{n}+2] \pi$-electrons, as well as uninterrupted conjugation and planarity, is aromatic. 
Numerous examples of porphyrin analogues have been reported, each of which exhibit different degrees of aromaticity. These properties are most easily probed by proton NMR spectroscopy. Through this characterization method, the resonances corresponding to the internal and external protons of these porphyrins can help to determine the level of aromaticity that they may possess, if they possess any at all.

The $\pi$-electron pathway in porphyrins produces a diamagnetic ring current which, when in the presence of an induced magnetic field, will deshield the external protons of the macrocycle and shield the internal protons (Figure 1). This causes the external protons to shift downfield in a ${ }^{1} \mathrm{H}$ NMR spectrum, and the internal protons to shift upfield. The extent to which these peaks are shifted in their respective directions gives insights into the level of aromaticity seen in these compounds. The degree of aromatic character that these macrocycles possess can fluctuate when changes to the structure are made. These changes in aromaticity can show how alterations including the meso- and pyrrole unit substituents, additional rings, and changes to the internal atoms can affect the flow of electrons around the porphyrin nucleus and, in turn, its stability.

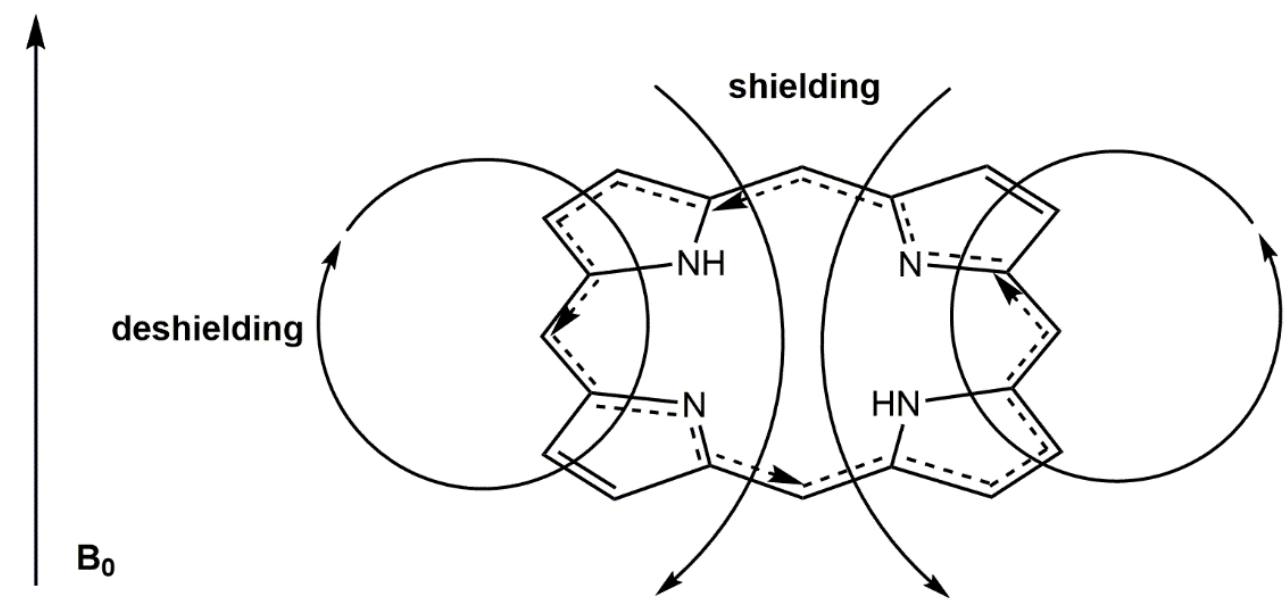

Figure 1. Shielding and deshielding regions due to porphyrin ring current 
Porphyrin 1 (Chart 1), without any structural modifications, can be used as a reference point for the comparison of core-modified and substituted porphyrins. Firstly, the two major tautomers for porphyrins have been found to rapidly interconvert, resulting in the pyrrole units all being equivalent on the NMR timescale at room temperature. ${ }^{76}$ In regards to the peaks seen in a proton NMR spectra for porphyrins, the external meso-protons have been found to resonate downfield near $10 \mathrm{ppm}$, while the internal NH protons give rise to a broad peak near $-4 \mathrm{ppm} .^{76}$ These two extremely different values help to show the effect of the diatropic ring current on these macrocycles. The substituents on the pyrrole rings are also affected by the ring current. The methyl units directly attached to the porphyrin pyrroles give singlets at relatively downfield values near $3.6 \mathrm{ppm} .{ }^{76}$ Upon modifying the core of the porphyrin to introduce an internal carbon, the degree of aromaticity may change, but other factors also play a role. Carbaporphyrinoids such as benzocarbaporphyrins $\mathbf{3}$, azuliporphyrins $\mathbf{4}$, tropiporphyrins $\mathbf{9}$, benziporphyrins $\mathbf{5}$ dimethoxybenziporphyrins 8 and oxybenziporphyrins 7a (Chart 1), show a wide range of aromatic properties and while $\mathbf{3}$ and $\mathbf{7 a}$ are strongly aromatic, $\mathbf{5}$ is essentially non-aromatic.

Benzocarbaporphyrins 3 possess global aromatic character due to the presence of an $18 \pi$ electron pathway within the structure. ${ }^{73}$ In the proton NMR spectrum of this carbaporphyrin, the internal $\mathrm{CH}$ gives a peak at $-6.74 \mathrm{ppm}$ and the internal $\mathrm{NH}$ protons show a broad resonance near $4.0 \mathrm{ppm}$, while the external meso-protons are observed downfield as two $2 \mathrm{H}$ singlets at 9.82 and $10.10 \mathrm{ppm}^{16,18}$ and the methyl groups resonate at $3.68 \mathrm{ppm}$ as a $6 \mathrm{H}$ singlet. ${ }^{73}$ The addition of trace amounts of TFA gave a monocationic species that retained comparable aromatic properties, showing the internal $\mathrm{CH}$ at $-6.75 \mathrm{ppm}$, the $\mathrm{NHs}$ as broad peaks at $-4.61 \mathrm{ppm}(1 \mathrm{H})$ and $-3.22 \mathrm{ppm}$ (2H), and the meso-protons at 10.06 and $10.33 \mathrm{ppm}$ (two 2H singlets). In 50\% TFA-CDCl 3 , a dicationic structure $\mathbf{3 H}_{2}{ }^{2+}$ (Scheme 5) was observed that also retained macrocyclic aromatic 
character, with the internal methylene and three $\mathrm{NH}$ protons resonating at -5.15 and $-1.5 \mathrm{ppm}$, respectively, and the external meso-protons giving peaks at 10.45 and $11.10 \mathrm{ppm}$, and the methyl protons showing up at $3.60 \mathrm{ppm} .^{77,78}$

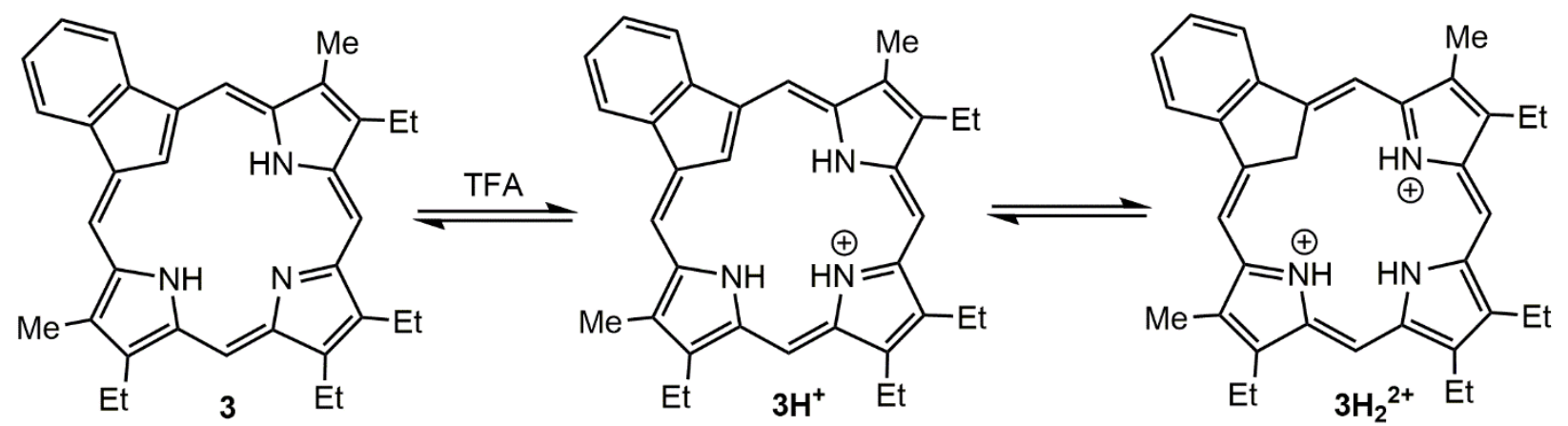

Scheme 5. Mono- and diprotonation of benzocarbaporphyrin 3

Tropiporphyrins 9 are carbaporphyrins containing a cycloheptatriene ring. ${ }^{25}$ These compounds exhibit slightly reduced aromaticity due to the presence of a strained seven-membered ring within the porphyrin structure. This reduced aromatic character results in upfield shifts to the proton resonances of the external meso-protons and methyl substituents, which give peaks at 8.0, 9.2 and 3.2 ppm, respectively. However, the internal $\mathrm{CH}$ is still strongly shielded and appears at 7 ppm. ${ }^{25}$ The results indicate that the diatropic ring current in tropiporphyrins is reduced compared to regular porphyrins or benzocarbaporphyrin. Tropiporphyrins are protonated with trace TFA to give monocationic species $\mathbf{9} \mathbf{H}^{+}$that exhibit slightly enhanced diatropic character. However, further protonation by the addition of more TFA leads to a non-aromatic dicationic species $\mathbf{9} \mathbf{H}_{\mathbf{2}}{ }^{\mathbf{2}}{ }^{\text {(Scheme }}$ $6) .^{25}$ 


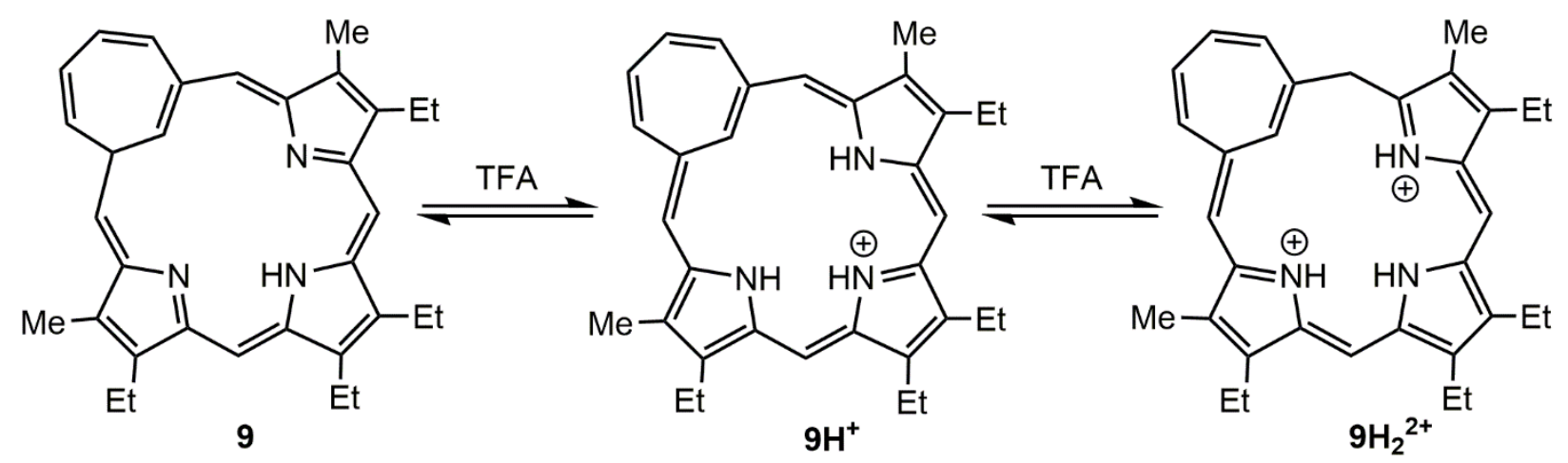

Scheme 6. Protonation of tropiporphyrin 9

Azuliporphyrins 4 also show reduced aromatic character due to the cross conjugated azulene unit that is present. ${ }^{73}$ Azuliporphyrin 4a is not very soluble in organic solvents, and the internal $\mathrm{CH}$ resonance could not be located in the proton NMR spectrum. However, tert-butyl subsitituted azuliporphyrins $\mathbf{4 b}$ were more soluble, and their proton NMR spectra showed the internal $\mathrm{CH}$ and $\mathrm{NH}$ peaks between 2.8 and $2.9 \mathrm{ppm}$, while the meso-protons were observed at 8.15 and 9.05 ppm. $^{79}$ Despite being cross conjugated, these carbaporphyrins still exhibit significant diatropic character. This observation was attributed to dipolar resonance contributors such as 4’ (Figure 2), which give the system its porphyrin-like delocalization pathway, as well as tropylium character. ${ }^{79-81}$ The significance of this dipolar resonance contributor is also supported by the polar nature of azuliporphyrins compared to benzocarbaporphyrins and benziporphyrins. ${ }^{73}$ 

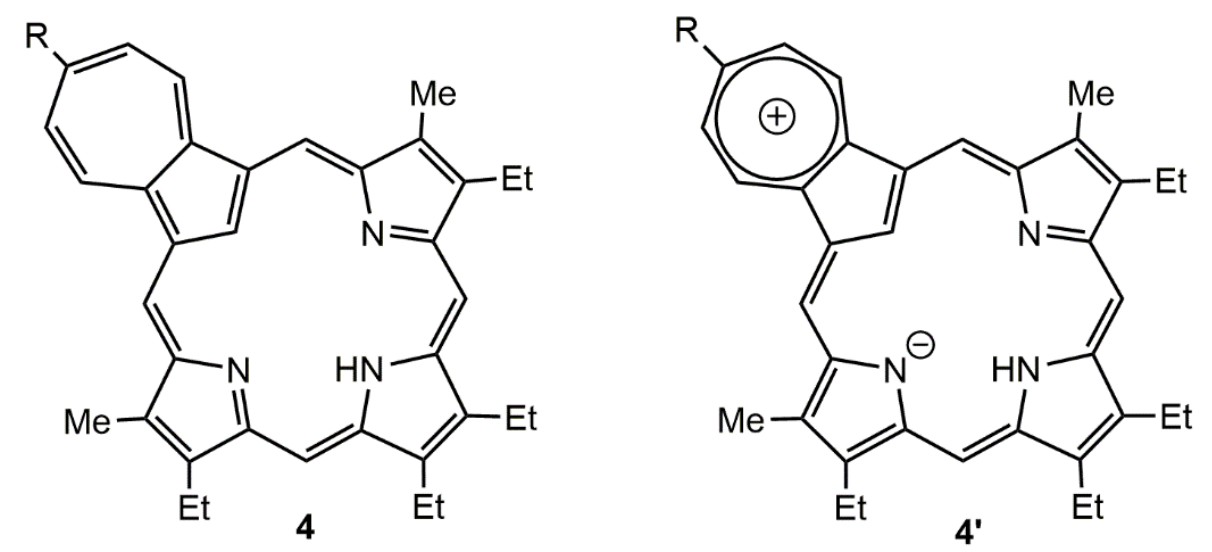

a. $\mathrm{R}=\mathrm{H}$

b. $R=t$-butyl

Figure 2. Dipolar resonance structure in azuliporphyrins

In contrast to azuliporphyrins, the cross conjugation resulting from the presence of a benzene ring in benziporphyrins 5 produces a non-aromatic system. The internal $\mathrm{CH}$ shows up as a poorly resolved triplet at $7.90 \mathrm{ppm}$, while the external benzene protons are observed as a triplet and doublet of doublets at 7.75 and 7.98 ppm, respectively, demonstrating the absence of a global aromatic ring current. ${ }^{23}$ In addition, the meso-protons gave two $2 \mathrm{H}$ singlets at 6.57 and $7.27 \mathrm{ppm}$, respectively, and the external methyls were observed as a $6 \mathrm{H}$ singlet at $2.43 \mathrm{ppm}$, values that are far upfield from those observed for a porphyrin. ${ }^{22}$ Benziporphyrins are non-aromatic because they would have to forfeit the aromaticity of the benzene ring in order for the entire system to be aromatic, and this is unlikely to be favorable. ${ }^{73}$ However, addition of TFA afforded a diprotonated dication $\mathbf{5 H}_{2}{ }^{2+}$, that displayed weakly diatropic character, shifting the meso-protons downfield to 7 and 8 ppm, while the internal $\mathrm{CH}$ was shifted upfield to near $5 \mathrm{ppm} .^{23}$ The observed diatropic character was proposed to arise from resonance contributors such as $\mathbf{5}^{\prime} \mathbf{H}_{\mathbf{2}}{ }^{\mathbf{2}}$ that enable delocalization of charge as well as the formation of the $18 \pi$-electron delocalization pathway (Scheme 7). ${ }^{73}$ 


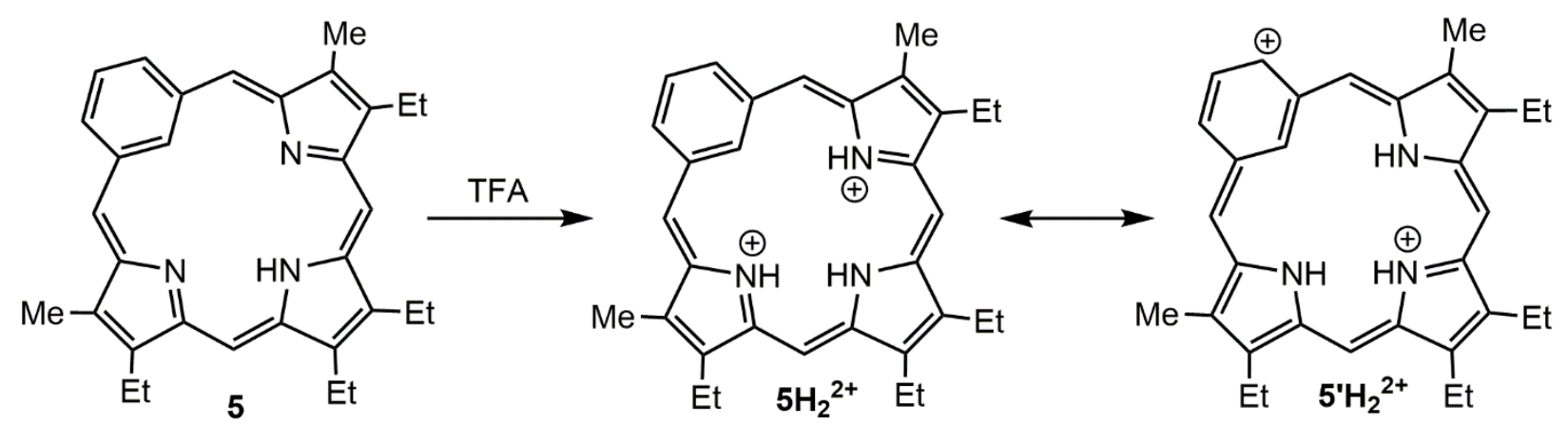

Scheme 7. Protonation of benziporphyrin 5

Closely related benziporphyrin-like structures have also been investigated, including dimethoxybenziporphyrin $\mathbf{8}$. Unlike 5, 8 was found to possess a weak diatropic ring current as a free base, but when the compound was diprotonated with TFA much larger shifts were seen in the proton NMR spectra. The interior $\mathrm{CH}$ for $\mathbf{8}$ was observed upfield at $-0.68 \mathrm{ppm}$, while the mesoprotons were shifted downfield to give two $2 \mathrm{H}$ singlets at 8.44 and $9.60 \mathrm{ppm} .^{82}$ This increase in diatropic character can be explained by the presence of the electron-donating methoxy groups, which provide favorable resonance contributors such as $\mathbf{8}^{\prime} \mathbf{H}_{\mathbf{2}}{ }^{2+}$ that possess $18 \pi$-electron delocalization pathways (Scheme 8). ${ }^{73}$

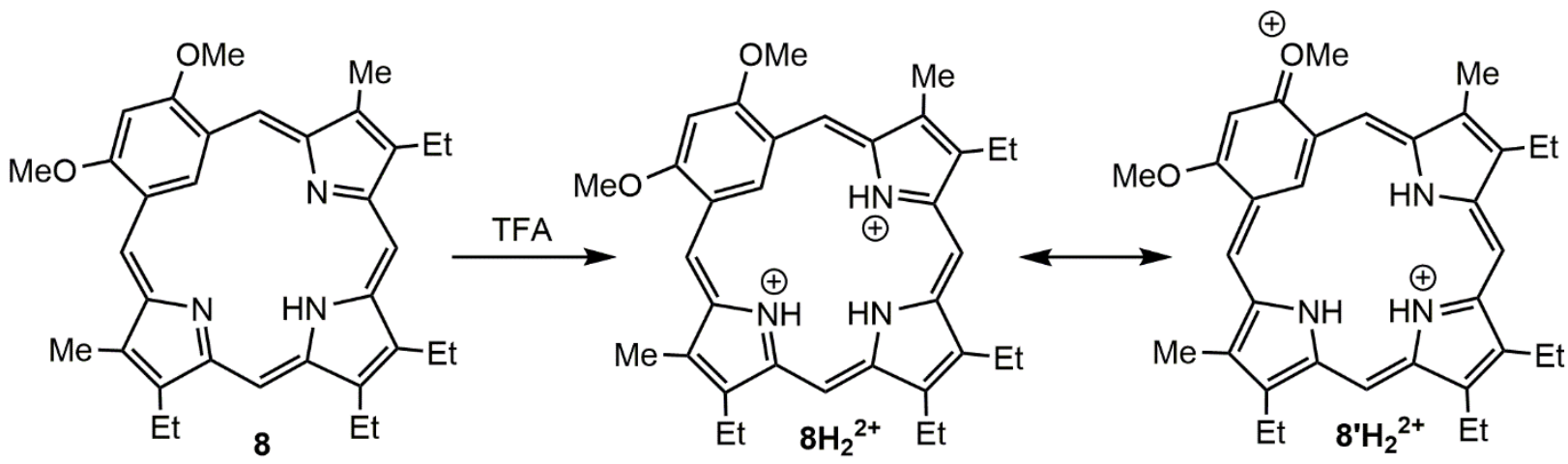

Scheme 8. Protonation of dimethoxybenziporphyrin 8 
Another variation on the benziporphyrin structure is oxybenziporphyrin 7a, which is highly diatropic because the six-membered ring favors a tautomer with a conjugated carbonyl group, allowing an aromatic delocalization pathway to be present in the system. ${ }^{22,23,73}$ Furthermore, related oxypyriporphyrins $\mathbf{7 b}$ are also well known and exhibit the necessary $18 \pi$-electron delocalization pathway to possess porphyrinoid aromaticity. ${ }^{23,77,78}$

Heteroporphyrins $\mathbf{2 8}$ (Chart 6) are porphyrin analogues in which one or more of the internal nitrogen atoms are replaced by a different atom. 21-Thia $\mathbf{2 8 b}$ and 21-oxaporphyrins 28a are examples of heteroporphyrins, and were first synthesized in 1971, along with related diheteroporphyrins 37 and $\mathbf{3 8}$ (Chart 6). ${ }^{57}$ It was found that 21-thiaporphyrin $\mathbf{2 5 b}$ showed the mesoproton resonances between 9.98-10.06 ppm, while the internal $\mathrm{NH}$ proton resonated at -4.98 ppm. ${ }^{57}$ The 21-oxaporphyrin 25a contained meso-protons that were observed between 9.69 and $10.12 \mathrm{ppm}$, but the internal $\mathrm{NH}$ proton could not be detected. ${ }^{57}$ Little data for meso-unsubstituted monoheteroporphyrins has been reported, although meso-substituted heteroporphyrins and diheteroporphyrins have been far better studied. When the proton NMR spectrum of porphyrin 37a was compared to that of the related 21,23-dioxa- 37b and 21,23-dithiaporphyrins 37c (Chart 6, page 99), it was found that the meso-proton peaks were shifted downfield from $9.55 \mathrm{ppm}$ to 10.98 and 10.71 ppm, respectively. ${ }^{83}$ Tetraphenylporphyrin 38a (TPP) was also compared to its 21,23diheteroatom derivatives, containing both oxygen 38b (2OTPP) and sulfur 35c (2STPP) atoms (Chart 6). The $\beta$-protons for TPP were observed at $8.72 \mathrm{ppm}$, while those for 2OTPP and 2STPP were seen at 9.37 and 9.63, respectively, showing similar downfield shifts. ${ }^{84}$ These results indicate that the diheteratom derivatives were more aromatic than the original porphyrins.

Porphyrins and porphyrin analogues possess varying diatropic characteristics that can easily be identified, in part, through proton NMR spectroscopy. Changes made to the porphyrin 
macrocycle can result in changes in the properties of these structures and are therefore important to investigate. Carbaporphyrins, in particular, are of interest because their structures can easily be varied using specific synthetic methods, and all of them include modified internal core atoms. Additional changes to these porphyrin analogues can provide important insights into how much change a porphyrin can undergo while maintaining its characteristic chemical properties.

Porphyrins have been synthesized in many ways over the years, and the route chosen for synthesis depends on the type of porphyrinoid being targeted and whether or not the macrocycle is symmetrical. The most common method of synthesizing simple symmetrical porphyrins is through the tetramerization of a monopyrrole. This route was first reported by Rothemund, and this methodology allowed tetraphenyl porphyrin (TPP) to be prepared by reacting pyrrole 39 with benzaldehyde ${ }^{84,85}$ (Scheme 9). Another example of this type of synthesis involves the reaction of 3,4-diethylpyrrole with formaldehyde to produce octaethylporphyrin 40 (OEP) ${ }^{86}$ (Scheme 9). Monopyrrole tetramerization works best with pyrroles that possess identical 3- and 4-substituents, otherwise mixtures will arise to due acid-catalyzed scrambling reactions. ${ }^{87,88}$ An example of this production of mixtures can be seen through the acid-catalyzed tetramerization of pyrrole $\mathbf{4 1}$, which gives four etioporphyrin type-isomers $\mathbf{4 2 - 4 5}$ (Scheme 10) ${ }^{89}$ 


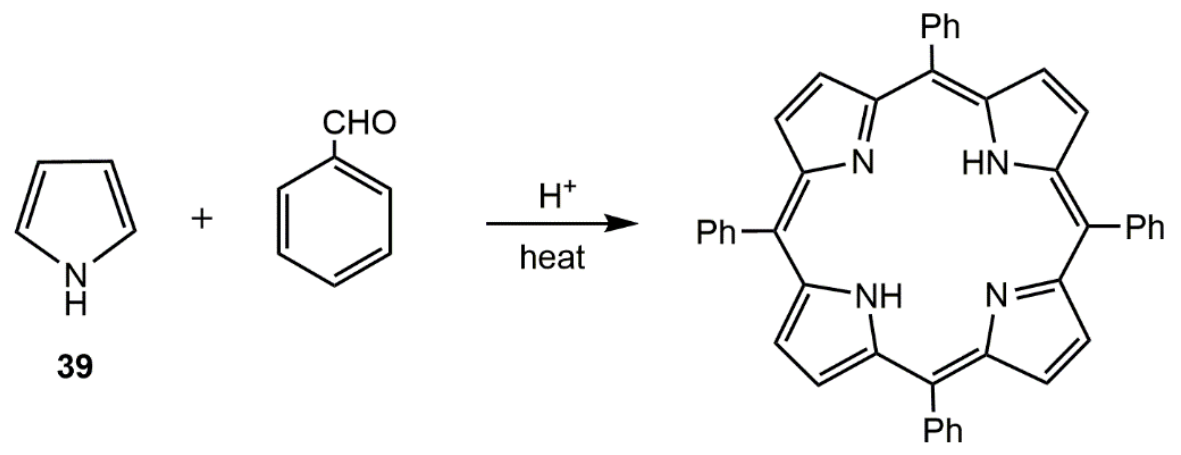

$38 a$
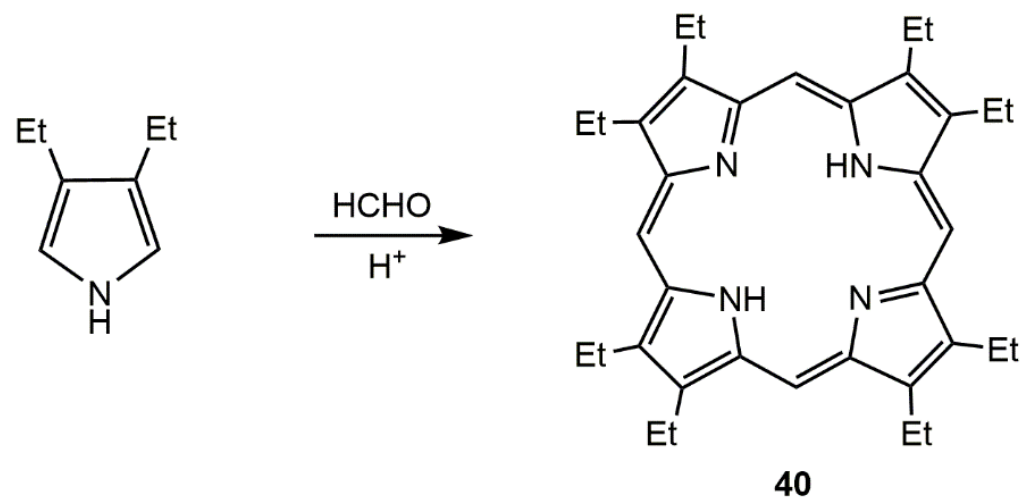

Scheme 9. Tetramerization of monopyrroles to produce porphyrins

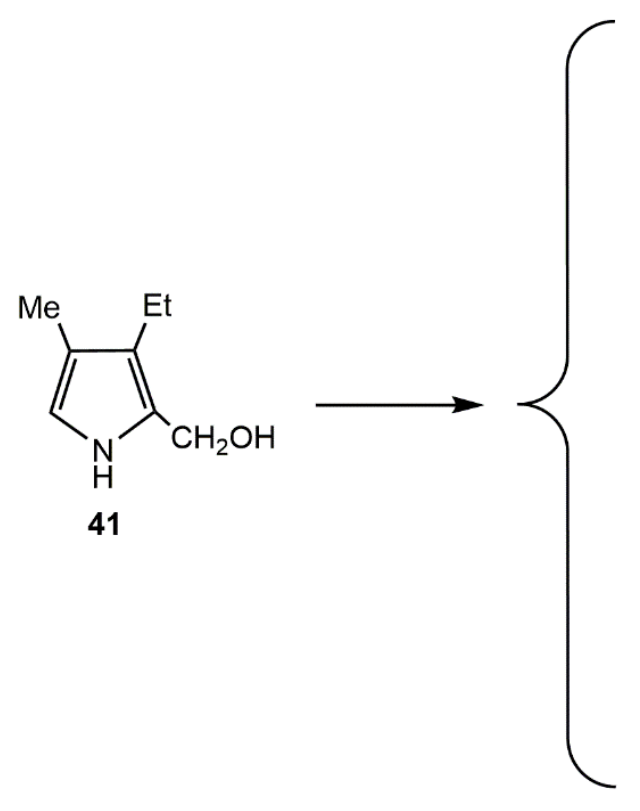

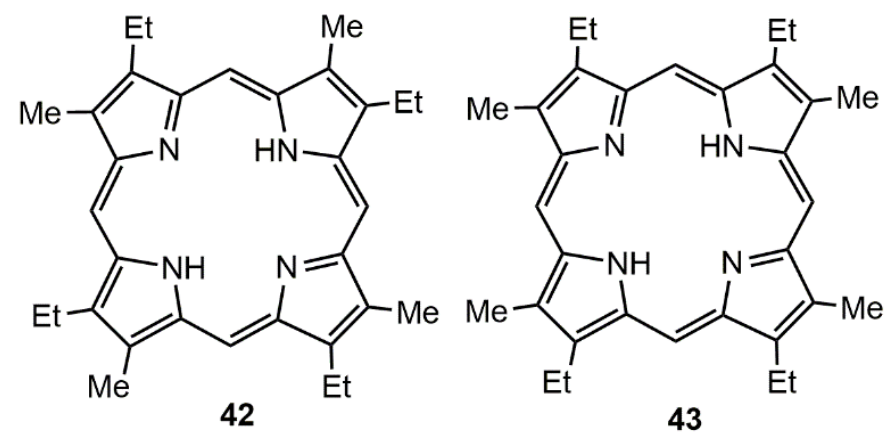<smiles>CCC1=C(C)c2cc3[nH]c(cc4nc(cc5[nH]c(cc1n2)c(C)c5C)C(C)=C4C)c(CC)c3C</smiles><smiles></smiles>

Scheme 10. Etioporphyrin type-isomers from the acid-catalyzed tetramerization of a pyrrole 
Another common method used for porphyrin synthesis is the MacDonald ' $2+2$ ' condensation. For this approach, two dipyrrolic components, commonly dipyrromethanes, are key intermediates. The use of two unsymmetrical dipyrromethanes would result in two different porphyrins, due to the possibility of one of the dipyrrolic units reacting in either of two orientations, related by a $180^{\circ}$ flip. ${ }^{89}$ However, it was found that this can be avoided by ensuring one of the dipyrromethane units is symmetrical. MacDonald and coworkers demonstrated this by reacting a symmetrical 1,9-diformyldipyrromethane 46 with a 1,9-di-unsubstituted dipyrromethane $\mathbf{4 7 a}$, or its corresponding dicarboxylic acid $\mathbf{4 7} \mathbf{b}$, in the presence of an acid catalyst to give pure porphyrin 48 in very high yields (Scheme 11). ${ }^{90}$<smiles>[R]c1[nH]c(Cc2[nH]c([R])c(CC)c2CC)c(C)c1C</smiles>

47 a. $\mathrm{R}=\mathrm{H}$

b. $\mathrm{R}=\mathrm{CO}_{2} \mathrm{H}$
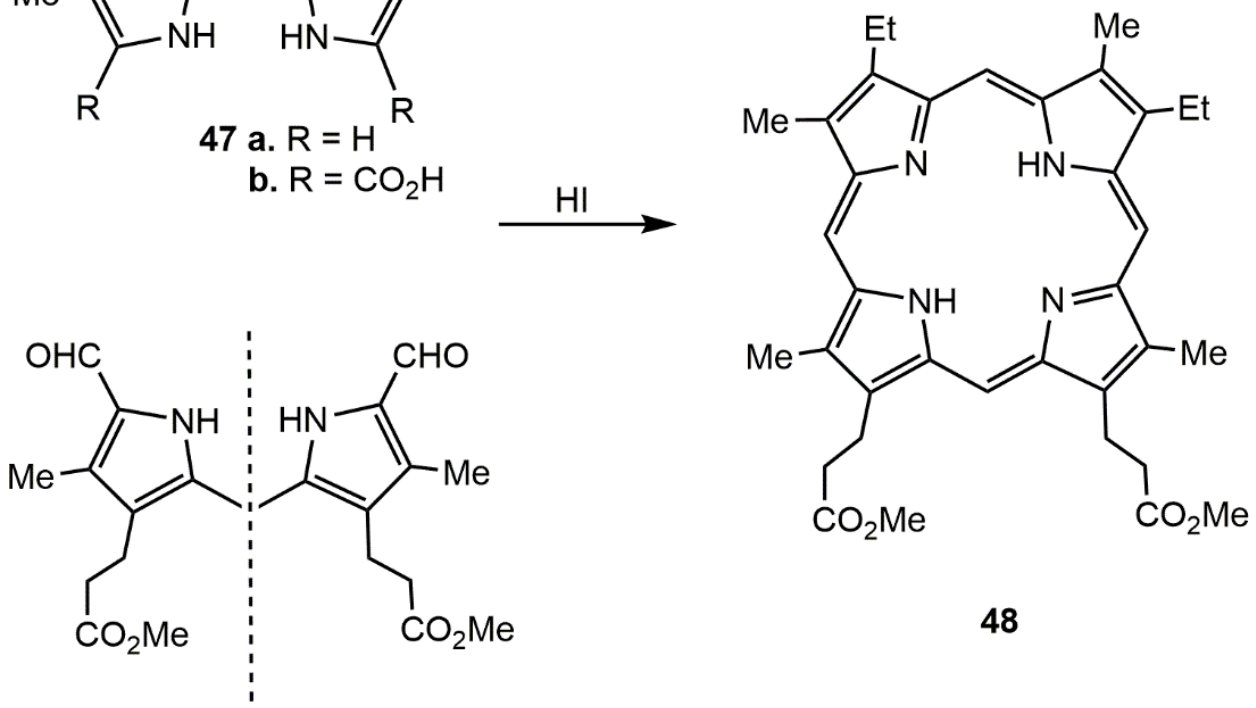

48

46

Scheme 11. '2+2’ MacDonald condensation reaction

A variant of the ' $2+2$ ' condensation, developed by Johnson and coworkers, ${ }^{57}$ and known as the ' $3+1$ ' route, involves the reaction of a tripyrrolic unit, such as tripyrrane 49 , with a monopyrrole bearing two aldehyde groups (e.g. 50) in the presences of an acid catalyst to give 
porphyrin $\mathbf{5 1}^{89}$ (Scheme 12). In most cases, the ' $3+1$ ' chemistry is carried out by reacting a tripyrrane unit with a monopyrrole in the form of a dialdehyde. However, there are examples where this is reversed, and a tripyrrane dialdehyde is condensed with a simple monopyrrole. ${ }^{91}$

The popularity of this method is, in part, due to the simplicity in constructing the aforementioned tripyrrane unit. Sessler and coworkers developed a convenient synthesis of tripyrranes, that afforded symmetrically substituted structures such as $\mathbf{4 9}^{92}$ (Scheme 12). Even though this approach is limited by the symmetry of the intermediates, the ease and success of the reactions, as well as the abundance of porphyrinoids it can produce, makes up for this deficiency.<smiles>[2H]c1c(Cc2[nH]c(Cc3[nH]c(C(=O)O)c(C)c3[2H])c(C)c2C)[nH]c(C(=O)O)c1[3H]</smiles>

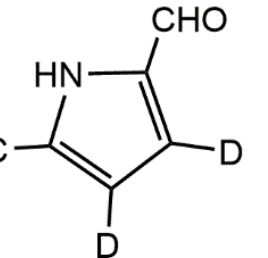

50

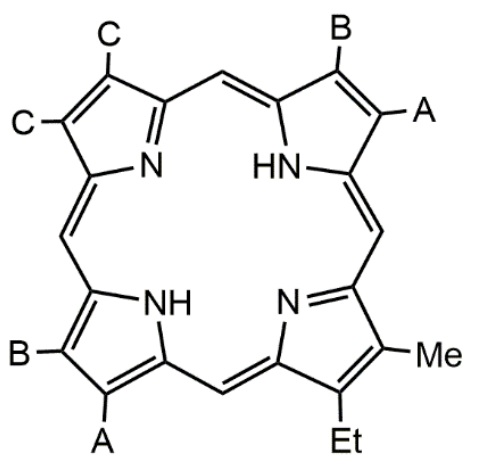

51

Scheme 12. '3+1'variant of the MacDonald Condensation

By reacting two equivalents of acetoxymethyl pyrrole 52 with one equivalent of diethylpyrrole 53a under acidic conditions, tripyrrane 54a can be isolated in $>70 \%$ yield (Scheme 13). Subsequent cleavage of the protective benzyl ester groups with Pd/C under an atmosphere of hydrogen gives the corresponding tripyrrane dicarboxylic acid 54b (Scheme 13), which can be 
used in the ' $3+1$ ' syntheses. It has also been found that the identity of the substituents on the tripyrrane can greatly influence the efficiency of these cyclization reactions. ${ }^{91,93,94}$
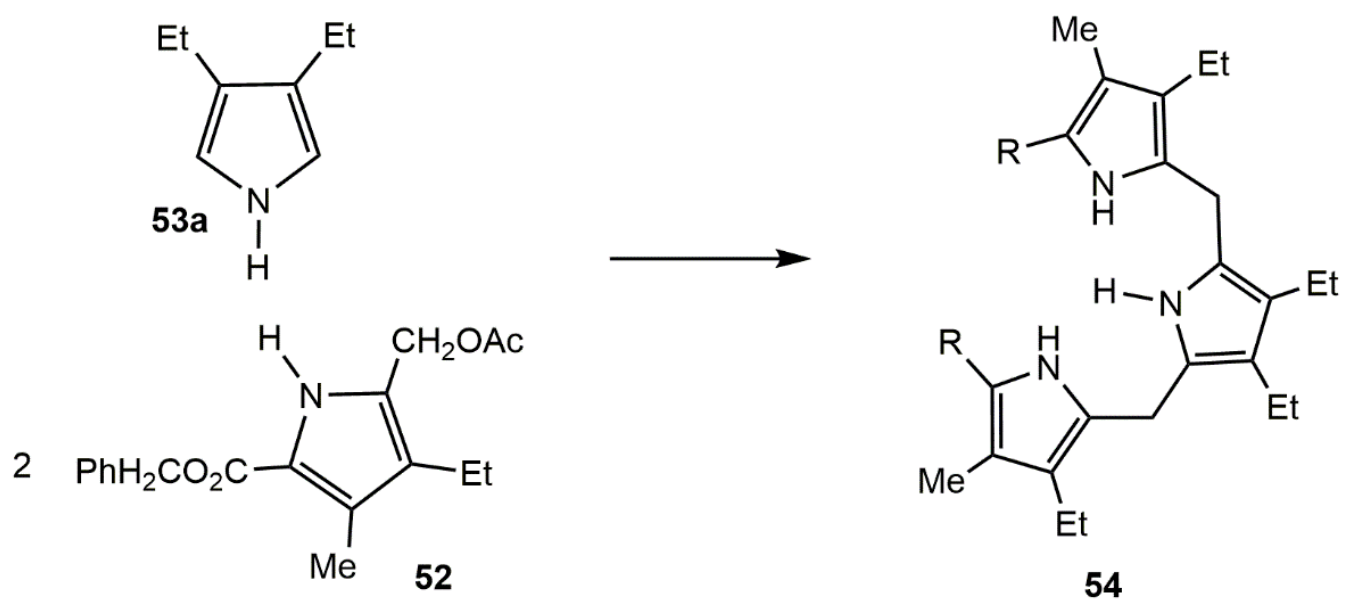
a. $\mathrm{R}=\mathrm{CO}_{2} \mathrm{CH}_{2} \mathrm{Ph}$
b. $\mathrm{R}=\mathrm{CO}_{2} \mathrm{H}$

Scheme 13. Synthesis of a tripyrrane

The ease in varying the dialdehyde unit is another factor that contributes to the popularity of the ' $3+1$ ' method. This paved the way for the synthesis of numerous porphyrinoids that possess unique functionality based on the identity of the inserted dialdehyde unit. Furthermore, the central pyrrole unit of the tripyrrane can be changed so that alternative heterocyclic or carbocyclic rings can be introduced. For example, in the study that first introduced the ' $3+1$ ' methodology, Johnson condensed tripyrrane 55a or heterotripyrrane 55b,c with furan dialdehyde 56a or thiophene dialdehyde $\mathbf{5 6 b}$ in the presence of $\mathrm{HBr}$ to give a series of heteroporphyrins 57a-d (Scheme 14). ${ }^{57}$ This method has also been very successful in the synthesis of carbaporphyrinoid systems, ${ }^{22}$ either by using carbocyclic dialdehydes or tripyrrance analogues that incorporate carbocyclic rings. This approach allows the synthesis of porphyrinoids that would not be accessible from the ' $2+2$ ' MacDonald reaction, therefore reinforcing the importance of this method for the development of novel porphyrin analogues. 

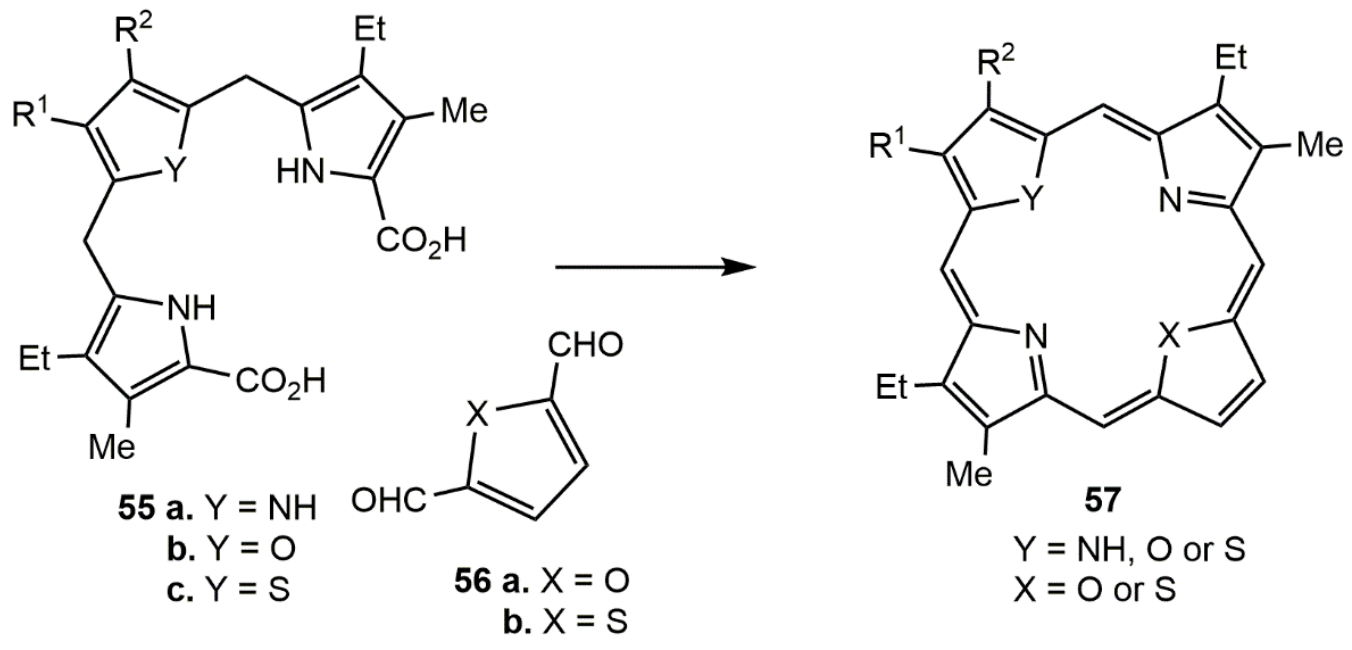

Scheme 14. Synthesis of heteroporphyrins through the ' $3+1$ ' variant of the MacDonald reaction

The internal cavity of porphyrinoids determines the abilities of these ligands to coordinate to metal centers. Often times, if the atoms and available bonding locations are altered within these macrocycles, their coordinating nature will be changed as well. Alkylation of carbaporphyrins has been well documented ${ }^{95-97,98}$ and this can greatly alter the coordination properties of these ligands. When N-confused porphyrin (NCP) $\mathbf{5 8}$ was reacted with methyl iodide in the presence of $\mathrm{Na}_{2} \mathrm{CO}_{3}$, the major product was $N, N^{\prime}$-dimethyl NCP 59 (Scheme 15) with methyl groups on the external nitrogen as well as the internal nitrogen at the 24-position. ${ }^{96}$ This alkylation reaction was interesting, as it introduced methyl groups on two nitrogen positions rather than just one, and demonstrated that not only are internally alkylated NCPs stable but also that the external nitrogen can easily be alkylated as well. This porphyrinoid was found to generate singlet oxygen after being irradiated at long wavelengths in the visible region, thereby showing that this compound has potential as a photosensitizer for photodynamic therapy (PDT). ${ }^{96}$ 


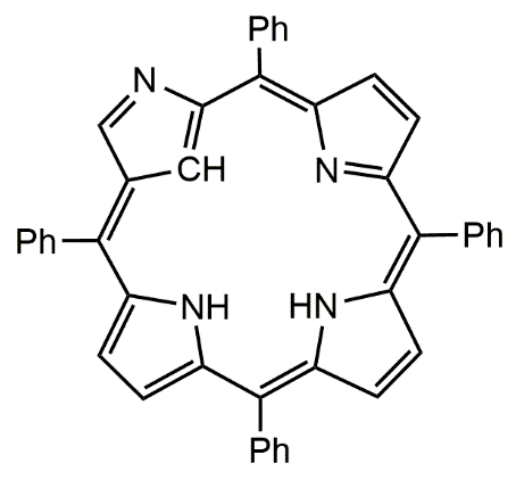

58

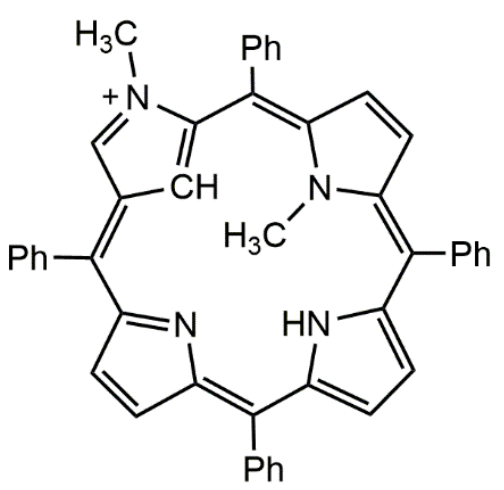

59

Scheme 15. Methylation of NCP to give a N,N-dimethyl NCP

Other work on NCPs by Latos-Grazynski and co-workers resulted in the preparation of outer $N$-methylated NCP 60, C-methylated NCP 61, C,N-dimethylated NCP 62 and their nickel(II) derivatives $^{95,98}$ (Chart 8).

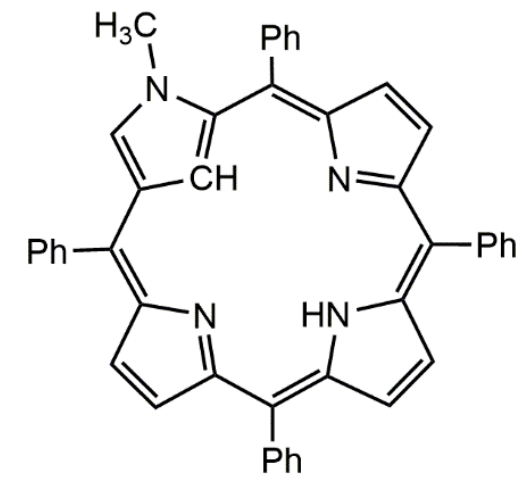

60<smiles>CC1=C(C(=C2C=CC(C(c3ccccc3)=c3ccc(=C(c4ccccc4)c4ccc(-c5ccccc5)[nH]4)[nH]3)=N2)c2ccccc2)C=NC1=C(c1ccccc1)c1ccccc1</smiles>

61

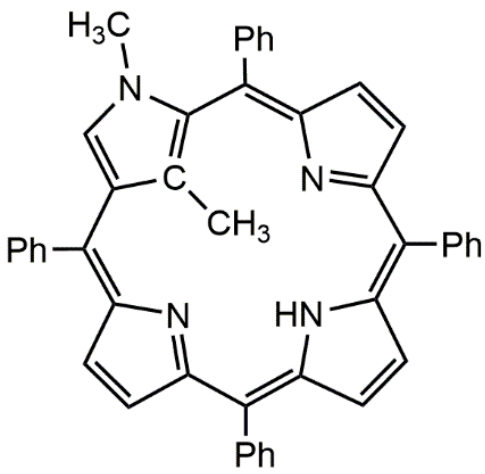

62

Chart 8. Examples of methylated N-confused porphyrins

An oxidized benziporphyrin derivative $\mathbf{6 3}$ was alkylated with methyl and ethyl iodide to give the corresponding $N$-methyl or $N$-ethyl products $\mathbf{6 4}$, with selective alkylation on the 23$\operatorname{position}^{97}$ (Scheme 16). Alkylation of the anionic palladium(II) complex of oxybenziporphyrin 65 
could take place on the oxygen as well as the internal carbon. ${ }^{99}$ When reacted with $n$-butyl iodide, a mixture of $O$-butyl 66 and $C$-butyl 67a products was obtained, but when reacted with methyl iodide only the internal carbon atom alkylated to give $\mathbf{6 7 b}^{99}$ (Scheme 17). $\mathrm{N}$-alkylporphyrins in general are of interest due to their role in a significant pathway for physiological porphyrin destruction. ${ }^{100}$ The $N$-alkylation of benzocarbaporphyrins resulted in $N$ - and $C$-alkylated products, and also allowed these systems to act as a dianionic ligands instead of exhibiting their usual trianionic character. ${ }^{101}$

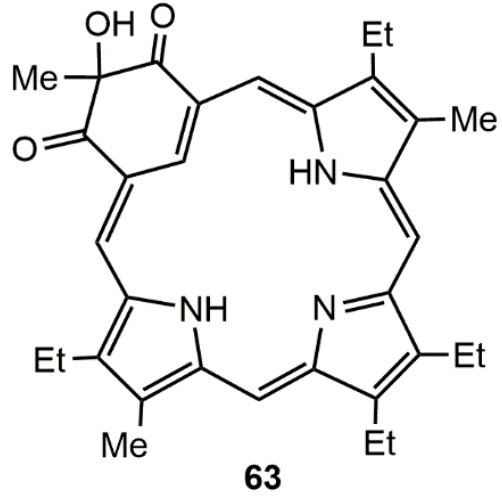

63

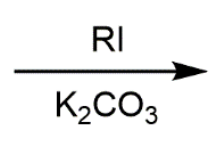

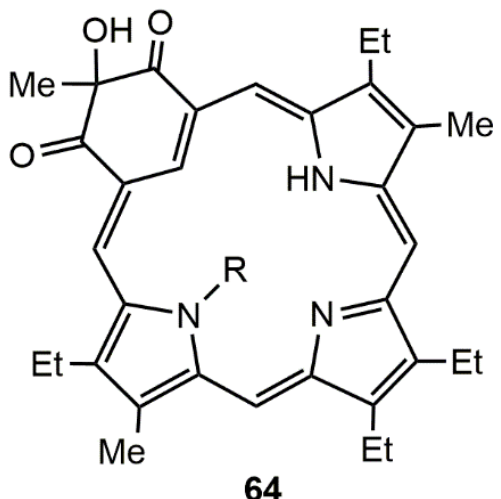

a. $\mathrm{R}=\mathrm{Me}$

b. $R=E t$

Scheme 16. Alkylation of an oxidized benziporphyrin derivative 

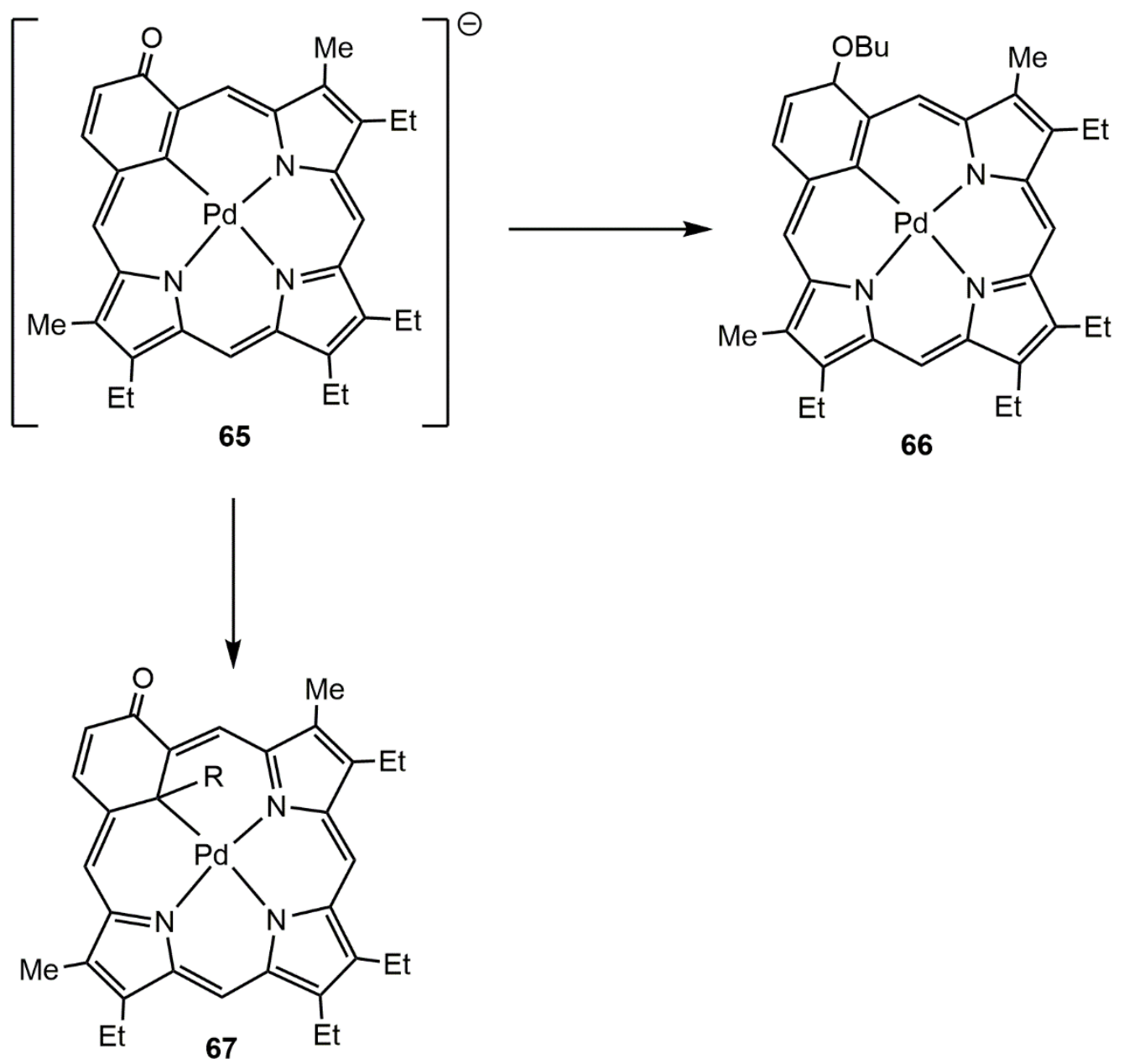
a. $\mathrm{R}=\mathrm{Bu}$
b. $\mathrm{R}=\mathrm{Me}$

Scheme 17. Alkylation of palladium(II) oxybenziporphyrin

The coordination chemistry of alkylated porphyrins and related macrocyclic systems involves a number of interesting reactions. For instance, metalated $N$-methylporphyrins have been observed to undergo demethylation of the pyrrolic nitrogen, and transfer of the methyl cation to another nitrogen base present in solution. ${ }^{102-105}$ Another case involved palladium(II) $p$ benziporphyrin complexes $\mathbf{6 8}$ undergoing a base promoted rearrangement to give the corresponding palladium(II) carbaporphyrins 69a,b (Scheme 18). ${ }^{106}$ Furthermore, the metalation 
of methylbenzocarbaporphyrin $\mathbf{7 0}$ with palladium(II) acetate resulted in an unexpected alkyl group migration, where the internal methyl was seen to move from the 22-position in $\mathbf{7 1}$ to the 21position in $\mathbf{7 2}^{101}$ (Scheme 19). The mechanism behind this migration is unknown, but was proposed to involve a [1,5]sigmatropic rearrangement which could, in part, be due to the proximity of the alkyl group on the 22-nitrogen to the internal 21-carbon atom. Similar rearrangements were also observed for carbaporphyrins without fused benzo-units ${ }^{107}$ and with naphthocarba[2,3b]porphyrins. ${ }^{108}$ Palladium-mediated rearrangements of vactaporphyrin ${ }^{109}$ and some expanded porphyrins have also been noted. ${ }^{110}$ Alongside palladium metal, rhodium(III) metal cations present within the cavity of benziporphyrins have been seen to promote the ring contraction of the benzene ring to a cyclopentadiene with the additional formation of a three-membered rhodacycle bridging the metal center and the internal carbon atom. ${ }^{68}$<smiles></smiles>

68

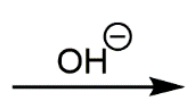
a. $\mathrm{R}=\mathrm{CHO}$
b. $\mathrm{R}=\mathrm{H}$

Scheme 18. Base-promoted rearrangement of palladium(II) p-benziporphyrin to palladium(II) carbaporphyrin 


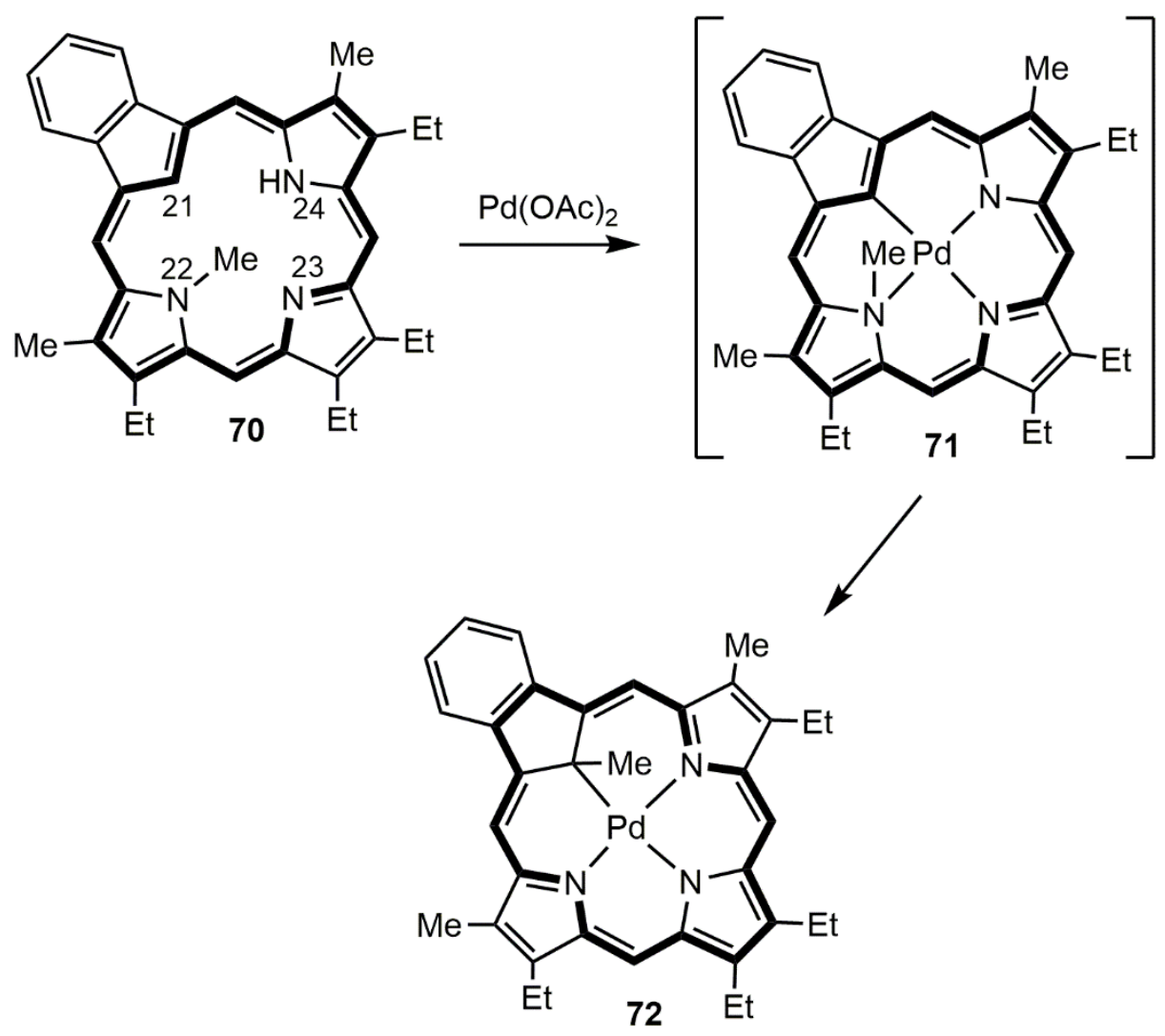

Scheme 19. Alkyl group migration in a palladium(II) benzocarbaporphyrin complex

Therefore, reactions of this type appear to have considerable significance and show that there is still much to investigate in regards to the coordination chemistry and reactivity of organometallic porphyrinoids. To further probe this chemistry, a 23-methylbenzocarbaporphyrin 73 was synthesized and its reactivity with palladium(II) acetate was compared to that of the 22methylbenzocarbaporphyrin 71. Metalation of the 23-methyl derivative provides insights into the significance of the alkyl group position within the porphyrinoid cavity, as well as the overall mechanism that is occurring. Furthermore, considering the C-H activation properties of rhodium(III) porphyrins, and the known formation of rhodacycle moieties within rhodium(III) carbaporphyrins, metalation of the 23-methylbenzocarbaporphyrin with rhodium complexes is also of interest. The introduction of internal alkyl groups may lead to deformation of porphyrinoid 
macrocycles and the influences of these structural changes on the spectroscopic properties of these systems is worthy of study. The convenience of the ' $3+1$ ' method allows for the construction of a series of $N$-methyl porphyrinoids. This not only allows investigations into the coordination chemistry of a multitude of internally methylated porphyrin analogues, but also enables variations in the electronic properties of these macrocycles to be assessed. 


\section{CHAPTER II: ALKYL GROUP MIGRATION IN INTERNALLY METHYLATED}

\section{PALLADIUM(II) BENZOCARBAPORPHYRINS}

\section{Introduction}

Carbaporphyrinoid systems form stable organometallic complexes with late transition metal cations. ${ }^{26}$ True carbaporphyrins such as $\mathbf{7 3}$ are trianionic ligands and have been shown to form stable $\mathrm{Ag}(\mathrm{III})$ and $\mathrm{Au}(\mathrm{III})$ derivatives. ${ }^{27-29}$ In order to modify the reactivity of this system, internal alkyl substituents have been introduced that could potentially enable this macrocyclic platform to act as a dianionic ligand. Benzocarbaporphyrin 73 was previously prepared by condensing a tripyrrane dicarboxylic acid 54b with indene dialdehyde $\mathbf{7 4}$ in the presence of TFA ${ }^{101}$ (Scheme 20). After purification by column chromatography and recrystallization, benzocarbaporphyrin 73 was reacted with methyl iodide in a suspension of potassium carbonate and refluxing acetone to give two alkylation products. The minor product $\mathbf{7 5}$ had the methyl group on the 21-position, while the major product $\mathbf{7 0}$ had the methyl located on the 22-position ${ }^{101}$ (Scheme 21). These two products were isolated in $10 \%$ and $62 \%$ yields, respectively, but no trace of the hypothetical 23-alkyl product was observed. ${ }^{101}$ 

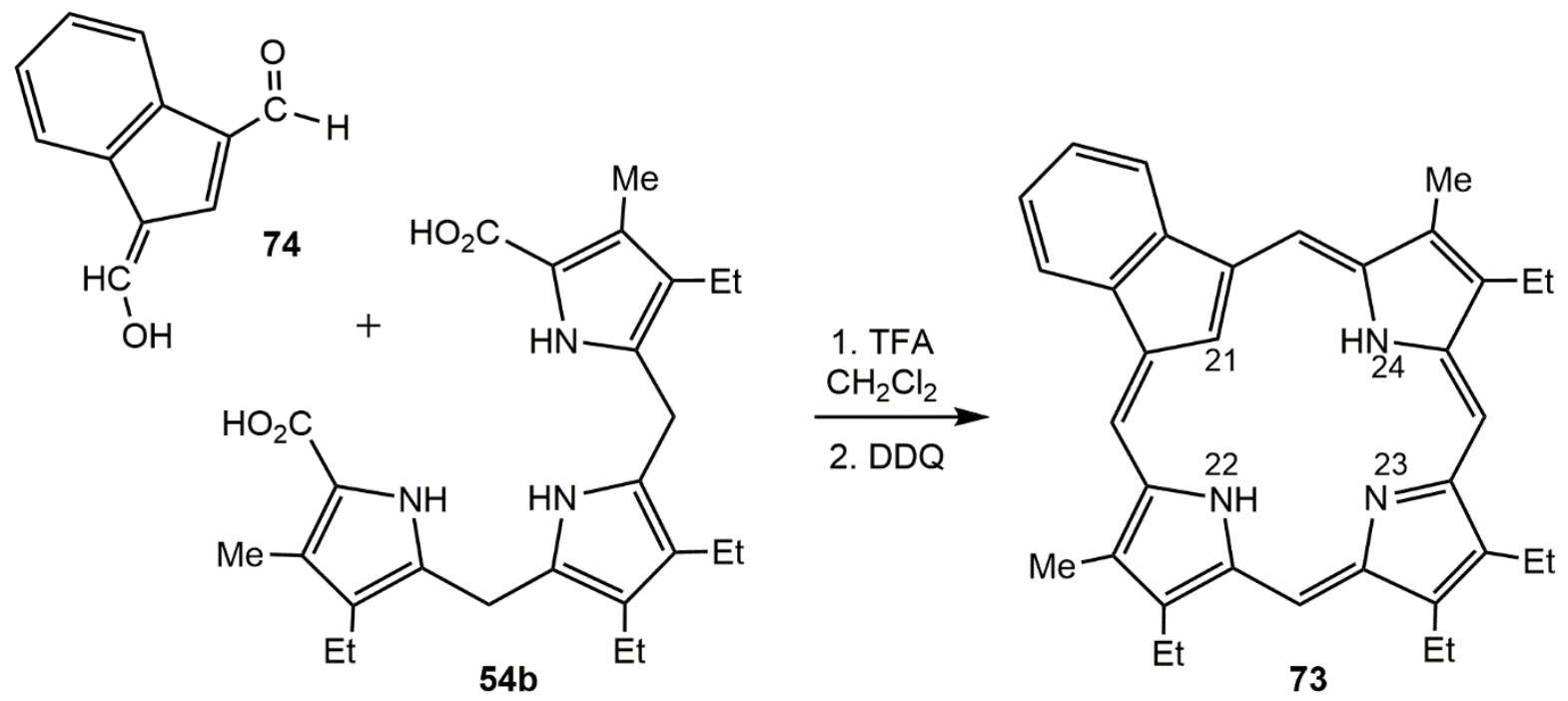

Scheme 20. Preparation of benzocarbaporphyrin via the ' $3+1$ ' MacDonald condensation
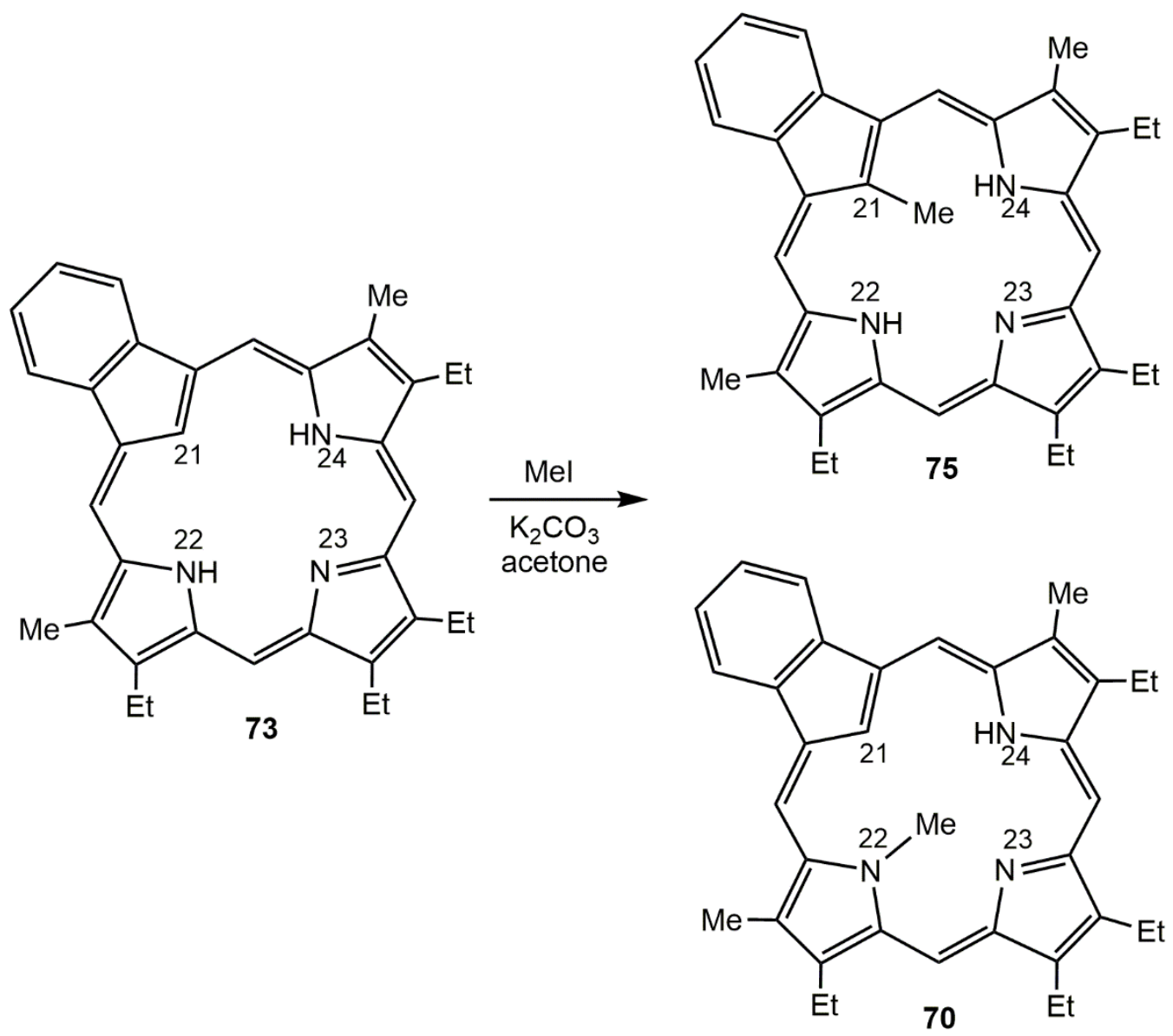

Scheme 21. Methylation of benzocarbaporphyrin 73 
Having replaced one of the inner hydrogens with a methyl group in $\mathbf{7 0}$ and $\mathbf{7 5}$, it was thought that the porphyrin analogues would now act as dianionic ligands. Reaction of $\mathbf{7 0}$ with palladium(II) acetate in refluxing acetonitrile initially gave $N$-methyl palladium(II) complex 71, but unexpectedly this rapidly rearranged to give the C-methyl palladium(II) complex 72 (Scheme 19). When the reaction time was reduced to 5 minutes the major product was $N$-methyl palladium(II) complex 71, but after 30 minutes virtually all of the material had converted into $\mathbf{7 2 .}$ Attempts to purify the $N$-methyl palladium(II) complex $\mathbf{7 1}$ by column chromatography were unsuccessful as this led to almost complete conversion to the C-methyl derivative 72. Therefore, this compound could not be isolated in pure form due to the persistence of alkyl group migration at room temperature. However, owing to the difference in symmetry between the two structures, it could be determined by proton NMR spectroscopy that C-methyl palladium complex 72 was being formed. The $N$-methyl palladium complex is asymmetrical, while the C-methyl palladium complex has a plane of symmetry, therefore the latter produces a smaller number of peaks in the NMR spectrum.

It was proposed that the alkyl group migration could have occurred through a Pd-alkyl intermediate, where the methyl group moves from one position to the other by hopping onto the metal center. This migration could have also occurred via a concerted [1,5]sigmatropic rearrangement due to the close proximity of the methyl group to the 21-position ${ }^{101}$ (Scheme 22). No intermediates were discovered to support either mechanism of migration, so it is unclear which of these was actually occurring. 


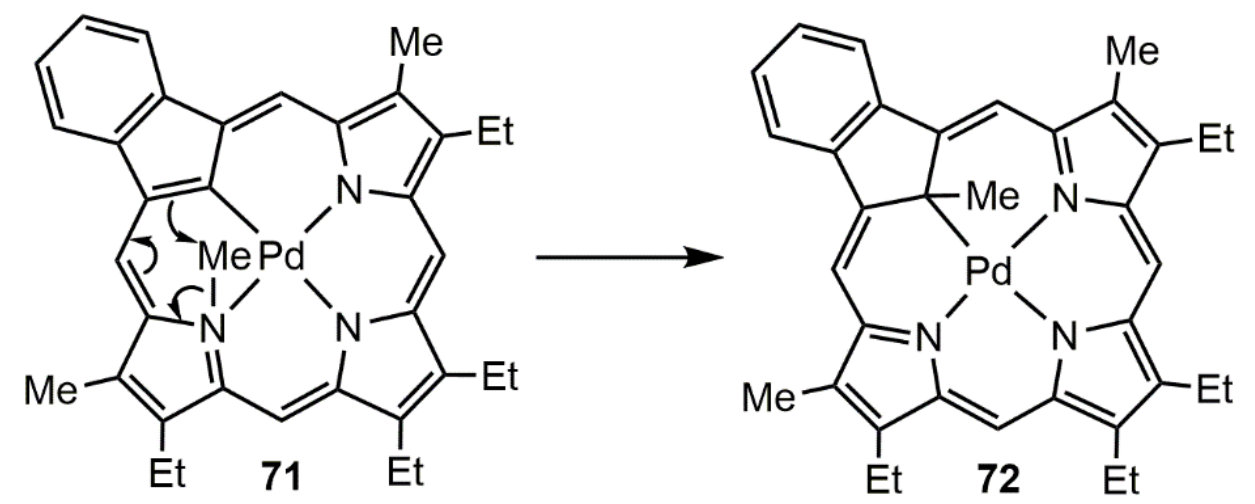

Scheme 22. Proposed [1,5] sigmatropic rearrangement for alkyl group migration

Rearrangements of this type could potentially be further investigated through the metalation of 23-methylbenzocarbaporphyrin $\mathbf{7 6}^{111}$ (Scheme 23). Ensuring the methyl is at a position further away from the 21-position would force the alkyl group to traverse the entire porphyrinoid cavity in order to migrate in a similar fashion. If the migration still occurs upon the insertion of a palladium(II) center into the carbaporphyrin cavity, it may be easier to determine which mechanism is taking place. Since a 23-alkyl product was not observed upon methylation of benzocarbaporphyrin $\mathbf{7 3}$, the desired carbaporphyrin $\mathbf{7 6}$ had to be constructed via the ' $3+1$ ' MacDonald-type condensation of a $N$-methylated tripyrrane 54d with indene dialdehyde $\mathbf{7 4}$ (Scheme 23). 

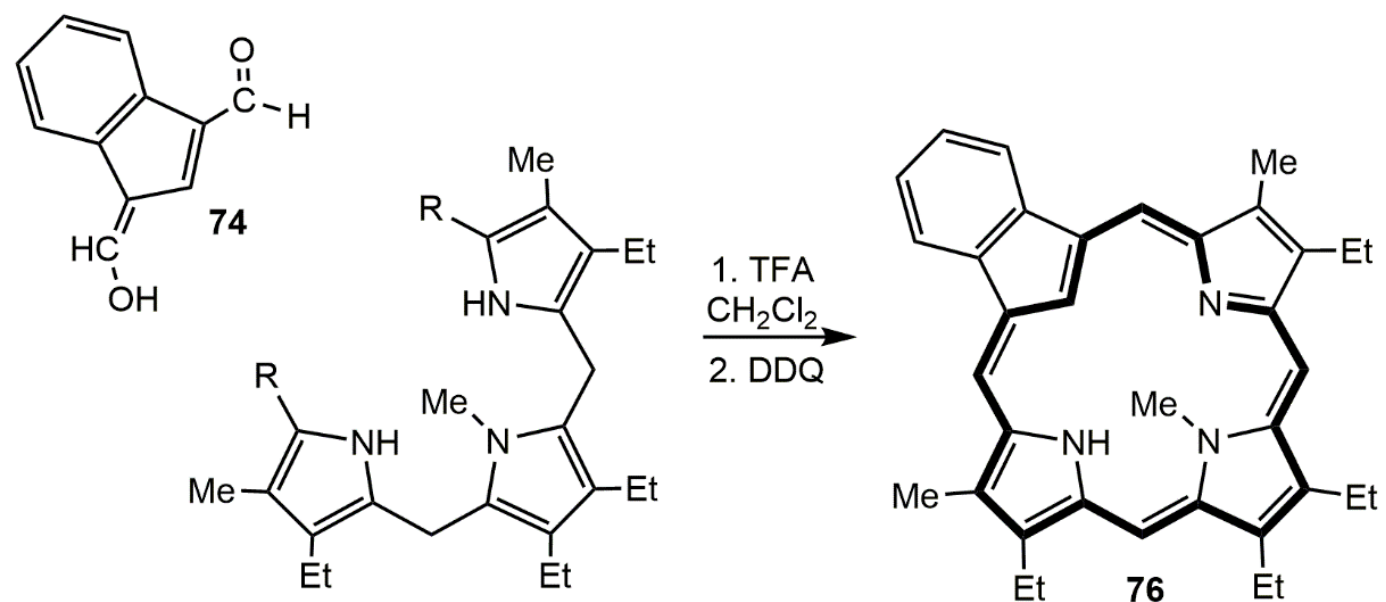
54 c. $\mathrm{R}=\mathrm{CO}_{2} \mathrm{CH}_{2} \mathrm{Ph}$
d. $\mathrm{R}=\mathrm{CO}_{2} \mathrm{H}$

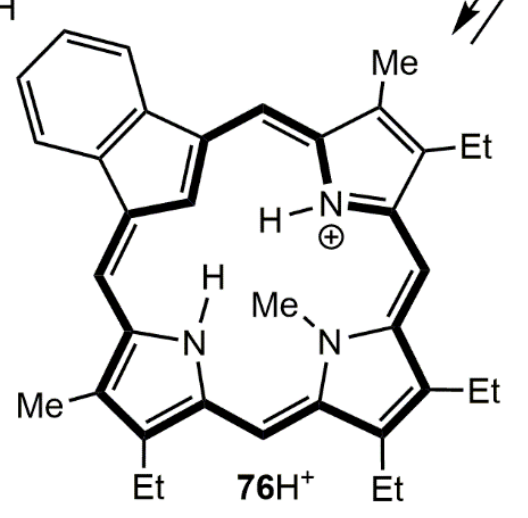

Scheme 23. Synthesis and protonation of 23-methylbenzocarbaporphyrin $\mathbf{7 6}$

\section{Results and Discussion}

The synthesis of tripyrrane 54d required the availability of two different pyrrolic precursors. The central pyrrole unit of 54d bears an $N$-methyl substituent, but initially it was necessary to prepare an $N$-unsubstituted pyrrole ethyl ester 78. Synthesis of this pyrrole ester began by reaction of ethyl isocyanoacetate with 3-acetoxy-4-nitrohexane and two equivalents of 1,8diazabicyclo[5.4.0]undec-7-ene (DBU) in THF-isopropyl alcohol (Barton-Zard condensation, Scheme 24). The product $\mathbf{7 8}$ was isolated in $70 \%$ yield but the proton NMR spectrum (Figure 3) showed that it was contaminated with approximately $3 \%$ of the corresponding isopropyl ester due to transesterification with the 2-propanol. However, as the ester is saponified at a later stage, 
pyrrole ethyl ester $\mathbf{7 8}$ was used in this form. Methylation of $\mathbf{7 8}$ was achieved by the dropwise addition of methyl iodide to a solution of the pyrrole ethyl ester and potassium hydroxide in dimethylsulfoxide (DMSO). The required $N$-methyl derivative $\mathbf{7 9}$ was obtained in $79 \%$ yield, but again the product was contaminated with a small amount of the related isopropyl ester. Subsequent saponification and decarboxylation of the pyrrole ester with sodium hydroxide in refluxing ethylene glycol at a temperature of $200^{\circ} \mathrm{C}$ for 2.5 hours produced 3,4-diethyl-1-methylpyrrole 53b in 84\% yield (Scheme 24). Pyrrole 53b had previously been prepared from 1,2-dimethylhydrazine using a variation on the Fischer indole synthesis. ${ }^{112}$ Initially, purification was carried out by vacuum distillation. However, it was later found that this was unnecessary, and pure 53b could be obtained in higher yields by simply extracting the product into hexanes.

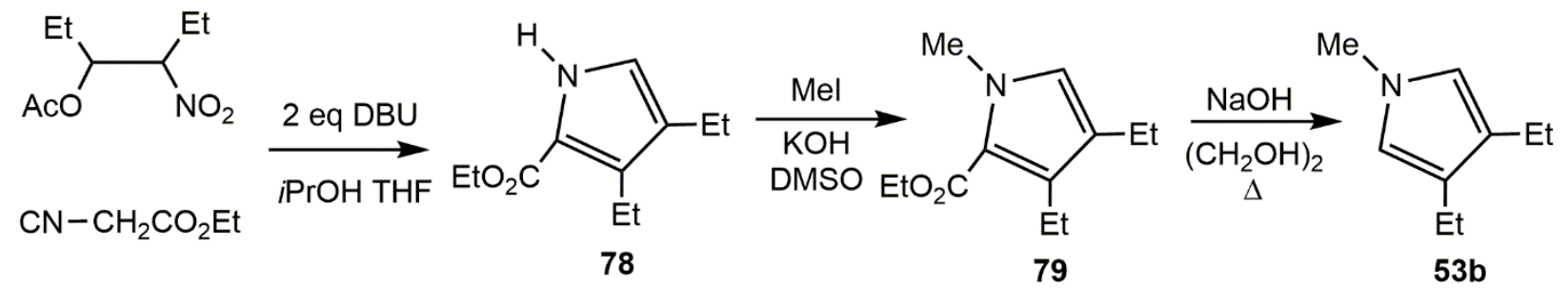

Scheme 24. Barton-Zard synthesis and methylation of a pyrrole precursor 


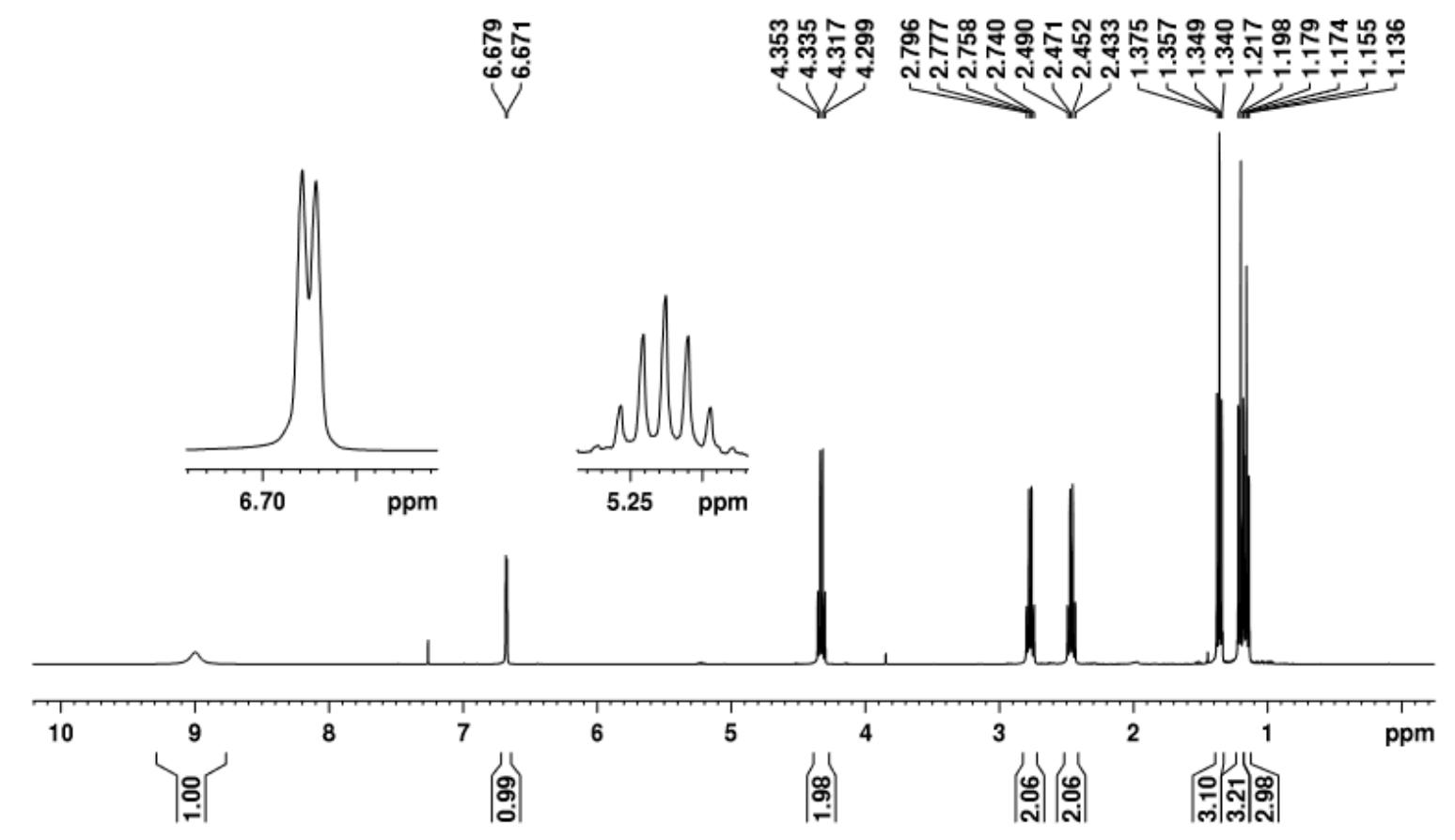

Figure 3. $500 \mathrm{MHz}{ }^{1} \mathrm{H}$ NMR spectrum of pyrrole ester 78 in $\mathrm{CDCl}_{3}$. The multiplet at $5.23 \mathrm{ppm}$ corresponds to a small amount of the related isopropyl ester pyrrole (ca. 3\%) that results from the transesterification with the solvent 2-propanol

A second pyrrole unit $\mathbf{8 0}$ was required to synthesized the desired tripyrrane. Diethyl aminomalonate $\mathbf{8 1}$ was reacted with 3-ethyl-2,4-pentanedione in gently boiling acetic acid for 2 hours to give the ethyl ester pyrrole 82. Subsequent transesterification with sodium benzyloxide in benzyl alcohol afforded the benzyl ester derivative 83, which was then reacted overnight with lead tetraacetate in acetic acid and acetic anhydride to give acetoxymethylpyrrole $\mathbf{8 0}$ (Scheme 25). 


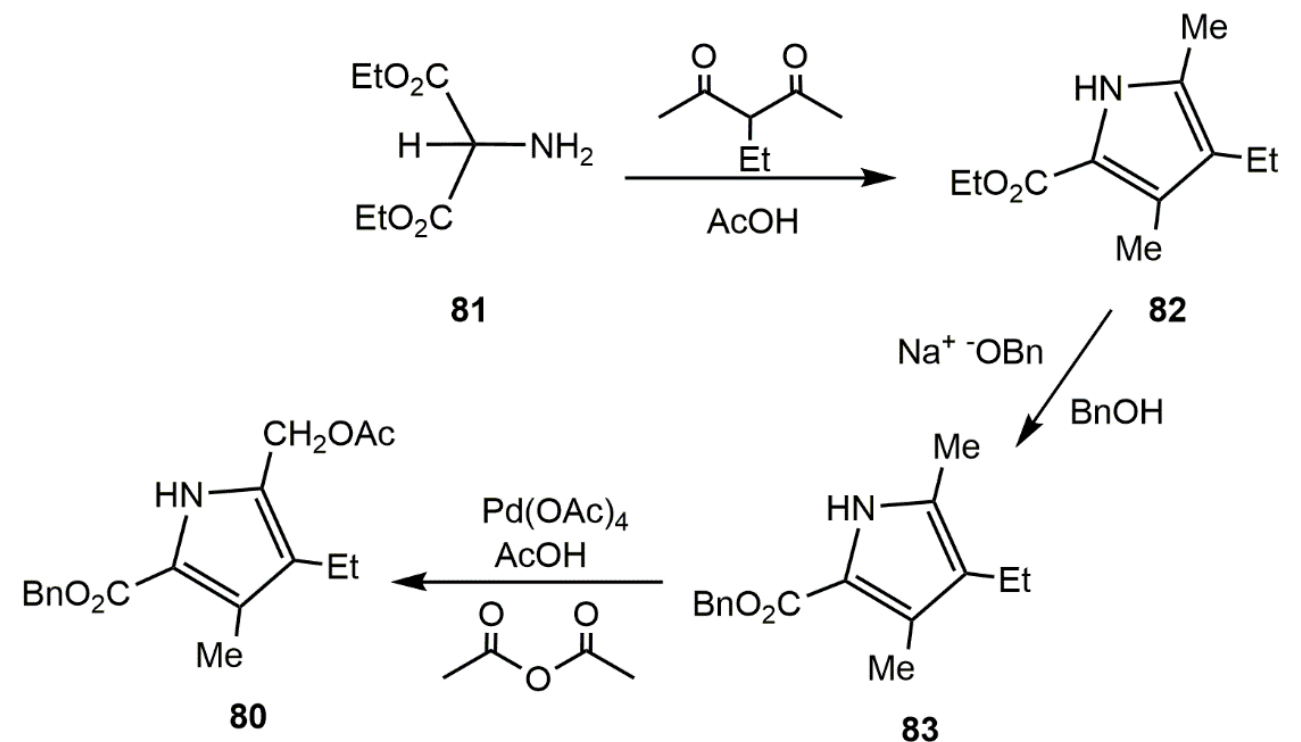

Scheme 25. Synthesis of acetoxymethylpyrrole $\mathbf{8 0}$

Tripyrranes can be prepared by condensing $\alpha, \alpha^{\prime}$-diunsubstituted pyrroles with two equivalents of an acetoxymethylpyrrole such as $\mathbf{8 0}$ in the presence of an acid catalyst. ${ }^{92}$ However, attempts to react $\mathbf{5 3 b}$ with $\mathbf{8 0}$ in the presences of Montmorillonite clay ${ }^{113}$ gave poor results, and reactions with acetic acid in ethanol or other alcohol solvents ${ }^{114}$ also gave low yields of the desire tripyrrolic product 54c (Scheme 26). It was speculated that ethanol might be replacing the acetoxy groups present on $\mathbf{8 0}$ to give ethyl ether by-products, which in turn lowered the yield of the desire product by consuming one of the intermediates. Superior results were obtained by reacting $\mathbf{5 3 \mathbf { b }}$ and $\mathbf{8 0}$ in a 1:2 ratio with acetic acid in refluxing acetonitrile under nitrogen. $N$-Unsubstituted tripyrranes commonly precipitate from solution, but this was not observed with $N$-methyl tripyrrane 54c and therefore the crude product had to be purified by column chromatography. Some tripyrranes have been known to decompose during chromatography, but in the case of 54c it could be isolated as an oil that solidified in the freezer (Scheme 26). However, the purification of this tripyrrane by column chromatography proved difficult. Pure samples were hard to acquire, even 
after several runs through silica columns eluting with $80 \%$ dichloromethane-hexanes. It was found that triethylamine aided in stabilizing the tripyrrane during column chromatography. When the reaction product was columned on silica gel and eluted with 80:20:1 dichloromethane/hexanes/triethylamine, pure samples of the tripyrrane were obtained after two chromatographic purifications. The benzyl ester protective groups of $\mathbf{5 4 c}$ were then cleaved by hydrogenolysis over Pd/C, to give the $N$-methyltripyrrane dicarboxylic acid 54d (Scheme 26).

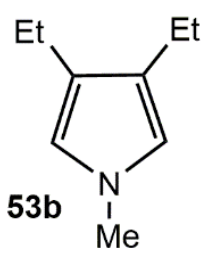

2

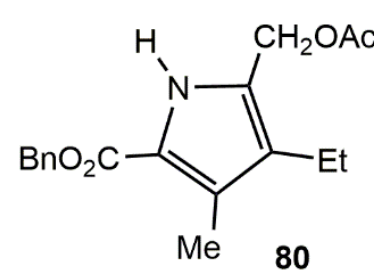

$\Delta$
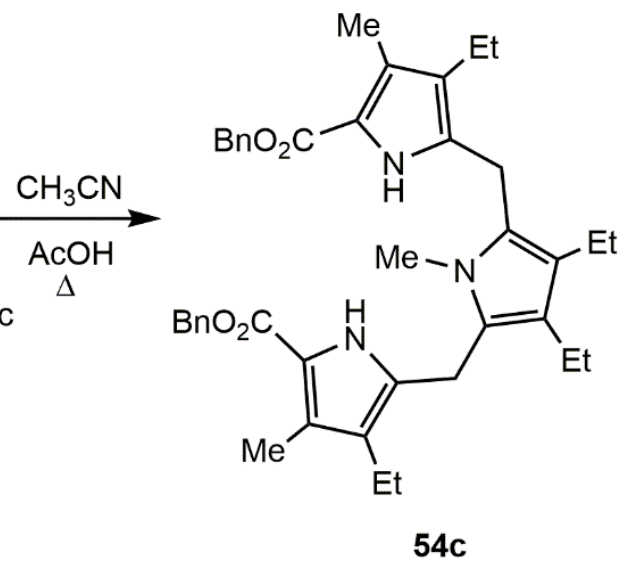

$54 c$

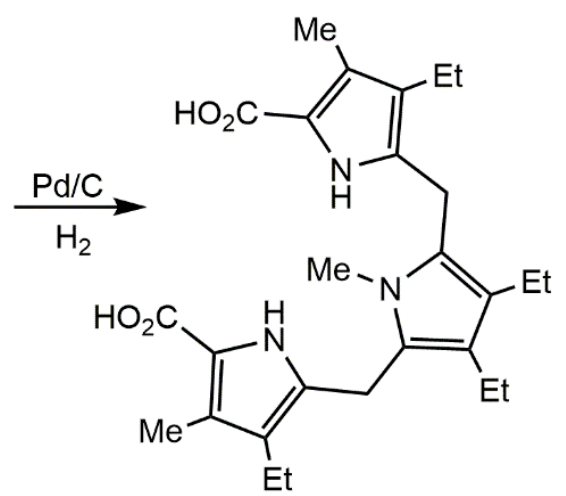

54d

Scheme 26. Synthesis of $N$-methyl tripyrrane $54 d$

$N$-Alkyl tripyrranes had not previously been applied to the preparation of porphyrinoid products. However, '2+2' MacDonald condensation of a $N$-methyldipyrrylmethane with a dipyrrylmethane dialdehyde had been used to synthesize a $N$-methylporphyrin, ${ }^{115}$ and related porphyrins were prepared from $a, c$-biladiene intermediates. ${ }^{116}$ The steric bulk associated with presence of an internal substituent might inhibit the cyclization reaction, and given the instability of tripyrranes under the acidic conditions used to carry out these reactions, there was a possibility that decomposition would ensue. Fortunately, acid catalyzed condensation of 54d with indene dialdehyde 74, followed by oxidation with 2,3-dichloro-5,6-dicyano-1,4-benzoquinone (DDQ) 
gave the desired 23-methylcarbaporphyrin $\mathbf{7 6}$ in 32\% yield (Scheme 23). Fairly concentrated conditions were used (less than $20 \mathrm{~mL}$ of dichloromethane for every $100 \mathrm{mg}$ of tripyrrane), but it was found that dilution did not afford any higher yields. The carbaporphyrin was chromatographed twice on grade 3 neutral alumina, eluting with dichloromethane and then chloroform, and a dark band was collected each time. The product was recrystallized from chloroform-methanol to yield purple-blue crystals. The $N$-substituted carbaporphyrin retains fully aromatic characteristics, and the proton NMR spectrum (Figure 4) showed that the macrocycle is symmetrical with relatively downfield meso-protons resonating as two $2 \mathrm{H}$ singlets at 9.67 and $10.03 \mathrm{ppm}$, while the benzoprotons were observed as multiplets at 7.67-7.71 and 8.79-8.83 ppm. The internal methyl group and $\mathrm{CH}$ were strongly shifted upfield to give a $3 \mathrm{H}$ singlet and $1 \mathrm{H}$ singlet at $-4.25 \mathrm{ppm}$ and -4.95 ppm, respectively, and the internal $\mathrm{NH}$ appeared as a broad peak between 0 and $-1 \mathrm{ppm}$. Also of note, the $\mathrm{CH}_{2}$ protons of the ethyl substituents appeared as multiplets due to complex coupling that stems from the diastereotopic nature of these protons. Apart from the coupling effects, these results are very similar to those obtained for $\mathrm{N}$-unsubstituted benzocarbaporphyrin. The UV-Vis of carbaporphyrin 76 (Figure 5) was typical of an aromatic porphyrinoid, showing a strong Soret absorption at $439 \mathrm{~nm}$ and Q bands at 538, 567, 624 and $683 \mathrm{~nm}$. Addition of trifluoroacetic acid (TFA) afforded a monoprotonated species $\mathbf{7 6} \mathrm{H}^{+}$that showed a diminished Soret band at $444 \mathrm{~nm}$ (Figure 5). Crystals of $\mathbf{7 6}$ that were suitable for X-ray crystallographic analysis were obtained and the results showed that the pyrrole ring bearing the internal methyl group was titled by $24.34(3)^{\circ}$ from the macrocyclic plane, defined by atoms C1, C2, C3, C4, C5, C6, C7, C8, C9, C10, C11, C14, C15, C16, C17, C18, C19, C20 and C21 (Figure 6). The remainder of the macrocyclic framework is rather flat with the two remaining pyrrole rings and the indene subunit only tilted $0.46(3)^{\circ}, 3.04(3)^{\circ}$ and $2.96(2)^{\circ}$, respectively, from the macrocyclic plane. In addition, the $N$ - 
methyl bond is significantly bent relative to the pyrrole ring plane as evidenced by the $139.2(1)^{\circ}$ C12-C11-N23-C25 and 137.8(1) ${ }^{\circ}$ C13-14-N23-C25 torsion angles. These results differ from the structure obtained for a benzocarbaporphyrin without the $\mathrm{N}$-alkyl substituent as this showed that the indene moiety was tilted by $15.5^{\circ}$ relative to the mean macrocyclic plane, whereas the pyrrole to mean macrocyclic plane tilts were $2.5^{\circ}, 4.4^{\circ}$, and $4.9^{\circ} .{ }^{18}$ With the exception of the C1-C2 bond length, a Mogul geometry check validated all bond distances, angles and torsions to be within

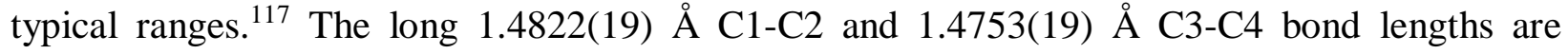
consistent with more single bond like character, which suggests the benzo $\pi$-system of the indene subunit is isolated from the main macrocyclic $\pi$-system.
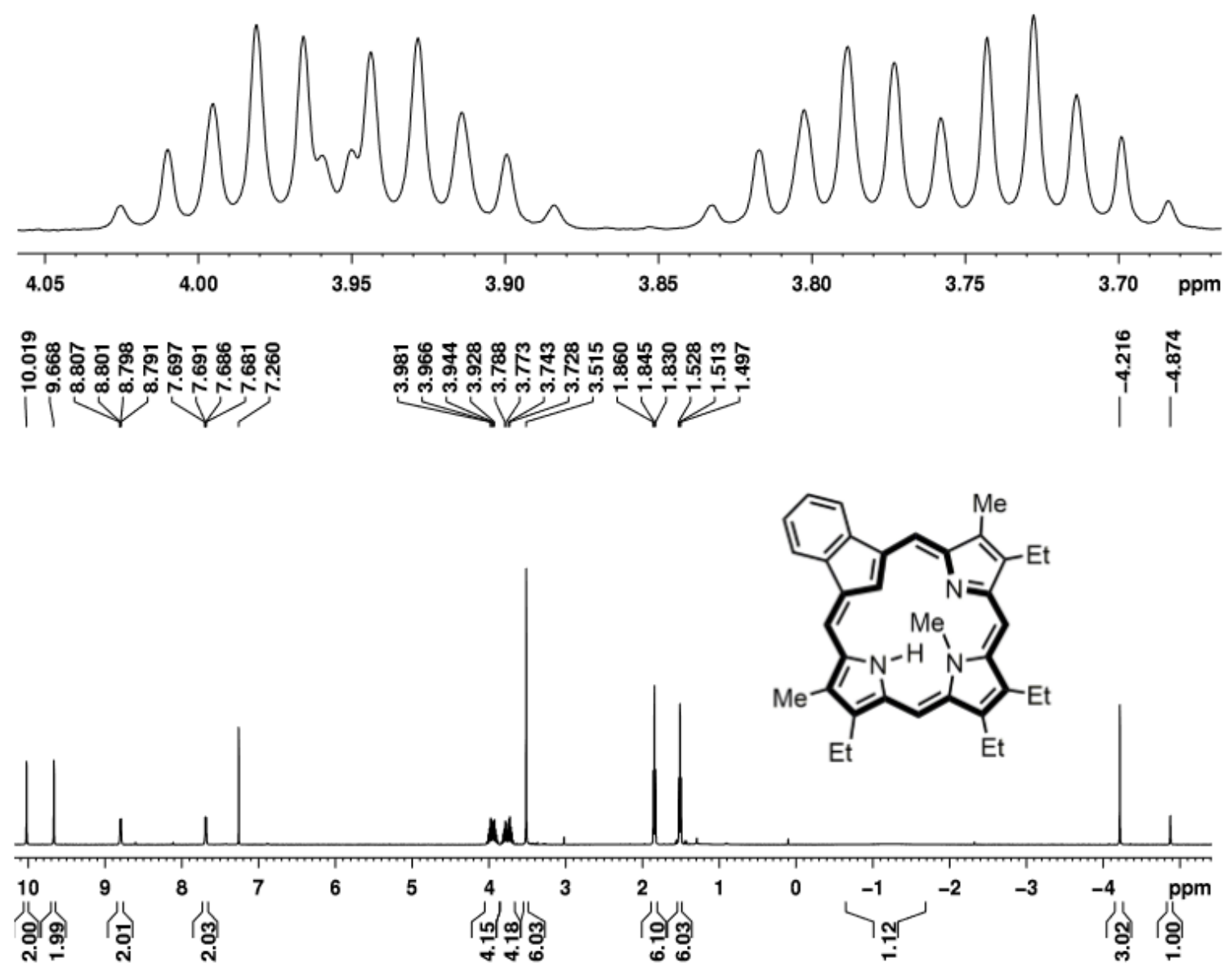

Figure 4. $500 \mathrm{MHz}{ }^{1} \mathrm{H}$ NMR spectrum of 23-methylbenzocarbaporphyrin 76 in $\mathrm{CDCl}_{3}$ 


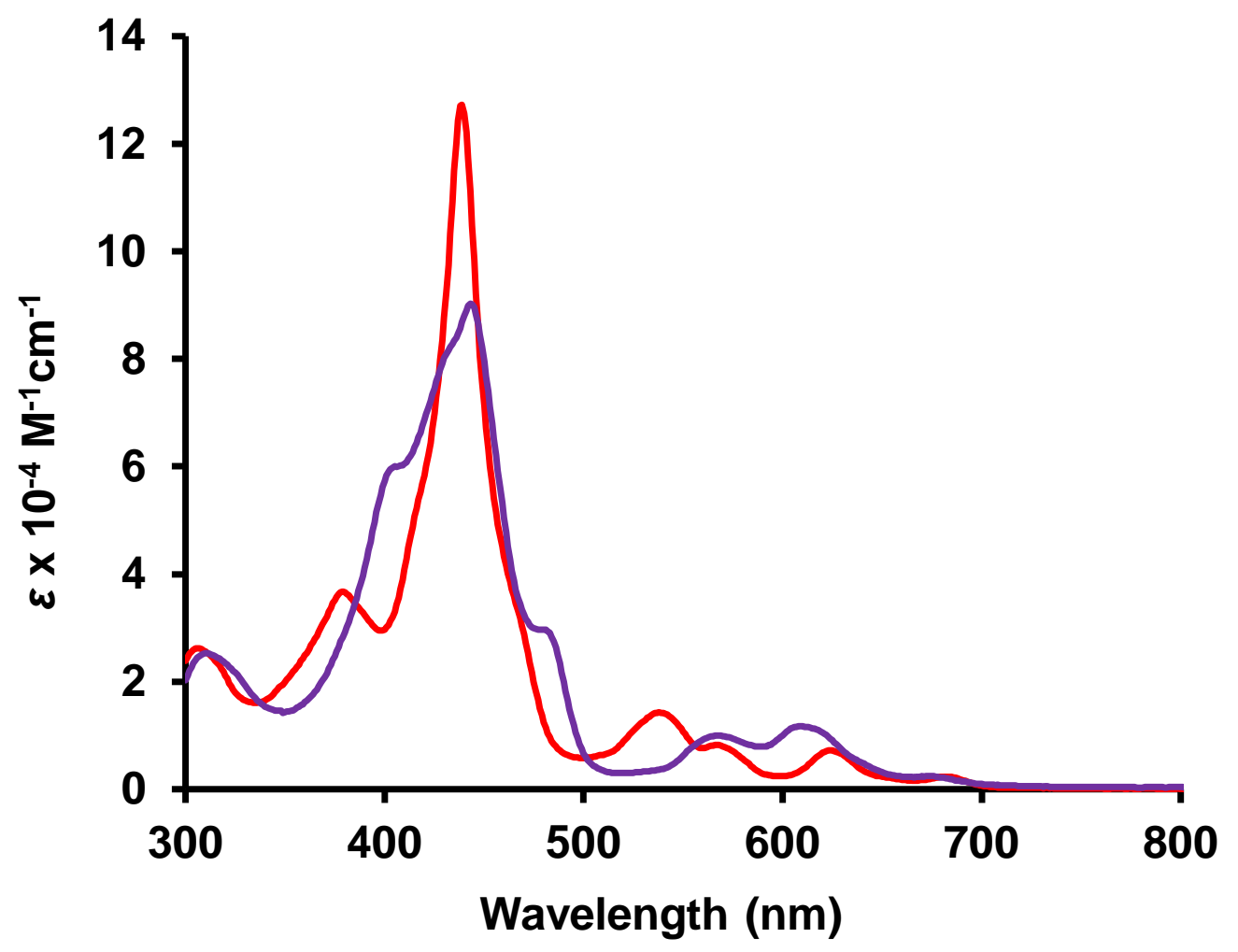

Figure 5. UV-vis spectra of 23-methylcarbaporphyrin 76 in 1\% triethylamine-dichloromethane (free base, red line) and 1\% TFA-dichloromethane (monocation $\mathbf{7 6} \mathrm{H}^{+}$, purple line)

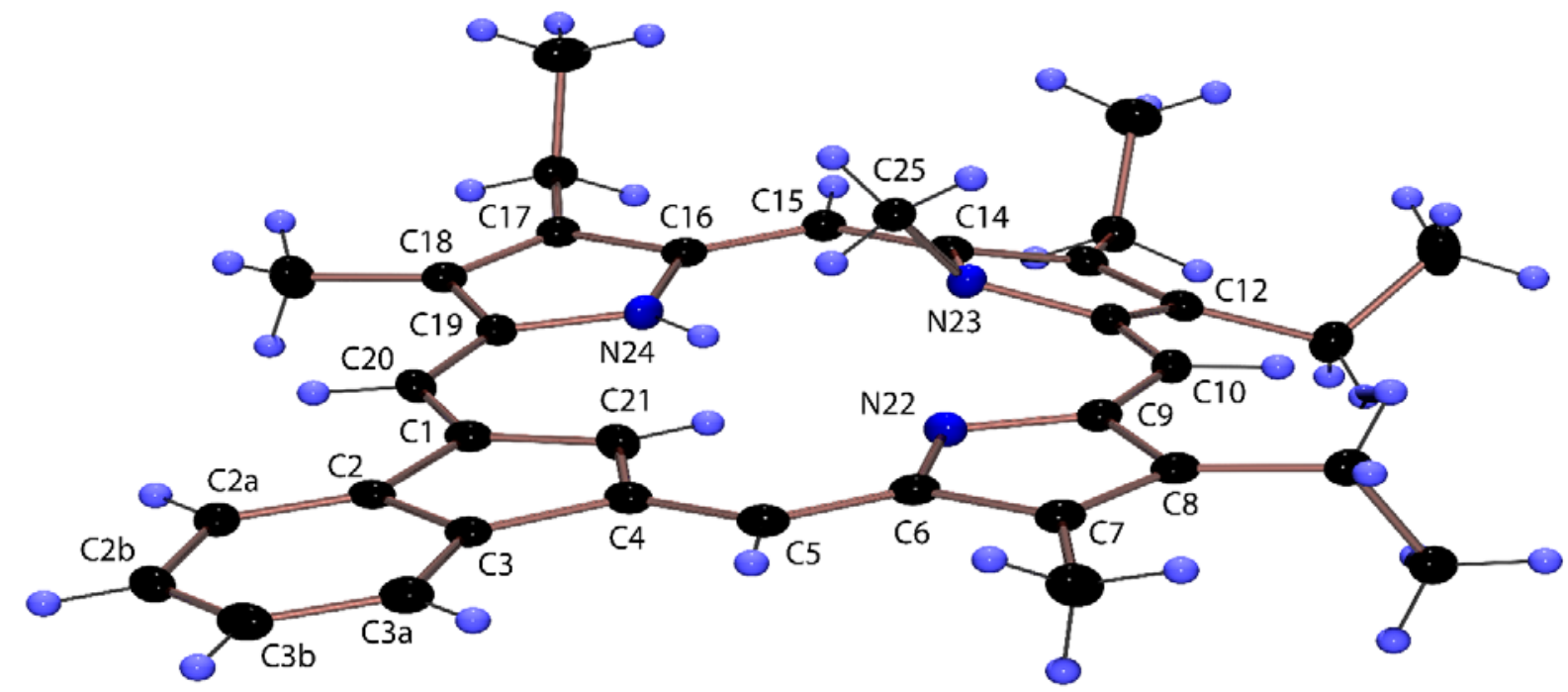

Figure 6. Color POV-Ray rendered ORTEP III drawing (50\% probability level, hydrogen atoms rendered arbitrarily small for clarity) of 23-methylcarbaporphyrin $\mathbf{7 6 .}$ 
Reaction of $\mathbf{7 6}$ with palladium(II) acetate in refluxing acetonitrile for 30 minutes gave a mixture of metalated products. When the reaction time was reduced to 5 minutes and the crude product purified by column chromatography and recrystallized from chloroform-methanol, a $\mathrm{N}$ methyl palladium complex $\mathbf{8 4}$ was isolated in $67 \%$ yield (Scheme 27). This compound retained strong aromatic character and the symmetry observed for the complex is evident in the NMR spectra. The meso-protons appeared downfield at 9.85 and $10.00 \mathrm{ppm}$ and the internal methyl gave a $3 \mathrm{H}$ singlet at $-2.86 \mathrm{ppm}$. The benzo-protons were observed as two $2 \mathrm{H}$ multiplets at 7.47 and 8.50 ppm, which demonstrates that this complex has a delocalized $\pi$-electron pathway similar to that of 76. The UV-Vis spectrum of $\mathbf{8 4}$ gave several strong absorptions between 300 and $520 \mathrm{~nm}$, followed by weaker, broad absorption peaks between 600 and $700 \mathrm{~nm}$ (Figure 7). When the reaction of 76 with palladium(II) acetate was carried out overnight, the initially formed $N$-methyl palladium complex 84 was completely converted to the C-methyl palladium(II) complex 72 (Scheme 27). Therefore, the alkyl group migration still occurs for 23-methylbenzocarbaporphyrin 76, but at a much slower rate. The C-methyl product $\mathbf{7 2}$ was identical to the previously isolated product from the reaction of 22-methylbenzocarbaporphyrin with palladium(II) acetate. ${ }^{102}$ The UV-Vis spectrum of $\mathbf{7 2}$ is very different from the UV-Vis spectrum of $\mathbf{8 4}$, displaying a Soret band at 421 $\mathrm{nm}$ as well as a fairly strong absorption at $697 \mathrm{~nm}$ (Figure 7). The difference in UV-Vis spectra is due to the presence of a redirected $\pi$-electron pathway in $\mathbf{7 2}$, where the conjugation passes through the middle of the indene unit. This relocated conjugation pathway also allows for the two palladium complexes to be easily distinguished from one another by proton NMR spectroscopy (Figure 8). 

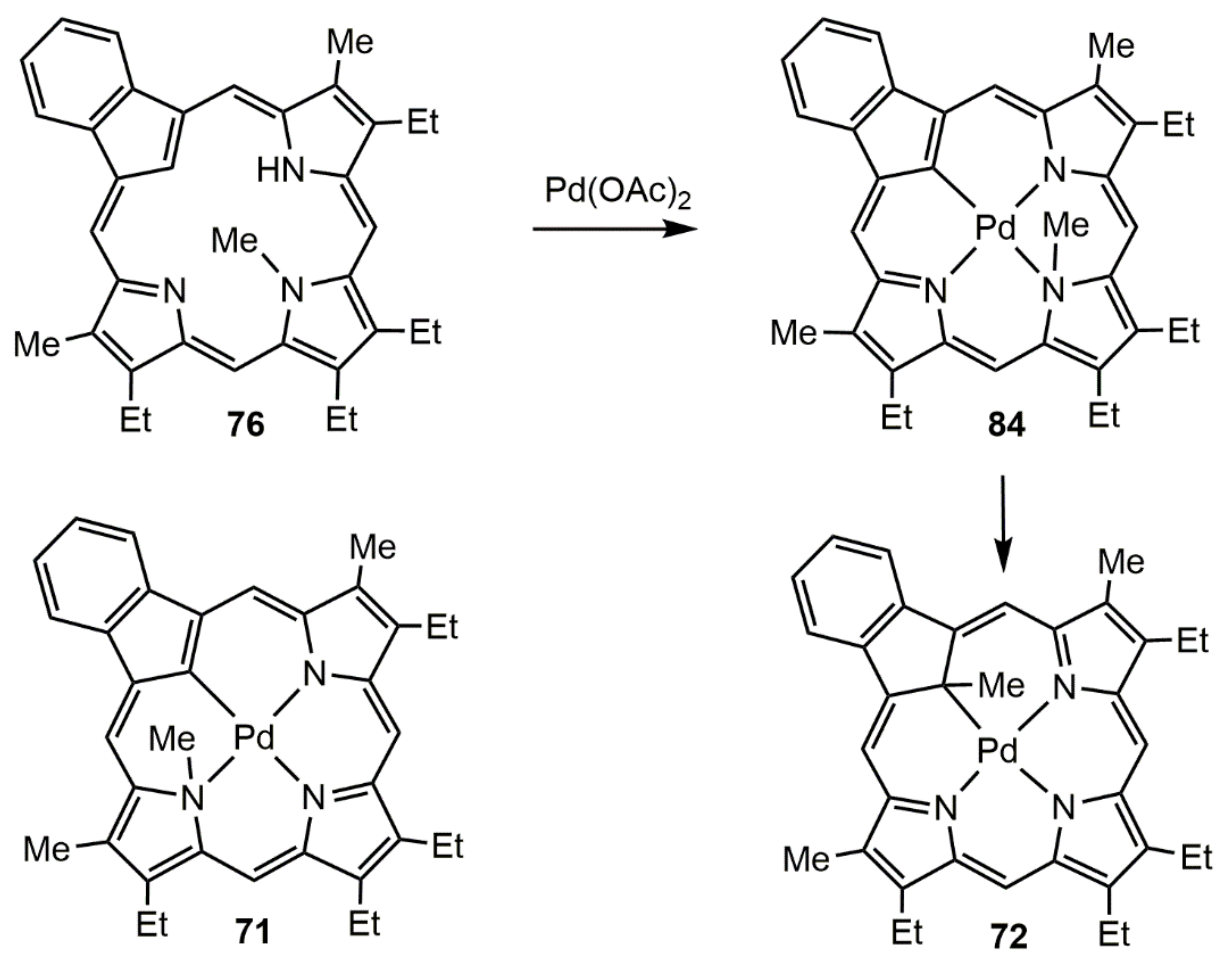

Scheme 27. Metalation of 23-methylbenzocarbaporphyrin to give 23- and 21-methyl palladium(II) complexes 


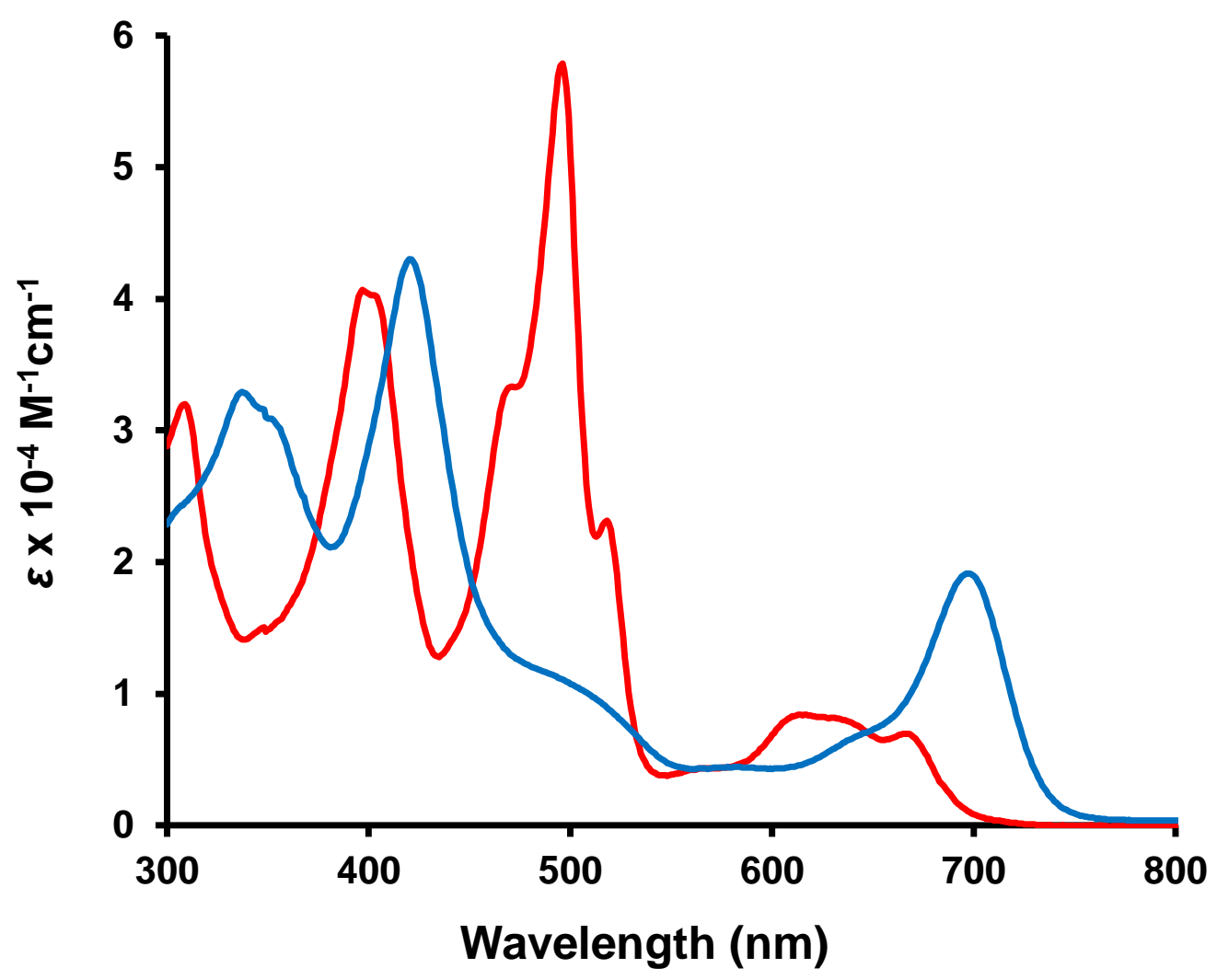

Figure 7. UV-vis spectra of palladium(II) carbaporphyrin complexes 84 (23-methyl, red line) and 72 (21-methyl, blue line)

Palladium complexes $\mathbf{7 2}$ and $\mathbf{8 4}$ both possess a plane of symmetry, but the downfield regions of their proton NMR spectra were quite different. For $\mathbf{8 4}$, the benzo-protons were observed at 7.45-7.49 ppm and 8.48-8.52 ppm, but in 21-methyl palladium complex 72 the protons resonate at 8.22-8.25 ppm and 9.39-9.43 ppm. These differences can be ascribed to the differing $\pi$-electron pathways (shown in bold, Figure 8) for these metalated carbaporphyrins. The downfield shifts for 72 are due to the $18 \pi$-electron pathway passing through the benzene unit, causing the benzoprotons to experience more of the deshielding effect due to the macrocyclic ring current. The mesoprotons of 72 were also identified as two $2 \mathrm{H}$ singlets resonating at 9.56 and $10.27 \mathrm{ppm}$. The internal 
methyl group was seen upfield at $-3.27 \mathrm{ppm}$, compared to $-2.86 \mathrm{ppm}$ in $\mathbf{8 4}$, but this is due to the methyl group being connected to a carbon atom rather than a nitrogen.
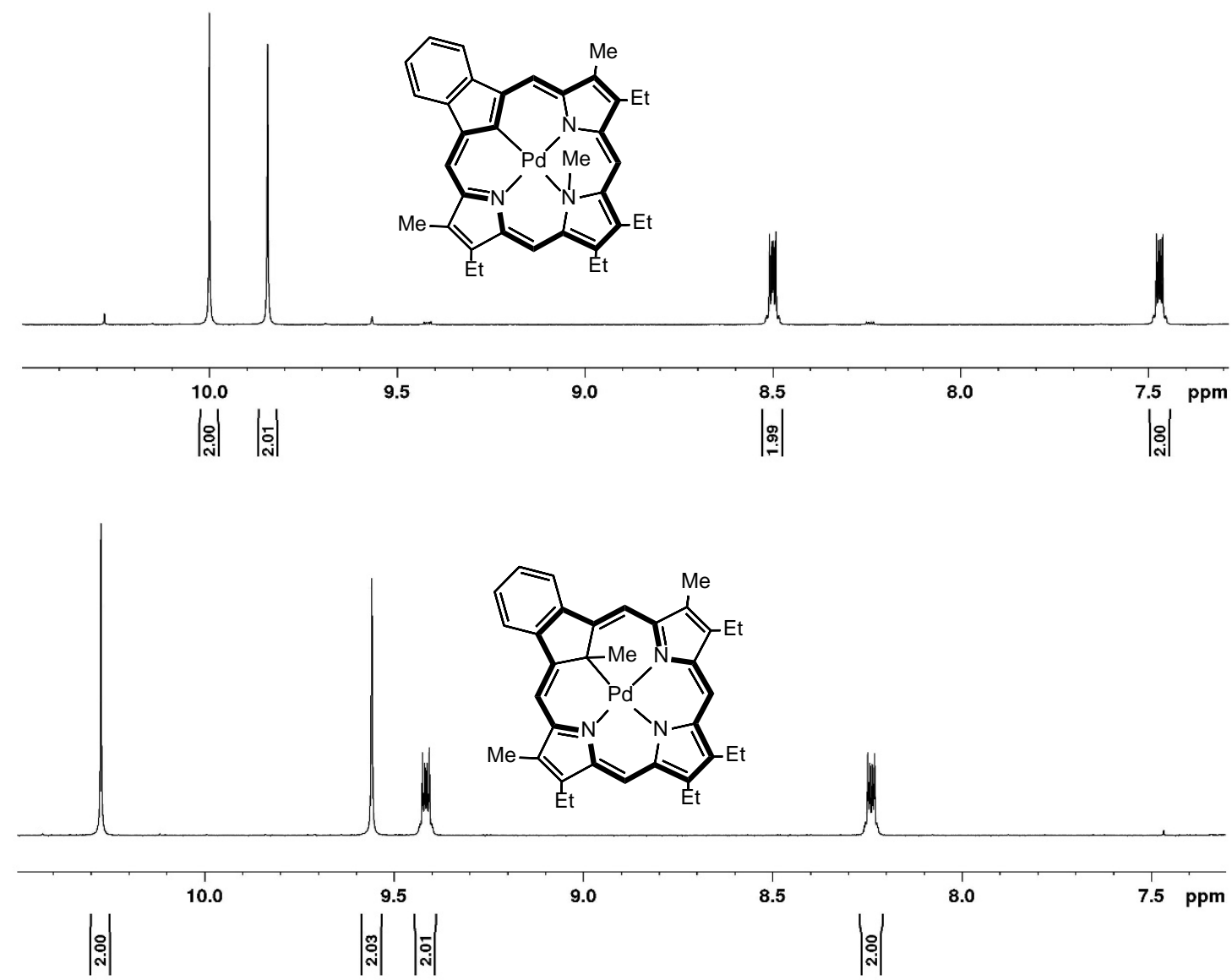

Figure 8. Downfield region for the ${ }^{1} \mathrm{H}$ NMR spectra of 23-methyl palladium complex 84 (top spectrum) and 21-methyl palladium complex 72 (bottom spectrum)

The structure of 72 was confirmed by X-ray crystallography (Figure 9). The framework of bond distances are consistent with an overall aromatic carbaporphyrin containing an $s p^{3}$ hybridized C21. The palladium(II) metal center resides in an approximately square planar coordination

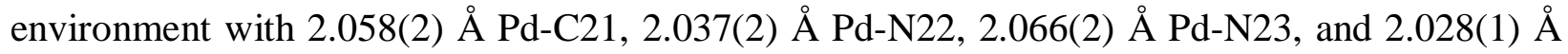
Pd-N24 bond lengths. Coordination of the palladium ion and the internal alkyl to C21 results in lengthened 1.491(2) $\AA$ and 1.498(2) $\AA s p^{3} \rightarrow s p^{2}$ C21-C1 and C21-C4 bond distances. This leads 
to C21 lying separate from the macrocycle’s $\pi$-system. This is further supported by C21 being located $0.360(2) \AA$ out of the $\mathrm{PdN}_{3}$ plane, and $0.196(2) \AA$ out of the plane defined by the other indene unit atoms. The indene unit itself is tilted $14.82(4)^{\circ}$ from the $\mathrm{PdN}_{3}$ plane. The carbon of the methyl group, C25, lies 1.935(2) Å out of the $\mathrm{PdN}_{3}$ plane and 1.707(2) $\AA$ out of the plane defined by the indene unit atoms. Furthermore, the C1-C2 and C3-C4 bond lengths are ca. $0.03 \AA$ shorter than those found in carbaporphyrin 76, which is consistent with the $\pi$-electron pathway being relocated through the indene unit in palladium complex 72.

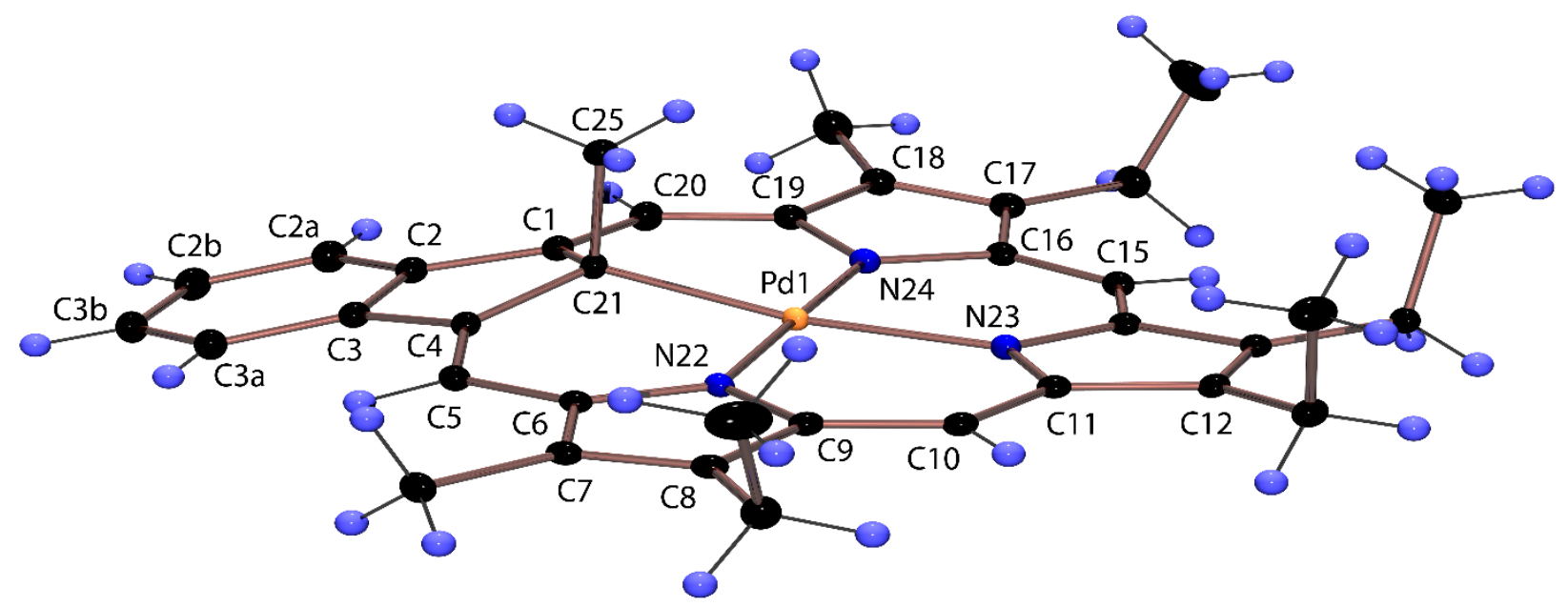

Figure 9. Color POV-Ray rendered ORTEP III drawing (50\% probability level, hydrogen atoms rendered arbitrarily for clarity) of 21-methyl palladium(II) complex 72

Two time course experiments were conducted to investigate the rate of methyl group migration. The first experiment consisted of timed reactions where $\mathbf{7 6}$ was refluxed with palladium(II) acetate in acetonitrile for time periods ranging from 5 minutes to overnight (ca. 16 hours). The relative proportions of the two palladium complexes were determined by comparing the integrations observed for the meso-, benzo-, and internal methyl protons of each compound. The percent compositions of each metal complex were graphed as a function of time (Figure 10a). 
Although the formation of $\mathbf{8 4}$ was very rapid, it was observed that the methyl group migration was only $50 \%$ complete after refluxing for 2.5 hours in acetonitrile. This is much slower compared to the almost full conversion to $\mathbf{7 2}$ seen after only 30 minutes for the 22-methyl derivative $\mathbf{7 1}$. It is possible that $\mathbf{7 1}$ could be an intermediate in the formation of $\mathbf{7 2}$ from 84 (Scheme 27). However, this species was not detected in any of the proton NMR spectra. As a slight excess of palladium(II) acetate is used in these reactions, there was also a possibility that this promoted the rearrangement. It was found that neither the absence or presence of palladium(II) acetate had a measurable effect on the rate of migration. This was determined by a second time course experiment where the 23methyl palladium(II) complex 84 was dissolved in $d_{3}$-acetonitrile in a NMR tube at $70{ }^{\circ} \mathrm{C}$ and spectra were collected using a $500 \mathrm{MHz}$ NMR spectrometer over a period of ca. 8 hours. The percent composition of each product was determined in the same fashion as the first experiment, and the values were plotted against increasing time. The results showed $50 \%$ conversion of $\mathbf{8 4}$ to 72 after 380 minutes (Figure 10b). This depicts a slower conversion due to the lower temperature but shows that the migration can occur without the presence of palladium(II) acetate. Methyl group migration without the presence of a palladium metal center was also tested by refluxing $\mathbf{7 6}$ in acetonitrile with no palladium(II) acetate added, and it was found that no migration occurred. Furthermore, it was found that this migration occurs even in the solid state. A crystalline sample of 84 that had been stored for approximately 1 year was found to have completely converted to the 21-methyl derivative 72. No intermediates could be seen in any of the NMR spectra obtained during the time course experiments, and therefore the mechanism remains ambiguous. However, these new results provide some insights into these rearrangements. It is possible that the 22-methyl palladium(II) complex is formed as an intermediate, as the conversion of $\mathbf{7 1}$ to $\mathbf{7 2}$ is fast compared 
to the rearrangement of $\mathbf{8 4}$ to $\mathbf{7 2}$. However, as the migration does not occur without the presence of a palladium metal center, the intermediacy of a palladium-methyl complex is more plausible.

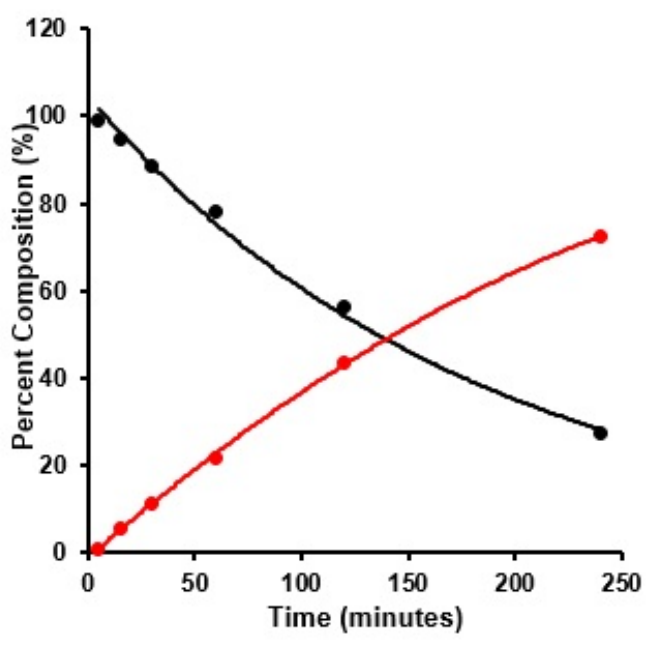

(a)

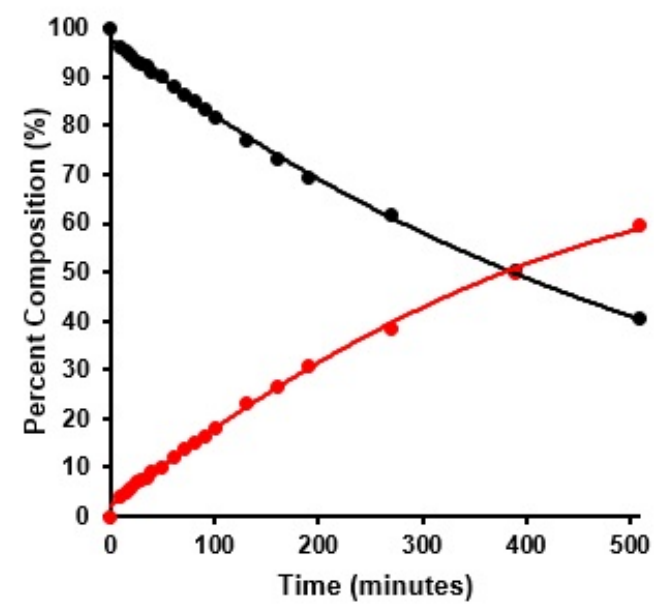

(b)

Figure 10. Timed experiments on the rate of alkyl group migration, showing the conversion of $\mathbf{8 4}$

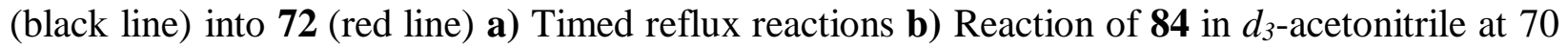
${ }^{\circ} \mathrm{C}$ in an NMR tube within a $500 \mathrm{MHz}$ NMR instrument

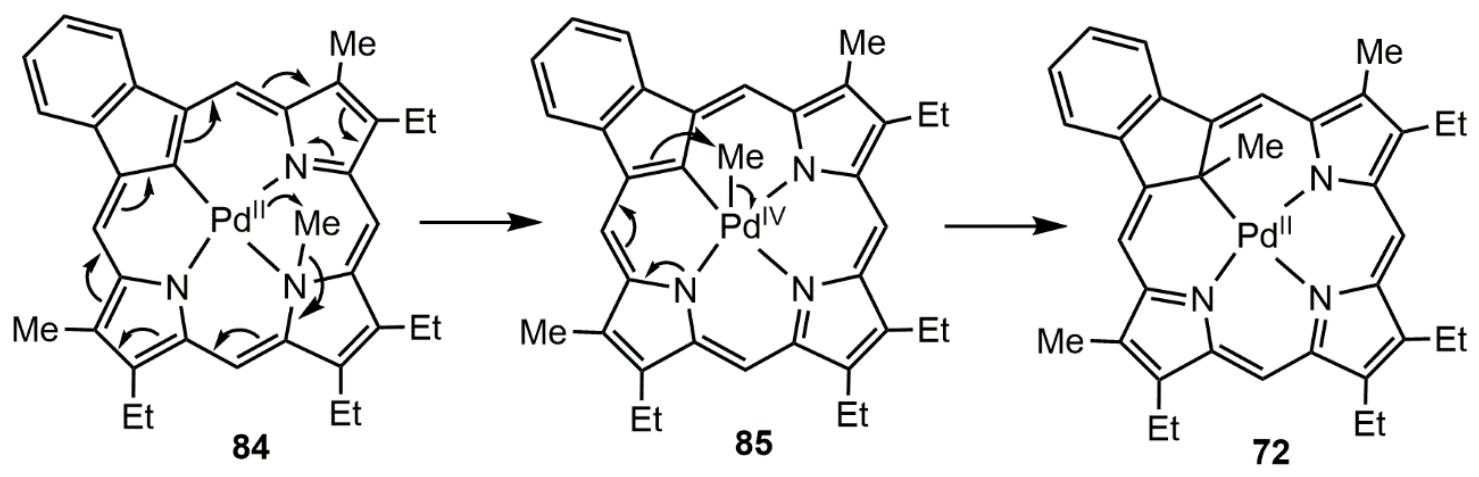

Scheme 28. Proposed mechanism for migration of an alkyl group from the 23- to 21-position of benzocarbaporphyrins 
It is proposed that a palladium-methyl intermediate is formed through an oxidative addition of the alkyl group onto the metal center forming a palladium(IV) species $\mathbf{8 5}$. Subsequent reductive elimination would lead to transfer of the methyl group to the 21-carbon, producing the observed product 72 and a reduction of the palladium to its original 2+ oxidation state (Scheme 28).

\section{Conclusion}

Introduction of internal methyl substituents results in benzocarbaporphyrins taking on dianonic character when reacted with metals such as palladium. Methyl group migration was also observed and investigated within these carbaporphyrinoids. It was found that methyl substituents located on two different internal nitrogens migrated to the internal carbon atom and produced the same 21-methyl palladium(II) benzocarbaporphyrin complex 72. Due to the dramatic effects that internal alkyl moieties have on carbaporphyrin coordination with palladium cations, the next chapter focuses on the introduction of rhodium into internally methylated carbaporphyrinoid systems. Rhodium(I) and rhodium(III) complexes of benzocarbaporphyrins, a carbaporphyrin diester and a naphthocarbaporphyrin are discussed. The metalation of the 23methylbenzocarbaporphyrin $\mathbf{7 6}$ with rhodium is also described, and the unexpected formation of a rhodium(III) bridged derivative is reported. 


\section{Experimental:}

Melting points are uncorrected. NMR spectra were recorded using a 400 or $500 \mathrm{MHz}$ NMR spectrometer and were run at $302 \mathrm{~K}$ unless otherwise indicated. ${ }^{1} \mathrm{H}$ NMR values are reported as chemical shifts $\delta$, relative integral, multiplicity ( $\mathrm{s}$, singlet; $\mathrm{d}$, doublet; $\mathrm{t}$, triplet; q, quartet; $\mathrm{m}$, multiplet; br, broad peak) and coupling constant $(J)$. Chemical shifts are reported in parts per million (ppm) relative to $\mathrm{CDCl}_{3}\left({ }^{1} \mathrm{H}\right.$ residual $\mathrm{CHCl}_{3}$ singlet $\delta 7.26,{ }^{13} \mathrm{C} \mathrm{CDCl}_{3}$ triplet $\left.\delta 77.23\right)$ or $d_{6}$-DMSO ( ${ }^{1} \mathrm{H}$ residual $d_{5}$-DMSO pentet $\delta 2.49,{ }^{13} \mathrm{C} d_{6}$-DMSO septet $\left.\delta 39.7\right)$ and coupling constants were taken directly from the spectra. NMR assignments were made with the aid of ${ }^{1} \mathrm{H}-$ ${ }^{1} \mathrm{H}$ COSY, HSQC, DEPT-135 and nOe difference proton NMR spectroscopy. 2D experiments were performed using standard software. High-resolution mass spectra (HRMS) were carried out using a double focusing magnetic sector instrument. Samples of X-ray quality crystals were suspended in mineral oil at ambient temperature and a suitable crystal was selected, mounted on a MiTeGen Micromount and transferred to a Bruker AXS SMART APEXII CCD X-ray diffractometer. The X-ray diffraction data were collected at $100(2) \mathrm{K}$ using Mo-K $\alpha(\lambda=0.71073$ Å) radiation.

Ethyl 3,4-diethyl-1-methylpyrrole-2-carboxylate (79). 3-Acetoxy-4-nitrohexane (52.5 g, $0.278 \mathrm{~mol})$ and ethyl isocyanoacetate (26.0 g, $0.230 \mathrm{~mol})$ were mixed with THF (165 mL) and 2propanol $(67 \mathrm{~mL})$ in a 3-neck round bottom flask fitted with a thermometer, addition funnel and drying tube. DBU (86.0 g, 0.565 mol) was added dropwise, maintaining the temperature between 20-30 ${ }^{\circ} \mathrm{C}$ with the aid of an salt-ice bath. After the addition was complete, the orange solution was stirred at room temperature overnight. The solvent was removed under reduced pressure and the residue poured into water $(225 \mathrm{~mL})$. Diethyl ether $(225 \mathrm{~mL})$ was added and the aqueous layer drawn off and extracted twice with $225 \mathrm{~mL}$ of diethyl ether. The combined ether layers were 
washed with $10 \%$ aqueous $\mathrm{HCl}(2 \times 300 \mathrm{~mL})$, dried over magnesium sulfate and the solvent removed on a rotary evaporator. The residue was vacuum distilled to yield ethyl 3,4diethylpyrrole-2-carboxylate (17a) ${ }^{43}$ (31.6 g, $0.162 \mathrm{~mol}, 70 \%$ ) as a pale yellow oil, bp 102-105 ${ }^{\circ} \mathrm{C}$ at 0.6 torr. ${ }^{1} \mathrm{H}$ NMR $\left(400 \mathrm{MHz}, \mathrm{CDCl}_{3}\right): \delta 1.16\left(3 \mathrm{H}, \mathrm{t},{ }^{3} \mathrm{~J}_{\mathrm{HH}}=7.5 \mathrm{~Hz}\right), 1.20\left(3 \mathrm{H}, \mathrm{t},{ }^{3} J_{\mathrm{HH}}=7.5 \mathrm{~Hz}\right)$ (3,4- $\left.\mathrm{CH}_{2} \mathrm{CH}_{3}\right), 1.36\left(3 \mathrm{H}, \mathrm{t},{ }^{3} J_{\mathrm{HH}}=7.2 \mathrm{~Hz}, \mathrm{OCH}_{2} \mathrm{CH}_{3}\right), 2.46\left(2 \mathrm{H}, \mathrm{q},{ }^{3} J_{\mathrm{HH}}=7.5 \mathrm{~Hz}, 4-\mathrm{CH}_{2} \mathrm{CH}_{3}\right)$, $2.77\left(2 \mathrm{H}, \mathrm{q},{ }^{3} J_{\mathrm{HH}}=7.5 \mathrm{~Hz}, 3-\mathrm{CH}_{2} \mathrm{CH}_{3}\right), 4.33\left(2 \mathrm{H}, \mathrm{q},{ }^{3} J_{\mathrm{HH}}=7.2 \mathrm{~Hz}, \mathrm{OCH}_{2}\right), 6.68\left(1 \mathrm{H}, \mathrm{d},{ }^{3} J_{\mathrm{HH}}=\right.$ $2.9 \mathrm{~Hz}, 5-\mathrm{H}), 9.00(1 \mathrm{H}, \mathrm{br} \mathrm{s}, \mathrm{NH})$. The proton NMR spectrum shows the presence of ca. $3 \%$ of the corresponding isopropyl ester due to transesterification with 2-propanol.

Potassium hydroxide pellets (3.84 g) were heated with dimethylsulfoxide $(15 \mathrm{~mL})$ until a yellow colored solution was obtained. The solution was cooled to room temperature and the foregoing pyrrole ester 17a (3.80 g, $19.5 \mathrm{mmol})$ was added. After the mixture had been stirred for $1 \mathrm{~h}$, methyl iodide (5.2 g) was added dropwise, keeping the temperature below $30{ }^{\circ} \mathrm{C}$ with the aid of a salt-ice bath. After the addition was completed, the solution was stirred for an additional $1 \mathrm{~h}$, keeping the temperature below $30{ }^{\circ} \mathrm{C}$. The solution was poured in ice/water $(200 \mathrm{~mL})$, and the aqueous layer was extracted with dichloromethane (2 x $200 \mathrm{~mL})$. The combined dichloromethane layers were washed with water ( 5 x $100 \mathrm{~mL})$, dried over magnesium sulfate and the solvent removed under reduced pressure. The residue was vacuum distilled to yield $N$-methylpyrrole ester 17b (3.20 g, $15.3 \mathrm{mmol}, 79 \%$ ) as a pale yellow oil, bp $98-100^{\circ} \mathrm{C}$ at 1 torr. ${ }^{1} \mathrm{H}$ NMR (500 MHz, $\left.\mathrm{CDCl}_{3}\right): \delta 1.12\left(3 \mathrm{H}, \mathrm{t},{ }^{3} \mathrm{~J}_{\mathrm{HH}}=7.6 \mathrm{~Hz}, 3-\mathrm{CH}_{2} \mathrm{CH}_{3}\right), 1.14\left(3 \mathrm{H}, \mathrm{t},{ }^{3}{ }_{\mathrm{HH}}=7.6 \mathrm{~Hz}, 4-\mathrm{CH}_{2} \mathrm{CH}_{3}\right), 1.30$ $\left(3 \mathrm{H}, \mathrm{t},{ }^{3} J_{\mathrm{HH}}=7.2 \mathrm{~Hz}, \mathrm{OCH}_{2} \mathrm{CH}_{3}\right), 2.37\left(2 \mathrm{H}, \mathrm{q},{ }^{3} \mathrm{~J}_{\mathrm{HH}}=7.6 \mathrm{~Hz}, 4-\mathrm{CH}_{2} \mathrm{CH}_{3}\right), 2.72\left(2 \mathrm{H}, \mathrm{q},{ }^{3} J_{\mathrm{HH}}=7.6\right.$ $\left.\mathrm{Hz}, 3-\mathrm{CH}_{2} \mathrm{CH}_{3}\right), 3.75$ (3H, s, N-Me), $4.24\left(2 \mathrm{H}, \mathrm{q},{ }^{3} \mathrm{JHH}_{\mathrm{HH}}=7.2 \mathrm{~Hz}, \mathrm{OCH}_{2}\right), 6.43(1 \mathrm{H}, \mathrm{s}, 5-\mathrm{H}) .{ }^{13} \mathrm{C}$ NMR (125 MHz, $\left.\mathrm{CDCl}_{3}\right): \delta 13.8\left(\mathrm{OCH}_{2} \mathrm{CH}_{3}\right), 14.5\left(4-\mathrm{CH}_{2} \mathrm{CH}_{3}\right), 15.2\left(3-\mathrm{CH}_{2} \mathrm{CH}_{3}\right), 17.4\left(4-\mathrm{CH}_{2}\right)$, 
18.5 (3- $\left.\mathrm{CH}_{2}\right), 36.5$ (N-Me), $58.7\left(\mathrm{OCH}_{2}\right)$, 118.6, 123.5, 125.8 (5-CH), 133.4, $161.2(\mathrm{C}=\mathrm{O})$. HRMS (EI) m/z: Calcd for $\mathrm{C}_{12} \mathrm{H}_{19} \mathrm{NO}_{2}$ 209.1416; found 209.1419.

3,4-Diethyl-1-methylpyrrole (53b). Pyrrole ester 79 (2.82 g, 13.5 mmol) was added to sodium hydroxide $(1.07 \mathrm{~g})$ in ethylene glycol $(11 \mathrm{~mL})$, and the mixture heated for $2.5 \mathrm{~h}$ at $200{ }^{\circ} \mathrm{C}$. After cooling the solution to room temperature, the mixture was partitioned between water (50 $\mathrm{mL})$ and hexanes $(50 \mathrm{~mL})$. The aqueous layer was drawn off and further extracted with hexanes (3 x $50 \mathrm{~mL}$ ). The combined hexanes layers were dried over magnesium sulfate and the solvent removed under reduced pressure to yield 3,4-diethyl-1-methylpyrrole (1.55 g, $11.3 \mathrm{mmol}, 84 \%$ ) as a pale yellow oil, b.p $78-80^{\circ} \mathrm{C}$ at 18 torr. ${ }^{1} \mathrm{H}$ NMR (500 MHz, $\left.\mathrm{CDCl} 3\right): \delta 1.46\left(6 \mathrm{H}, \mathrm{t},{ }^{3} \mathrm{~J}_{\mathrm{HH}}=7.6\right.$ $\mathrm{Hz}, 2 \times \mathrm{CH}_{2} \mathrm{CH}_{3}$ ), $2.71\left(4 \mathrm{H}, \mathrm{q},{ }^{3} \mathrm{~J}_{\mathrm{HH}}=7.6 \mathrm{~Hz}, 2 \times \mathrm{CH}_{2} \mathrm{CH}_{3}\right.$ ), 3.80 (3H, s, N-Me), 6.58 (2H, s, 2,5H). ${ }^{13} \mathrm{C}$ NMR (125 MHz, $\left.\mathrm{CDCl}_{3}\right): \delta 14.6\left(2 \times \mathrm{CH}_{2} \mathrm{CH}_{3}\right), 18.3\left(2 \times \mathrm{CH}_{2} \mathrm{CH}_{3}\right), 35.1(\mathrm{~N}-\mathrm{Me}), 118.2$ (2,5-CH), 124.1 (3,4-C). HR-MS (EI) m/z: Calcd for $\mathrm{C}_{9} \mathrm{H}_{15} \mathrm{~N}$ 137.1204; found 137.1204. 2,5-Bis(5-benzyloxycarbonyl-3-ethyl-4-methyl-2-pyrrolylmethyl)-3,4-diethyl-1methylpyrrole (54c). Benzyl 5-acetoxymethyl-4-ethyl-3-methylpyrrole-2-carboxylate (1.28 g, $4.10 \mathrm{mmol})$ and 3,4-diethyl-1-methylpyrrole (0.279 g, $2.04 \mathrm{mmol})$ were dissolved in acetonitrile $(15 \mathrm{~mL})$ and acetic acid $(1 \mathrm{~mL})$. The mixture was refluxed overnight under nitrogen. The solution was then cooled to room temperature, diluted with dichloromethane (50 mL), and then washed with water $(100 \mathrm{~mL})$ and aqueous $10 \%$ sodium bicarbonate solution $(100 \mathrm{~mL})$. The dichloromethane layer was dried over sodium sulfate and the solvent removed under reduced pressure. The residue was purified by column chromatography on silica gel, eluting with 80:20:1 dichloromethane-hexanes-triethylamine, and afforded the $N$-methyl tripyrrane $(0.624 \mathrm{~g}, 0.964$ mmol, 48\%) as an orange oil that slowly solidified when stored in the freezer. ${ }^{1} \mathrm{H}$ NMR (500 MHz, $\left.\mathrm{CDCl}_{3}\right): \delta 1.05\left(6 \mathrm{H}, \mathrm{t},{ }^{3} \mathrm{~J}_{\mathrm{HH}}=7.6 \mathrm{~Hz}, 3{ }^{\prime}, 3{ }^{\prime}{ }^{-}-\mathrm{CH}_{2} \mathrm{CH}_{3}\right), 1.09\left(6 \mathrm{H}, \mathrm{t},{ }^{3} \mathrm{~J}_{\mathrm{HH}}=7.6 \mathrm{~Hz}, 3,4-\mathrm{CH}_{2} \mathrm{CH}_{3}\right)$, 
2.27 (6H, s, 4',4'”- $\mathrm{CH}_{3}$ ), 2.39-2.45 (8H, m, 4 x $\mathrm{CH}_{2} \mathrm{CH}_{3}$ ), 2.98 (3H, s, N-Me), 3.81 (4H, s, 2 x bridge- $\left.\mathrm{CH}_{2}\right)$, $5.24\left(4 \mathrm{H}, \mathrm{s}, 2 \times \mathrm{OCH}_{2}\right), 7.28-7.39(10 \mathrm{H}, \mathrm{m}, 2 \mathrm{xPh}), 8.23(2 \mathrm{H}, \mathrm{br} \mathrm{s}, 2 \times \mathrm{NH}) .{ }^{13} \mathrm{C}$ NMR (125 MHz, CDCl $)$ ): $\delta 10.7$ (4',4'’-Me), 15.4 (3',3'”- $\left.\mathrm{CH}_{2} \mathrm{CH}_{3}\right), 17.24$ (3,4- $\left.\mathrm{CH}_{2} \mathrm{CH}_{3}\right), 17.28$, 18.0 (4 x $\mathrm{CH}_{2} \mathrm{CH}_{3}$ ), 21.9 (2 x bridge- $\mathrm{CH}_{2}$ ), 30.4 (N-Me), 65.6 (2 x $\left.\mathrm{OCH}_{2}\right), 117.1,122.2,122.9$, 123.4, $128.1(\mathrm{Ph}), 128.7(\mathrm{Ph}), 131.1,136.9,161.6\left(2\right.$ x C=O). HR-MS (ESI) m/z: [M + H] ${ }^{+}$Calcd for $\mathrm{C}_{41} \mathrm{H}_{50} \mathrm{~N}_{3} \mathrm{O}_{4}$ 648.3801; found 648.3792.

$N$-methyl tripyrrane dicarboxylic acid 54d. $N$-methyl tripyrrane 54c (0.703 g, $1.09 \mathrm{mmol})$ was placed in a hydrogenation vessel and dissolved in acetone $(100 \mathrm{~mL})$, methanol (34 mL) and triethylamine (14 drops). The mixture was purged with nitrogen, 10\% palladium-charcoal (67 mg) was added and the solution shaken under an atmosphere of hydrogen at $40 \mathrm{psi}$ at room temperature overnight. The catalyst was removed by suction filtration and the solvent removed on a rotary evaporatory while maintaining the bath temperature below $30{ }^{\circ} \mathrm{C}$. The residue was dissolved in $5 \%$ aqueous ammonium and diluted to a final volume of $50 \mathrm{~mL}$ with water. The resulting solution was cooled to $0-5{ }^{\circ} \mathrm{C}$, and then acidified by adding acetic acid dropwise while maintaining the temperature below $5{ }^{\circ} \mathrm{C}$. The mixture was allowed to sit in a salt-ice bath for $30 \mathrm{~min}$ and the precipitate was then collected by suction filtration and washed multiple times with water to remove all traces of acid. The solid was dried overnight in a vacuum desiccator to yield the dicarboxylic acid (0.419 g, $0.897 \mathrm{mmol}, 83 \%)$ as an unstable pale purple powder that was used without further purification. ${ }^{1} \mathrm{H}$ NMR (500 MHz, $d_{6}$-DMSO): $\delta 0.73\left(6 \mathrm{H}, \mathrm{t},{ }^{3} J_{\mathrm{HH}}=7.5 \mathrm{~Hz}\right), 0.97\left(6 \mathrm{H}, \mathrm{t},{ }^{3} \mathrm{~J}_{\mathrm{HH}}=7.5\right.$ $\mathrm{Hz})\left(4 \times \mathrm{CH}_{2} \mathrm{CH}_{3}\right), 2.12\left(6 \mathrm{H}, \mathrm{s}, 4^{\prime}, 4^{\prime}{ }^{\prime}-\mathrm{CH}_{3}\right), 2.11\left(4 \mathrm{H}, \mathrm{q},{ }^{3} J_{\mathrm{HH}}=7.5 \mathrm{~Hz}\right), 2.35\left(4 \mathrm{H}, \mathrm{q},{ }^{3} J_{\mathrm{HH}}=7.5\right.$ $\mathrm{Hz}$ ) (4 x $\left.\mathrm{CH}_{2} \mathrm{CH}_{3}\right), 2.99$ (3H, s, N-Me), 3.81 (4H, s, 2 x bridge- $\left.\mathrm{CH}_{2}\right), 10.14$ (2H, br s, 2 x NH), 11.53 (2H, v br, 2 x CO $2 \mathrm{H}) .{ }^{13} \mathrm{C}$ NMR (125 MHz, $\left.\mathrm{CDCl}_{3}\right): \delta 9.9$ (4',4'’-Me), 14.8, 16.3, 16.5, 17.2, 
21.6 (2 x bridge- $\left.\mathrm{CH}_{2}\right), 30.1$ (N-Me), 116.5, 119.6, 122.6, 123.1, 125.5, 130.4, 162.1 (2 x C=O). HR-MS (ESI) m/z: Calcd for $\mathrm{C}_{27} \mathrm{H}_{37} \mathrm{~N}_{3} \mathrm{O}_{4} 490.2682$; found 490.2672 .

8,12,13,17-Tetraethyl-7,18,23-trimethylbenzo[b]carbaporphyrin (76). $N$-methyl tripyrrane dicarboxylic acid 54d (0.144 g, $0.308 \mathrm{mmol})$ was dissolved in TFA $(1.5 \mathrm{~mL})$ and stirred under nitrogen for $2 \mathrm{~min}$. Dichloromethane $(27 \mathrm{~mL})$ was added, followed immediately by indene dialdehyde (54.0 mg, $0.314 \mathrm{mmol}$ ), and the solution stirred under nitrogen for $2 \mathrm{~h}$. The mixture was neutralized by the dropwise addition of triethylamine, DDQ (72.0 mg, $0.317 \mathrm{mmol})$ was added, and the mixture stirred for a further $1 \mathrm{~h}$. The solution was washed with water $(100 \mathrm{~mL})$ and then sodium bicarbonate $(100 \mathrm{~mL})$, and the solvent removed under reduced pressure. The residue was chromatographed twice on grade 3 neutral alumina eluting with dichloromethane and then chloroform, and a dark brown band was collected. The product was recrystallized from chloroform-methanol to yield the 23-methylbenzocarbaporphyrin (52.5 mg, $0.102 \mathrm{mmol}$, 32\%) as dark purple-blue crystals, mp $>300{ }^{\circ} \mathrm{C}$. UV-vis $\left(1 \% \mathrm{Et}_{3} \mathrm{~N}-\mathrm{CH}_{2} \mathrm{Cl}_{2}\right): \lambda_{\max } / \mathrm{nm}(\log \varepsilon) 306$ (4.42), 379 (4.56), 439 (5.10), 538 (4.15), 567 (3.91), 624 (3.86), 683 (3.36). UV-vis (1\% TFA- $\mathrm{CH}_{2} \mathrm{Cl}_{2}$ ): $\lambda_{\max } / \mathrm{nm}(\log \varepsilon) 310$ (4.42), 403 (sh, 4.77), 444 (4.97), 481 (4.47), 568 (4.01), 608 (4.08), 677 (3.26). ${ }^{1} \mathrm{H}$ NMR ( $\left.500 \mathrm{MHz}, \mathrm{CDCl}_{3}\right): \delta-4.95(1 \mathrm{H}, \mathrm{s}, 21-\mathrm{H}),-4.25$ (3H, s, N-Me), -0.63 (1H, v br, $\mathrm{NH}), 1.47\left(6 \mathrm{H}, \mathrm{t},{ }^{3} J_{\mathrm{HH}}=7.7 \mathrm{~Hz}, 12,13-\mathrm{CH}_{2} \mathrm{CH}_{3}\right), 1.83\left(6 \mathrm{H}, \mathrm{t},{ }^{3} \mathrm{~J}_{\mathrm{HH}}=7.7 \mathrm{~Hz}, 8,17-\mathrm{CH}_{2} \mathrm{CH}_{3}\right), 3.51$ (6H, s, 7,18-Me), 3.67-3.75 (2H, m), 3.76-3.84 (2H, m) (12,13- $\left.\mathrm{CH}_{2}\right)$, 3.88-4.02 (4H, m, 8,17- $\left.\mathrm{CH}_{2}\right)$, 7.67-7.71 (2H, m, $\left.2^{2}, 3^{2}-\mathrm{H}\right), 8.79-8.83\left(2 \mathrm{H}, \mathrm{m}, 2^{1}, 3^{1}-\mathrm{H}\right), 9.67$ (2H, s, 10,15-H), 10.03 (2H, s, 5,20H). ${ }^{1} \mathrm{H}$ NMR (500 MHz, TFA-CDCl $): ~ \delta-6.53(1 \mathrm{H}, \mathrm{br} \mathrm{s}, 21-\mathrm{H}),-4.79(2 \mathrm{H}, \mathrm{s}, 2 \mathrm{x} \mathrm{NH}),-4.51(3 \mathrm{H}$, s, N-Me), $1.50\left(6 \mathrm{H}, \mathrm{t},{ }^{3} \mathrm{~J}_{\mathrm{HH}}=7.7 \mathrm{~Hz}, 12,13-\mathrm{CH}_{2} \mathrm{CH}_{3}\right), 1.77\left(6 \mathrm{H}, \mathrm{t},{ }^{3} J_{\mathrm{HH}}=7.7 \mathrm{~Hz}, 8,17-\mathrm{CH}_{2} \mathrm{CH}_{3}\right)$, 3.60 (6H, s, 7,18-Me), 3.69-3.80 (4H, m, 12,13- $\left.\mathrm{CH}_{2}\right), 4.05$ (4H, q, $\left.{ }^{3} J_{\mathrm{HH}}=7.7 \mathrm{~Hz}, 8,17-\mathrm{CH}_{2}\right), 7.70-$ 7.80 (2H, br, $\left.2^{2}, 3^{2}-\mathrm{H}\right), 8.64-8.72\left(2 \mathrm{H}, \mathrm{m}, 2^{1}, 3^{1}-\mathrm{H}\right), 10.00(2 \mathrm{H}, \mathrm{s}, 10,15-\mathrm{H}), 10.26$ (2H, s, 5,20-H). 
${ }^{13} \mathrm{C}$ NMR (125 MHz, CDCl $)$ : $\delta 11.5$ (7,18-Me), 16.9 (12,13- $\left.\mathrm{CH}_{2} \mathrm{CH}_{3}\right), 17.4\left(8,17-\mathrm{CH}_{2} \mathrm{CH}_{3}\right), 20.0$ (8,17- $\left.\mathrm{CH}_{2}\right), 20.1$ (12,13- $\left.\mathrm{CH}_{2}\right), 31.4$ (N-Me), 96.4 (10,15-CH), 103.4 (5,20-CH), 118.8 (21-CH), $120.4\left(2^{1}, 3^{1}-\mathrm{CH}\right), 126.5\left(2^{2}, 3^{2}-\mathrm{CH}\right), 134.2,135.2,136.3,140.7,144.0,147.6 .{ }^{13} \mathrm{C}$ NMR $(125 \mathrm{MHz}$, TFA-CDCl $): \delta 11.7$ (7,18-Me), $16.5\left(12,13-\mathrm{CH}_{2} \mathrm{CH}_{3}\right), 16.9\left(8,17-\mathrm{CH}_{2} \mathrm{CH}_{3}\right), 20.13,20.15(4 \mathrm{x}$ $\left.\mathrm{CH}_{2} \mathrm{CH}_{3}\right), 30.7$ (N-Me), 95.8 (10,15-CH), 103.6 (5,20-CH), 116.5 (21-CH), 121.9 (2 $\left.{ }^{1}, 3^{1}-\mathrm{CH}\right)$, $128.7\left(2^{2}, 3^{2}-\mathrm{CH}\right), 136.2,136.8,138.8,138.9,140.6,141.5,141.7,150.6$. HR-MS (ESI) m/z: [M + $\mathrm{H}]^{+}$Calcd for $\mathrm{C}_{36} \mathrm{H}_{40} \mathrm{~N}_{3}$ 514.3222; found 514.3198.

[8,12,13,17-Tetraethyl-7,18,23-trimethylbenzo[b]carbaporphyrinato]palladium(II)

(84). Palladium(II) acetate (10.0 mg, $0.0450 \mathrm{mmol})$ was added to a solution of 23methylbenzocarbaporphyrin 76 (10.0 mg, $0.0195 \mathrm{mmol})$ in acetonitrile $(10 \mathrm{~mL})$ and heated under reflux in a pre-heated oil bath for $5 \mathrm{~min}$. The solution was diluted with chloroform (30 mL) and washed with water $(50 \mathrm{~mL})$. The aqueous solution was back extracted with chloroform and the combined organic layers were evaporated under reduced pressure. The residue was purified by column chromatography on silica gel eluting with dichloromethane-hexanes (70:30) and a dark green band was collected. The solvent was removed on a rotary evaporator and the residue recrystallized from chloroform-methanol to give the palladium complex $(8.0 \mathrm{mg}, 0.013 \mathrm{mmol}$, 67\%) as dark green crystals, $\mathrm{mp}>300{ }^{\circ} \mathrm{C}$. UV-vis $\left(\mathrm{CH}_{2} \mathrm{Cl}_{2}\right): \lambda_{\max } / \mathrm{nm}(\log \varepsilon) 309$ (4.50), 397 (4.61), 404 (sh, 4.60), 470 (sh, 4.52), 496 (4.76), 518 (4.36), 561 (3.63), 613 (3.93), 635 (sh, 3.90), 667 (3.84). ${ }^{1} \mathrm{H}$ NMR (500 MHz, $\left.\mathrm{CDCl}_{3}\right): \delta-2.86(3 \mathrm{H}, \mathrm{s}, \mathrm{N}-\mathrm{Me}), 1.67$ (6H, t, ${ }^{3} \mathrm{~J}_{\mathrm{HH}}=7.7 \mathrm{~Hz}, 12,13-$ $\left.\mathrm{CH}_{2} \mathrm{CH}_{3}\right), 1.81\left(6 \mathrm{H}, \mathrm{t},{ }^{3} \mathrm{JHH}_{\mathrm{HH}}=7.7 \mathrm{~Hz}, 8,17-\mathrm{CH}_{2} \mathrm{CH}_{3}\right), 3.48$ (6H, s, 7,18-Me), 3.84-4.00 (8H, m, 4 x $\left.\mathrm{CH}_{2} \mathrm{CH}_{3}\right), 7.45-7.49$ (2H, m, $\left.2^{2}, 3^{2}-\mathrm{H}\right), 8.48-8.52\left(2 \mathrm{H}, \mathrm{m}, 2^{1}, 3^{1}-\mathrm{H}\right), 9.85$ (2H, s, 10,15-H), 10.00 (2H, s, 5,20-H). ${ }^{13} \mathrm{C}$ NMR (125 MHz, $\left.\mathrm{CDCl}_{3}\right): \delta 11.7$ (7,18-Me), $16.5\left(12,13-\mathrm{CH}_{2} \mathrm{CH}_{3}\right), 17.6(8,17-$ $\mathrm{CH}_{2} \mathrm{CH}_{3}$ ), 20.16, 20.21 (4 x $\left.\mathrm{CH}_{2} \mathrm{CH}_{3}\right), 45.3$ (N-Me), 98.9 (10,15-CH), 109.6 (5,20-CH), 118.8 
$\left(2^{1}, 3^{1}-\mathrm{CH}\right), 126.0\left(2^{2}, 3^{2}-\mathrm{CH}\right), 133.8,135.1,136.3,138.9,140.7,142.7,145.6,149.8$. HR-MS (ESI) m/z: Calcd for $\mathrm{C}_{36} \mathrm{H}_{37} \mathrm{~N}_{3} \mathrm{Pd}$ 617.2022; found 617.2044.

[8,12,13,17-Tetraethyl-7,18,21-trimethylbenzo[b]carbaporphyrinato]palladium(II)

Palladium(II) acetate (10 mg, $0.0450 \mathrm{mmol})$ was added to a solution of 23methylbenzocarbaporphyrin 76 (10 mg, $0.0195 \mathrm{mmol})$ in acetonitrile $(10 \mathrm{~mL})$ and the mixture refluxed overnight. The solvent was removed under reduced pressure and the residue chromatographed on a silica gel column eluting with dichloromethane-hexanes (70:30). A brown band was collected and the solvent removed under reduced pressure to give 72 (10.2 mg, 0.0165 mmol, 85\%) as a dark brown solid, mp $>300{ }^{\circ} \mathrm{C} .{ }^{1} \mathrm{H}$ NMR (500 MHz, $\left.\mathrm{CDCl}_{3}\right): \delta$-3.21 (3H, s, 21$\left.\mathrm{CH}_{3}\right), 1.74\left(6 \mathrm{H}, \mathrm{t},{ }^{3} J_{\mathrm{HH}}=7.7 \mathrm{~Hz}\right), 1.75\left(6 \mathrm{H}, \mathrm{t},{ }^{3} J_{\mathrm{HH}}=7.7 \mathrm{~Hz}\right)\left(4 \times \mathrm{CH}_{2} \mathrm{CH}_{3}\right), 3.33(6 \mathrm{H}, \mathrm{s}), 3.71-$ $3.78\left(8 \mathrm{H}, \mathrm{m}, 4 \times \mathrm{CH}_{2} \mathrm{CH}_{3}\right), 8.22-8.25\left(2 \mathrm{H}, \mathrm{m}, 2^{2}, 3^{2}-\mathrm{H}\right), 9.39-9.43\left(2 \mathrm{H}, \mathrm{m}, 2^{1}, 3^{1}-\mathrm{H}\right), 9.56(2 \mathrm{H}, \mathrm{s}$, 10,15-H), 10.27 (2H, s, 5,20-H). The sample had identical spectroscopic properties to a previously prepared sample obtained by reacting $\mathbf{7 0}$ with $\mathrm{Pd}(\mathrm{OAc})_{2}{ }^{101}$ 


\section{CHAPTER III: RHODIUM COMPLEXES OF CARBAPORPHYRINOID SYSTEMS}

\section{Introduction}

Benzocarbaporphyrin 3 typically acts as a trianionic ligand, forming stable organometallic complexes 10a and 10b with silver(III) $)^{27,28}$ and gold(III) ${ }^{28}$, respectively (Scheme 29). However, attempts to react 3 with nickel(II), palladium(II) or platinum(II) salts were unsuccessful. ${ }^{27}$ Azuliporphyrins, which are similar in structure, readily generated nickel(II), palladium(II) and platinum(II) complexes 13a-c ${ }^{32}$ and have also been seen to form stable rhodium(III) derivatives 15a-c and 16 as well as iridium(III) complexes 17a-d (Chart 9). Rhodium porphyrins have been widely investigated for their catalytic properties and can promote C-H and C-C bond activation, ${ }^{1}$ and rhodium(I) and rhodium(III) benzocarbaporphyrins have recently been reported. ${ }^{30}$ Carbaporphyrin 3 reacted with $\left[\mathrm{Rh}(\mathrm{CO})_{2} \mathrm{Cl}\right]_{2}$ in refluxing dichloromethane to afford rhodium(I) complex 86 in 90\% yield (Scheme 29). The rhodium metal coordinates with the 22- and 23nitrogen atoms of the benzocarbaporphyrin and possesses two terminal carbonyl ligands. Subsequent reaction in refluxing pyridine led to the formation of rhodium(III) complex 11a in 50\% yield. This rhodium(III) derivative incorporates two axial pyridine ligands coordinating to the metal center on opposite faces of the carbaporphyrin system. It is of interest to investigate the effect internal alkylation has on the coordination chemistry of benzocarbaporphyrins with rhodium metal. In this chapter, the syntheses and characterization of rhodium carbaporphyrin complexes are discussed. Metalation studies of 21-, 22-, and 23-methylbenzocarbaporphyrins with a rhodium(I) reagent were performed and the results were compared to those of $N$-unsubstituted 
benzocarbaporphyrin 3. Furthermore, a carbaporphyrin diester and naphthocarbaporphyrin also underwent metalation with $\left[\mathrm{Rh}(\mathrm{CO})_{2} \mathrm{Cl}\right]_{2}$ and the results of these reactions were also analyzed.

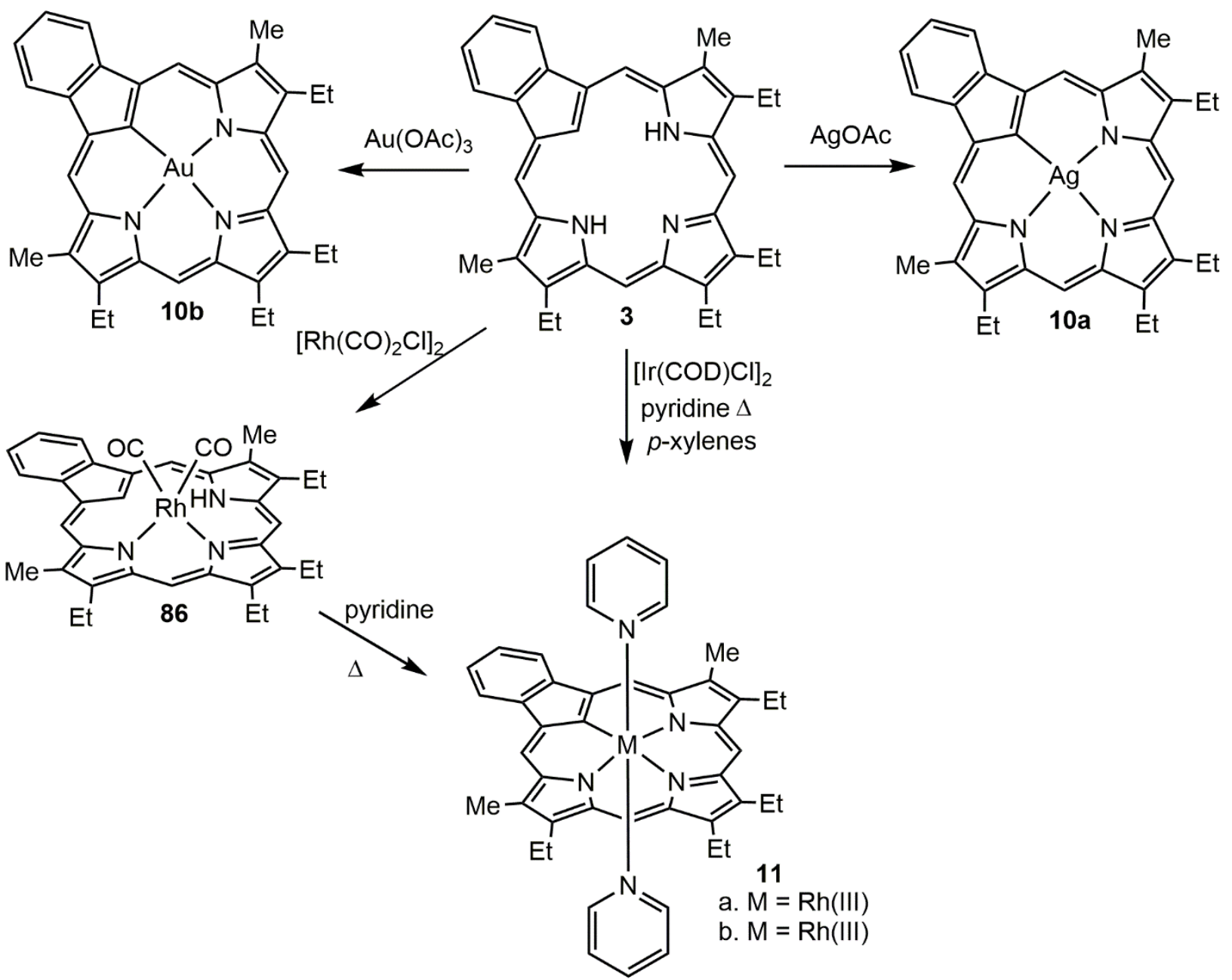

Scheme 29. Metalation of benzocarbaporphyrin 3 to give rhodium(I) and rhodium(III) derivatives 


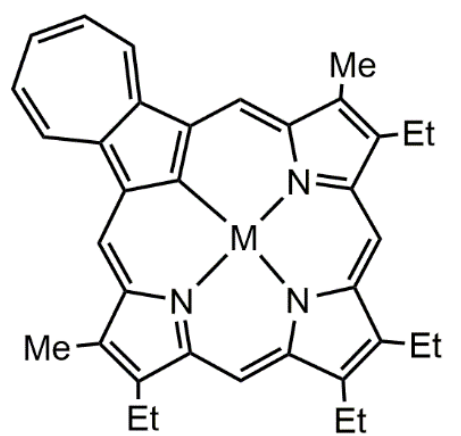

13 a. $\mathrm{M}=\mathrm{Ni}(\mathrm{II})$

b. $M=P d(I I)$

c. $M=P t(I I)$

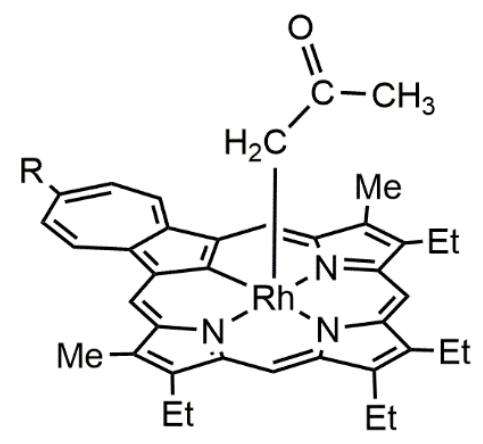

16

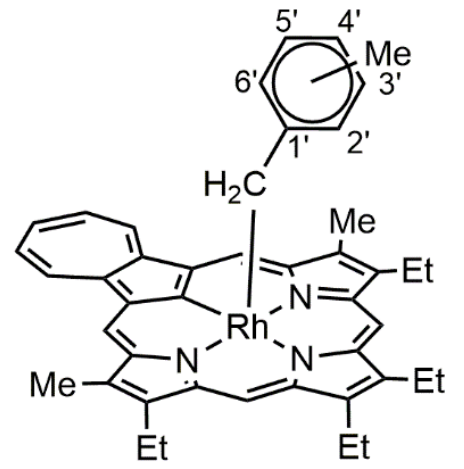

15

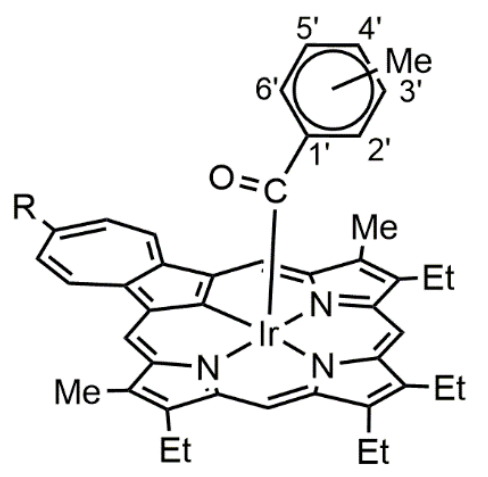

17

$\mathrm{R}=\mathrm{H}$ or $t-\mathrm{Bu}$

a. 2'-Me, R = H; b. 4'-Me, R = H

c. $2^{\prime}-\mathrm{Me}, \mathrm{R}=t-\mathrm{Bu}$; d. 4'-Me, $\mathrm{R}=t-\mathrm{Bu}$

Chart 9. Examples of metalated azuliporphyrins

\section{Results and Discussion}

Recently, syntheses of naphthocarbaporphyrin $\mathbf{8 7 ^ { 1 0 8 }}$ and carbaporphyrin diester $\mathbf{8 8}{ }^{29}$ have been developed. Carbaporphyrin diester 88 was reacted with $\left[\mathrm{Rh}(\mathrm{CO})_{2} \mathrm{Cl}\right]_{2}$ in refluxing dichloromethane in the presence of anhydrous sodium acetate to give the corresponding rhodium(I) complex 89 (Scheme 30). The crude product was chromatographed on grade 3 neutral alumina eluting with chloroform and collected as a dark green band. The fraction was 
recrystallized from chloroform-hexanes to give the pure rhodium(I) carbaporphyrin diester $\mathbf{8 9}$ in up to $99 \%$ yield.
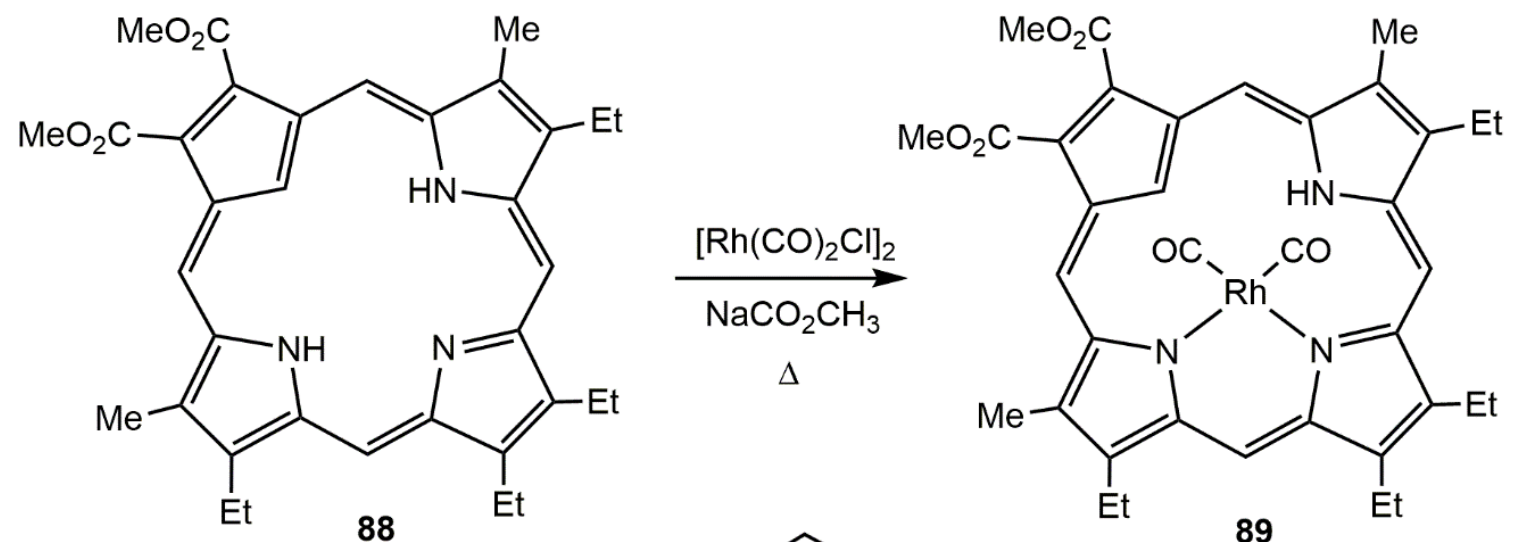

88

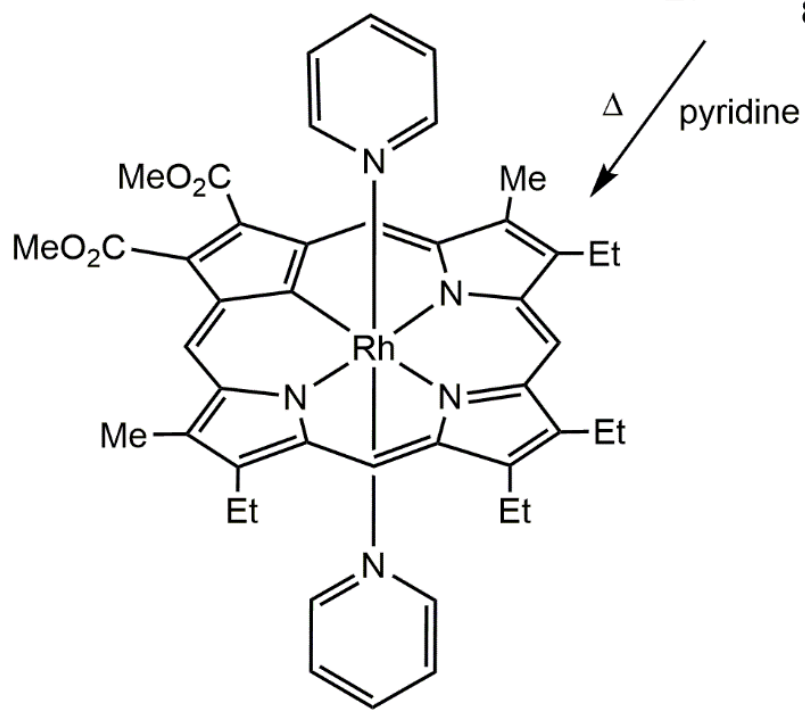

90

Scheme 30. Rhodium(I) and rhodium(III) complexes of carbaporphyrin diester $\mathbf{8 8}$

Carbaporphyrin $\mathbf{8 8}$ is a symmetrical molecule, but this symmetry is lost upon introduction of the rhodium(I) metal center. This can be seen by comparison of the proton NMR spectra of the free base carbaporphyrin diester and the rhodium(I) complex. The free base (88) had two peaks for the meso-protons resonating at 9.51 and $10.24 \mathrm{ppm}$. The internal $\mathrm{CH}$ was observed at -6.27 ppm and the $\mathrm{NH}$ protons gave a broad $2 \mathrm{H}$ singlet at $-3.12 \mathrm{ppm}$. The two external methyl groups 
appeared as a $6 \mathrm{H}$ singlet at $3.51 \mathrm{ppm}$ and the two methyl ester groups gave a $6 \mathrm{H}$ singlet at 4.27 ppm. ${ }^{29}$ These results not only demonstrate that $\mathbf{8 8}$ possesses a plane of symmetry but also confirms the macrocyclic aromaticity for the system. When rhodium(I) is introduced into the center of the cavity, the loss of symmetry can be seen due to the duplication of many of the resonances. For instance, the meso-protons now produce four separate $1 \mathrm{H}$ singlets at 9.40, 9.44, 10.02 and 10.37 ppm. Similarly, the methyl groups afford two $3 \mathrm{H}$ singlets at 3.35 and $3.45 \mathrm{ppm}$, instead of the single peak observed in free base $\mathbf{8 8}$. The internal $\mathrm{CH}$ and $\mathrm{NH}$ peaks for $\mathbf{8 9}$ were observed at $\mathbf{- 4 . 9 4}$ and -1.09 ppm, respectively (Figure 11). These values are shifted upfield compared to the equivalent peaks seen for free base $\mathbf{8 8}$, but still indicate that $\mathbf{8 9}$ retains a high degree of macrocyclic aromaticity. The carbon-13 NMR spectrum for $\mathbf{8 9}$ further supported this lack of symmetry and gave four resonances for the meso-carbons at 92.4, 102.8, 107.5 and $116.7 \mathrm{ppm}$. The carbon atoms of the terminal carbonyl ligands were observed as two doublets at 177.0 and $178.7 \mathrm{ppm}\left({ }^{1} J_{\mathrm{Rh}-\mathrm{C}}=\right.$ 69.9 and $67.7 \mathrm{~Hz}$, respectively) (Figure 12). The splitting of these peaks is due to the presence of the NMR active nucleus rhodium-103 $(I=1 / 2)$. The IR spectrum for $\mathbf{8 9}$ showed two strong peaks at 2066 and $2011 \mathrm{~cm}^{-1}$, which correspond to the two terminal carbonyl ligands of the rhodium(I) metal center. An additional absorption at $1698 \mathrm{~cm}^{-1}$ was observed due to the methyl ester carbonyl groups (Figure 13). The UV-Vis spectrum of $\mathbf{8 9}$ gave two relatively weak Soret-like bands at 357 and $490 \mathrm{~nm}$ with smaller Q bands at 569 and $611 \mathrm{~nm}$ (Figure 14). 


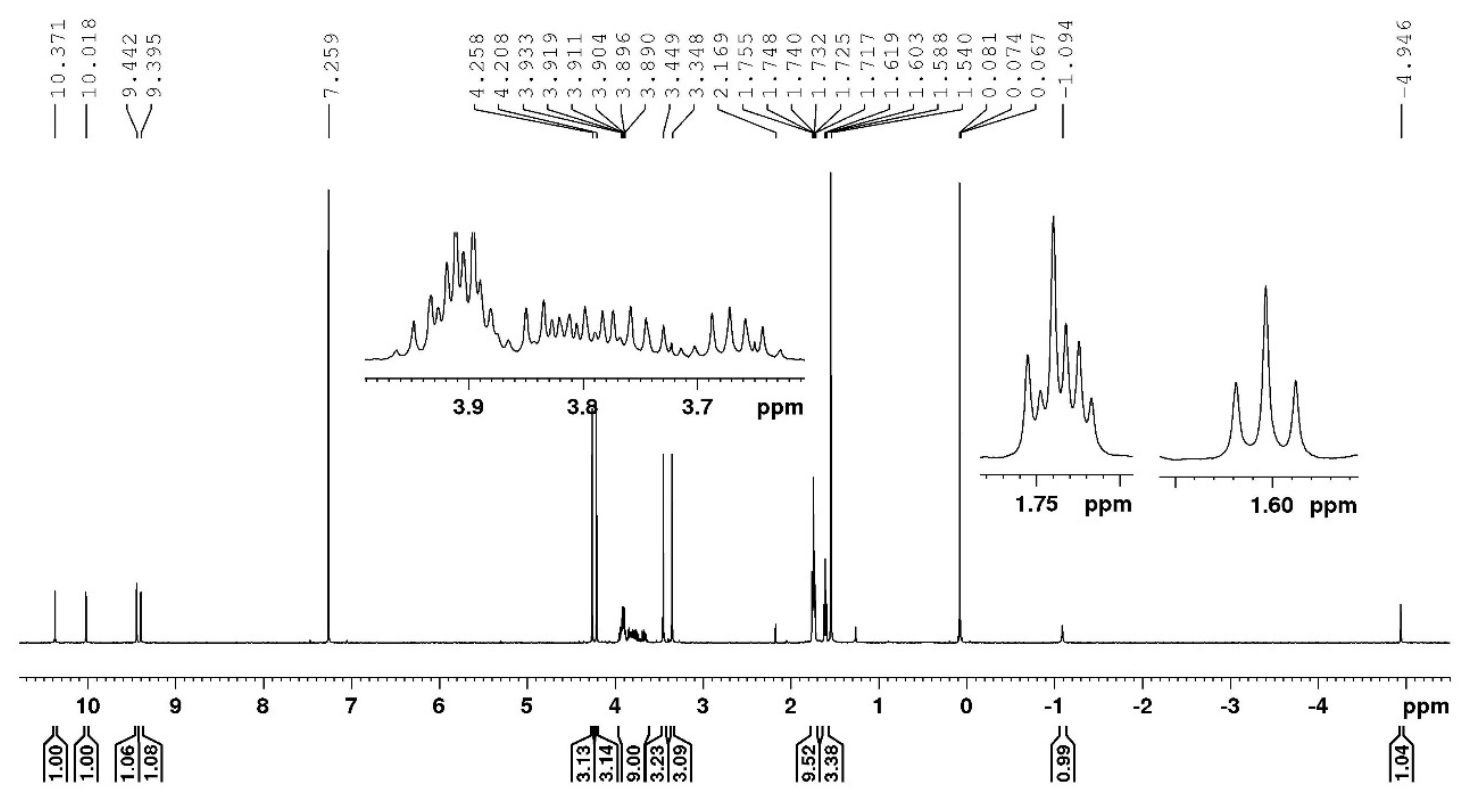

Figure 11. $500 \mathrm{MHz}{ }^{1} \mathrm{H}$ NMR spectrum of rhodium(I) carbaporphyrin diester 89 in $\mathrm{CDCl}_{3}$

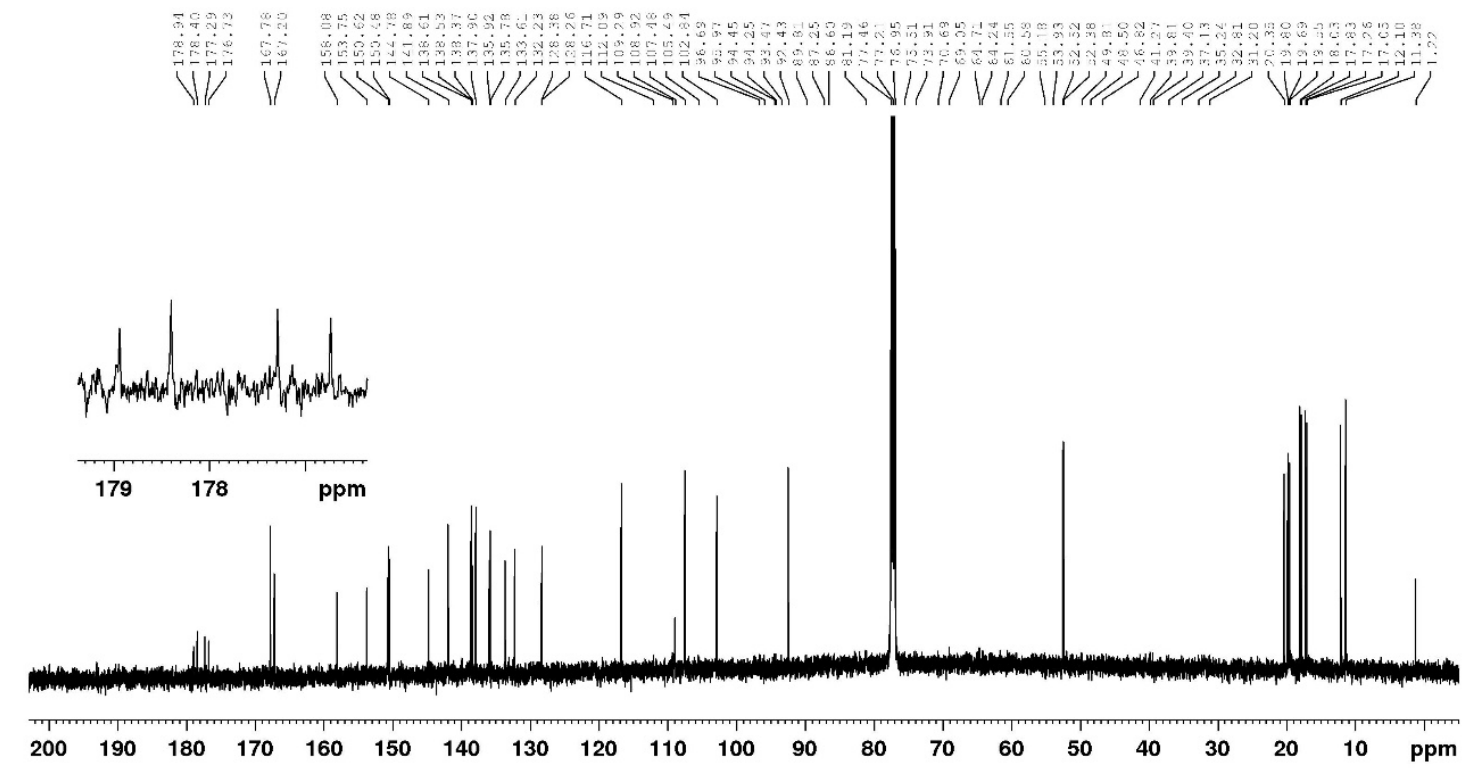

Figure 12. $125 \mathrm{MHz}{ }^{13} \mathrm{C}$ NMR spectrum of rhodium(I) carbaporphyrin diester 89 in $\mathrm{CDCl}_{3}$ 


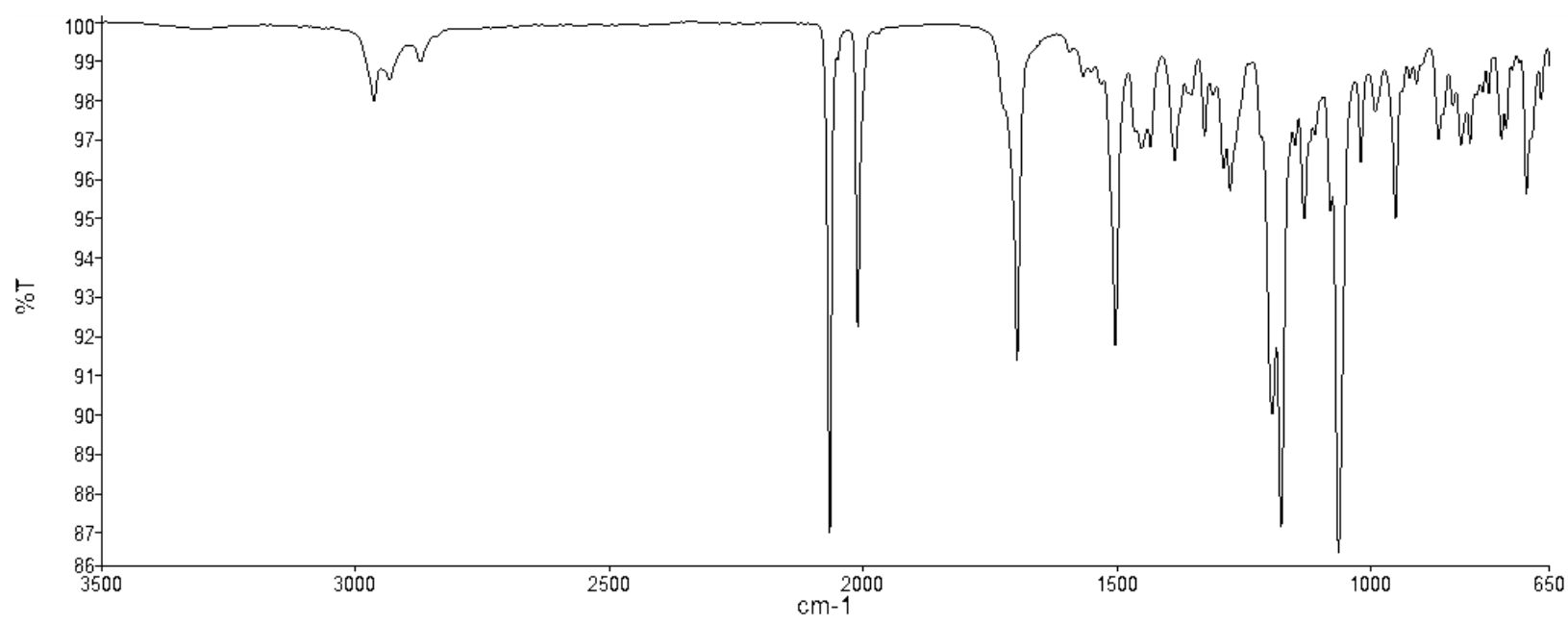

Figure 13. IR spectrum of rhodium(I) carbaporphyrin diester $\mathbf{8 9}$

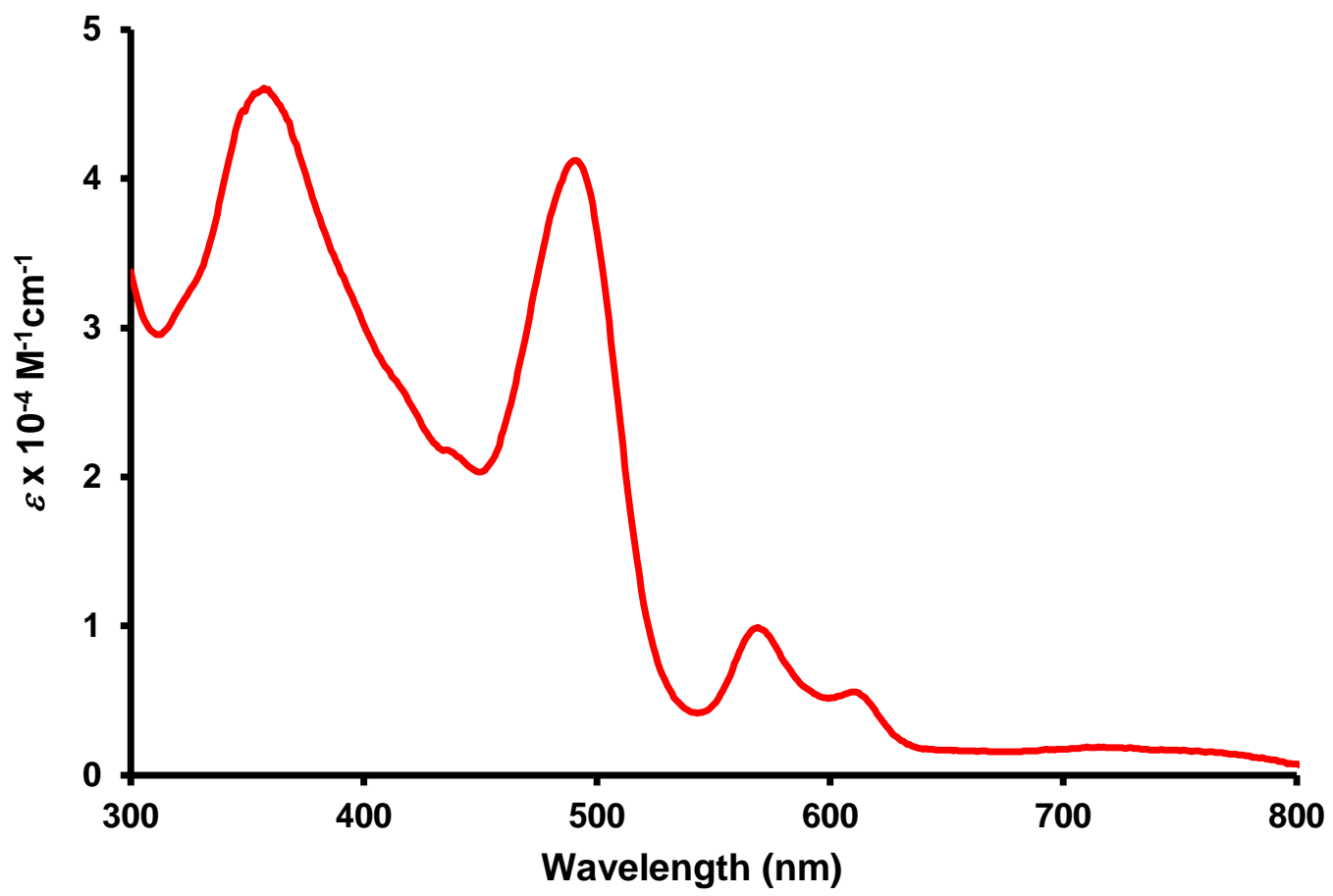

Figure 14. UV-Vis spectrum of rhodium(I) carbaporphyrin diester $\mathbf{8 9}$

The structure for this complex was also confirmed by X-ray crystallography (Figure 15). The framework of bond lengths shows that this carbaporphyrin is aromatic with a delocalized $\pi$ electron pathway passing through the C21 atom. This is evidenced by the longer bond lengths of 
1.462(3) $\AA$ and 1.471(3) $\AA$ for the C1-C2 and C3-C4 bonds, respectively. The bond lengths for C1-C21 and C4-C21 are found to be comparatively shorter, showing lengths of 1.407(3) $\AA$ and 1.401(3) $\AA$, respectively, which demonstrates that the $\pi$-electron pathway is passing through these bonds. The bonds directly connected to the meso-carbons, C4-C5, C5-C6, C9-C10, C10-C11, C14C15, C15-C16, C19-C20 and C20-C1, varied from 1.384-1.410 $\AA$ in reasonable agreement with expected aromatic bond lengths. The rhodium(I) metal center is coordinated to two adjacent pyrrolic nitrogen atoms in a typical square planar $\mathrm{L}_{2} \mathrm{Rh}(\mathrm{CO})_{2}$ coordination geometry, with the rhodium(I) atom displaced by 1.5623(6) $\AA$ from the 24-atom mean macrocylic plane of the porphyrinoid. Similarities in the crystal structure parameters were observed between $\mathbf{8 9}$ and rhodium(I) benzocarbaporphyrin $\mathbf{8 6}$. The previously reported $\mathbf{8 6}^{30}$ has its rhodium(I) metal center displaced by $1.5958(2) \AA$, which is only slightly larger than the displacement seen for $\mathbf{8 9}$. Furthermore, the dihedral angle that the Rh1, N23, N24, C25, C26 plane makes with the mean macrocylic plane in 89 is $60.32(4)^{\circ}$, which is similar to the corresponding $61.78(2)^{\circ}$ dihedral angle observed for $\mathbf{8 6}$. The $0.188 \AA$ rms distance the framework atoms lie from their mean plane indicate that $\mathbf{8 9}$ is relatively planar. However, the need to accommodate the two internal hydrogen atoms leads to some distortion, which is noted through the $13.8(1)^{\circ}, 9.3(1)^{\circ}, 13.0(1){ }^{\circ}$, and $12.0(1)^{\circ}$ dihedral angles between the framework plane and the respective cyclopentadiene and three pyrrolic mean planes. The internal carbon and uncoordinated pyrrolic nitrogen protons were easily identifiable in the difference Fourier of the X-ray structure, allowing for a clear assignment of oxidation state to the rhodium metal center. 

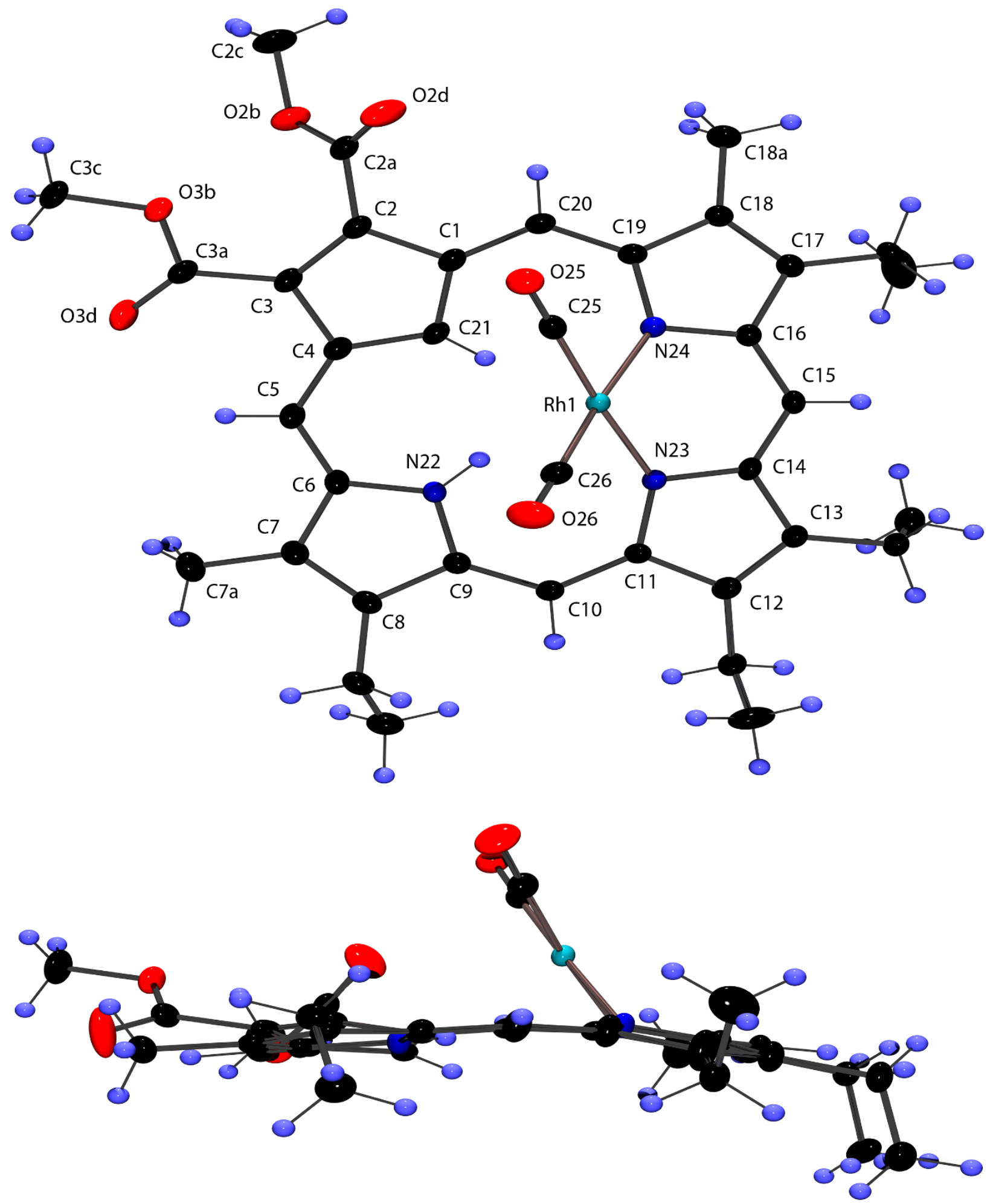

Figure 15. Color POV-Ray rendered ORTEP III drawing (50\% probability level, hydrogen atoms rendered arbitrarily small for clarity) of rhodium(I) carbaporphyrin $\mathbf{8 9}$ 
Rhodium(I) complex 89 was refluxed in pyridine for 30 minutes and gave a rhodium(III) complex 90 containing two axial pyridine ligands coordinating through the nitrogen to the metal center (Scheme 30). This compound was chromatographed on grade 3 neutral alumina eluting with toluene and a dark band was collected. Recrystallization from chloroform-hexanes gave the pure product 90 in 14\% yield. The carbaporphyrin ring regains its symmetry in $\mathbf{9 0}$. This rhodium(III) complex is rather insoluble in chloroform but gave reasonable NMR data in $d_{6}$-benzene, and the proton NMR spectrum confirmed the symmetry and aromatic character of the macrocycle. Two relatively downfield $2 \mathrm{H}$ singlets for the meso-protons were observed at 9.46 and $10.59 \mathrm{ppm}$. The $\alpha$-protons of the two pyridine rings were shielded by the ring current of the carbaporphyrin and gave an upfield $4 \mathrm{H}$ multiplet at $2.33 \mathrm{ppm}$. The $\beta$ - and $\gamma$-protons were less shielded by the ring current, but were still shifted upfield to 4.10 and $4.70 \mathrm{ppm}$, respectively (Figure 16). The large shielding effect the pyridine ligands are experiencing can be further appreciated through a comparison to pyridine, as this shows the $\alpha$-proton resonances downfield near 9 ppm. The UV-Vis spectrum of 90 gave a strong Soret band at $481 \mathrm{~nm}$ and Q bands at 603 and $680 \mathrm{~nm}$ (Figure 17).

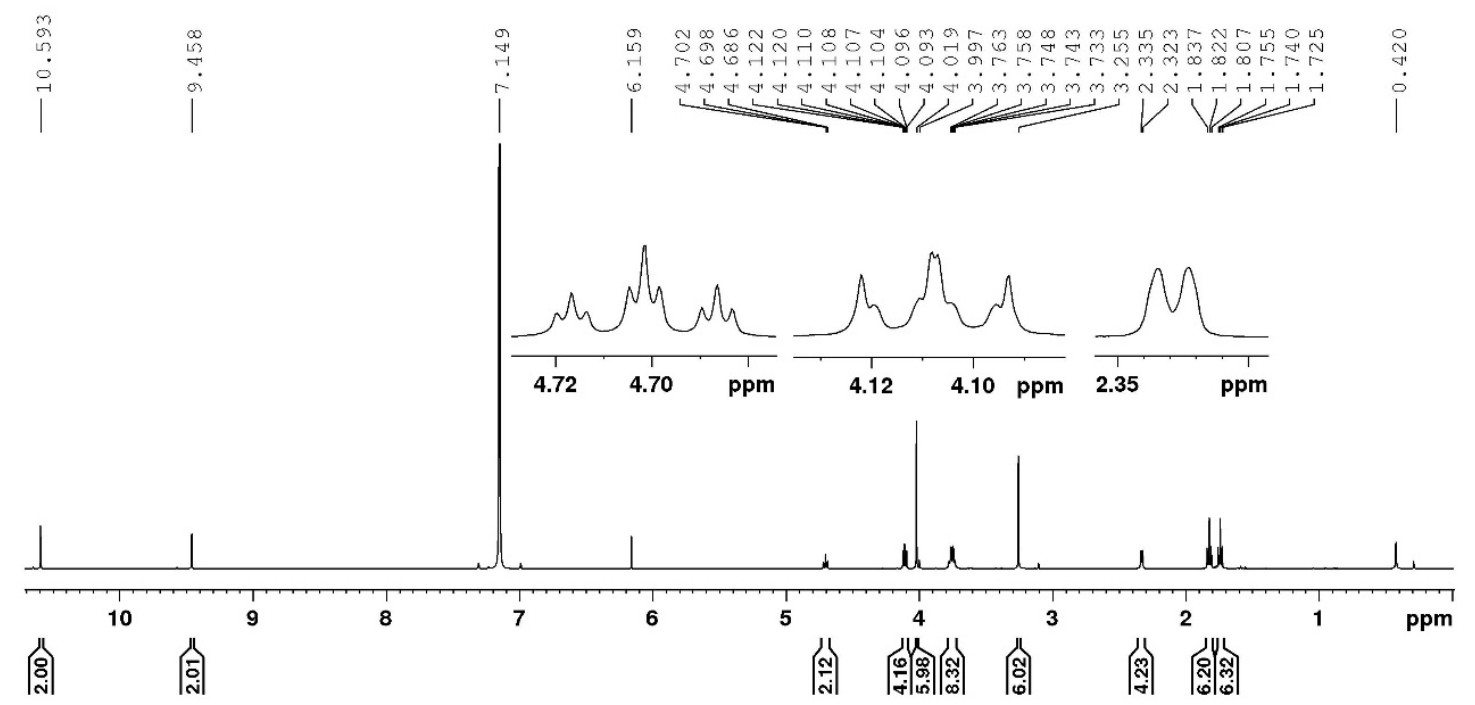

Figure 16. $500 \mathrm{MHz}{ }^{1} \mathrm{H}$ NMR spectrum of rhodium(III) carbaporphyrin diester 90 in $d_{6}$-benzene 


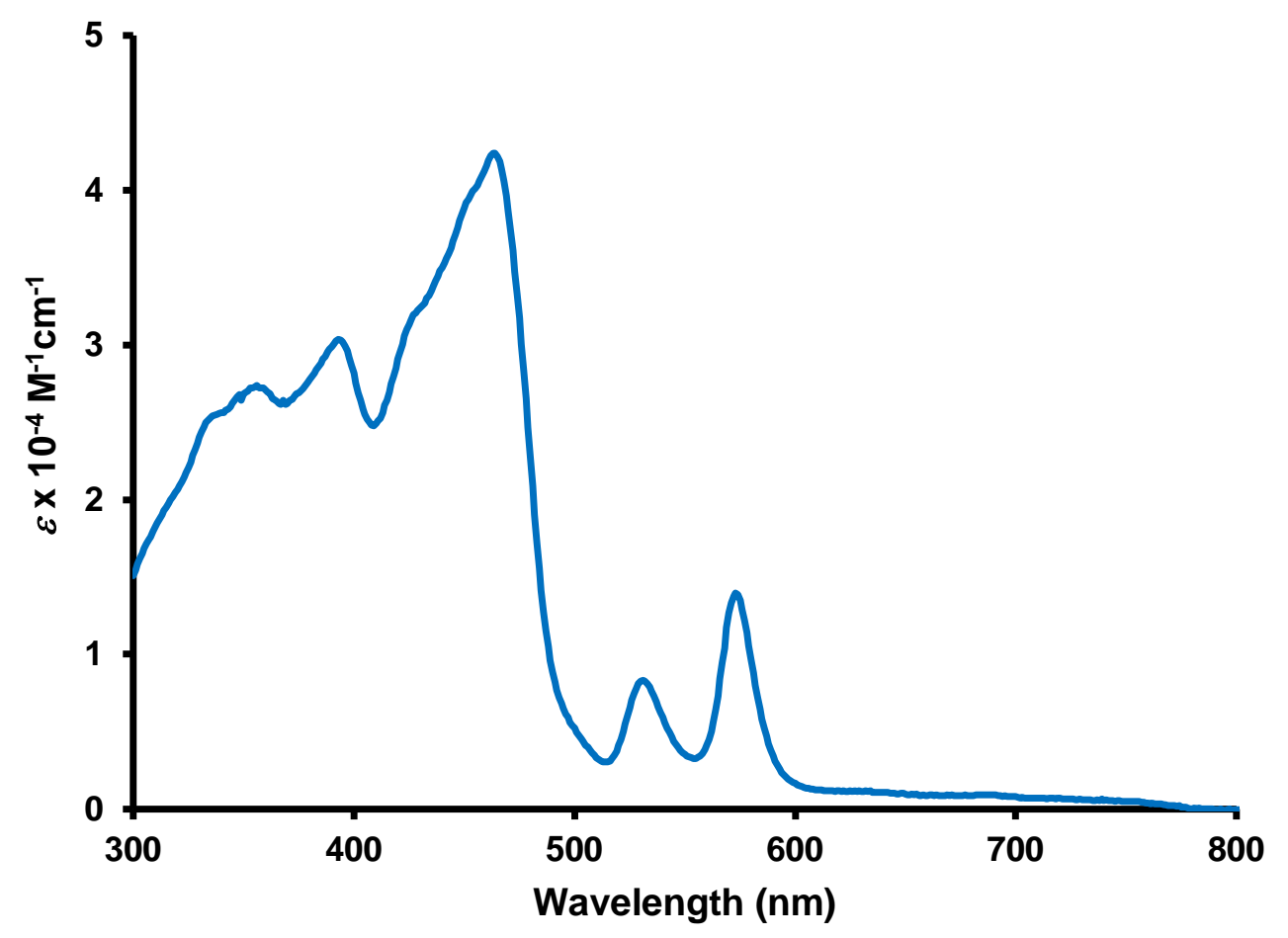

Figure 17. UV-Vis spectrum of rhodium(III) carbaporphyrin diester $\mathbf{9 0}$

Crystals suitable for X-ray crystallography were obtained, and the structure of $\mathbf{9 0}$ was further confirmed (Figure 18). The X-ray structure demonstrated that the rhodium(III) atom is sixcoordinate with the pyrrolic nitrogens and the cyclopentadienyl internal carbon atom occupying the equatorial plane, while the pyridine ligands took up each of the axial positions, resulting in a nearly idealized octahedral coordination environment. Unlike the rhodium atom seen in $\mathbf{8 9}$, the rhodium(III) atom of $\mathbf{9 0}$ is only displaced 0.0810(5) $\AA$ from the 24-atom mean macrocyclic plane of the porphyrinoid. The $0.077 \AA$ rms distance the framework atoms lie from their mean plane indicate the macrocycle is essentially planar, and this is further supported by the $5.0(1)^{\circ}, 3.5(1)^{\circ}$, $5.8(1)^{\circ}$, and $2.7(1)^{\circ}$ dihedral angles of the cyclopentadiene and three pyrrolic mean planes with the mean macrocyclic plane. The framework of bond lengths also show that this carbaporphyrin is aromatic with the delocalized $\pi$-electron pathway passing through the internal C21 atom. This is 
supported by the longer bond lengths of 1.464(3) and 1.469(3) $\AA$ seen for the C1-C2 and C3-C4 bonds, respectively. Comparatively shorter are the more aromatic-like bond lengths of 1.416(3) and $1.413 \AA$ that are observed for the C1-C21 and C4-C21 bonds, respectively. Furthermore, the bonds directly connected to the meso-carbons varied from 1.382-1.404 $\AA$, which is expected for the bonding seen within an aromatic systems. 

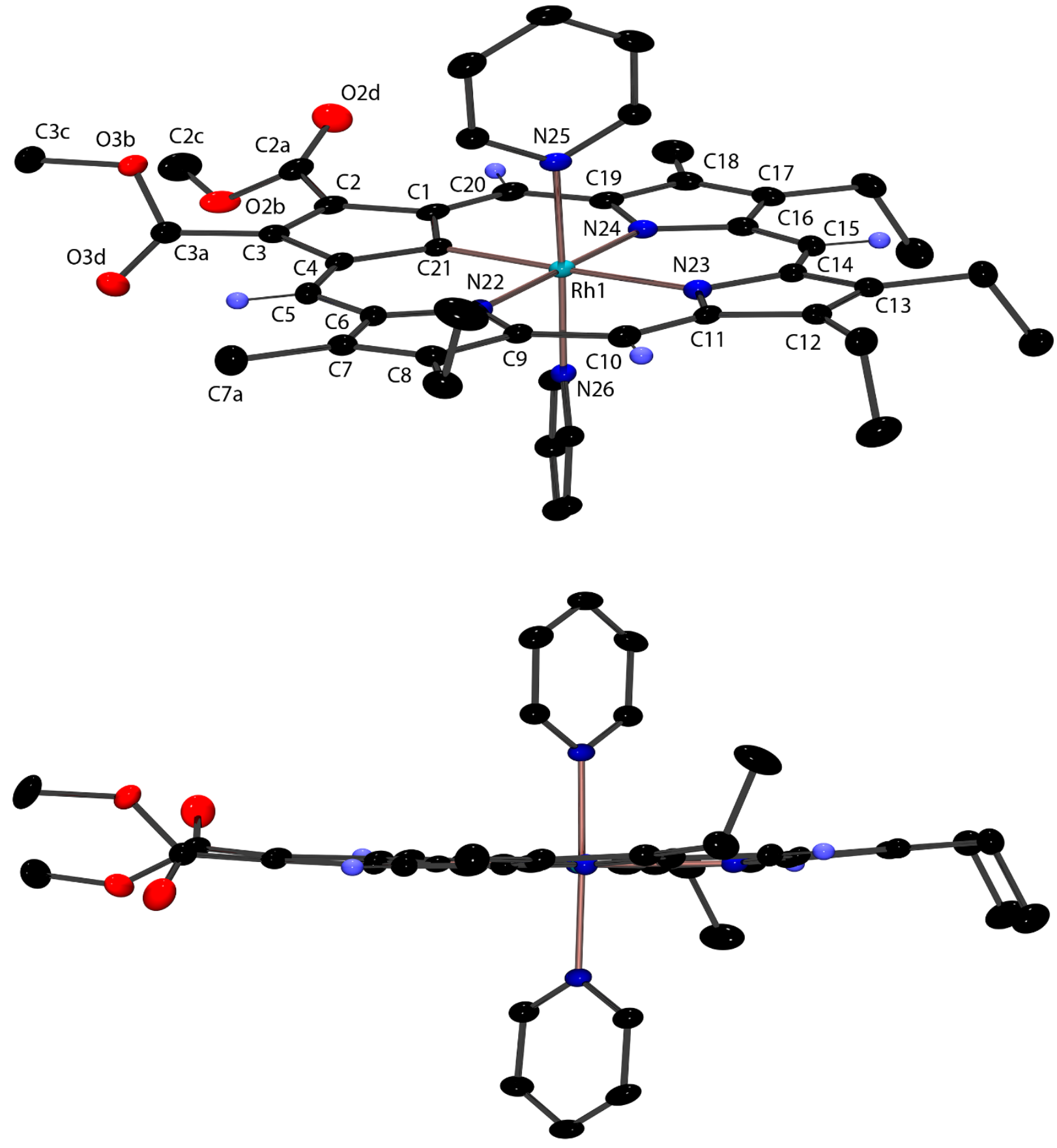

Figure 18. Color POV-Ray rendered ORTEP III drawing (50\% probability level, hydrogen atoms rendered arbitrarily small or omitted for clarity) of rhodium(III) complex $\mathbf{9 0}$ 
Naphtho[2.3-b]carbaporphyrin 87 was also reacted with $\left[\mathrm{Rh}(\mathrm{CO})_{2} \mathrm{Cl}\right]_{2}$ in refluxing dichloromethane to give the rhodium(I) complex 91 (Scheme 31). The crude material was purified using a silica gel column, eluting with 20:80 dichloromethane-hexanes, and a green band was collected. Subsequent recrystallization from chloroform-hexanes gave pure $\mathbf{9 1}$ in $72 \%$ yield.

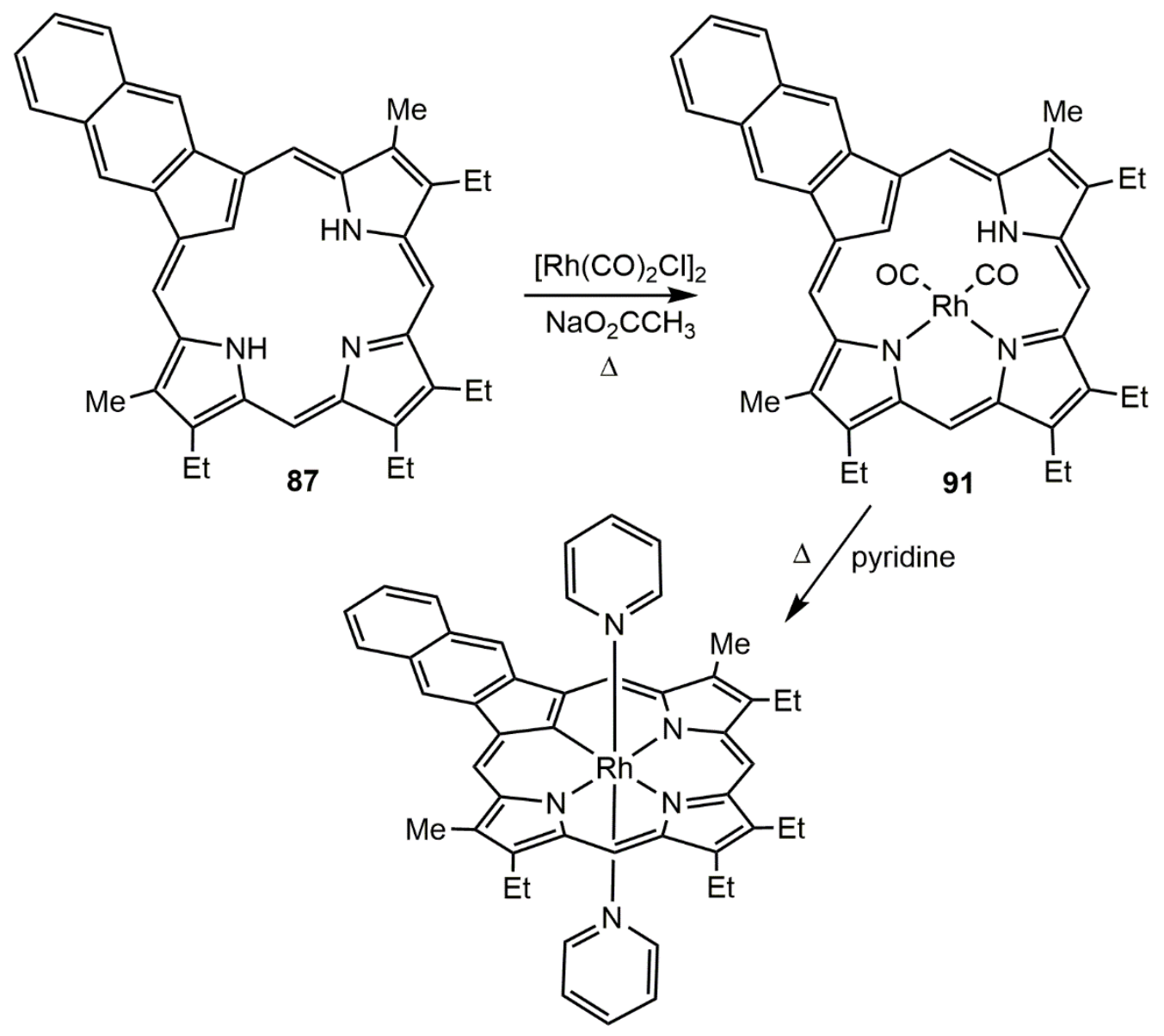

92

Scheme 31. Rhodium(I) and rhodium(III) complexes of naphthocarbaporphyrin 87

Free base naphthocarbaporphyrin $\mathbf{8 7}$ is symmetrical and the proton NMR spectrum shows the meso-protons as two $2 \mathrm{H}$ singlets at 9.76 and $10.00 \mathrm{ppm}$, and the methyl substituents as a $6 \mathrm{H}$ singlet at $3.61 \mathrm{ppm}$. In addition, the internal $\mathrm{CH}$ for $\mathbf{8 7}$ gives a singlet at $-6.55 \mathrm{ppm}$ and the two NH protons afford a broad 2H singlet at $-3.87 \mathrm{ppm} .{ }^{108}$ Rhodium(I) complex 91, in contrast, showed 
four $1 \mathrm{H}$ singlets for the meso-protons between 9.78 and $10.34 \mathrm{ppm}$, demonstrating the loss of symmetry in this metalated derivative. The inner $\mathrm{NH}$ and $\mathrm{CH}$ resonances appeared at -2.48 and $5.19 \mathrm{ppm}$, respectively (Figure 19). When comparing the upfield and downfield shifts seen for free base 87 and rhodium(I) derivative 91, it is evident that they exhibit comparable diamagnetic ring currents. The carbon-13 NMR spectrum further demonstrates the loss of symmetry seen in $\mathbf{9 5}$ (Figure 20). The four meso-protons were identified at 93.8, 97.5, 103.1 and $105.0 \mathrm{ppm}$, while the internal $\mathrm{CH}$ gave a peak at $123.0 \mathrm{ppm}$. The carbonyl resonances appear as two doublets at 177.6 and $178.4 \mathrm{ppm}\left({ }^{1} J_{\mathrm{Rh}-\mathrm{C}}=69.0\right.$ and $67.1 \mathrm{~Hz}$, respectively). Interestingly, the IR spectrum for $\mathbf{9 1}$ gave three peaks near $2000 \mathrm{~cm}^{-1}$ for carbonyl ligand stretching, rather than the two that might have been expected (Figure 21). The lower frequency bands appear to be a Fermi doublet that results from the carbonyl peak interacting with an aromatic overtone. The UV-vis spectrum for $\mathbf{9 1}$ was porphyrin-like and displayed a strong Soret band at $481 \mathrm{~nm}$ and several Q bands between 566 and $680 \mathrm{~nm}$ (Figure 22). 

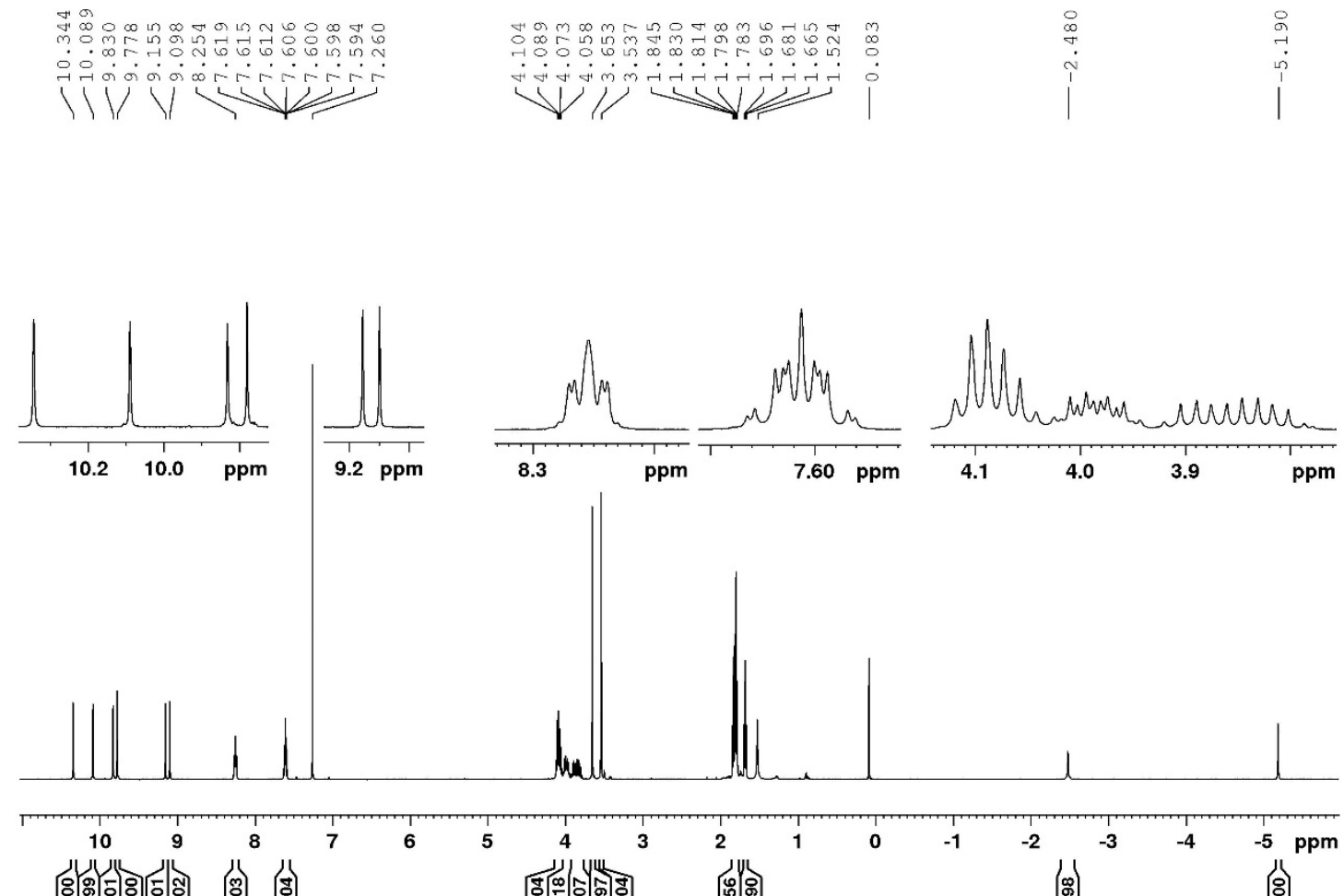

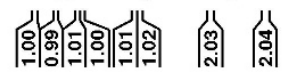

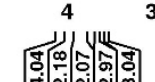

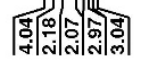
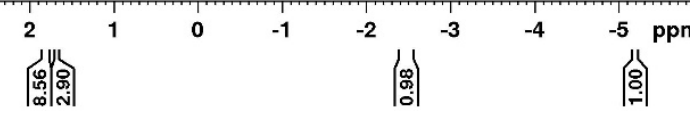

Figure 19. $500 \mathrm{MHz}{ }^{1} \mathrm{H}$ NMR spectrum of rhodium(I) naphthocarbaporphrin 91 in $\mathrm{CDCl}_{3}$

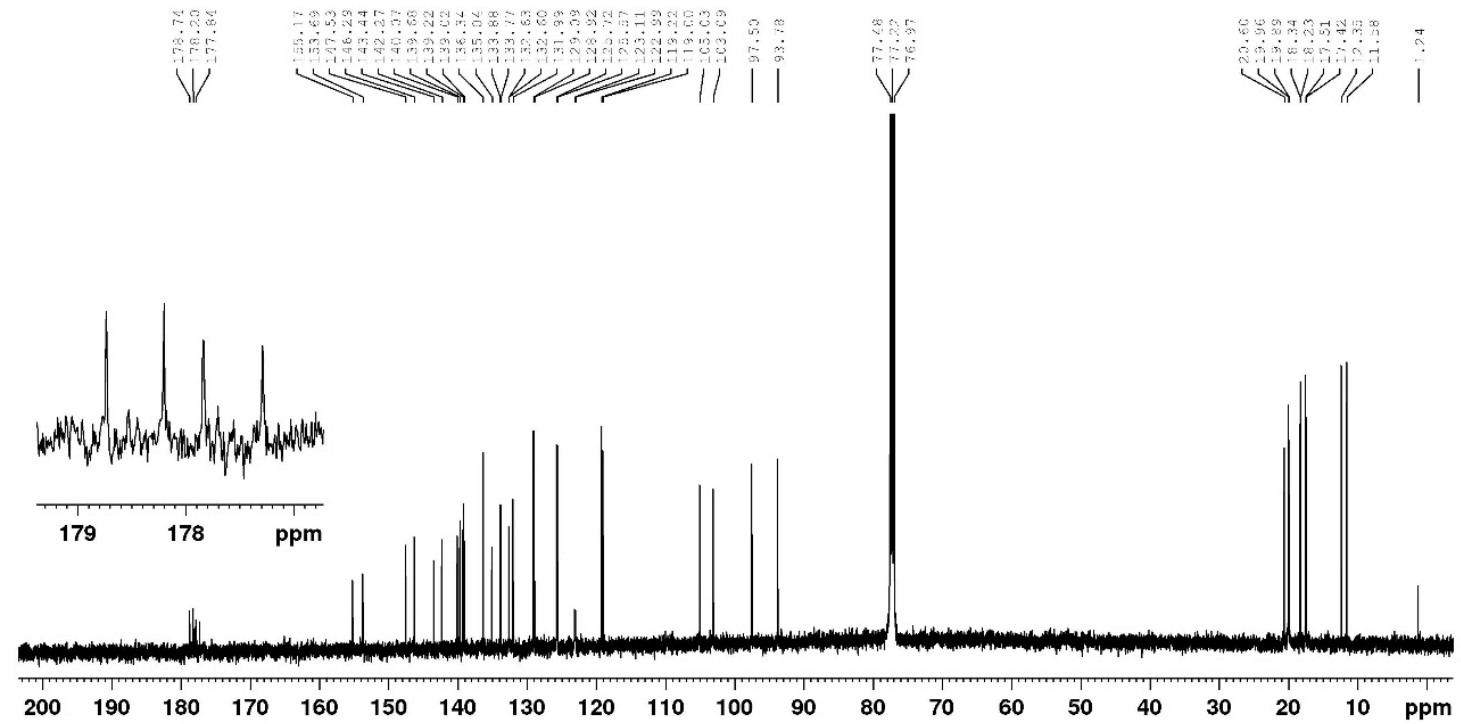

Figure 20. $125 \mathrm{MHz}{ }^{13} \mathrm{C}$ NMR spectrum of rhodium(I) naphthocarbaporphyrin 91 in $\mathrm{CDCl}_{3}$ 


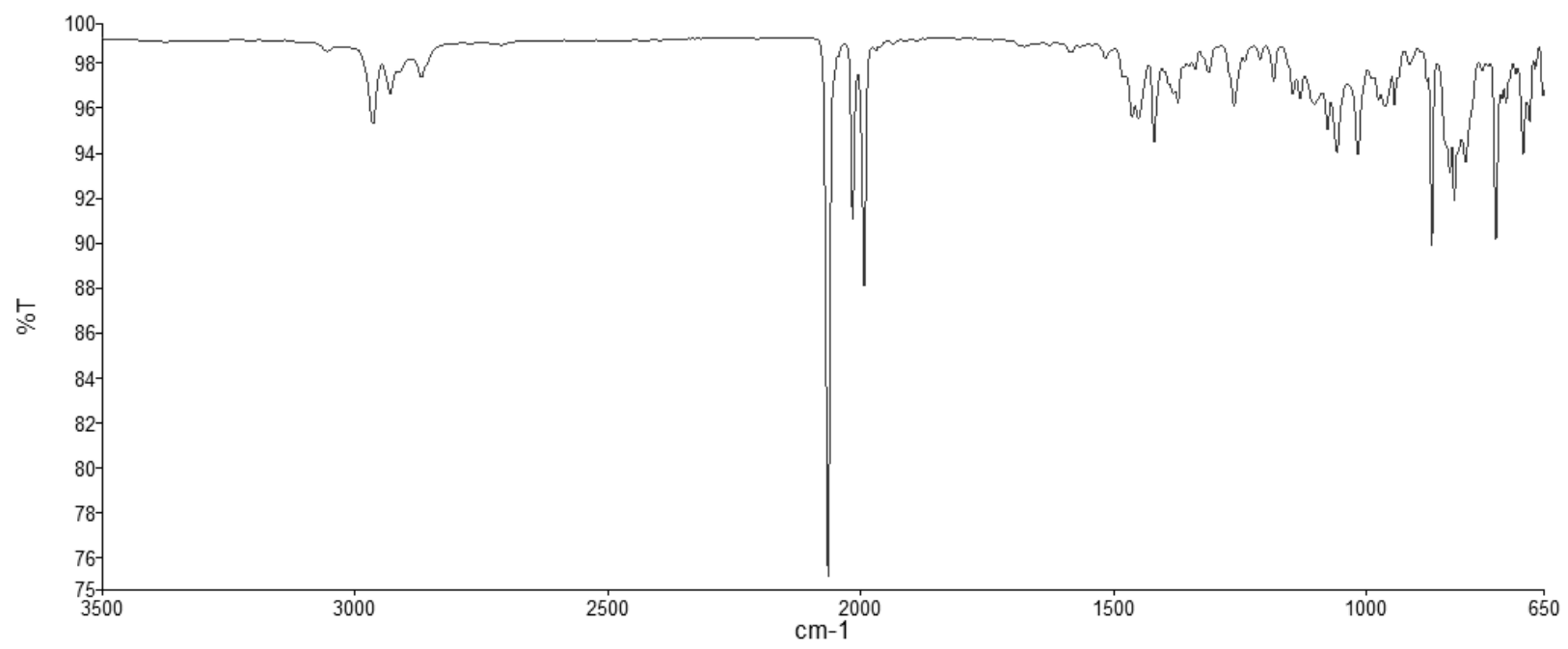

Figure 21. IR spectrum of rhodium(I) naphthocarbaporphyrin 91

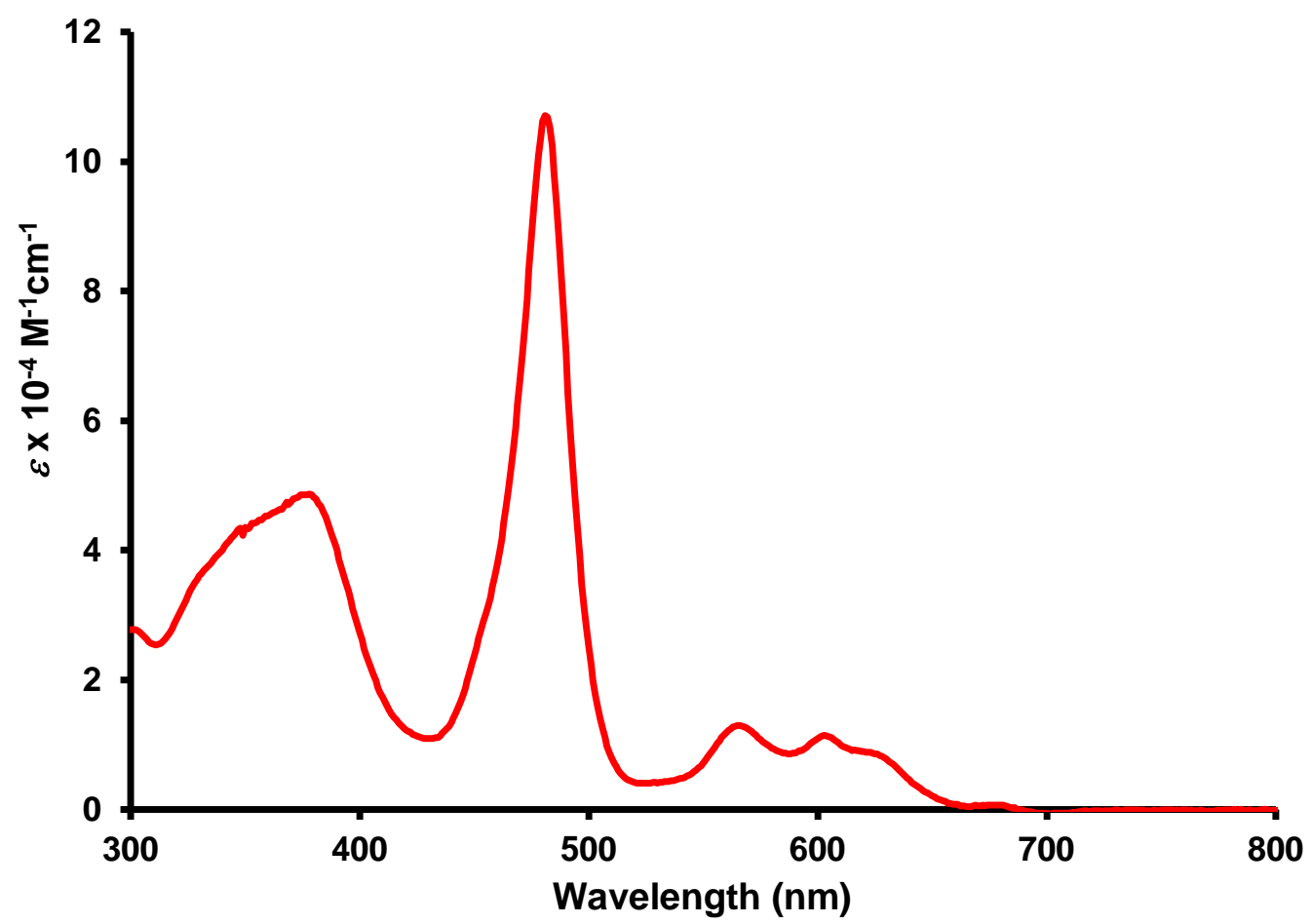

Figure 22. UV-Vis spectrum of rhodium(I) naphthocarbaporphyrin 91

The structure of 91 was also confirmed by X-ray crystallography (Figure 23), which verified the presence of a dicarbonyl-rhodium(I) moiety coordinating to two adjacent nitrogens. 
This coordination is analogous to the coordination seen in rhodium(I) benzocarbaporphyrin $\mathbf{8 6}$ and rhodium(I) carbaporphyrin diester $\mathbf{8 9}$. Square planar $\mathrm{L}_{2} \mathrm{Rh}(\mathrm{CO})_{2}$ geometry is observed, with the rhodium(I) atom displaced by $1.5544(4) \AA$ from the 24 -atom mean macrocyclic plane. The dihedral angle the Rh, N23, N24, C25, C26 plane makes with the mean macrocyclic plane is $59.70(5)^{\circ}$ and similar to the values seen in related rhodium(I) carbaporphyrin systems, as are the $\mathrm{Rh}-\mathrm{N}$ and $\mathrm{Rh}-\mathrm{C}_{(\mathrm{CO})}$ distances. The $0.210 \AA \AA$ rms distance the framework atoms lie from their mean plane indicate reasonable planarity. However, there is some distortion observed through the $14.03(3)^{\circ}, 12.85(7)^{\circ}, 12.34(7)^{\circ}$, and $13.45(7)^{\circ}$ dihedral angles of the benz[f]indene and three pyrrolic mean planes, respectively. This is likely due to accomodation of the two internal hydrogen atoms. Overall, the framework bond distances are consistent with an aromatic system containing a delocalized $\pi$-bonding model where the bonds directly connected to the meso-carbons fall within a typical aromatic bond length range of 1.389-1.409 A. The bond lengths for C1-C2 and C3-C4 are relatively long, 1.476 and $1.479 \AA$, respectively, indicating that the delocalized $\pi$-system runs through the internal C21. 

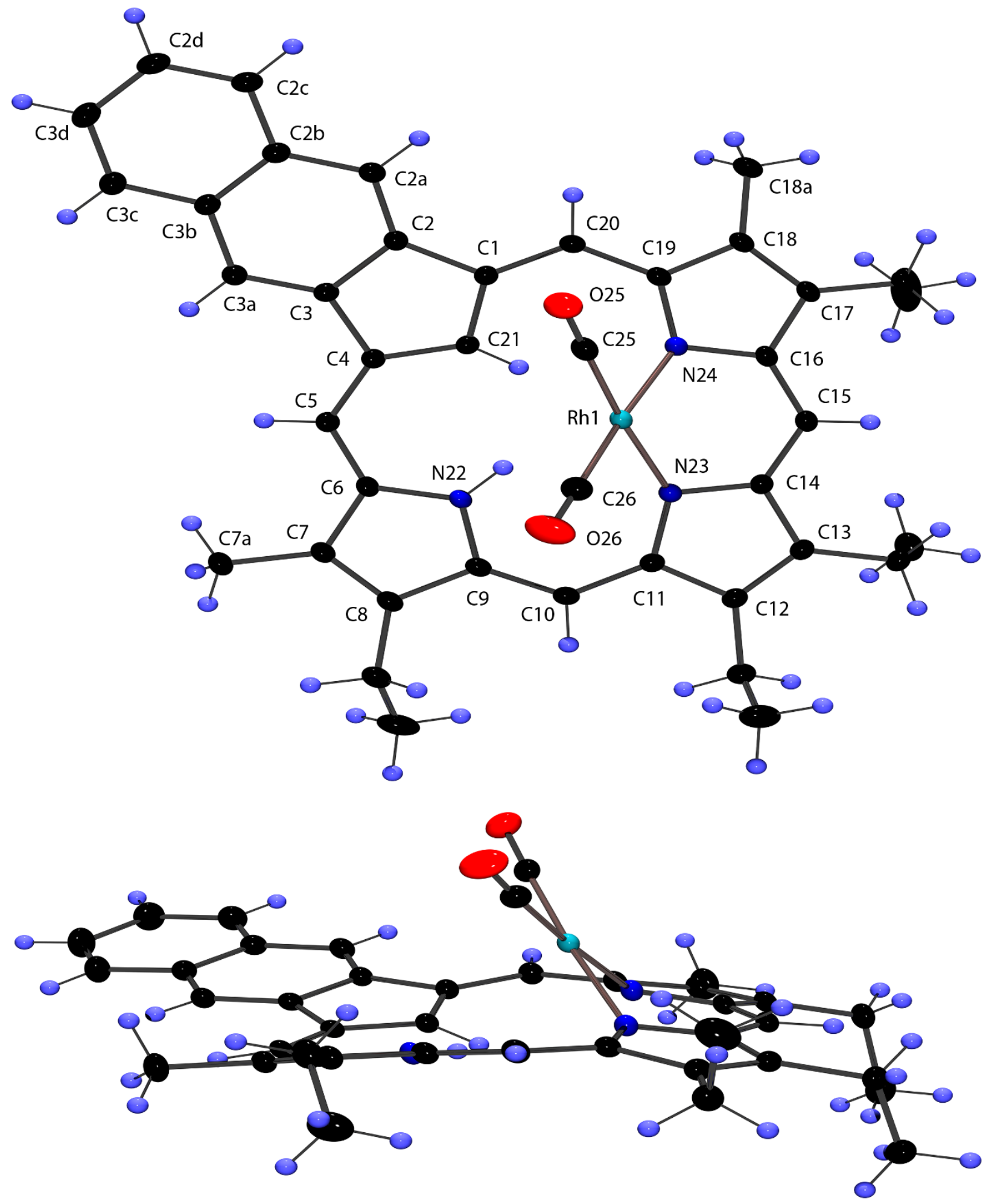

Figure 23. Color POV-Ray rendered ORTEP III drawing (50\% probability level, hydrogen atoms rendered arbitrarily small for clarity) of rhodium(I) naphthocarbaporphyrin $\mathbf{9 1}$ 
When a solution of 91 in pyridine was refluxed for one hour, rhodium(III) complex 92 was formed (Scheme 31). This compound also incorporated two axial pyridine ligands coordinating to the rhodium metal center. Purification through chromatography on alumina with 50:50 toluenehexanes gave a light brown band that was collected and then further purified through recrystallization from chloroform-hexanes to give pure $\mathbf{9 2}$ in 21\% yield. Like the rhodium(III) complex 90, this compound was also insoluble in chloroform and was therefore dissolved in $d_{6^{-}}$ benzene to collect NMR spectra (Figure 24). The symmetry that was lost in the rhodium(I) complex is regained in the rhodium(III) complex, showing similarity to the free base naphthocarbaporphyrin $\mathbf{8 7}$. This symmetry is supported by the presence of two $2 \mathrm{H}$ singlets for the meso-protons resonating at 10.07 and $10.56 \mathrm{ppm}$, and the external methyl groups giving a $6 \mathrm{H}$ singlet at $3.59 \mathrm{ppm}$. The $\alpha$-, $\beta$ - and $\gamma$-protons of the axial pyridine ligands are observed as a $4 \mathrm{H}$ doublet at $1.44 \mathrm{ppm}$, a $4 \mathrm{H}$ triplet at $3.92 \mathrm{ppm}$ and a $2 \mathrm{H}$ triplet at $4.58 \mathrm{ppm}$, respectively. The large upfield shifts that these pyridine resonances demonstrate the high degree of aromatic character present in 92. Further evidence for the aromaticity and symmetry of this compound is evident in the carbon-13 NMR spectrum (Figure 25). The meso-protons were observed at 94.7 and $102.8 \mathrm{ppm}$ and the $\alpha$-, $\beta$ - and $\gamma$ - pyridine resonances at 148.1, 120.6 and $133.1 \mathrm{ppm}$, respectively. The UVVis spectrum of 92 showed a strong Soret band at $447 \mathrm{~nm}$, followed by several Q bands between 530 and $606 \mathrm{~nm}$ (Figure 26). The X-ray structure for this compound was not obtained, as suitable crystals for analysis could not be produced. 


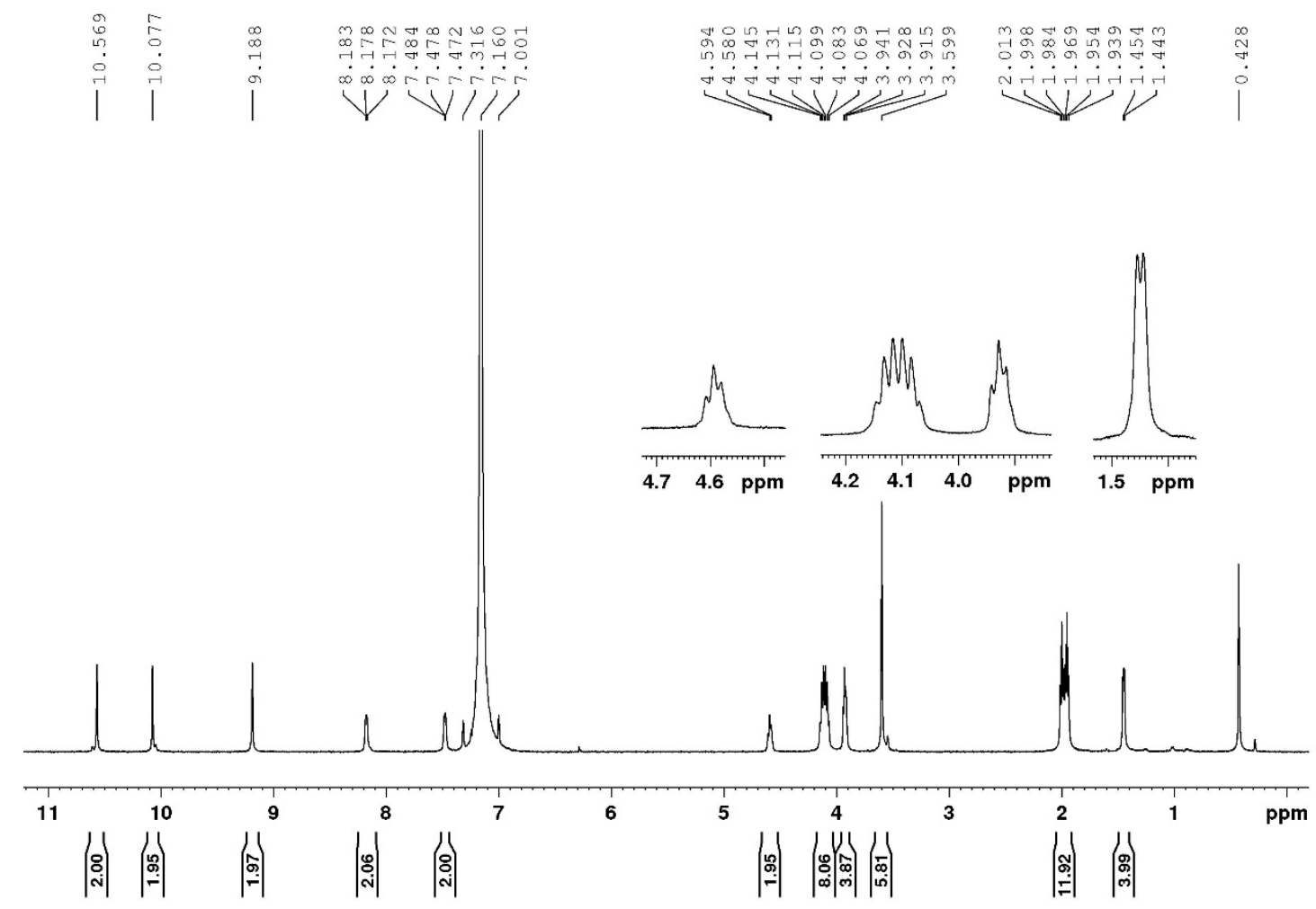

Figure 24. $500 \mathrm{MHz}{ }^{1} \mathrm{H}$ NMR spectrum of rhodium(III) naphthocarbaporphyrin 92 in $d_{6}$-benzene

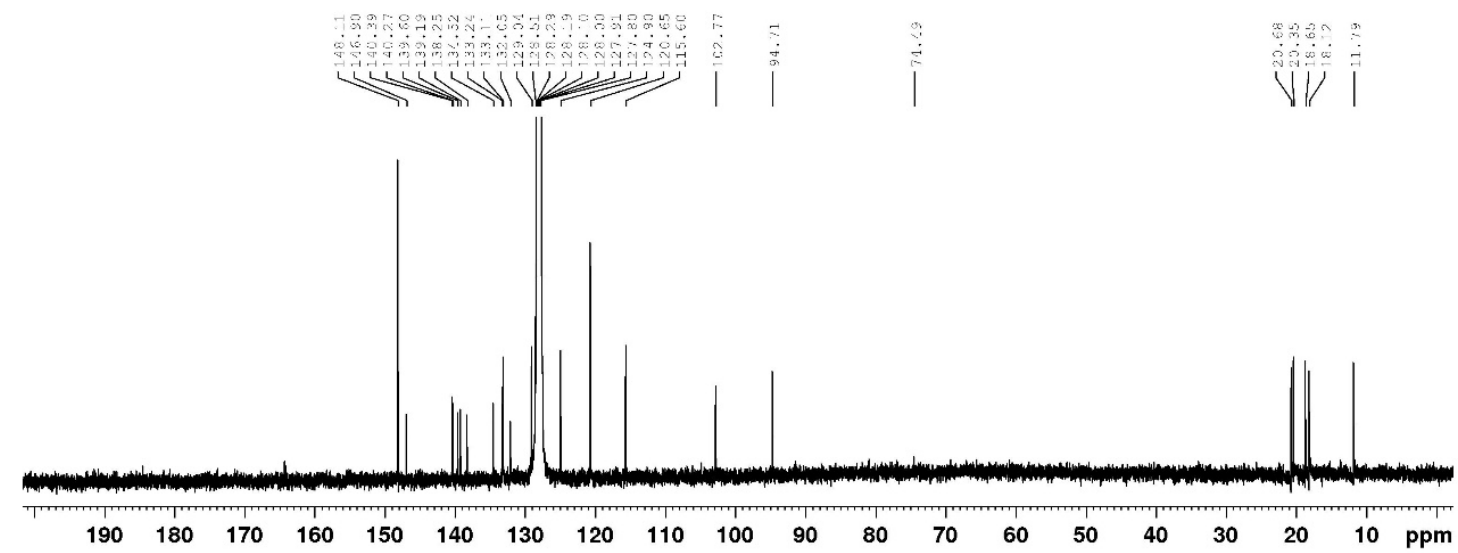

Figure 25. $125 \mathrm{MHz}{ }^{13} \mathrm{C}$ NMR spectrum of rhodium(III) naphthocarbaporphyrin 92 in $d_{6}$-benzene 


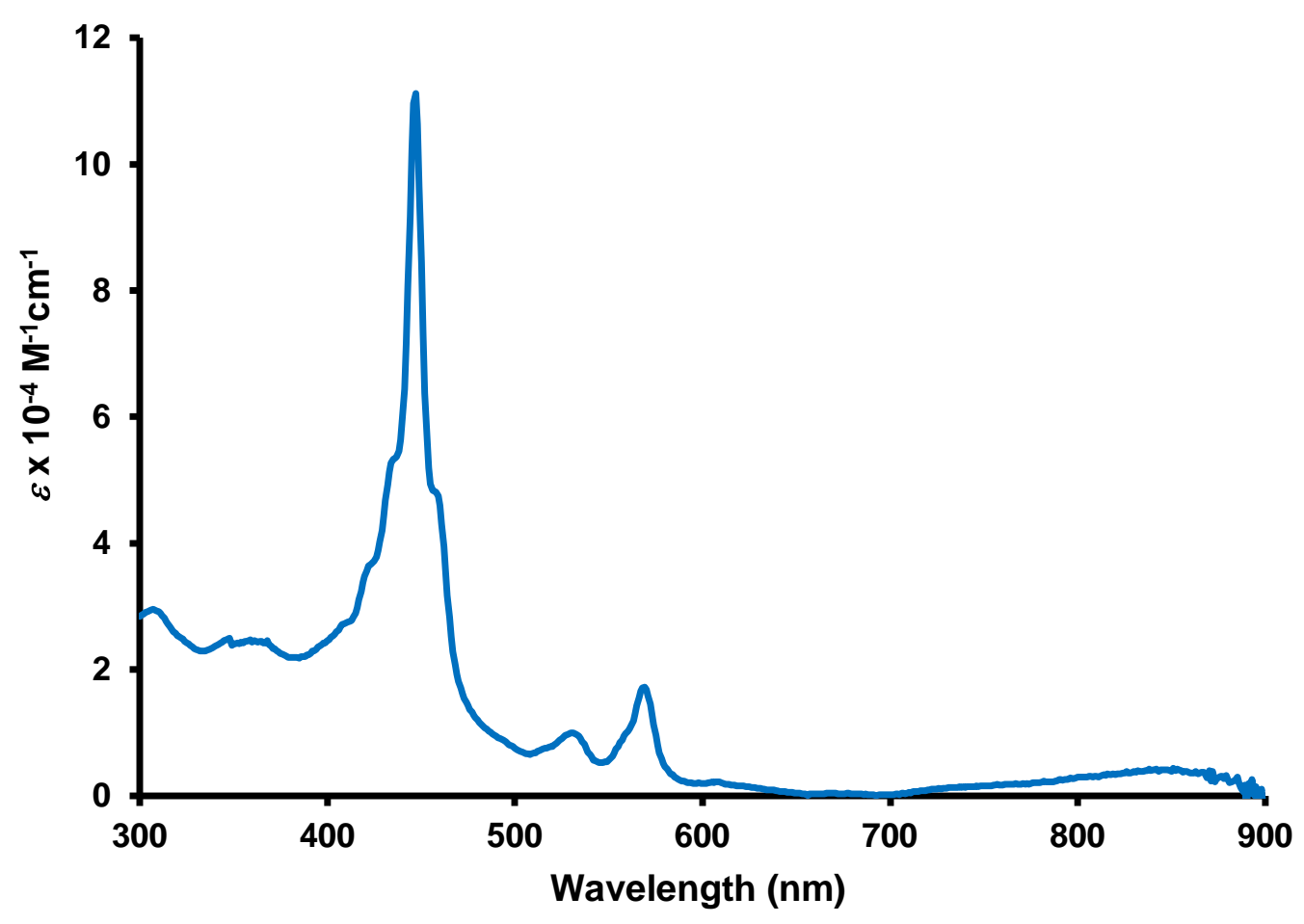

Figure 26. UV-Vis spectrum of rhodium(III) naphthocarbaporphyrin 92

Benzocarbaporphyrin $\mathbf{3}$ was shown to form stable rhodium(I) and rhodium(III) derivatives ${ }^{30}$, while internally methylated benzocarbaporphyrins $\mathbf{7 0}$ and $\mathbf{7 6}$ gave rise to unexpected alkyl group migrations when metalated with palladium(II) acetate. ${ }^{111,101}$ The ability of the internally alkylated benzocarbaporphyrins to form palladium(II) complexes, as well as the observed methyl group migrations within these complexes, make systems such as $\mathbf{7 0 , 7 5}$, and $\mathbf{7 6}$ important metalation targets. Internally methylated compounds such as these have the potential to undergo C-H activation upon the introduction of a rhodium(III) metal center. However, in the 23methylcarbaporphyrin $\mathbf{7 6}$ the internal alkyl residue on N23 may block coordination to a rhodium(I) metal cation and it was questionable whether the formation of a rhodium(I) derivative of $\mathbf{7 6}$ could be possible. 
In order to investigate the effects of internal alkyl substituents, 21- and 22methylbenzocarbaporphyrins 75 and 70, respectively, were reacted with $\left[\mathrm{Rh}(\mathrm{CO})_{2} \mathrm{Cl}\right]_{2}$ in refluxing dichloromethane overnight. ${ }^{111}$ 22-Methylcarbaporphyrin $\mathbf{7 0}$ reacted under these conditions to give the corresponding rhodium(I) complex 93 (Scheme 32). The rhodium complex was purified through an alumina column, eluting with 50\% dichloromethane-hexanes, and collected as a brown band. The fractions were recrystallized from chloroform-hexanes to give the pure rhodium(I) 22methylbenzocarbaporphyrin complex 93 in 50\% yield.
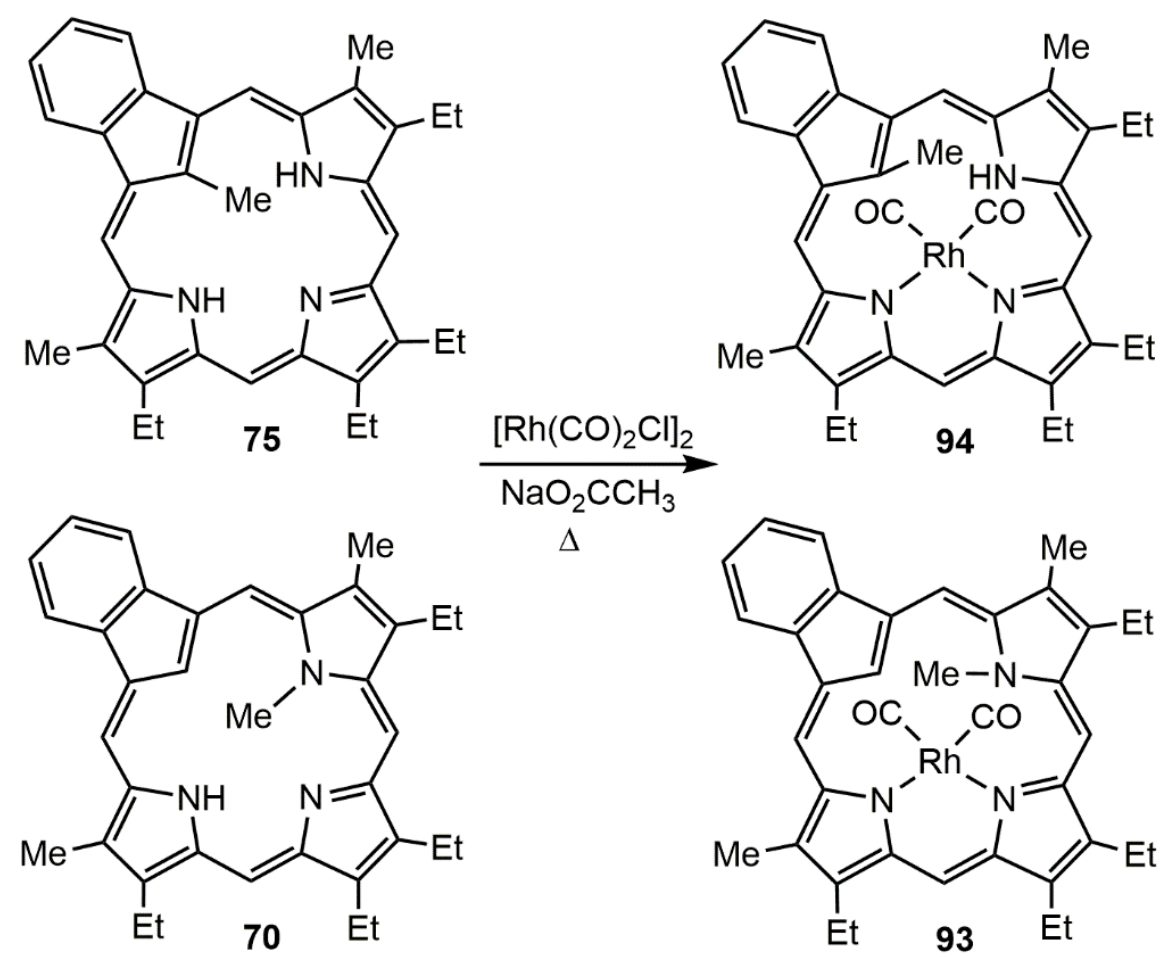

Scheme 32. Formation of 21- and 22-methyl rhodium(I) derivatives 94 and 93

The rhodium(I) complex retains the strong aromatic character seen in $\mathbf{7 0}$ and the proton NMR spectrum shows the meso-protons downfield as four $1 \mathrm{H}$ singlets between 9.64 and 10.16 ppm. Furthermore, the external methyl substituents were significantly deshielded and were observed as two $3 \mathrm{H}$ singlets at 3.36 and $3.69 \mathrm{ppm}$, while the internal methyl group was strongly 
shielded and identified as a $3 \mathrm{H}$ singlet at an upfield value of $-4.50 \mathrm{ppm}$. The internal $\mathrm{C}-\mathrm{H}$ was also relatively upfield at $-4.68 \mathrm{ppm}$, resonating as a $1 \mathrm{H}$ singlet (Figure 27). The carbon-13 NMR spectrum showed the carbonyl resonances as doublets at 178.8 and $179.4 \mathrm{ppm}\left({ }^{1} J_{\mathrm{Rh}-\mathrm{C}}=\mathrm{ca} .69 \mathrm{~Hz}\right)$ due to coupling with rhodium-103 (I = 1/2). The meso-carbons gave rise to four peaks at 97.1, 102.5, 104.6 and 106.6 ppm, while the internal indene carbon appeared at $121.2 \mathrm{ppm}$ (Figure 28). The presence of the terminal CO ligands is also evident from the IR spectrum, which showed two strong peaks at 1985 and $2057 \mathrm{~cm}^{-1}$ (Figure 29). The UV-vis spectrum for 93 displayed a Soretlike band at $481 \mathrm{~nm}$ and smaller absorptions at 561 and $637 \mathrm{~nm}$ (Figure 30). 

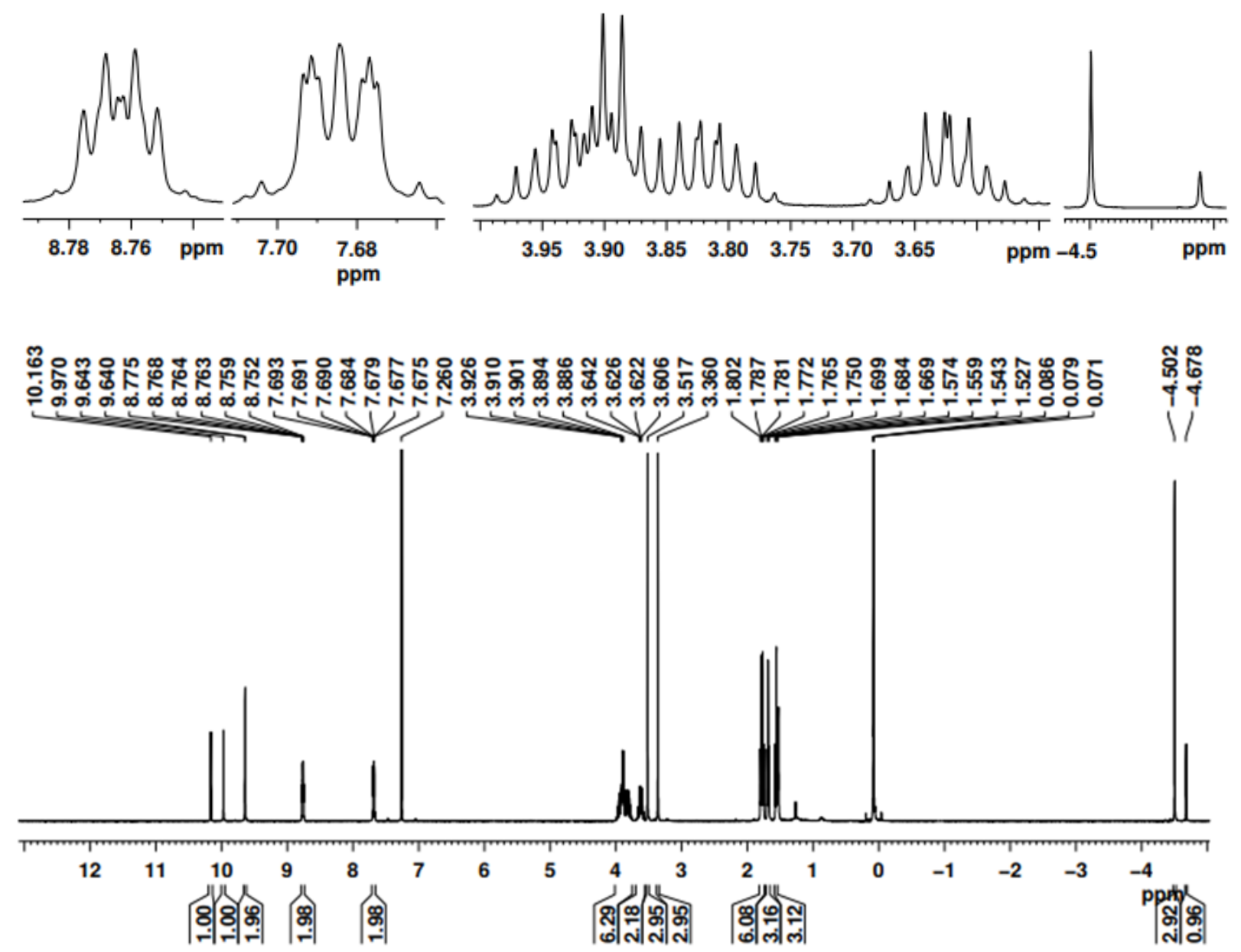

Figure 27. $500 \mathrm{MHz}{ }^{1} \mathrm{H}$ NMR spectrum of rhodium(I) 22-methylbenzocarbaporphyrin 93 in $\mathrm{CDCl}_{3}$ 


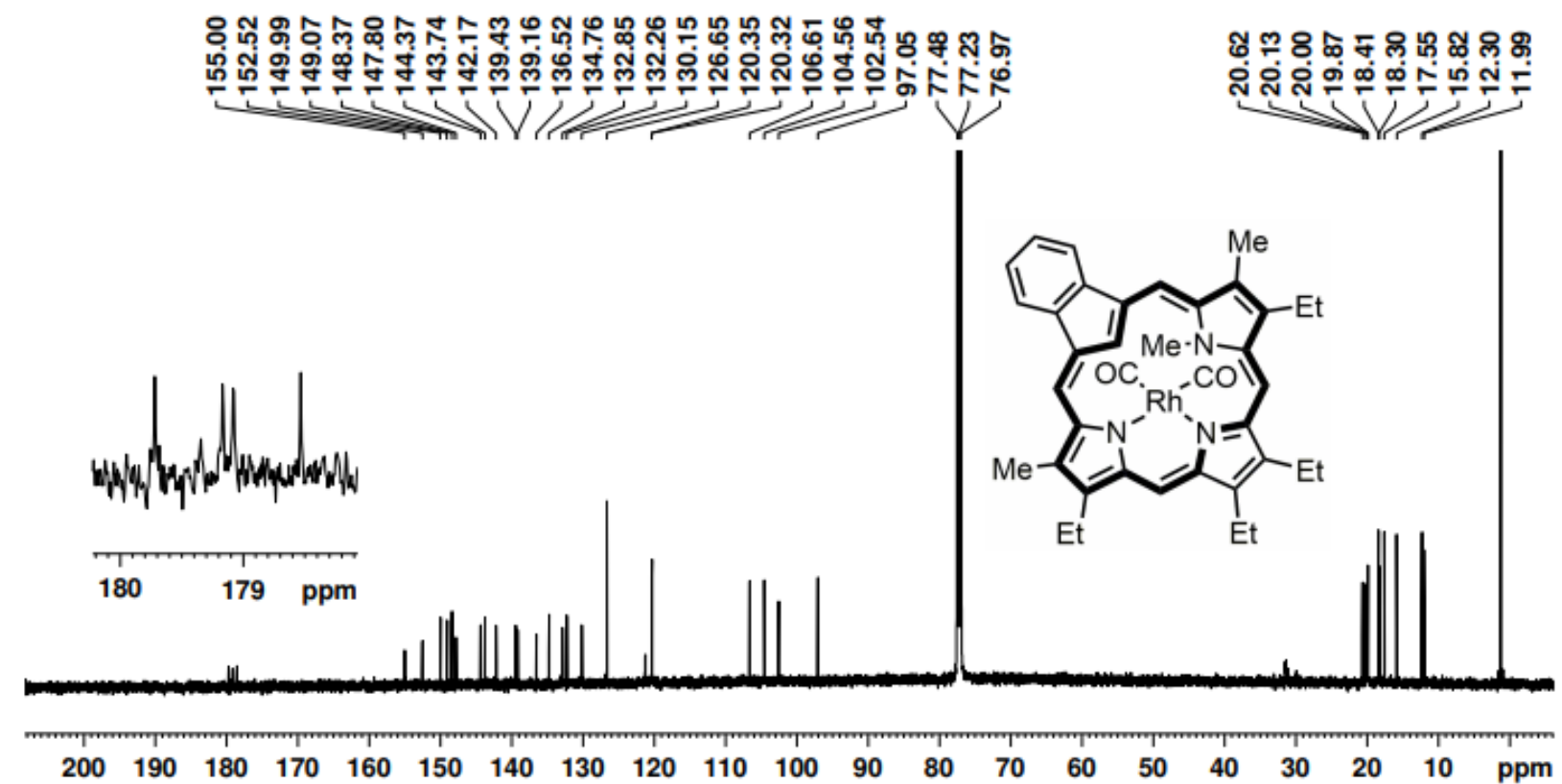

Figure 28. $125 \mathrm{MHz}{ }^{13} \mathrm{C}$ NMR spectrum of rhodium(I) 22-methylbenzocarbaporphyrin 93 in $\mathrm{CDCl}_{3}$

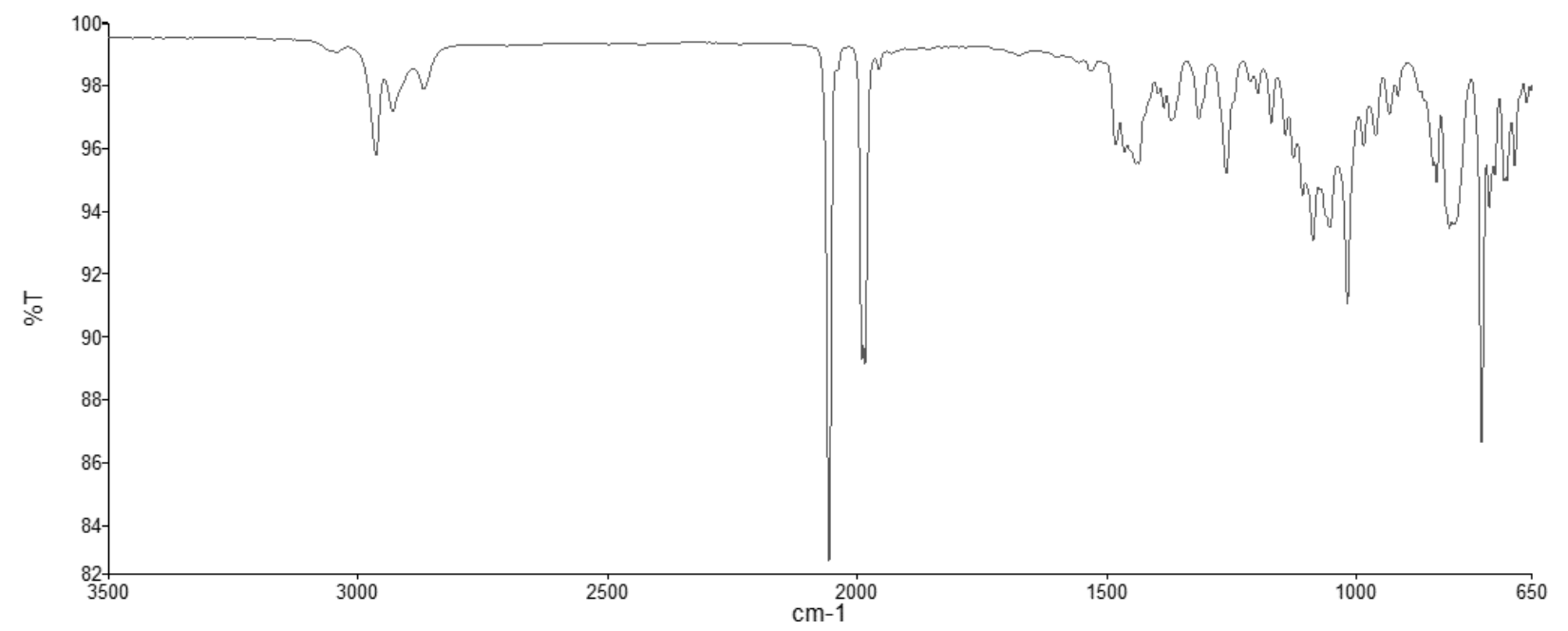

Figure 29. IR spectrum of rhodium(I) 22-methylbenzocarbaporphyrin 93 


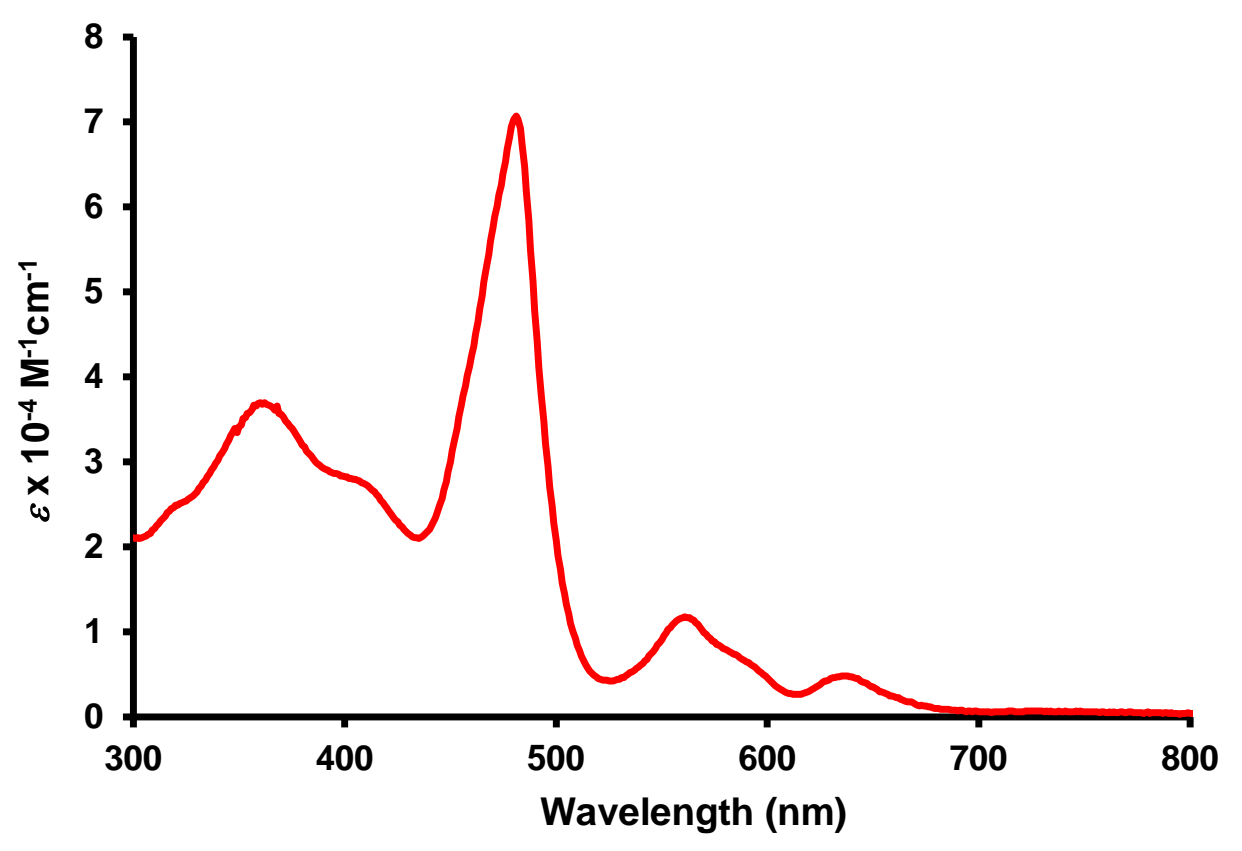

Figure 30. UV-Vis spectrum of rhodium(I) 22-methylbenzocarbaporphyrin 93

21-Methylcarbaporphyrin 75 reacted similarly to give rhodium(I) complex 94 (Scheme 32). The crude product was purified by column chromatography on neutral alumina eluting with 50\% dichloromethane-hexanes, and a green band was collected. Recrystallization from chloroform-hexanes gave the pure product $\mathbf{9 4}$ in 67\% yield. The loss of symmetry when $\mathbf{7 5}$ was converted to the rhodium(I) derivative is evident in the proton NMR spectrum of $\mathbf{9 4}$, where the meso-protons give rise to four $1 \mathrm{H}$ singlets at 9.52, 9.56, 9.69 and $9.82 \mathrm{ppm}$, and the internal methyl and $\mathrm{NH}$ protons appear upfield at -5.66 and $-2.83 \mathrm{ppm}$, respectively (Figure 31). Alongside the display of asymmetry, the large downfield and upfield shifts in the spectrum provide evidence that the strong aromatic character of the macrocycle is retained. The carbon-13 NMR spectrum shows two doublets for the terminal CO ligands between 177 and 179 ppm, while the meso-carbons appear at 93.0, 99.9, 103.5 and 108.6 ppm (Figure 32). The IR spectrum of 94 also gave two strong peaks for the carbonyl ligands at 1996 and $2063 \mathrm{~cm}^{-1}$ (Figure 33). The UV-Vis spectrum for 94 
showed similarities to the spectrum of $\mathbf{9 3}$, with a Soret-like band at $476 \mathrm{~nm}$ and weaker absorptions at 562, 604 and $627 \mathrm{~nm}$ (Figure 34). Attempts to form rhodium(III) derivatives from 94 and 93 in refluxing pyridine were unsuccessful.
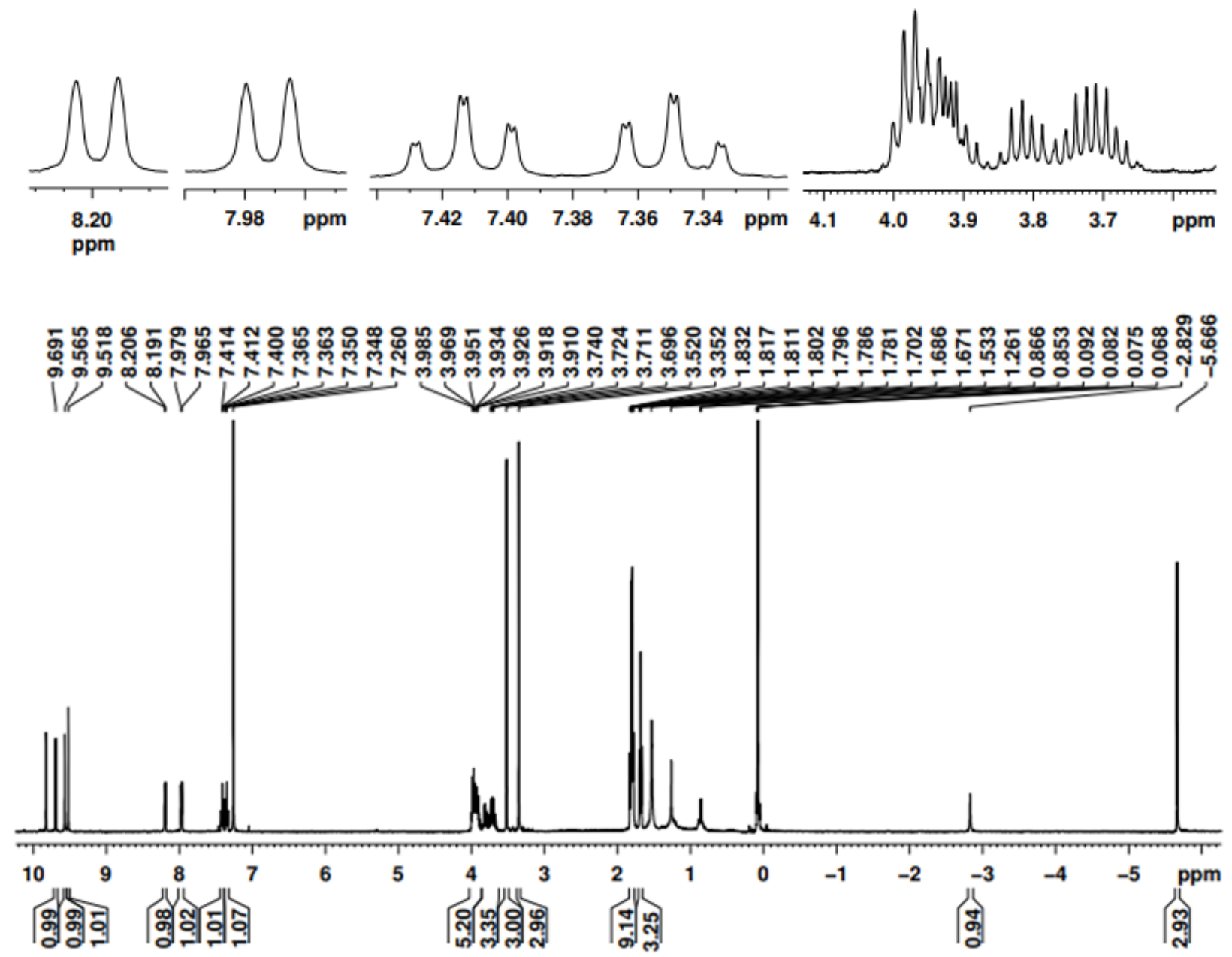

Figure 31. $500 \mathrm{MHz}{ }^{1} \mathrm{H}$ NMR spectrum of rhodium(I) 21-methylbenzocarbaporphyrin 94 in $\mathrm{CDCl}_{3}$ 


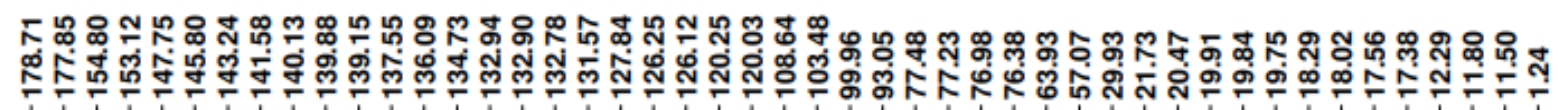
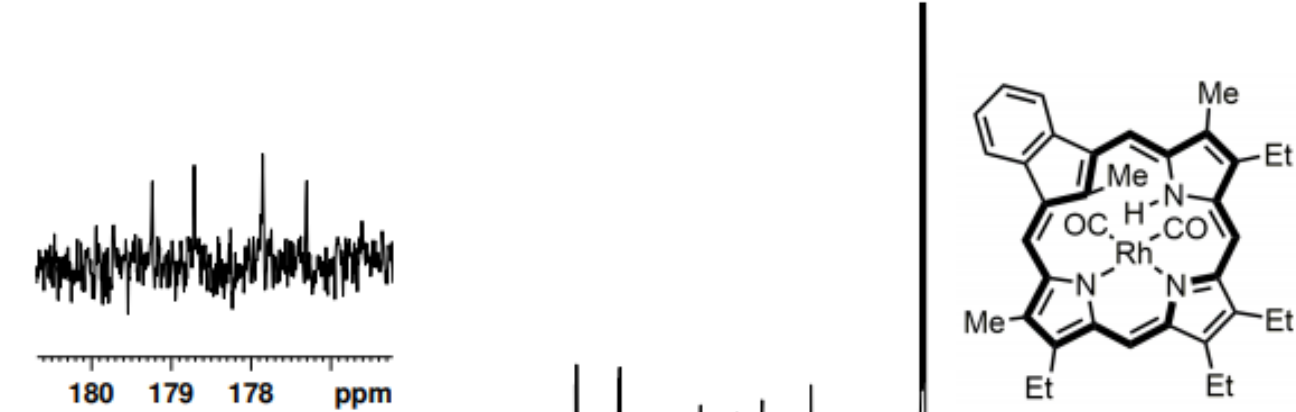

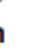

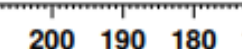

Figure 32. $125 \mathrm{MHz}{ }^{13} \mathrm{C}$ NMR spectrum of rhodium(I) 21-methylbenzocarbaporphyrin 94 in $\mathrm{CDCl}_{3}$

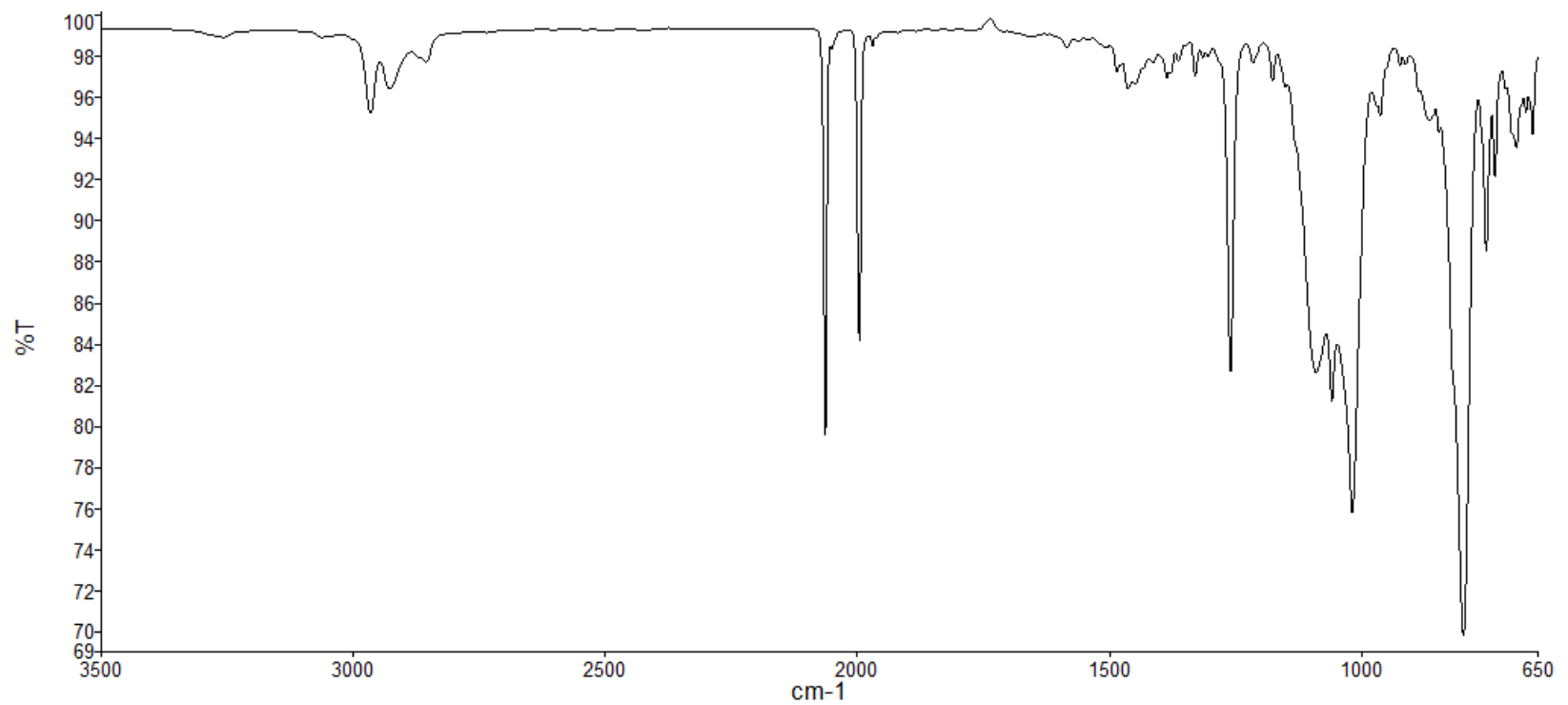

Figure 33. IR spectrum of rhodium(I) 21-methylbenzocarbaporphyrin 94 


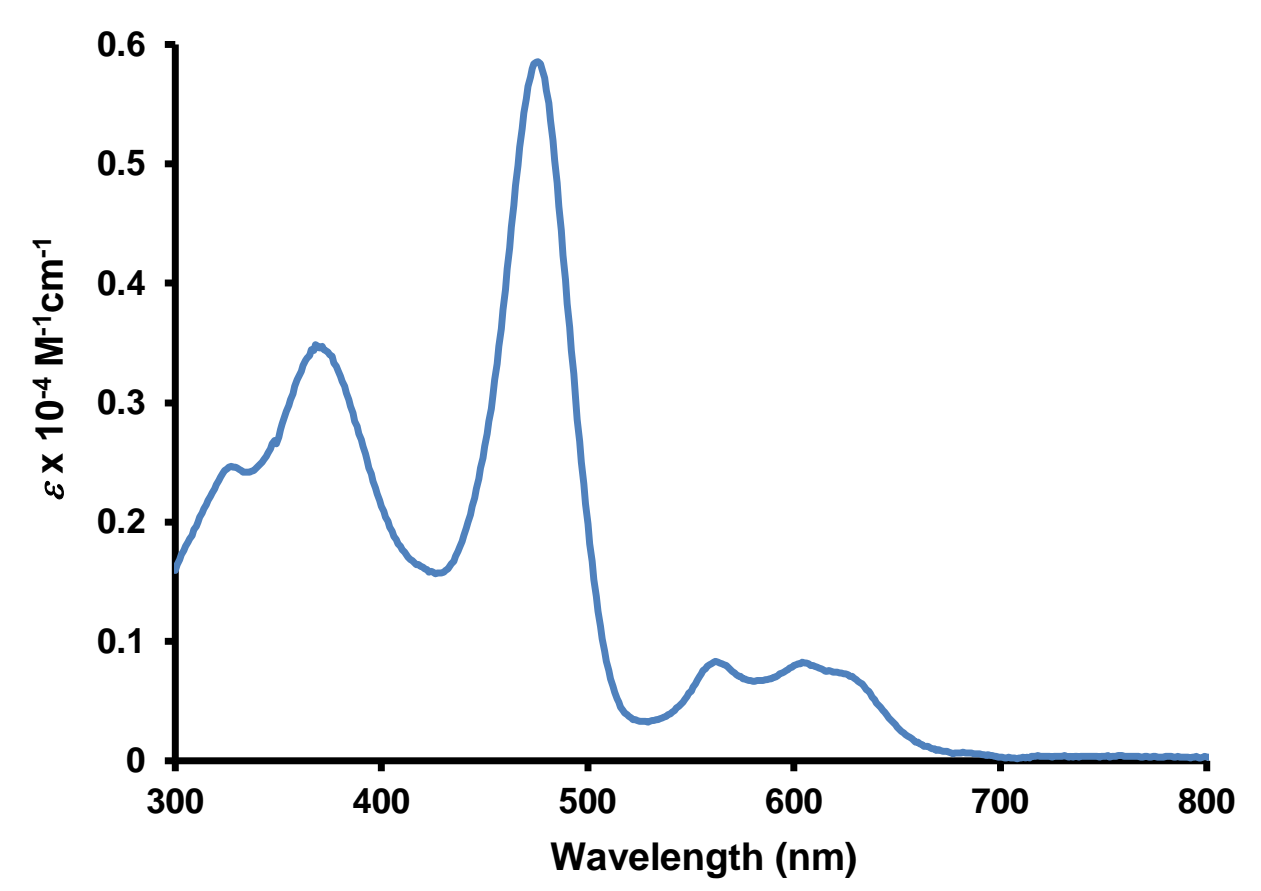

Figure 34. UV-Vis spectrum of rhodium(I) 21-methylbenzocarbaporphyrin 94

When 23-methylbenzocarbaporphyrin $\mathbf{7 6}$ was reacted under the same conditions with $\left[\mathrm{Rh}(\mathrm{CO})_{2} \mathrm{Cl}\right]_{2}$, a rhodium(I) complex of the type described above was not formed. ${ }^{111}$ This was due to the placement of the internal methyl substituent in a site that prevented coordination to two adjacent nitrogens. Nevertheless, when $\mathbf{7 6}$ was reacted with $\left[\mathrm{Rh}(\mathrm{CO})_{2} \mathrm{Cl}\right]_{2}$ in refluxing toluene, a bridged rhodium(III) complex 95 was generated in 31\% yield (Scheme 33). The crude compound was chromatographed twice on silica gel, eluting with dichloromethane, followed by recrystallization from chloroform-hexanes to give the pure product. The formation of $\mathbf{9 5}$ from $\mathbf{7 6}$ was unexpected but may involve a similar rearrangement to the one observed for palladium(II) complex 84, where the methyl group seemingly somersaults over the rhodium center. Related bridged rhodium(III) complexes were previously obtained by the ring contraction of benziporphyrin complexes. ${ }^{68}$ 

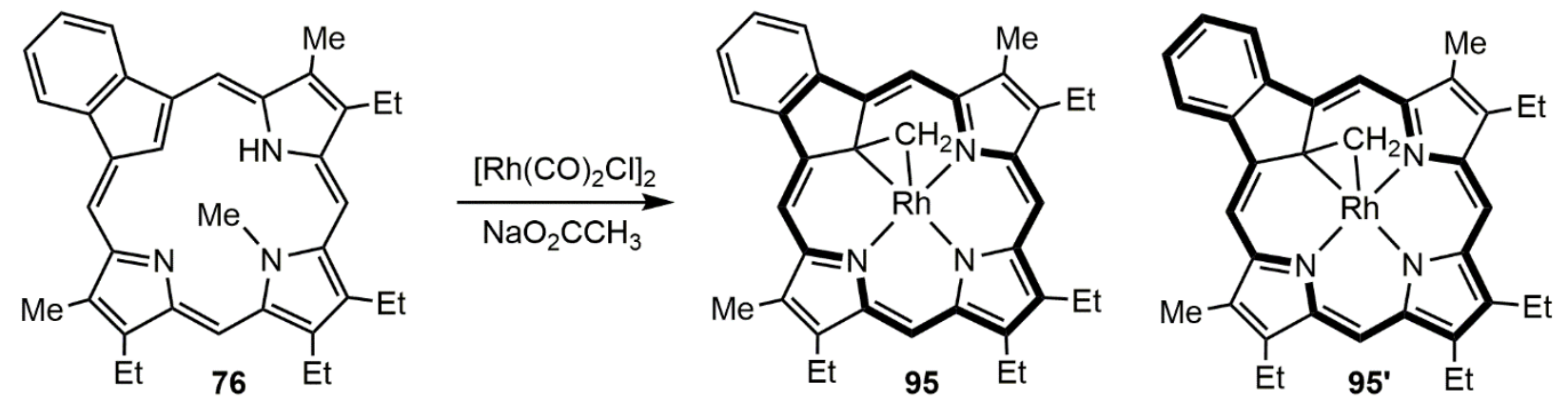

Scheme 33. Formation of rhodium derivative $\mathbf{9 5}$ of 23-methylcarbaporphyrin $\mathbf{7 6}$

The proton NMR spectrum of $\mathbf{9 5}$ shows a plane of symmetry, with the meso-proton resonances appearing as two $2 \mathrm{H}$ singlets at 9.51 and $10.03 \mathrm{ppm}$ (Figure 35). The benzo-protons are observed as two downfield multiplets at 8.16-8.20 and 9.37-9.41 ppm. The external methyl substituents were also shifted downfield and resonated as a $6 \mathrm{H}$ singlet at $3.34 \mathrm{ppm}$. The bridging $\mathrm{CH}_{2}$ unit appears as a weakly split $2 \mathrm{H}$ doublet at $-3.22 \mathrm{ppm}\left({ }^{2} J_{\mathrm{RhH}}=1.0 \mathrm{~Hz}\right)$ due to coupling with rhodium-103 $(I=1 / 2)$. The observed chemical shifts are characteristic of a strong diatropic ring current, showing that the aromaticity of the rhodium(III) bridged complex is retained. An $18 \pi-$ electron pathway is emphasized in structure 95, but a 22 $\pi$-electron circuit (see 95’ in Scheme 33) may also potentially contribute to this structure. This consideration helps to explain the pronounced downfield shifts of the benzo-protons observed in the proton-NMR spectrum. The carbon-13 NMR spectrum further demonstrates the symmetry of $\mathbf{9 5}$, and the meso-carbons gave rise to two peaks at 103.7 and $104.2 \mathrm{ppm}$ (Figure 36). The internal methylene and 21-carbon peaks gave doublets at $46.3 \mathrm{ppm}\left({ }^{1} J_{\mathrm{RhC}}=21.4 \mathrm{~Hz}\right)$ and $54.5 \mathrm{ppm}\left({ }^{1} J_{\mathrm{RhC}}=14.2 \mathrm{~Hz}\right)$, respectively. The UV-Vis spectrum of 95 (Figure 37) is very different from rhodium(I) complexes 94 and 93, showing a peak at $333 \mathrm{~nm}$, followed by a series of broad absorptions ending with a moderately strong band centered on $682 \mathrm{~nm}$. This spectrum was also dissimilar from the rhodium(I) and rhodium(III) derivatives of both naphthocarbaporphyrin $\mathbf{8 7}$ and carbaporphyrin diester $\mathbf{8 8}$. This is 
most likely due to the 18 or $22 \pi$-electron pathway being relocated through the periphery of the indene unit while bypassing C21.

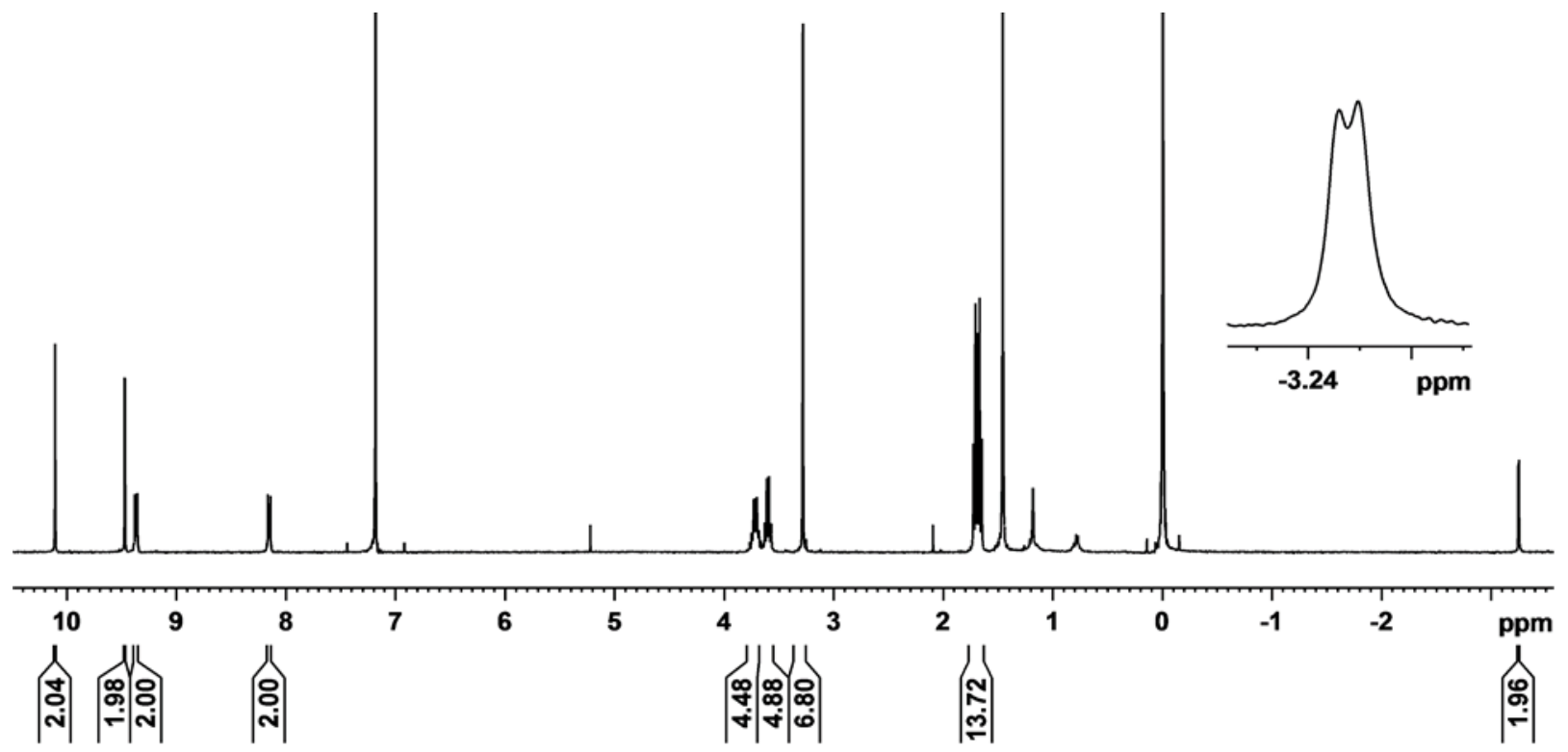

Figure 35. $400 \mathrm{MHz}{ }^{1} \mathrm{H}$ NMR spectrum of bridged rhodium(III) complex 95 in $\mathrm{CDCl}_{3}$

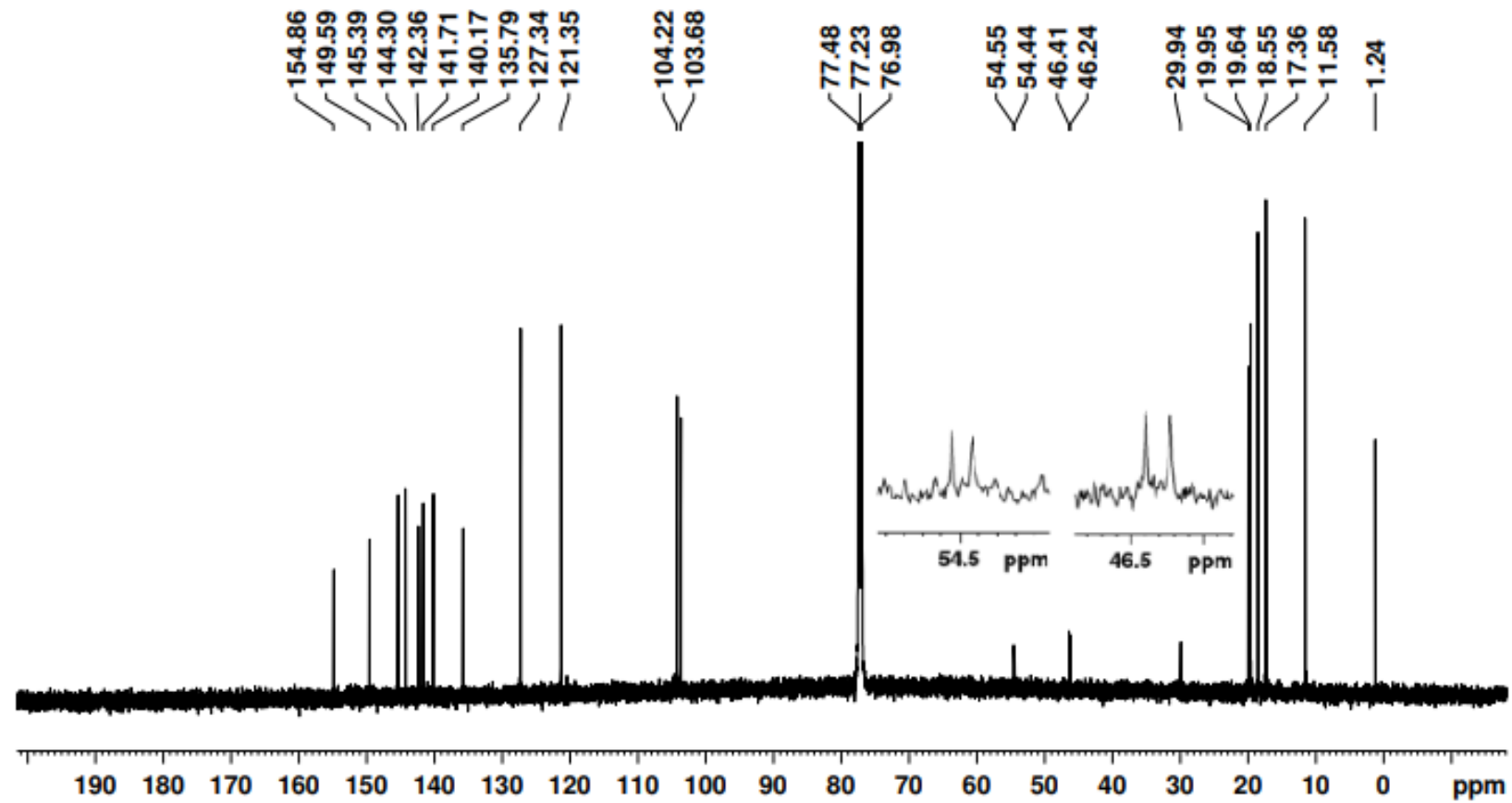

Figure 36. $100 \mathrm{MHz}{ }^{13} \mathrm{C}$ NMR spectrum of bridged rhodium(III) complex 95 in $\mathrm{CDCl}_{3}$ 


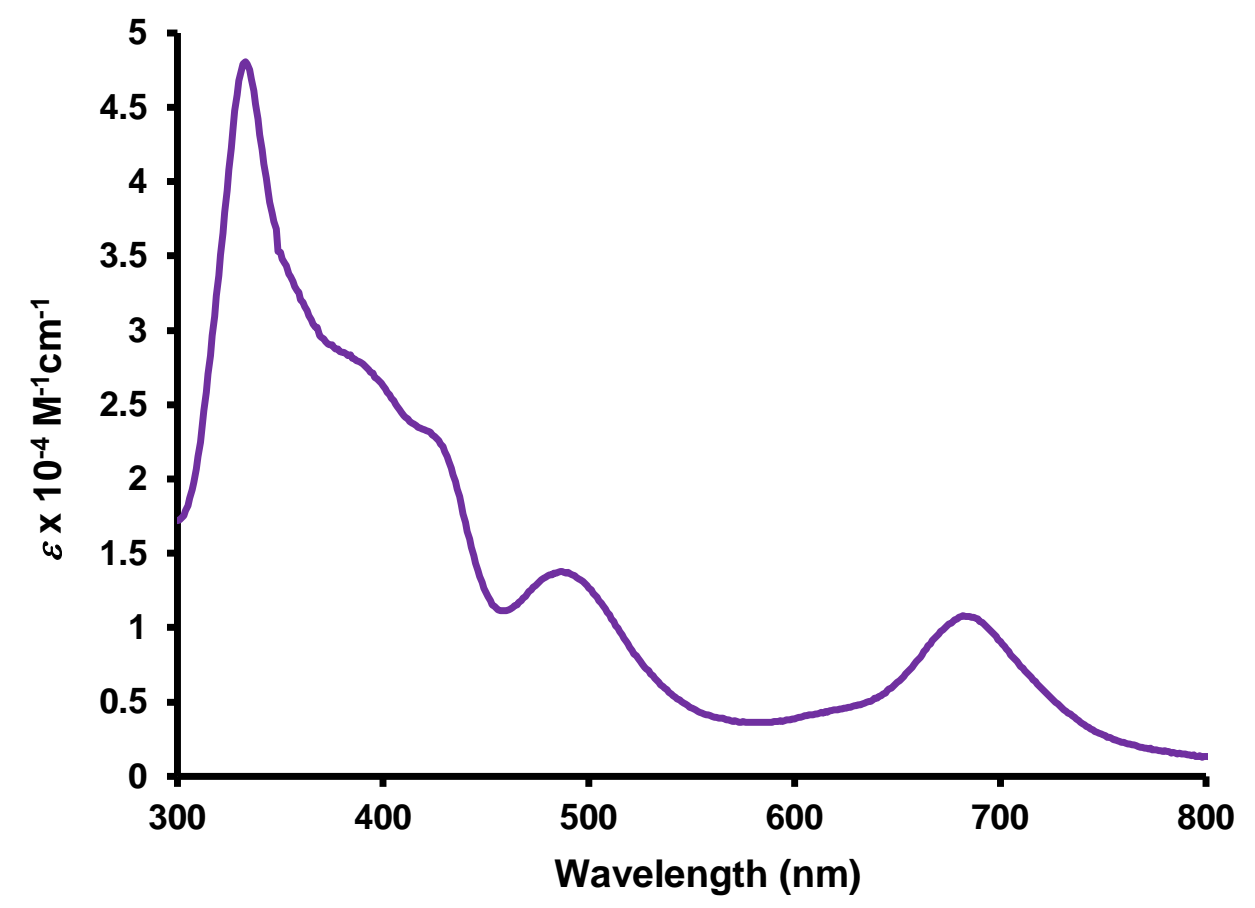

Figure 37. UV-Vis spectrum of bridged rhodium(III) complex 95

The structure of 95 was confirmed by X-ray crystallography (Figure 38). The porphyrinoid framework is remarkably similar to that of C-methyl palladium complex $\mathbf{7 2}$, with the main difference being the presence of a bridging $\mathrm{CH}_{2}$ unit. The overall framework of bond distances is consistent with an aromatic compound possessing a $\pi$-delocalization pathway that passes through the indene unit due to the presence of the $s p^{3}$ hybridized C21 atom. The bond lengths of C1-C2 and C3-C4 are consistent with this and give smaller values of ca. $1.447 \AA$ compared to the larger bond lengths of 1.463(2) and 1.462(2) A that are observed for the C1-C21 and C4-C21 bonds. The rhodium(III) center resides in a distorted square pyramidal coordination environment with the equatorial plane consisting of the C21 and the three pyrrole nitrogen atoms. The bond lengths between Rh-C21, Rh-N22, Rh-N23 and Rh-N24 are 2.1329(11) $\AA$, 2.0369(9) $\AA$, 2.0431(9) $\AA$ and 2.0410(9) Å, respectively. Additionally, the C1-C21-C25 and C4-C21-C25 bond angles are both $120.5(1)^{\circ}$, while the $\mathrm{C} 25-\mathrm{C} 21-\mathrm{Rh}$ bond angle is $64.09(6)^{\circ}$, and the methylene unit C25 lies 
1.585(1) $\AA$ out of the $\mathrm{RhN}_{3}$ plane. The rhodium(III) metal center of $\mathbf{9 5}$ has a similar coordination environment to the one seen in the related tetraphenyl rhodium(III) carbaporphyrin complex that was formed through a ring contraction of $p$-benziporphyrins. ${ }^{68}$ However, the methylene unit is separated from the Rh atom by 1.9932(1) $\AA$ in 95, which is $0.04 \AA$ shorter than was observed in the tetraphenyl rhodium(III) carbaporphyrin complex. 

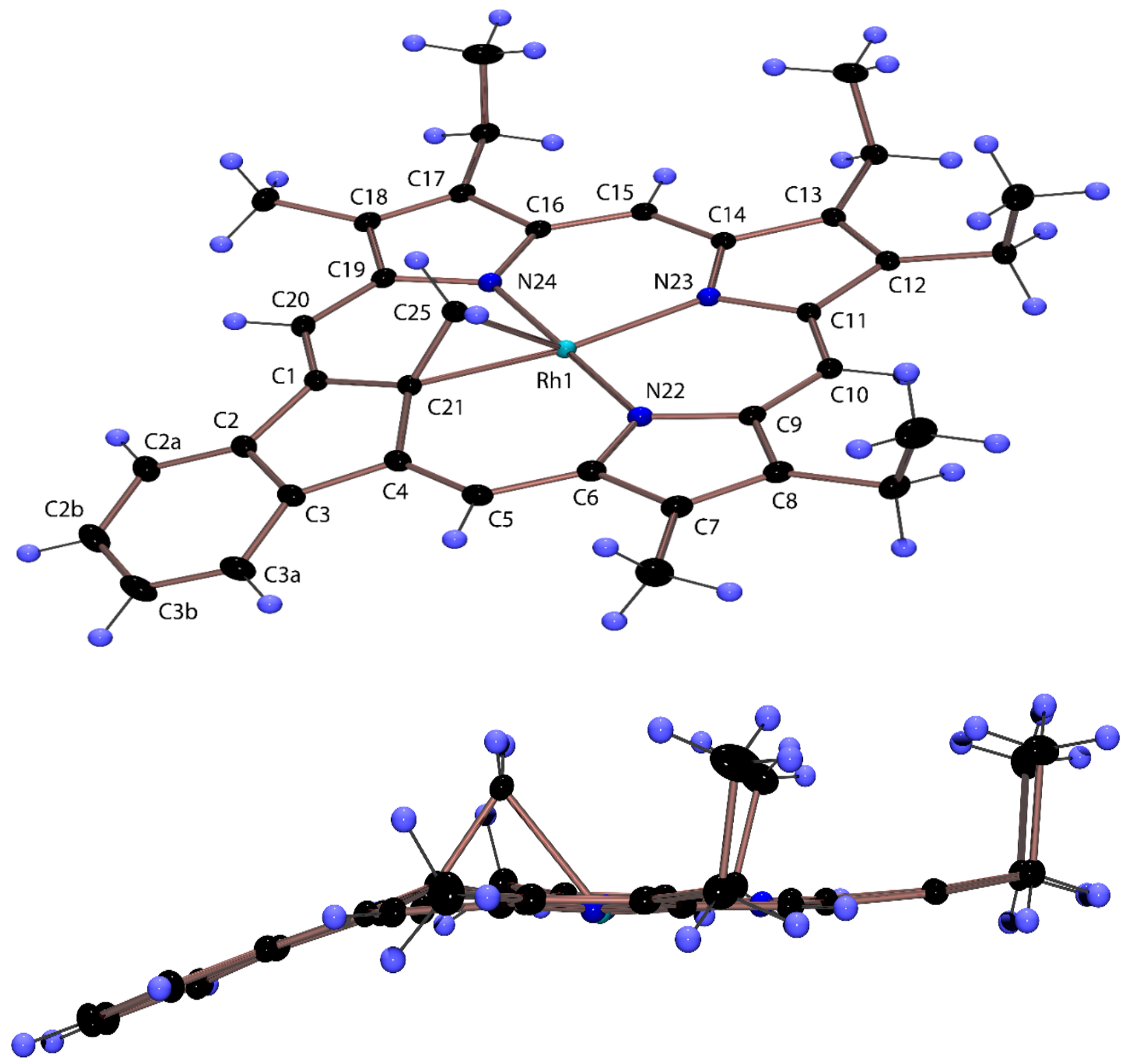

Figure 38. Color POV-Ray rendered ORTEP III drawing (50\% probability level, hydrogen atoms rendered arbitrarily small for clarity) of methylene-bridged rhodium(III) carbaporphyrin 95 


\section{Conclusion}

The rhodium(I) and rhodium(III) complexes reported in this chapter greatly expand the number of carbaporphyrinoids that can form stable complexes with rhodium metal. Carbaporphyrin diester $\mathbf{8 8}$ and naphthocarbaporphyrin $\mathbf{8 7}$ formed stable rhodium(I) and rhodium(III) complexes in a similar fashion to that of benzocarbaporphyrin 3 . In contrast, it was found that internally methylated benzocarbaporphyrins reacted differently than $\mathbf{3}$ when rhodium metal was introduced into the macrocycle cavity. 21- and 22-methylbenzocarbaporphyrins 75 and 70, respectively, formed stable rhodium(I) complexes but could not be converted into rhodium(III) derivatives. 23-Methylbenzocarbaporphyrin $\mathbf{7 6}$ did not even form this type of rhodium(I) complex, but instead rearranged to give a bridged rhodium(III) derivative $\mathbf{9 5}$. These investigations show that the inner substituent greatly impacts the coordination chemistry of carbaporphyrin systems. In the following chapter, a series of $N$-methylated porphyrinoids are described and their properties are compared to their respective $N$-unsubstituted counterparts, in order to further demonstrate the effects internal alkylation has on a number of porphyrinoid macrocycles. 


\section{Experimental:}

Melting points are uncorrected. NMR spectra were recorded using a 400 or $500 \mathrm{MHz}$ NMR spectrometer and were run at $302 \mathrm{~K}$ unless otherwise indicated. ${ }^{1} \mathrm{H}$ NMR values are reported as chemical shifts $\delta$, relative integral, multiplicity ( $\mathrm{s}$, singlet; $\mathrm{d}$, doublet; $\mathrm{t}$, triplet; q, quartet; $\mathrm{m}$, multiplet; br, broad peak) and coupling constant $(J)$. Chemical shifts are reported in parts per million (ppm) relative to $\mathrm{CDCl}_{3}\left({ }^{1} \mathrm{H}\right.$ residual $\mathrm{CHCl}_{3}$ singlet $\delta 7.26,{ }^{13} \mathrm{C} \mathrm{CDCl}_{3}$ triplet $\left.\delta 77.23\right)$ or $d_{6}$-DMSO ( ${ }^{1} \mathrm{H}$ residual $d_{5}$-DMSO pentet $\delta 2.49,{ }^{13} \mathrm{C} d_{6}$-DMSO septet $\left.\delta 39.7\right)$ and coupling constants were taken directly from the spectra. NMR assignments were made with the aid of ${ }^{1} \mathrm{H}-$ ${ }^{1} \mathrm{H}$ COSY, HSQC, DEPT-135 and nOe difference proton NMR spectroscopy. 2D experiments were performed using standard software. High-resolution mass spectra (HRMS) were carried out using a double focusing magnetic sector instrument. Samples of X-ray quality crystals were suspended in mineral oil at ambient temperature and a suitable crystal was selected, mounted on a MiTeGen Micromount and transferred to a Bruker AXS SMART APEXII CCD X-ray diffractometer. The X-ray diffraction data were collected at $100(2) \mathrm{K}$ using Mo-K $\alpha(\lambda=0.71073$ Å) radiation.

[8,12,13,17-Tetraethyl-2,3-dimethoxycarbonyl-7,18-dimethyl-21-

carbaporphyrinato](dicarbonyl)rhodium(I) (89). Carbaporphyrin diester $\mathbf{8 8}^{29}$ (60.2 mg, 0.106 mmol) was dissolved in dichloromethane $(111 \mathrm{~mL})$ under nitrogen. Anhydrous sodium acetate (84.7 mg) was then added, followed by tetracarbonyldi- $\mu$-chlorodirhodium(I) (40.4 mg, 0.10 mmol), and the mixture refluxed overnight under nitrogen. The solvent was removed under reduce pressure and the residue was chromatographed on grade 3 alumina eluting with chloroform. A dark green band was collected, and recrystallized with chloroform-hexanes to afford the rhodium(I) carbaporphyrin (76.2 mg, $0.105 \mathrm{mmol}$, 99\%) as dark crystals, $\mathrm{mp}>300{ }^{\circ} \mathrm{C}$. IR (ZnSe): $v_{\mathrm{Co}} / \mathrm{cm}^{-1}$ 
2066, 2011, 1698. UV-vis $\left(\mathrm{CH}_{2} \mathrm{Cl}_{2}\right): \lambda_{\max } / \mathrm{nm}$ (log $\left.\varepsilon\right) 357$ (4.66), 490 (4.61), 569 (4.00), 611 (3.74), 723 (3.27). ${ }^{1} \mathrm{H}$ NMR (500 MHz, $\left.\mathrm{CDCl}_{3}\right): \delta-4.94$ (1H, s, 21-H), -1.09 (1H, s, NH), 1.60 (3H, t, $J=$ 7.7 Hz), 1.72-1.76 (9H, m) (4 x CH $\left.2 \mathrm{CH}_{3}\right), 3.35$ (3H, s, 7-Me), 3.45 (3H, s, 18-Me), 3.63-3.71 (1H, m), 3.72-3.86 (3H, m), 3.87-3.96 (4H, m) $\left(4 \times \mathrm{CH}_{2} \mathrm{CH}_{3}\right), 4.21$ (3H, s, 3- $\left.\mathrm{CO}_{2} \mathrm{Me}\right), 4.26(3 \mathrm{H}, \mathrm{s}, 2-$ CO $\left.{ }_{2} \mathrm{Me}\right), 9.40(1 \mathrm{H}, \mathrm{s}), 9.44(1 \mathrm{H}, \mathrm{s})(10,15-\mathrm{H}), 10.02(1 \mathrm{H}, \mathrm{s}, 20-\mathrm{H}), 10.37$ (1H, s, 5-H). ${ }^{13} \mathrm{C}$ NMR (125 MHz, $\left.\mathrm{CDCl}_{3}\right): \delta 11.4$ (18-Me), 12.1 (7-Me), 17.1, 17.3, 17.8, 18.0, 19.6, 19.7, 19.8, 20.4, 52.4 (3- $\left.\mathrm{CO}_{2} \mathrm{CH}_{3}\right), 52.5$ (2- $\left.\mathrm{CO}_{2} \mathrm{CH}_{3}\right), 92.4$ (10- or 15-CH), 102.8 (10- or 15- $\left.\mathrm{CH}\right), 107.5$ (20-CH), 108.9 (21-CH), 116.7 (5-CH), 128.3, 128.4, 132.2, 133.6, 135.8, 135.9, 137.9, 138.4, 138.55, 138.63, 141.9, 144.8, 150.5, 150.6, 158.8, 158.1, 167.2 (ester C=O), 167.8 (ester C=O), 177.0 (d, $\left.{ }^{1} J_{\mathrm{Rh}-\mathrm{C}}=69.9 \mathrm{~Hz}, \mathrm{CO}\right), 178.7\left(\mathrm{~d},{ }^{1} J_{\mathrm{Rh}-\mathrm{C}}=67.7 \mathrm{~Hz}, \mathrm{CO}\right)$. HR-MS $(\mathrm{ESI}) \mathrm{m} / \mathrm{z}:[\mathrm{M}+\mathrm{H}]^{+}$calcd for $\mathrm{C}_{37} \mathrm{H}_{39} \mathrm{~N}_{3} \mathrm{O}_{6} \mathrm{Rh} 724.1894$, found 724.1887.

[8,12,13,17-Tetraethyl-7,18-dimethyl-21-carbanaphtho[2,3-

b]porphyrinato](dicarbonyl)rhodium(I) (91). Naphthocarbaporphyrin 87 $^{108}$ (20.1 mg, 0.0365 mmol) was reacted with $\left[\mathrm{Rh}(\mathrm{CO})_{2} \mathrm{Cl}\right]_{2}(15.2 \mathrm{mg}, 0.039 \mathrm{mmol})$ and sodium acetate $(29.8 \mathrm{mg})$ under the foregoing conditions. The product was purified on a silica gel column eluting with 20:80 dichloromethane-hexanes and a green band was collected. The solvent was evaporated and the residue recrystallized from chloroform-hexanes to yield the naphthocarbaporphyrin complex (18.5 mg, $0.0261 \mathrm{mmol}, 72 \%$ ) as dark crystals, $\mathrm{mp}>300^{\circ} \mathrm{C}$. IR (ZnSe): $v_{\mathrm{co}} / \mathrm{cm}^{-1} 2065,2017,1993$. UVvis $\left(\mathrm{CH}_{2} \mathrm{Cl}_{2}\right): \lambda_{\max } / \mathrm{nm}$ (log $\left.\varepsilon\right) 378$ (4.69), 481 (5.03), 566 (4.11), 603 (4.06), 624 (sh, 3.94), 680 (2.73). ${ }^{1} \mathrm{H}$ NMR (500 MHz, $\left.\mathrm{CDCl}_{3}\right): \delta-5.19(1 \mathrm{H}, \mathrm{s}, 21-\mathrm{H}),-2.48(1 \mathrm{H}, \mathrm{s}, \mathrm{NH}), 1.68(3 \mathrm{H}, \mathrm{t}, J=7.7$ $\mathrm{Hz}), 1.78-1.85$ (9H, 3 overlapping triplets) $\left(4 \times \mathrm{CH}_{2} \mathrm{CH}_{3}\right), 3.54$ (3H, s), 3.65 (3H, s) (7,18- $\left.\mathrm{CH}_{3}\right)$, 3.78-3.92 (2H, m), 3.94-4.02 (2H, m), 4.04-4.12 (4H, m) $\left(4 \times \mathrm{CH}_{2} \mathrm{CH}_{3}\right), 7.58-7.63\left(2 \mathrm{H}, \mathrm{m}, 2^{3}, 3^{3}-\right.$ H), 8.23-8.28 (2H, m, $\left.2^{2}, 3^{2}-H\right), 9.10(1 \mathrm{H}, \mathrm{s}), 9.15(1 \mathrm{H}, \mathrm{s})\left(2^{1}, 3^{1}-\mathrm{H}\right), 9.78(1 \mathrm{H}, \mathrm{s}), 9.83(1 \mathrm{H}, \mathrm{s})$ 
(10,15-H), 10.09 (1H, s), 10.34 (1H, s) (5,20-H). ${ }^{13} \mathrm{C}$ NMR (125 MHz, CDCl $): \delta 11.6,12.3,17.4$ 17.5, 18.2, 18.3, 19.9, 19.96, 19.98, 20.6, 93.8, 97.5, 103.1, 105.0, 119.0, 119.2, 123.0 (21-CH), 125.6, 125.7, 128.9, 129.1, 132.0, 132.61, 132.63, 133.8, 133.9, 135.0. 136.3, 139.0, 139.2, 139.7, 140.1, 142.3, 143.4, 146.3, 147.5, 153.69, 153.71, 155.2, 177.6 (d, $\left.{ }^{1} J_{\mathrm{Rh}-\mathrm{C}}=69.0 \mathrm{~Hz}, \mathrm{CO}\right), 178.4$ (d, ${ }^{1} J_{\mathrm{Rh}-\mathrm{C}}=67.1 \mathrm{~Hz}, \mathrm{CO}$ ). HR-MS (ESI) m/z: $[\mathrm{M}+\mathrm{H}]^{+}$calcd for $\mathrm{C}_{41} \mathrm{H}_{39} \mathrm{~N}_{3} \mathrm{O}_{2} \mathrm{Rh}$ 708.2097, found 708.2089.

[8,12,13,17-Tetraethyl-2,3-dimethoxycarbonyl-7,18-dimethyl-21-

carbaporphyrinato](dipyridine)rhodium(III) (90). Rhodium(I) complex 89 (10.0 mg, 0.0138 mmol) was dissolved in pyridine $(10 \mathrm{~mL})$ and refluxed under nitrogen in a preheated oil bath for 30 min. The solvent was removed under reduced pressure and the residue chromatographed on grade 3 alumina eluting with toluene. A dark band was collected and recrystallized with chloroform-hexanes to yield rhodium(III) carbaporphyrin 90 (3.2 mg, $0.0039 \mathrm{mmol}, 28 \%$ ) as dark crystals, mp $>300{ }^{\circ} \mathrm{C}$. UV-vis $\left(\mathrm{CH}_{2} \mathrm{Cl}_{2}\right): \lambda_{\max } / \mathrm{nm}$ (log $\left.\varepsilon\right) 356$ (4.44), 393 (4.43), 427 (sh, 4.50), 450 (sh, 4.59), 463 (4.63), 531 (3.92), 573 (4.14). ${ }^{1} \mathrm{H}$ NMR (500 MHz, $\mathrm{C}_{6} \mathrm{D}_{6}$ ): $\delta 1.74$ (6H, t, $J=7.5$ Hz), 1.82 (6H, t, $J=7.5 \mathrm{~Hz}$ ), 2.33 (4H, d, $J=5.9$ Hz, 4 x $\alpha$-pyridine-H), 3.25 (6H, s, 17,18-Me), 3.73-3.80 (8H, m, 4 x CH $\mathrm{CH}_{2} \mathrm{CH}_{3}$ ), 4.02 (6H, s, 2 x OMe), 4.09-4.12 (4H, m, 4 x $\beta$-pyridine-H), 4.70 (2H, dt, J = 1.6, 7.6 Hz, 2 x $\gamma$-pyridine-H), 9.46 (2H, s, 10,15-H), 10.59 (2H, s, 5,20-H). ${ }^{13} \mathrm{C}$ NMR (125 MHz, $\left.\mathrm{CDCl}_{3}\right): \delta 11.3$ (7,18-Me), 17.6, 18.3, 19.9, 20.2, 51.4 (2 x OMe), 94.2 (10.15-CH), 113.1 (5,20-CH), 121.2 (4 x $\beta$-pyridine-CH), 129.5, 133.6 (2 x $\gamma$-pyridine-CH), 136.2, 138.4, 139.1, 139.2, 141.8, 142.2, 142.5, 148.5 (4 x $\alpha$-pyridine-CH), 167.7 (2 x C=O). HR-MS (ESI) m/z: calcd for $\mathrm{C}_{45} \mathrm{H}_{46} \mathrm{~N}_{5} \mathrm{O}_{4} \mathrm{Rh}$ 823.2605, found 823.2638. 
[8,12,13,17-Tetraethyl-7,18-dimethyl-21-carbanaphtho[2,3-

b]porphyrinato](dipyridine)rhodium(III) (92). Rhodium(I) naphthocarbaporphyrin 91 (18.5 mg, $0.0261 \mathrm{mmol})$ was dissolved in pyridine $(17 \mathrm{~mL})$ and refluxed under nitrogen for $1 \mathrm{~h}$. The solvent was removed under reduced pressure and the residue purified on a grade 3 alumina column eluting with 50:50 toluene-hexanes. A light brown band was collected and recrystallized with chloroformhexanes to yield the rhodium(III) complex (4.5 mg, $0.0056 \mathrm{mmol}$, 21\%) as dark crystals, mp >300 ${ }^{\circ} \mathrm{C}$. UV-vis $\left(\mathrm{CH}_{2} \mathrm{Cl}_{2}\right): \lambda_{\max } / \mathrm{nm}$ (log $\left.\varepsilon\right) 348$ (4.40), 422 (sh, 4.56), 434 (sh, 4.68), 447 (5.04), 458 (sh, 4.68), 530 (3.99), 569 (4.24), 606 (3.47), 851 (3.65). ${ }^{1} \mathrm{H}$ NMR (500 MHz, $\mathrm{C}_{6} \mathrm{D}_{6}, 60{ }^{\circ} \mathrm{C}$ ): $\delta 1.44$ (4H, d, $J=6.2 \mathrm{~Hz}, 4 \mathrm{x} \alpha$-pyridine-H), $1.94(6 \mathrm{H}, \mathrm{t}, J=7.6 \mathrm{~Hz}), 1.99(6 \mathrm{H}, \mathrm{t}, J=7.6 \mathrm{~Hz})(4 \mathrm{x}$ $\mathrm{CH}_{2} \mathrm{CH}_{3}$ ), 3.59 (6H, s, 7,18-Me), 3.92 (4H, t, $J=7.1 \mathrm{~Hz}, 4$ x $\beta$-pyridine-H), 4.06-4.14 (8H, m, 4 x $\left.\mathrm{CH}_{2} \mathrm{CH}_{3}\right), 4.58\left(2 \mathrm{H}, \mathrm{t}, J=7.1 \mathrm{~Hz}, 2\right.$ x $\gamma$-pyridine-H), 7.45-7.48 (2H, m, $\left.2^{3}, 3^{3}-\mathrm{H}\right), 8.15-8.18(2 \mathrm{H}$, m, $\left.2^{2}, 3^{2}-\mathrm{H}\right), 9.18\left(2 \mathrm{H}, \mathrm{s}, 2^{1}, 3^{1}-\mathrm{H}\right), 10.07$ (2H, s, 10,15-H), 10.56 (2H, s, 5,20-H). ${ }^{13} \mathrm{C}$ NMR $(125$ $\left.\mathrm{MHz}, \mathrm{C}_{6} \mathrm{D}_{6}, 60{ }^{\circ} \mathrm{C}\right): \delta 11.8,18.1,18.6,20.3,20.7,94.7,102.8,115.6,120.6$ (4 x $\beta$-pyridine-CH), 14.9, 129.0, 132.1, 133.1 (2 x $\gamma$-pyridine-CH), 133.2, 134.5, 138.2, 139.2, 139.6, 140.3, 140.4, 146.9, 148.1 (4 x $\alpha$-pyridine-CH). HR-MS (ESI) m/z: $[\mathrm{M}+\mathrm{H}]^{+}$calcd for $\mathrm{C}_{49} \mathrm{H}_{46} \mathrm{~N}_{5} \mathrm{Rh}$ 807.2808, found 807.2798.

[8,12,13,17-Tetraethyl-7,18,22-trimethylbenzo[b]carbaporphyrinato](dicarbonyl)rhodium(I) (93). 22-Methylbenzocarbaporphyrin 70 (10.4 mg, $0.0203 \mathrm{mmol}$ ) was dissolved in dichloromethane $(22 \mathrm{~mL})$ under nitrogen. Anhydrous sodium acetate $(16.3 \mathrm{mg})$ was added, followed by $\left[\mathrm{Rh}(\mathrm{CO})_{2} \mathrm{Cl}\right]_{2}(7.8 \mathrm{mg}, 0.0201 \mathrm{mmol})$, and the mixture was refluxed overnight under nitrogen. The solvent was removed under reduced pressure, and the residue purified by column chromatography on silica gel eluting with dichloromethane-hexanes (50:50). The rhodium complex was collected as a brown band. Recrystallization from chloroform- 
hexanes afforded the 93 (6.8 $\mathrm{mg}, 0.010 \mathrm{mmol}, 50 \%)$ as dark crystals, $\mathrm{mp}>300{ }^{\circ} \mathrm{C}$. UV-vis $\left(\mathrm{CH}_{2} \mathrm{Cl}_{2}\right): \lambda_{\max } / \mathrm{nm}$ (log $\left.\varepsilon\right) 319$ (sh, 4.39), 360 (4.57), 407 (sh, 4.44), 481 (4.85), 561 (4.07), 586 (sh, 3.83), 637 (3.68). IR (ZnSe): $v_{\mathrm{CO}}$ 1985, $2057 \mathrm{~cm}^{-1} .{ }^{1} \mathrm{H}$ NMR (500 MHz, $\left.\mathrm{CDCl}_{3}\right): \delta$-4.68 (1H, s, 21-H), -4.50 (3H, s, N-Me), $1.56\left(3 \mathrm{H}, \mathrm{t},{ }^{3} J_{\mathrm{HH}}=7.7 \mathrm{~Hz}\right), 1.68\left(3 \mathrm{H}, \mathrm{t},{ }^{3} J_{\mathrm{HH}}=7.7 \mathrm{~Hz}\right), 1.76(3 \mathrm{H}, \mathrm{t}$, $\left.{ }^{3} J_{\mathrm{HH}}=7.7 \mathrm{~Hz}\right), 1.79\left(3 \mathrm{H}, \mathrm{t},{ }^{3} J_{\mathrm{HH}}=7.7 \mathrm{~Hz}\right)\left(4 \times \mathrm{CH}_{2} \mathrm{CH}_{3}\right), 3.36(3 \mathrm{H}, \mathrm{s}), 3.52$ (3H, s) (7,18-Me), 3.56-3.69 (2H, m), 3.76-3.99 (6H, m) $\left(4 \times \mathrm{CH}_{2} \mathrm{CH}_{3}\right), 7.66-7.71\left(2 \mathrm{H}, \mathrm{m}, 2^{2}, 3^{2}-\mathrm{H}\right), 8.74-8.78(2 \mathrm{H}$, m, $\left.2^{1}, 3^{1}-\mathrm{H}\right), 9.640(1 \mathrm{H}, \mathrm{s}), 9.643(1 \mathrm{H}, \mathrm{s})(10,15-\mathrm{H}), 9.97(1 \mathrm{H}, \mathrm{s}), 10.16(1 \mathrm{H}, \mathrm{s})(5,20-\mathrm{H}) .{ }^{13} \mathrm{C}$ NMR (125 MHz, $\left.\mathrm{CDCl}_{3}\right): \delta$ 12.0, 12.3, 15.8, 17.6, 18.3, 18.4, 19.9, 20.0, 20.1, 20.6, 97.1, 102.5, 104.6, 106.6, 120.32, 120.35, 121.2 (br), 126.6, 130.1, 132.3, 132.9, 134.8, 136.5, 139.2, 139.4, 142.2, 143.7, 144.4, 147.8, 148.4, 149.1, 150.0, 152.5, 155.0, $178.8\left(\mathrm{~d},{ }^{1} J_{\mathrm{RhC}}=68.5 \mathrm{~Hz}, \mathrm{CO}\right), 179.4(\mathrm{~d}$, ${ }^{1} J_{\mathrm{RhC}}=69.4 \mathrm{~Hz}, \mathrm{CO}$ ). HR-MS (ESI) $\mathrm{m} / \mathrm{z}$ : $[\mathrm{M}+\mathrm{H}]^{+}$Calcd for $\mathrm{C}_{38} \mathrm{H}_{39} \mathrm{~N}_{3} \mathrm{O}_{2} \mathrm{Rh}$ 672.2097; found 672.2104 .

\section{$[8,12,13,17-$ Tetraethyl-7,18,21-}

trimethylbenzo[b]carbaporphyrinato](dicarbonyl)rhodium(I) (94). Anhydrous sodium acetate (11.4 mg) was added, followed by $\left[\mathrm{Rh}(\mathrm{CO})_{2} \mathrm{Cl}\right]_{2}(5.5 \mathrm{mg}, 0.0142 \mathrm{mmol})$, to a solution of 21methylbenzocarbaporphyrin $75(7.0 \mathrm{mg}, 0.0136 \mathrm{mmol})$ in dichloromethane $(15 \mathrm{~mL})$ under nitrogen. The resulting mixture was heated under reflux overnight. The solvent was removed under reduced pressure and the residue purified by column chromatography on silica gel eluting with dichloromethane-hexanes (50:50). The complex eluted as a green band. Recrystallization from chloroform-hexanes gave the rhodium(I) complex (6.2 $\mathrm{mg}, 0.00924 \mathrm{mmol}, 67 \%)$ as as dark crystals, mp $>300{ }^{\circ} \mathrm{C}$. UV-vis $\left(\mathrm{CH}_{2} \mathrm{Cl}_{2}\right): \lambda_{\max } / \mathrm{nm}$ (log $\left.\varepsilon\right) 327$ (4.18), 368 (4.34), 476 (4.56), 562 (3.71), 604 (3.71), 627 (3.64). IR (ZnSe): $v_{\mathrm{CO}}$ 1996, $2063 \mathrm{~cm}^{-1} .{ }^{1} \mathrm{H}$ NMR (500 MHz, $\left.\mathrm{CDCl}_{3}\right): \delta-$ 5.66 (3H, s, N-Me), -2.83 (1H, br s, NH), 1.69 (3H, t, $\left.{ }^{3} J_{\mathrm{HH}}=7.7 \mathrm{~Hz}\right), 1.78-1.83$ (9H, 3 overlapping 
triplets) (4 x CH $\left.2 \mathrm{CH}_{3}\right), 3.35$ (3H, s), 3.52 (3H, s) (7,18-Me), 3.67-3.85 (3H, m), 3.88-4.01 (5H, m) (4 x CH $\left.\mathrm{CH}_{3}\right), 7.35\left(1 \mathrm{H}, \mathrm{dt},{ }^{4} J_{\mathrm{HH}}=0.9 \mathrm{~Hz},{ }^{3} J_{\mathrm{HH}}=7.3 \mathrm{~Hz}\right), 7.41\left(1 \mathrm{H}, \mathrm{dt},{ }^{4} J_{\mathrm{HH}}=0.9 \mathrm{~Hz},{ }^{3} J_{\mathrm{HH}}=7.4\right.$ Hz) $\left(2^{2}, 3^{2}-\mathrm{H}\right), 7.97\left(1 \mathrm{H}, \mathrm{d},{ }^{3} J_{\mathrm{HH}}=7.2 \mathrm{~Hz}\right), 8.20\left(1 \mathrm{H}, \mathrm{d},{ }^{3} J_{\mathrm{HH}}=7.3 \mathrm{~Hz}\right)\left(2^{1}, 3^{1}-\mathrm{H}\right), 9.52(1 \mathrm{H}, \mathrm{s}), 9.56$ (1H, s) (10,15-H), $9.69(1 \mathrm{H}, \mathrm{s}), 9.82(1 \mathrm{H}, \mathrm{s})(5,20-\mathrm{H}) .{ }^{13} \mathrm{C}$ NMR $\left(125 \mathrm{MHz}, \mathrm{CDCl}_{3}\right): \delta 11.5,11.8$ (21-Me), 12.3, 17.4, 17.6, 18.0, 18.3, 19.75, 19.84, 19.91, 20.5, 93.0, 99.9, 103.5, 108.6, 120.0, 120.2, 126.1, 126.2, 127.8, 131.5, 132.8, 132.89, 132.94, 134.7, 136.1, 137.5, 139.1, 139.9, 140.1, 141.6, 143.2, 145.8, 147.7, 153.1, 154.8, $177.6\left(\mathrm{~d},{ }^{1} J_{\mathrm{RhC}}=67.0 \mathrm{~Hz}, \mathrm{CO}\right), 179.0\left(\mathrm{~d},{ }^{1} J_{\mathrm{RhC}}=66.2\right.$ Hz, CO). HR-MS (ESI) m/z: [M + H] $]^{+}$Calcd for $\mathrm{C}_{38} \mathrm{H}_{39} \mathrm{~N}_{3} \mathrm{O}_{2} \mathrm{Rh}$ 672.2097; found 672.2088.

[8,12,13,17-Tetraethyl-7,18-dimethyl-21-methylene-

benzo[b]carbaporphyrinato]rhodium(III) (95). 23-Methylbenzocarbaporphyrin 76 (20.0 mg, $0.0390 \mathrm{mmol}$ ) was dissolved in toluene $(40 \mathrm{~mL})$ under nitrogen. Anhydrous sodium acetate (33.3 mg) was added, followed by $\left[\mathrm{Rh}(\mathrm{CO})_{2} \mathrm{Cl}\right]_{2}(14.3 \mathrm{mg}, 0.0368 \mathrm{mmol})$, and the mixture refluxed overnight. The solvent was removed under reduced pressure and the residue was purified twice by column chromatography on silica gel eluting with dichloromethane. The product eluted as a dark band. Recrystallization from chloroform-methanol gave the rhodium(III) complex (7.4 mg, 0.0121 mmol, 31\%) as dark crystals, $\mathrm{mp}>300{ }^{\circ} \mathrm{C}$. UV-vis $\left(\mathrm{CH}_{2} \mathrm{Cl}_{2}\right): \lambda_{\max } / \mathrm{nm}(\log \varepsilon) 333$ (4.68), 386 (sh, 4.45), 424 (sh, 4.36), 486 (4.14), 682 (4.03). ${ }^{1} \mathrm{H}$ NMR (400 MHz, $\left.\mathrm{CDCl}_{3}\right): \delta-3.22$ (2H, br d, ${ }^{2} J_{\mathrm{RhH}}$ $\left.=1.0 \mathrm{~Hz}, \mathrm{Rh}-\mathrm{CH}_{2}\right), 1.74\left(6 \mathrm{H}, \mathrm{t},{ }^{3} J_{\mathrm{HH}}=7.7 \mathrm{~Hz}, 8,17-\mathrm{CH}_{2} \mathrm{CH}_{3}\right), 1.78\left(6 \mathrm{H}, \mathrm{t},{ }^{3} \mathrm{~J}_{\mathrm{HH}}=7.7 \mathrm{~Hz}, 12,13-\right.$ $\mathrm{CH}_{2} \mathrm{CH}_{3}$ ), 3.34 (6H, s, 7,18-Me), 3.62-3.70 (4H, m, 8,17- $\left.\mathrm{CH}_{2}\right)$, 3.72-3.83 (4H, m, 12,13- $\mathrm{CH}_{2}$ ), 8.16-8.20 (2H, m, $\left.2^{2}, 3^{2}-\mathrm{H}\right), 9.37-9.41\left(2 \mathrm{H}, \mathrm{m}, 2^{1}, 3^{1}-\mathrm{H}\right), 9.51(2 \mathrm{H}, \mathrm{s}, 10,15-\mathrm{H}), 10.12$ (2H, s, 5,20H). ${ }^{13} \mathrm{C}$ NMR (100 MHz, $\left.\mathrm{CDCl}_{3}\right): \delta 11.6(7,18-\mathrm{Me}), 17.4\left(8,17-\mathrm{CH}_{2} \mathrm{CH}_{3}\right), 18.5\left(12,13-\mathrm{CH}_{2} \mathrm{CH}_{3}\right)$, $19.6\left(12,13-\mathrm{CH}_{2}\right), 19.9\left(8,17-\mathrm{CH}_{2}\right), 46.3\left(\mathrm{~d},{ }^{1} J_{\mathrm{RhC}}=21.4 \mathrm{~Hz}, \mathrm{Rh}-\mathrm{CH}_{2}\right), 54.5\left(\mathrm{~d},{ }^{1} J_{\mathrm{RhC}}=14.2 \mathrm{~Hz}\right.$, 21-C), 103.7 (10,15-CH), 104.2 (5,20-CH), 121.3 ( $\left.2^{1}, 3^{1}-\mathrm{CH}\right), 127.3\left(2^{2}, 3^{2}-\mathrm{CH}\right), 135.8,140.2$, 
141.7, 142.4, 144.3, 145.4, 149.6, 154.9. HR-MS (EI) m/z: Calcd for $\mathrm{C}_{36} \mathrm{H}_{36} \mathrm{~N}_{3} \mathrm{Rh}$ 613.1964; found 613.1659. 


\section{CHAPTER IV: SYNTHESIS AND CHARACTERIZATION OF N-METHYLATED PORPHYRINOIDS}

\section{Introduction}

Upon the successful synthesis and metalation of 23-methylbenzocarbaporphyrin 76, attempts were made to generate a series of porphyrinoids from $N$-methyl tripyrrane unit 54d. Using the ' $3+1$ ' variant of the MacDonald condensation, a number of dialdehyde units were condensed with 54d to give porphyrinoid systems containing an internal methyl group. It is of interest to compare the spectroscopic properties of the methylated derivatives with the previously reported $\mathrm{N}$-unsubstituted macrocycles in order to determine how the internal alkyl substituent affects these porphyrinoid systems. In this chapter, the synthesis and characterization of $N$-methylated porphyrinoids is discussed. NMR and UV-vis spectra are used to identify differences in the properties of the macrocycles due to the presence of internal alkylation.

\section{Results and Discussion}

The synthesis of oxa- or thiaporphyrins containing an internal methyl group at the 23position will simultaneously test the effect of introducing both a heteroatom (O or S) and an $\mathrm{N}$ alkyl substituent. 21-Oxaporphyrin 96a and the related $N$-methylated derivative $\mathbf{9 6 \mathbf { b }}$ were both prepared and their properties contrasted. Reaction of tripyrrane dicarboxylic acid 54b with furan dialdehyde 56a under dilute conditions (99 $\mathrm{mL}$ of dichloromethane for $100 \mathrm{mg}$ of tripyrrane) in the presence of TFA, followed by oxidation with ferric chloride, gave oxaporphyrin 96a (Scheme 34). Tripyrrane 54d similarly reacted with $\mathbf{5 6 a}$ to afford the $N$-methyl analogue $\mathbf{9 6 b}$ (Scheme 34), 
although better results were obtained under fairly concentrated conditions $(<20 \mathrm{~mL}$ dichloromethane for $100 \mathrm{mg}$ of tripyrrane). Crude $N$-methyl derivative $\mathbf{9 6 \mathbf { b }}$ was chromatographed twice on neutral alumina eluting with chloroform, and a dark band was collected each time. The fractions were recrystallized from chloroform-hexanes to give the pure product as dark crystals in 42.3\%. $N$-Unsubstituted oxaporphyrin 96a was purified by chromatography on neutral alumina, eluting with chloroform, and a bright red/pink band was collected. The fractions were recrystallized from chloroform-hexanes to give $N$-unsubstituted oxaporphyrin 96a in 66.2\% yield. A related 21-oxaporphyrin 96c was synthesized by Johnson et al. ${ }^{57}$ that differed only by one of the external substituents and was isolated as a monocationic species.

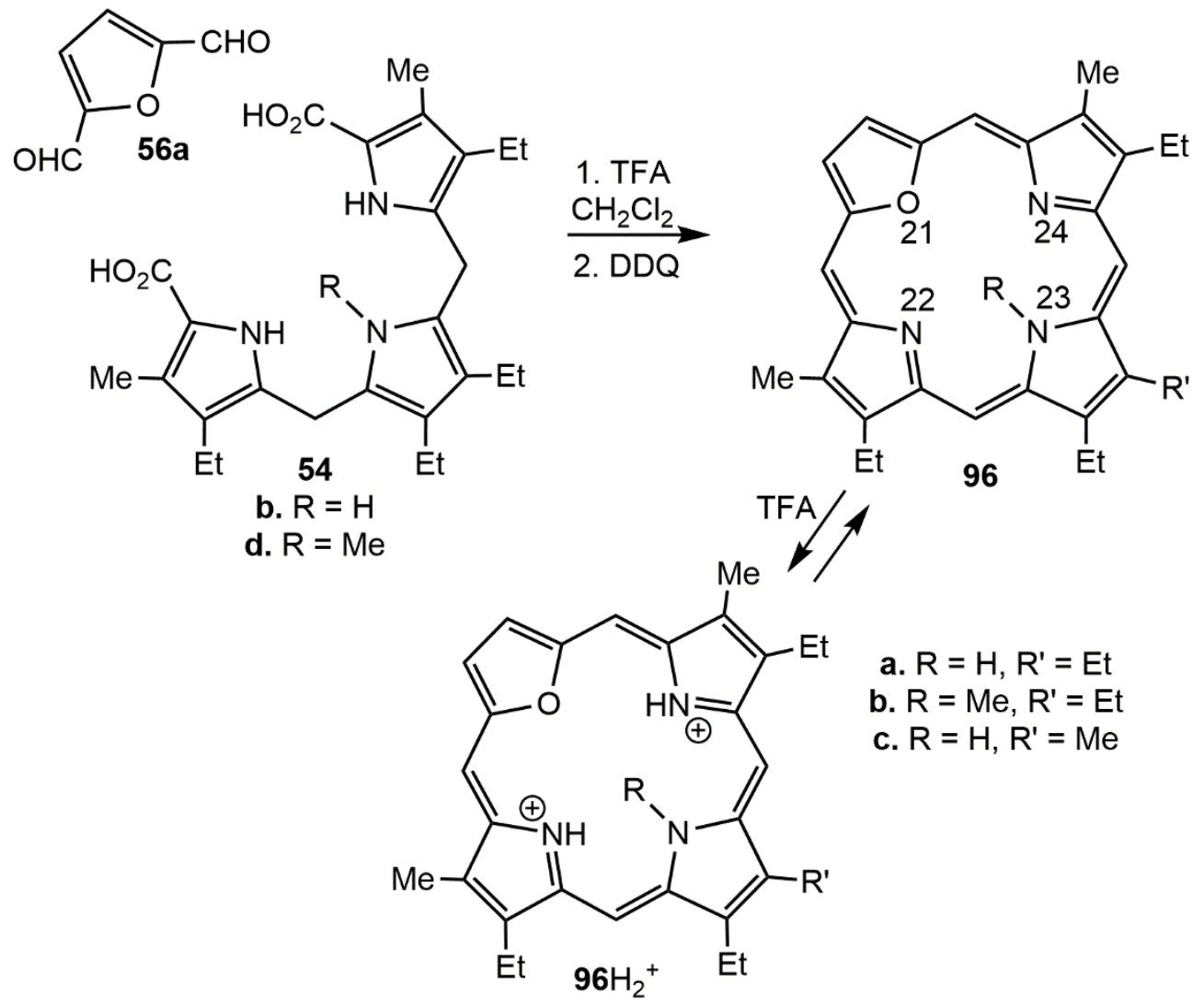

Scheme 34. '3+1' Synthesis of 23-methyl-21-oxaporphyrin 96 
The proton NMR spectrum of $N$-methyl derivative $96 \mathbf{b}$ indicated that this was a symmetrical molecule showing two $2 \mathrm{H}$ singlets for the meso-protons at 10.34 and $11.10 \mathrm{ppm}$, as well as an additional $2 \mathrm{H}$ singlet for the two furan ring protons at $10.69 \mathrm{ppm}$. The external methyl groups were observed as a $6 \mathrm{H}$ singlet at $3.70 \mathrm{ppm}$, while the internal methyl group gave an upfield singlet at $-5.21 \mathrm{ppm}$ (Figure 39). These resonances indicate that the system possesses a strong diatropic ring current, demonstrating that the macrocycle retains strongly aromatic properties despite the introduction of an internal substituent. The carbon-13 NMR spectrum of $\mathbf{9 6 \mathbf { b }}$ also demonstrates the symmetry of this system, showing the meso-carbons at 101.46 and $102.65 \mathrm{ppm}$, while the furan $\mathrm{CH}$ carbons appear further downfield at $132.53 \mathrm{ppm}$ (Figure 40). The internal methyl group can be seen at 32.34 ppm as a broad singlet, possibly due to incomplete decoupling of the upfield proton resonance. When $\mathbf{9 6} \mathbf{b}$ was run in TFA-CDCl 3 , a dicationic species $\mathbf{9 6 \mathbf { b }} \mathrm{H}_{2}{ }^{2+}$ was observed (Scheme 34). The two internal NH protons appeared as a $2 \mathrm{H}$ singlet at $-4.28 \mathrm{ppm}$, which was not present in the free base $\mathbf{9 6 b}$. The internal methyl group of the dication resonated as a 3H singlet at $-5.60 \mathrm{ppm}$, which was further upfield compared to the free base, while the mesoprotons and furan protons were identified as $2 \mathrm{H}$ singlets downfield at 10.82, 11.10 and $10.56 \mathrm{ppm}$, respectively. $\mathrm{N}$-Unsubstituted oxaporphyrin 96 a required the addition of TFA in $\mathrm{CDCl}_{3}$ in order to obtain high quality spectra. This data showed the meso-protons and furan protons as two $2 \mathrm{H}$ singlets at 11.04, 11.18 and 10.61, respectively, while the external methyl groups resonated as a $6 \mathrm{H}$ singlet at $3.82 \mathrm{ppm}$. These values are shifted slightly downfield from those seen in the dication species of the $N$-methyl derivative, but overall the protonated versions of $\mathbf{9 6 a}$ and $\mathbf{9 6 \mathbf { b }}$ appear to possess similar macrocyclic ring currents.

The UV-Vis spectrum of $\mathbf{9 6 b}$ shows bathochromic shifts to longer wavelengths when compared to the $\mathrm{N}$-unsubstituted counterpart 96a (Figure 41). The non-methylated compound gave 
two Soret bands at 371 and $392 \mathrm{~nm}$, while $N$-methyl derivative $\mathbf{9 6 \mathbf { b }}$ afforded two Soret bands at 378 and $407 \mathrm{~nm}$. The weaker Q bands of each species further demonstrates the shift to longer wavelengths. For 96a, these absorptions are observed at 489, 520, 585 and $643 \mathrm{~nm}$, whereas the bands for $\mathbf{9 6 b}$ are broadened and centered at higher values of 501, 527, 604 and $664 \mathrm{~nm}$. Addition of $5 \%$ trifluoracetic acid (TFA) to a solution of $\mathbf{9 6 \mathbf { b }}$ gave the related dicationic species $\mathbf{9 6 \mathbf { b }} \mathrm{H}_{2}{ }^{2+}$ and this produced a single, much stronger Soret band at $395 \mathrm{~nm}$ followed by weaker Q bands at 527, 561 and $611 \mathrm{~nm}$ (Figure 42). The dication for the non-methylated counterpart 96a similarly afforded a Soret band at $394 \mathrm{~nm}$, with two weaker absorptions centered at 523 and $551 \mathrm{~nm}$ (Figure 42). Hence, the Q bands for $\mathbf{9 6 b}$ are bathochromically shifted, although the Soret band is only slightly modified.

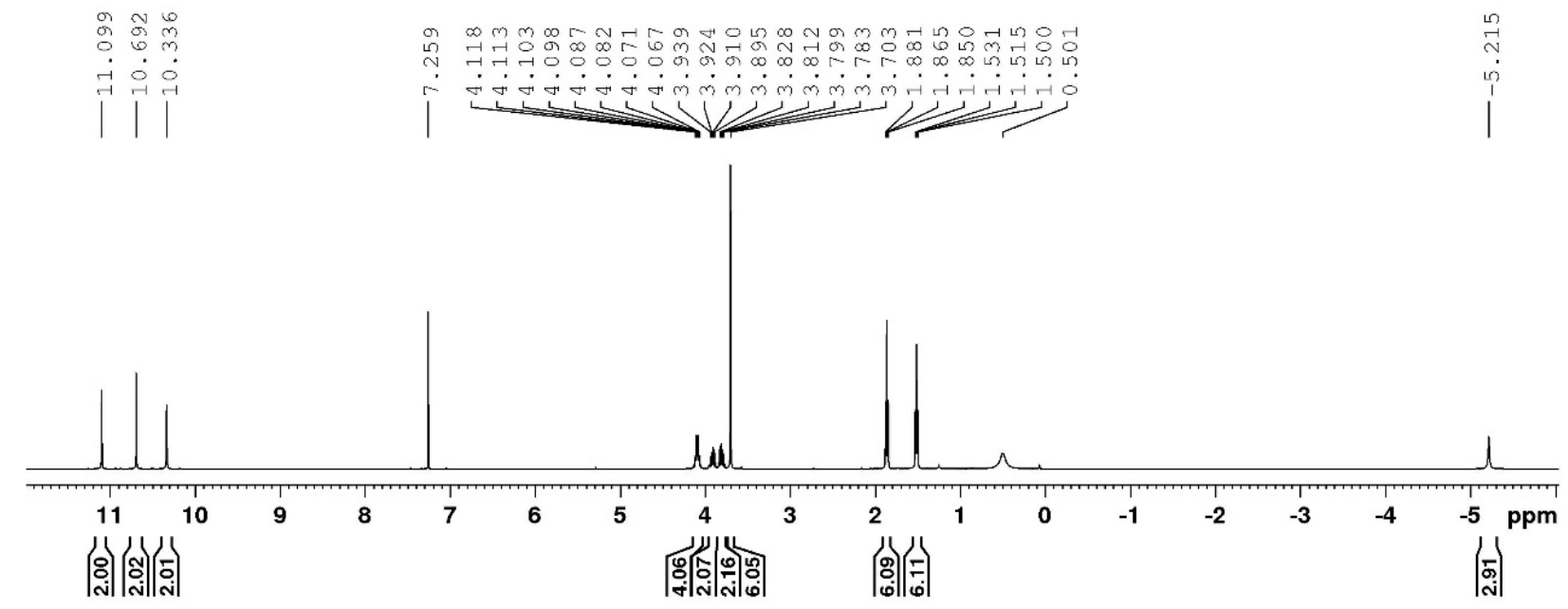

Figure 39. $500 \mathrm{MHz}{ }^{1} \mathrm{H}$ NMR spectrum of 23-methyl-21-oxaporphyrin 96b in $\mathrm{CDCl}_{3}$ 


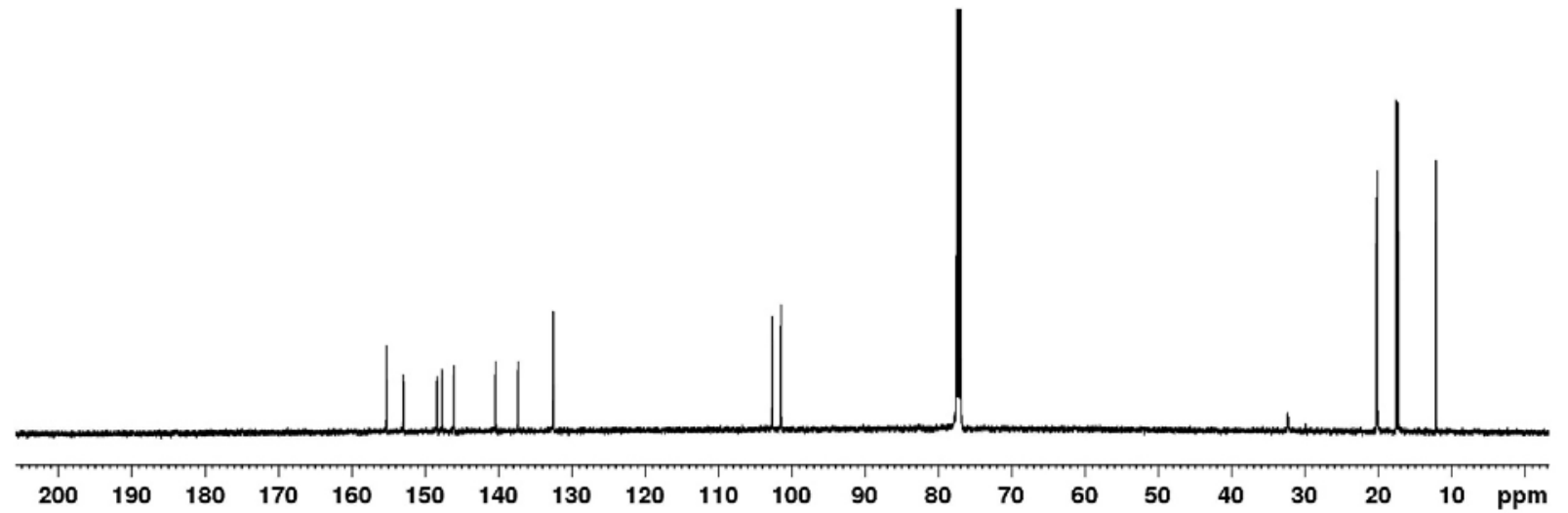

Figure 40. $125 \mathrm{MHz}{ }^{13} \mathrm{C}$ NMR spectrum of 23-methyl-21-oxaporphyrin $96 \mathbf{b}$ in $\mathrm{CDCl}_{3}$

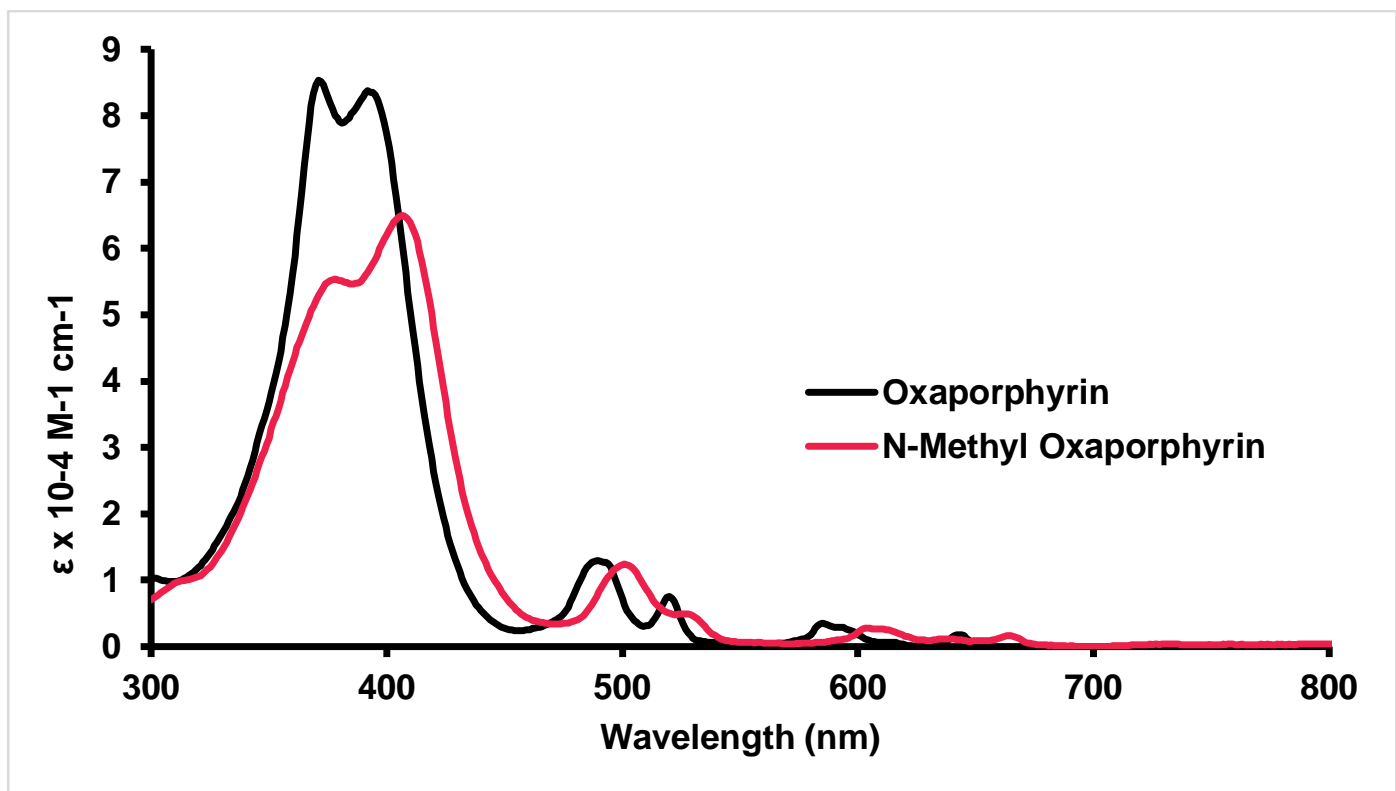

Figure 41. UV-Vis spectra of $\mathbf{9 6} \mathbf{b}$ in 1\% triethylamine-dichloromethane (free base, pink line) and 96a in 1\% triethylamine-dichloromethane (free base, black line) 


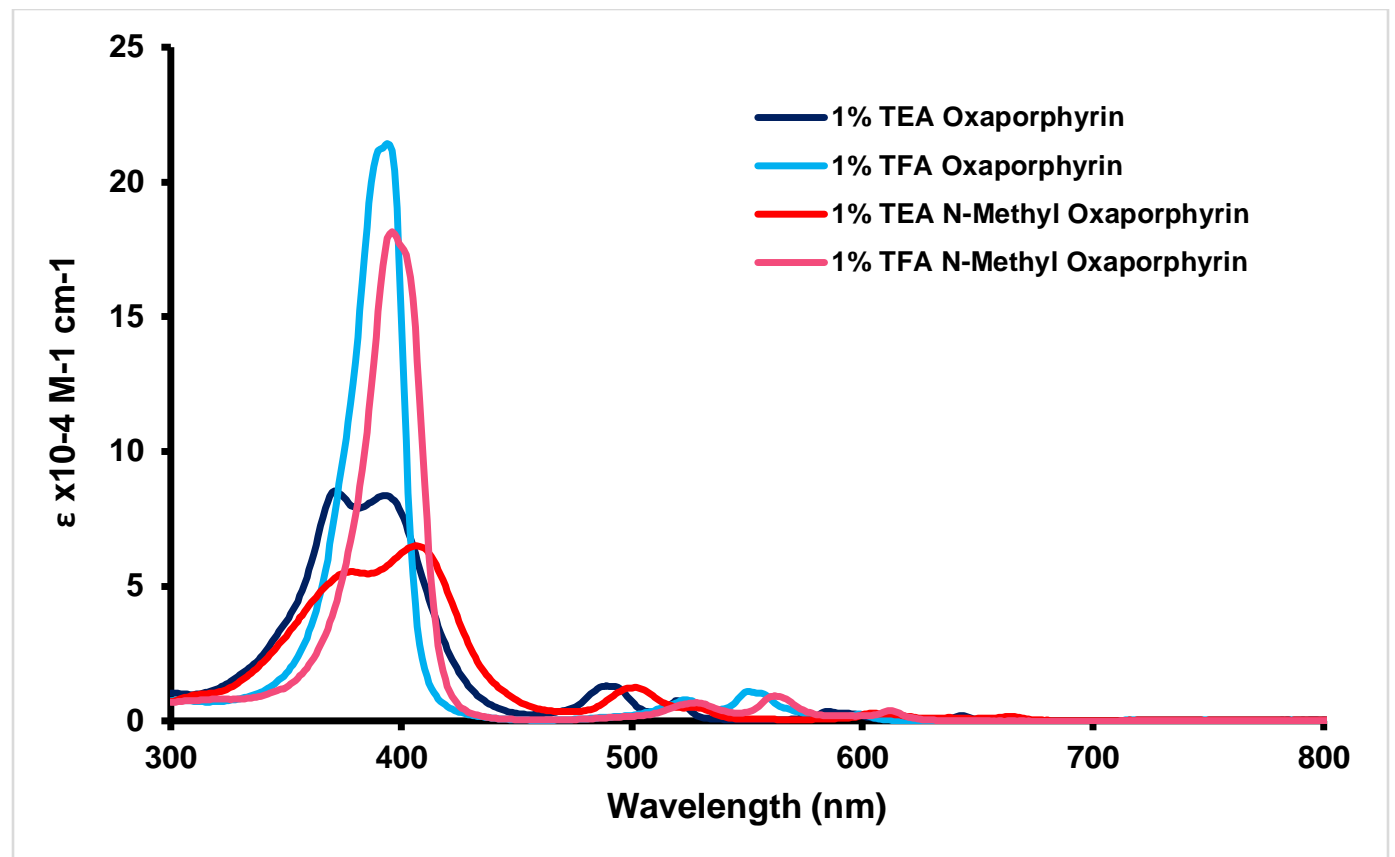

Figure 42. UV-Vis spectra of $\mathbf{9 6 a}$ in 1\% triethylamine-dichloromethane (free base, dark blue line) or $1 \%$ TFA-dichloromethane (dication, light blue line), and $\mathbf{9 6 b}$ in $1 \%$ triethylaminedichloromethane (free base, red line) or 1\% TFA-dichloromethane (dication, pink line)

21-Thiaporphyrin 97a and its $N$-methyl derivative $\mathbf{9 7} \mathbf{b}$ were similarly synthesized and their properties contrasted. Condensation of tripyrrane $\mathbf{5 4 b}$ with thiophene dialdehyde $\mathbf{5 6 b}$ under dilute conditions ( $99 \mathrm{~mL}$ of dichloromethane for $100 \mathrm{mg}$ of tripyrrane) in the presence of TFA, followed by oxidation with ferric chloride, gave $N$-unsubstituted thiaporphyrin 97 a in $42 \%$ yield (Scheme 35). 23-Methyl-21-thiaporphyrin 97b was also synthesized by condensing $N$-methyl tripyrrane 54d with thiophene dialdehyde 56b, although concentrated conditions $(<20 \mathrm{~mL}$ of dichloromethane for $100 \mathrm{mg}$ tripyrrane) and oxidation with DDQ gave better results, and $\mathbf{9 7 \mathbf { b }}$ was isolated in 17\% yield (Scheme 35). Both thiaporphyrin derivatives were chromatographed twice on grade 3 neutral alumina, eluting with dichloromethane. 21-Thiaporphyrin 97a was collected as a green/yellow band while $N$-methyl thiaporphyrin $\mathbf{9 7 b}$ was collected as a yellow/brown band. 
Both compounds were highly soluble in organic solvents and were characterized without further purification. The related $N$-unsubstituted thiaporphyrin $97 \mathbf{c}$, which differs by the placement of one external substituent, was prepared in a similar fashion by Johnson and coworkers. ${ }^{57}$ However, the authors noted that thiaporphyrin 97c was probably obtained as a mixture of isomers, due to the presence of additional peaks in the NMR spectrum. In contrast, 97a and 97b were both isolated as single isomers.

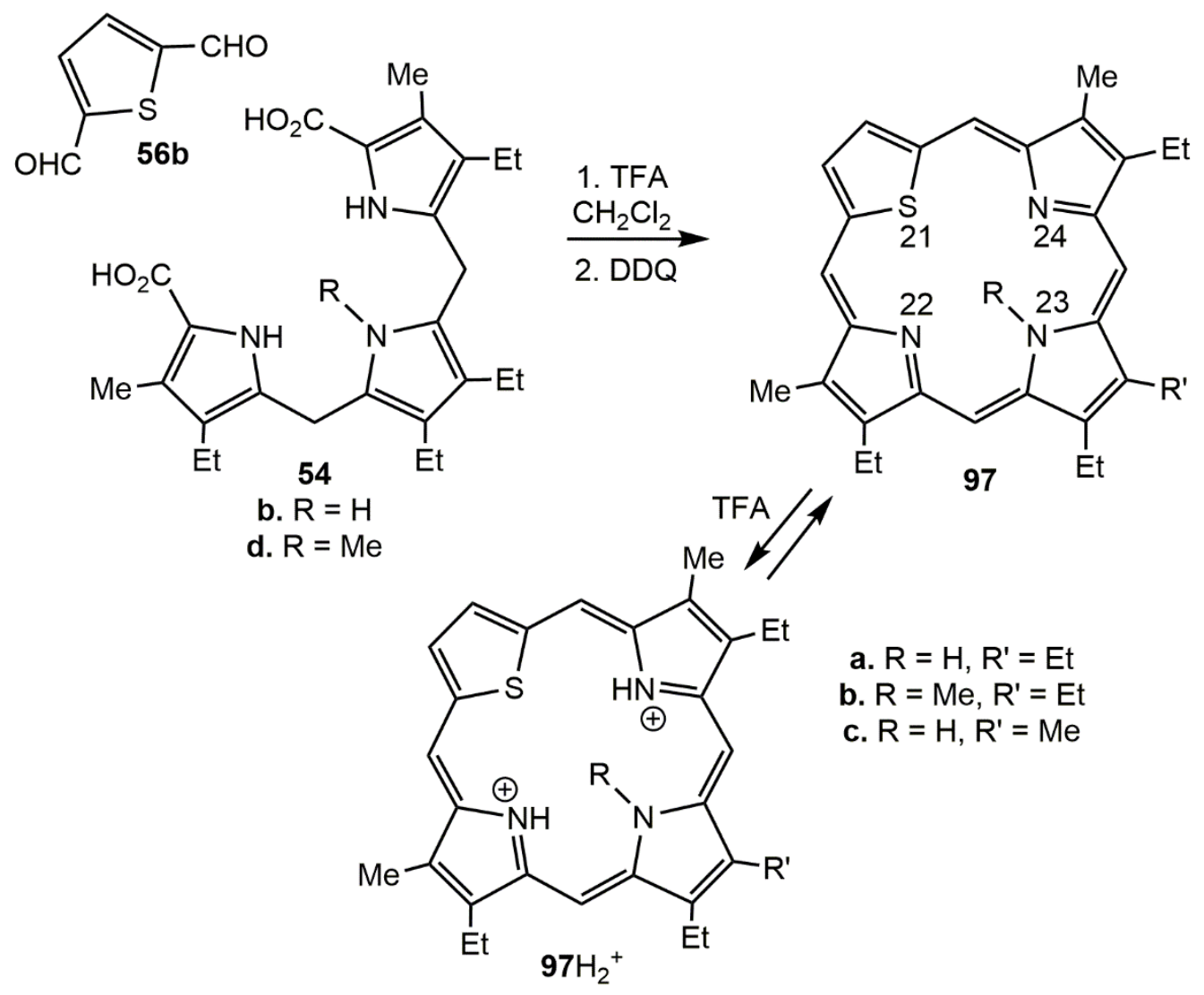

Scheme 35. '3+1' Synthesis of 23-methyl-21-thiaporphyrin 97

The proton NMR spectrum of $\mathbf{9 7 b}$ displayed the internal methyl as a $3 \mathrm{H}$ singlet at an upfield value of $-4.39 \mathrm{ppm}$, while the two $2 \mathrm{H}$ singlets corresponding to the meso-protons were located downfield at 9.96 and 10.57 ppm (Figure 43). The protons on the thiophene ring also 
resonated downfield as a $2 \mathrm{H}$ singlet at $10.00 \mathrm{ppm}$. The shifts and the number of peaks observed are consistent with a highly aromatic and symmetrical molecule. The carbon-13 NMR spectrum also supports this as the meso-carbons and the $\mathrm{CH}$ carbons of the thiophene ring resonate at 103.41, 113.15, and 133.88 ppm, respectively (Figure 44). When TFA was added to the sample, the proton NMR spectrum of dicationic species $\mathbf{9 7} \mathbf{b H}_{2}{ }^{2+}$ was obtained and this showed significant shifts in the proton resonances of the macrocycle. The meso-protons were identified as two $2 \mathrm{H}$ singlets at 10.72 and $11.48 \mathrm{ppm}$ and the thiophene protons were observed as a $2 \mathrm{H}$ singlet at $10.39 \mathrm{ppm}$. The

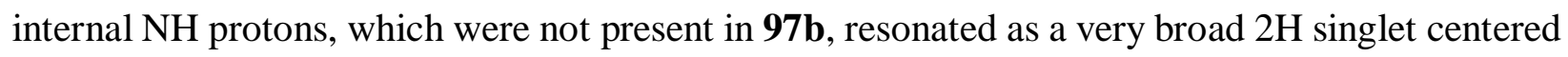
at $-4.12 \mathrm{ppm}$, while the internal methyl was identified as a $3 \mathrm{H}$ singlet at $-4.84 \mathrm{ppm}$.

The proton NMR spectrum of non-methylated 97a showed the meso-protons as two $2 \mathrm{H}$ singlets at 10.15 and $10.72 \mathrm{ppm}$, while the thiophene $\mathrm{CH}$ resonated at $10.13 \mathrm{ppm}$. The external methyl groups were identified as a $6 \mathrm{H}$ singlet at $3.45 \mathrm{ppm}$, and the internal $\mathrm{NH}$ proton was present as a broad $1 \mathrm{H}$ singlet at $-3.87 \mathrm{ppm}$. These values suggest that $\mathbf{9 7 a}$ possesses a slightly larger diatropic ring current than $N$-methyl derivative $\mathbf{9 7 b}$, although they are both strongly aromatic structures. The carbon-13 spectrum of 97a further indicates the symmetry of the molecule, as the meso-carbons gave two peaks at 99.16 and $112.68 \mathrm{ppm}$. When TFA was added to $\mathbf{9 7 a}$, the protonNMR spectrum of the resulting dication showed the meso-protons as two $2 \mathrm{H}$ singlets at 10.97 and $11.66 \mathrm{ppm}$, the thiophene protons as a $2 \mathrm{H}$ singlet at $10.60 \mathrm{ppm}$, and the external methyl groups as a $6 \mathrm{H}$ singlet at $3.79 \mathrm{ppm}$. Once again, the downfield shifts are slightly larger for the $\mathrm{N}$ unsubstituted version.

The UV-Vis spectra of $N$-methyl thiaporphyrin $97 \mathbf{b}$ and $N$-unsubstituted thiaporphyrin 97a were compared (Figure 45). The non-methylated compound 97a gave a Soret band at $398 \mathrm{~nm}$, followed by several weaker Q bands from $495 \mathrm{~nm}$ to $649 \mathrm{~nm}$. $N$-methyl derivative $\mathbf{9 7 b}$ gave a 
Soret band at $412 \mathrm{~nm}$ while the Q bands were identified between $505 \mathrm{~nm}$ and $667 \mathrm{~nm}$. These values confirm that bathochromic shifts are observed for the methylated derivative, demonstrating the effect the internal alkyl group has on the chromophore. The UV-Vis spectra of the free base and dication forms of both $\mathbf{9 7 a}$ and $\mathbf{9 7 b}$ were also compared (Figure 46). It can be observed from these spectra that the addition of TFA affords shifts to longer wavelengths in both $\mathbf{9 7 b}$ and $\mathbf{9 7 a}$, where 97b and $\mathbf{9 7} \mathbf{b H}_{2}{ }^{2+}$ gave Soret bands at 412 and $423 \mathrm{~nm}$, respectively, while $\mathbf{9 7 a}$ and $\mathbf{9 7} \mathbf{a H}_{2}{ }^{2+}$ gave Soret bands at 398 and $418 \mathrm{~nm}$, respectively.
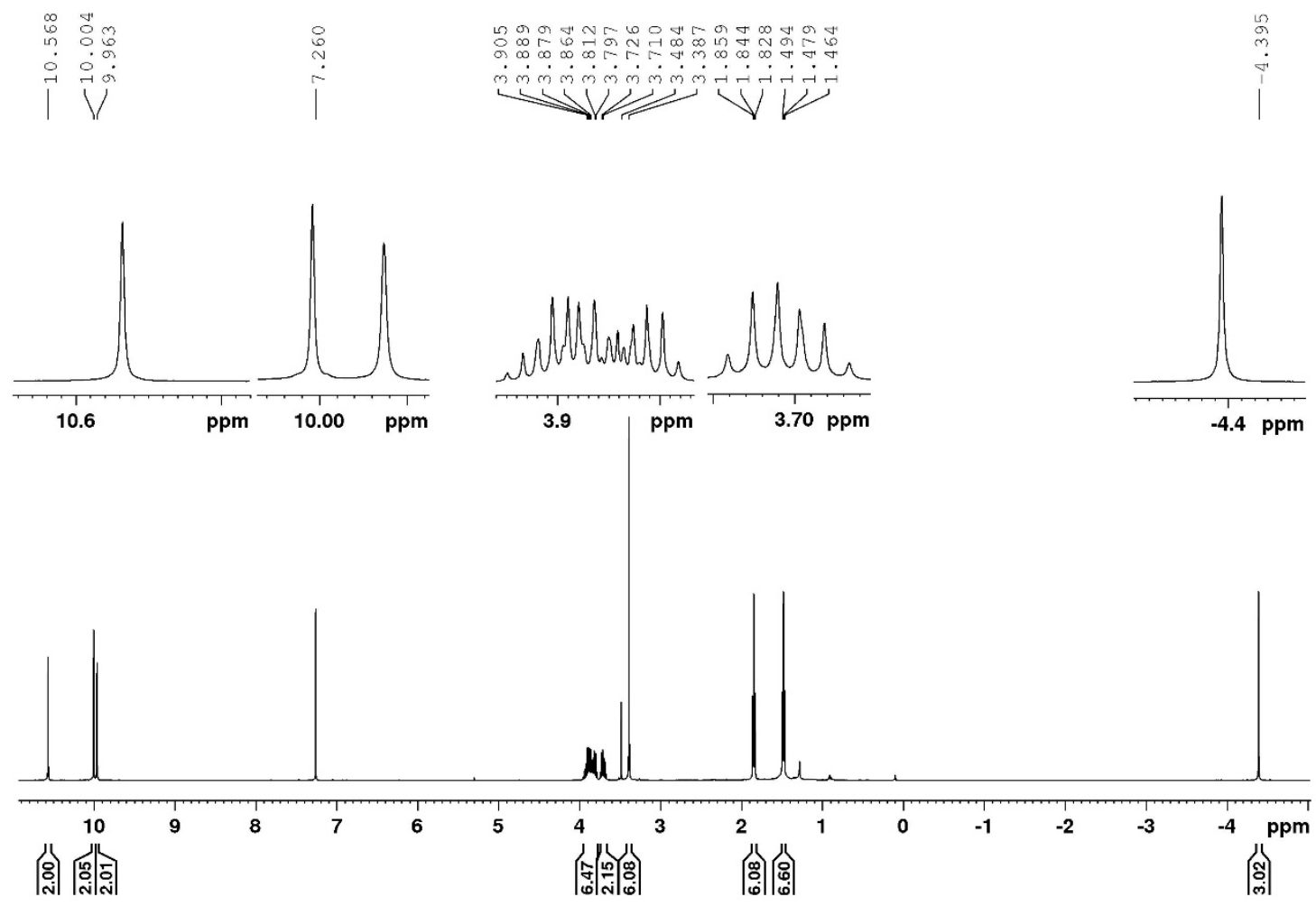

Figure 43. $500 \mathrm{MHz}{ }^{1} \mathrm{H}$ NMR spectrum of $N$-methyl thiaporphyrin 97b in $\mathrm{CDCl}_{3}$ 


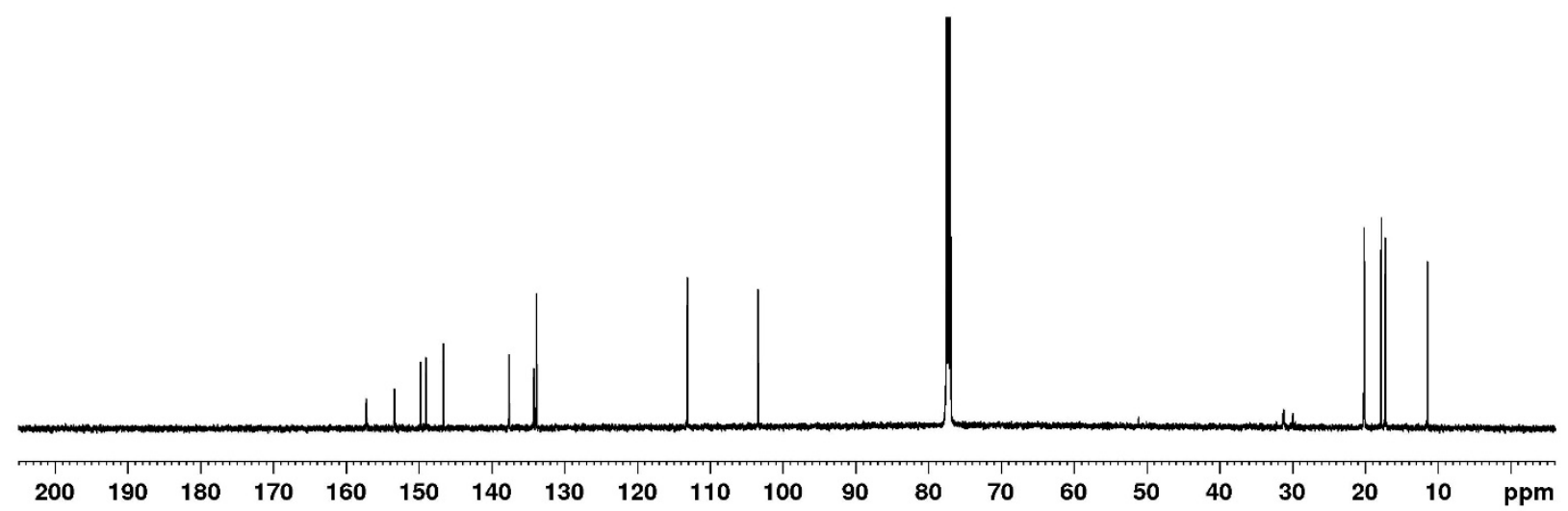

Figure 44. $125 \mathrm{MHz}{ }^{13} \mathrm{C}$ NMR spectrum of $\mathrm{N}$-methyl thiaporphyrin 97b in $\mathrm{CDCl}_{3}$

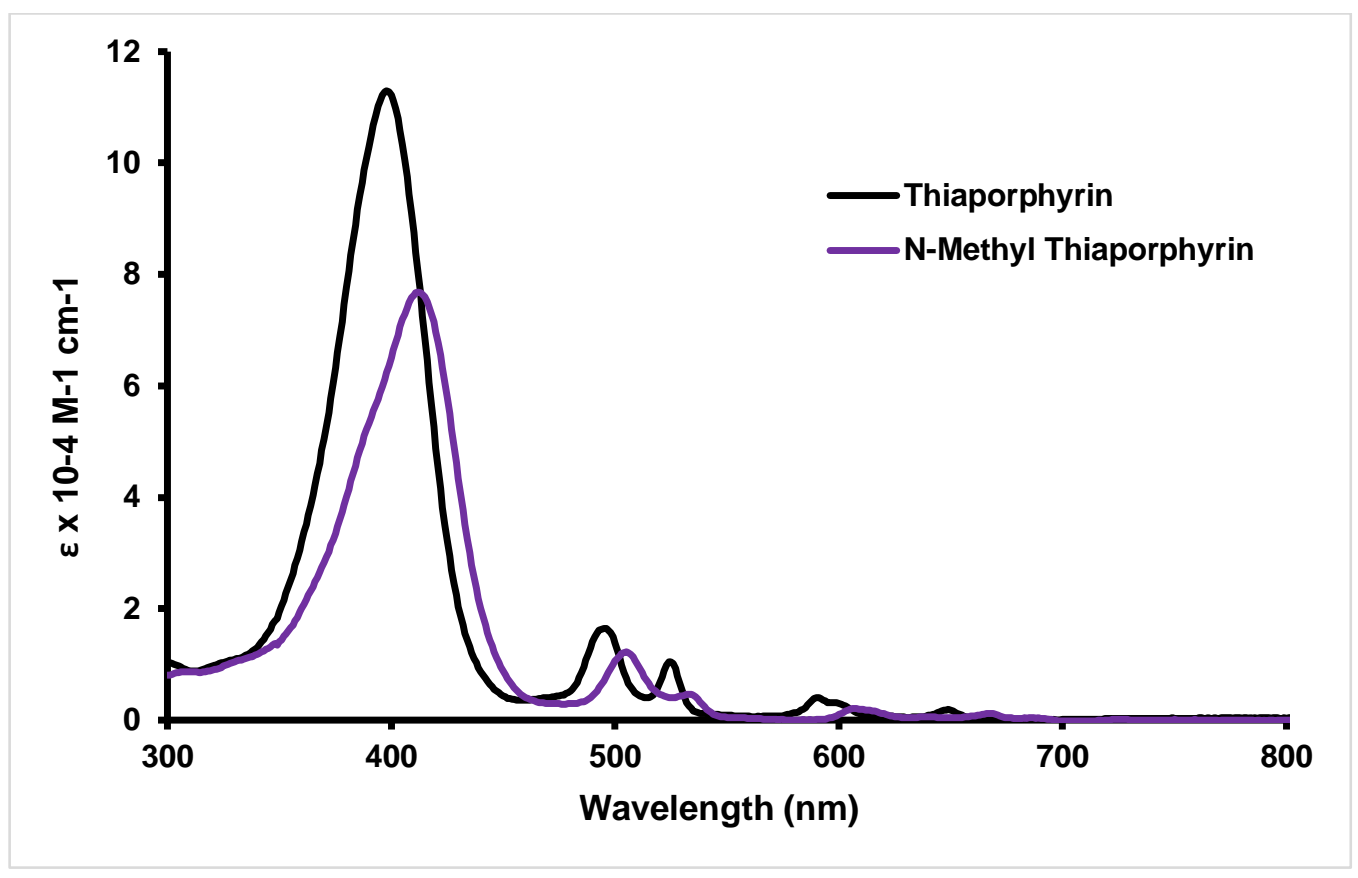

Figure 45. UV-Vis spectra of $\mathbf{9 7 b}$ in $1 \%$ triethylamine-dichloromethane (free base, purple line) and 97a in 1\% triethylamine-dichloromethane (free base, black line) 


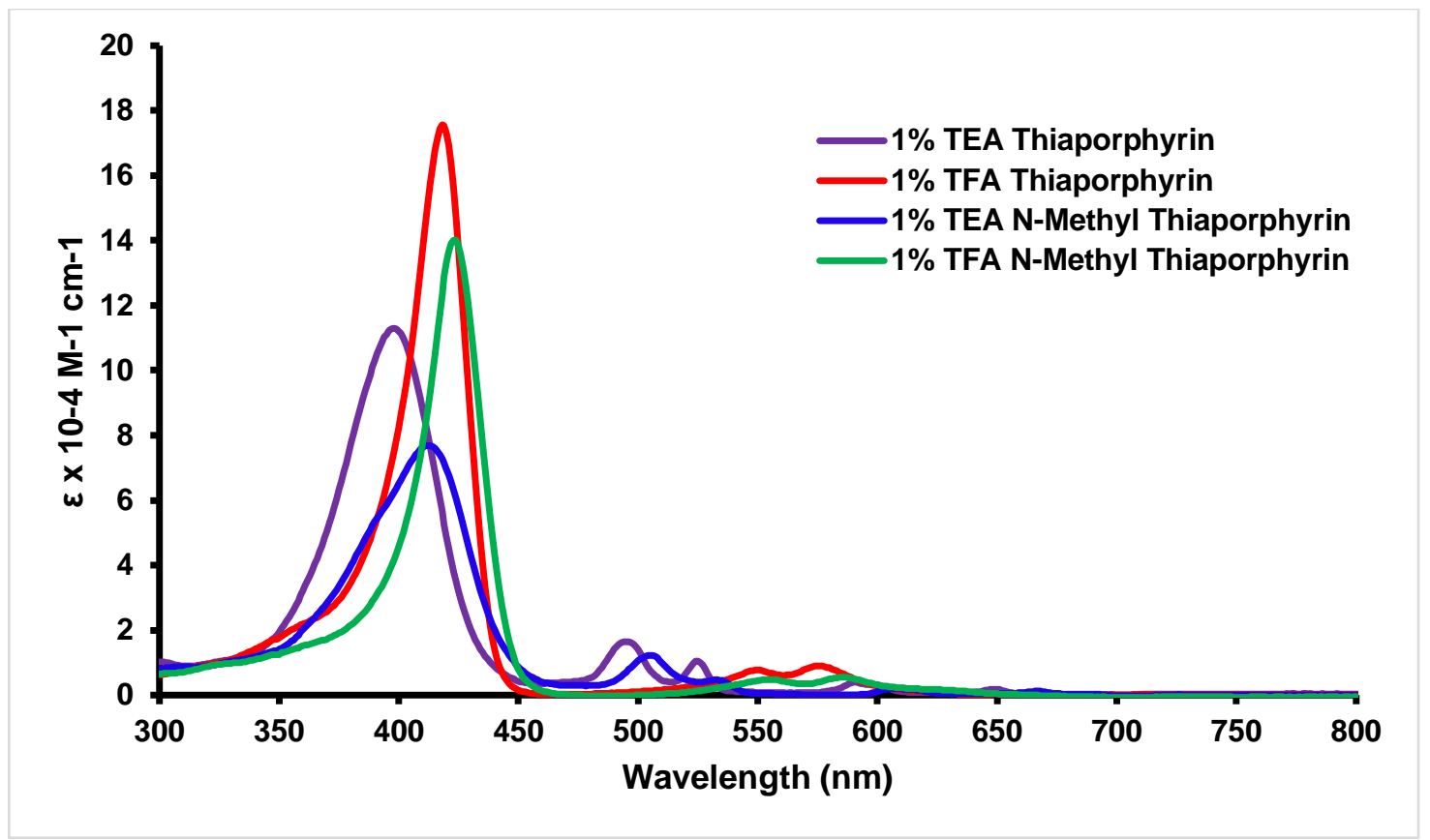

Figure 46. UV-Vis spectra of $\mathbf{9 7 \mathbf { b }}$ in $1 \%$ triethylamine-dichloromethane (free base, blue line) and 1\% TFA-dichloromethane (dication, green line), 97a in 1\% triethylamine-dichloromethane (free base, purple line) and 1\% TFA-dichloromethane (dication, red line)

In addition to heteroporphyrins, it was also of interest to investigate how internally methylated porphyrins differ from regular $N$-unsubstituted porphyrins. Condensation of $N$-methyl tripyrrane 54d with diethyl pyrrole dialdehyde 98 gave $N$-methylporphyrin $\mathbf{9 9 b}$ (Scheme 36). The compound was purified by column chromatography on alumina, eluting with chloroform. A dark red/pink band was collected and recrystallized from chloroform-hexanes to give the pure product as dark purple/blue crystals in $50 \%$ yield. 
<smiles>CCc1c(C=O)[nH]c(C=O)c1CC</smiles><smiles>[R]n1c(CC)c(CC)c(CC)c1Cc1[nH]c(C(=O)O)c(C)c1CC</smiles>

b. $\mathrm{R}=\mathrm{H}$

d. $\mathrm{R}=\mathrm{Me}$

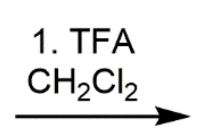

2. $\mathrm{DDQ}$

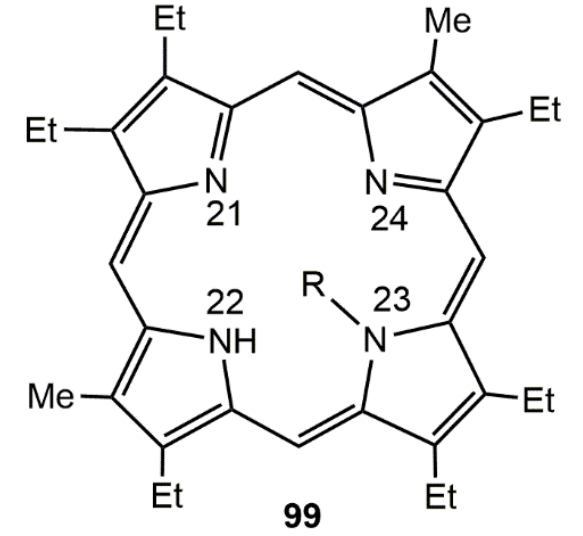

a. $\mathrm{R}=\mathrm{H}$

b. $\mathrm{R}=\mathrm{Me}$

Scheme 36. '3+1' Synthesis of 23-methylporphyrin 99

The proton NMR spectrum of $\mathbf{9 9} \mathbf{b}$ demonstrated the symmetry and aromatic character of the internally methylated porphyrin (Figure 47). The meso-protons were identified as two $2 \mathrm{H}$ singlets at 9.90 and $9.94 \mathrm{ppm}$, and the external methyl groups were observed as a $6 \mathrm{H}$ singlet at $3.49 \mathrm{ppm}$, while the internal methyl group was seen far upfield as a $3 \mathrm{H}$ singlet at $-4.76 \mathrm{ppm}$. These large shifts are consistent with an aromatic system and are also observed in the $N$-unsubstituted counterpart 99a, demonstrating the preservation of aromaticity upon the insertion of an internal substituent. The meso-protons and the external methyl groups of 99a were seen as a $4 \mathrm{H}$ singlet and a $6 \mathrm{H}$ singlet at 10.10 and $3.64 \mathrm{ppm}$, respectively. ${ }^{118}$ The carbon-13 NMR spectrum of the $N$ methylated derivative (99b) further indicates the symmetrical nature of this compound (Figure 48). The meso-carbons resonate as two peaks at 96.72 and 101.59 ppm, while the internal methyl appears at $32.80 \mathrm{ppm}$. When TFA was added to the sample, a diprotonated species $\mathbf{9 9} \mathbf{b} \mathrm{H}_{2}{ }^{2+}$ was formed. The proton NMR spectrum of the dication displayed the meso-protons as two $2 \mathrm{H}$ singlets at 10.61 and $10.73 \mathrm{ppm}$, while the internal methyl and $\mathrm{NH}$ protons appeared as a $3 \mathrm{H}$ singlet at - 
$5.59 \mathrm{ppm}$ and a $2 \mathrm{H}$ broad singlet at $-3.52 \mathrm{ppm}$, respectively. For comparison, the proton NMR spectrum of 99a in TFA-CDCl 3 gave two 2H singlets at 10.69 and $10.70 \mathrm{ppm}$ for the meso-protons, while the four internal $\mathrm{NH}$ protons appeared as two separate broad $2 \mathrm{H}$ singlets at -4.02 and -4.17 ppm. These data indicate that 99a and 99b have comparable diatropic properties.

The UV-Vis spectra of $N$-methyl porphyrin 99b and $N$-unsubstituted porphyrin 99a were also compared (Figure 49). Bathochromic shifts were observed for the methylated derivative, although overall the spectra were similar. $N$-Methyl porphyrin 99 b displayed a Soret band at 410 nm, while $N$-unsubstituted porphyrin 99a gave a Soret band at a significantly shorter wavelength of $397 \mathrm{~nm}$. Both displayed four Q bands, although the bands of $\mathbf{9 9 \mathbf { b }}$ are slightly broader and shifted towards the red. The UV-Vis spectra of the free base $N$-methyl porphyrin $\mathbf{9 9 \mathbf { b }}$ and the dication $\mathbf{9 9} \mathbf{b H}_{2}{ }^{2+}$ were also compared (Figure 50). Free base $\mathbf{9 9 b}$ shows a slight shoulder at $379 \mathrm{~nm}$ and a strong Soret band at $410 \mathrm{~nm}$, followed by a number of weaker absorptions between 505 and 641 $\mathrm{nm}$. However, dication $\mathbf{9 9} \mathrm{bH}_{2}{ }^{2+}$ gave a much stronger Soret band at $404 \mathrm{~nm}$ and two Q bands at 551 and $596 \mathrm{~nm}$. 

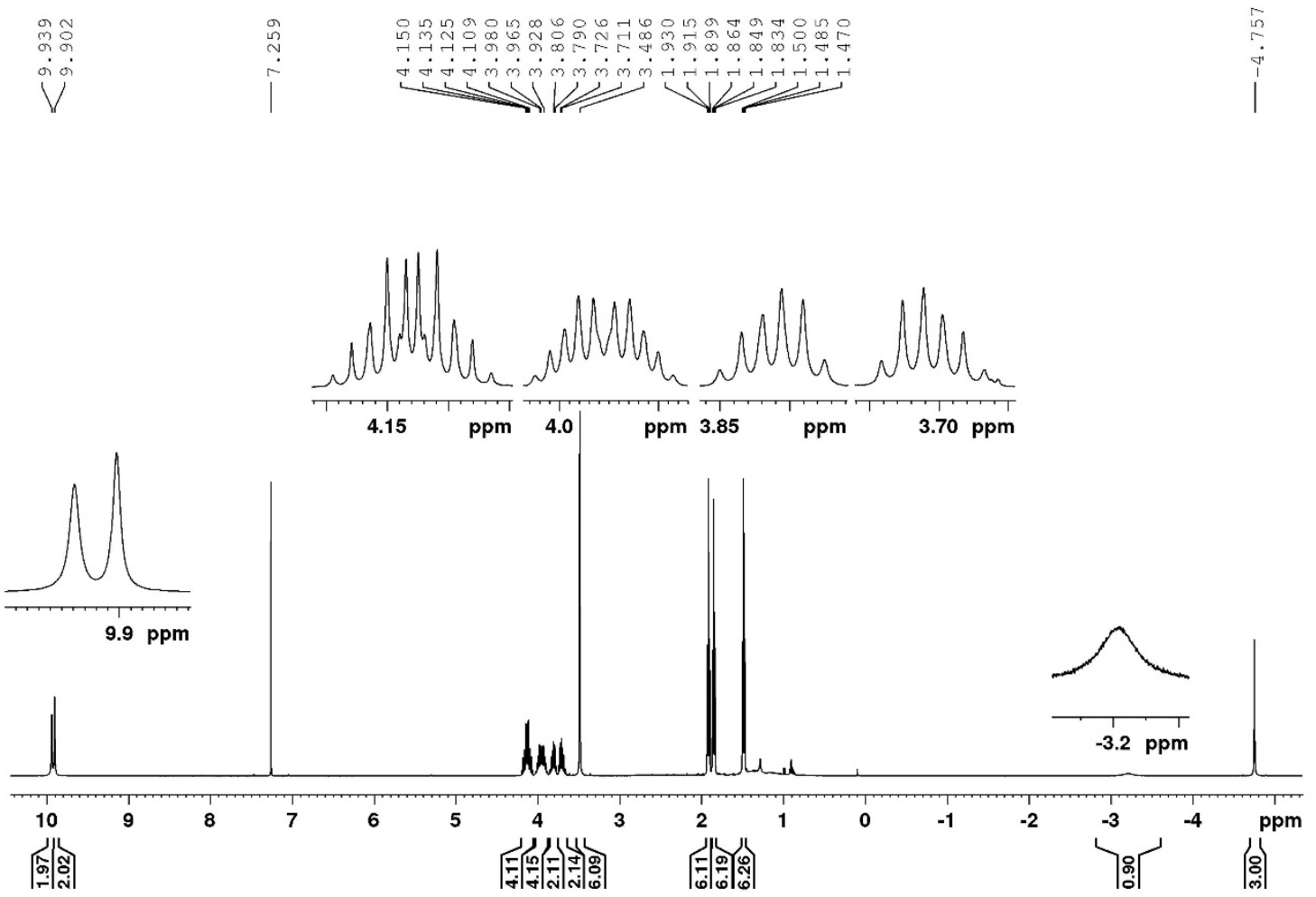

Figure 47. $500 \mathrm{MHz}{ }^{1} \mathrm{H}$ NMR spectrum of $N$-methyl porphyrin 99b in $\mathrm{CDCl}_{3}$

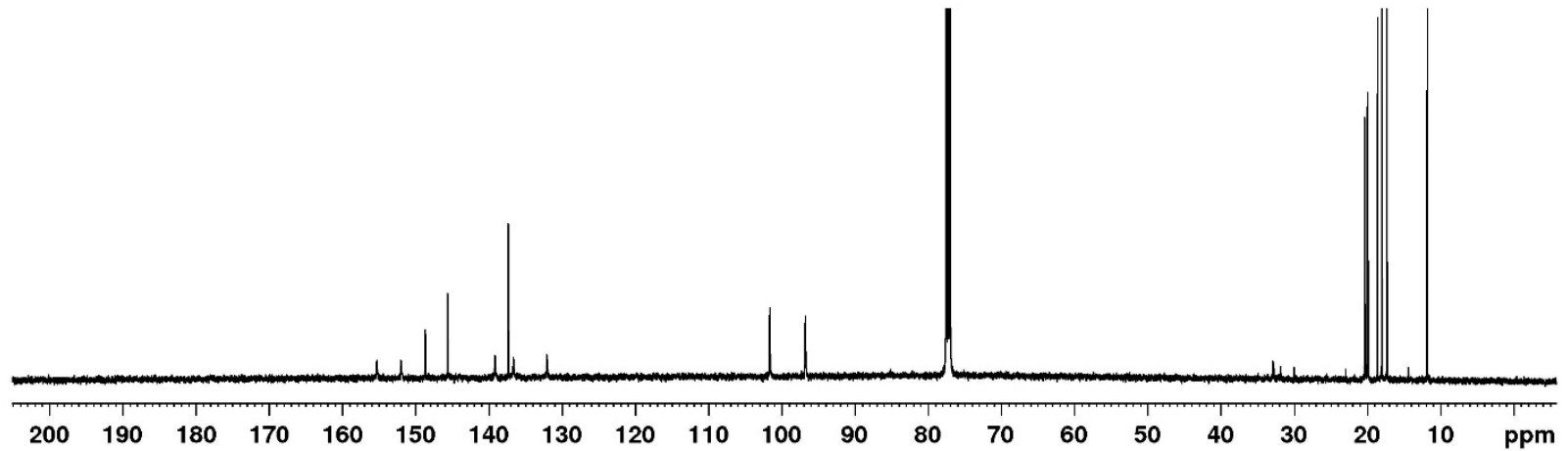

Figure 48. $125 \mathrm{MHz}{ }^{13} \mathrm{C}$ NMR spectrum of $N$-methyl porphyrin 99b in $\mathrm{CDCl}_{3}$ 


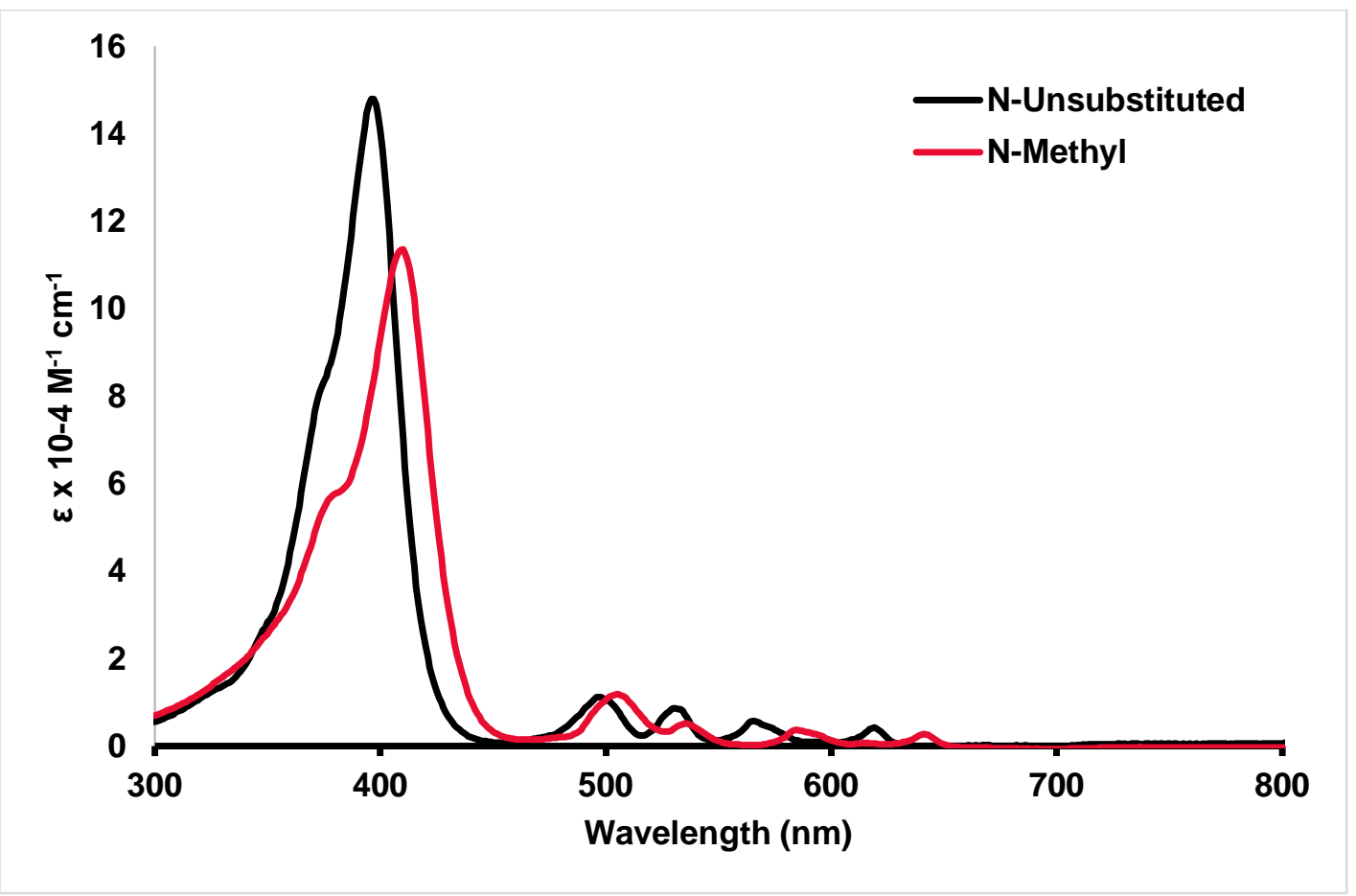

Figure 49. UV-Vis spectra of $N$-methyl porphyrin 99b (red line) and $N$-unsubstituted porphyrin 99a (black line)

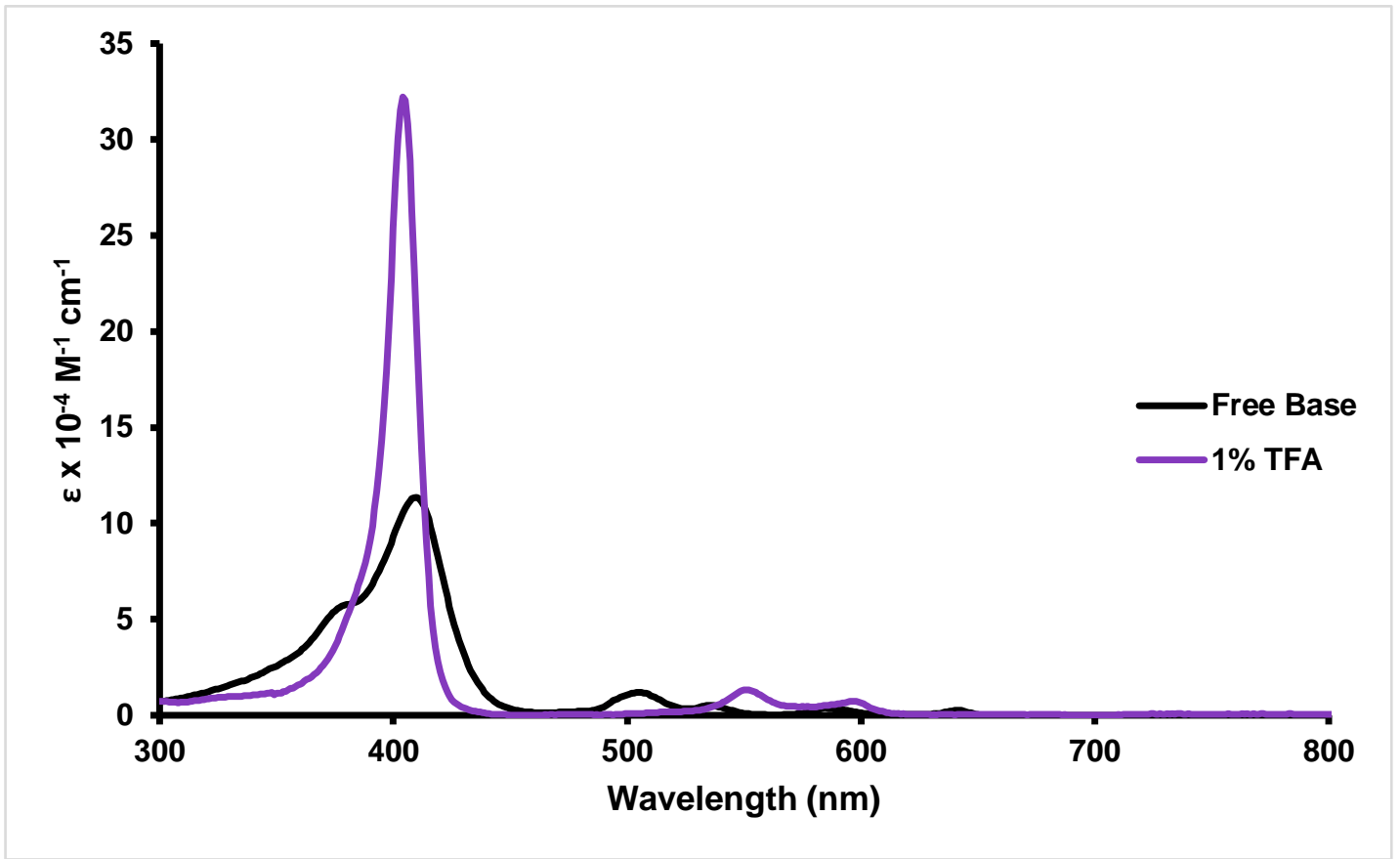

Figure 50. UV-Vis spectra of $\mathbf{9 9 b}$ in 1\% triethylamine-dichloromethane (free base, black line) and 1\% TFA-dichloromethane (dication, purple line) 
The $N$-methyl derivative 100a of oxybenziporphyrin $7 \mathbf{a}$ was also synthesized. This compound was formed by reaction of $N$-methyl tripyrrane 54d in dichloromethane and TFA with dialdehyde 101a (Scheme 37). Purification on an alumina column, eluting first with dichloromethane and then $50 \%$ chloroform-dichloromethane, followed by recrystallization from chloroform-hexanes, gave the pure product in $13 \%$ yield.

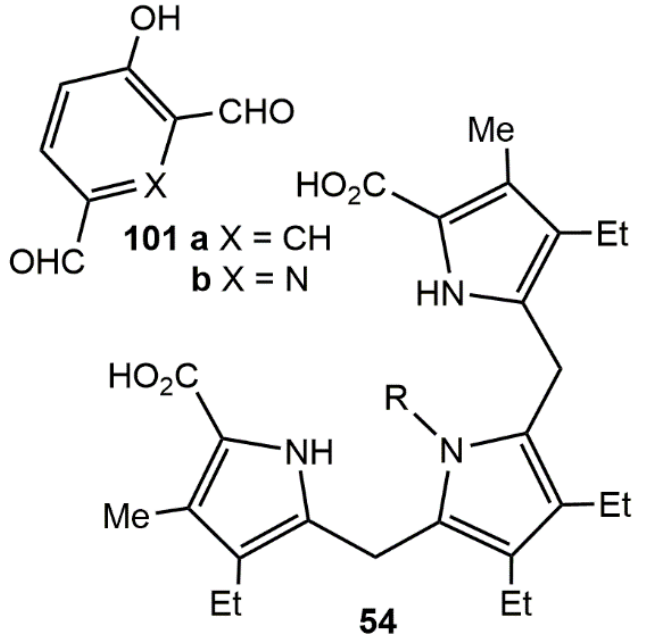

b. $\mathrm{R}=\mathrm{H}$

d. $R=M e$

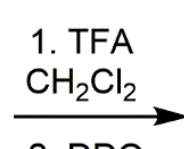

2. DDQ

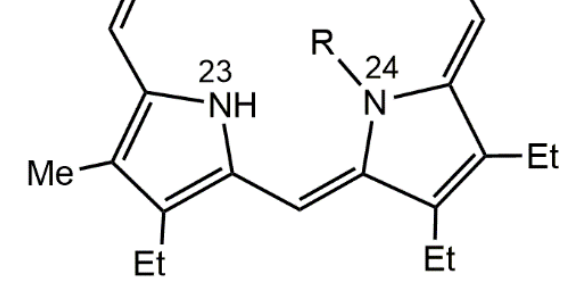

7

a. $X=\mathrm{CH}, \mathrm{R}=\mathrm{H}$ a. $\mathrm{X}=\mathrm{CH}, \mathrm{R}=\mathrm{Me}$

b. $X=N, R=H \quad$ b. $X=N, R=M e$

Scheme 37. ' $3+1$ ' Synthesis of $N$-methylated and $N$-unsubstituted oxybenzi- and oxypyriporphyrins

The proton NMR spectrum of 100a confirmed the asymmetry of this compound by showing an increased number of peaks for the protons on the structure (Figure 51). The four mesoprotons present in this molecule correspond to the two $1 \mathrm{H}$ singlets at 10.50 and $9.20 \mathrm{ppm}$, and the $2 \mathrm{H}$ singlet at $9.37 \mathrm{ppm}$. Furthermore, the proton on the $\beta$-carbon relative to the carbonyl unit resonates as a doublet of doublets at $8.72 \mathrm{ppm}$, showing evidence for long-range coupling to the internal $\mathrm{CH}$ proton of the six membered ring. Two $3 \mathrm{H}$ singlets at 3.33 and $3.43 \mathrm{ppm}$ correspond to the two external methyl substituents, while the internal methyl group resonated as a $3 \mathrm{H}$ singlet 
at $-3.63 \mathrm{ppm}$ and the internal $\mathrm{CH}$ was observed at $-4.14 \mathrm{ppm}$ as a broad $1 \mathrm{H}$ singlet. In comparison, $N$-unsubstituted oxybenziporphyrin 7a has been reported to show the meso-protons as four separate $1 \mathrm{H}$ singlets between 8.85 and $10.29 \mathrm{ppm}$, the internal $\mathrm{CH}$ as a weakly coupled doublet $(J=2 \mathrm{~Hz})$ at $-7.3 \mathrm{ppm}$, and the internal $\mathrm{NH}$ protons as a broad $2 \mathrm{H}$ peak near $-4 \mathrm{ppm} .{ }^{23}$ The shifts seen in the upfield region for the $N$-methyl derivative 100a are slightly weaker than those seen in its $N$-unsubstituted counterpart 7a, but the downfield regions for these porphyrinoids are very similar. The carbon-13 NMR spectrum of 100a (Figure 52) shows the carbonyl carbon at 189.33 ppm, while the meso-carbons appear at 96.7, 99.0, 111.2 and 118.9 ppm, and the internal methyl was identified at $33.3 \mathrm{ppm}$. The carbon-13 NMR spectrum of $N$-unsubstituted oxybenziporphyrin 7a showed the carbon of the carbonyl present on the six-membered ring at $187.94 \mathrm{ppm}$ and the meso-carbons were located at 93.3, 94.9, 105.7 and $111.1 \mathrm{ppm} .{ }^{23} \mathrm{~N}$-methylated oxybenziporphyrin 100a appears to possess a similar aromatic ring current to $7 \mathbf{a}$, and potential distortion of the macrocycle due to the presence of the $N$-methyl group does not appear to significantly affect macrocyclic diatropicity.

However, the UV-Vis spectrum of $N$-unsubstituted oxybenziporphyrin 7a is significantly different from its $N$-methyl derivative 100a (Figure 53). These closely related carbaporphyrinoids possess the same chromophore, but the UV-Vis spectrum for 100a is much weaker and broader, as well as slightly shifted towards longer wavelengths, compared to its $N$-unsubstituted counterpart. A weak absorption is seen at $331 \mathrm{~nm}$ in 100a, while two absorptions at 313 and 335 $\mathrm{nm}$ are identified for 7a. The Soret band for 100a is observed at $441 \mathrm{~nm}$ with another band shortly after at $473 \mathrm{~nm}$. The same pattern is seen in $7 \mathbf{a}$ but these two bands are much stronger and are observed at 426 and $452 \mathrm{~nm}$. Furthermore, the four Q bands for 100a are much broader and less distinguishable than those of 7a. The strongest absorption within the Q bands is seen at $588 \mathrm{~nm}$ 
for 7a but at a much larger wavelength of $621 \mathrm{~nm}$ for the $N$-methyl derivative 100a. Addition of TFA to 100a results in the formation of a dicationic species $100 \mathrm{aH}_{2}{ }^{2+}$ that gives shifts in the absorption values compared to that of free base 100a (Figure 54). The Soret band for the free base is observed at $441 \mathrm{~nm}$ with a second absorption close after at $473 \mathrm{~nm}$, while the dication gives a single Soret band at $433 \mathrm{~nm}$ that is slightly weaker than that for the free base. Furthermore, the free base shows two Q bands centered at 621 and $724 \mathrm{~nm}$, while the spectrum for the dication produced broader, less distinguishable Q bands between 550 and $770 \mathrm{~nm}$. The IR spectrum for the $N$-methyloxybenziporphyrin 100a gave a strong peak at $1618 \mathrm{~cm}^{-1}$ for the carbonyl stretching frequency (Figure 55), which is similar to the frequency of $1624 \mathrm{~cm}^{-1}$ that was observed for $7 \mathbf{a}^{23}$

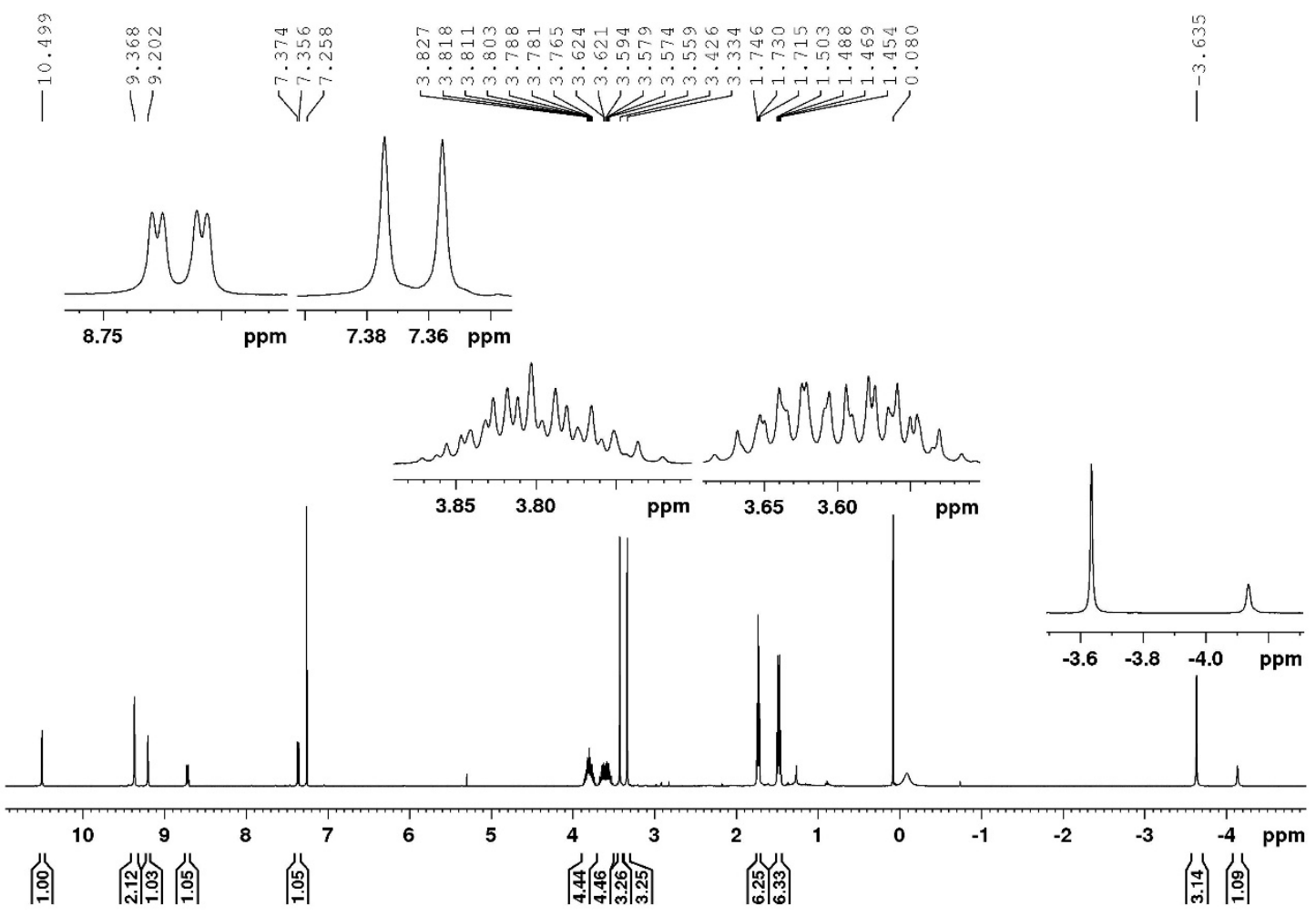

Figure $51.500 \mathrm{MHz}{ }^{1} \mathrm{H}$ NMR spectrum of $N$-methyloxybenziporphyrin 100a in $\mathrm{CDCl}_{3}$ 


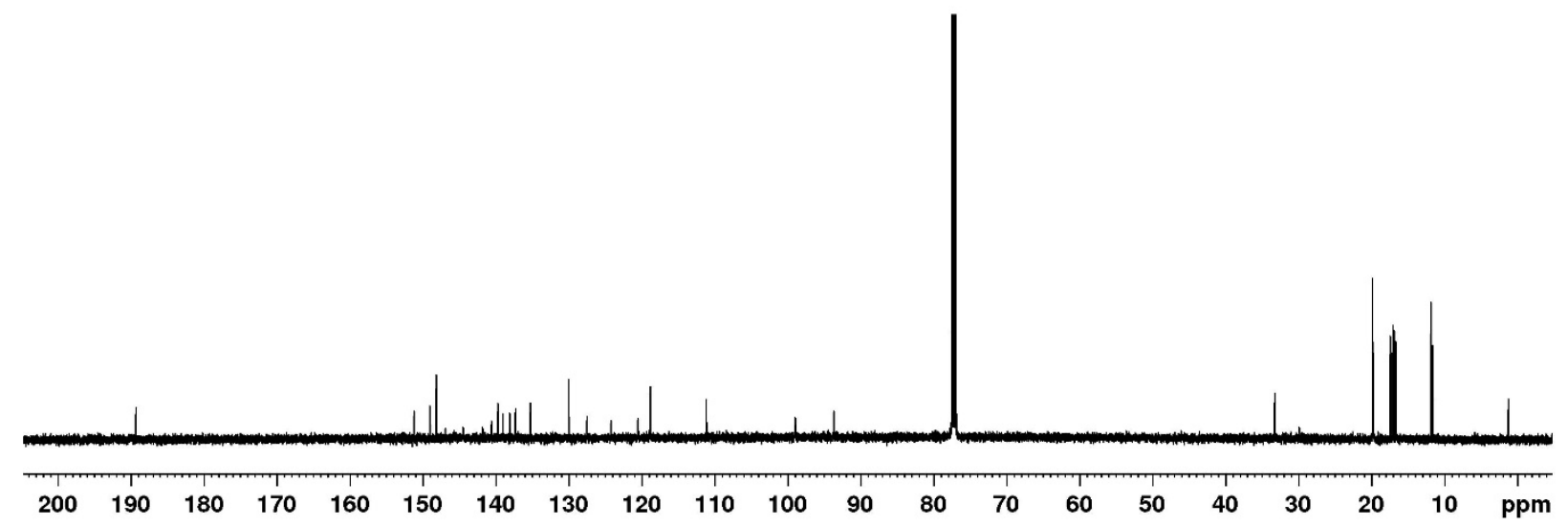

Figure 52. $125 \mathrm{MHz}{ }^{13} \mathrm{C}$ NMR spectrum of $\mathrm{N}$-methyloxybenziporphyrin 100a in $\mathrm{CDCl}_{3}$

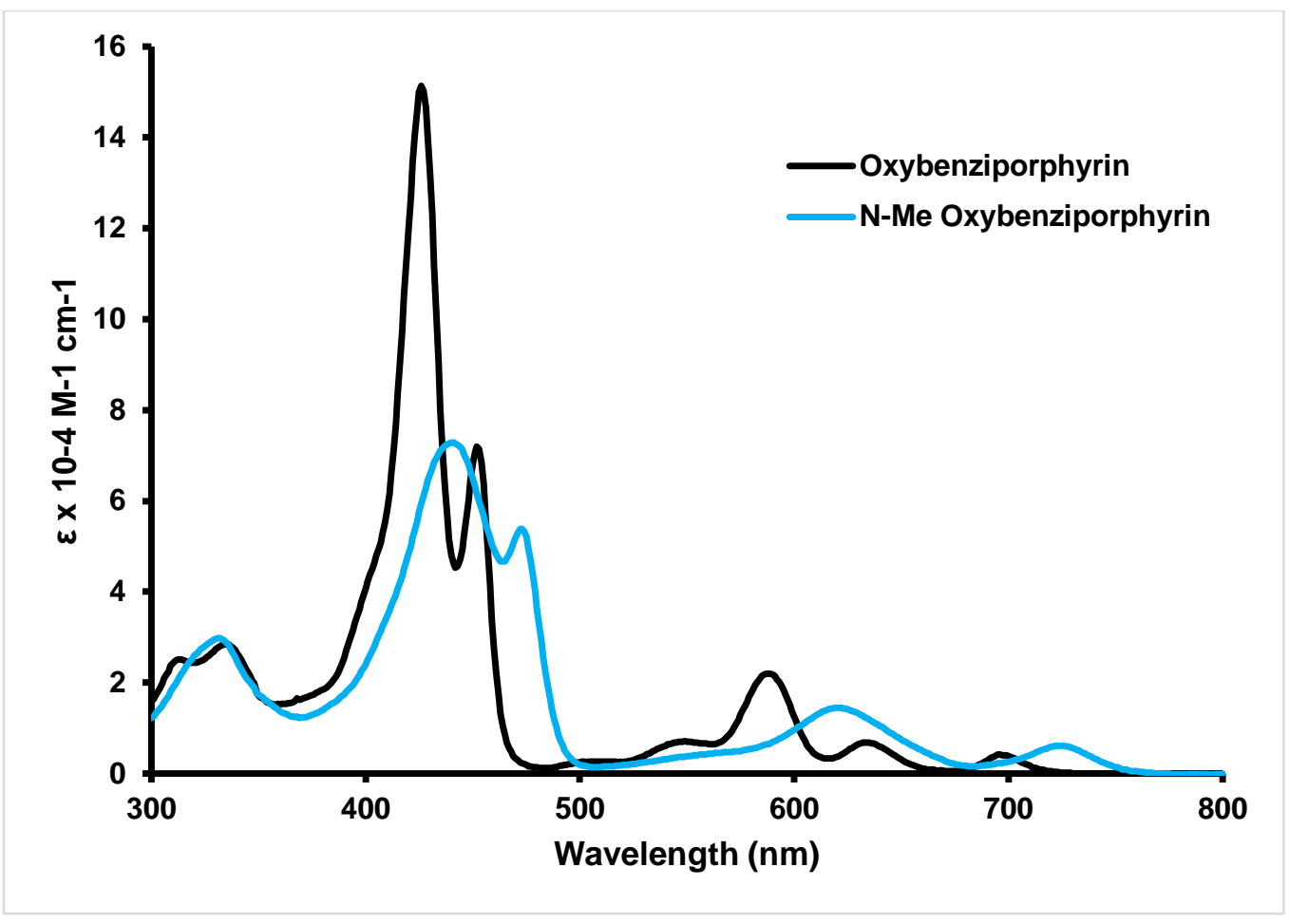

Figure 53. UV-Vis spectra of $N$-methyloxybenziporphyrin 100a (blue line) and $N$-unsubstituted oxybenziporphyrin 7a (black line) 


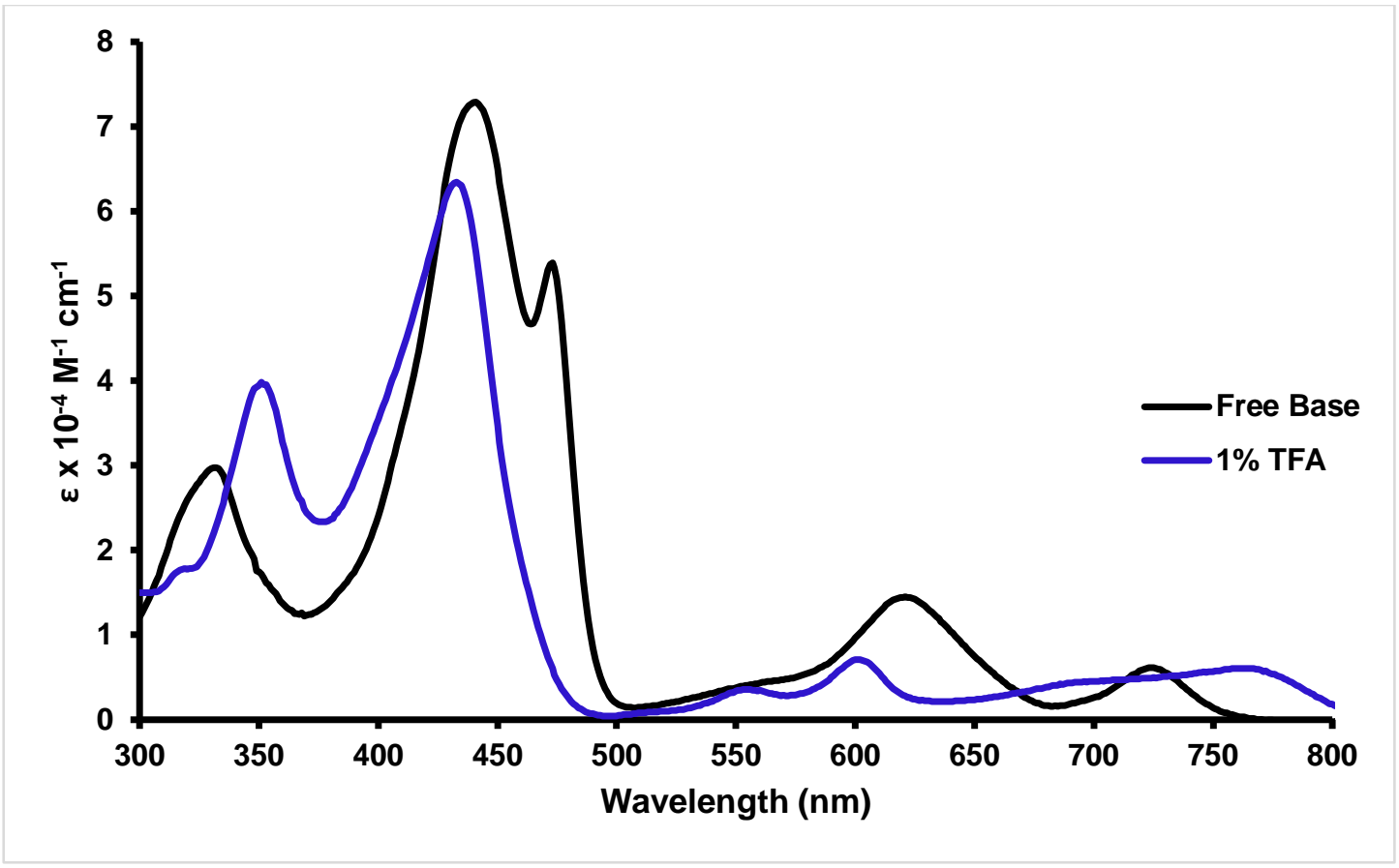

Figure 54. UV-Vis spectra of 100a in 1\% triethylamine-dichloromethane (free base, black line) and 1\% TFA-dichloromethane (dication, blue line)

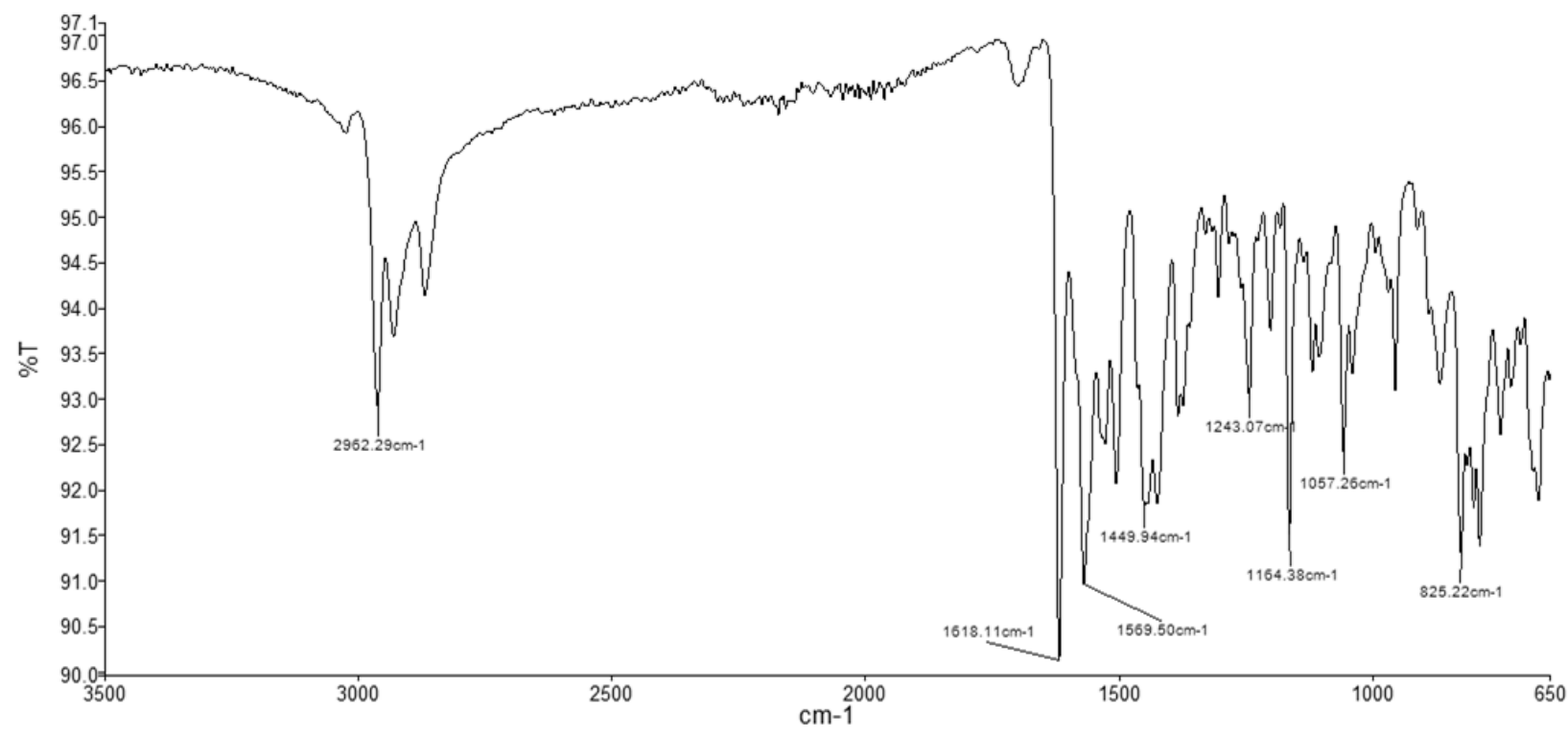

Figure 55. IR spectrum of $N$-methyloxybenziporphyrin 100a 
$\mathrm{N}$-methyloxybenziporphyrin 100a was reacted with palladium(II) acetate in refluxing acetonitrile to give the related palladium(II) complex 102 (Scheme 38), which was purified by column chromatography on grade 3 alumina eluting with chloroform. Following recrystallization from chloroform-hexanes, the pure metalated product 102 was isolated as green crystals in 36.4\% yield. The proton NMR spectrum of the complex shows very similar shifts to the proton NMR spectrum of the free base compound 100a (Figure 56). The meso-protons resonated as four $1 \mathrm{H}$ singlets at 9.22, 9.46, 9.52 and 10.58 ppm, while the external methyl substituents were observed as two $3 \mathrm{H}$ singlets at 3.27 and $3.32 \mathrm{ppm}$. The internal methyl was the only upfield shifted peak and was observed as a $3 \mathrm{H}$ singlet at $-2.36 \mathrm{ppm}$. The carbon-13 NMR spectrum of 102 displayed the meso-carbons at 98.7, 100.2, 123.6 and 125.8 ppm, while the internal methyl and the carbonyl carbon were observed at 46.3 ppm and 190.2 ppm, respectively (Figure 57). The proton NMR resonances of $\mathbf{1 0 2}$ are shifted slightly further downfield compared to those for 100a, indicating that a slightly stronger diatropic ring current is present within the metalated complex 102. The IR spectrum for palladium(II) complex 102 gave a strong absorption at $1555 \mathrm{~cm}^{-1}$ for the carbonyl stretching frequency (Figure 58).
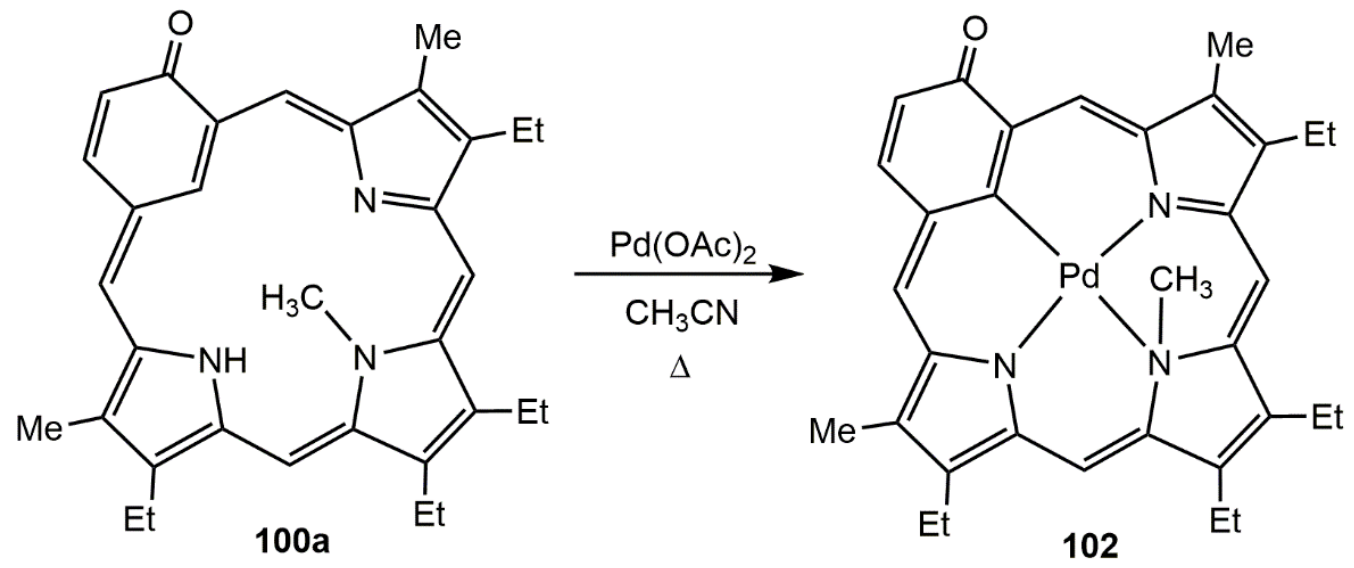

Scheme 38. Metalation of $N$-methyloxybenziporphyrin 100a with palladium(II) acetate 

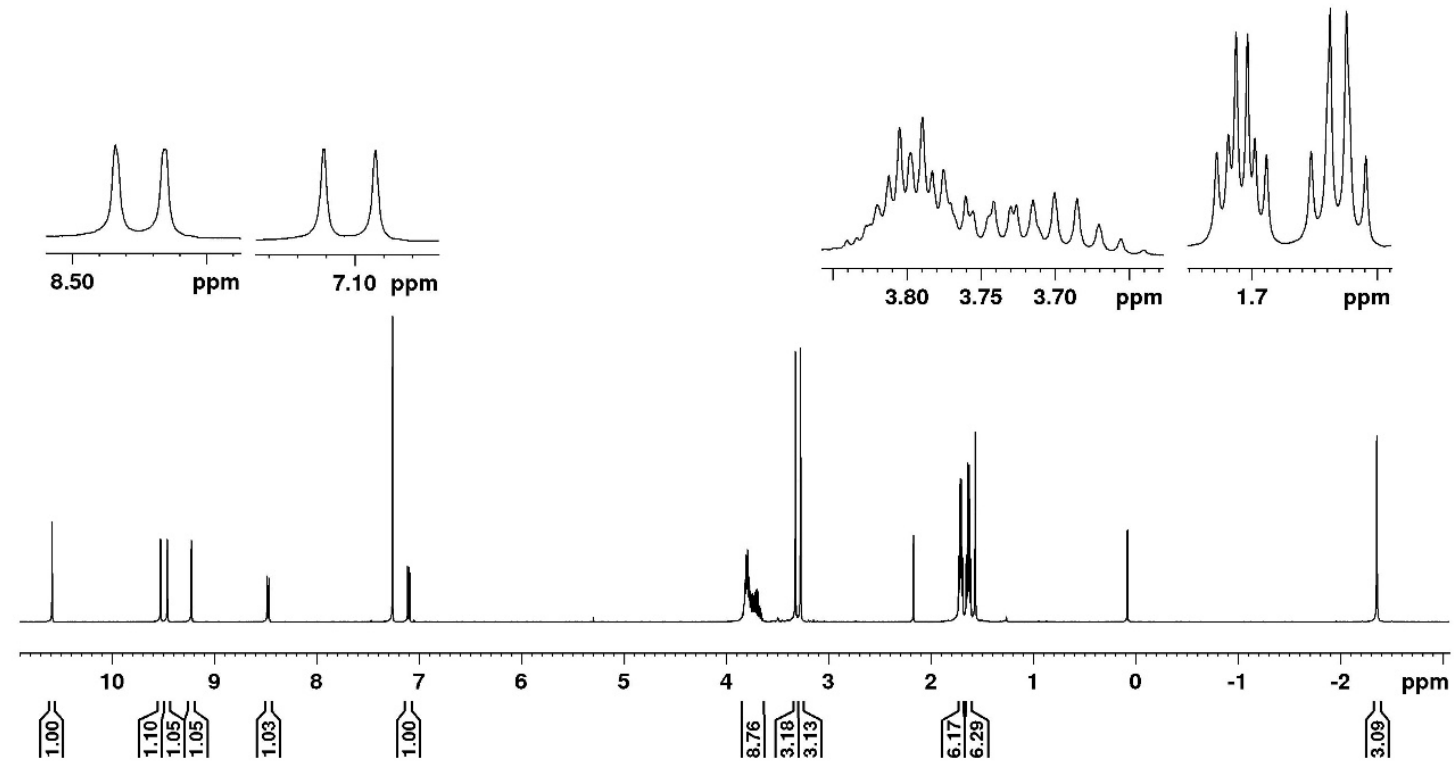

Figure 56. $500 \mathrm{MHz}{ }^{1} \mathrm{H}$ NMR spectrum of palladium(II) oxybenziporphyrin complex 102 in $\mathrm{CDCl}_{3}$

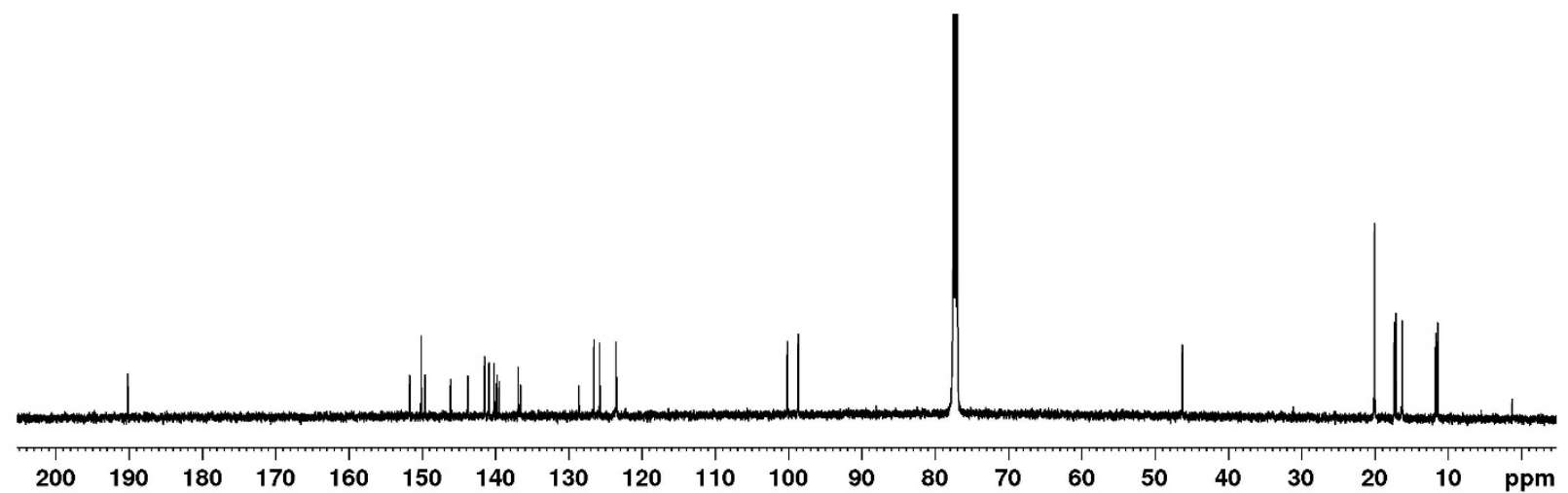

Figure 57. $125 \mathrm{MHz}{ }^{13} \mathrm{C}$ NMR spectrum of palladium(II) oxybenziporphyrin complex 102 in $\mathrm{CDCl}_{3}$ 


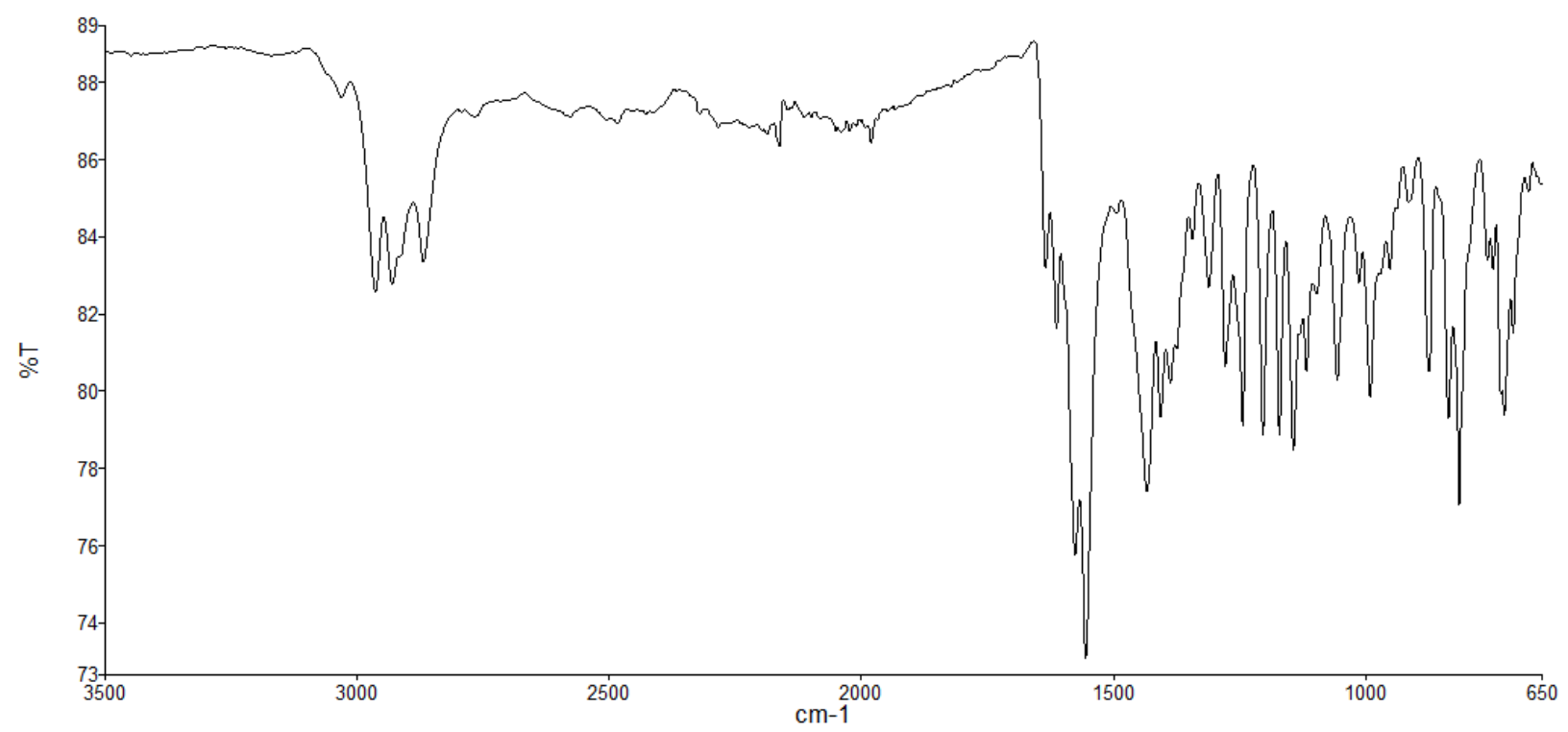

Figure 58. IR spectrum of palladium(II) oxybenziporphyrin complex 102

$N$-methyloxypyriporphyrin 100b was synthesized by reaction of $N$-methyl tripyrrane $\mathbf{5 4 d}$ with dialdehyde 101b in dichloromethane and TFA (Scheme 37, page 116). The crude material was chromatographed with neutral alumina, eluting with chloroform. The compound did not recrystallize well, as it was very soluble in organic solvents, and was therefore collected in $35.8 \%$ yield and characterized without further purification. The proton NMR spectrum of this compound suggests it is fairly aromatic, due to the internal $\mathrm{N}$-methyl and $\mathrm{NH}$ protons appearing as a $3 \mathrm{H}$ singlet and $1 \mathrm{H}$ broad singlet at -4.20 and $-1.26 \mathrm{ppm}$, respectively (Figure 59). The four mesoprotons were observed as $1 \mathrm{H}$ singlets between 9.64 and $10.89 \mathrm{ppm}$ and the external methyl groups were identified as two $3 \mathrm{H}$ singlets at downfield values of 3.44 and $3.58 \mathrm{ppm}$. For comparison, the proton NMR spectrum of $N$-unsubstituted oxypyriporphyrin ${ }^{77} \mathbf{7 b}$ has previously been reported to display the meso-protons as four $1 \mathrm{H}$ singlets between 9.48 and $10.96 \mathrm{ppm}$ and the external methyl groups as two $3 \mathrm{H}$ singlets at 4.01 and $4.04 \mathrm{ppm}$. Two broad singlets near $-3.6 \mathrm{ppm}$ were also observed for the NH protons, since there are two internal protons compared to the single proton 
within the cavity of $\mathbf{1 0 0 b}$. The carbon-13 spectrum of 100b (Figure 60) showed the carbonyl carbon resonating at $185.53 \mathrm{ppm}$ and the meso-carbons at 96.8, 97.3, 113.3 and $119.8 \mathrm{ppm}$, while the carbonyl carbon of $N$-unsubstituted oxypyriporphyrin $7 \mathbf{b}$ resonated at $184.9 \mathrm{ppm}$ and the four meso-carbons were observed between 96 and $108 \mathrm{ppm}$. The IR spectrum for $\mathrm{N}$ methyloxypyriporphyrin 100b (Figure 61) shows a strong peak at $1626 \mathrm{~cm}^{-1}$, which is similar to the peak observed at $1629 \mathrm{~cm}^{-1}$ for $N$-unsubstituted oxypyriporphyrin $\mathbf{7 b}$.

Although the NMR spectrum of $\mathbf{1 0 0 b}$ are not significantly altered by the presence of an internal methyl group, comparison of the UV-Vis spectra of $N$-methyloxypyriporphyrin $100 \mathbf{b}$ and the $N$-unsubstituted oxypyriporphyrin $\mathbf{7 b}$ showed drastic differences when the internal methyl group was present (Figure 62). There are similar bathochromic shifts in the spectrum for $\mathbf{1 0 0 b}$ to those seen for all of the methylated derivatives discussed above. However, much more significantly, the absorptions for $\mathbf{1 0 0 b}$ were much weaker and broader than those observed for $\mathbf{7 b}$. These observations suggest that the internal methyl group induces a significant amount of distortion to the chromophore. The Soret band for the $N$-unsubstituted system was observed at 420 $\mathrm{nm}$, while the Soret band for the $N$-methyl derivative was a broad band centered on $434 \mathrm{~nm}$. When TFA was added to $\mathbf{1 0 0 b}$ a dicationic species was formed and the peaks in the resulting UV-Vis spectrum (Figure 63) were seen to sharpen and increase in intensity, with the Soret band centered on $426 \mathrm{~nm}$. 


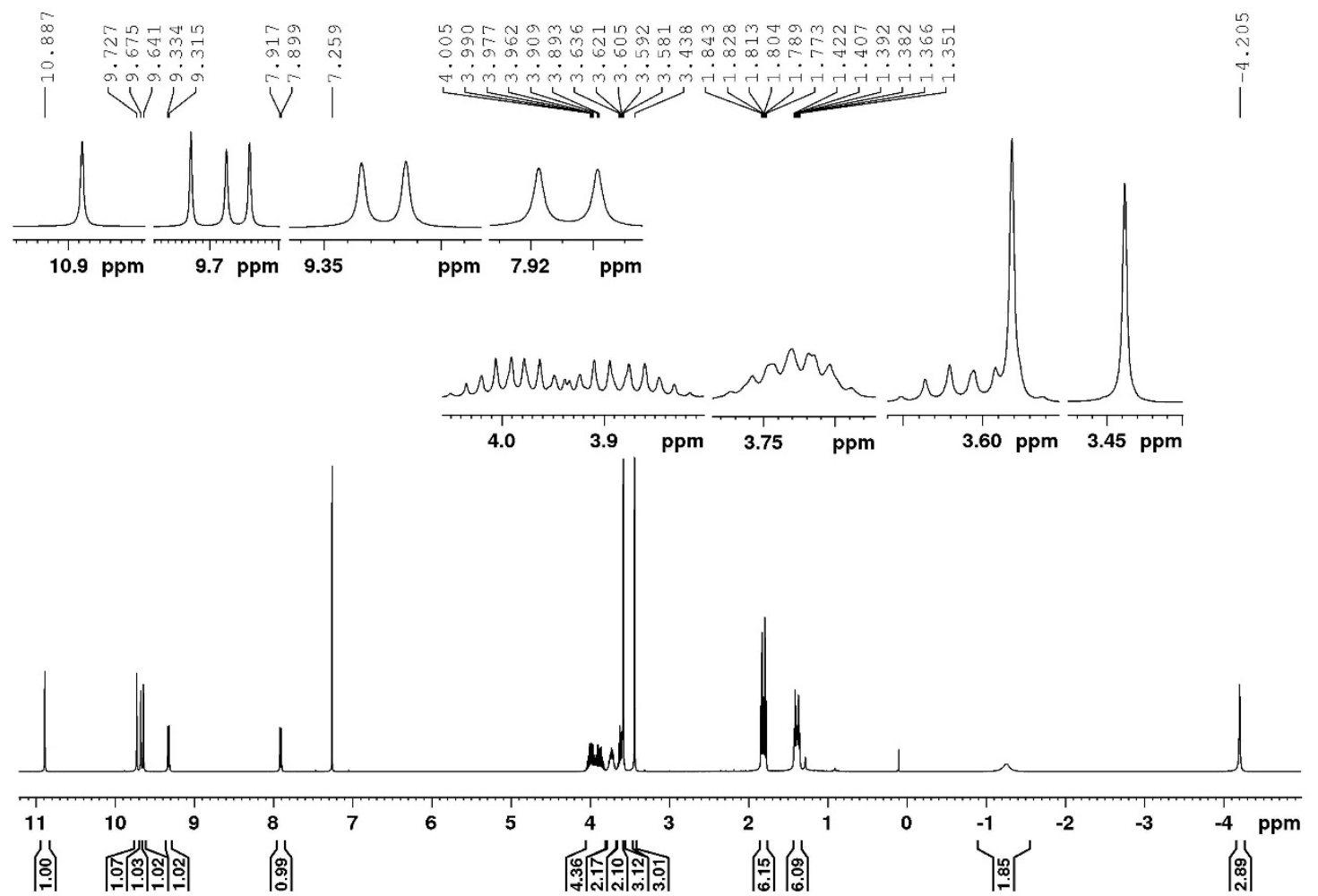

Figure 59. $500 \mathrm{MHz}{ }^{1} \mathrm{H}$ NMR spectrum of $N$-methyloxypyriporphyrin $\mathbf{1 0 0 b}$ in $\mathrm{CDCl}_{3}$

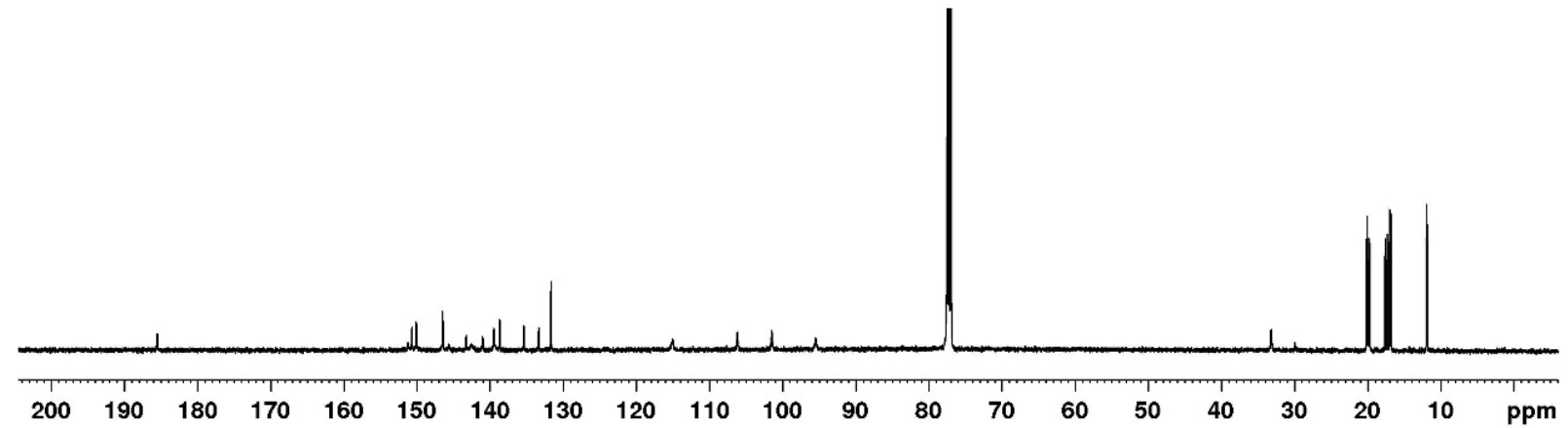

Figure 60. $125 \mathrm{MHz}{ }^{13} \mathrm{C}$ NMR spectrum of $N$-methyloxypyriporphyrin $100 \mathrm{~b}$ in $\mathrm{CDCl}_{3}$ 


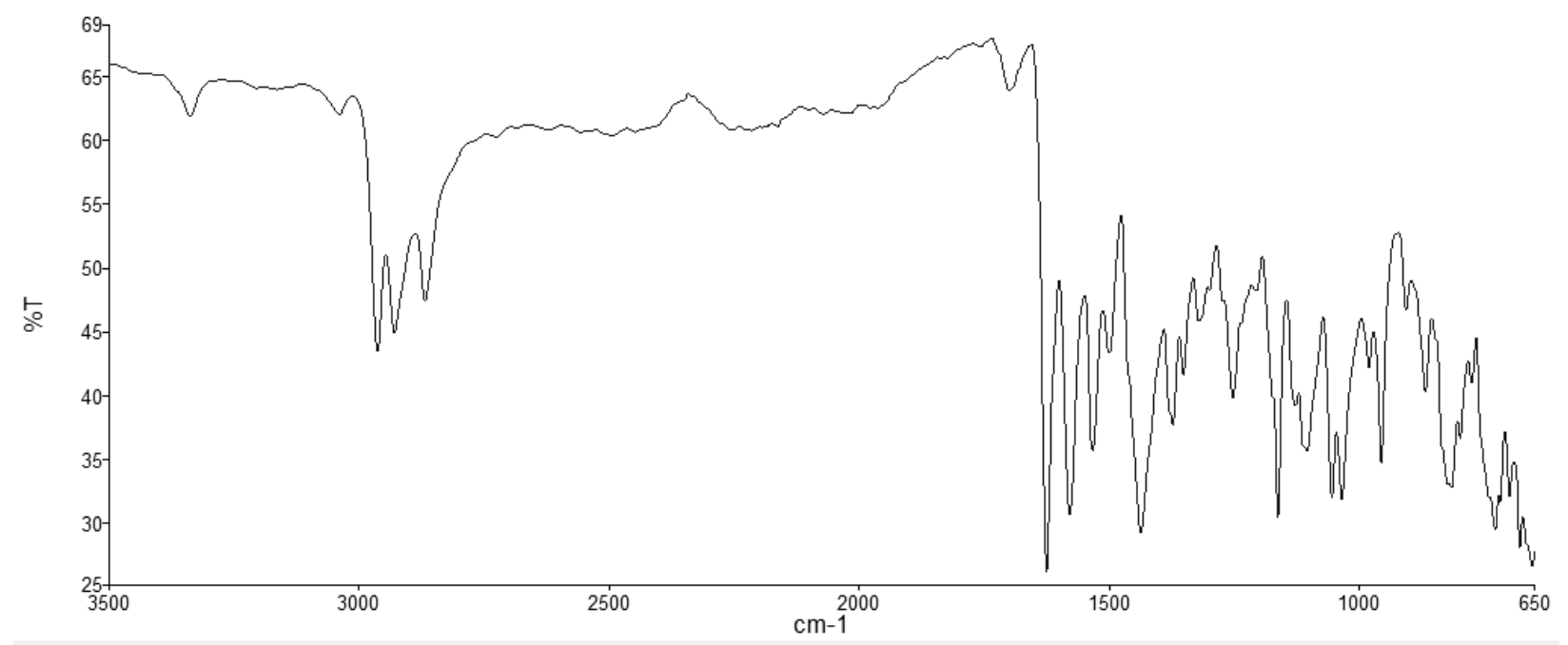

Figure 61. IR spectrum of $N$-methyloxypyriporphyrin 100b

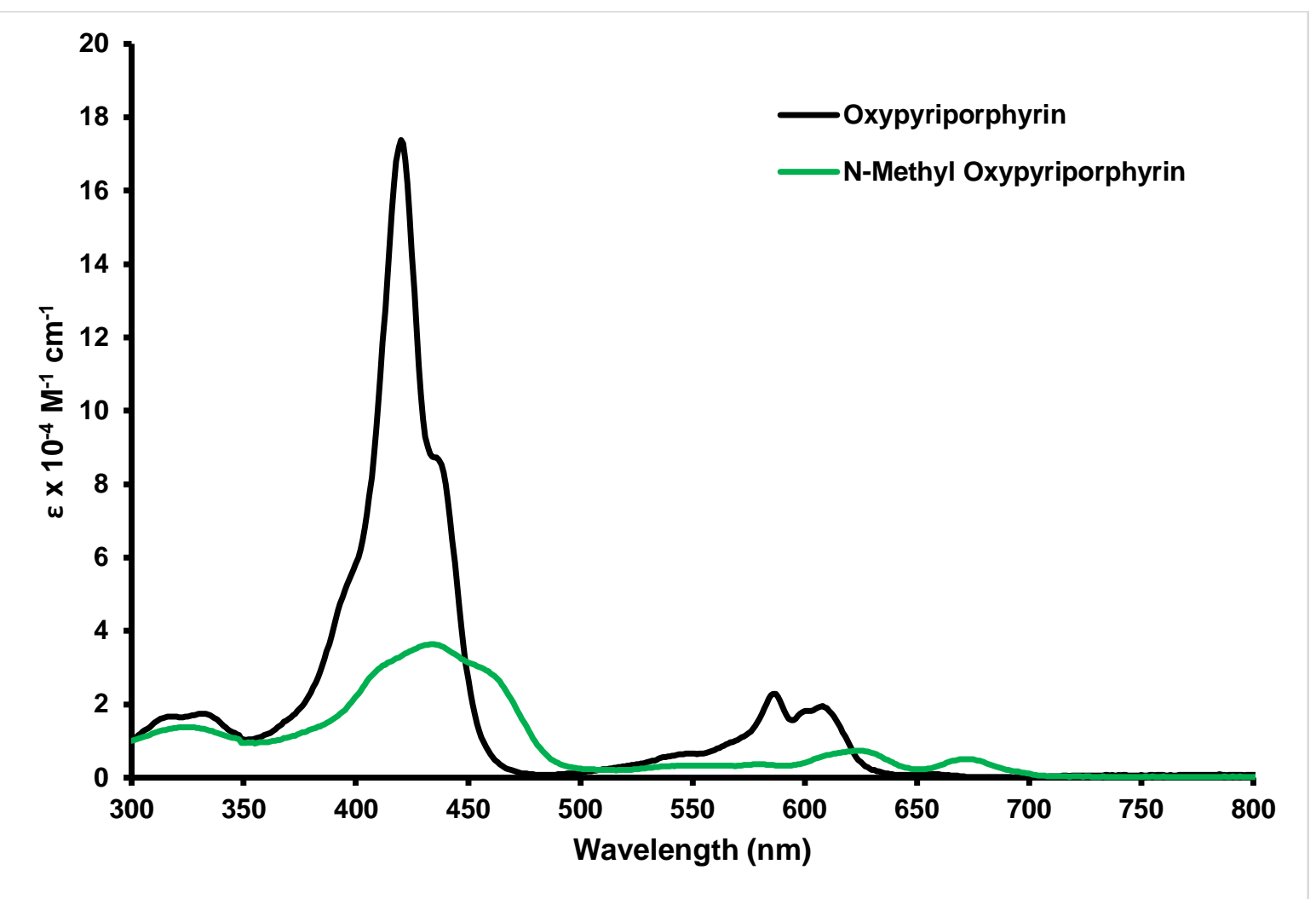

Figure 62. UV-Vis spectra of $N$-methyloxypyriporphyrin 100b (green line) and the $N$ unsubstituted oxypyriporphyrin $7 \mathbf{b}$ (black line) 


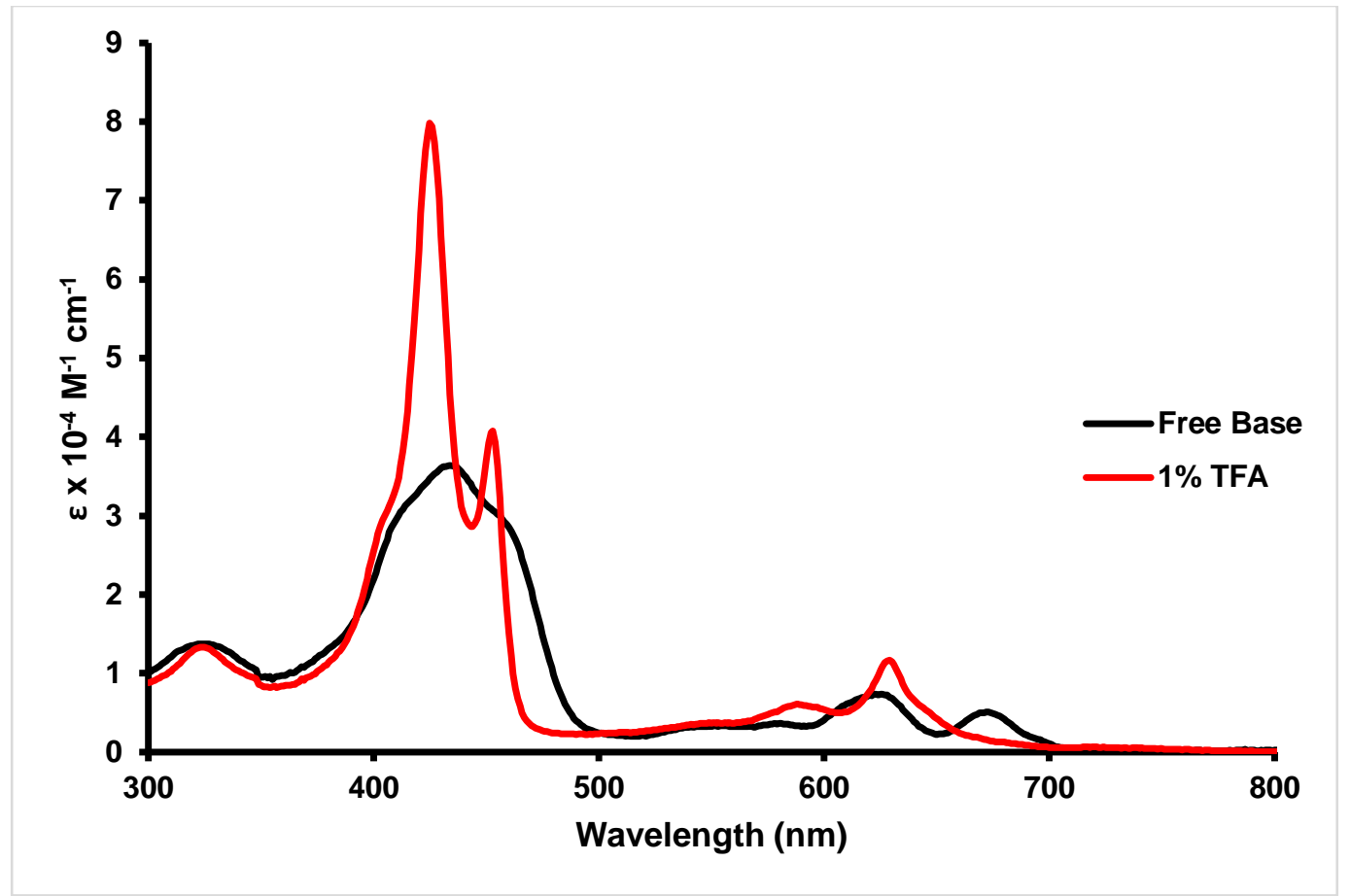

Figure 63. UV-Vis spectra of $\mathbf{1 0 0 b}$ in $1 \%$ triethylamine-dichloromethane (free base, black line) and $1 \%$ TFA-dichloromethane (dication, green line)

An internally methylated azuliporphyrin $\mathbf{1 0 3 \mathbf { b }} \mathrm{H}_{2}{ }^{2+}$ was also synthesized (Scheme 39). It

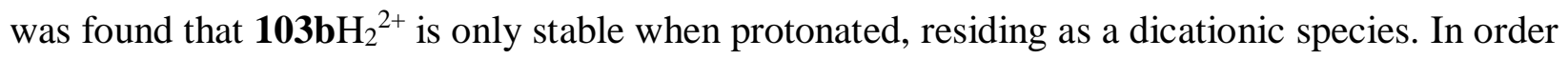
to optimized yields, $N$-methyl tripyrrane $\mathbf{5 4 d}$ was condensed overnight with azulene dialdehyde 104 in dichloromethane in the presence of TFA under dilute conditions. Oxidation by ferric chloride, followed by a wash with $10 \%$ aqueous hydrochloric acid, gave the crude dihydrochloride. Recrystallization twice from chloroform-hexanes gave $\mathbf{1 0 3 b \mathrm { b } _ { 2 }}{ }^{2+}$ in $78 \%$ yield. 


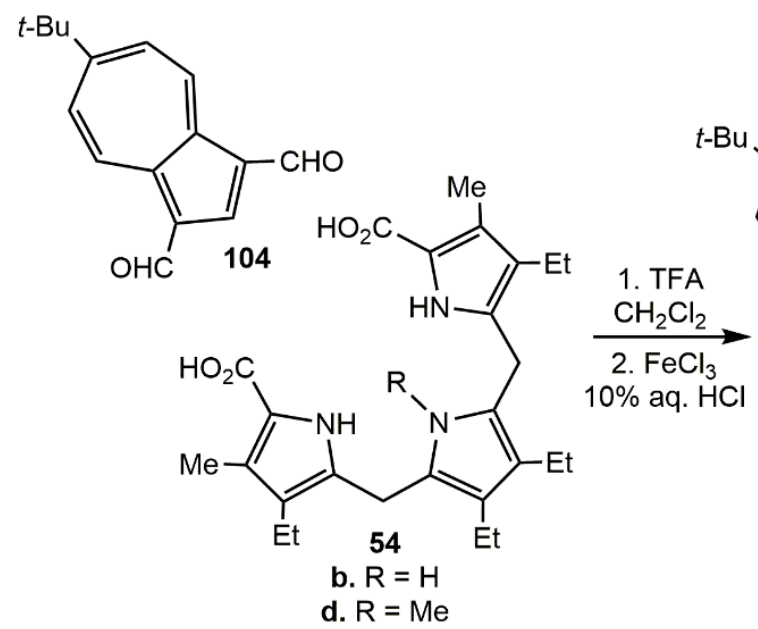

b. $R=H$

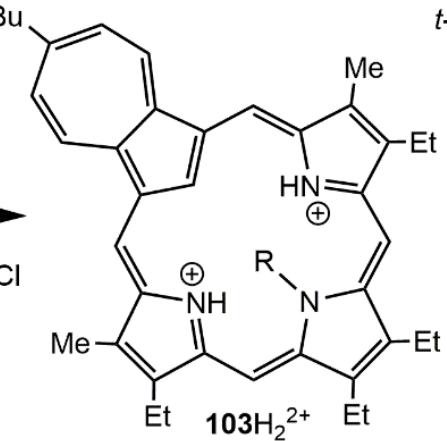

a. $\mathrm{R}=\mathrm{H}$

b. $\mathrm{R}=\mathrm{Me}$

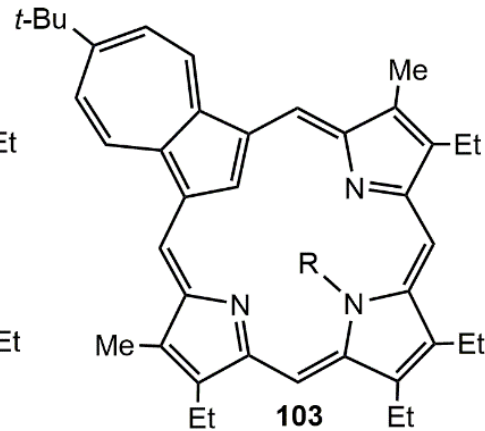

a. $\mathrm{R}=\mathrm{H}$

b. $\mathrm{R}=\mathrm{Me}$

Scheme 39. Synthesis of $N$-methylazuliporphyrin $\mathbf{1 0 3 b \mathrm { b } _ { 2 }}{ }^{2+}$

The proton NMR spectrum for $\mathbf{1 0 3 b H}_{2}{ }^{2+}$ demonstrated that the macrocycle posseses a plane of symmetry. This is evident in part from the two $2 \mathrm{H}$ singlets at 9.66 and $10.72 \mathrm{ppm}$ for the meso-protons and the two $2 \mathrm{H}$ doublets at 8.89 and $10.39 \mathrm{ppm}$ for the four external azulene protons (Figure 64). The external methyl groups were also observed as a $6 \mathrm{H}$ singlet at $3.46 \mathrm{ppm}$. These

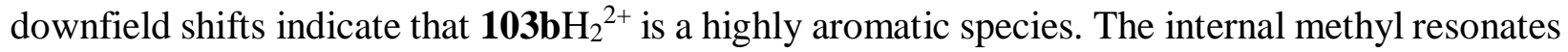
as a $3 \mathrm{H}$ singlet at $-3.36 \mathrm{ppm}$, while the internal $\mathrm{CH}$ and $\mathrm{NH}$ protons appear as a $1 \mathrm{H}$ singlet at -3.38 ppm and a $2 \mathrm{H}$ broad singlet at $-0.36 \mathrm{ppm}$, respectively, again confirming the aromatic nature of this system. The proton NMR spectrum reported for a $N$-unsubstituted azuliporphyrin dication $103 \mathbf{a H}_{2}{ }^{2+}$ gave similar chemical shifts. ${ }^{81}$ In that case, the meso-protons were reported as two $2 \mathrm{H}$ singlets at 9.93 and $10.39 \mathrm{ppm}$, while the external methyl groups were observed as a $6 \mathrm{H}$ singlet at 3.47 ppm. Furthermore, the internal peaks of the non-methylated counterpart were similar to those of the $\mathrm{N}$-methyl derivative, where the internal $\mathrm{CH}$ is observed as a $1 \mathrm{H}$ singlet at $-3.19 \mathrm{ppm}$, and the internal $\mathrm{NH}$ protons resonate as broad $1 \mathrm{H}$ and $2 \mathrm{H}$ singlets at -2.0 and $-0.25 \mathrm{ppm}$, respectively. This data indicates that the methyl group does not undermine the aromaticity of the tert-butyl 
azuliporphyrin dication. However, while azuliporphyrin 103a lacking an internal methyl could be isolated as a free base and characterized, this was not possible for $\mathbf{1 0 3 b}$. Further evidence of the

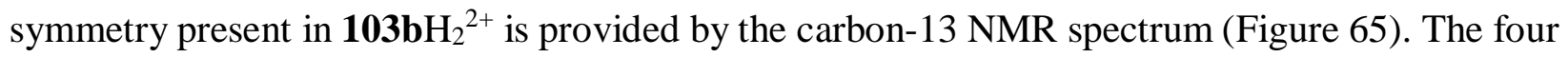
meso-carbons gave two peaks at 97.2 and $111.0 \mathrm{ppm}$, while the internal methyl carbon and the quartnerary tert-butyl carbon resonated at 32.3 and $40.8 \mathrm{ppm}$, respectively. The three carbons of the methyl groups on the tert-butyl substituent appeared at $31.5 \mathrm{ppm}$.

The UV-Vis data for $\mathbf{1 0 3} \mathbf{b}$ were obtained for the free base and the dication, and the free base spectrum in $1 \%$ triethylamine-dichloromethane was compared to the one previously reported for the $N$-unsubstituted counterpart 103a (Figure 66). Overall, this data shows bathochromic shifts in the methylated derivative compared to its non-methylated counterpart. Each spectrum contains multiple bands, the strongest for 103b appearing at $363 \mathrm{~nm}$, while the strongest band for 103a is observed at a slightly shorter wavelength of $358 \mathrm{~nm}$. These results indicate that the presence of an internal methyl substituent does alter the tert-butylazuliporphyrin chromophore in a similar fashion to the other methylated porphyrinoids described above, and the effects are nowhere near as drastic as those seen for oxybenzi- and oxypyriporphyrins. The UV-Vis spectra of the $N$-methyl derivative in $1 \%$ triethylamine and $1 \%$ trifluoroacetic acid were also contrasted (Figure 67). These results show that the spectrum of the protonated species possesses stronger and sharper peaks than the spectrum of the free base, demonstrating that protonation considerably alters the azuliporphyrin chromophore. The protonated form shows a strong absorbance at $370 \mathrm{~nm}$, followed by a Soret band at $468 \mathrm{~nm}$. A third reasonably strong band is also seen at $647 \mathrm{~nm}$. In contrast, the free base shows several mid-level absorptions between 363 and $482 \mathrm{~nm}$, followed by a very broad, weaker band centered at $669 \mathrm{~nm}$. 


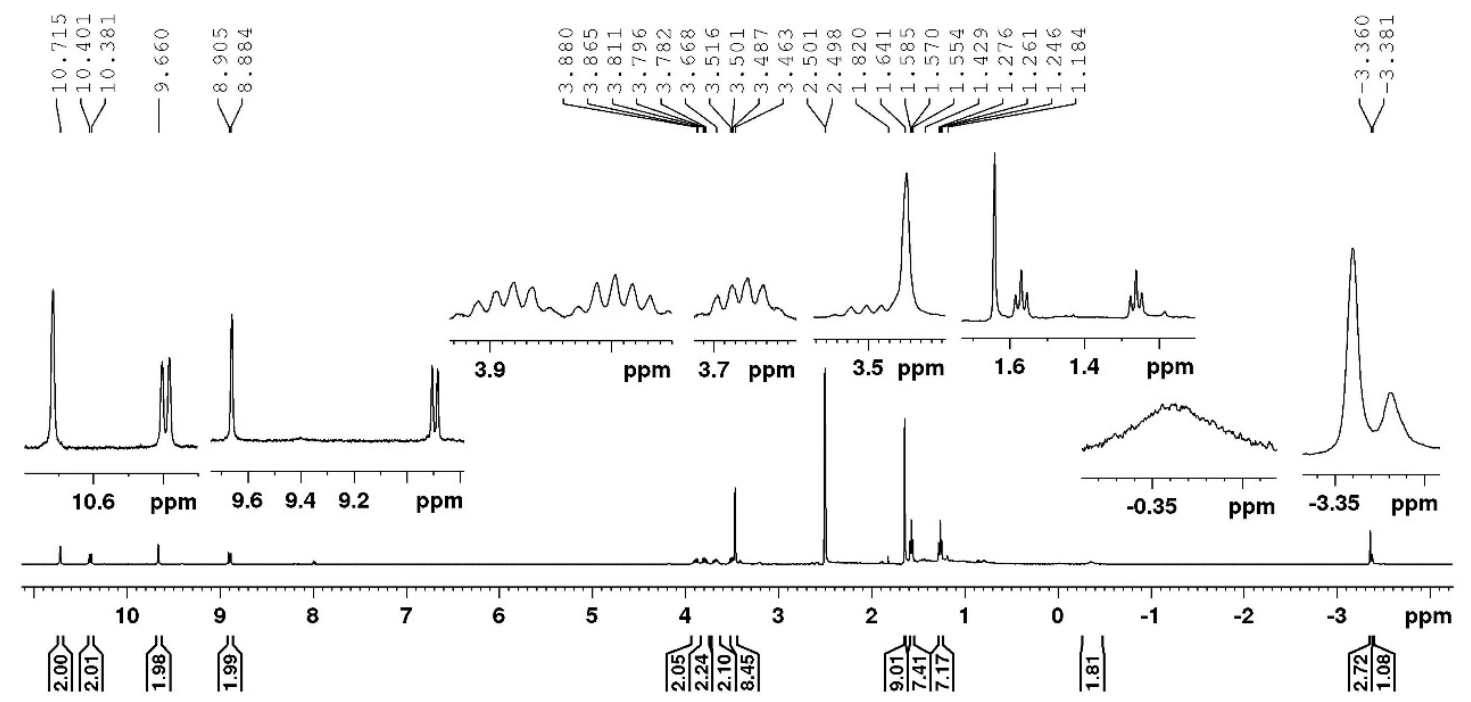

Figure 64. $500 \mathrm{MHz}{ }^{1} \mathrm{H}$ NMR spectrum of tert-butyl azuliporphyrin $\mathbf{1 0 3 b H}_{2}{ }^{2+}$ in TFA- $d_{6}$-DMSO

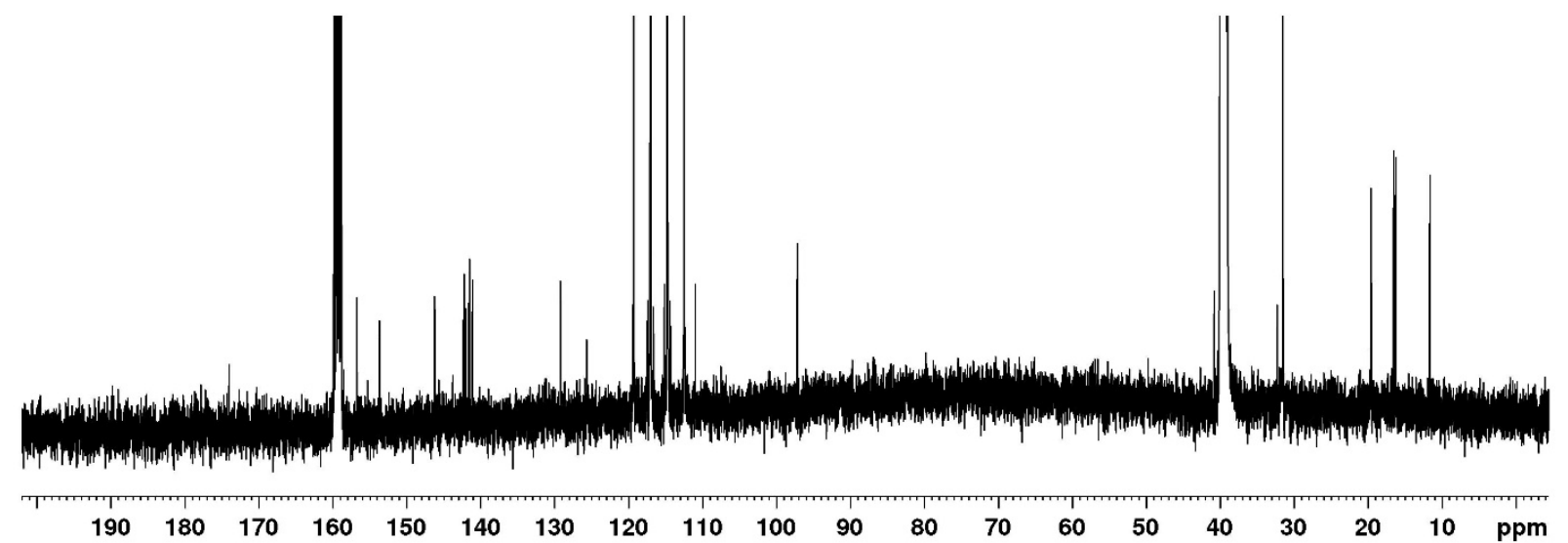

Figure 65. $125 \mathrm{MHz}{ }^{13} \mathrm{C}$ NMR spectrum of tert-butyl azuliporphyrin $\mathbf{1 0 3 b H}_{2}{ }^{2+}$ in TFA-d ${ }_{6}$-DMSO 


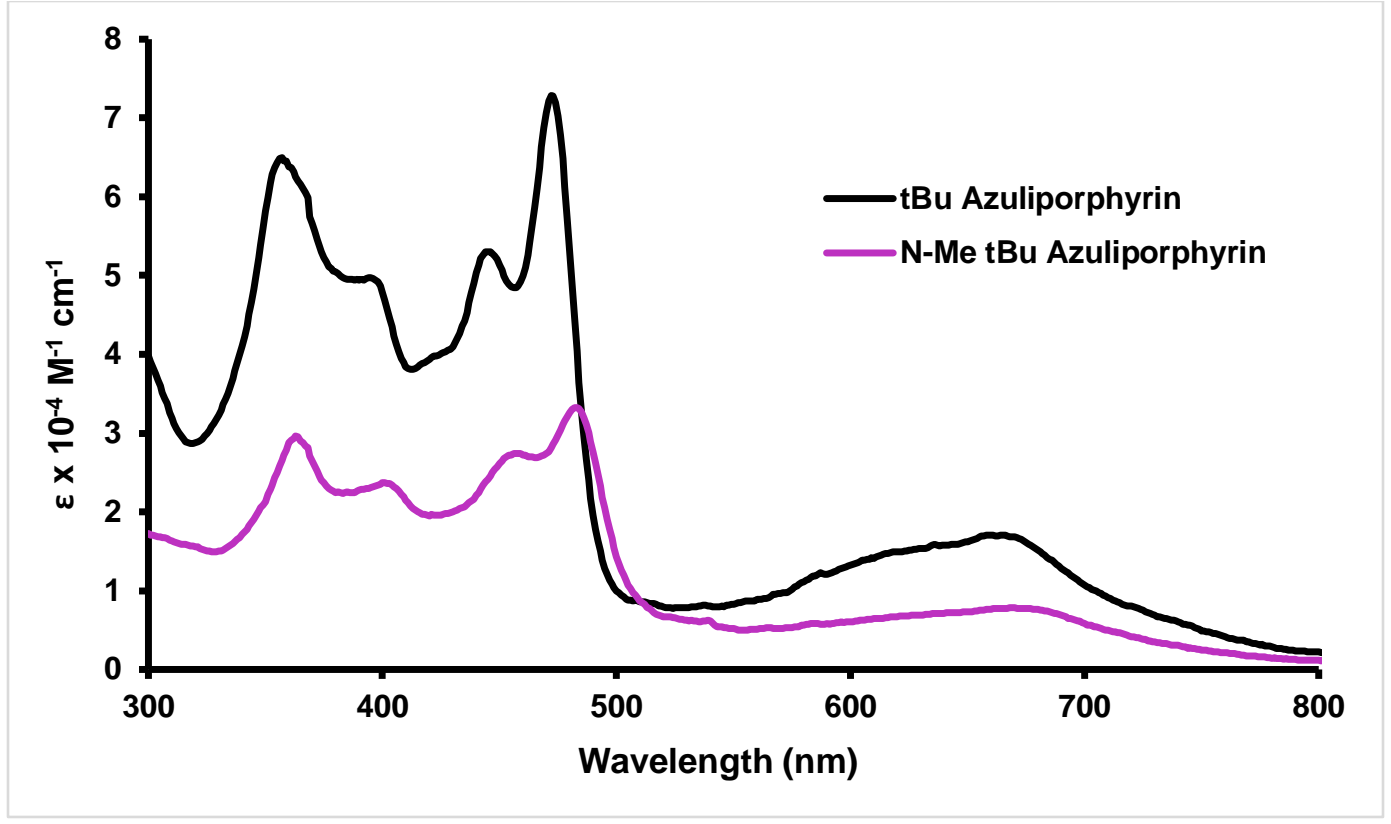

Figure 66. UV-Vis spectra of 103a in 1\% triethylamine-dichloromethane (free base, black line) and $\mathbf{1 0 3 b}$ in $1 \%$ triethylamine-dichloromethane (free base, purple line).

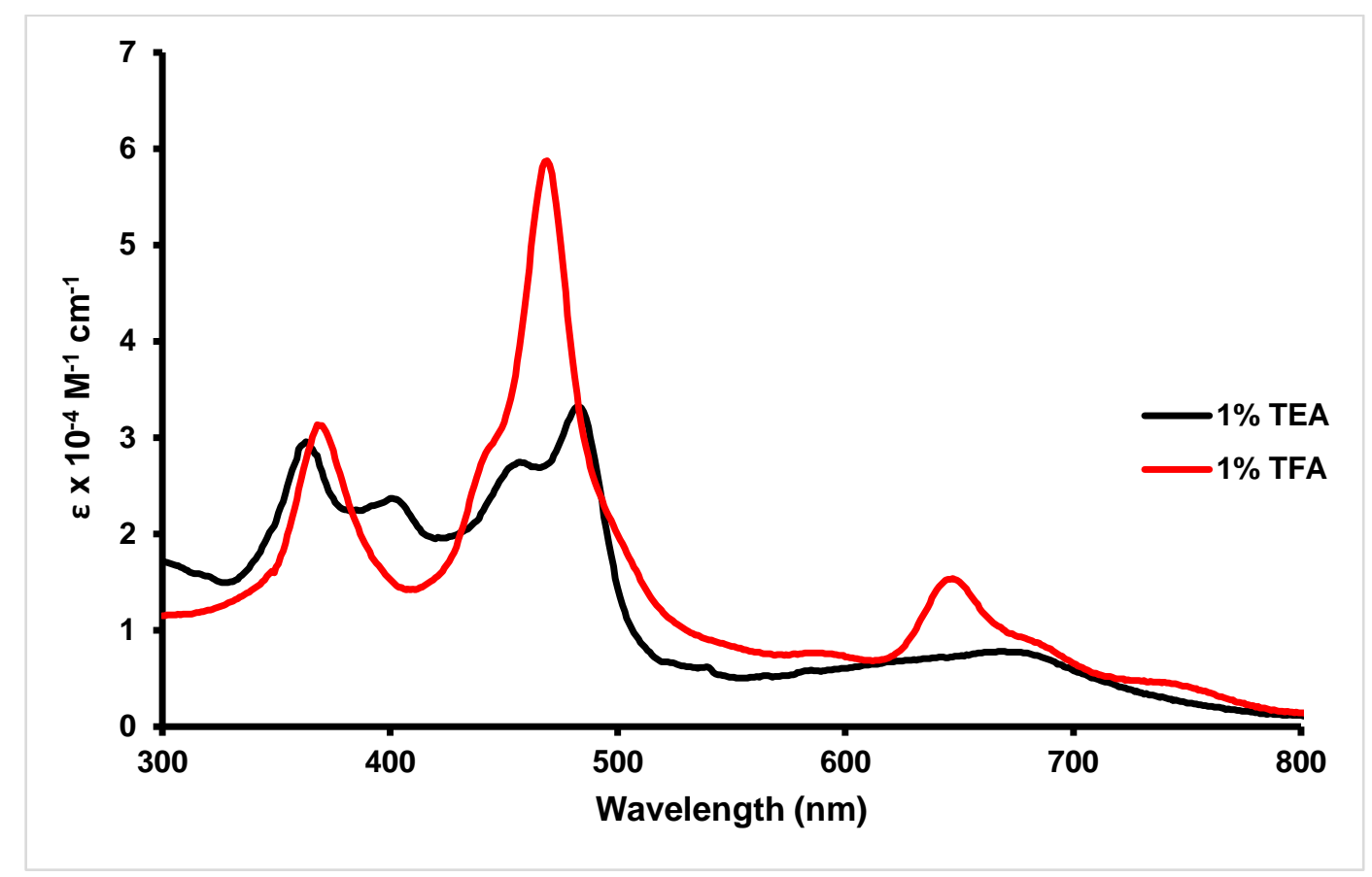

Figure 67. UV-Vis spectra of $\mathbf{1 0 3 b}$ in 1\% triethylamine-dichloromethane (free base, black line) and in 1\% TFA-dichloromethane (dication, red line). 


\section{Conclusion}

By comparing a series of porphyrin analogues with their $N$-methylated derivatives, it has been found that the introduction of an internal methyl group significantly affects the porphyrinoid chromophore, which consequently alters some of the electronic properties of these macrocycles. These effects are primarily evident in the UV-Vis spectra, where bathochromic shifts and peak broadening are observed for each of the reported $N$-methyl porphyrinoid systems. Other spectroscopic properties are not as clearly affected by the internal substituent, and only slight shifts are seen in the NMR spectra of the $N$-methylated compounds. This series of macrocycles also demonstrates that the versatile ' $3+1$ ' method can successfully introduce an internal substituent into a number of different porphyrin analogues. Furthermore, the introduction of a $N$-methyl group can alter the coordination chemistry of these structures, and this was demonstrated by the formation of a palladium(II) $N$-methyloxybenziporphyrin. In this case, not only does the internal substituent not inhibit metal coordination, it also switches the carbaporphyrinoid cavity from being a trianionic ligand to a dianionic ligand. 


\section{Experimental:}

Melting points are uncorrected. NMR spectra were recorded using a 400 or $500 \mathrm{MHz}$ NMR spectrometer and were run at $302 \mathrm{~K}$ unless otherwise indicated. ${ }^{1} \mathrm{H}$ NMR values are reported as chemical shifts $\delta$, relative integral, multiplicity ( $\mathrm{s}$, singlet; $\mathrm{d}$, doublet; $\mathrm{t}$, triplet; q, quartet; $\mathrm{m}$, multiplet; br, broad peak) and coupling constant $(J)$. Chemical shifts are reported in parts per million (ppm) relative to $\mathrm{CDCl}_{3}\left({ }^{1} \mathrm{H}\right.$ residual $\mathrm{CHCl}_{3}$ singlet $\delta 7.26,{ }^{13} \mathrm{C} \mathrm{CDCl}_{3}$ triplet $\left.\delta 77.23\right)$ or $d_{6}$-DMSO ( ${ }^{1} \mathrm{H}$ residual $d_{5}$-DMSO pentet $\delta 2.49,{ }^{13} \mathrm{C} d_{6}$-DMSO septet $\left.\delta 39.7\right)$ and coupling constants were taken directly from the spectra. NMR assignments were made with the aid of ${ }^{1} \mathrm{H}-$ ${ }^{1} \mathrm{H}$ COSY, HSQC, DEPT-135 and nOe difference proton NMR spectroscopy. 2D experiments were performed using standard software. High-resolution mass spectra (HRMS) were carried out using a double focusing magnetic sector instrument.

8,12,13,17-Tetraethyl-7,18-dimethyl-21-oxaporphyrin (96a). Tripyrrane dicarboxylic acid 54b (100 mg, $0.221 \mathrm{mmol})$ was dissolved in TFA (1 mL) and stirred under nitrogen for 2 minutes. The mixture was diluted with dichloromethane $(99 \mathrm{~mL})$, followed by the addition of 2,5furandicarboxaldehyde (28.2 $\mathrm{mg}, 0.227 \mathrm{mmol})$, and was left to stir overnight under nitrogen. The solution was washed with ferric chloride $(0.1 \%, 200 \mathrm{~mL})$, water and then aqueous sodium bicarbonate solution. The solvent was removed under reduced pressure and the crude material chromatographed on neutral grade 3 alumina, eluting with chloroform. A bright red/pink band was collected and recrystallized from chloroform-hexanes to give $\mathbf{9 6 a}(66.0 \mathrm{mg}, 0.133 \mathrm{mmol}, 66 \%)$ as dark purple/blue crystals, $\mathrm{mp}>300{ }^{\circ} \mathrm{C}$. UV-Vis (1\% Et $\mathrm{t}_{3} \mathrm{~N}$-dichloromethane): $\lambda_{\max } / \mathrm{nm}$ (log $\left.\varepsilon\right) 371$ (4.93), 392, (4.92), 489 (4.11), 520 (3.88), 585 (3.54), 643 (3.26). UV-Vis (1\% TFAdichloromethane): $\lambda_{\max } / \mathrm{nm}$ (log $\left.\varepsilon\right) 394$ (5.33), 523 (3.90), 551 (4.04), 599 (3.40). ${ }^{1} \mathrm{H}$ NMR (500 $\left.\mathrm{MHz}, \mathrm{TFA}-\mathrm{CDCl}_{3}\right): \delta-4.53\left(3 \mathrm{H}\right.$, br s, 2 x NH), 1.85-1.91 (12H, m, 4 x $\mathrm{CH}_{2} \mathrm{CH}_{3}$, overlapping 
triplets), 3.83 (6H, s, 7,18-Me), 4.26-4.33 (8H, m, 4 x CH $2 \mathrm{CH}_{3}$ ), 10.61 (2H, s), 11.04 (2H, s), 11.18 (2H, s). ${ }^{13} \mathrm{C}$ NMR (125 MHz, TFA-CDCl $): ~ \delta 11.89,16.82,17.63,20.33,20.50,100.42,101.30$, 133.92, 140.92, 141.48, 142.68, 143.39, 146.30, 146.32, 147.39, 147.42, 155.31. HR-MS (ESI) $\mathrm{m} / \mathrm{z}:[\mathrm{M}+\mathrm{H}]+$ calcd for $\mathrm{C}_{30} \mathrm{H}_{34} \mathrm{~N}_{3} \mathrm{O} 452.2702$, found 452.2696 .

8,12,13,17-Tetraethyl-7,18,23-trimethyl-21-oxaporphyrin (96b). $N$-methyl tripyrrane 54d (200 mg, $0.428 \mathrm{mmol}$ ) was dissolved in TFA (2 mL) and stirred under nitrogen for 2 minutes. The solution was diluted with dichloromethane (38 mL), followed by immediate addition of 2,5furandicarboxaldehyde (54.5 mg, $0.441 \mathrm{mmol}$ ), and left to stir for another 2 hours under nitrogen. The mixture was neutralized by the dropwise addition of triethylamine, DDQ (100 mg) was added, and the solution stirred for 1 hour. The mixture was washed with water and then with aqueous sodium bicarbonate solution, and the solvent removed under reduced pressure. The crude material was chromatographed twice on neutral grade 3 alumina, eluting with chloroform, and a dark purple band was collected each time. Recrystallization from chloroform-hexanes gave pure 23-methyl21-oxaporphyrin (84.2 mg, $0.181 \mathrm{mmol}, 42 \%$ ) as dark purple/blue crystals, $\mathrm{mp}>300{ }^{\circ} \mathrm{C}$. UV-Vis (1\% Et 3 N-dichloromethane): $\lambda_{\max } / \mathrm{nm}$ (log $\left.\varepsilon\right) 378$ (4.74), 407 (4.81), 501 (4.09), 527 (3.69), 604 (3.45), 638 (3.08), 664 (3.23). UV-Vis (1\% TFA-dichloromethane): $\lambda_{\max } / \mathrm{nm}$ (log $\left.\varepsilon\right) 396$ (5.26), 528 (3.83), 562 (3.97), 612 (3.58). UV-Vis (5\% TFA-dichloromethane): $\lambda_{\max } / \mathrm{nm}$ (log $\varepsilon$ ) 395 (5.27), 528 (3.86), 561 (3.99), 611 (3.64), 730 (3.29). ${ }^{1} \mathrm{H}$ NMR (500 MHz, $\mathrm{CDCl}_{3}$ ): $\delta$-5.21 (3H, s, N-Me), $1.52\left(6 \mathrm{H}, \mathrm{t},{ }^{3} J_{\mathrm{HH}}=7.7 \mathrm{~Hz}, 12,13-\mathrm{CH}_{2} \mathrm{CH}_{3}\right), 1.87\left(6 \mathrm{H}, \mathrm{t},{ }^{3} \mathrm{~J}_{\mathrm{HH}}=7.7 \mathrm{~Hz}, 8,17-\mathrm{CH}_{2} \mathrm{CH}_{3}\right)$, 3.70 (6H, s, 7,18-Me), 3.77-3.84 (2H, m), 3.88-3.96 (2H, m), 4.05-4.13 (4H, m) $\left(4 \mathrm{x} \mathrm{CH}_{2} \mathrm{CH}_{3}\right)$ 10.34 (2H, s, 10,15-H), 10.69 (2H, s, 2,3-H), 11.10 (2H, s, 5,20-H). ${ }^{1} \mathrm{H}$ NMR (500 MHz, TFA$\left.\mathrm{CDCl}_{3}\right): \delta-5.60(3 \mathrm{H}, \mathrm{s}, \mathrm{N}-\mathrm{Me}),-4.28(2 \mathrm{H}, \mathrm{s}, 2 \mathrm{x} \mathrm{NH}), 1.52\left(6 \mathrm{H}, \mathrm{t},{ }^{3} \mathrm{~J}_{\mathrm{HH}}=7.7 \mathrm{~Hz}, 12,13-\mathrm{CH}_{2} \mathrm{CH}_{3}\right)$, $1.88\left(6 \mathrm{H}, \mathrm{t},{ }^{3} \mathrm{~J}_{\mathrm{HH}}=7.7 \mathrm{~Hz}, 8,17-\mathrm{CH}_{2} \mathrm{CH}_{3}\right), 3.79(6 \mathrm{H}, \mathrm{s}, 7,18-\mathrm{Me}), 3.81-3.92\left(4 \mathrm{H}, \mathrm{m}, 12,13-\mathrm{CH}_{2}\right)$, 
4.23-4.28 (4H, m, 8,17-CH $), 10.56$ (2H, s, 2,3-H), 10.82 (2H, s, 10,15-H), 11.10 (2H, s, 5,20-H).

${ }^{13} \mathrm{C}$ NMR (125 MHz, $\left.\mathrm{CDCl}_{3}\right): \delta 12.14,17.28,17.55,20.13,20.27,32.34$ (br, $\left.\mathrm{N}-\mathrm{CH}_{3}\right), 101.46$, 102.65, 132.53, 137.31, 140.38, 146.10, 147.71, 148.39, 152.96, 155.26. ${ }^{13} \mathrm{C}$ NMR (125 MHz, TFA-CDCl $): \delta 11.70,16.59,16.70,20.27,20.60,30.56\left(\mathrm{~N}-\mathrm{CH}_{3}\right), 100.61,102.47,134.42,140.45$, 141.23, 141.97, 142.94, 147.40, 156.05, 156.95. HR-MS (ESI) $\mathrm{m} / \mathrm{z}$ : $[\mathrm{M}+\mathrm{H}]^{+}$calcd for $\mathrm{C}_{31} \mathrm{H}_{36} \mathrm{~N}_{3} \mathrm{O}$ 466.2858, found 466.2855 .

8,12,13,17-Tetraethyl-7,18-dimethyl-21-thiaporphyrin (97a). Tripyrrane dicarboxylic acid 54b (100 mg, $0.221 \mathrm{mmol})$ was dissolved in TFA (1 mL) and stirred under nitrogen for 2 minutes. The mixture was diluted with dichloromethane $(99 \mathrm{~mL})$, followed by the addition of 2,5thiophenedicarboxaldehyde (31.7 mg, $0.226 \mathrm{mmol}$ ), and left to stir overnight under nitrogen. The mixture was washed with ferric chloride $(0.1 \%, 200 \mathrm{~mL})$, water and then aqueous sodium bicarbonate solution. The solvent was removed under reduced pressure and the crude material was then chromatographed on neutral grade 3 alumina, eluting with dichloromethane. A red/pink band was collected, which was chromatographed a second time on neutral alumina, eluting with dichloromethane, and a green/brown band was collected. The solid was vacuum dried overnight to give 21-thiaporphyrin $97 \mathrm{a}$ (43.0 $\mathrm{mg}, 0.0921 \mathrm{mmol}, 42 \%$ ) as a dark solid, $\mathrm{mp}>300{ }^{\circ} \mathrm{C}$. UV-Vis (1\% Et 3 N-dichloromethane): $\lambda_{\max } / \mathrm{nm}$ (log $\left.\varepsilon\right) 398$ (5.05), 495 (4.22), 525 (4.02), 590 (3.60), 597 (3.50), 649 (3.28). UV-Vis (1\% TFA-dichloromethane): $\lambda_{\max } / \mathrm{nm}(\log \varepsilon) 418$ (5.24), 549 (3.89), 576 (3.96). ${ }^{1} \mathrm{H}$ NMR (500 MHz, $\left.\mathrm{CDCl}_{3}\right): \delta-3.87(1 \mathrm{H}$, br s, NH), $1.86(6 \mathrm{H}, \mathrm{t}, J=7.7 \mathrm{~Hz}, 8,17-$ $\left.\mathrm{CH}_{2} \mathrm{CH}_{3}\right), 1.95\left(6 \mathrm{H}, \mathrm{t}, J=7.7 \mathrm{~Hz}, 12,13-\mathrm{CH}_{2} \mathrm{CH}_{3}\right), 3.45(6 \mathrm{H}, \mathrm{s}, 7,18-\mathrm{Me}), 3.93(4 \mathrm{H}, \mathrm{q}, J=7.7 \mathrm{~Hz}$, 8,17- $\mathrm{CH}_{2} \mathrm{CH}_{3}$ ), $4.23\left(4 \mathrm{H}, \mathrm{q}, J=7.7 \mathrm{~Hz}, 12,13-\mathrm{CH}_{2} \mathrm{CH}_{3}\right), 10.13(2 \mathrm{H}, \mathrm{s}, 2,3-\mathrm{H}), 10.15$ (2H, s, 10,15H), $10.72(2 \mathrm{H}, \mathrm{s}, 5,20-\mathrm{H}) .{ }^{1} \mathrm{H}$ NMR (500 MHz, TFA-CDCl $): \delta 1.84$ (6H, t, $J=7.8 \mathrm{~Hz}, 8,17-$ $\left.\mathrm{CH}_{2} \mathrm{CH}_{3}\right), 1.89$ (6H, t, $\left.J=7.8 \mathrm{~Hz}, 12,13-\mathrm{CH}_{2} \mathrm{CH}_{3}\right), 3.79(6 \mathrm{H}, \mathrm{s}, 7,18-\mathrm{Me}), 4.23(4 \mathrm{H}, \mathrm{q}, J=7.8 \mathrm{~Hz}$, 
8,17- $\left.\mathrm{CH}_{2} \mathrm{CH}_{3}\right), 4.32\left(4 \mathrm{H}, \mathrm{q}, J=7.8 \mathrm{~Hz}, 12,13-\mathrm{CH}_{2} \mathrm{CH}_{3}\right), 10.60(2 \mathrm{H}, \mathrm{s}, 2,3-\mathrm{H}), 10.97$ (2H, s, 10,15H), 11.66 (2H, s, 5,20-H). ${ }^{13} \mathrm{C}$ NMR (125 MHz, $\left.\mathrm{CDCl}_{3}\right): \delta 11.55,17.85,18.57,20.05,20.14$, 99.16, 112.68, 133.11, 136.76, 138.28, 140.47, 145.98, 146.80, 153.76, 156.48. ${ }^{13} \mathrm{C}$ NMR (125 MHz, TFA-CDCl $): \delta 12.05,16.50,17.65,20.36,20.47,100.90,115.56,139.31,142.98,142.99$, 143.70, 145.34, 146.14, 147.18, 147.56, 147.57, 150.19. HR-MS (ESI) m/z: [M + H]+ calcd for $\mathrm{C}_{30} \mathrm{H}_{34} \mathrm{~N}_{3} \mathrm{~S} 468.2473$, found 468.2481 .

8,12,13,17-Tetratethyl-7,18,23-trimethyl-21-thiaporphyrin (97b). $N$-methyl tripyrrane 54d (200 mg, $0.428 \mathrm{mmol}$ ) was dissolved in TFA (2 mL) and stirred under nitrogen for 2 minutes. The solution was diluted with dichloromethane ( $38 \mathrm{~mL}$ ), followed by the immediate addition of 2,5thiophendicarboxaldehyde (62.0 mg, $0.443 \mathrm{mmol}$ ), and left to stir for another 2 hours under nitrogen. The mixture was neutralized by the dropwise addition of triethylamine, DDQ (100 mg) was added, and the solution stirred for 1 hour. The mixture was washed with water and then with aqueous sodium bicarbonate solution, and the solvent removed under reduced pressure. The crude material was chromatographed twice on neutral grade 3 alumina eluting with dichloromethane and a dark brown band was collected each time. The product was vacuum dried overnight to give 23methyl-21-thiaporphyrin (34.6 mg, $0.0718 \mathrm{mmol}, 17 \%$ ) as a dark blue/purple solid, mp > $300{ }^{\circ} \mathrm{C}$. UV-Vis (1\% Et 3 N-dichloromethane): $\lambda_{\max } / \mathrm{nm}$ (log $\left.\varepsilon\right) 412$ (4.89), 505 (4.09), 533 (3.67), 607 (3.31), 640 (2.76), 668 (3.08). UV-Vis (1\% TFA -dichloromethane): $\lambda_{\max } / \mathrm{nm}(\log \varepsilon) 425$ (5.13), 556 (3.67), 587 (3.73). UV-Vis (5\% TFA -dichloromethane): $\lambda_{\max } / \mathrm{nm}$ (log $\left.\varepsilon\right) 423$ (5.15), 554 (3.68), 584 (3.74). ${ }^{1} \mathrm{H}$ NMR (500 MHz, $\left.\mathrm{CDCl}_{3}\right): \delta-4.39$ (3H, s, N-Me), $1.48\left(6 \mathrm{H}, \mathrm{t},{ }^{3} J_{\mathrm{HH}}=7.7 \mathrm{~Hz}\right.$, 12,13- $\left.\mathrm{CH}_{2} \mathrm{CH}_{3}\right), 1.84\left(6 \mathrm{H}, \mathrm{t},{ }^{3} \mathrm{~J}_{\mathrm{HH}}=7.7 \mathrm{~Hz}, 8,17-\mathrm{CH}_{2} \mathrm{CH}_{3}\right), 3.39$ (6H, s, 7,18-Me), 3.67-3.74 (2H, m), 3.78-3.95 (6H, m) (4 x CH $\left.\mathrm{CH}_{3}\right) 9.96(2 \mathrm{H}, \mathrm{s}, 10,15-\mathrm{H}), 10.00$ (2H, s, 2,3-H), 10.57 (2H, s, 5,20-H). ${ }^{1} \mathrm{H}$ NMR (500 MHz, TFA-CDCl $): ~ \delta-4.84$ (3H, s, N-Me), -4.12 (2H, br s, 2 x NH), 1.40 
$\left(6 \mathrm{H}, \mathrm{t},{ }^{3} \mathrm{~J}_{\mathrm{HH}}=7.7 \mathrm{~Hz}, 12,13-\mathrm{CH}_{2} \mathrm{CH}_{3}\right), 1.84\left(6 \mathrm{H}, \mathrm{t},{ }^{3} \mathrm{~J}_{\mathrm{HH}}=7.7 \mathrm{~Hz}, 8,17-\mathrm{CH}_{2} \mathrm{CH}_{3}\right), 3.75(6 \mathrm{H}, \mathrm{s}$, 7,18-Me), 3.76-3.82 (2H, m), 3.85-3.92 (2H, m), 4.14-4.22 (4H, m) (4 x $\left.\mathrm{CH}_{2} \mathrm{CH}_{3}\right) 10.39$ (2H, s, 2,3-H), 10.72 (2H, s, 10,15-H), $11.48(2 \mathrm{H}, \mathrm{s}, 5,20-\mathrm{H}) .{ }^{13} \mathrm{C}$ NMR (125 MHz, CDCl $): \delta 11.44$, 17.22, 17.78, 20.11, 20.14, $31.20\left(\mathrm{~N}_{-} \mathrm{CH}_{3}\right), 103.41,113.15,133.88,134.25,137.64,146.66$, 149.05, 149.81, 153.39, 157.25. ${ }^{13} \mathrm{C}$ NMR (125 MHz, TFA-CDCl 3$): \delta 12.05,16.40,16.53,20.45$, 20.50, $30.46\left(\mathrm{~N}-\mathrm{CH}_{3}\right), 102.32,111.07,113.34,114.79,115.60,117.87,139.03,142.30,142.97$, 145.06, 145.81, 147.44, 150.99, 158.27. HR-MS (ESI) m/z: $[\mathrm{M}+\mathrm{H}]^{+}$calcd for $\mathrm{C}_{31} \mathrm{H}_{36} \mathrm{~N}_{3} \mathrm{~S}$ 482.2630, found 482.2616.

2,3,8,12,13,17-Hexaethyl-7,18,21-trimethylporphyrin (99b). $N$-methyl tripyrrane 54d (265 mg, $0.567 \mathrm{mmol})$ was dissolved in TFA $(2.5 \mathrm{~mL})$ and stirred under nitrogen for 2 minutes. The solution was diluted with dichloromethane $(50 \mathrm{~mL})$, followed by the immediate addition of 2,5pyrroledicarboxaldehyde (104.7 mg, $0.585 \mathrm{mmol}$ ), and left to stir for another 2 hours under nitrogen. The mixture was neutralized by the dropwise addition of triethylamine, DDQ (132.5 mg) was added, and the solution stirred for 1 hour. The mixture was washed with water, and then with aqueous sodium bicarbonate solution, and the solvent removed under reduced pressure. The crude material was chromatographed on neutral grade 3 alumina, eluting with chloroform, and a red/pink band was collected. Recrystallization from chloroform-hexanes gave pure 23-methylporphyrin (148 mg, $0.284 \mathrm{mmol}, 50 \%)$ as dark purple/blue crystals, mp $238-242^{\circ} \mathrm{C}$. UV-Vis $\left(1 \% \mathrm{Et}_{3} \mathrm{~N}-\right.$ dichloromethane): $\lambda_{\max } / \mathrm{nm}$ (log $\varepsilon$ ) 379 (4.76), 410 (5.06), 505 (4.07), 535 (3.71), 585 (3.57), 615 (2.85), 641 (3.43). UV-Vis (1\% TFA-dichloromethane): $\lambda_{\max } / \mathrm{nm}$ (log $\left.\varepsilon\right) 404$ (5.51), 550 (4.14), 596 (3.91). UV-Vis (5\% TFA-dichloromethane): $\lambda_{\max } / \mathrm{nm}$ (log $\varepsilon$ ) 404 (5.51), 551 (4.12), 596 (3.86). ${ }^{1} \mathrm{H}$ NMR (500 MHz, $\left.\mathrm{CDCl}_{3}\right): \delta-4.76(3 \mathrm{H}, \mathrm{s}, \mathrm{N}-\mathrm{Me}),-3.22(1 \mathrm{H}, \mathrm{br} \mathrm{s}, \mathrm{NH}), 1.48\left(6 \mathrm{H}, \mathrm{t},{ }^{3} J_{\mathrm{HH}}\right.$ $=7.7 \mathrm{~Hz}), 1.85\left(6 \mathrm{H}, \mathrm{t},{ }^{3} J_{\mathrm{HH}}=7.7 \mathrm{~Hz}\right), 1.92\left(6 \mathrm{H}, \mathrm{t},{ }^{3} \mathrm{~J}_{\mathrm{HH}}=7.7 \mathrm{~Hz}\right)\left(6 \times \mathrm{CH}_{2} \mathrm{CH}_{3}\right), 3.49(6 \mathrm{H}, \mathrm{s}, 7,18-$ 
Me), 3.67-3.74 (2H, m), 3.78-3.85 (2H, m), 3.88-4.02 (4H, m), 4.07-4.19 (4H, m) (6 x $\left.\mathrm{CH}_{2} \mathrm{CH}_{3}\right)$, 9.90 (2H, s, 5,20-H), 9.94 (2H, s, 10,15-H). ${ }^{1} \mathrm{H}$ NMR (500 MHz, TFA-CDCl $)$ ): $\delta$-5.59 (3H, s, NMe), -3.52 (2H, br s, 2 x NH), $1.41\left(6 \mathrm{H}, \mathrm{t},{ }^{3} J_{\mathrm{HH}}=7.7 \mathrm{~Hz}\right), 1.80\left(6 \mathrm{H}, \mathrm{t},{ }^{3} \mathrm{~J}_{\mathrm{HH}}=7.8 \mathrm{~Hz}\right), 1.88(6 \mathrm{H}, \mathrm{t}$, $\left.{ }^{3} J_{\mathrm{HH}}=7.8 \mathrm{~Hz}\right)\left(6 \times \mathrm{CH}_{2} \mathrm{CH}_{3}\right), 3.73(6 \mathrm{H}, \mathrm{s}, 7,18-\mathrm{Me}), 3.70-3.86(4 \mathrm{H}, \mathrm{m}), 4.13-4.27(8 \mathrm{H}, \mathrm{m})(6 \mathrm{x}$ $\left.\mathrm{CH}_{2} \mathrm{CH}_{3}\right), 10.61$ (2H, s, 5,20-H), 10.73 (2H, s, 10,15-H). ${ }^{13} \mathrm{C}$ NMR (125 MHz, $\left.\mathrm{CDCl}_{3}\right): \delta 11.75$, 17.27, 17.97, 18.54, 19.87, 19.96, 20.94, $32.80\left(\mathrm{~N}-\mathrm{CH}_{3}\right), 96.72,101.59,132.03,136.61,137.30$, 139.13, 145.58, 148.65, 151.96, 155.28. ${ }^{13} \mathrm{C}$ NMR (125 MHz, TFA-CDCl $): \delta 12.01,16.53,16.62$, 17.70, 20.13, 20.20, 20.54, $29.96\left(\mathrm{~N}-\mathrm{CH}_{3}\right)$, 99.94, 100.86, 138.59, 140.03, 141.19, 142.15, 143.02, 145.75, 146.06, 154.55. HR-MS (ESI) $m / z$ : $[\mathrm{M}+\mathrm{H}]^{+}$calcd for $\mathrm{C}_{35} \mathrm{H}_{45} \mathrm{~N}_{4}$ 521.3644, found 521.3641.

9,13,14,18-Tetraethyl-8,19,24-trimethyloxybenziporphyrin (100a). $N$-methyl tripyrrane 54d (185 mg, $0.396 \mathrm{mmol})$ was dissolved in TFA ( $2 \mathrm{~mL}$ ) and stirred under nitrogen for 2 minutes. The solution was diluted with dichloromethane $(33 \mathrm{~mL})$, followed by immediate addition of 5formylsalicylaldehyde (58 mg, $0.386 \mathrm{mmol}$ ), and left to stir for another 2 hours under nitrogen. The mixture was neutralized by the dropwise addition of triethylamine, DDQ (88 mg) was added, and the solution stirred for 1 hour. The mixture was washed with water and then with aqueous sodium bicarbonate solution, and the solvent removed under reduced pressure. The crude material was chromatographed on neutral grade 3 alumina, eluting with dichloromethane and then $50 \%$ chloroform-dichloromethane, and a dark green band was collected. Recrystallization from chloroform-hexanes gave pure 24-methyloxybenziporphyrin (24.8 $\mathrm{mg}, 0.0504 \mathrm{mmol}, 13 \%)$ as dark blue/purple crystals, mp > 300 ${ }^{\circ}$. IR: $v_{\mathrm{CO}} / \mathrm{cm}^{-1} 1618(\mathrm{C}=\mathrm{O})$. UV-Vis $\left(1 \% \mathrm{Et}_{3} \mathrm{~N}-\right.$ dichloromethane): $\lambda_{\max } / \mathrm{nm}$ (log $\left.\varepsilon\right) 331$ (4.47), 441 (4.86), 473 (4.73), 564 (3.65), 621 (4.16), 725 (3.79). UV-Vis (1\% TFA-dichloromethane): $\lambda_{\max } / \mathrm{nm}(\log \varepsilon) 317$ (4.24), 351 (4.60), 434 (4.80), 
556 (3.54), 602 (3.84), 698 (3.65), 760 (3.77). UV-Vis (5\% TFA-dichloromethane): $\lambda_{\max } / \mathrm{nm}$ (log ع) 319 (4.25), 351 (4.60), 433 (4.80), 511 (2.93), 554 (3.55), 601 (3.85), 693 (3.65), 764 (3.78). ${ }^{1} \mathrm{H}$ NMR (500 MHz, $\left.\mathrm{CDCl}_{3}\right): \delta-4.14$ (1H br s, 22-H), -3.63 (3H, s, N-Me), 1.45-1.50 (6H, m, 13,14$\mathrm{CH}_{2} \mathrm{CH}_{3}$ ), $1.73\left(6 \mathrm{H}, \mathrm{t},{ }^{3} \mathrm{~J}_{\mathrm{HH}}=7.7 \mathrm{~Hz}, 9,18-\mathrm{CH}_{2} \mathrm{CH}_{3}\right.$ ), 3.33 (3H, s, 8-Me), 3.43 (3H, s, 19-Me), 3.52-3.68 (4H, m, 13,14-CH $)$, 3.74-3.86 (4H, m, 9,18- $\left.\mathrm{CH}_{2}\right), 7.36\left(1 \mathrm{H}, \mathrm{d},{ }^{3} J_{\mathrm{HH}}=9.4 \mathrm{~Hz}, 3-\mathrm{H}\right)$, $8.72\left(1 \mathrm{H}, \mathrm{dd},{ }^{3} J_{\mathrm{HH}}=9.5 \mathrm{~Hz},{ }^{4} J_{\mathrm{HH}}=2.2 \mathrm{~Hz}, 4-\mathrm{H}\right), 9.20(1 \mathrm{H}, \mathrm{s}, 16-\mathrm{H}), 9.37(2 \mathrm{H}, \mathrm{s}, 6,11-\mathrm{H}), 10.50$ (1H, s, 21-H). ${ }^{1} \mathrm{H}$ NMR (500 MHz, TFA-CDCl 3 ): $\delta-0.52$ (3H, s, N-Me), 0.82 (1H, s, 22-H), 1.33 (6H, q, $J=7.7 \mathrm{~Hz}$, ), 1.43-1.47 (6H, m) (4 x CH $\left.2 \mathrm{CH}_{3}\right), 2.95$ (3H, s), 2.96 (3H, s) (8,19-Me), 3.023.13 (4H, m), 3.23-3.35 (4H, m) (4 x CH $\left.\mathrm{CH}_{3}\right), 5.49(1 \mathrm{H}, \mathrm{s}), 5.52(1 \mathrm{H}, \mathrm{s})(2 \mathrm{x} \mathrm{NH}), 7.62(1 \mathrm{H}, \mathrm{d}, J$ = $8.8 \mathrm{~Hz}, 3-\mathrm{H}), 7.93(1 \mathrm{H}, \mathrm{s}), 7.99(1 \mathrm{H}, \mathrm{s})(11,16-\mathrm{H}), 8.58(1 \mathrm{H}, \mathrm{dd}, J=8.9 \mathrm{~Hz}, J=2.0 \mathrm{~Hz}, 4-\mathrm{H})$, 8.80 (1H, s, 6-H), 9.46 (1H, s, 21-H). ${ }^{13} \mathrm{C}$ NMR (125 MHz, $\left.\mathrm{CDCl}_{3}\right): \delta 11.61,11.85,16.69,16.84$, 17.08, 17.44, 19.78, 19.79, 19.87, $33.25\left(\mathrm{~N}_{-} \mathrm{CH}_{3}\right)$, 93.69, 98.97, 111.18, 118.86, 120.52, 120.56, 124.21, 127.50, 130.03, 135.28, 137.33, 138.09, 139.04, 139.72, 140.60, 141.82, 144.53, 145.72 , 145.76, 146.92, 148.18, 149.02, 151.22, 189.33. ${ }^{13} \mathrm{C}$ NMR (125 MHz, TFA-CDCl $): \delta 11.16$, 11.21, 15.33, 15.36, 15.42, 18.96, 19.14, 19.17, $37.17\left(\mathrm{~N}_{-} \mathrm{CH}_{3}\right), 94.30,95.66,116.55,119.76$, 120.51, 122.20, 123.85, 127.46, 140.13, 140.57, 140.69, 141.18, 143.85, 146.49, 147.52, 149.71, 154.36, 155.90, 159.26, 172.76. HR-MS (ESI) $\mathrm{m} / \mathrm{z}:[\mathrm{M}+\mathrm{H}]^{+}$calcd for $\mathrm{C}_{33} \mathrm{H}_{38} \mathrm{~N}_{3} \mathrm{O} 492.3015$, found 492.3025 .

[9,13,14,18-Tetraethyl-8,19,24-trimethyloxybenziporphyrinato] palladium(II) (102). Palladium(II) acetate (10 $\mathrm{mg}, 0.0445 \mathrm{mmol})$ was added to a solution of 23methyloxybenziporphyrin $(10 \mathrm{mg}, 0.0203 \mathrm{mmol})$ in acetonitrile $(10 \mathrm{~mL})$ and the solution was reacted under reflux for 30 minutes. The mixture was then diluted with chloroform and washed with water, and the solvent was removed under reduced pressure. The crude material was 
chromatographed on grade 3 neutral alumina eluting with chloroform, and a green band was collected and recrystallized from chloroform-methanol to give palladium(II) 23methyloxybenziporphyrin (4.4 mg, $0.00738 \mathrm{mmol}$, 36\%) as emerald green crystals, mp 180-185 ${ }^{\circ} \mathrm{C}$, dec. IR: $v_{\mathrm{CO}} / \mathrm{cm}^{-1} 1555(\mathrm{C}=\mathrm{O}) .{ }^{1} \mathrm{H}$ NMR $\left(500 \mathrm{MHz}, \mathrm{CDCl}_{3}\right): \delta-2.36$ (3H, s, N-Me), 1.61-1.65 (6H, m), 1.69-1.73 (6H, m) (4 x CH $\left.\mathrm{CH}_{3}\right), 3.27$ (3H, s, 8-Me), 3.32 (3H, s, 19-Me), 3.64-3.84 (8H, m, $\left.4 \times \mathrm{CH}_{2} \mathrm{CH}_{3}\right), 7.10\left(1 \mathrm{H}, \mathrm{d},{ }^{3} J_{\mathrm{HH}}=9.0 \mathrm{~Hz}, 3-\mathrm{H}\right), 8.47\left(1 \mathrm{H}, \mathrm{d},{ }^{3} J_{\mathrm{HH}}=9.2 \mathrm{~Hz}, 4-\mathrm{H}\right), 9.22(1 \mathrm{H}, \mathrm{s}$, 6-H), $9.46(1 \mathrm{H}, \mathrm{s}), 9.52(1 \mathrm{H}, \mathrm{s})(11,16-\mathrm{H}), 10.58(1 \mathrm{H}, \mathrm{s}, 21-\mathrm{H}) .{ }^{13} \mathrm{C}$ NMR $\left(125 \mathrm{MHz}, \mathrm{CDCl}_{3}\right)$ : $\delta$ 11.42, 11.70, 16.24, 16.30, 17.12, 17.33, 20.01, 20.03, 20.05, $46.28\left(\mathrm{~N}_{-} \mathrm{CH}_{3}\right), 98.70,100.16$, 123.45, 123.56, 125.78, 126.57, 128.61, 136.58, 136.89, 139.47, 139.78, 140.19, 140.87, 140.93, 141.40, 141.50, 143.78, 146.14, 149.64, 150.14, 151.70, 190.17. HR-MS (ESI) $m / z:[M+H]^{+}$ calcd for $\mathrm{C}_{33} \mathrm{H}_{36} \mathrm{~N}_{3} \mathrm{OPd}$ 596.1893, found 596.1874.

9,13,14,18-Tetraethyl-8,19,24-trimethyloxypyriporphyrin (100b). $N$-methyl tripyrrane 54d (177 mg, 0.379 mmol) was dissolved in TFA ( $2 \mathrm{~mL}$ ) and stirred under nitrogen for 2 minutes. The solution was diluted with dichloromethane (33 mL), followed by immediate addition of 5formylsalicylaldehyde (57.4 mg, $0.380 \mathrm{mmol})$, and left to stir for another 2 hours under nitrogen. The mixture was neutralized by the dropwise addition of triethylamine, DDQ (86 mg) was added, and the solution stirred for 1 hour. The mixture was washed with water and then with aqueous sodium bicarbonate solution, and the solvent removed under reduced pressure. The crude material was chromatographed twice on neutral grade 3 alumina, eluting with chloroform and then $2 \%$ methanol-chloroform, and a dark green band was collected each time. The product was dried overnight under vacuum to give 24-methyloxybenziporphyrin (66.7 $\mathrm{mg}, 0.135 \mathrm{mmol}, 36 \%$ ) as a dark blue/purple solid, $\mathrm{mp}>300^{\circ} \mathrm{C}$. IR: $v_{\mathrm{CO}} / \mathrm{cm}^{-1} 1625$ (C=O). UV-Vis $\left(1 \% \mathrm{Et}_{3} \mathrm{~N}-\right.$ dichloromethane): $\lambda_{\max } / \mathrm{nm}$ (log $\left.\varepsilon\right) 324$ (4.14), 434 (4.56), 546 (3.51), 581 (3.56), 625 (3.87), 673 
(3.70). UV-Vis (1\% TFA-dichloromethane): $\lambda_{\max } / \mathrm{nm}(\log \varepsilon) 324$ (4.13), 425 (4.90), 453 (4.61), 549 (3.57), 588 (3.78), 629 (4.07). UV-Vis (5\% TFA-dichloromethane): $\lambda_{\max } / \mathrm{nm}$ (log ع) 323 (4.19), 426 (4.82), 550 (3.61), 599 (3.74), 627 (3.93), 648 (3.80). ${ }^{1} \mathrm{H}$ NMR (500 MHz, $\left.\mathrm{CDCl}_{3}\right): \delta$ $4.20(3 \mathrm{H}, \mathrm{s}, \mathrm{N}-\mathrm{Me}),-1.26(1 \mathrm{H}, \mathrm{br} \mathrm{s}, \mathrm{NH}), 1.37\left(3 \mathrm{H}, \mathrm{t}, J=7.7 \mathrm{~Hz}, 14-\mathrm{CH}_{2} \mathrm{CH}_{3}\right) 1.41(3 \mathrm{H}, \mathrm{t}, J=7.7$ $\left.\mathrm{Hz}, 13-\mathrm{CH}_{2} \mathrm{CH}_{3}\right), 1.79\left(3 \mathrm{H}, \mathrm{t}, J=7.7 \mathrm{~Hz}, 9-\mathrm{CH}_{2} \mathrm{CH}_{3}\right), 1.83\left(3 \mathrm{H}, \mathrm{t}, J=7.7 \mathrm{~Hz}, 18-\mathrm{CH}_{2} \mathrm{CH}_{3}\right), 3.44$ (3H, s, 8-Me), 3.58 (3H, s, 19-Me), 3.59-3.64 (2H, m, 13- $\left.\mathrm{CH}_{2}\right)$, 3.69-3.77 (2H, m, 14-CH $)$, 3.82$3.93\left(2 \mathrm{H}, \mathrm{m}, 9-\mathrm{CH}_{2}\right), 3.94-4.02\left(2 \mathrm{H}, \mathrm{m}, 18-\mathrm{CH}_{2}\right), 7.91(1 \mathrm{H}, \mathrm{d}, J=9.4 \mathrm{~Hz}), 9.33(1 \mathrm{H}, \mathrm{d}, J=9.4$ Hz), 9.64 (1H, s, 16-H), 9.68 (1H, s, 6-H), 9.73 (1H, s, 11-H), 10.89 (1H, s, 21-H). ${ }^{1} \mathrm{H}$ NMR (500 MHz, TFA-CDCl $\left.{ }_{3}\right): \delta$-2.92 (3H, s, N-Me), 0.30 (2H, br s, 2 x NH), 1.41-1.46 (6H, m, 13,14$\left.\mathrm{CH}_{2} \mathrm{CH}_{3}\right)$ 1.63-1.66 (6H, m, 9,18- $\left.\mathrm{CH}_{2} \mathrm{CH}_{3}\right), 3.35$ (3H, s), 3.38 (3H, s) (8,19-Me), 3.39-3.50 (4H, m, 13,14- $\left.\mathrm{CH}_{2}\right), 3.71-3.79\left(4 \mathrm{H}, \mathrm{m}, 9,18-\mathrm{CH}_{2}\right), 8.57(1 \mathrm{H}, \mathrm{d}, J=9.1 \mathrm{~Hz}, 3-\mathrm{H}), 9.24(1 \mathrm{H}, \mathrm{s}), 9.63(1 \mathrm{H}$, d, $J=9.1 \mathrm{~Hz}, 4-\mathrm{H}), 9.78(1 \mathrm{H}, \mathrm{s}, 6-\mathrm{H}), 10.70(1 \mathrm{H}, \mathrm{s}, 21-\mathrm{H}) .{ }^{13} \mathrm{C}$ NMR $\left(125 \mathrm{MHz}, \mathrm{CDCl}_{3}\right): \delta 11.83$, 11.93, 16.82, 16.98, 17.32, 17.61, 19.71, 19.83, 20.08, 20.11, $33.19\left(\mathrm{~N}_{-} \mathrm{CH}_{3}\right), 95.49,101.51$, 106.21, 115.07, 131.69, 133.37, 135.40, 138.72, 139.52, 141.04, 143.29, 146.50, 150.10, 150.71, 151.24, 185.23. ${ }^{13} \mathrm{C}$ NMR (125 MHz, TFA-CDCl $): \delta 11.49,11.58,15.93,16.11,19.62,34.54$ (N-CH 3$), 96.83,97.33,113.31,119.83,127.91,127.93,139.37,139.62,140.17,140.58,142.31$, 142.60, 142.93, 144.78, 145.64, 147.79, 149.46, 149.74, 159.10, 159.63, 173.83 (br). HR-MS (ESI) $m / z:[M+H]^{+}$calcd for $\mathrm{C}_{32} \mathrm{H}_{37} \mathrm{~N}_{4} \mathrm{O}$ 493.2967, found 493.2965.

tert-Butyl 23-methylazuliporphyrin dihydrochloride $\left(\mathbf{1 0 3 H}_{2}{ }^{2+}\right) \cdot \mathrm{N}$-methyl tripyrrane $\mathbf{5 4 d}$ (103 mg, $0.221 \mathrm{mmol}$ ) was dissolved in TFA (1 mL) and stirred for 2 minutes under nitrogen. The mixture was diluted with dichloromethane $(99 \mathrm{~mL})$, followed by the immediate addition of 6-tertbutylazulene-1,3-dicarbaldehyde (57.6 mg, $0.240 \mathrm{mmol})$, and the solution was left to stir overnight under nitrogen. The mixture was oxidized by vigorous washing with ferric chloride $(0.1 \%, 200$ 
$\mathrm{mL}$ ), and then washed with $10 \%$ aqueous hydrochloric acid. The solvent was evaporated off under reduced pressure and the residue was recrystallized twice from chloroform-hexanes to give tertbutyl 23-methylazuliporphyrin dihydrochloride (113 mg, $0.194 \mathrm{mmol}, 78 \%$ ) as dark crystals, mp $>300{ }^{\circ} \mathrm{C}$. UV-Vis (1\% Et ${ }_{3} \mathrm{~N}-$ dichloromethane): $\lambda_{\max } / \mathrm{nm}$ (log $\left.\varepsilon\right) 363$ (4.47), 401 (4.37), 459 (4.44), 482 (4.52) 669 (3.89). UV-Vis (1\% TFA-dichloromethane): $\lambda_{\max } / \mathrm{nm}$ (log $\left.\varepsilon\right) 368$ (4.50), 443 (sh, 4.46), 469 (4.77), 587 (3.88), 647 (4.19), 686 (sh, 3.93), 750 (3.62). ${ }^{1} \mathrm{H}$ NMR (500 MHz, TFA- $d_{6^{-}}$ DMSO): $\delta-3.38$ (1H, s, CH), -3.36 (3H, s, N-Me), -0.36 (2H, br s, 2 x NH), 1.26 (6H, t, $J=7.7$ Hz) $1.57(6 \mathrm{H}, \mathrm{t}, J=7.7 \mathrm{~Hz})\left(4 \times \mathrm{CH}_{2} \mathrm{CH}_{3}\right), 1.64\left(9 \mathrm{H}, \mathrm{s}, 2^{3}-\mathrm{C}\left(\mathrm{CH}_{3}\right)_{3}\right), 3.46$ (6H, s, 7,18-Me), 3.44$3.53(2 \mathrm{H}, \mathrm{m}), 3.64-3.71(2 \mathrm{H}, \mathrm{m}), 3.75-3.83(2 \mathrm{H}, \mathrm{m}), 3.85-3.92(2 \mathrm{H}, \mathrm{m})\left(4 \mathrm{x} \mathrm{CH}_{2} \mathrm{CH}_{3}\right), 8.89(2 \mathrm{H}$, d, $\left.J=10 \mathrm{~Hz}, 2^{2}, 3^{2}-\mathrm{CH}\right), 9.66$ (2H, s), 10.39 (2H, d, $\left.J=10 \mathrm{~Hz}, 2^{1}, 3^{1}-\mathrm{CH}\right), 10.72(2 \mathrm{H}, \mathrm{s}) .{ }^{13} \mathrm{C}$ NMR (125 MHz, TFA- $d_{6}$-DMSO): $\delta$ 11.62, 16.25, 16.51, 19.56, 19.59, $\left.31.50\left(\mathrm{C}_{(\mathrm{CH}}\right)_{3}\right), 32.25\left(\mathrm{~N}-\mathrm{CH}_{3}\right)$, $40.78\left(C\left(\mathrm{CH}_{3}\right)_{3}\right), 97.16,110.95,125.63,129.15,141.07,141.46,141.53,142.01,142.19,142.31$, 146.18, 153.68, 156.74. HR-MS (ESI) m/z: $[\mathrm{M}+\mathrm{H}]+$ calcd for $\mathrm{C}_{41} \mathrm{H}_{48} \mathrm{~N}_{3}$ 582.3848, found 582.3847. 


\section{CHAPTER V: CONCLUSIONS}

Investigations into the modification of porphyrinoid systems are of interest in order to analyze the effects they may have on these macrocyclic molecules. By altering atoms within the porphyrinoid cavity, changing the external substituents, or inserting one or more different rings into the system, changes in the electronic properties, structure stability and reactivity can be assessed. Carbaporphyrinoids are a common type of porphyrin analogue that involve a core modification where one or more of the internal nitrogen atoms are replaced by carbon atoms. Metalation of carbaporphyrins has been widely studied, demonstrating that these compounds can still act as versatile ligands and bind with a number of late transition metals, despite the inclusion of internal carbon atoms.

Among the carbaporphyrinoid systems that form stable metal complexes are benzocarbaporphyrins, which have previously been shown to form organometallic complexes with Rh(III), Ir(III), Au(III) and Ag(III) metal cations. The work reported in this thesis demonstrates that internal alkylation alters the coordination chemistry of benzocarbaporphyrins and allows them to form stable complexes with metals in the $2+$ oxidation state. When reacted with $\mathrm{Pd}(\mathrm{OAc})_{2}, 23-$ methylbenzocarbaporphyrin formed a 23-methyl palladium(II) complex that rearranged to give a C-methyl palladium(II) complex. This was proposed to occur via an oxidative addition of the alkyl group onto the palladium metal center, followed by a reductive elimination that transfers the methyl onto the 21-carbon. Internally methylated benzocarbaporphyrins, as well as a carbaporphyrin diester and a naphthocarbaporphyrin, were also shown to form rhodium complexes. It was found that 21- and 22-methylbenzocarbaporphyrins reacted with $\left[\mathrm{Rh}(\mathrm{CO})_{2} \mathrm{Cl}\right]_{2}$ 
to give stable rhodium(I) complexes, but reaction with a 23-methylbenzocarbaporphyrin gave a very different result. Specifically, the 23-methyl compound did not produce a rhodium(I) species, but instead formed a rhodium(III) complex accompanied by the unexpected formation of an internal methylene bridge. Hence, the introduction of the internal methyl substituent drastically affects how benzocarbaporphyrins coordinate to rhodium. A carbaporphyrin diester and a naphthocarbaporphyrin were also shown to react with $\left[\mathrm{Rh}(\mathrm{CO})_{2} \mathrm{Cl}\right]_{2}$ to give rhodium(I) complexes. However, unlike the rhodium(I) derivatives of 21- and 22-methylbenzocarbaporphyrins, these compounds generated rhodium(III) complexes in refluxing pyridine, thereby demonstrating that the carbaporphyrin platform can bind rhodium in two oxidation states. Four of the rhodium complexes were characterized by X-ray crystallography, and this provided information on the bonding frameworks that were obtained. Furthermore, the formation of the rhodium(III) system with a bridging methylene unit demonstrates how a 23-methylbenzocarbaporphyrin can rearrange when rhodium is incorporated into the macrocycle. This rearrangement also highlights the capability of rhodium(III) porphyrinoids to promote C-H bond activation.

Carbaporphyrinoid systems are commonly synthesized using the ' $3+1$ ' variant of the MacDonald Condensation. This methodology allows for alteration of either the tripyrrane unit or the dialdehyde unit, making it simple to design a multitude of modified porphyrinoids. The insertion of an internal methyl group had dramatic effects on benzocarbaporphyrin systems, and therefore it was of interest to investigate the effects of internal substituents on other porphyrinoids. A series of $N$-methylated porphyrin analogues were synthesized using the ' $3+1$ ' approach, and the characterization of these modified porphyrinoids is reported in this thesis. The introduction of an internal methyl group affected all of the porphyrinoid systems, where the most dramatic of changes were observed in the UV-Vis spectra. The alkyl substituent may distort the $\pi$-system of the 
macrocycle, and consequently this results in bathochromic shifts to the absorption bands for the methylated derivatives compared to their non-methylated counterparts. In the case of the 24methyloxybenzi- and oxypyriporphyrins, the UV-Vis spectra show drastic weakening and broadening in the absorption bands. For the other methylated derivatives, the shift to longer wavelengths was the most evident change, with a slight broadening of the bands in some cases. Metalation of $\mathrm{N}$-methyloxybenziporphyrin with $\mathrm{Pd}(\mathrm{OAc})_{2}$ gave a stable palladium(II) complex, where the internal methyl remained in its original position. This result demonstrated that the presence of an internal methyl group facilitates the formation of a stable palladium(II) complex.

Although the introduction of an internal alkyl substituent greatly affected the chromophores of porphyrinoid systems, the porphyrin analogues fully retained their aromatic characteristics. The global aromatic properties of these systems were assessed by proton NMR spectroscopy and in every case the diamagnetic ring currents were not significantly altered by introducing internal alkyl substituents. Nevertheless, the dramatic changes observed in the electronic spectra upon alkylation, and the modification of the systems' ability to form organometallic derivatives, demonstrates that incorporation of $\mathrm{N}$-alkyl substituents provides a valuable strategy for adjusting the properties of porphyrinoid structures. 


\section{REFERENCES}

1. Thompson, S. J.; Brennan, M. R.; Lee, S. Y.; Dong, G. Synthesis and applications of rhodium porphyrin complexes. Chem. Soc. Rev. 2018, 47, 929-981.

2. Fleischer, E. B. Structure of porphyrins and metalloporphyrins. Acc. Chem. Res., 1970, 3, 105-112.

3. Smith, K. M. Porphyrins and Metalloporphyrins: A New Edition Based on the Original Volume by J. E. Falk, Elsevier, 1975.

4. Wasielewski, M. R. Photoinduced electron transfer in supramolecular systems for artificial photosynthesis. Chem. Rev. 1992, 92, 435-461.

5. Sheldon, R. A. Metalloporphyrins in Catalytic Oxidations; Taylor \& Francis: United Kingdom, 1994.

6. Lash, T. D. Synthesis of novel porphyrinoid chromophores. In The Porphyrin Handbook, Kadish, K. M.; Smith, K. M.; Guilard, R., Eds.; Academic Press: New York, 2000; Vol. 2, pp. 125

7. The Porphyrin Handbook, Kadish, K. M.; Smith, K. M.; Guilard, R., Eds.; Academic Press: New York, 2000; Vol. 6.

8. Bonnett, R. Photosensitizers of the porphyrin and phthalocyanine series for photodynamic therapy. Chem. Soc. Rev. 1995, 24, 19-33.

9. Ogoshi, H.; Mizutani, T. Multifunctional and Chiral Porphyrins: Model Receptors for Chiral Recognition. Acc. Chem. Res. 1998, 31, 81-89. 
10. Chandra, R. M.; Tiwari, P.; Kaur, M.; Sharma, Jain, R.; Dass, S. MetalloporphyrinsApplications and clinical significance. Indian J. Clin. Biochem. 2000, 15, 183-199.

11. Suslick, K. S.; Rakow, N. A.; Kosal, M. E.; Chou, J-H. The materials chemistry of porphyrins and metalloporphyrins. J. Porphyrins Phthalocyanines 2000, 4, 407-413.

12. (a) Brown, S. B.; Truscott, T. G. New light on cancer therapy. Chem. Br. 1993, 29, 955.

(b) Milgrom, L. R.; MacRobert, S. Light years ahead. Chem. Br. 1998, 34 (35), 45.

13. Mironov, A. Transition Metal Complexes of Porphyrins and Porphyrinoids. Handbook of Porphyrin Science- With Applications to Chemistry, Physics, Material Science, Engineering, Biology and Medicine, Kadish, K. M.; Smith, K. M.; Guilard, R., eds.; World Scientific Publishing, 2012, Vol. 18, pp. 303-413.

14. Sanders, J. K. M.; Bampos, N.; Clyde-Watson, Z.; Darling, S. L.; Hawley, J. C.; Kim, HJ.; Mak, C. C.; Webb, S. J. Introduction, In The Porphyrin Handbook, Kadish, K. M.; Smith, K. M.; Guilard, R., Eds.; Academic Press: New York, 2000; Vol. 3, pp. 3.

15. Lash, T.D. Carbaporphyrins and Related Systems. Synthesis, Characterization, Reactivity and Insights into the Nature of Porphyrinoid Aromaticity. Handbook of Porphyrin ScienceWith Applications to Chemistry, Physics, Material Science, Engineering, Biology and Medicine, Kadish, K. M.; Smith, K. M.; Guilard, R., eds.; World Scientific Publishing, 2012, Vol. 16, pp. 1-329.

16. Lash, T. D.; Hayes, M. J. Conjugated macrocycles related to porphyrins. Part 9. Carbaporphyrins. Angew. Chem. Int. Ed. Engl. 1997, 36, 840-842.

17. Lash, T. D. Recent advances on the synthesis and chemistry of carbaporphyrins and related porphyrinoid systems. Eur. J. Org. Chem. 2007, 33, 5461-5481. 
18. Lash, T .D.; Hayes, M. J.; Spence, J. D.; Muckey, M. A.; Ferrence, G. M.; Szczepura, L. F. Conjugated Macrocycles Related to the Porphyrins. 21. Synthesis, Spectroscopy, Electrochemistry, and Structural Characterization of Carbaporphyrins. J. Org. Chem. 2002, 67, 4860-4874.

19. Lash, T. D. Out of the Blue! Azuliporphyrins and Related Carbaporphyrinoid Systems. Acc. Chem. Res. 2016, 49, 471-482.

20. Lash, T. D. Benziporphyrins, a Unique Platform for Exploring the Aromatic Characteristics of Porphyrinoid Systems. Org. Biomol. Chem. 2015, 9, 682-705.

21. (a) Srinivasan, A.; Furuta, H. Confusion Approach to Porphyrinoid Chemistry. Acc. Chem. Res. 2005, 38, 10-20. (b) Toganoh, M.; Furuta, H. Blooming of Confused Porphyrinoids - Fusion, Expansion, Contraction, and More Confusion. Chem. Commun. 2012, 48, 937954.

22. Lash, T. D. Oxybenziporphyrin, a fully aromatic semiquinone porphyrin analog with

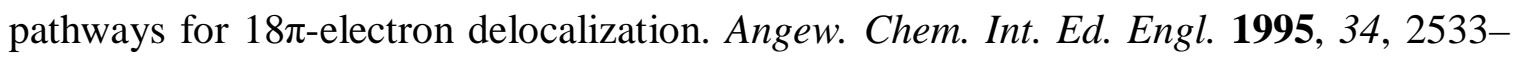
2535.

23. Lash, T. D.; Chaney, S. T.; Richter, D. T. Conjugated Macrocycles Related to Pophyrins. 12. Oxybenzi- and Oxypyriporphyrins: Aromaticity and Conjugation in Highly Modified Porphyrinoid Structures. J. Org. Chem. 1998, 63, 9076-9088.

24. Richter, D. T.; Lash T. D. Conjugated macrocycles related to the porphyrins. Part 18: Synthesis and spectroscopic characterization of electron-rich benzi- and oxybenziporphyrins: influence of steric and electronic factors on porphyrinoid aromaticity. Tetrahedron 2001, 57, 3659-3673. 
25. (a) Lash, T. D.; Chaney, S. T. Conjugated macrocycles related to the porphyrins. Part 7. Tropiporphyrin: tropylium versus porphyrinoid aromaticity. Tetrahedron Lett. 1996, 37, 8825- 8828. (b) Bergman, K. M.; Ferrence, G. M.; Lash, T. D. Conjugated Macrocycles Related to the Porphyrins. Part 36. Tropiporphyrins, Cycloheptatrienyl Analogues of the Porphyrins: Synthesis, Spectroscopy, Chemistry, and Structural Characterization of a Silver(III) Derivative. J. Org. Chem. 2004, 69, 7888-7897.

26. Lash, T. D. Metal Complexes of Carbaporphyrinoid Systems. Chem. Asian J. 2014, 9, 682705.

27. Muckey, M. A.; Szczepura, L. F.; Ferrence, G. M.; Lash, T. D. Silver(III) Carbaporphyrins: The First Organometallic Complexes of True Carbaporphyrins. Inorg. Chem. 2002, 41, 4840-4842.

28. Lash, T. D.; Colby, D. A.; Szczepura, L. F. New Riches in Carbaporphyrin Chemistry: Silver and Gold Organometallic Complexes of Benzocarbaporphyrins. Inorg. Chem. 2004, 43, 5258-5267.

29. Sahota, N.; Ferrence, G. M.; Lash, T. D. Synthesis and Properties of Carbaporphyrin and Carbachlorin Dimethyl Esters Derived from Cyclopentanedialdehydes. J. Org. Chem. 2017, 82, 9715-9730.

30. Adiraju, V. A. K.; Ferrence, G. M.; Lash, T. D. Rhodium(I), Rhodium(III) and Iridium(III) Carbaporphyrins. Dalton Trans. 2016, 45, 13691-13694.

31. Stepien, M.; Latos-Grazynski, L. Benziporphyrins: Exploring Arene Chemistry in s Macrocyclic Environment. Acc. Chem. Res. 2005, 38, 88-98. 
32. (a) Graham, S. R.; Ferrence, G. M.; Lash, T. D. Organometallic Chemistry of Carbaporphyrinoids: Synthesis and Characterization of Nickel(II) and Palladium (II) Azuliporphyrins. Chem. Commun. 2002, 38, 894-895. (b) Lash, T. D.; Colby, D. A.; Graham, S. R.; Ferrence, G. M.; Szczepura, L. F. Organometallic Chemistry of Azuliporphyrins: Synthesis, Spectroscopy, Electrochemistry and Structural Characterization of Nickel(II), Palladium(II) and Platinum(II) Complexes of Azuliporphyrins. Inorg. Chem. 2003, 42, 7326-7338.

33. Bialek, M. J.; Latos-Grazynski, L. Merging of Inner and Outer Ruthenium Organometallic Coordination Motifs within an Azuliporphyrin Framework. Chem. Commun. 2014, 50, 9270-9272.

34. Stateman, L. M.; Ferrence, G. M.; Lash, T. D. Rhodium(III) Azuliporphyrins. Organometallics 2015, 34, 3842-3848.

35. Lash, T. D.; Pokharel, K.; Zeller, M.; Ferrence, G. M. Iridium(III) azuliporphyrins. Chem. Commun. 2012, 48, 11793-11795.

36. (a) Toganoh, M.; Furuta, H. In Handbook of Porphyrin Science - With Applications to Chemistry, Physics, Material Science, Engineering, Biology and Medicine, Kadish, K. M.; Smith, K. M.; Guilard, R., eds.; World Scientific Publishing: Singapore, 2010, Vol. 2, pp 295-367. (b) Harvey, J. D.; Ziegler, C. J. Developments in the metal chemistry of Nconfused porphyrin. Coord. Chem. Rev. 2003, 247, 1-19.

37. Chmielewski, P. J.; Latos-Grazynski, L.; Rachlewicz, K.; Glowiak, T. Tetra-ptolylporphyrin with an Inverted Pyrrole Ring: A Novel Isomer of Porphyrin. Angew. Chem. Int. Ed. Engl. 1994, 33, 779 - 781. 
38. Furuta, H.; Ogawa, T.; Uwatoko, Y.; Araki, K. N-Confused TetraphenylporphyrinSilver(III) Complex. Inorg. Chem. 1999, 38, 2676 - 2682.

39. Lash, T. D.; Rasmussen, J. M.; Bergman, K. M.; Colby, D. A. Conjugated Macrocycles Related to the Porphyrins. Part 33. Carbaporphyrinoid Chemistry Has a Silver Lining! Silver(III) Oxybenzi-, Oxynaphthi-, Tropi-, and Benzocarbaporphyrins. Org. Lett. 2004, 6, 549.

40. Stepien, M.; Latos-Grazynski, L.; Lash, T. D.; Szterenberg, L. Palladium(II) Complexes of Oxybenziporphyrin. Inorg. Chem. 2001, 40, 6892.

41. Pokharel, K. Synthesis and Metalation of Porphyrin Analogues. M.S. Thesis, Illinois State University, Normal, IL, May 2007.

42. El-Beck, J. A.; Lash, T. D. Tetraphenyloxybenziporphyrin, a New Organometallic Ligand for Silver(III) and Gold(III). Org. Lett. 2006, 8, 5263-5266.

43. Szymanski, J. T.; Lash, T. D. Dimethoxytetraphenylbenziporphyrins. Tetrahedron Lett. 2003, 44, 8613-8616.

44. Lash, T. D.; Szymanski, J. T.; Ferrence, G. M. Tetraaryldimethoxybenziporphyrins at the Edge of Carbaporphyrinoid Aromaticity. J. Org. Chem. 2007, 72, 6481-6492.

45. Fosu, S. C.; Ferrence, G. M.; Lash, T. D. Synthesis and Metalation of Dimethoxybenziporphyrins, Thiabenziporphyrins and Dibenziporphyrins. J. Org. Chem. 2014, 79, 11061-11074.

46. Lash, T. D.; Ferrence, G. M. Metalation and Selective Oxidation of Diphenyl-23-oxa-, thia, and Selena-21-carbaporphyrins. Inorg. Chem. 2017, 56, 11426-11434.

47. Chatterjee, T.; Shetti, V. S.; Sharma, R.; Ravikanth, M. Heteroatom-Containing Porphyrin Analogues. Chem. Rev. 2017, 117, 3254-3328. 
48. Latos-Grazyniski, L. Core modified Heteroanalogues of Porphyrins and Metalloporphyrins. In The Porphyrin Handbook; Kadish, K. M., Smith, K. M., Guilard, R., Eds.; Academic Press: New York, 2000; Vol. 2, pp 361-416.

49. Latos-Grazyñski, L.; Lisowski, J.; Olmstead, M. M.; Balch, A. L. Five-Coordinate Complexes of 21-Thiaporphyrin. Preparation, Spectra, and Structures of Iron(II), Nickel (II) and Copper(II) Complexes. Inorg. Chem. 1989, 28, 1183-1188.

50. Latos-Grazyñski, L.; Lisowski, J.; Olmstead, M. M.; Balch, A. L. Preparation and Structural Characterization of a Six-Coordinate 21- Thiaporphyrin Complex: RhIII(STPP)Cl2 (STPP = Tetraphenyl-21- thiaporphyrin Anion). Inorg. Chem. 1989, 28, $3328-3331$.

51. Chuang, C. H.; Ou, C. K.; Liu, S. T.; Kumar, A.; Ching, W. M.; Chiang, P. C.; Dela Rosa, M. A. C.; Hung, C. H. Ruthenium Complexes of Thiaporphyrin and Dithiaporphyrin. Inorg. Chem. 2011, 50, 11947- 11957.

52. Gebauer, A.; Schmidt, J. A. R.; Arnold, J. Synthesis, Characterization, and Properties of a Lithium 21-Thiaporphyrin Complex. Inorg. Chem. 2000, 39, 3424-3427.

53. Tung, J. Y.; Liau, B. C.; Elango, S.; Chen, J. H.; Hsieh, H. Y.; Liao, F. L.; Wang, S. L.; Hwang, L. P. Preparation and Structural Characterization of mercury 21-thiaporphyrin complex: HgII(Stpp)CI (Stpp = tetraphenyl-21-thiaporphyrin anion). Inorg. Chem. Commun. 2002, 5, 150-155.

54. Ghosh, A.; Ravikanth, M. Rhenium (I) Tricarbonyl Complexes of 5,10,15,20Tetraphenyl-21-thia and 21-Oxaporphyrins. Inorg. Chem. 2012, 51, 6700-6709. 
55. Chmielewski, P. J.; Latos-Grazyñski, L.; Olmstead, M. M.; Balch, A. L. Nickel Complexes of 21-Oxaporphyrin and 21, 23-Dioxaporphyrin. Chem. Eur. J. 1997, 3, $268-278$.

56. Pawlicki, M.; Latos-Grazyñski, L. Iron Complexes of 5,10,15,20- Tetraphenyl-21oxaphyrin. Inorg. Chem. 2002, 41, 5866-5873.

57. Broadhurst, M. J.; Grigg, R.; Johnson, A. W. Synthesis of Porphin Analogues Containing Furan and/or Thiophen Rings. J. Chem. Soc. C 1971, 0, 3681-3690.

58. Ambre, R.; Yu, C. Y.; Mane, S. B.; Yao, C. F.; Hung, C. H. Toward carboxylate group functionalized A4, A2B2, A3B oxaporphyrins and zinc complex of oxaporphyrins. Tetrahedron 2011, 67, 4680-4688.

59. Stute, S.; Gotzke, L.; Meyer, D.; Merroun, M. L.; Rapta, P.; Kataeva, O.; Seichter, W.; Gloe, K.; Dunsch, L.; Gloe, K. Molecular Structure, UV/Vis Spectra, and Cyclic Voltammograms of Mn(II), Co(II), and Zn(II) 5,10,15,20-Tetraphenyl-21-oxaporphyrins. Inorg. Chem. 2013, 52, 1515-1524.

60. Bond, A. D.; Sanders, J. K. M.; Stulz, E. Ruthenium(II) and rhodium(III) porphyrin phosphine complexes: influence of substitution pattern on structure and electronic properties. New J. Chem. 2011, 35, 2691-2696.

61. Olguin, J.; Muller-Bunz, H.; Albrecht, M. Springloaded porphyrin NHC hybrid rhodium(III) complexes: carbene dissociation and oxidation catalysis. Chem. Commun., 2014, 50, 3488-3490.

62. Anding, B. J.; Ellern, A.; Woo, L. K. Comparative Study of Rhodium and Iridium Porphyrin Diaminocarbene and N-Heterocyclic Carbene Complexes. Organometallics 2014, 33, 2219-2229. 
63. Chan, K. S.; Lau, C. M. Syntheses of Acyl Rhodium Porphyrins by Aldehydic CarbonHydrogen Bond Activation with Rh(III) Porphyrin Chloride and Methyl. Organometallics 2006, 25, 260-265.

64. Chan, K. S.; Chiu, P. F.; Choi, K. S. Base-Promoted Selective Activation of Benzylic Carbon-Hydrogen Bonds of Toluenes by Rhodium(III) Porphyrins. Organometallics 2007, 26, 1117-1119.

65. (a) Chan, Y. W.; Chan, K. S. Base-promoted carbon-hydrogen bond activation of alkanes with rhodium(III) porphyrin complexes. Organometallics 2008, 27, 4625-4635. (b) Fung, H. S.; Chan, Y. W.; Cheung, C. W.; Choi, K. S.; Lee, S. Y.; Qian, Y. Y.; Chan, K. S. Reactivity Studies of Rhodium(III) Porphyrins with Methanol in Alkaline Media. Organometallics 2009, 28, 3981-3989.

66. Aoyama, Y.; Yoshida, T.; Sakurai, K.; and Ogoshi, H. Activation of arene carbonhydrogen bonds. Direct electrophilic aromatic metalation with a rhodium-porphyrin complex. J. Chem. Soc., Chem. Commun. 1983, 8, 478-479.

67. Aoyama, Y.; Yoshida, T.; Sakurai, K.; and Ogoshi, H. Activation of arene carbonhydrogen bonds. Highly regioselective, electrophilic aromatic metalation with rhodium(III) porphyrin and subsequent cleavage of carbon-rhodium bond. Organometallics 1986, 5, 168-173.

68. Hurej, K.; Pawlicki, M.; Szterenberg, L.; Latos-Grazynski, L. A Rhodium-Mediated Contraction of Benzene to Cyclopentadiene: Transformations of Rhodium(III) mBenziporphyrins. Angew. Chem. Int. Ed. 2016, 55, 1427-1431. 
69. Sondheimer F, Wolovsky R and Amiel Y. Unsaturated Macrocyclic Compounds. XXIII. The Synthesis of the Fully Conjugated Macrocyclic Polyenes Cyclooctadecanonaene ([18]Annulene), Cyclotetracosadodecaene ([24]Annulene), and Cyclotriacontapentadecaene([30]Annulene). J. Am. Chem. Soc. 1962, 84, 274-284.

70. Vogel, E.; Pretzer, W.; Boll, W. A. 1.6-imino-cyclodecapentaen (1.6-imino-[10]-annulen). Tetrahedron Lett. 1965, 6, 3613-3617.

71. Vogel E. J. Novel porphyrinoid macrocycles and their metal complexes. Heterocyclic Chem. 1996, 33, 1461- 1487.

72. Cyranksi, M. K.; Krygowski, T. N.; Wisiorowski, M.; Hommes, N. J. R.; Schleyer, P. V. R. Global and Local Aromaticity in Porphyrins: An Analysis Based on Molecular Geometries and Nucleus-Independent Shifts. Angew. Chem. Int. Ed. 1998, 37, 177-180.

73. Lash, T. D. Origin of aromatic character in porphyrinoid systems. J. Porphyrins Phthalocyanines 2011, 15, 1094-1115.

74. Webb, L. E.; Fleisher, E. B. The Structure of Porphine. J. Am. Chem. Soc. 1965, 87, 667669.

75. (a) Vogel, E.; Röhrig, P.; Sicken, M.; Knipp, B.; Herrmann, A.; Pohl, M.; Schmickler, H.; Lex, J. The Thiophene Analogue of Porphyrin: Tetrathiaporphyrin Dication. Angew. Chem. Int. Ed. Engl. 1989, 28, 1651-1654. (b) Vogel, E.; Pohl, M.; Herrmann, A.; Wiss, T.; König, C.; Lex, J.; Gross, M.; Gisselbrecht, J. P. Porphyrinoid Macrocycles Based on Thiophene-The Octaethyltetrathiaporphyrin Dication. Angew. Chem. Int. Ed. Engl. 1996, 35, 1520-1524. 
76. Medforth, C. J. NMR Spectroscopy of Diamagnetic Porphyrins. In The Porphyrin Handbook, Kadish K. M., Smith, K. M., Guilard, R., Eds.; Academic Press: New York, 2000; Vol. 5, pp 1-80.

77. Lash, T. D.; Chaney, S. T. Conjugated macrocycles related to the porphyrins. 6. Oxypyriporphyrin, the first fully aromatic porphyrinoid macrocycle with a pyridine subunit. Chem. Eur. J. 1996, 2, 944-948.

78. (a) Adams K. R.; Bonnett, R.; Burke, P. J.; Salgado, A.; Vallés, M. A. Cleavage of (octaethyl-2,3-dihydroxychlorinato)nickel(II) to give the novel 2,3-dioxo-2,3-secochlorin system. J. Chem. Soc., Perkin Trans. I 1997, 0, 1769-1772. b) Kozyrev, A. N.; Alderfer, J. L.; Dougherty, T. J.; Pandey, R. K. Synthesis of Mono- and Di (oxopyri) porphyrins: A New Approach through Ring Enlargement with Diazomethane. Angew. Chem. Int. Ed. 1999, 38, 126-128. (c) Ryppa, C.; Niedzwiedzki, D.; Morozowich, N. L.; Srikanth, R.; Zeller, M.; Frank, H. A.; Brückner, C. Stepwise Conversion of Two Pyrrole Moieties of Octaethylporphyrin to Pyridin-3-ones: Synthesis, Mass Spectral, and Photophysical Properties of Mono and Bis(oxypyri)porphyrins. Chem. Eur. J. 2009, 15, 5749-5762.

79. Lash, T. D.; El-Beck, J. A.; Ferrence, G. M. Synthesis and Reactivity of mesoUnsubstituted Azuliporphyrins Derived from 6-tert-Butyl- and 6-Phenylazulene. J. Org. Chem. 2007, 72, 8402-8415.

80. Lash, T. D.; Chaney, S. T. Azuliporphyrin: A Case of Borderline Aromaticity. Angew. Chem. Int. Ed. Engl. 1997, 36, 839-840. 
81. Lash, T. D.; Colby, D. A.; Graham, S. R.; Chaney, S. T. Synthesis, Spectroscopy and Reactivity of meso-Unsubstituted Azuliporphyrins and Their Heteroanalogues. Oxidative Ring Contractions to Carba-, Oxacarba-, Thiacarba-, and Selenacarbaporphyrins. J. Org. Chem. 2004, 69, 8851-8864.

82. Richter, D. T.; Lash, T. D. Conjugated macrocycles related to the porphyrins. Part 18: Synthesis and spectroscopic characterization of electron-rich benzi- and oxybenziporphyrins: influence of steric and electronic factors on porphyrinoid aromaticity. Tetrahedron 2001, 57, 3659-3673.

83. Campomanes, P.; Menéndez, M. I.; Cárdenas-Jirón, G. I.; Sordo, T. L. Molecular magnetic properties of heteroporphyrins: a theoretical analysis. Phys. Chem. Chem. Phys. 2007, 9, $5644-5648$.

84. Rothemund, P.; Menotti, A. R. The synthesis of $\alpha, \beta, \gamma, \delta$-tetraphenylporphine. J. Am. Chem. Soc. 1941, 63, 267-270.

85. Rothemund, P. Formation of porphyrins from pyrrole and aldehydes. J. Am. Chem. Soc. 1935, 57, 2010-2011.

86. Sessler, J. L.; Mozaffari, A.; Johnson, M. R. 3,4-Diethylpyrrole and 2,3,7,8,12,13,17,18octaethylporphyrin. Org. Synth. 1991, 70, 68-77.

87. Taniguchi, M.; Lindsey, J. S. Enumeration of isomers of substituted tetrapyrrole macrocycles: from classical problems in biology to modern combinatorial libraries. In Handbook of Porphyrin Science; Kadish, K. M, Smith, K. M, Guilard R., Eds.; World Scientific Press; Singapore, 2012; Vol. 23, pp 3-80.

88. Mauzerall, D. The thermodynamic stability of porphyrinogens. J. Am. Chem. Soc. 1960, 82, 2601-2603. 
89. Vicente, M. D.; Smith, K. M. Syntheses and Functionalizations of Porphyrin Macrocycles. Curr Org Synth. 2014, 11, 3-28.

90. Arsenault, G. P.; Bullock, E.; MacDonald, S. F. Pyrromethanes and porphyrins therefrom. J. Am. Chem. Soc. 1960, 82, 4384-4389.

91. Lash, T. D. What's in a Name? The MacDonald Condensation. J. Porphyrins Phthalocyanines 2016, 20, 855-888.

92. (a) Sessler, J. L.; Lynch, V.; Johnson, M. R. Synthesis and crystal structure of a novel tripyrrane-containing porphyrinogen-like macrocycle. J. Org. Chem. 1987, 52, 4394-4397. (b) Sessler, J.L.; Cyr, M. J.; Lynch, V. Synthetic and structural studies of sapphyrin, a $22 \pi-$ electron pentapyrrolic "expanded porphyrin". J. Am. Chem. Soc. 1990, 112, 2810-2813.

93. Jiao,W.; Lash, T. D. tert-Butyl-Substituted Tripyrranes: Insights into the Steric and Conformational Factors that Influence Porphyrinoid Ring Formation in the " $3+1$ " Methodology. J. Org. Chem. 2003, 68, 3896-3901.

94. Lash, T. D.; Bergman, K. M. Further Observations on Conformational and Substituent Effects in Acid-Catalyzed “3 + 1” Cyclizations of Tripyrranes with Aromatic Dialdehydes. J. Org. Chem. 2012, 77, 9774-9783.

95. Chmielewski, P. J.; Latos-Grazynski, L. N-methyltetraphenylporphyrin with an inverted $\mathrm{N}$-methylpyrrole ring: the first isomer of $N$-methyltetraphenylporphyrin. J. Chem. Soc. Perkin Trans. 2 1995, 0, 503-509.

96. Xiao, Z.; Dolphin, D. Facile synthesis of $N, N^{\prime}$-dimethylated $N$-confused porphyrins. Tetrahedron 2002, 58, 9111-9116. 
97. Lash, T. D.; Miyake, K.; Xu, L.; Ferrence, G. M. Synthesis of a Series of Aromatic Benziporphyrins and Heteroanalogues via Tripyrrane-Like Intermediates Derived from Resorcinol and 2-Methylresorcinol. J. Org. Chem. 2011, 76, 6295-6308.

98. Chmielewski, P. J.; Latos-Grazynski, L.; Glowiak, T. Reactions of Nickel(II) 2-Aza5,10,15,20-tetraphenyl-21- carbaporphyrin with Methyl Iodide. The First Structural Characterization of a Paramagnetic Organometallic Nickel(II) Complex. J. Am. Chem. Soc. 1996, 118, 5690-5701.

99. Lash, T. D. Carbaporphyrinoid Systems. Chem. Rev. 2017, 117, 2313-2446.

100. Lavallee, D. K. The Chemistry and Biochemistry of N-Substituted Porphyrins, VCH Verlagsgesellschaft: Weinheim, Germany,1987; p. 313

101. Lash, T. D. Unexpected Alkyl Group Migration in Palladium(II) Benzocarbaporphyrins. Org. Lett. 2011, 13, 4632-4635.

102. Shears, B.; Hambright, P. A novel metal ion catalyzed demethylation of an N-methyl etioporphyrin. Inorg. Nucl. Chem. Lett. 1970, 6, 679-680.

103. Lavallee, D. K. Metal ion promoted demethylation of N-methyltetraphenylporphinatocopper(II). Inorg. Chem. 1976, 15, 691-694.

104. Lavallee, D. K. Metal ion promoted demethylation of N-Methyltetraphenyl-porphyrin by nickel(II), zinc(II) and manganese(II). Inorg. Chem. 1977, 16, 955-957.

105. Kuila, D.; Lavallee, D. K. Kinetics and mechanisms of dealkylation reactions of Nmethylporphyrin complexes. 3. Effects of porphyrin ring substituents and reaction media. Inorg. Chem. 1983, 22, 1095-1099. 
106. Szyszko, B.; Latos-Grazynski, L.; Szterenberg, L. A Facile Palladium-Mediated Contraction of Benzene to Cyclopentadiene: Transformations of Palladium(II) $p$ Benziporphyrin. Angew. Chem., Int. Ed. 2011, 50, 6587-6591.

107. Li, D.; Lash, T. D. Synthesis and Reactivity of Carbachlorins and Carbaporphyrins. J. Org. Chem. 2014, 79, 7112-7121.

108. Grabowski, E. Y.; AbuSalim, D. I.; Lash, T. D. Naphtho[2,3-b]carbaporphyrins. J. Org. Chem. 2018, 83, 11825-11838

109. Pacholska-Dudziak, E.; Skonieczny, J.; Pawlicki, M.; Szterenberg, L.; Ciunik, Z.; LatosGrazẏnśki, L. Palladium Vacataporphyrin Reveals Conformational Rearrangements Involving Hückel and Möbius Macrocyclic Topologies. J. Am. Chem. Soc. 2008, 130, 6182-6195.

110. Yoneda, T.; Saito, S.; Yorimitsu, H.; Osuka, A. Palladium(II)-Triggered Rearrangement of Heptaphyrins to N-Confused Porphyrins. Angew. Chem., Int. Ed. 2011, 50, 3475-3478.

111. Latham, A. N.; Ferrence, G. M.; Lash, T. D. Metalation and Methyl Group Migration in 21-, 22-, and 23-Methylcarbaporphyrins: Synthesis and Characterization of Palladium(II), Rhodium(I), and Rhodium(III) Derivatives. Organometallics 2019, 38, 575-585.

112. Chapelle, J. P.; Elguero, J.; Jacquier, R.; Tarrago, G. Enehydrazines [(alk-1-en-1yl)hydrazines]. III. Reaction of 1,2-Dimethylhydrazine with Carbonyl Compounds; Formation of N-Methylpyrroles and 3-Pyrazolines. Bull. Soc. Chim. Fr. 1970, 3147-3155.

113. Boudif, A.; Momenteau, M. A New Convergent Method for Porphyrin Synthesis Based on a ' $3+1$ ' condensation. J. Chem. Soc., Perkin Trans. 1 1996, 1235-1242.

114. Lash, T. D. Porphyrins with Exocyclic Rings. Part 9. Porphyrin Synthesis by the " $3+1$ ” Approach. J. Porphyrins Phthalocyanines 1997, 1, 29-44. 
115. Jackson, A. H.; Pandey R. K.; Roberts, E. Total Synthesis of an N-Methylporphyrin. J. Chem. Soc., Chem. Commun. 1985, 470-471.

116. Pandey, R. K.; Jackson A. H.; Smith, K. M. Ring Syntheses of the Four NMethylprotoporphyrin Isomers and of Related $N$-Methyl and $N, N$ '-Dimethylporphyrins. $J$. Chem. Soc., Perkin Trans. 1 1991, 1211-1220.

117. Bruno, I. J.; Cole, J. C.; Kessler, M.; Luo, J.; Motherwell, W. D. S.; Purkis, L. H.; Smith, B. R.; Taylor, R.; Cooper, R. I.; Harris, S. E.; Orpen, A. G. Retrieval of Crystallographically-derived Molecular Geometry Information. J. Chem. Inf. Comput. Sci. 2004, 44, 2133-2144. 


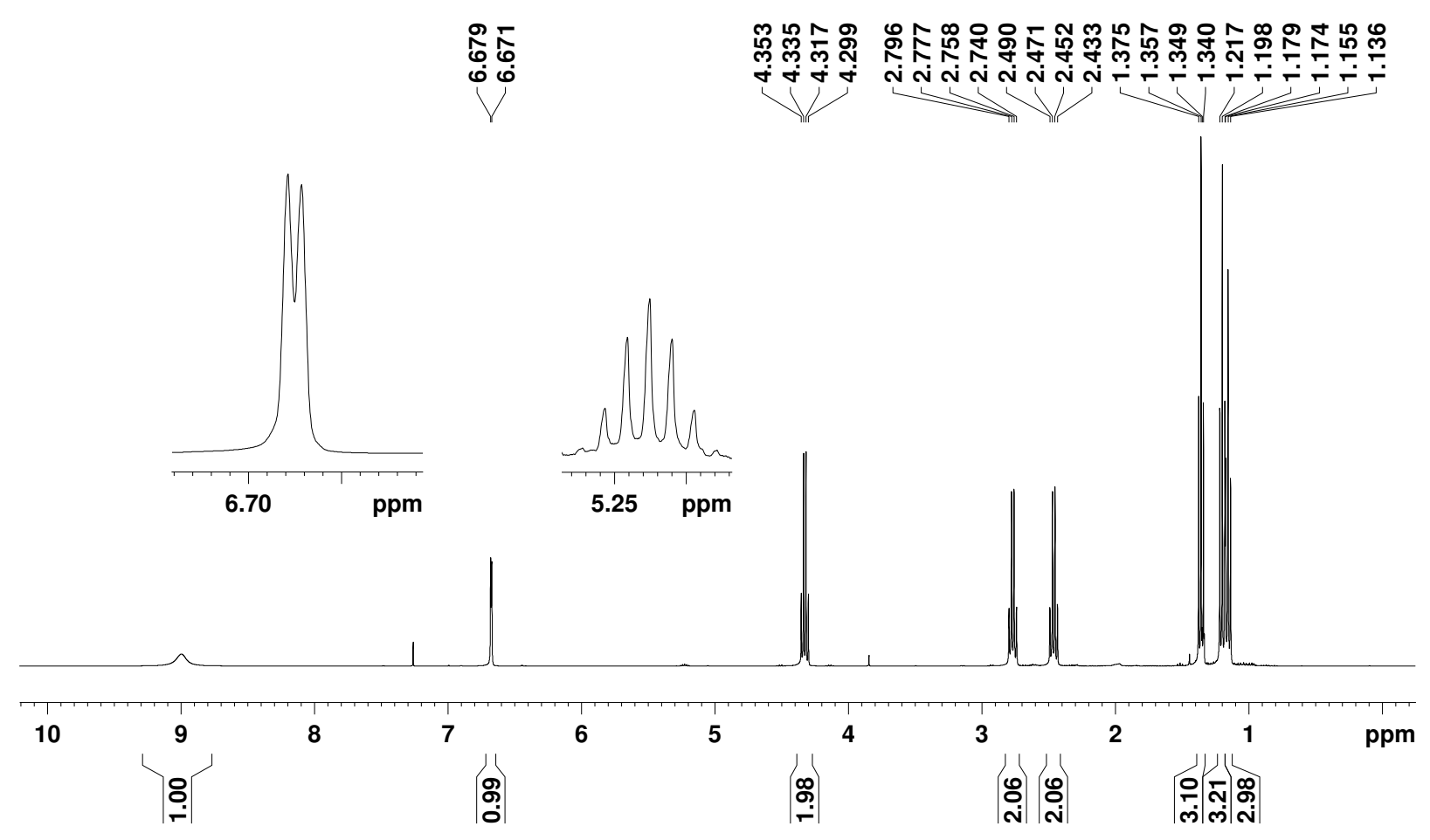

Figure A-1. $400 \mathrm{MHz}{ }^{1} \mathrm{H}$ NMR spectrum of pyrrole ethyl ester 79 in $\mathrm{CDCl}_{3}$. The small multiplet at $5.23 \mathrm{ppm}$ corresponds to a small amount of the related isopropyl ester (ca. 3\%) that results from transesterification with the solvent 2-propanol. 


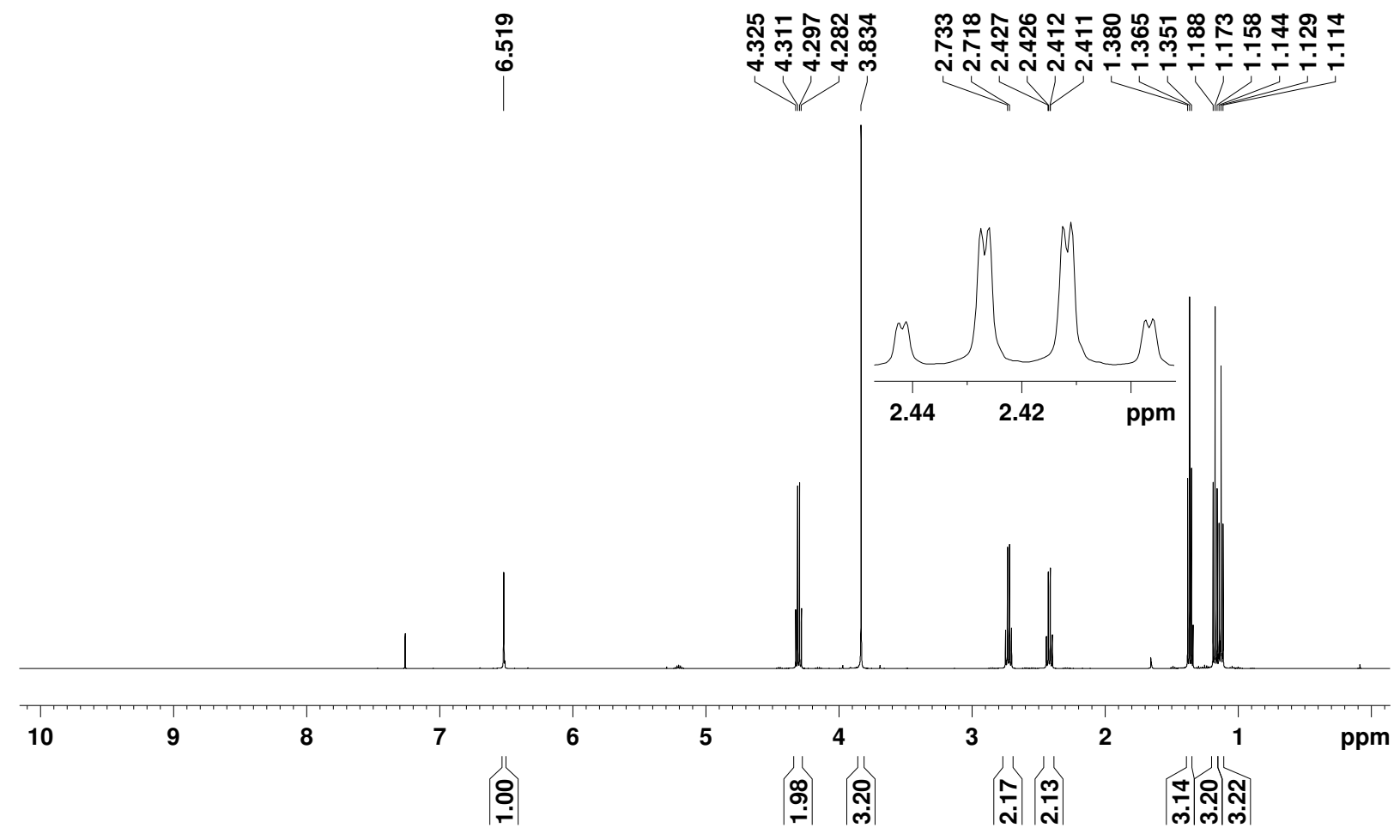

Figure A-2. ${ }^{1} \mathrm{H}$ NMR spectrum of $\mathrm{N}$-methylpyrrole ethyl ester 79 in $\mathrm{CDCl}_{3}$. 


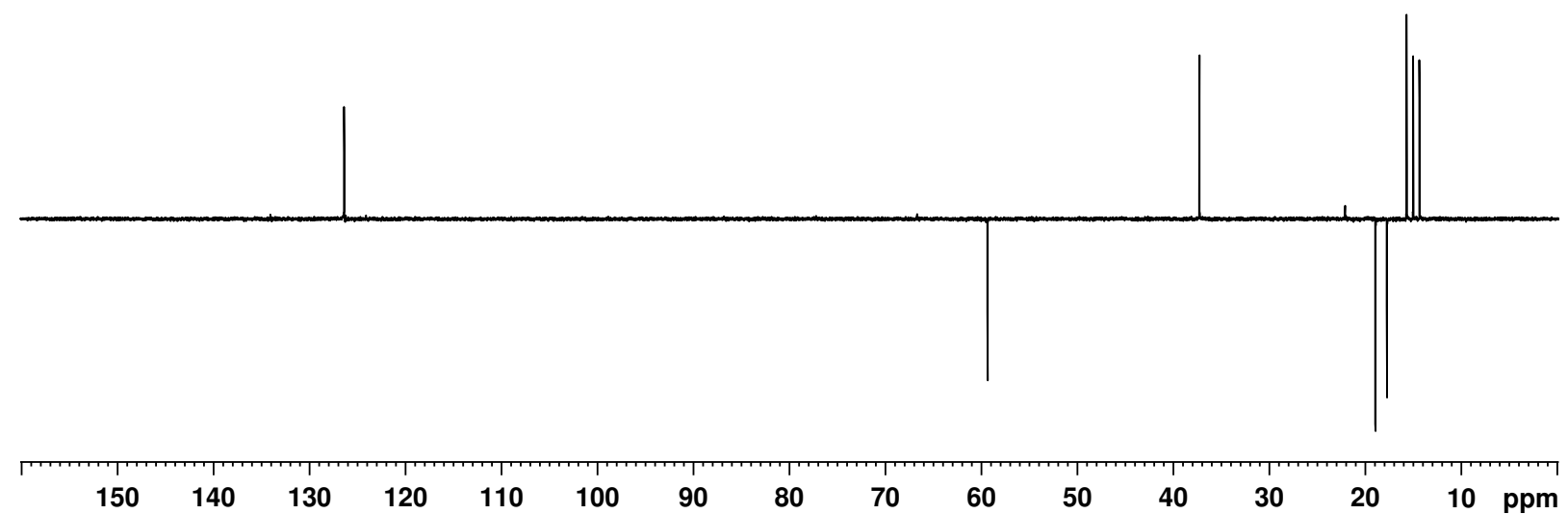

Figure A-3. DEPT-135 NMR spectrum of $N$-methylpyrrole ester $\mathbf{1 7 b}$.

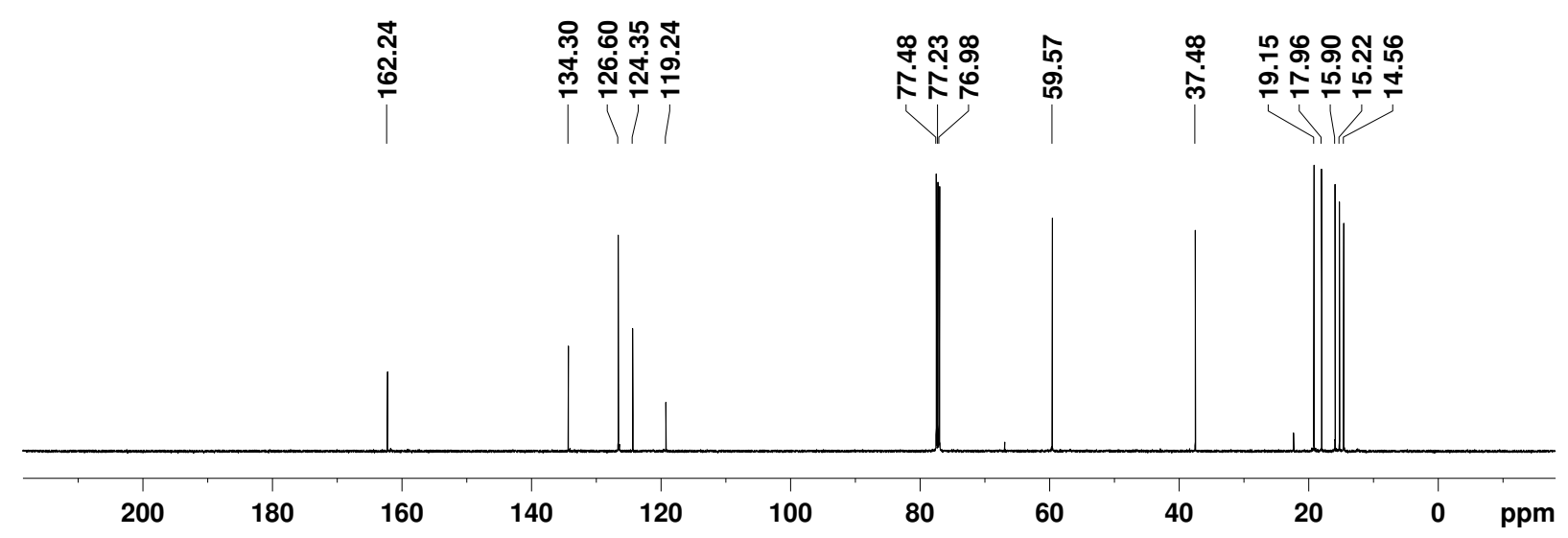

Figure A-4. $125 \mathrm{MHz}{ }^{13} \mathrm{C}$ NMR spectrum of $N$-methylpyrrole ethyl ester 79 in $\mathrm{CDCl}_{3}$. The small peaks showing up at 22.3 and 66.9 ppm correspond to the presence of the related isopropyl ester. 


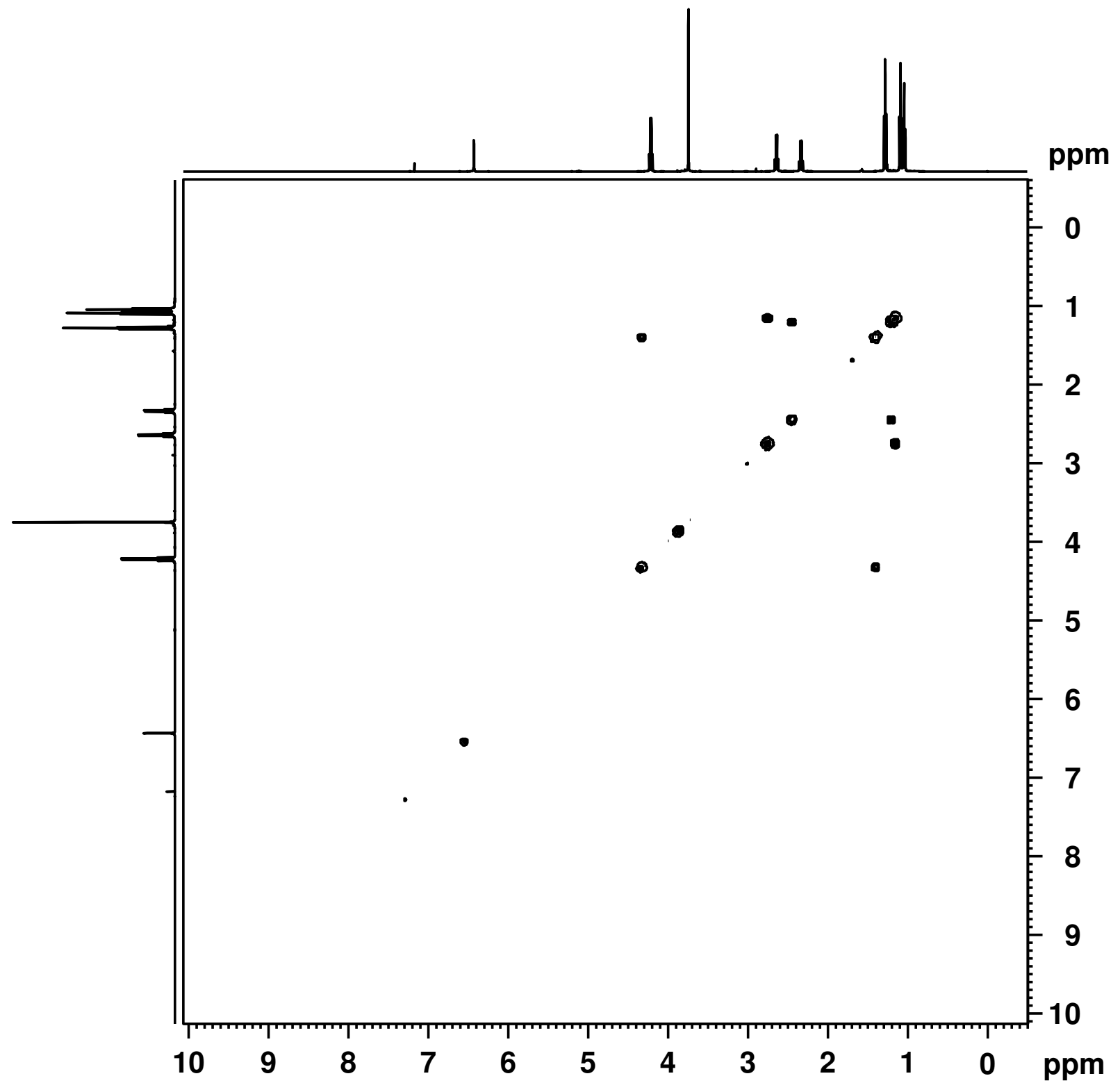

Figure A-5. ${ }^{1} \mathrm{H}-{ }^{1} \mathrm{H}$ COSY NMR spectrum of $N$-methylpyrrole ethyl ester 79 in $\mathrm{CDCl}_{3}$. 


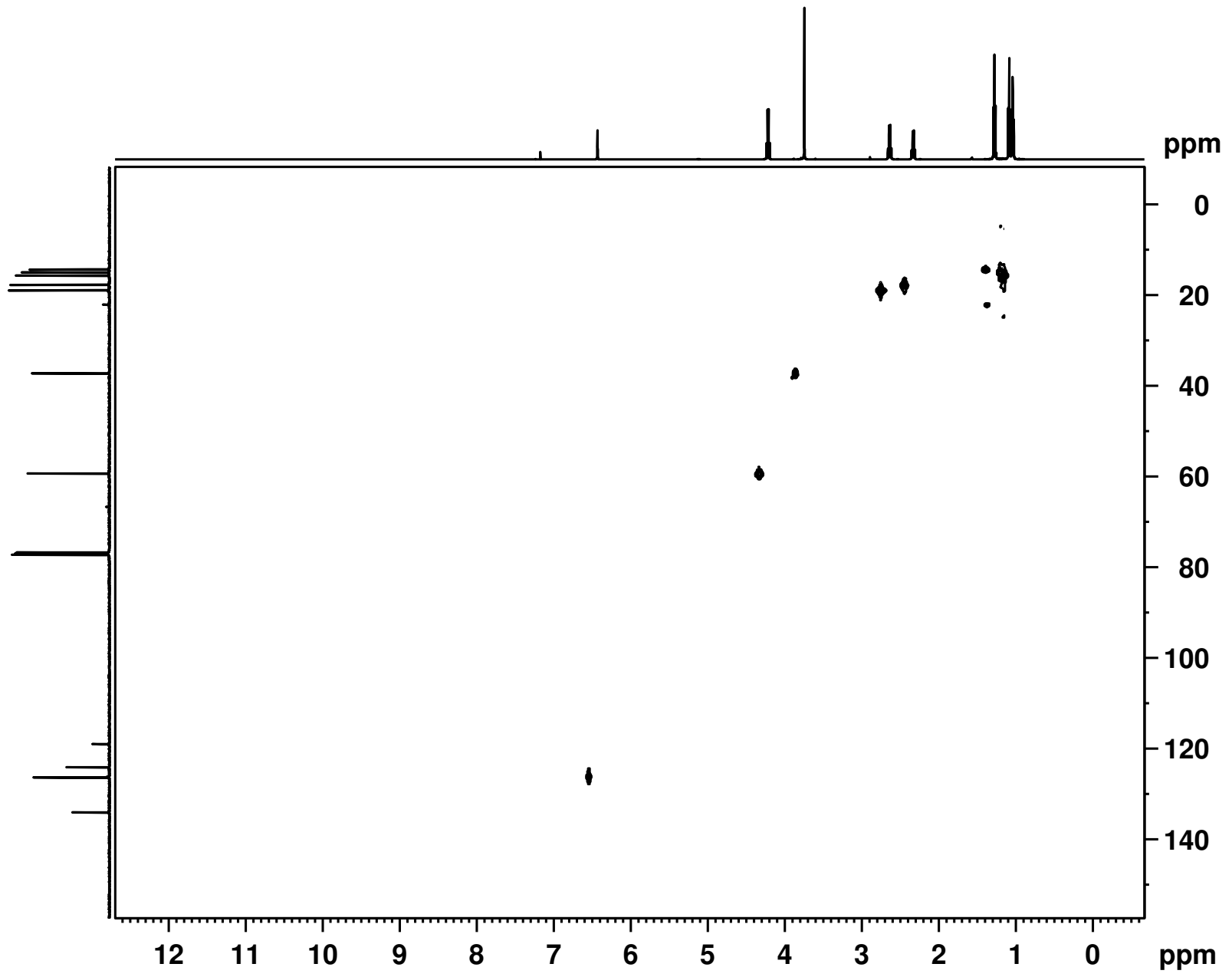

Figure A-6. HSQC NMR spectrum of $N$-methyl pyrrole ester 79. 


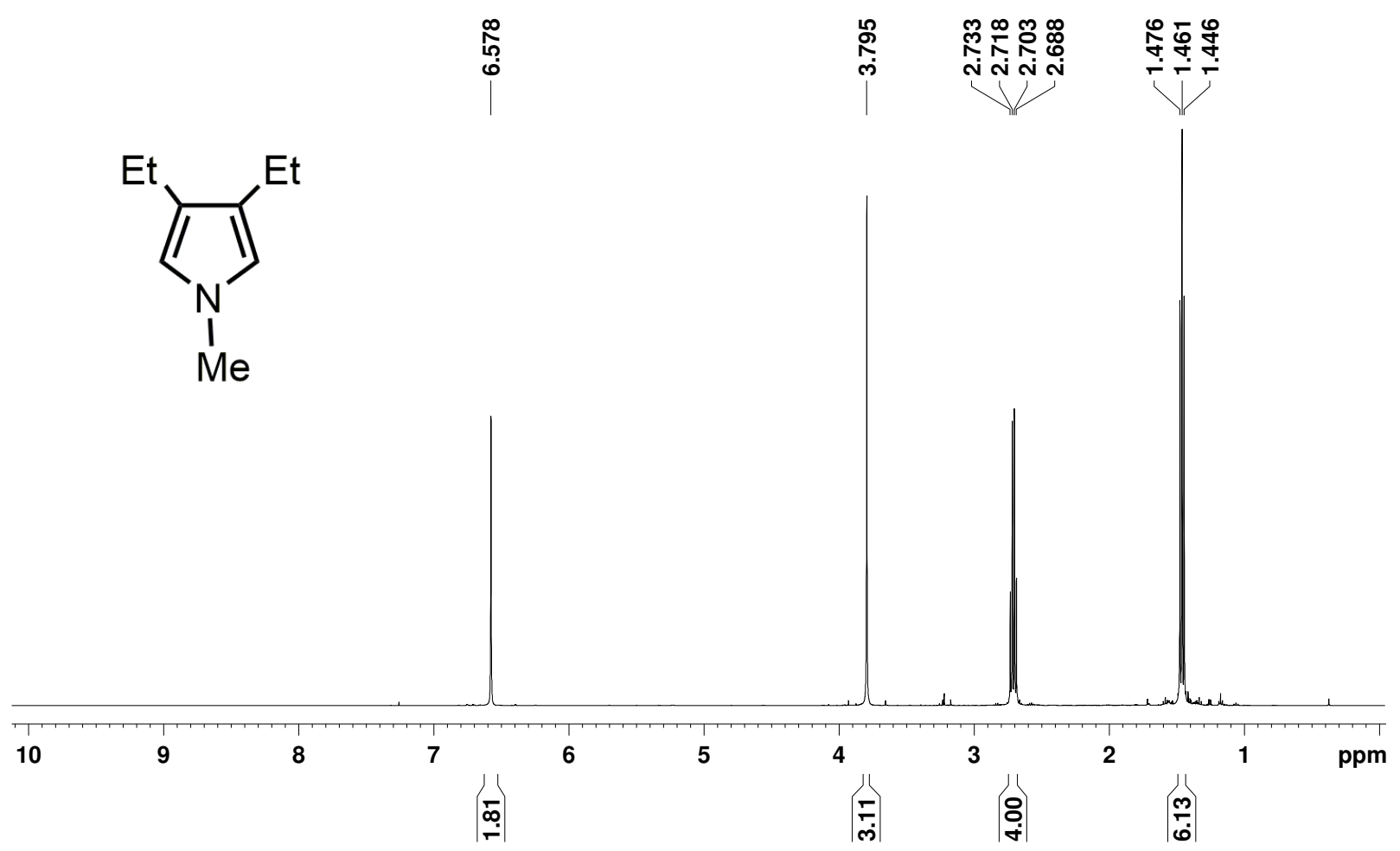

Figure A-7. $500 \mathrm{MHz}{ }^{1} \mathrm{H}$ NMR spectrum of 3,4-diethyl-1-methylpyrrole (53b) in $\mathrm{CDCl}_{3}$.

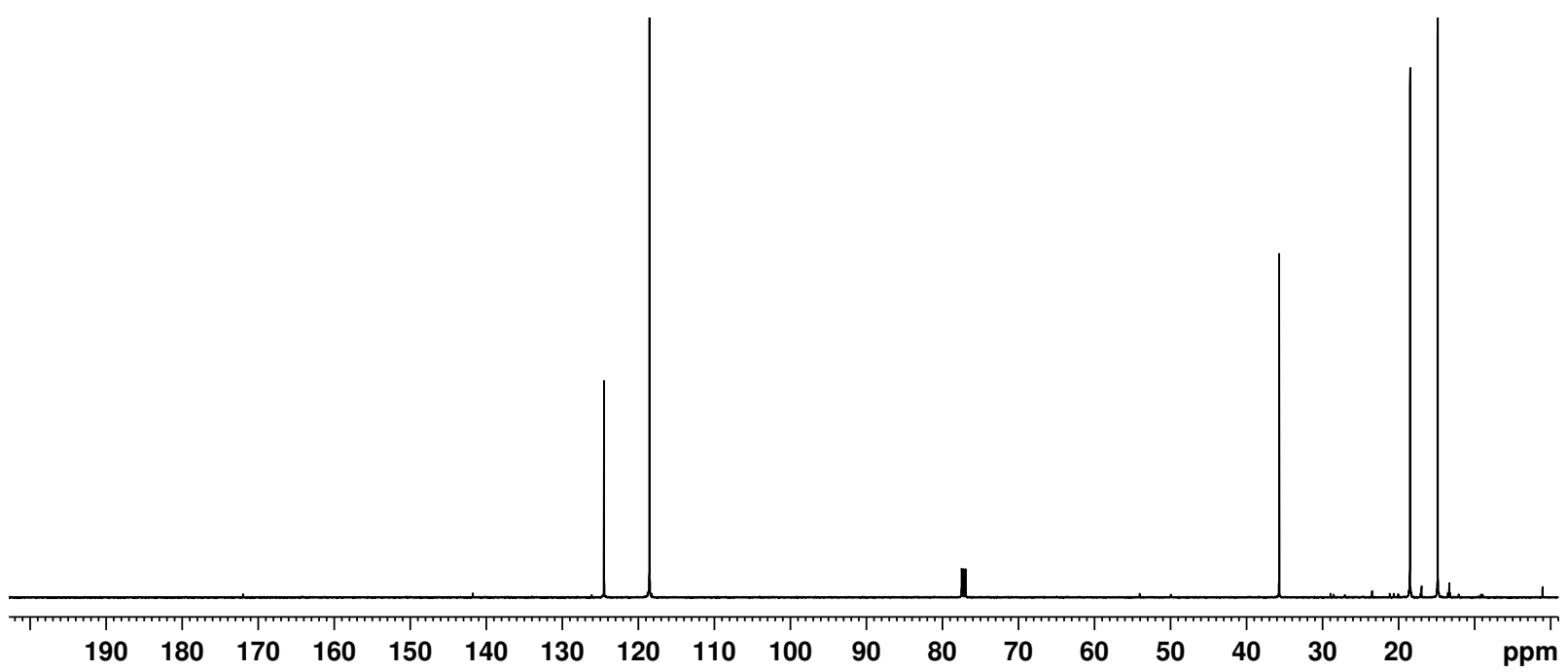

Figure A-8. $125 \mathrm{MHz}{ }^{13} \mathrm{C}$ NMR spectrum of 3,4-diethyl-1-methylpyrrole (53b) in $\mathrm{CDCl}_{3}$. 


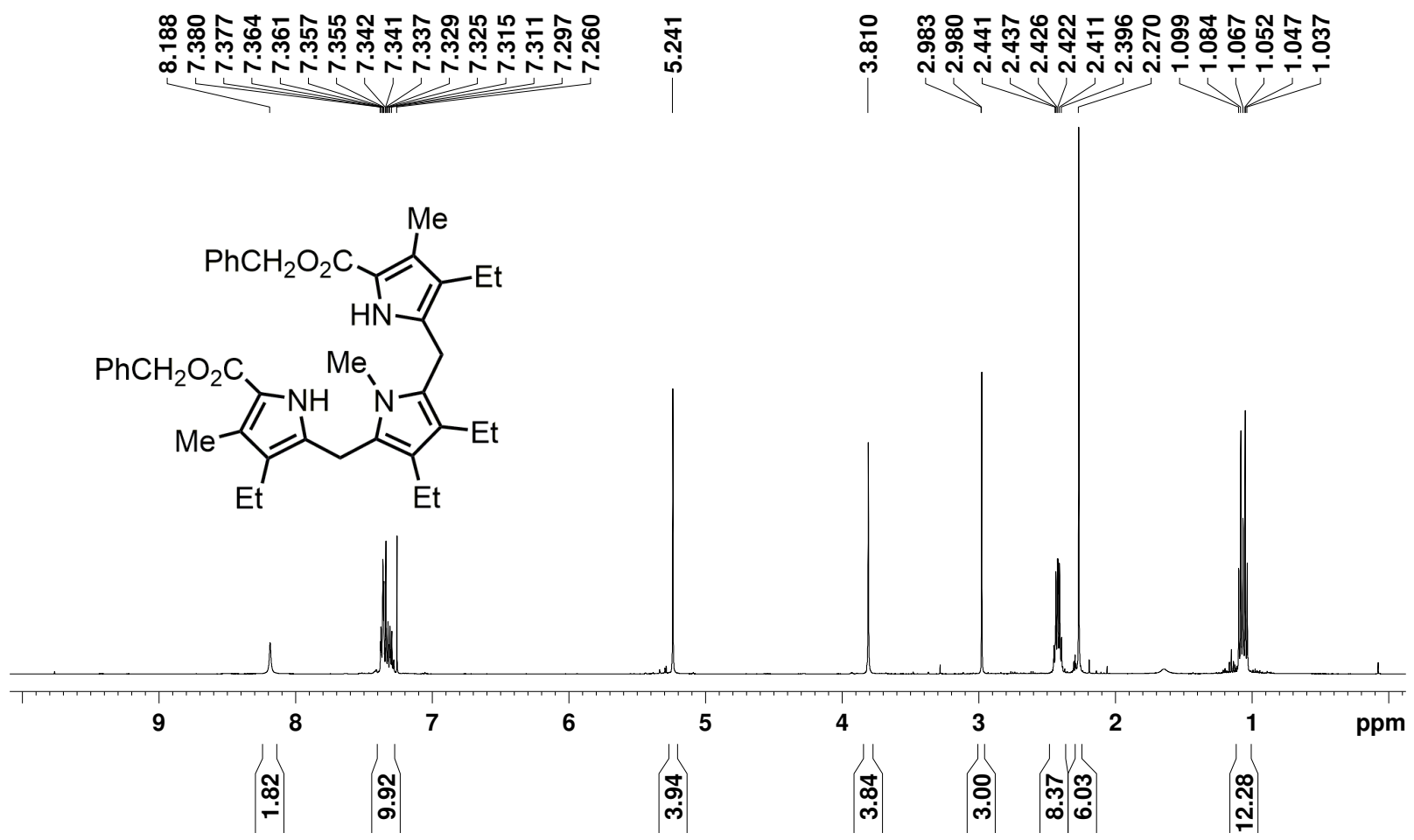

Figure A-9. $500 \mathrm{MHz}{ }^{1} \mathrm{H}$ NMR spectrum of $N$-methyltripyrrane dibenzyl ester 54c in $\mathrm{CDCl} 3$. 


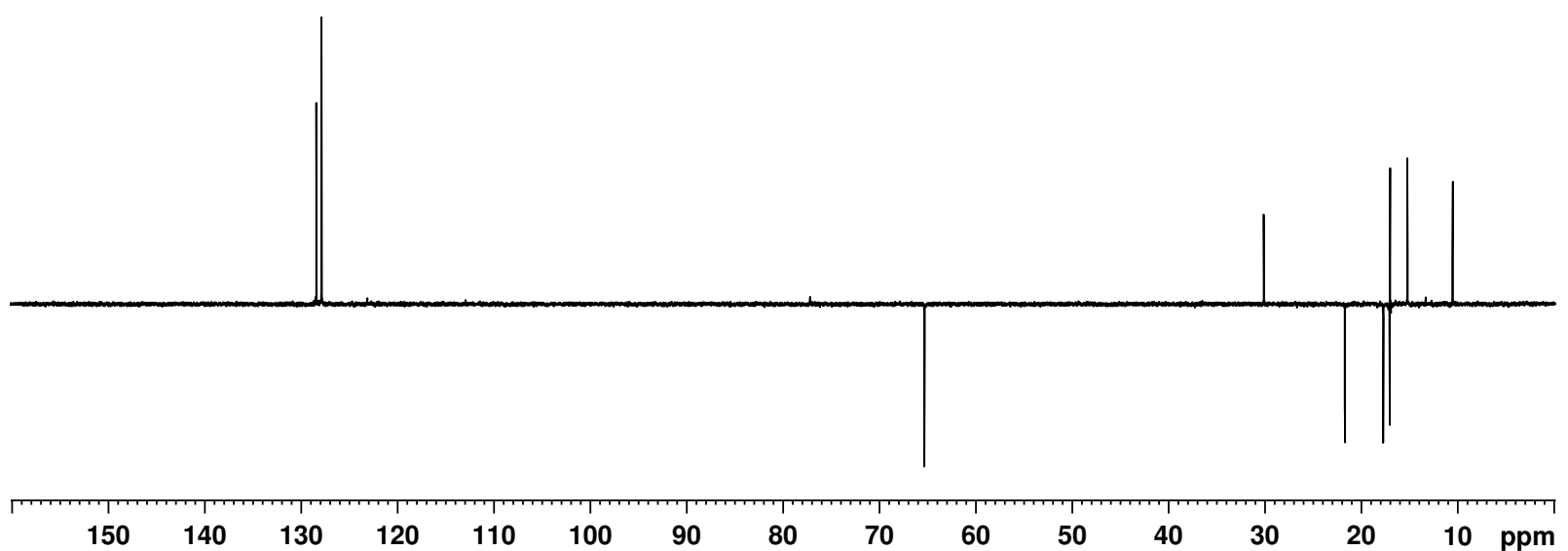

Figure A-10. $125 \mathrm{MHz}{ }^{1} \mathrm{H}$ NMR spectrum of $N$-methyltripyrrane dibenzyl ester $54 \mathbf{c}$ in $\mathrm{CDCl}_{3}$.

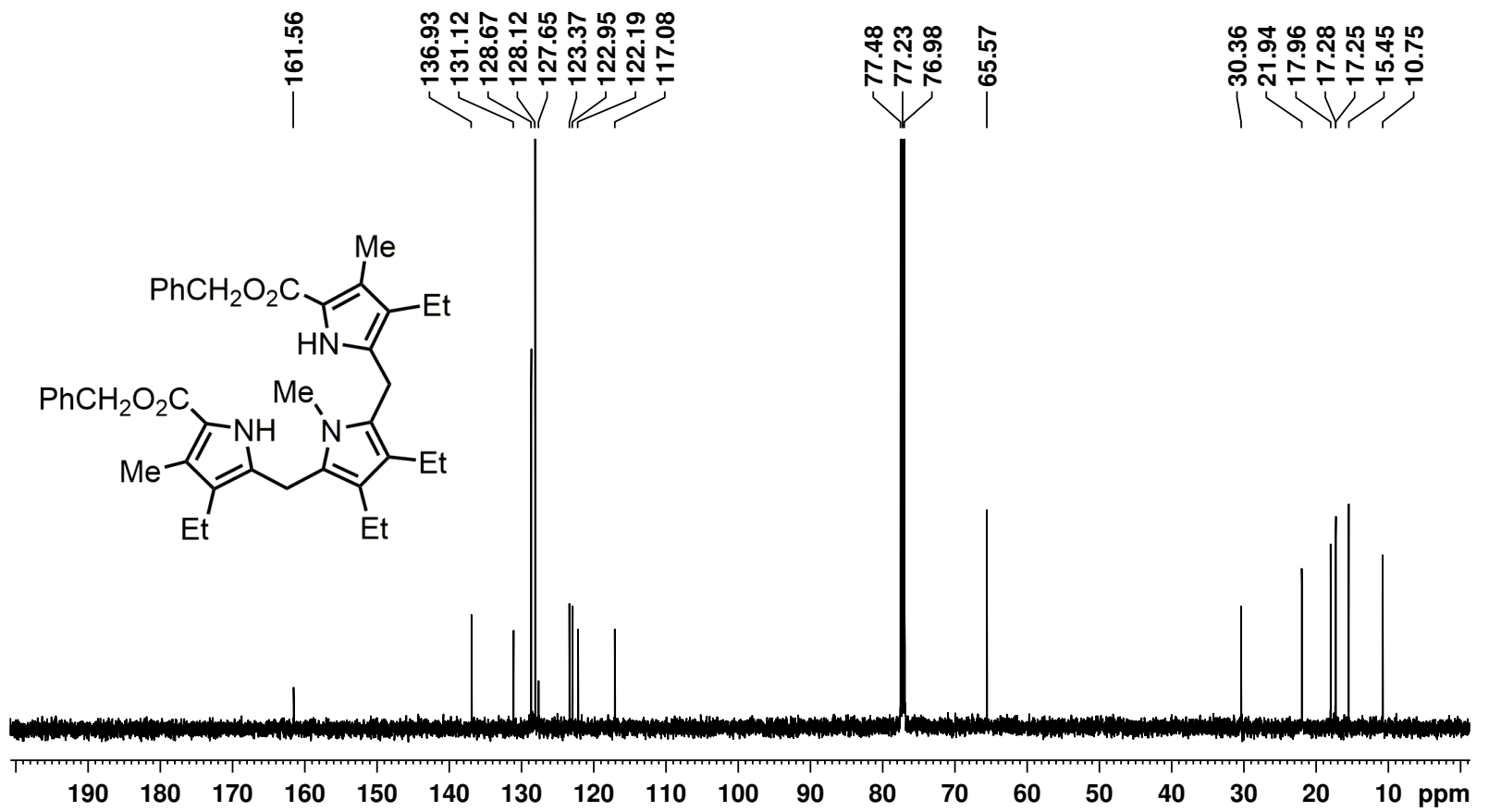

Figure A-11. $125 \mathrm{MHz}{ }^{1} \mathrm{H}$ NMR spectrum of $N$-methyltripyrrane dibenzyl ester $\mathbf{5 4 c}$ in $\mathrm{CDCl}_{3}$. 


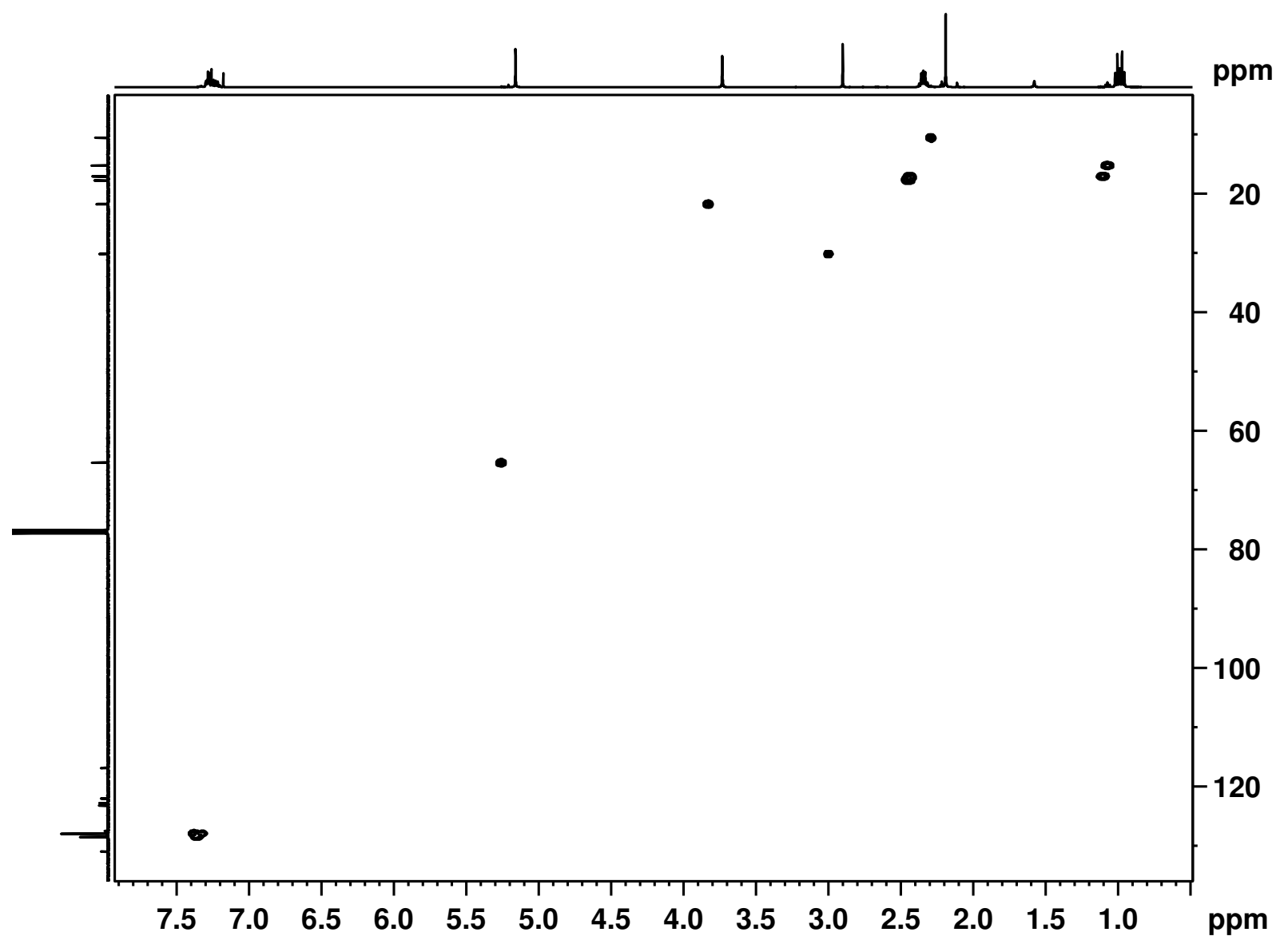

Figure A-12. HSQC NMR spectrum of $N$-methyltripyrrane dibenzyl ester $\mathbf{5 4} \mathbf{c}$ in $\mathrm{CDCl}_{3}$. 


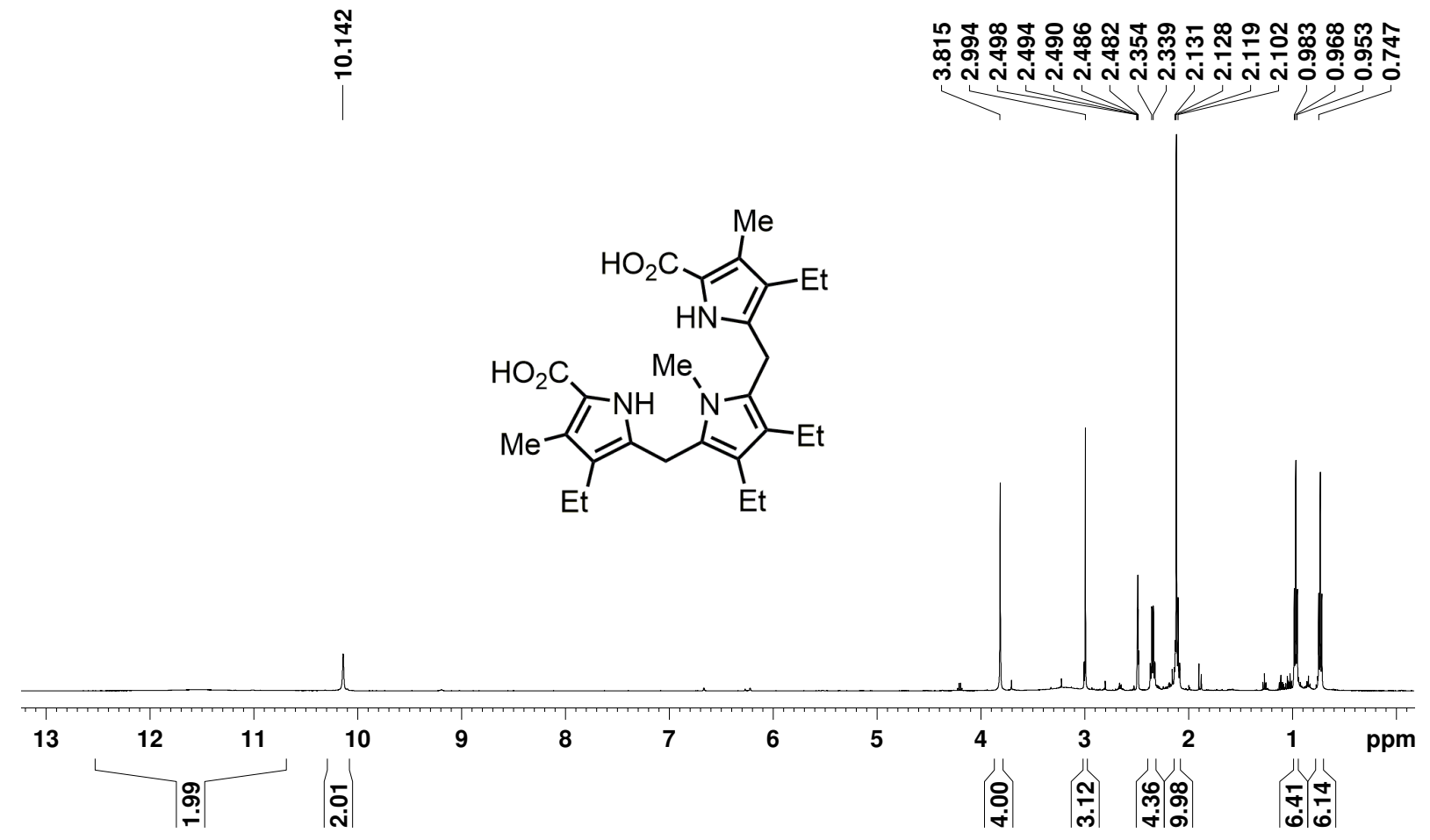

Figure A-13. $500 \mathrm{MHz}{ }^{1} \mathrm{H}$ NMR spectrum of $N$-methyltripyrrane dicarboxylic acid 54d in $d_{6^{-}}$ DMSO. 


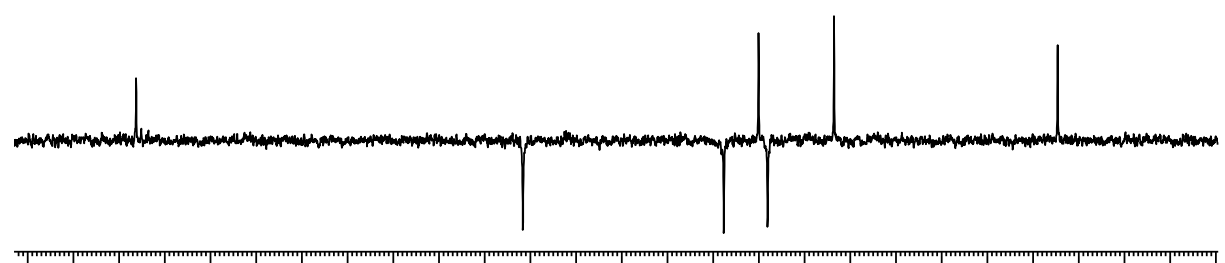

$\begin{array}{lllllllllllllllllllllllll}32 & 31 & 30 & 29 & 28 & 27 & 26 & 25 & 24 & 23 & 22 & 21 & 20 & 19 & 18 & 17 & 16 & 15 & 14 & 13 & 12 & 11 & 10 & 9 & \mathrm{ppm}\end{array}$

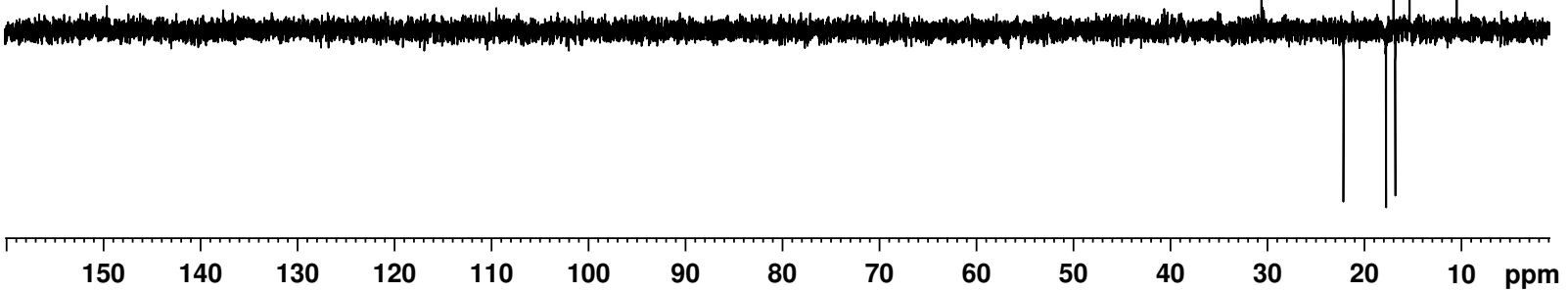

Figure A-14. DEPT-135 NMR spectrum of $N$-methyltripyrrane dicarboxylic acid 54d in $d_{6^{-}}$ DMSO.

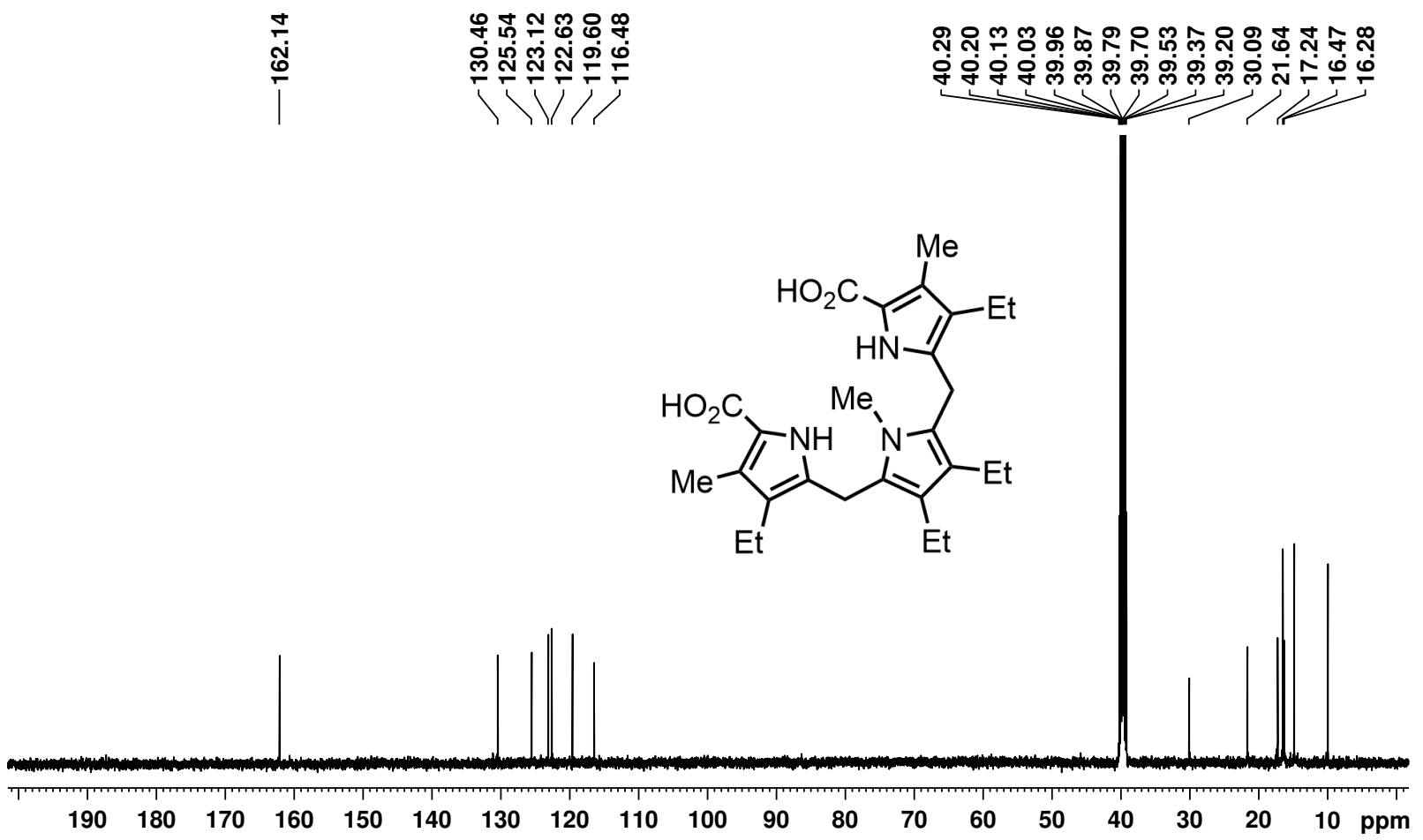

Figure A-15. $125 \mathrm{MHz}{ }^{13} \mathrm{C}$ NMR spectrum of $N$-methyltripyrrane dicarboxylic acid 54d in $d_{6^{-}}$ DMSO. 


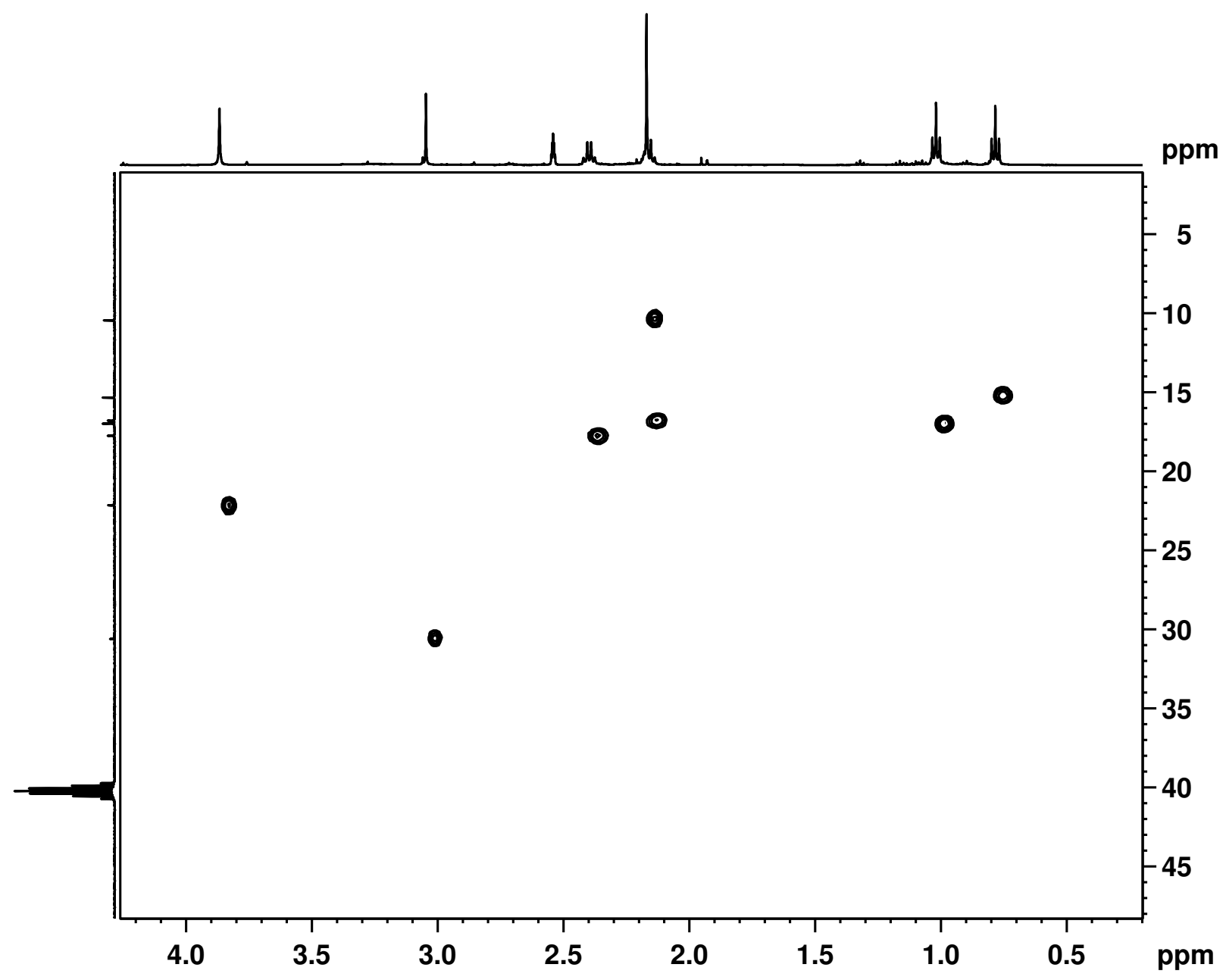

Figure A-16. HSQC NMR spectrum of $N$-methyltripyrrane dicarboxylic acid 54d in $d_{\sigma^{-}}$ DMSO. 


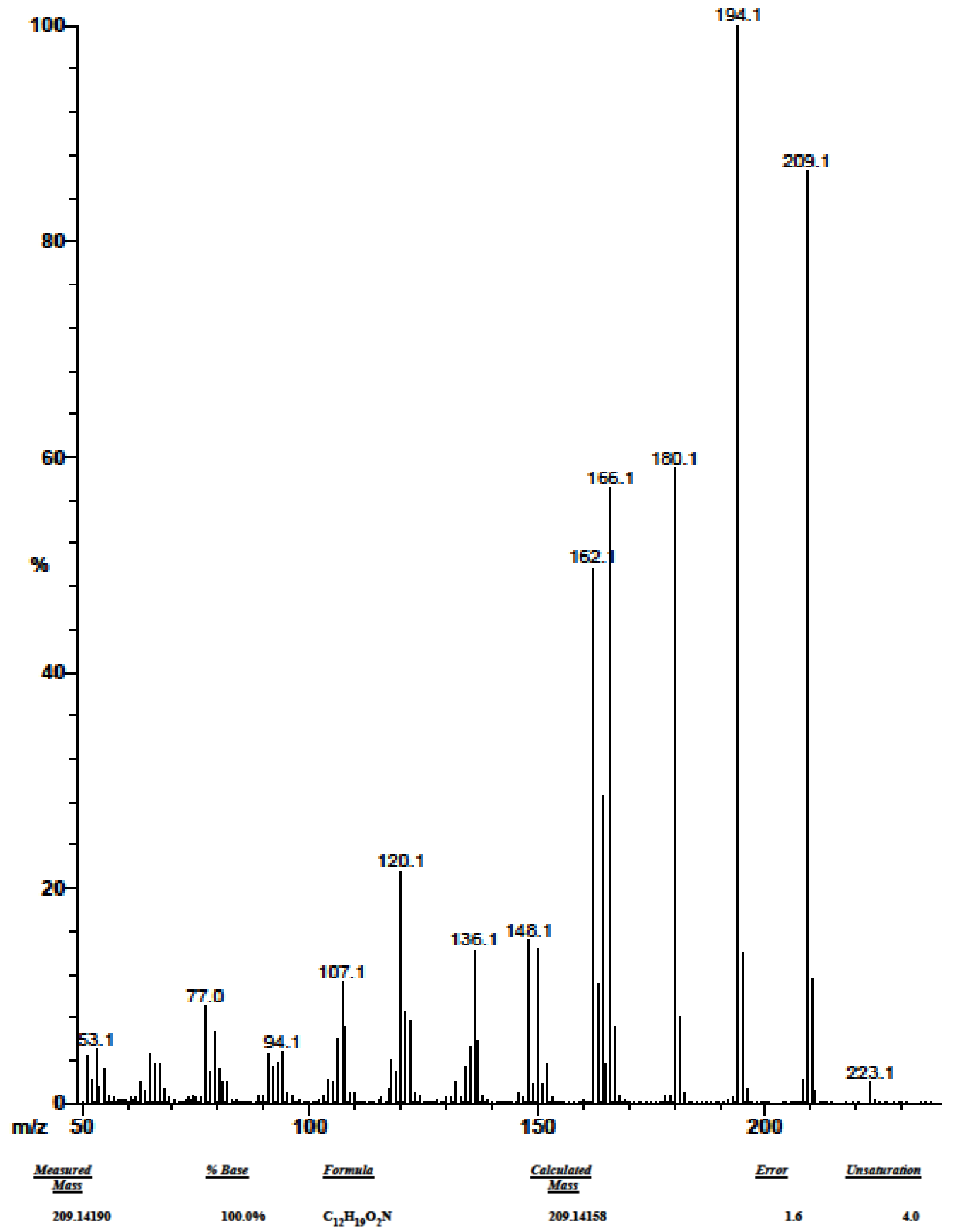

Figure A-17. EI MS of $N$-methylpyrrole ester 79. 


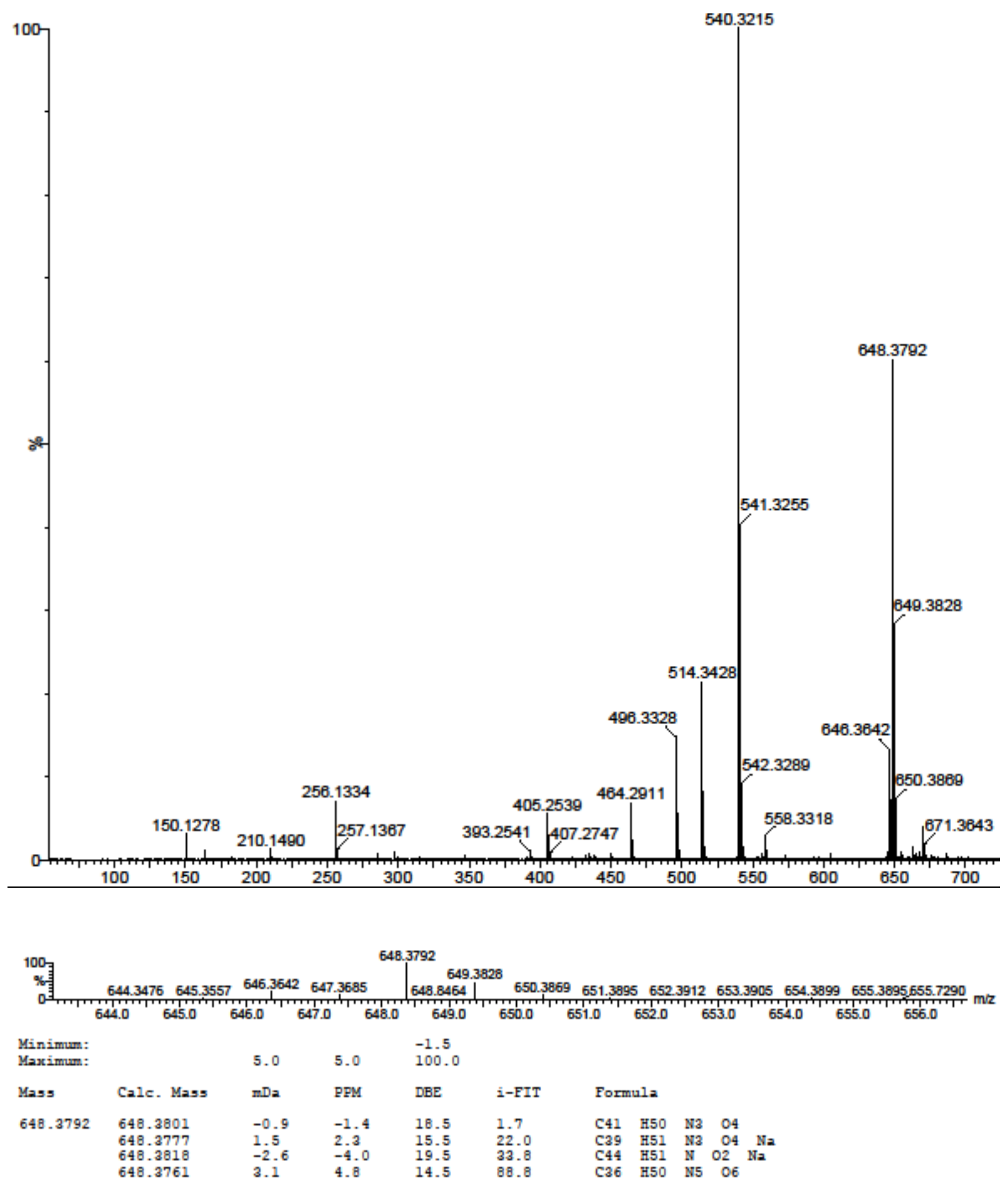

Figure A-18. ESI MS of $N$-methyltripyrrane dibenzyl ester $\mathbf{5 4 c}$. 


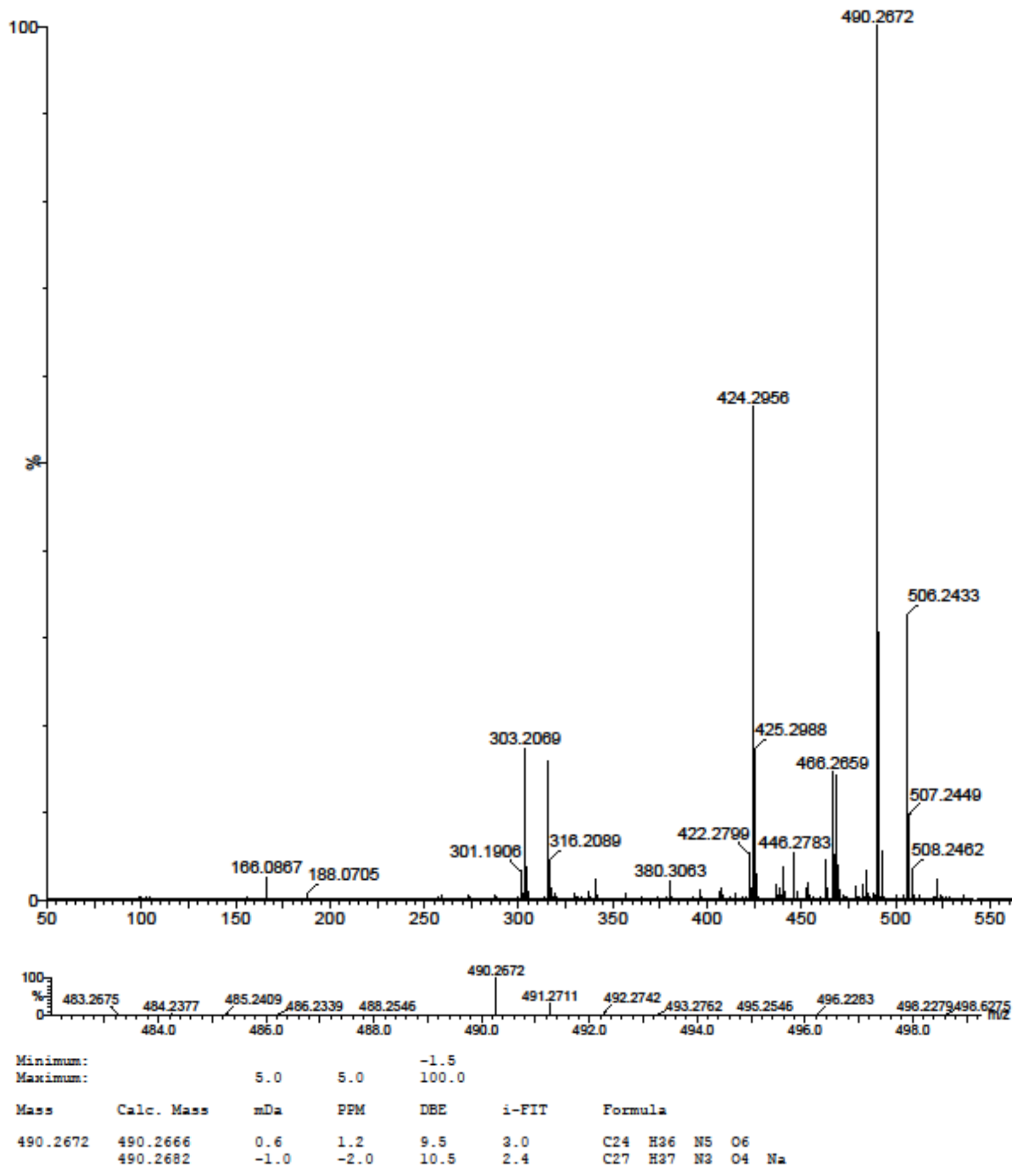

Figure A-19. ESI MS of $N$-methyltripyrrane dicarboxylic acid 54d. 


\section{APPENDIX B: SPECTROSCOPIC DATA FOR 23-METHYLBENZOCARBAPORPHYRIN}

\section{AND PALLADIUM(II) COMPLEXES}

Table B-1. Summary of framework bond distances $(\AA)$ and angles $\left(^{\circ}\right)$ for $\mathbf{7 2}$ and $\mathbf{7 6 .}$
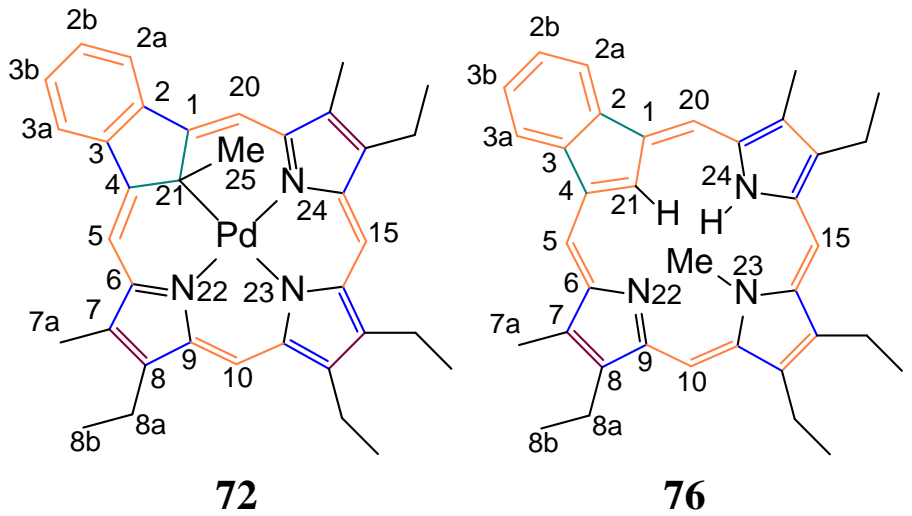

\begin{tabular}{|l|c|c|}
\hline & $\mathbf{7 2}$ & $\mathbf{7 6}$ \\
\hline C21-C25 & $1.577(2)$ & -- \\
\hline M-C21 & $2.0576(16)$ & -- \\
\hline M-N22 & $2.0363(14)$ & -- \\
\hline M-N23 & $2.0697(14)$ & -- \\
\hline M-N24 & $2.0352(14)$ & -- \\
\hline M-C25 & -- & -- \\
\hline C1-C21 & $1.491(2)$ & $1.406(2)$ \\
\hline C1-C2 & $1.450(2)$ & $1.4822(19)$ \\
\hline C2-C3 & $1.414(2)$ & $1.4136(19)$ \\
\hline C3-C4 & $1.449(2)$ & $1.4753(19)$ \\
\hline C4-C21 & $1.498(2)$ & $1.4077(19)$ \\
\hline C4-C5 & $1.367(2)$ & $1.3977(19)$ \\
\hline C5-C6 & $1.412(2)$ & $1.4015(19)$ \\
\hline C6-N22 & $1.364(2)$ & $1.3668(17)$ \\
\hline C6-C7 & $1.446(2)$ & $1.4590(19)$ \\
\hline C7-C8 & $1.361(2)$ & $1.3574(19)$ \\
\hline C8-C9 & $1.455(2)$ & $1.4577(19)$ \\
\hline C9-N22 & $1.387(2)$ & $1.3638(17)$ \\
\hline C9-C10 & $1.378(2)$ & $1.4001(19)$ \\
\hline C10-C11 & $1.402(2)$ & $1.3928(19)$ \\
\hline C11-N23 & $1.363(2)$ & $1.3991(17)$ \\
\hline C11-C12 & $1.448(2)$ & $1.4302(18)$ \\
\hline C12-C13 & $1.374(2)$ & $1.3784(19)$ \\
\hline C13-C14 & $1.446(2)$ & $1.4301(18)$ \\
\hline
\end{tabular}




\begin{tabular}{|l|c|c|}
\hline C14-N23 & $1.368(2)$ & $1.4037(17)$ \\
\hline C14-C15 & $1.396(2)$ & $1.3924(19)$ \\
\hline C15-C16 & $1.384(2)$ & $1.3968(19)$ \\
\hline C16-N24 & $1.390(2)$ & $1.3711(17)$ \\
\hline C16-C17 & $1.451(2)$ & $1.4369(19)$ \\
\hline C17-C18 & $1.363(2)$ & $1.3781(19)$ \\
\hline C18-C19 & $1.449(2)$ & $1.4422(19)$ \\
\hline C19-N24 & $1.365(2)$ & $1.3724(17)$ \\
\hline C19-C20 & $1.412(2)$ & $1.3937(19)$ \\
\hline C1-C20 & $1.366(2)$ & $1.3971(19)$ \\
\hline C1-C21-C4 & $103.80(13)$ & $111.55(13)$ \\
\hline C21-M-N22 & $89.67(6)$ & -- \\
\hline C21-M-N23 & $170.06(6)$ & -- \\
\hline C21-M-N24 & $90.12(6)$ & -- \\
\hline N22-M-N23 & $90.34(6)$ & -- \\
\hline N22-M-N24 & $177.98(6)$ & -- \\
\hline N23-M-N24 & $90.22(6)$ & -- \\
\hline C25-C21-M & $98.06(10)$ & -- \\
\hline C25-C21-C1 & $107.20(13)$ & -- \\
\hline C25-C21-C4 & $107.75(13)$ & -- \\
\hline
\end{tabular}

Purple more double bond like ( $\leq 1.37 \AA$ ), orange - more aromatic (1.38-1.41 $\AA$ ), blue - more single bond like (1.42-1.45 Å), green - single bond limit ( $\geq 1.46 \AA)$. 

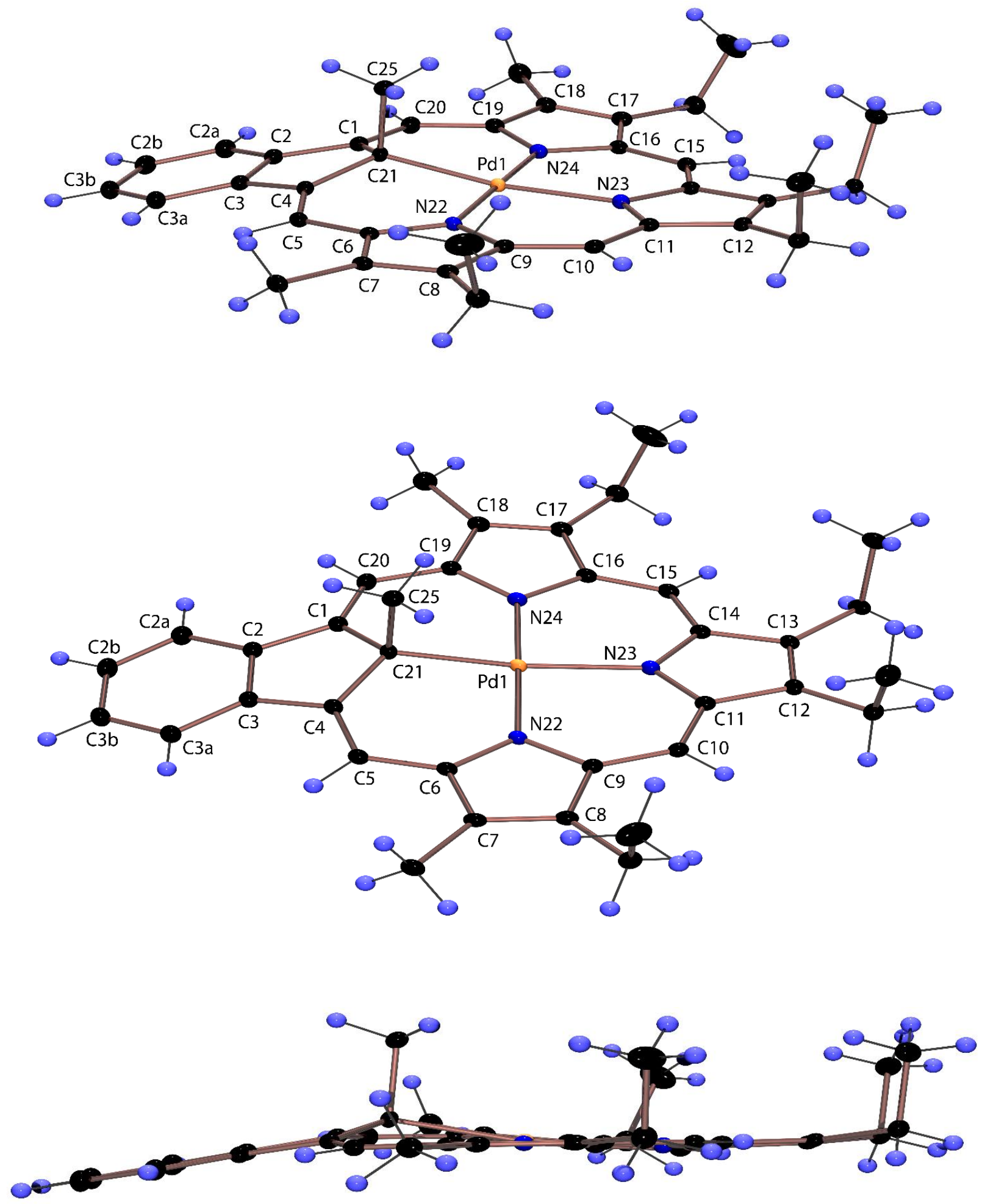

Figure B-1. Color POV-Ray rendered ORTEP III drawings (50\% probability level, hydrogen atoms rendered arbitrarily small for clarity) showing three views of palladium(II) derivative 72. 

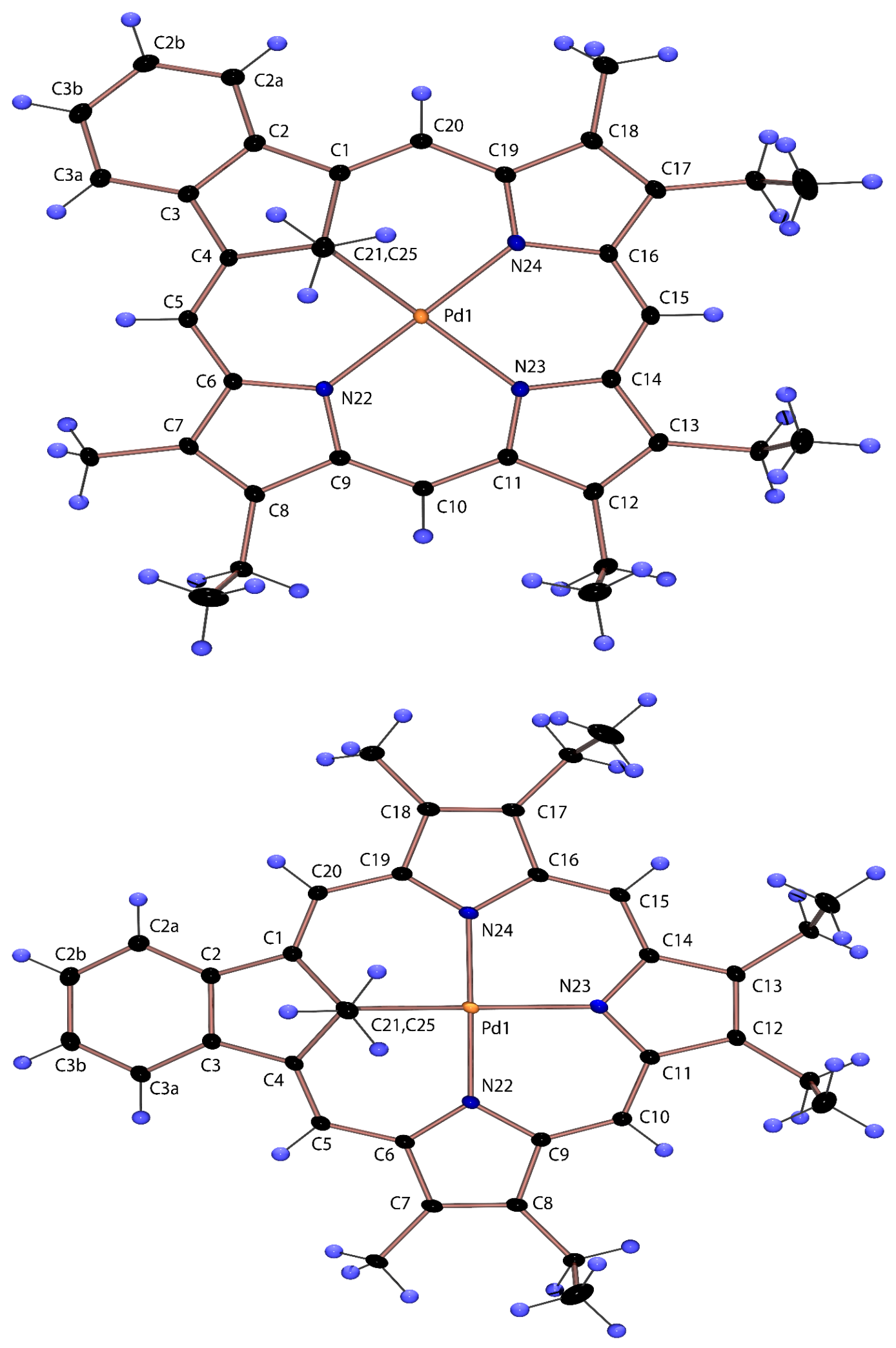

Figure B-2. Color POV-Ray rendered ORTEP III drawing (50\% probability level, hydrogen atoms rendered arbitrarily small for clarity) showing two additional views of compound $\mathbf{7 2}$. 

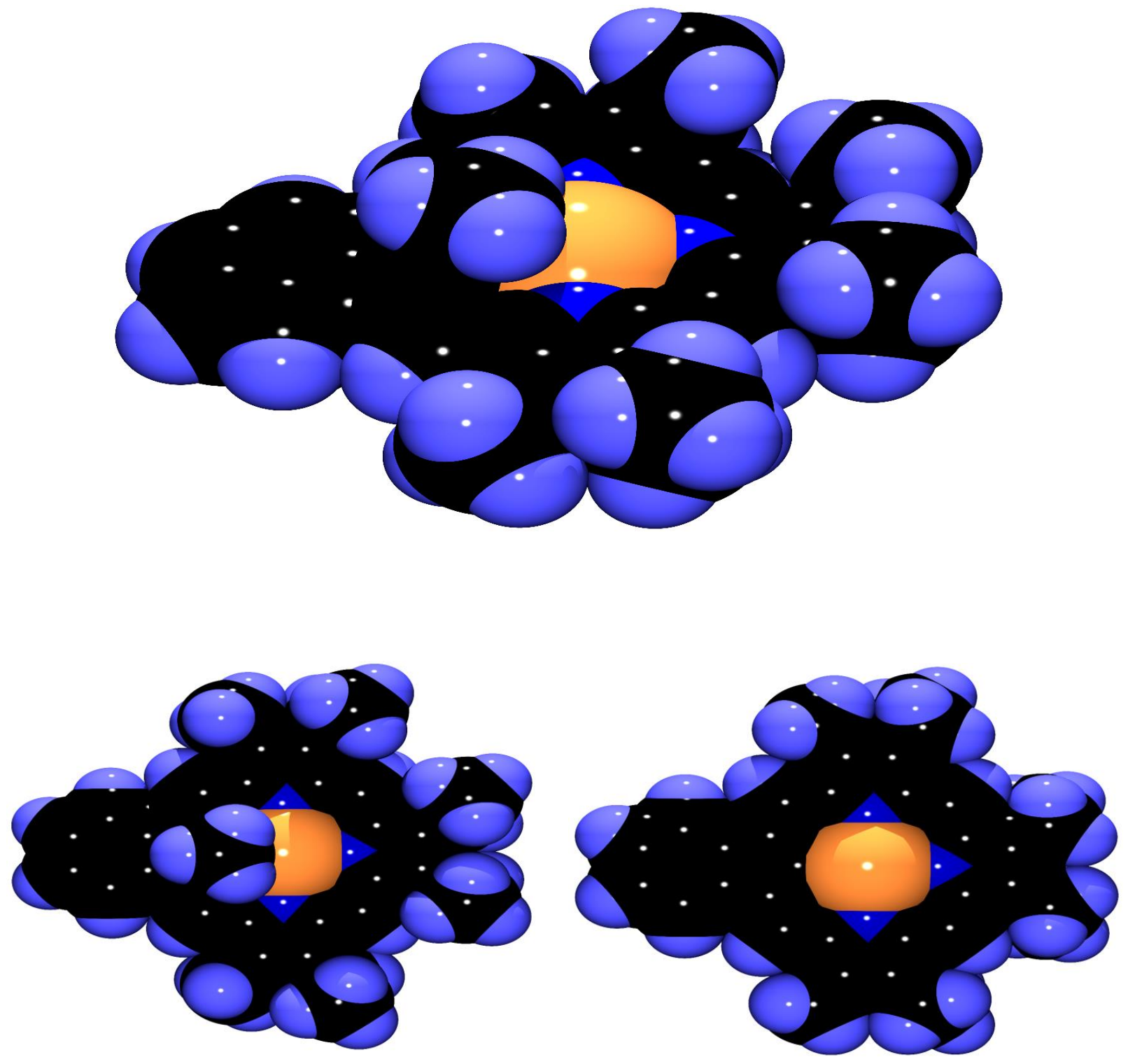

Figure B-3. Color POV-Ray rendered space-filling drawings of palladium complex 72. 

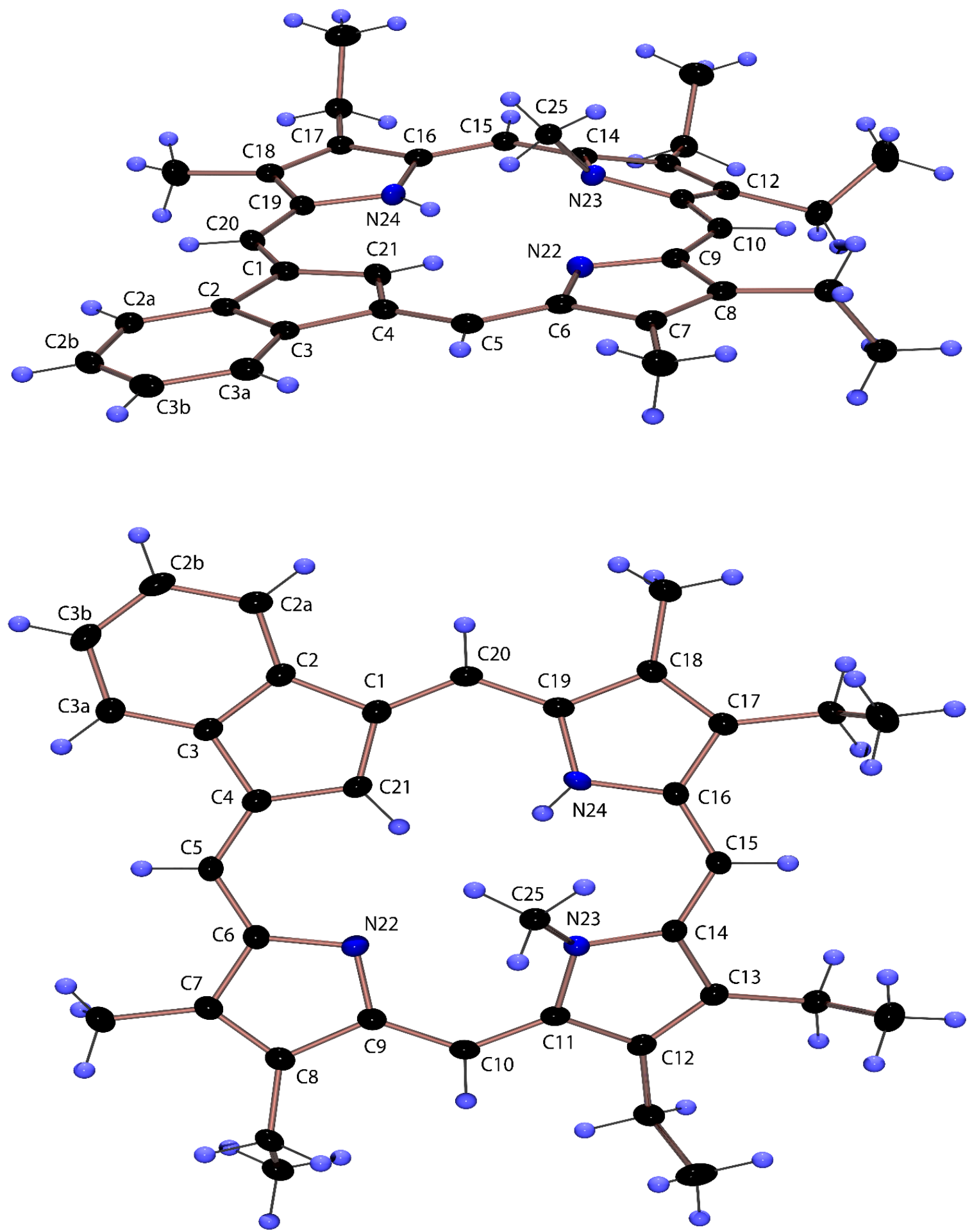

Figure B-4. Color POV-Ray rendered ORTEP III drawings (50\% probability level, hydrogen atoms rendered arbitrarily small for clarity) showing two views of 23-methylcarbaporphyrin 76. 

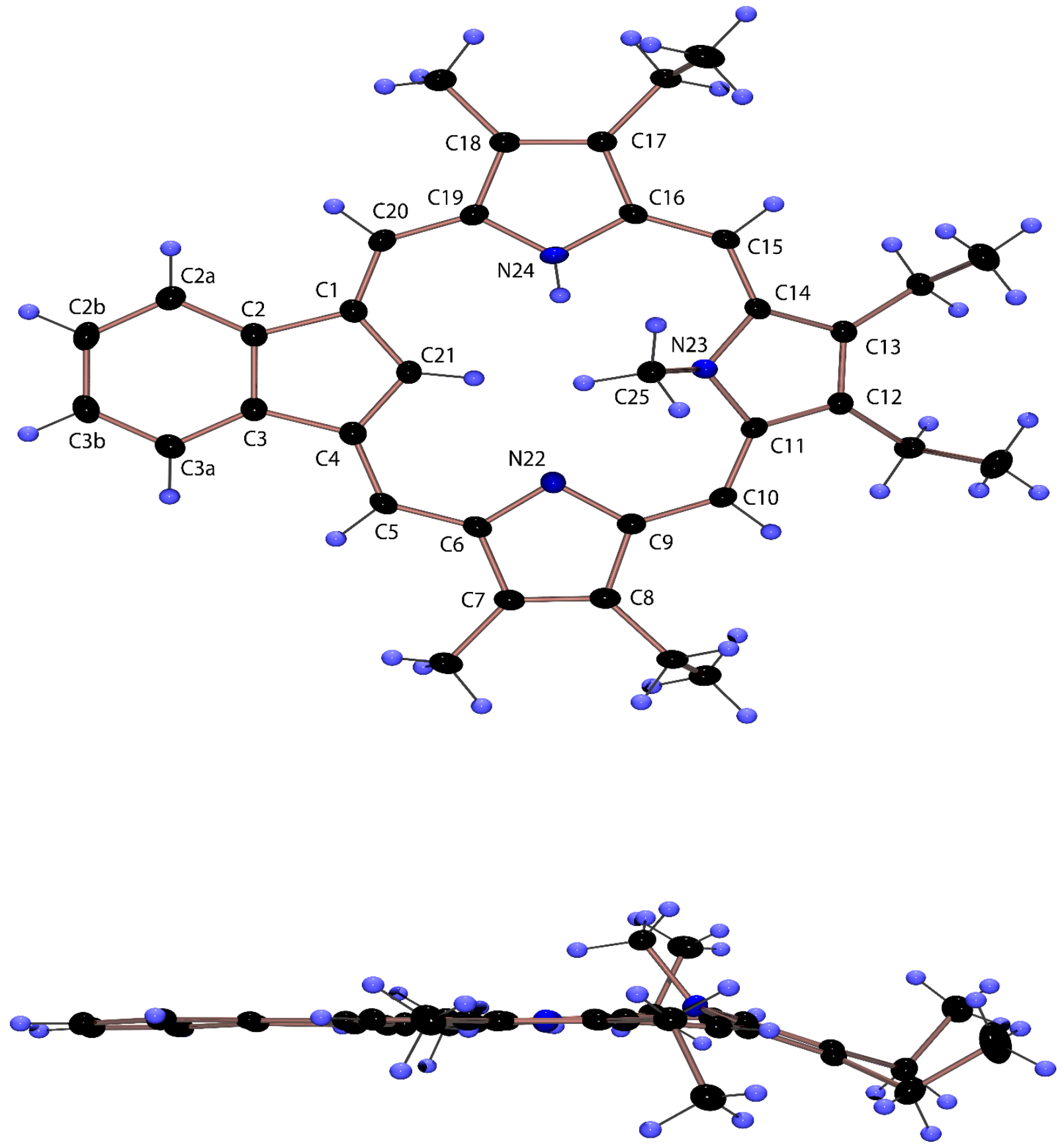

Figure B-5. Color POV-Ray rendered ORTEP III drawings (50\% probability level, hydrogen atoms rendered arbitrarily small for clarity) showing two further views of 23methylcarbaporphyrin $\mathbf{7 6}$. 

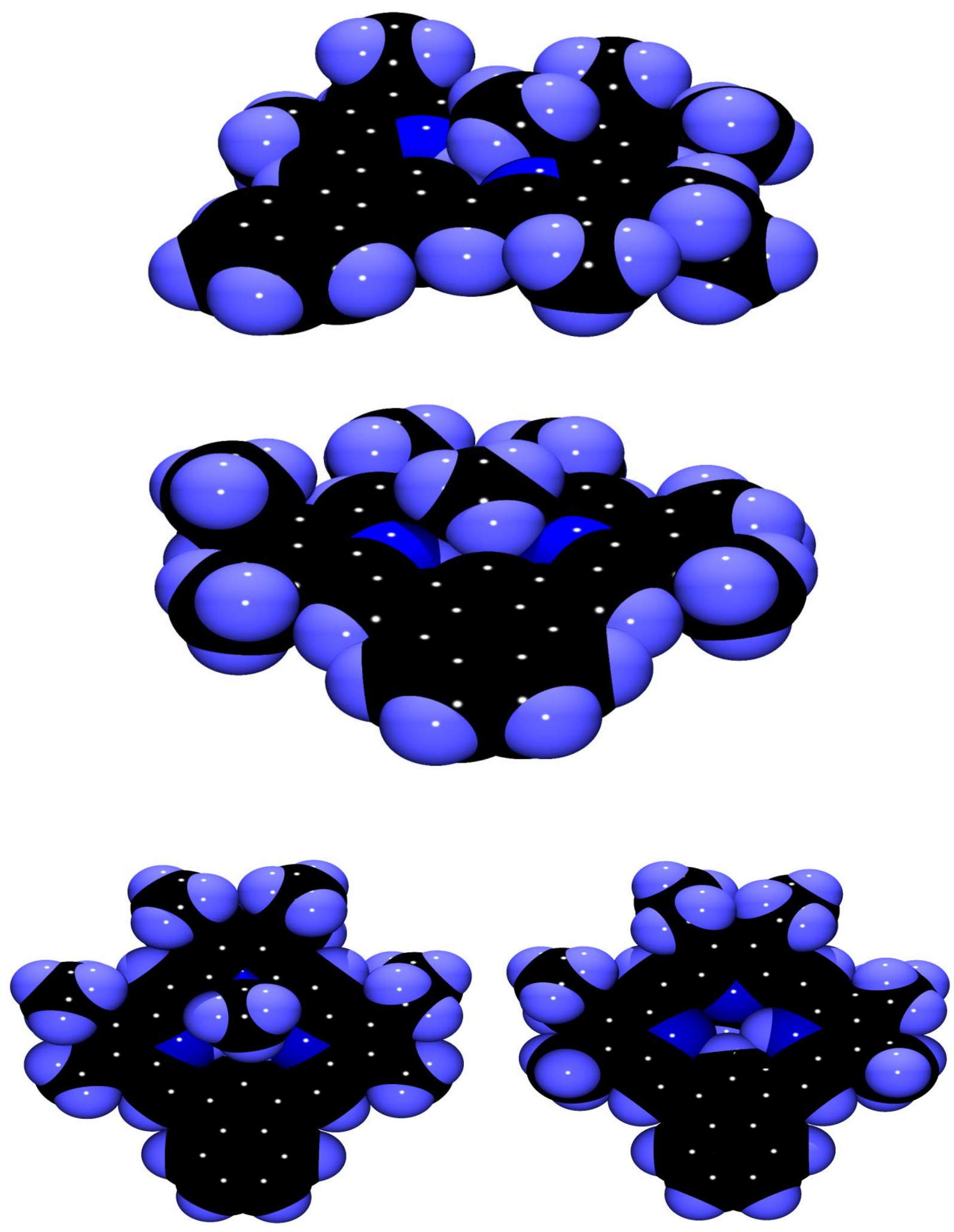

Figure B-6. Color POV-Ray rendered space-filling drawings showing 4 views of compound 23-methylcarbaporphyrin 76. 


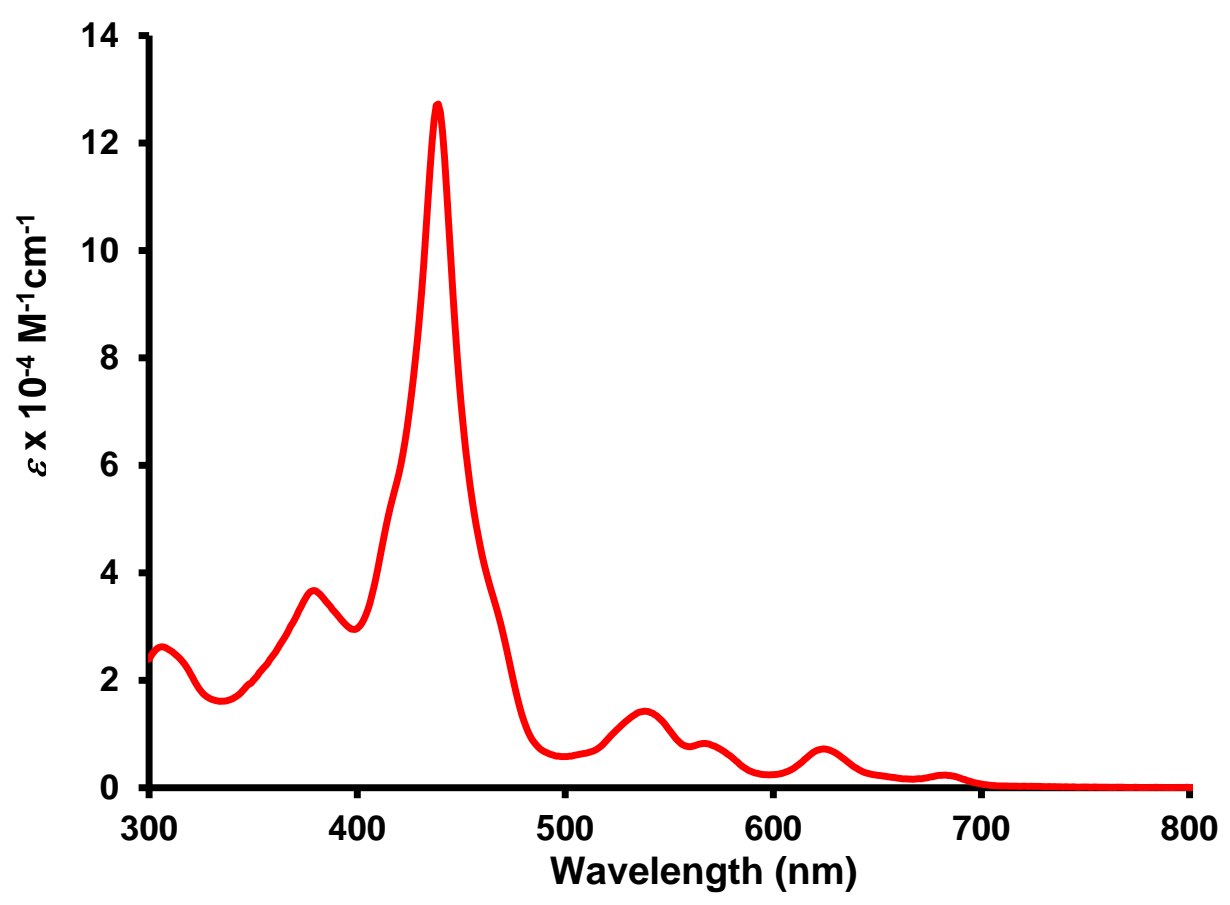

Figure B-7. UV-vis spectrum of 23-methylcarbaporphyrin 76 in 1\% Et3 N-dichloromethane.

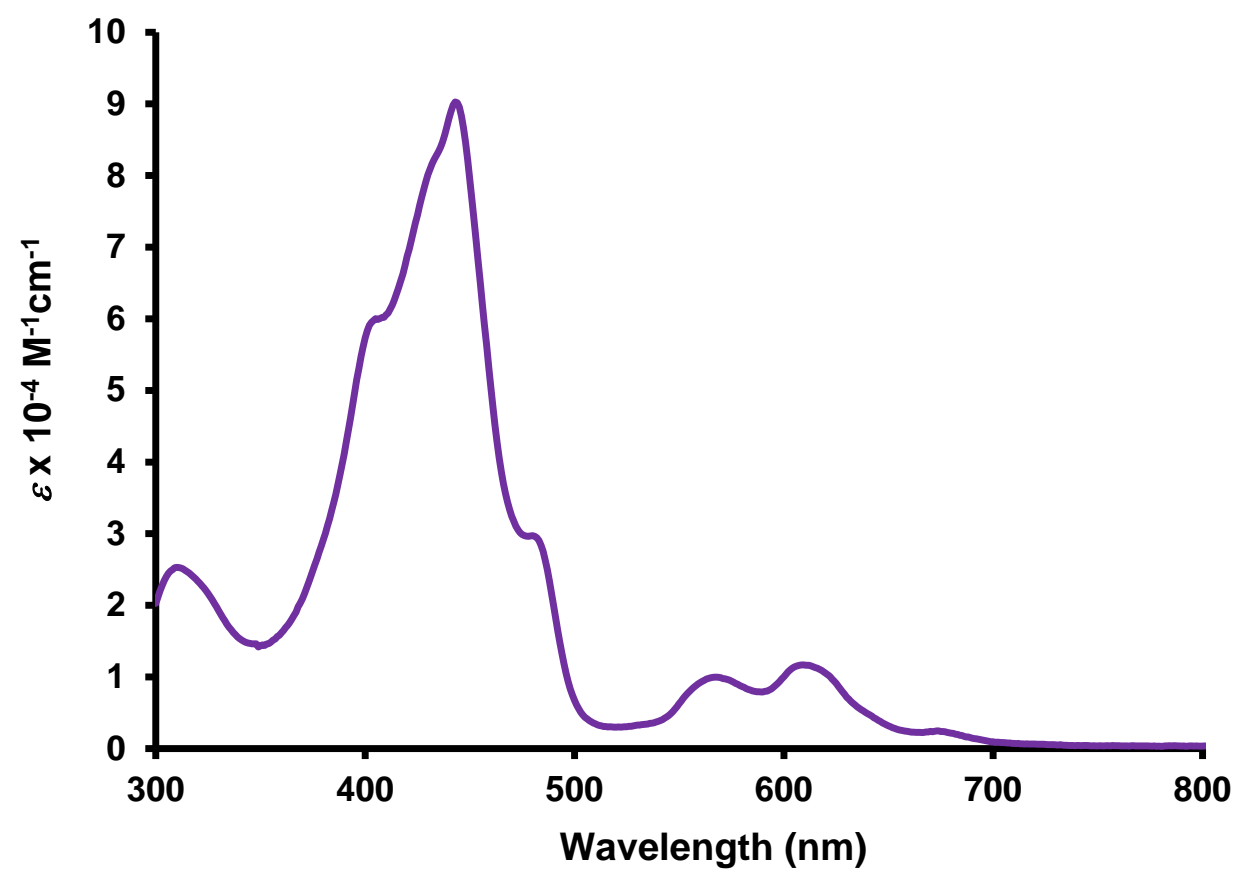

Figure B-8. UV-vis spectrum of monocation $\mathbf{7 6} \mathrm{H}^{+}$in $1 \%$ TFA-dichloromethane. 


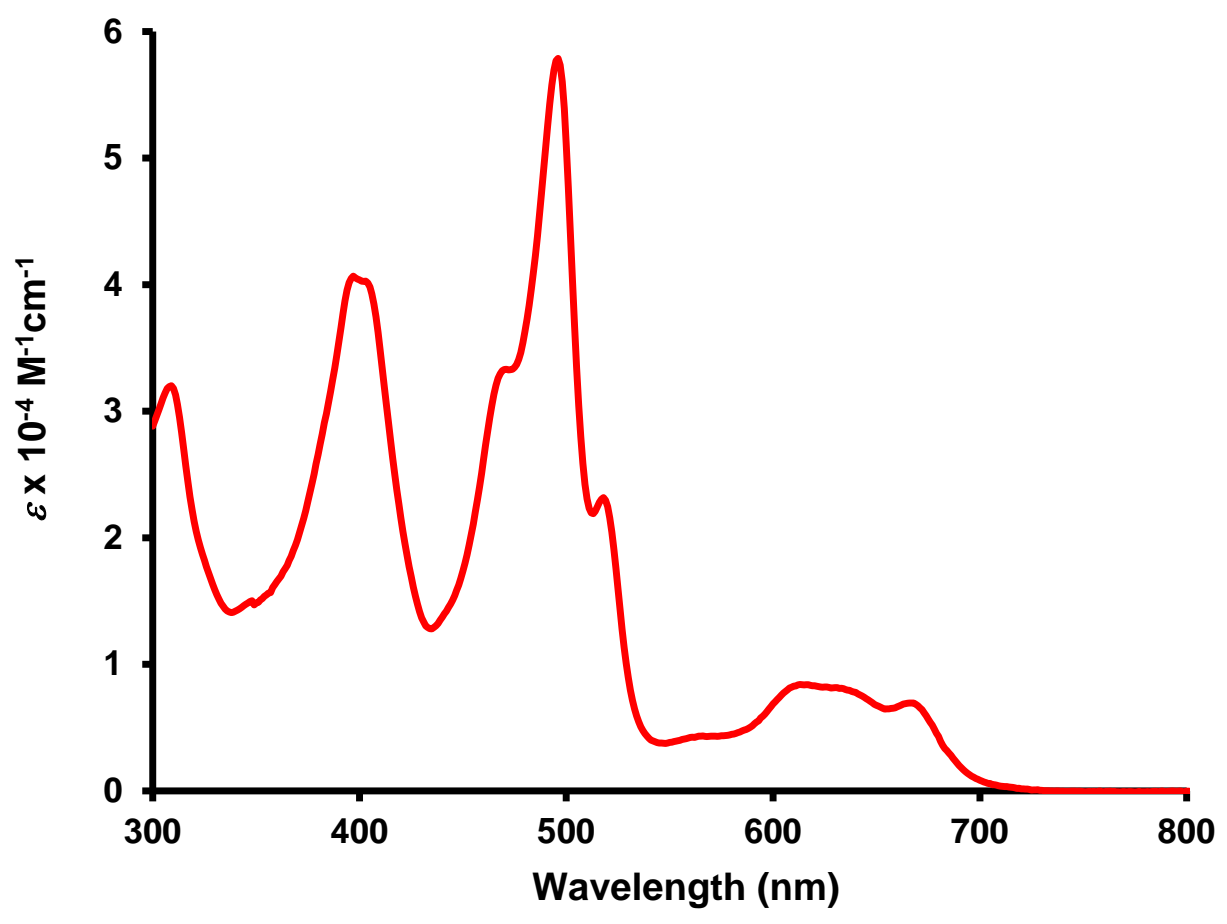

Figure B-9. UV-vis spectrum of palladium(II) complex 84 in dichloromethane. 


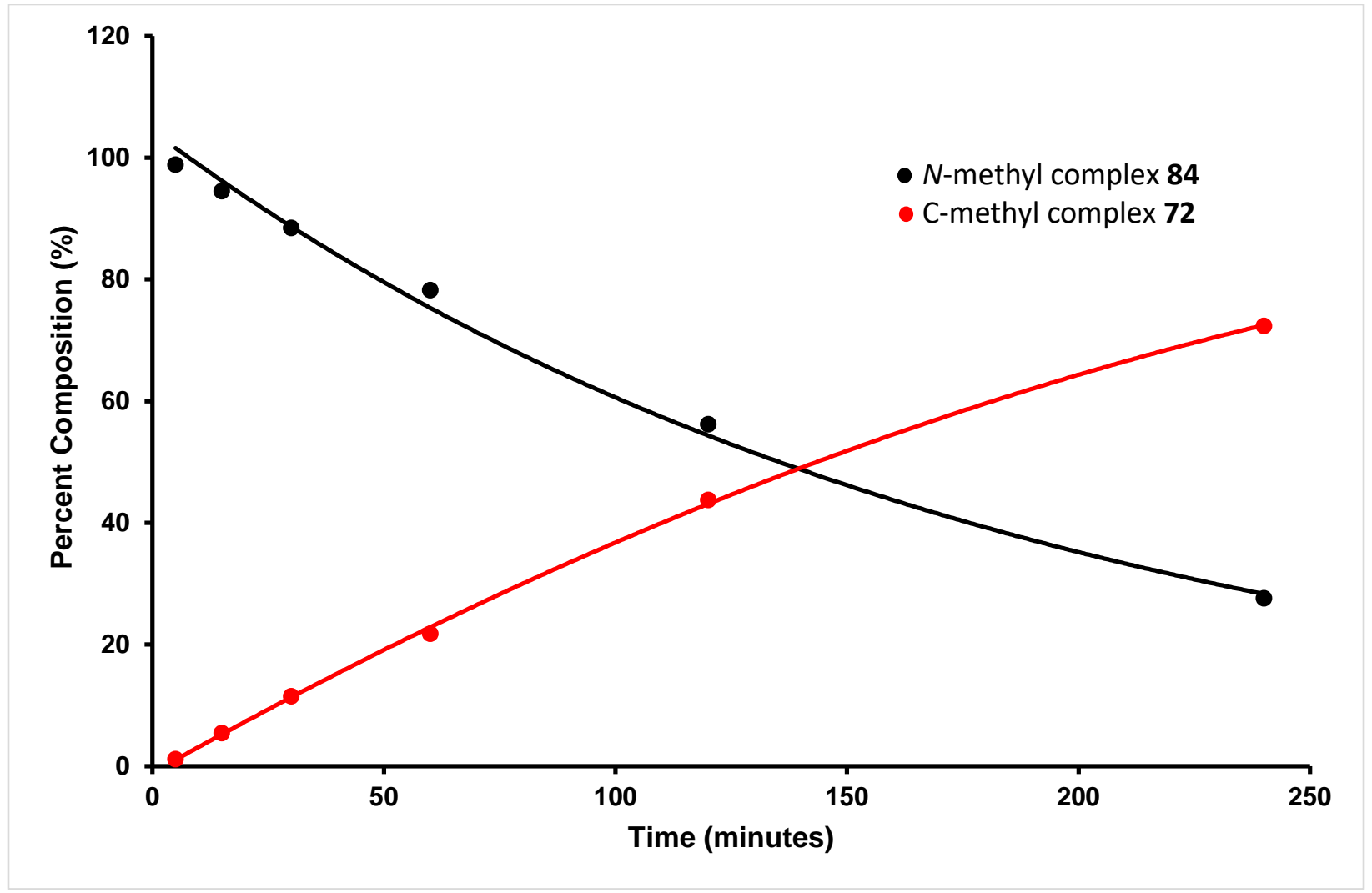

Figure B-10. Reflux reactions time course study for the metalation of $\mathbf{7 6}$ with palladium(II) acetate showing the conversion of $\mathbf{8 4}$ into $\mathbf{7 2 .}$ 


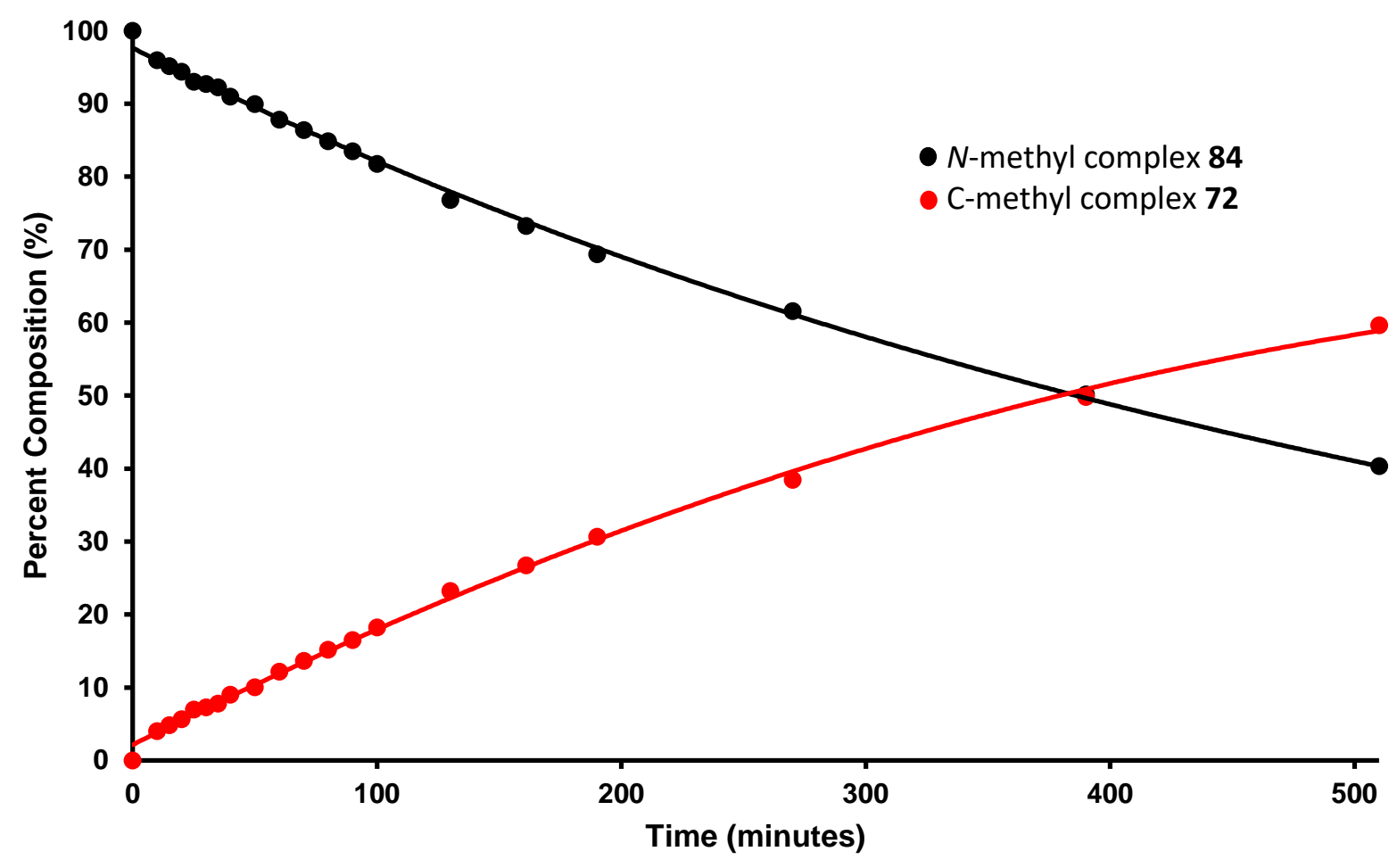

Figure B-11. Proton-NMR time course study for the metalation of $\mathbf{7 6}$ with palladium(II) acetate showing the conversion of $\mathbf{8 4}$ into $\mathbf{7 2 .}$ 


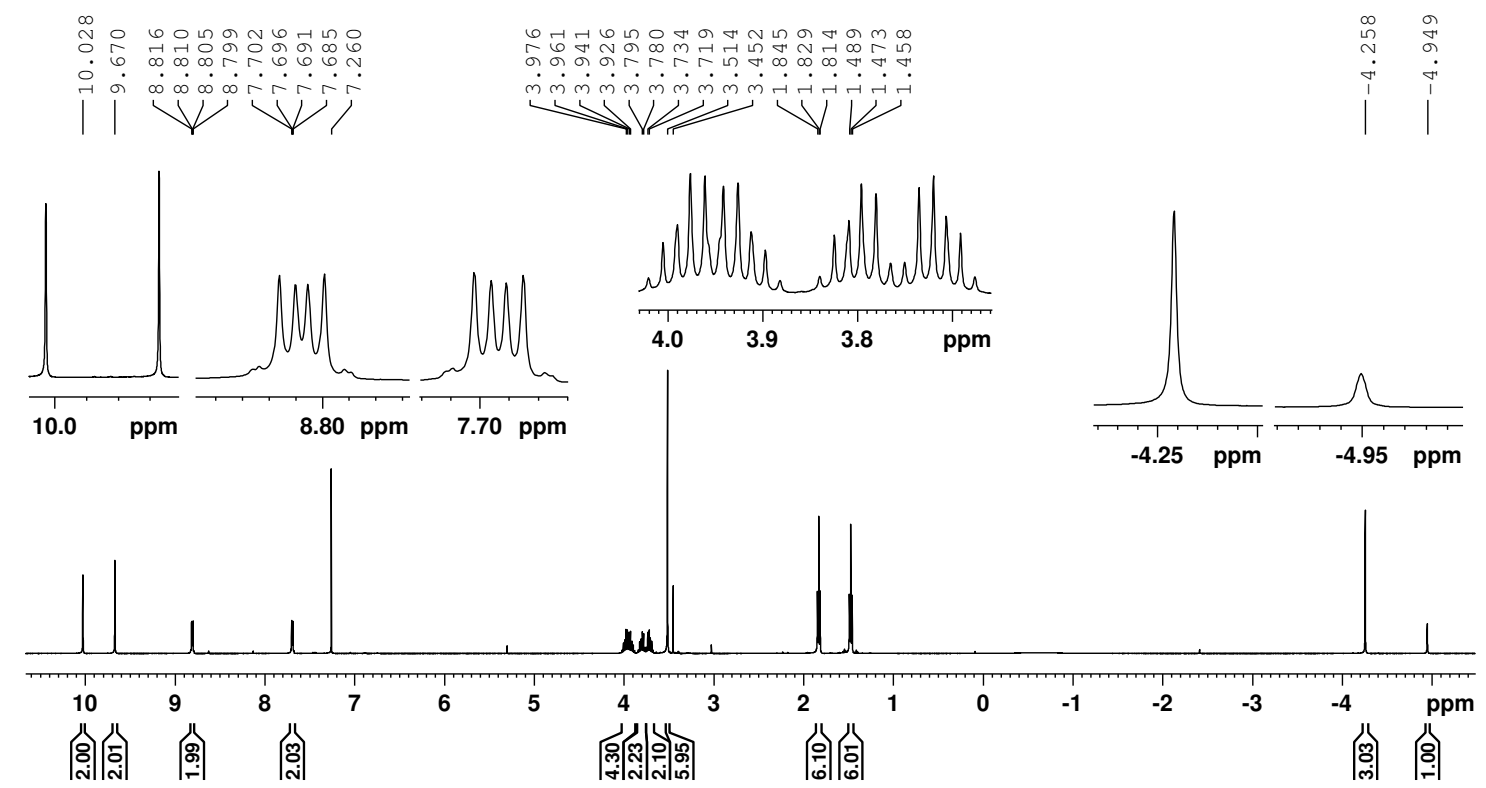

Figure B-12. $500 \mathrm{MHz}{ }^{1} \mathrm{H}$ NMR spectrum of 23-methylbenzocarbaporphyrin 76 in $\mathrm{CDCl}_{3}$. 


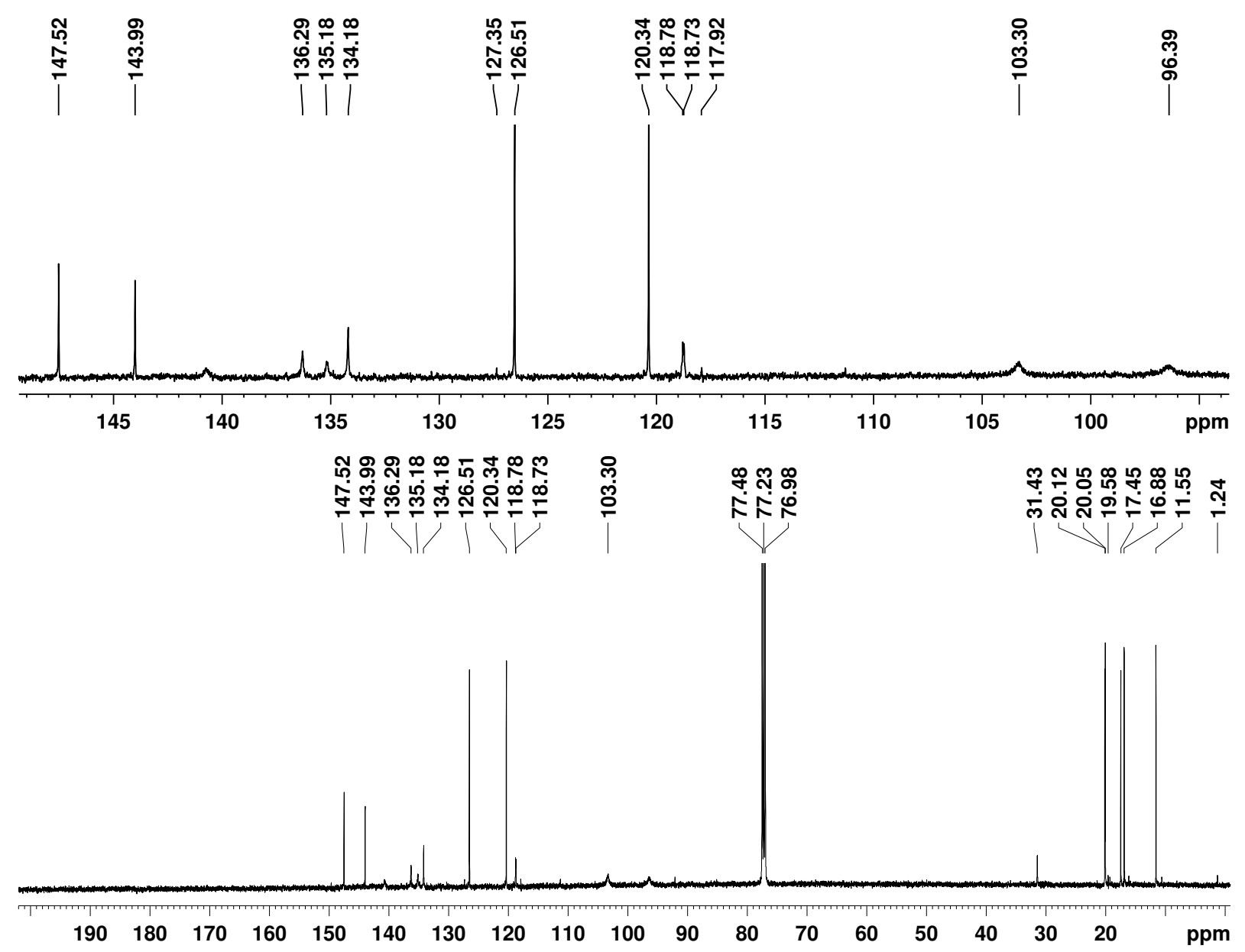

Figure B-13. $125 \mathrm{MHz}{ }^{13} \mathrm{C}$ NMR spectrum of 23-methylcarbaporphyrin 76 in $\mathrm{CDCl}_{3}$. 


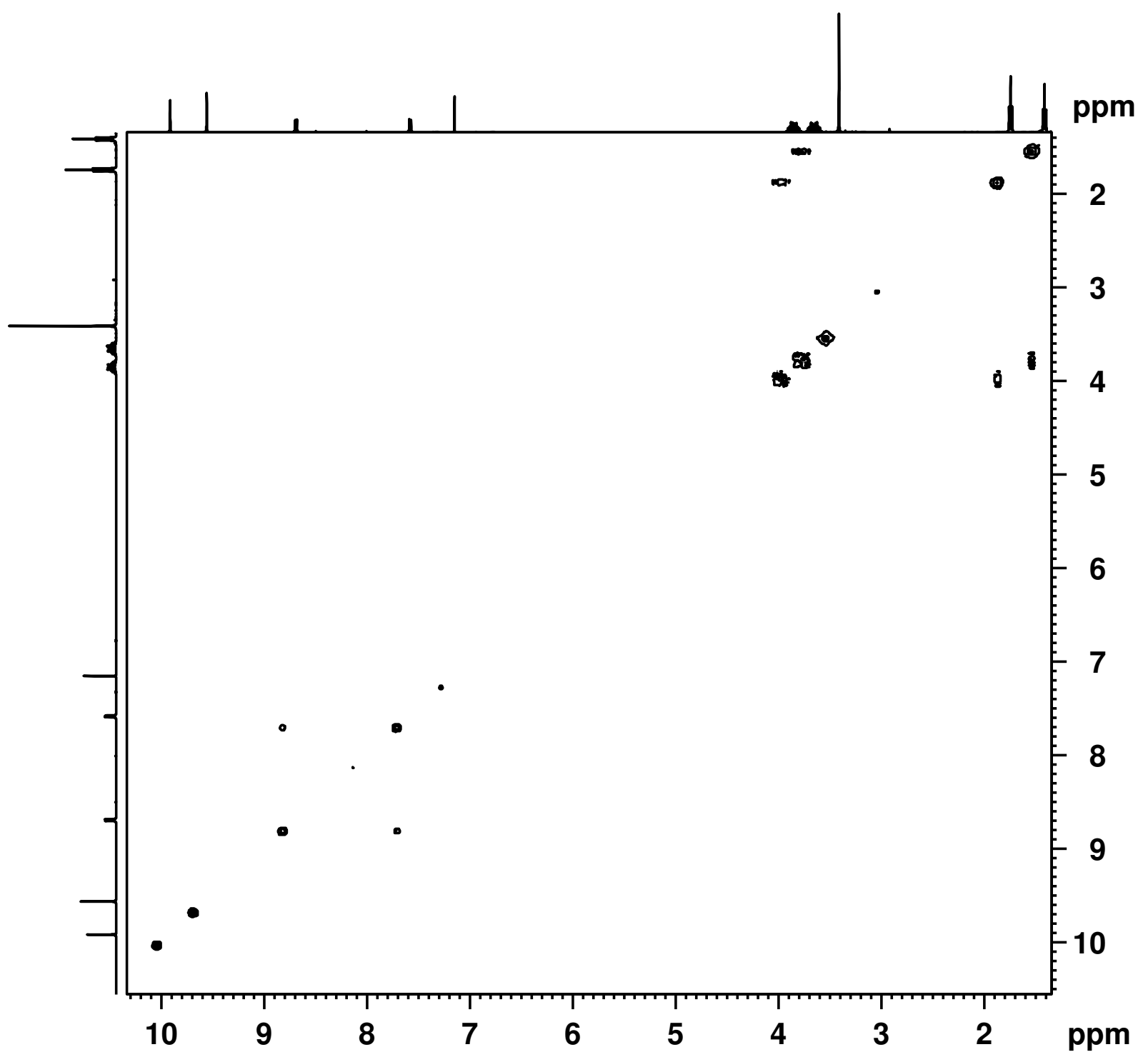

Figure B-14. ${ }^{1} \mathrm{H}-{ }^{1} \mathrm{H}$ COSY NMR spectrum of 23-methylcarbaporphyrin 76 in $\mathrm{CDCl}_{3}$. 


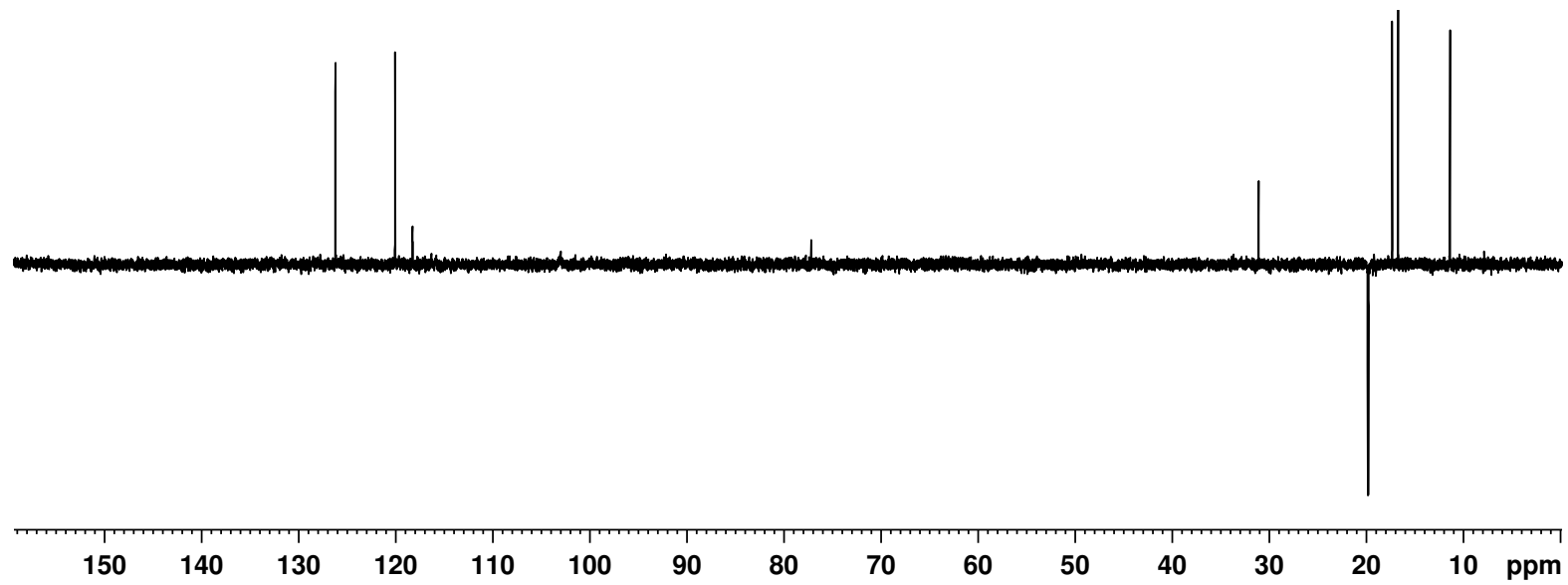

Figure B-15. DEPT-135 NMR spectrum of 23-methylcarbaporphyrin 76 in $\mathrm{CDCl}_{3}$.

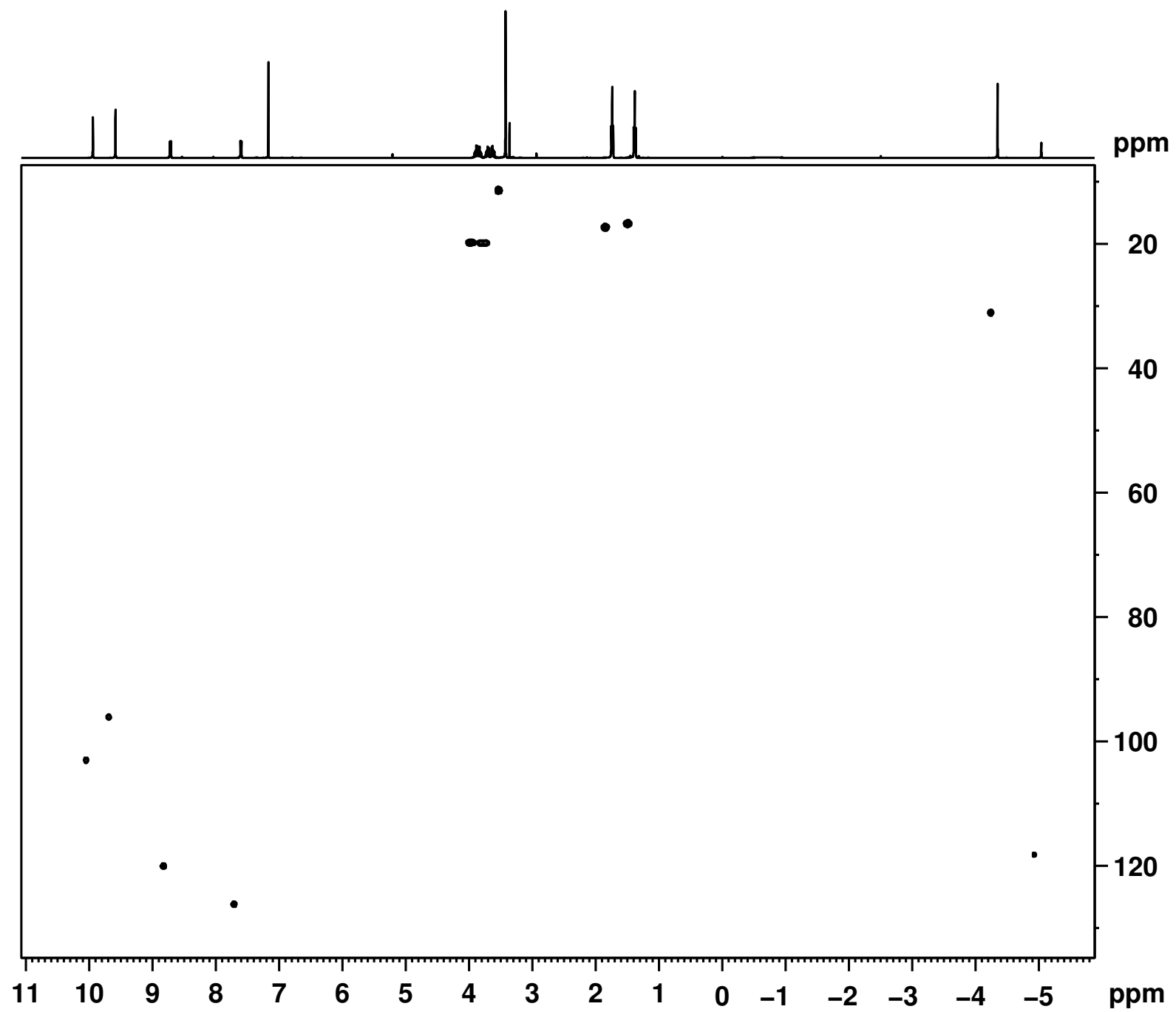

Figure B-16. HSQC NMR spectrum of 23-methylcarbaporphyrin 76 in $\mathrm{CDCl}_{3}$. 


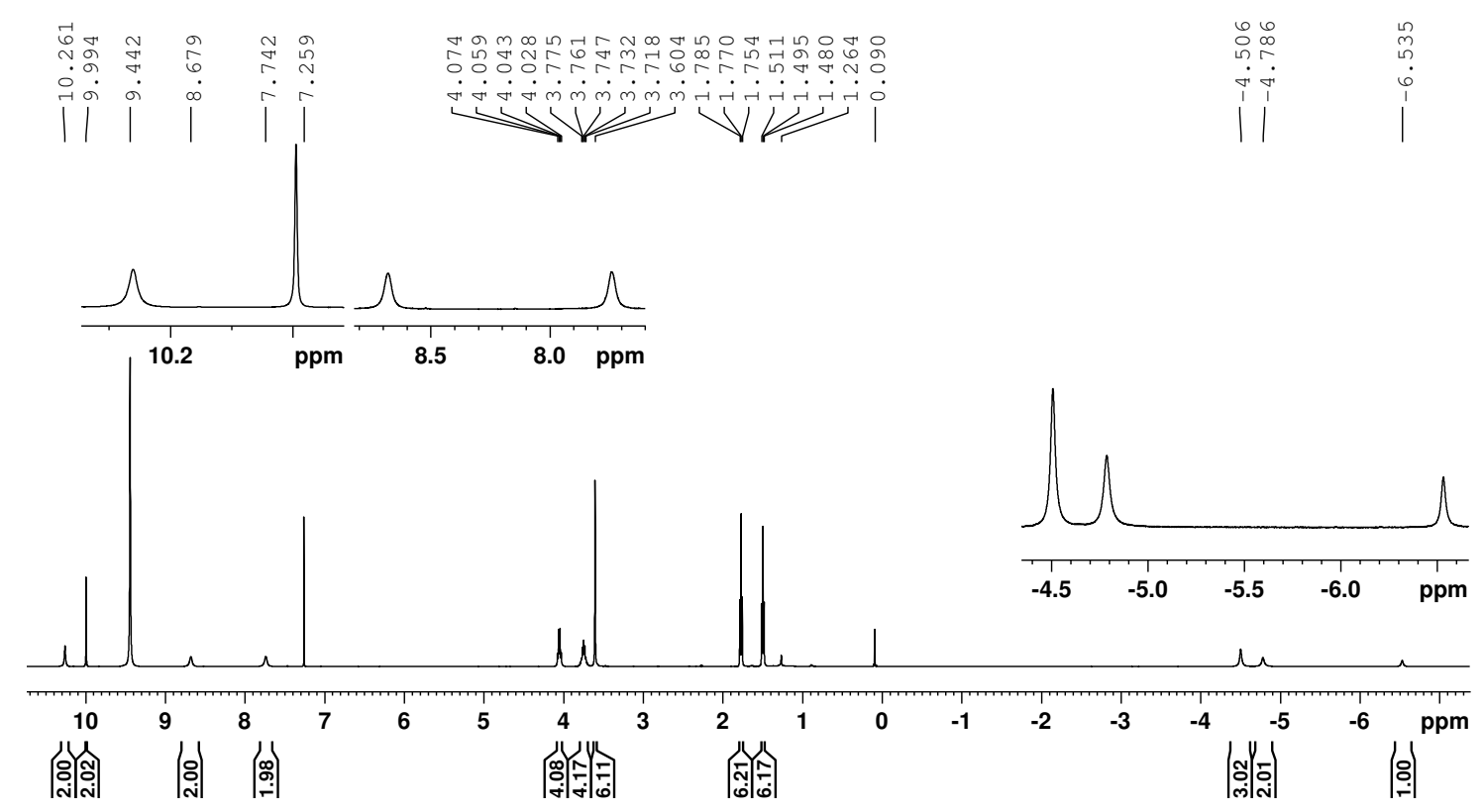

Figure B-17. $500 \mathrm{MHz}{ }^{1} \mathrm{H}$ NMR spectrum of 23-methylbenzocarbaporphyrin monocation $76 \mathrm{H}^{+}$in $\mathrm{TFA}-\mathrm{CDCl}_{3}$. 


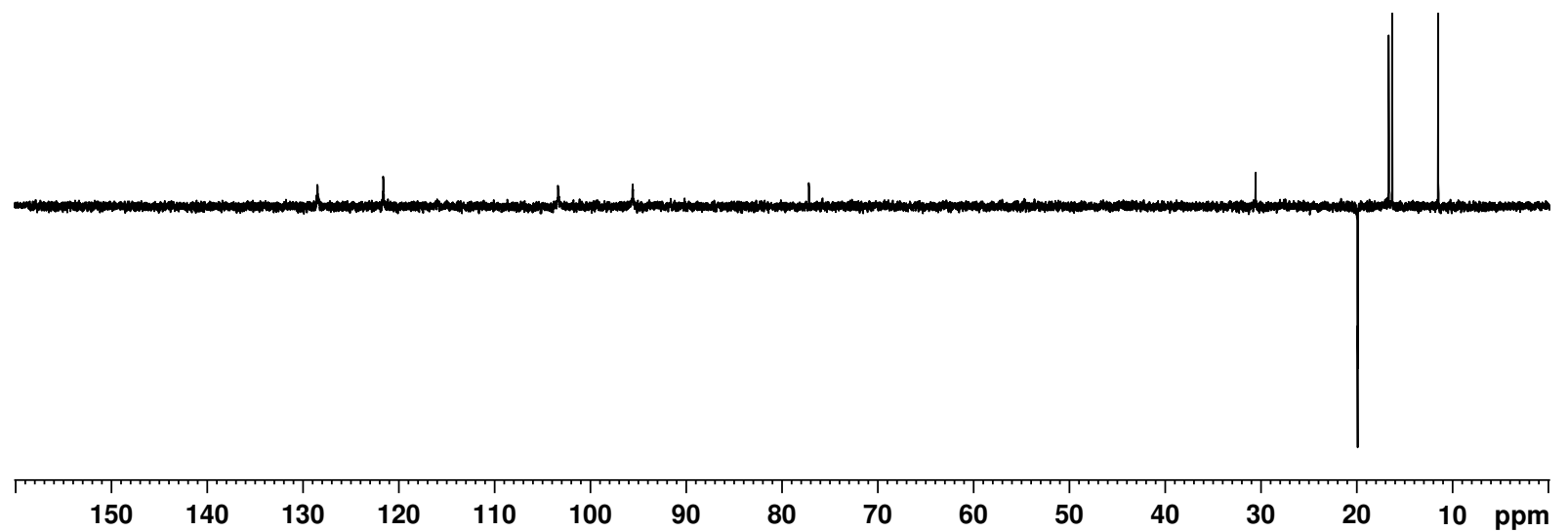

Figure B-18. DEPT-135 NMR spectrum of 23-methylcarbaporphyrin monocation $76 \mathrm{H}^{+}$in TFA-CDCl ${ }_{3}$.

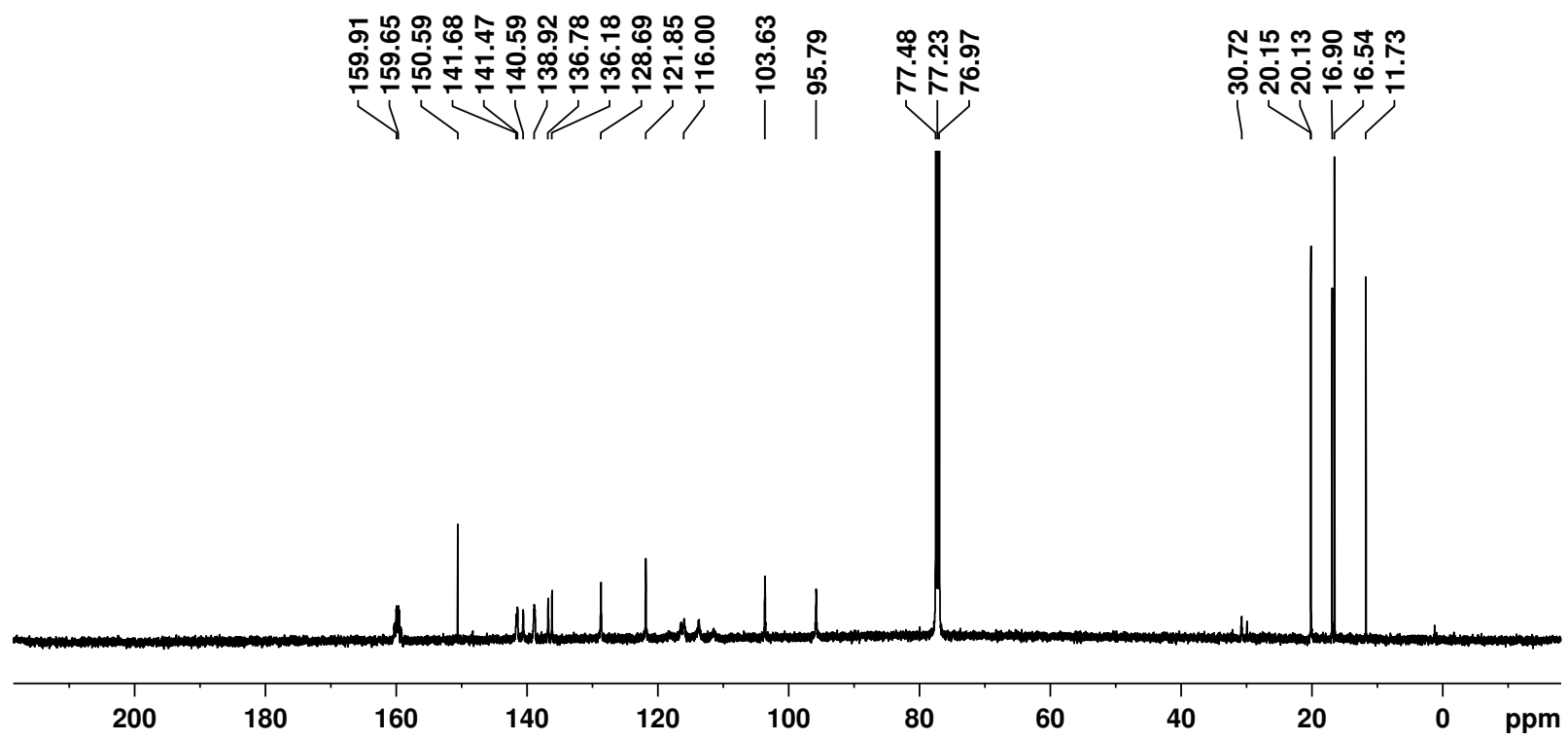

Figure B-19. $125 \mathrm{MHz}{ }^{13} \mathrm{C}$ NMR spectrum of 23-methylcarbaporphyrin monocation $76 \mathrm{H}^{+}$in TFA-CDCl ${ }_{3}$. 


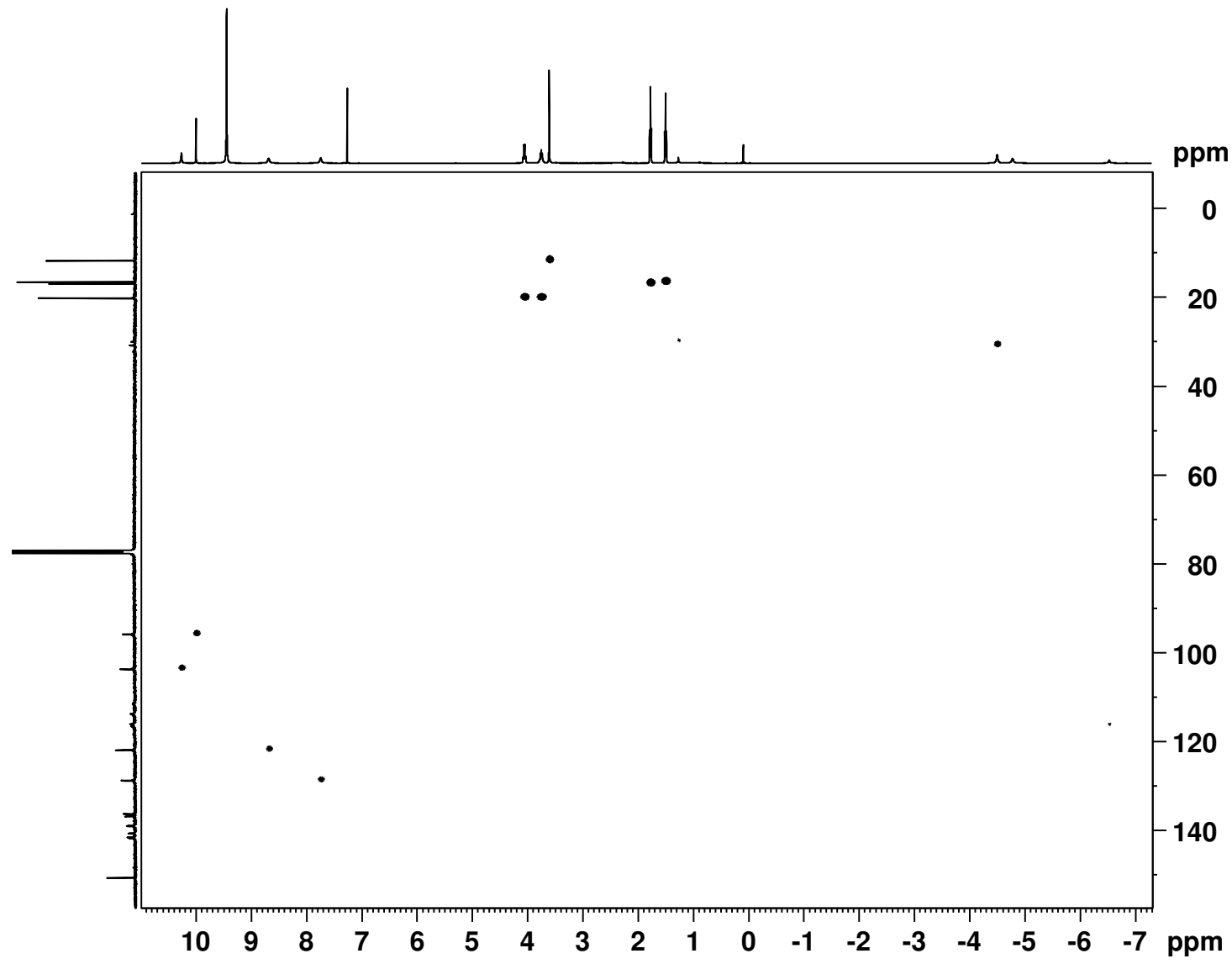

Figure B-20. HSQC NMR spectrum of 23-methylbenzocarbaporphyrin monocation $76 \mathrm{H}^{+}$in TFA-CDCl ${ }_{3}$. 


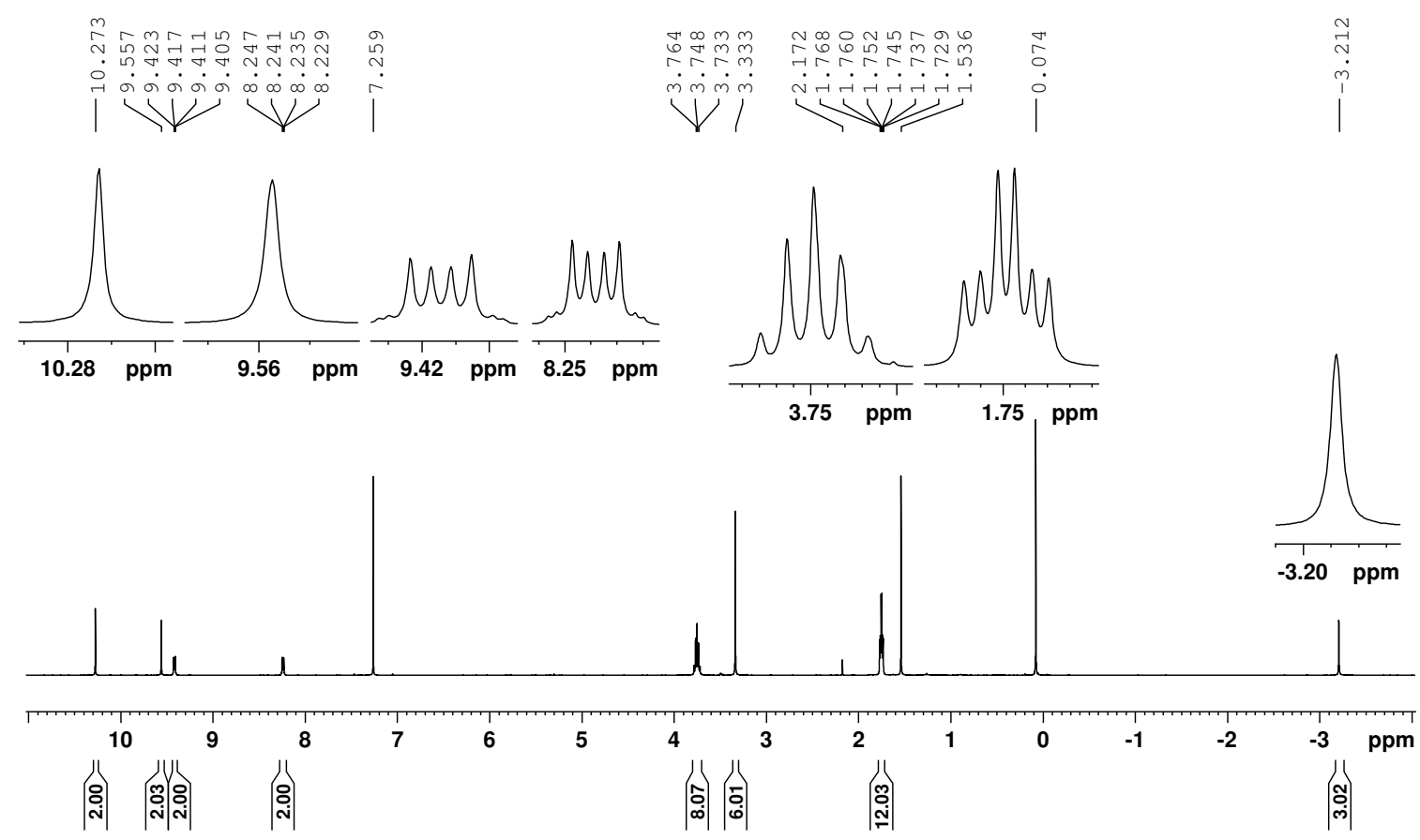

Figure B-21. $500 \mathrm{MHz}{ }^{1} \mathrm{H}$ NMR spectrum of palladium(II) 21-methylcarbaporphyrin 72 in $\mathrm{CDCl}_{3}$. 

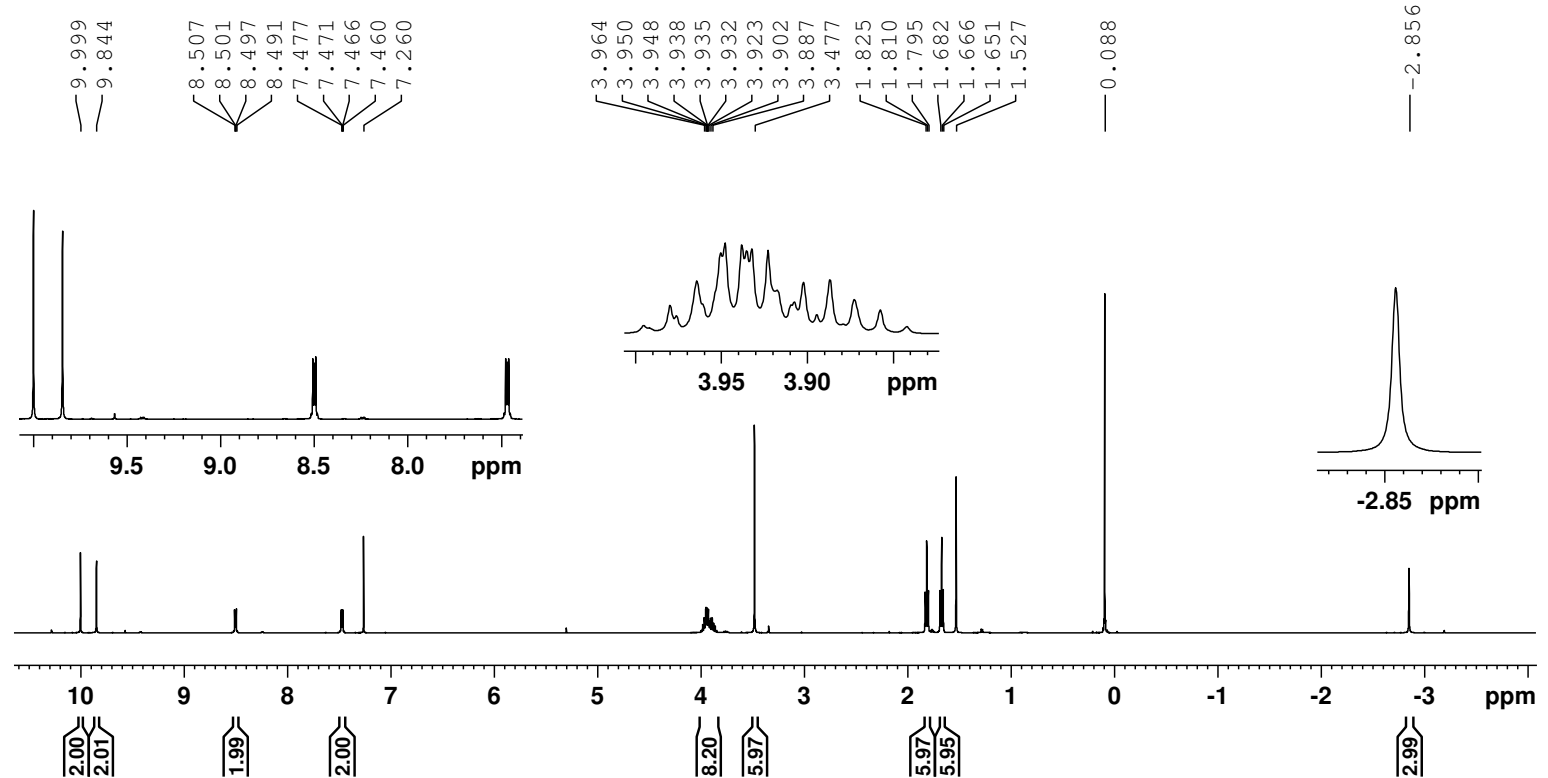

Figure B-22. $500 \mathrm{MHz}{ }^{1} \mathrm{H}$ NMR spectrum of palladium(II) 23-methylcarbaporphyrin 84 in $\mathrm{CDCl}_{3}$. 


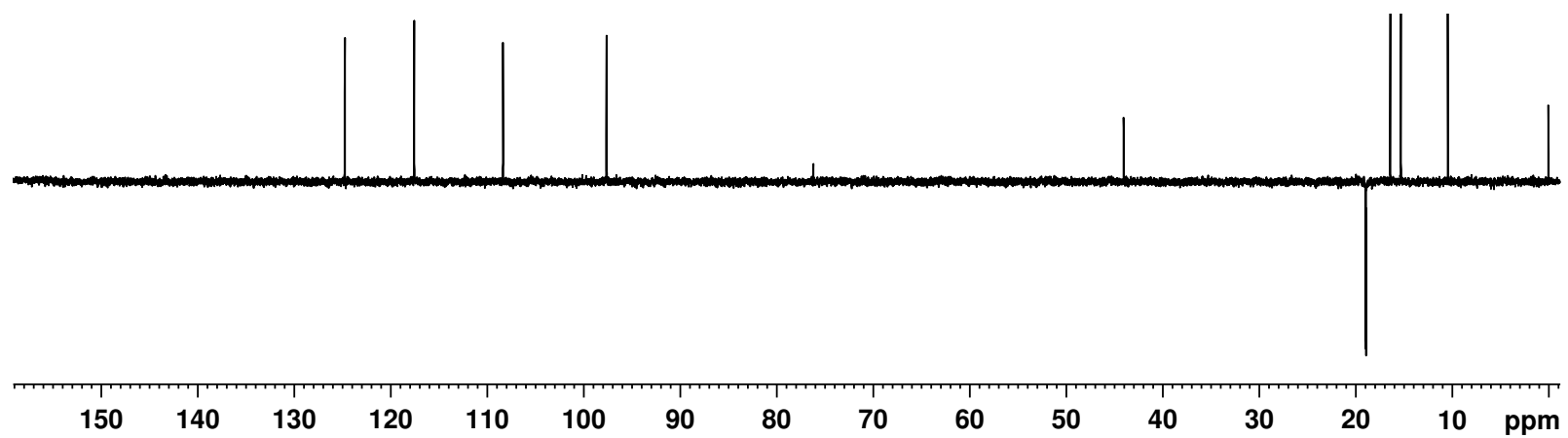

Figure B-23. DEPT-135 NMR spectrum of palladium(II) 23-methylcarbaporphyrin 84 in $\mathrm{CDCl}_{3}$.

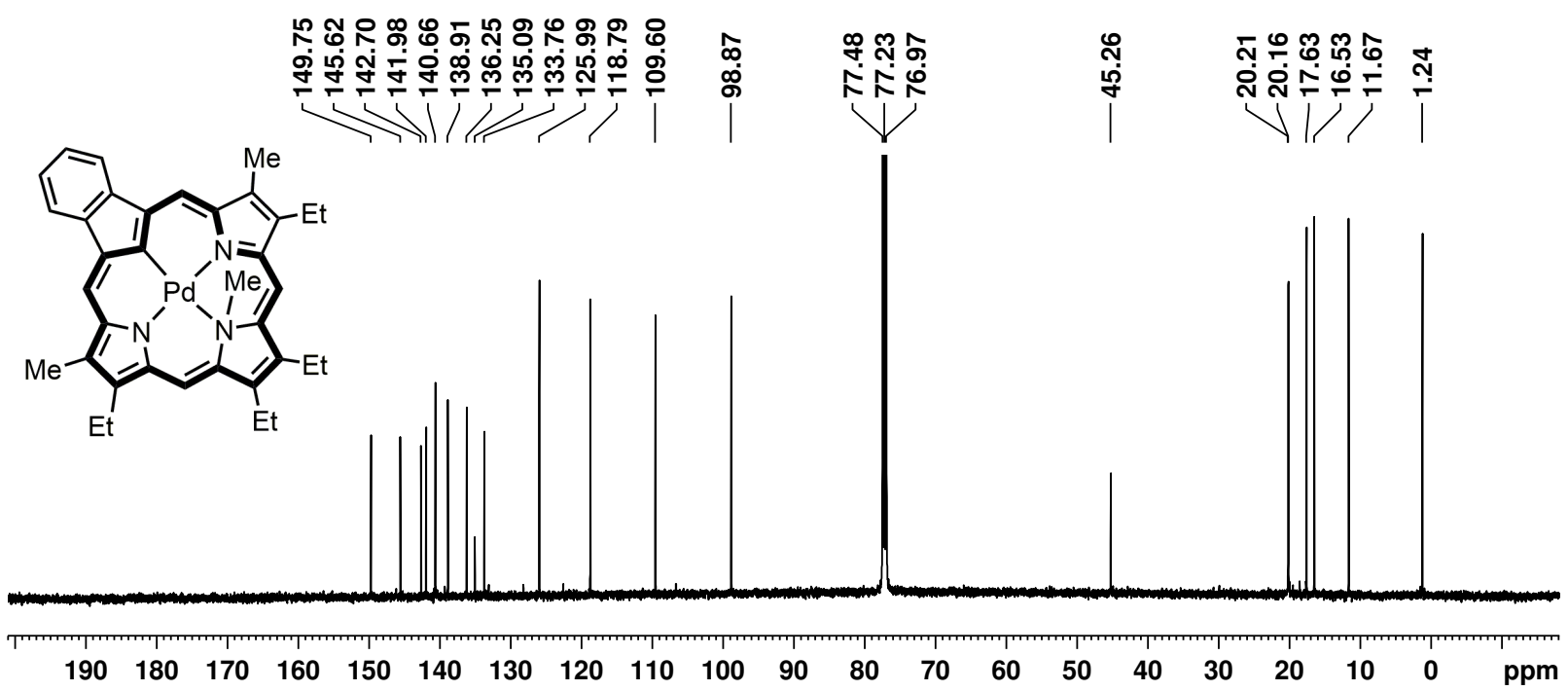

Figure B-24. $125 \mathrm{MHz}{ }^{13} \mathrm{C}$ NMR spectrum of palladium(II) 23-methylcarbaporphyrin 84 in $\mathrm{CDCl}_{3}$. 


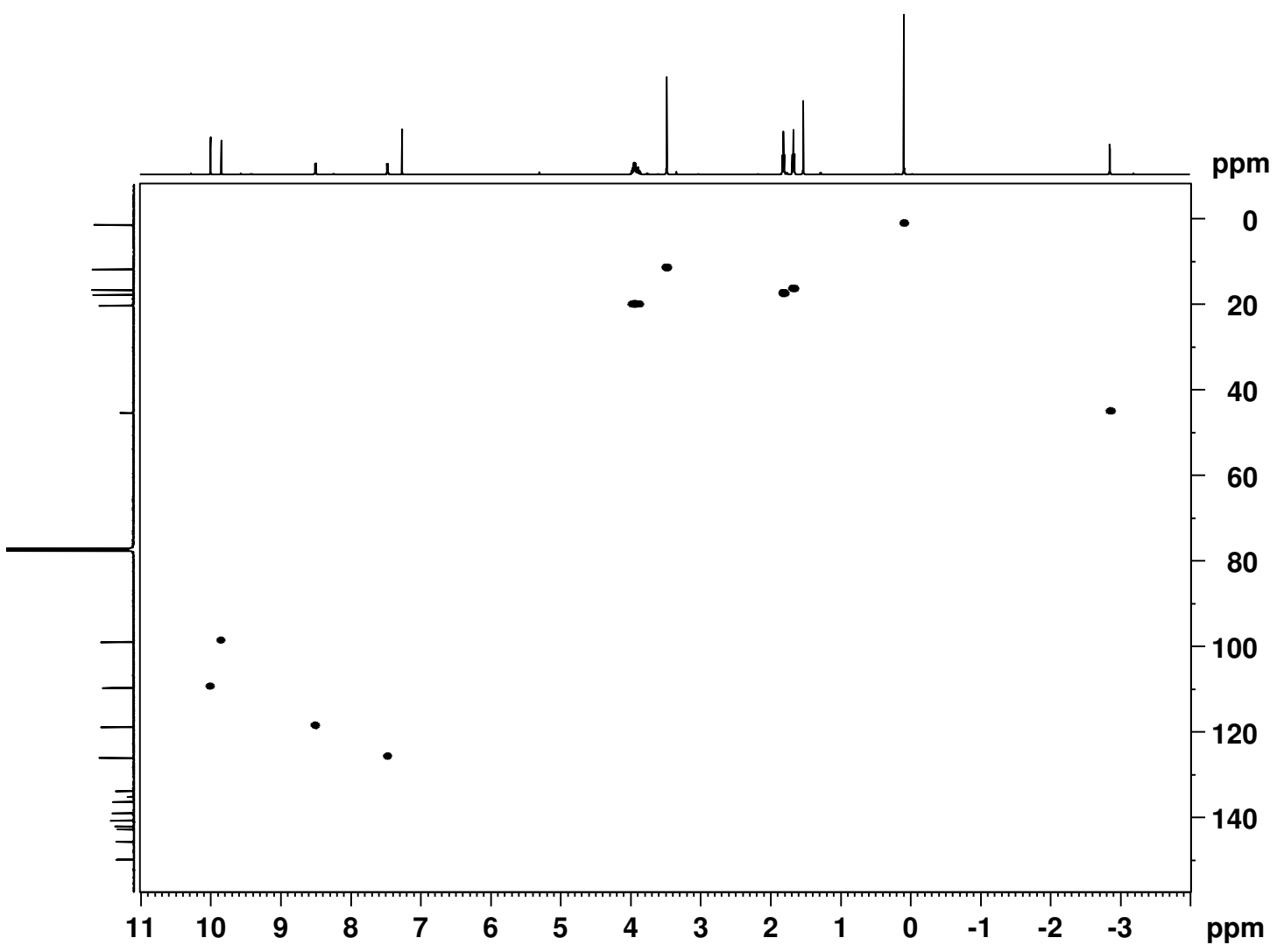

Figure B-25. HSQC NMR spectrum of palladium(II) 23-methylcarbaporphyrin 84 in $\mathrm{CDCl}_{3}$. 

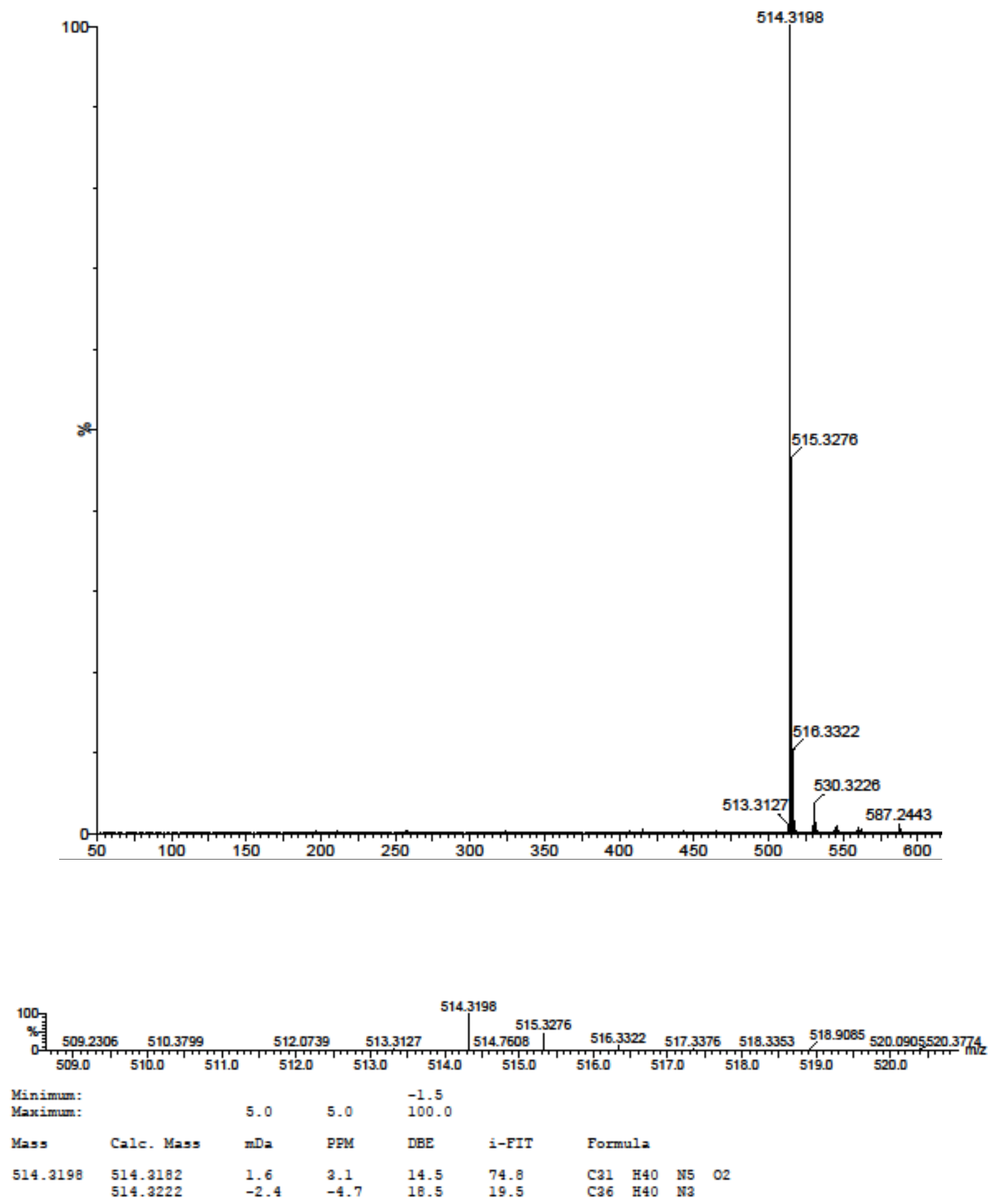

Figure B-26. ESI MS of $N$-methylcarbaporphyrin 76. 

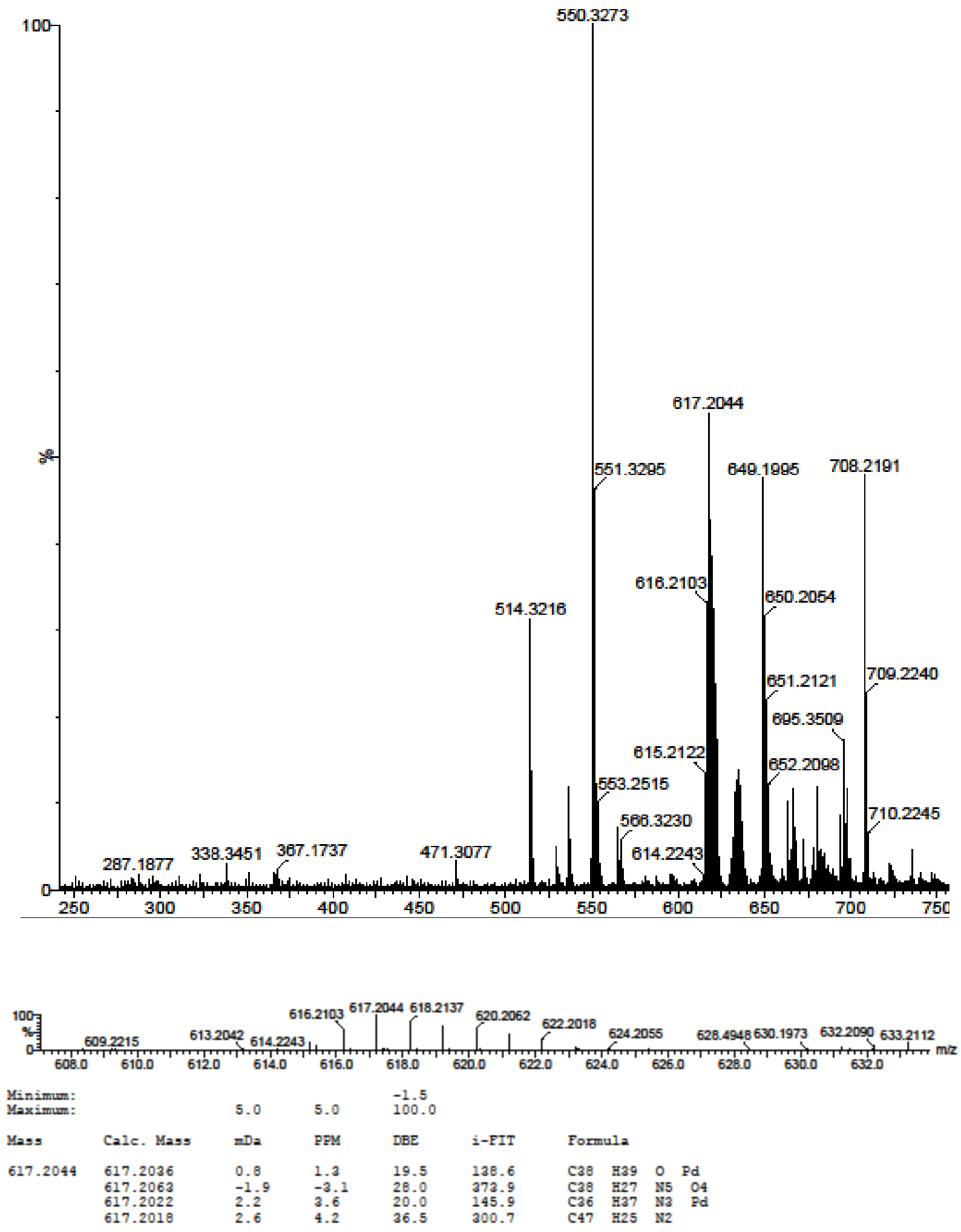

Figure B-27. ESI MS of palladium(II) complex 84. 


\section{APPENDIX C: SPECTROSCOPIC DATA FOR RHODIUM COMPLEXES}

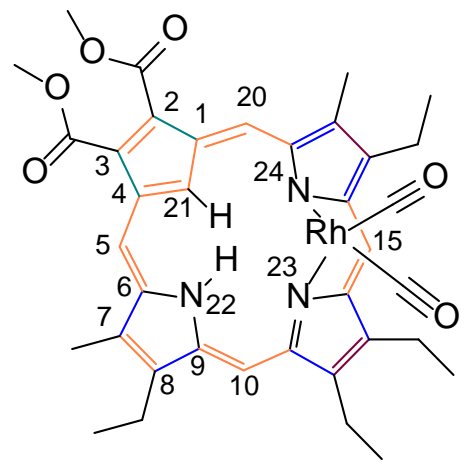

89

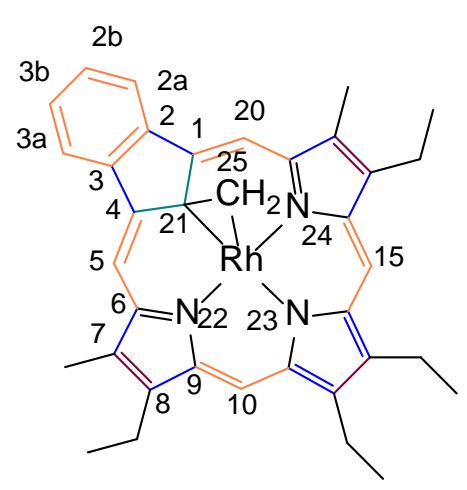

95

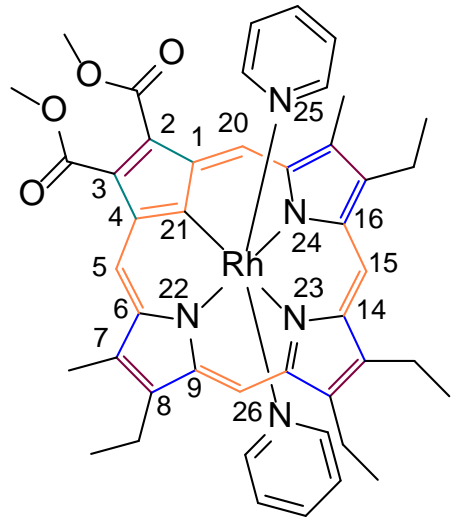

90

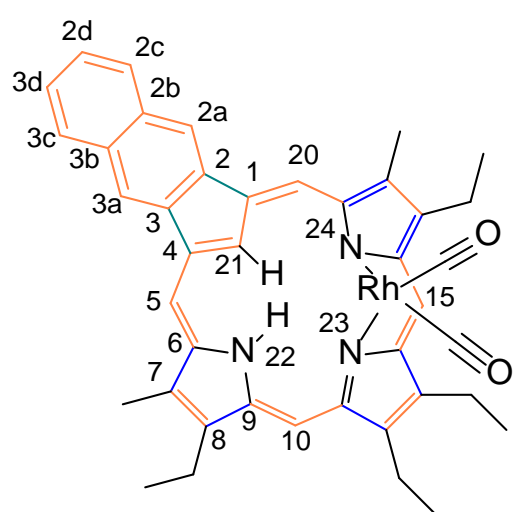

91

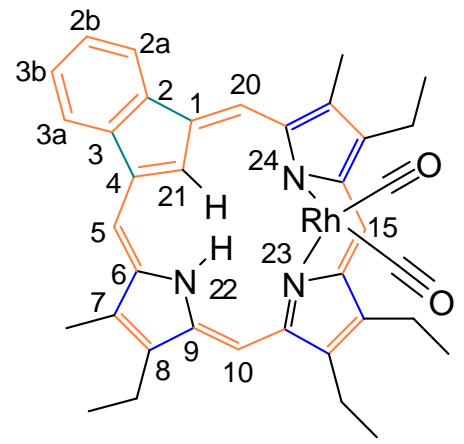

86

Table C-1. Summary of framework bond distances $(\AA)$ and angles $\left(^{\circ}\right)$ for $\mathbf{8 6},{ }^{30} \mathbf{8 9}, \mathbf{9 0}, \mathbf{9 1}$ and 95.

\begin{tabular}{|l|c|c|c|c|c|}
\hline & $\begin{array}{c}\mathbf{8 9 \bullet 0 . 5} \\
\mathbf{C}_{\mathbf{5}} \mathbf{H}_{\mathbf{1 2}}\end{array}$ & $\mathbf{9 0 \bullet C H C l} \mathbf{3}$ & $\mathbf{9 1}$ & $\mathbf{9 5}$ & $\mathbf{8 6}^{30}$ \\
\hline C25-O25 & $1.133(3)$ & -- & $1.1372(19)$ & -- & $1.1356(14)$ \\
\hline C26-O26 & $1.138(3)$ & -- & $1.134(2)$ & -- & $1.1370(14)$ \\
\hline Rh-C25(orN25) & $1.866(2)$ & $2.0641(18)$ & $1.8529(16)$ & $1.9932(11)$ & $1.8636(12)$ \\
\hline Rh-C26(orN26) & $1.856(2)$ & $2.0720(18)$ & $1.8677(16)$ & -- & $1.8536(11)$ \\
\hline Rh-C21 & -- & $1.986(2)$ & -- & $2.1329(11)$ & -- \\
\hline Rh-N22 & -- & $2.0454(18)$ & -- & $2.0369(9)$ & -- \\
\hline Rh-N23 & $2.0895(18)$ & $2.1046(19)$ & $2.0894(12)$ & $2.0431(9)$ & $2.0920(9)$ \\
\hline Rh-N24 & $2.0806(18)$ & $2.0485(18)$ & $2.0836(12)$ & $2.0410(9)$ & $2.0847(9)$ \\
\hline C1-C21 & $1.407(3)$ & $1.416(3)$ & $1.4130(19)$ & $1.4632(15)$ & $1.4098(14)$ \\
\hline C1-C2 & $1.462(3)$ & $1.464(3)$ & $1.4751(19)$ & $1.4468(15)$ & $1.4744(13)$ \\
\hline C2-C3 & $1.377(3)$ & $1.362(3)$ & $1.4385(19)$ & $1.4178(16)$ & $1.4141(14)$ \\
\hline C2-C2a & -- & -- & $1.370(2)$ & -- & $1.3901(14)$ \\
\hline C2a-C2b & -- & -- & $1.425(2)$ & -- & $1.3997(14)$ \\
\hline C2b-C3b & -- & -- & $1.428(2)$ & -- & $1.3909(16)$ \\
\hline C3b-C3a & -- & -- & $1.422(2)$ & -- & $1.3986(16)$ \\
\hline
\end{tabular}




\begin{tabular}{|c|c|c|c|c|c|}
\hline C3a-C3 & -- & -- & $1.3686(19)$ & -- & $1.3900(14)$ \\
\hline C3-C4 & $1.471(3)$ & $1.469(3)$ & $1.4760(19)$ & $1.4470(15)$ & 1.4762(14) \\
\hline C4-C21 & $1.401(3)$ & $1.413(3)$ & 1.3995(19) & 1.4624(15) & 1.4054(14) \\
\hline C4-C5 & $1.410(3)$ & $1.397(3)$ & $1.4076(19)$ & $1.3779(15)$ & 1.4038(15) \\
\hline C5-C6 & $1.384(3)$ & $1.391(3)$ & $1.3916(19)$ & $1.4085(15)$ & 1.3899(15) \\
\hline C6-N22 & $1.380(3)$ & $1.387(3)$ & $1.3785(17)$ & $1.3777(14)$ & $1.3742(13)$ \\
\hline C6-C7 & $1.440(3)$ & $1.443(3)$ & $1.445(2)$ & 1.4496(15) & $1.4372(14)$ \\
\hline C7-C8 & $1.378(3)$ & $1.364(3)$ & $1.377(2)$ & $1.3644(16)$ & 1.3812(14) \\
\hline С8-C9 & $1.445(3)$ & $1.452(3)$ & $1.444(2)$ & $1.4540(15)$ & $1.4383(14)$ \\
\hline C9-N22 & $1.359(3)$ & $1.376(3)$ & $1.3695(18)$ & $1.3802(13)$ & $1.3668(13)$ \\
\hline C9-C10 & 1.391(3) & 1.404(3) & $1.389(2)$ & 1.3864(15) & 1.3914(14) \\
\hline C10-C11 & $1.401(3)$ & $1.382(3)$ & $1.4081(19)$ & 1.3983(15) & $1.4028(14)$ \\
\hline C11-N23 & $1.386(3)$ & $1.368(3)$ & $1.3811(18)$ & $1.3718(13)$ & $1.3814(13)$ \\
\hline C11-C12 & $1.455(3)$ & $1.459(3)$ & $1.4447(19)$ & $1.4510(15)$ & $1.4480(14)$ \\
\hline C12-C13 & $1.362(3)$ & $1.362(3)$ & $1.372(2)$ & $1.3702(15)$ & 1.3698(14) \\
\hline C13-C14 & $1.443(3)$ & $1.458(3)$ & $1.4414(19)$ & $1.4485(15)$ & 1.4427(14) \\
\hline C14-N23 & $1.386(3)$ & $1.367(3)$ & $1.3859(18)$ & $1.3665(14)$ & $1.3859(12)$ \\
\hline C14-C15 & $1.387(3)$ & $1.393(3)$ & $1.395(2)$ & $1.3949(15)$ & $1.3931(14)$ \\
\hline C15-C16 & $1.397(3)$ & $1.402(3)$ & $1.395(2)$ & 1.5353(17) & 1.3991(14) \\
\hline C16-N24 & $1.376(3)$ & $1.366(3)$ & $1.3770(18)$ & $1.3791(14)$ & $1.3756(12)$ \\
\hline C16-C17 & $1.447(3)$ & $1.455(3)$ & $1.442(2)$ & $1.4524(15)$ & $1.4389(14)$ \\
\hline C17-C18 & $1.370(3)$ & $1.359(3)$ & $1.375(2)$ & $1.3614(15)$ & 1.3772(14) \\
\hline C18-C19 & $1.441(3)$ & $1.440(3)$ & $1.4440(19)$ & $1.4538(15)$ & $1.4460(13)$ \\
\hline C19-N24 & $1.388(3)$ & 1.391(3) & $1.3782(18)$ & $1.3791(14)$ & $1.3813(13)$ \\
\hline C19-C20 & $1.397(3)$ & $1.387(3)$ & $1.409(2)$ & $1.4051(15)$ & 1.4055(13) \\
\hline C1-C20 & $1.404(3)$ & $1.394(3)$ & $1.3930(19)$ & 1.3791(15) & $1.3950(14)$ \\
\hline C1-C21-C4 & $110.26(19)$ & $107.78(19)$ & 111.63(12) & 107.31(9) & -- \\
\hline C6-N22-C9 & $111.53(18)$ & 106.65(18) & $111.43(12)$ & -- & -- \\
\hline C21-Rh-N22 & -- & $90.10(8)$ & -- & 89.43(4) & -- \\
\hline C21-Rh-N23 & -- & 178.59(8) & -- & $168.60(4)$ & -- \\
\hline C21-Rh-N24 & -- & $90.57(8)$ & -- & $89.05(4)$ & -- \\
\hline C21-Rh-N25 & -- & $89.29(8)$ & -- & -- & -- \\
\hline C21-Rh-N26 & -- & $89.09(8)$ & -- & -- & -- \\
\hline N22-Rh-N23 & -- & $89.97(7)$ & -- & $90.46(4)$ & -- \\
\hline N22-Rh-N24 & -- & 179.27(8) & -- & 177.62(3) & -- \\
\hline N22-Rh-N25 & -- & $89.00(7)$ & -- & -- & -- \\
\hline N22-Rh-N26 & -- & $90.21(7)$ & -- & -- & -- \\
\hline N23-Rh-N24 & $85.43(7)$ & $89.35(7)$ & $85.79(5)$ & $90.66(4)$ & $85.90(3)$ \\
\hline N24-Rh-C25(c & $91.43(8)$ & $90.70(7)$ & $91.96(6)$ & -- & $91.86(4)$ \\
\hline N24-Rh-C26(or] & 175.36(9) & $90.11(7)$ & $176.68(6)$ & -- & 173.99(4) \\
\hline N23-Rh-C25(orN25) & $176.27(8)$ & $89.30(7)$ & $175.90(6)$ & -- & 177.11(4) \\
\hline N23-Rh-C26(orN26) & 94.62(9) & $92.32(7)$ & $93.41(6)$ & -- & $92.26(4)$ \\
\hline C25-Rh-C26 & $88.35(10)$ & -- & $88.64(7)$ & -- & $89.78(5)$ \\
\hline C25-C21-Rh & -- & -- & -- & $64.09(6)$ & -- \\
\hline C25-C21-C1 & -- & -- & -- & $120.47(10)$ & -- \\
\hline
\end{tabular}




\begin{tabular}{|l|c|c|c|c|c|}
\hline C25-C21-C4 & -- & -- & -- & $120.52(10)$ & -- \\
\hline N25-Rh-N26 & -- & $178.19(8)$ & -- & -- & -- \\
\hline Rh-C25-O25 & $177.3(2)$ & -- & $177.43(15)$ & -- & $178.59(11)$ \\
\hline Rh-C26-O26 & $176.2(2)$ & -- & $176.25(15)$ & -- & $176.81(10)$ \\
\hline
\end{tabular}

Purple more double bond like ( $\leq 1.37)$, orange - more aromatic (1.38-1.41), blue more single bond like (1.42-1.45), green - single bond limit ( $\geq 1.46)$. 

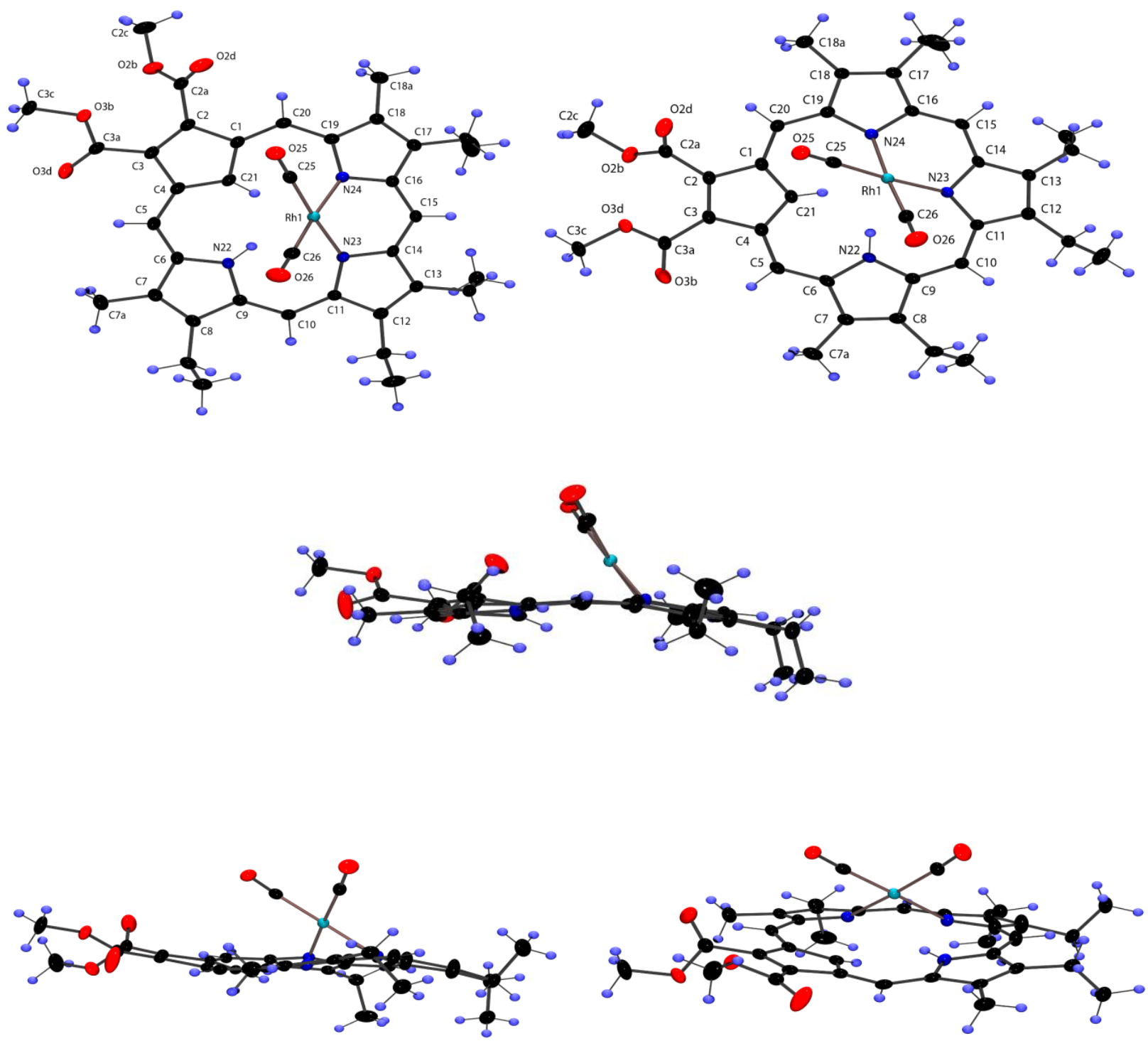

Figure C-1. Color POV-Ray rendered ORTEP III drawings (50\% probability level, hydrogen atoms rendered arbitrarily small for clarity) showing a variety of views for rhodium(I) carbaporphyrin complex 89. 

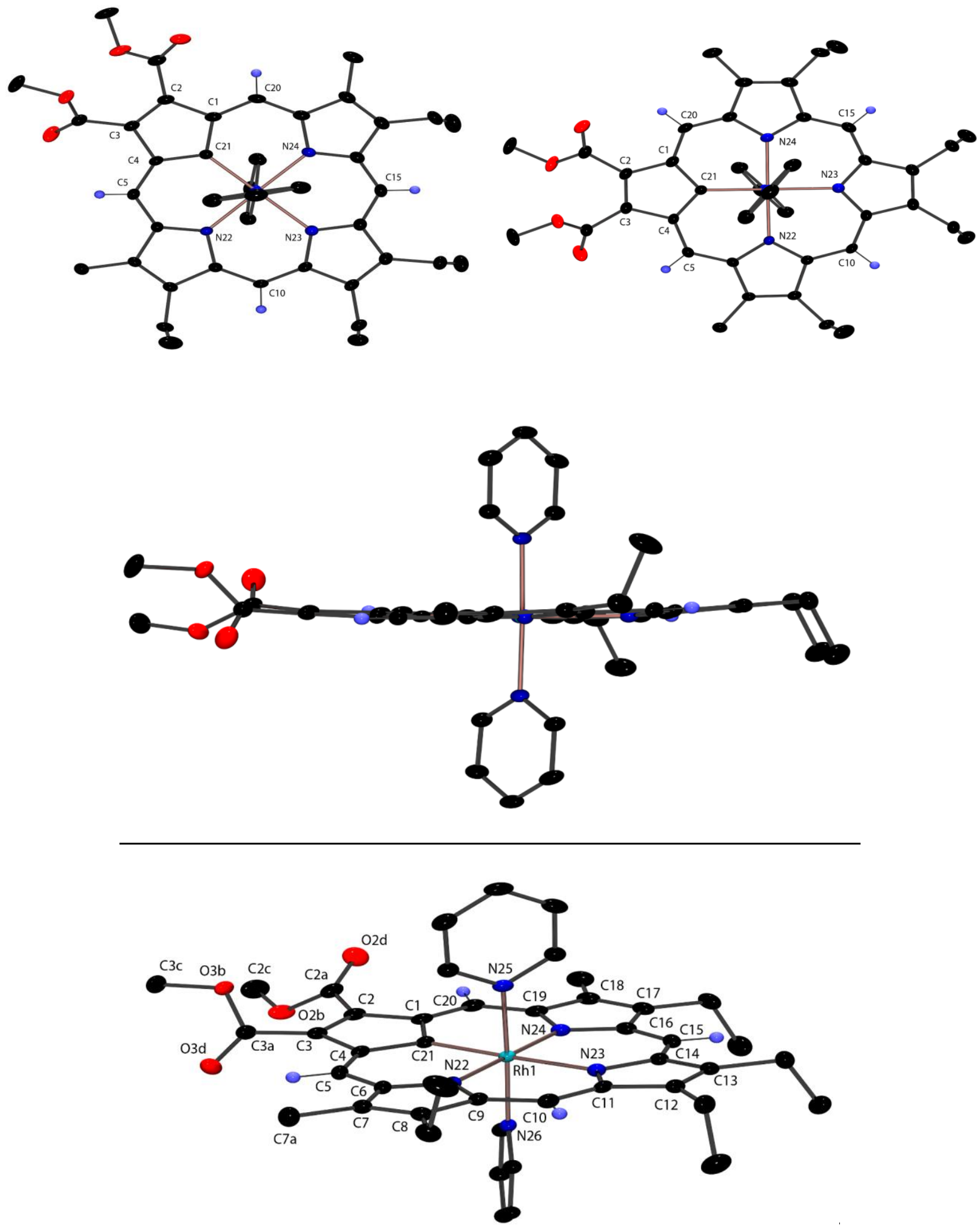

Figure C-2. Color POV-Ray rendered ORTEP III drawing (50\% probability level, hydrogen atoms rendered arbitrarily small or omitted for clarity) showing a variety of views for rhodium(III) carbaporphyrin $\mathbf{9 0}$. 


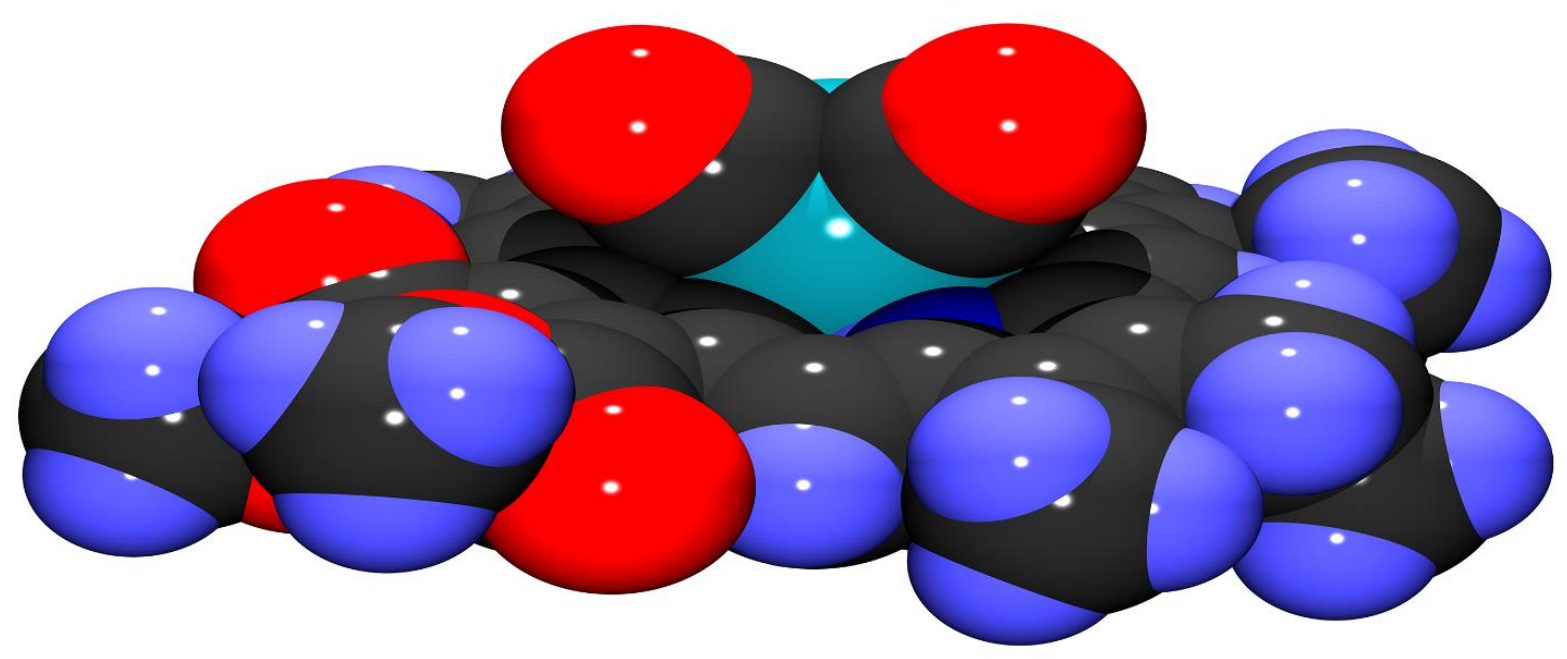

Figure C-3. Color POV-Ray rendered ORTEP III drawing (50\% probability level, hydrogen atoms rendered arbitrarily small for clarity) showing a space-filling representation of rhodium(I) carbaporphyrin complex 89.

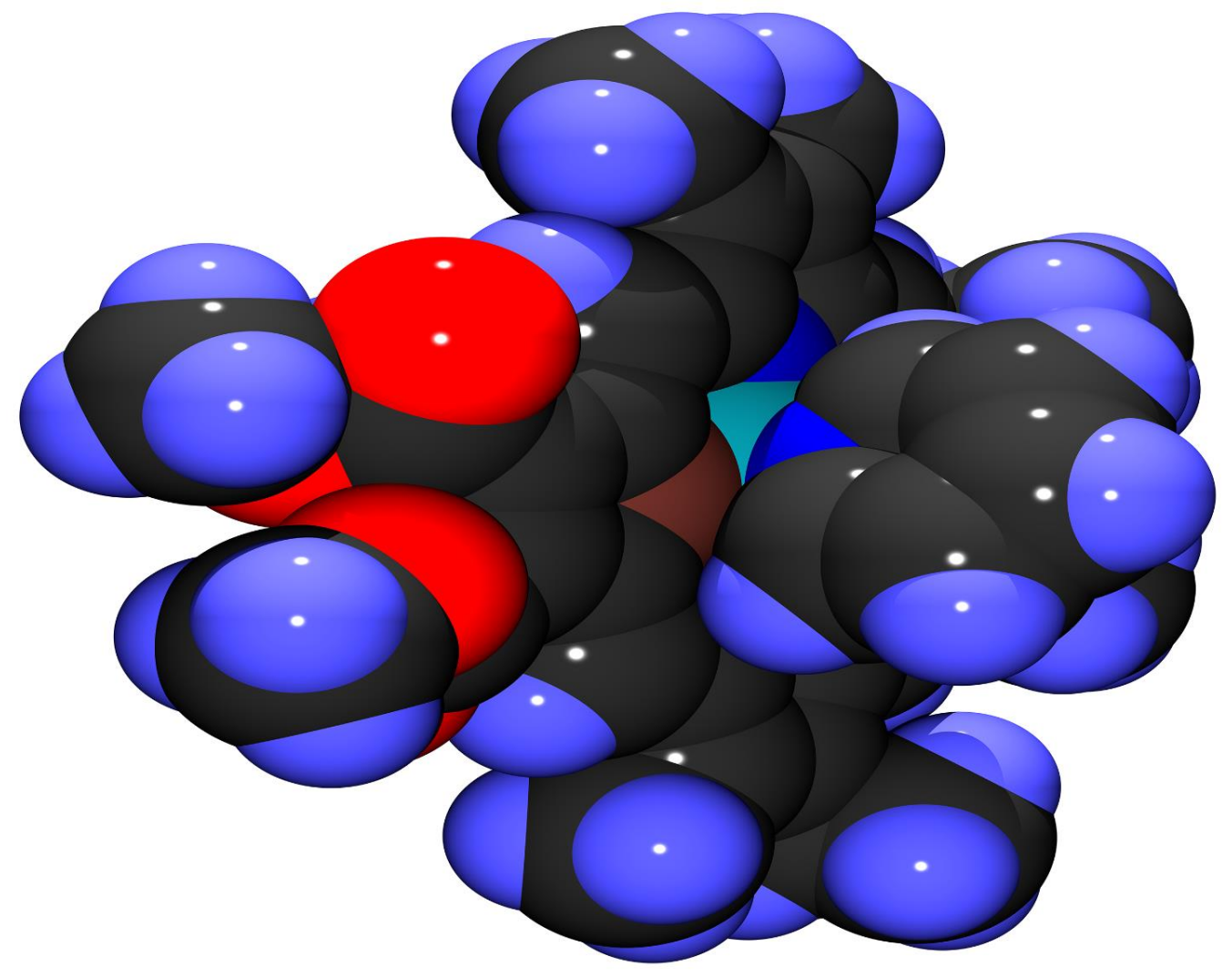

Figure C-4. Color POV-Ray rendered space-filling drawing of rhodium(III) carbaporphyrin 90, carbon atom attached to rhodium highlighted in brown. 

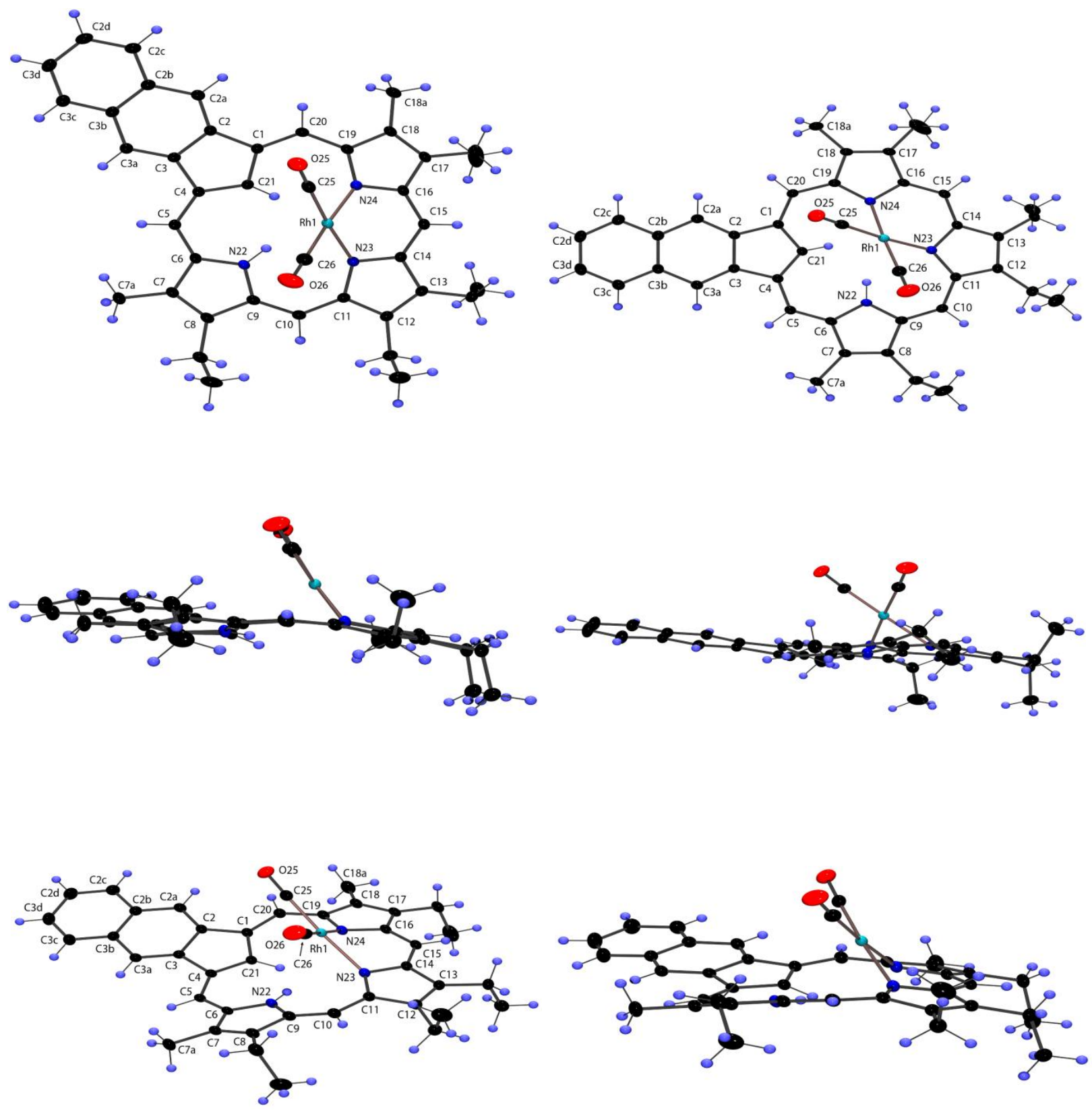

Figure C-5. Color POV-Ray rendered ORTEP III drawing (50\% probability level, hydrogen atoms rendered arbitrarily small for clarity) showing a variety of views for rhodium(I) naphthocarbaporphyrin $\mathbf{9 1 .}$ 

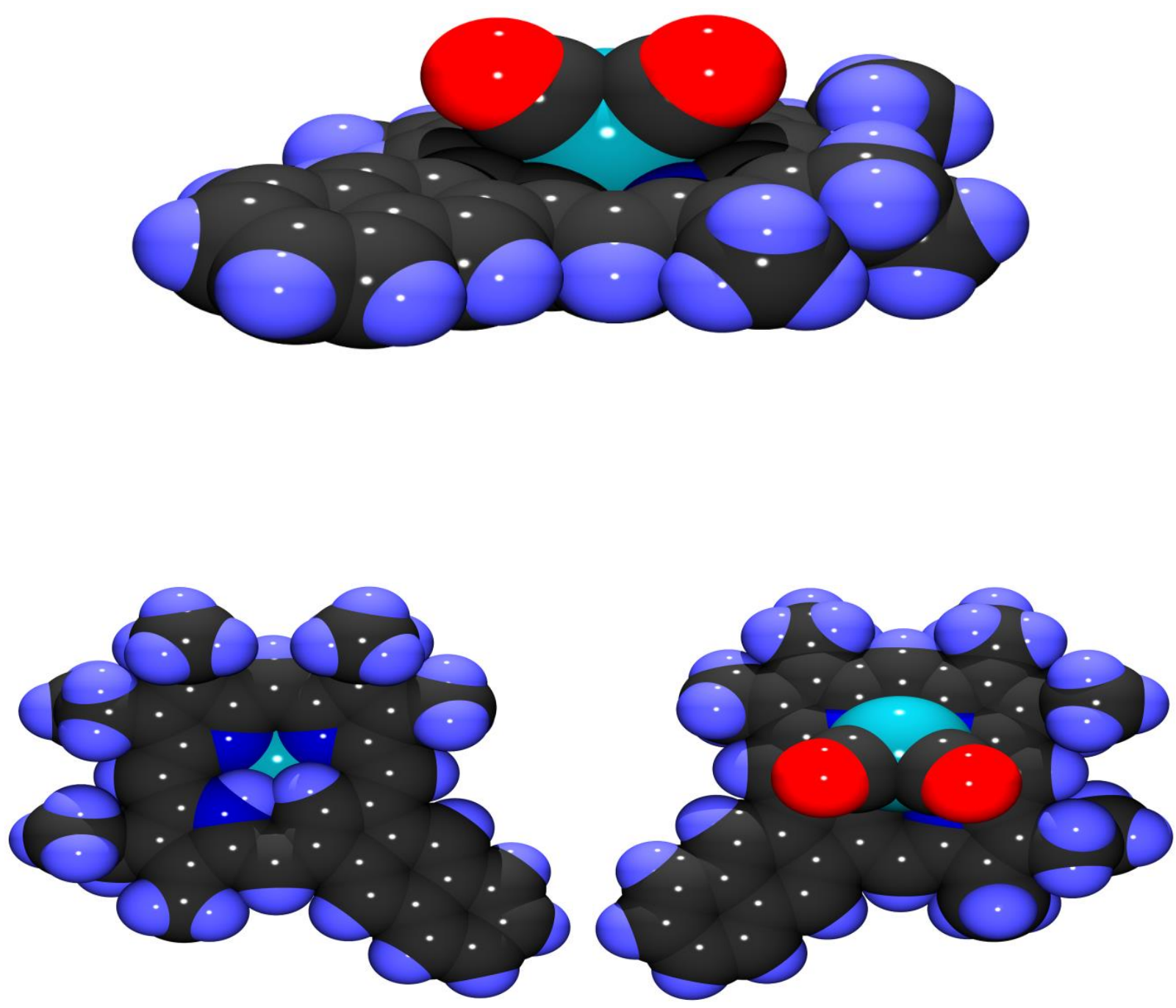

Figure C-6. Color POV-Ray rendered space-filling drawings of rhodium(I) naphthocarbaporphyrin 91. 

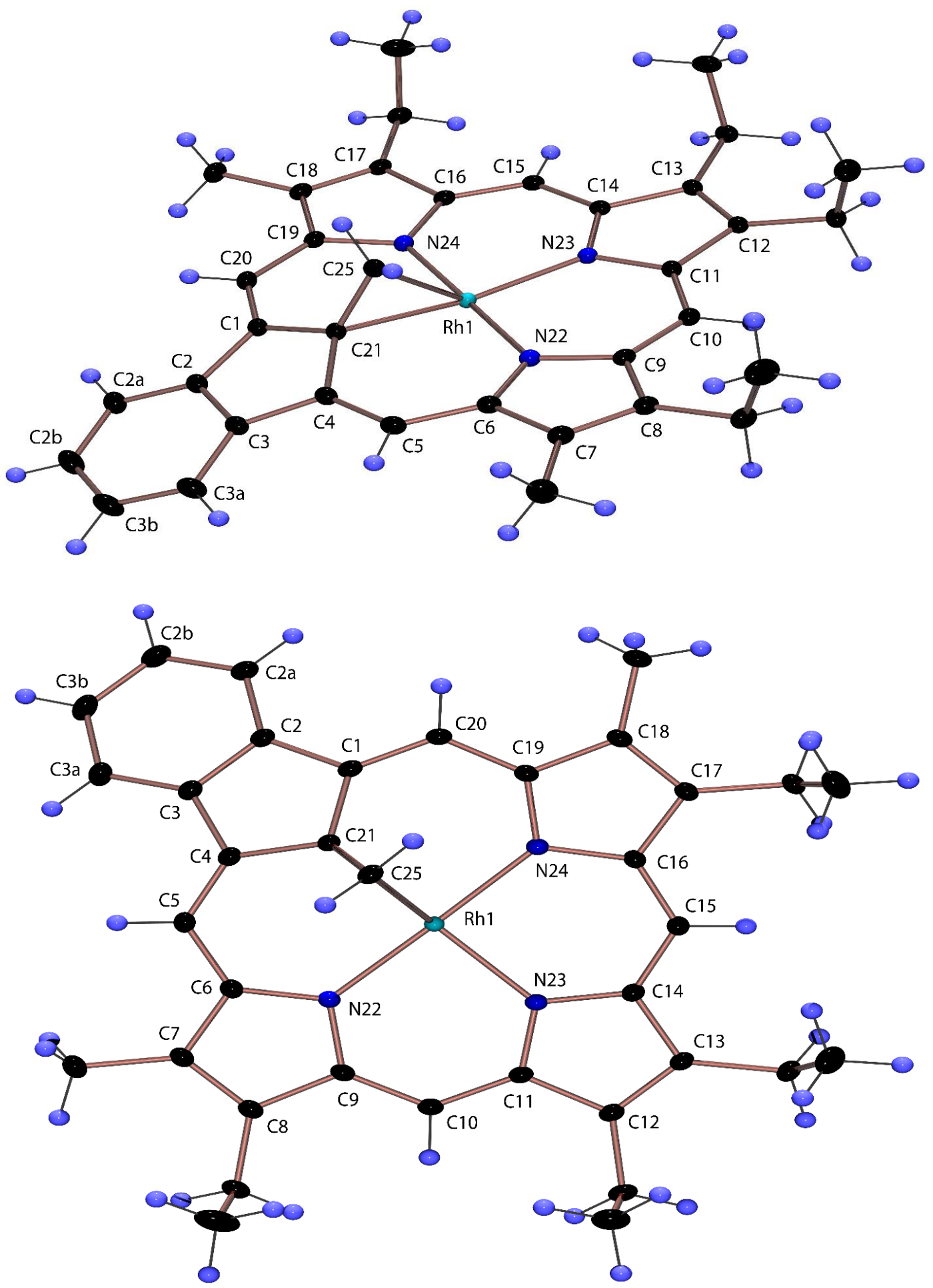

Figure C-7. Color POV-Ray rendered ORTEP III drawing (50\% probability level, hydrogen atoms rendered arbitrarily small for clarity) showing two views of rhodium(III) complex 95. 

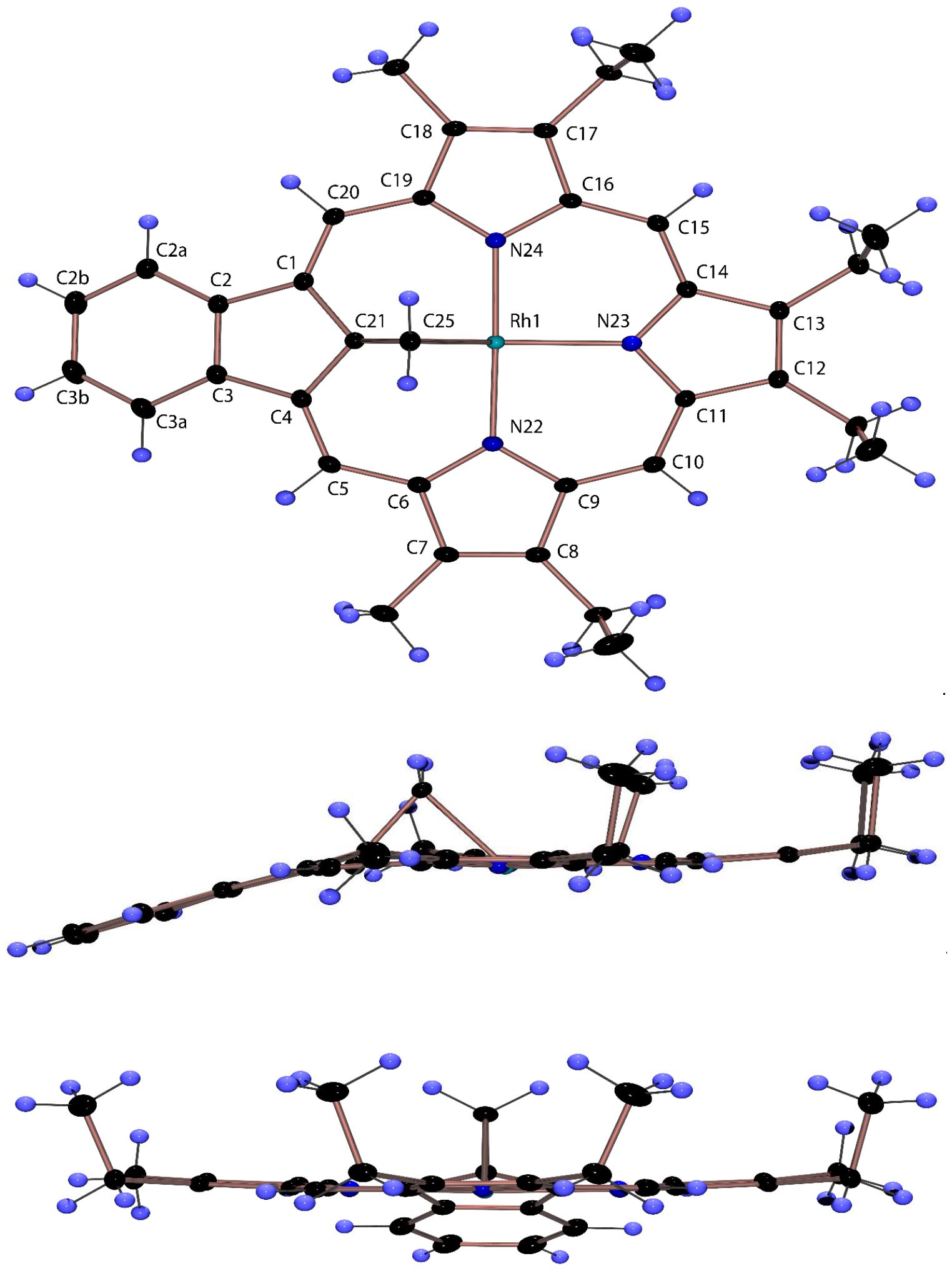

Figure C-8. Color POV-Ray rendered ORTEP III drawing (50\% probability level, hydrogen atoms rendered arbitrarily small for clarity) showing three further views of rhodium(III) complex 95. 

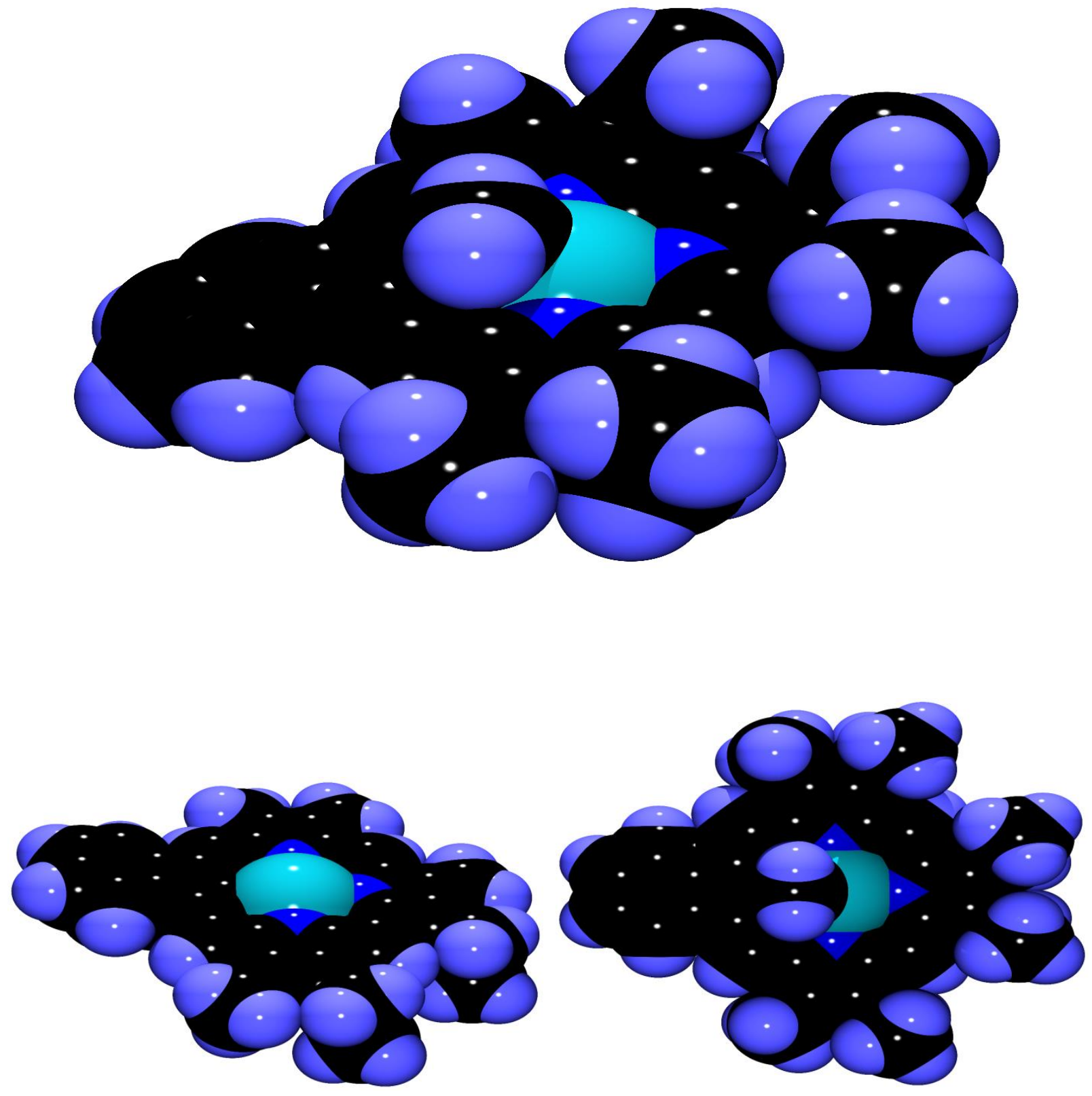

Figure C-9. Color POV-Ray rendered space-filling drawings showing three different views of methylene-bridged rhodium(III) complex 95. 


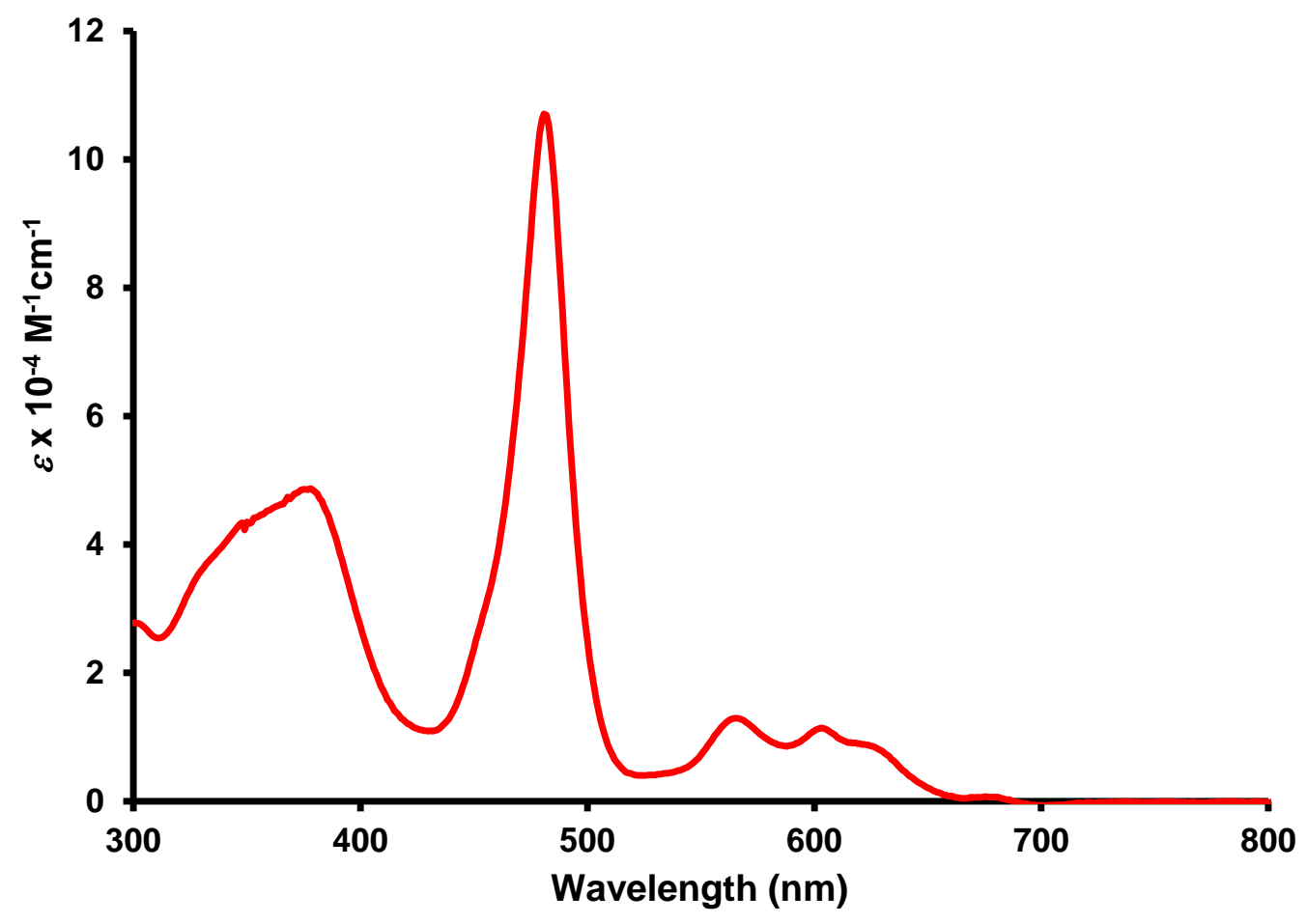

Figure C-10. UV-vis spectrum of rhodium(I) naphthocarbaporphyrin 91 in $\mathrm{CH}_{2} \mathrm{Cl}_{2}$.

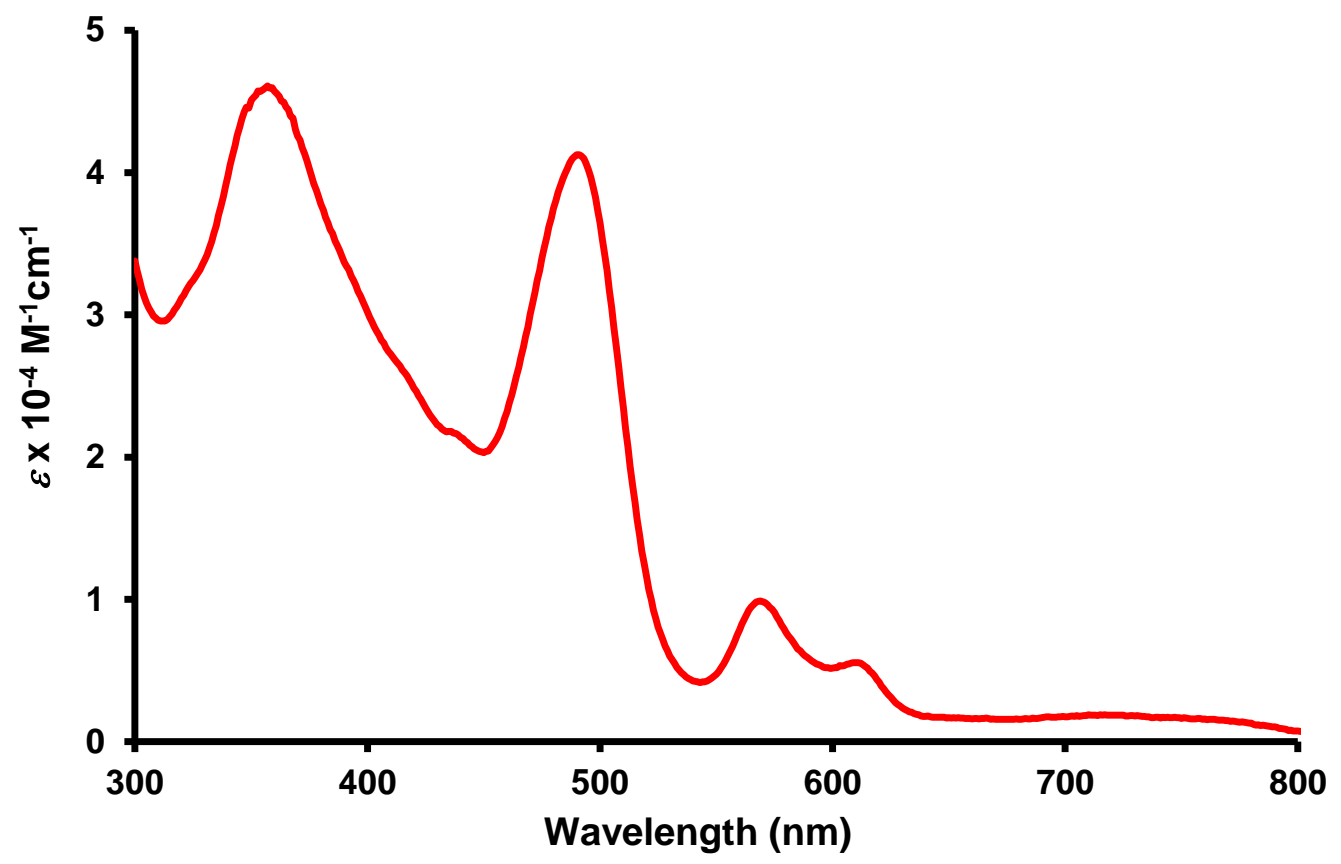

Figure C-11. UV-vis spectrum of rhodium(I) carbaporphyrin 89 in $\mathrm{CH}_{2} \mathrm{Cl}_{2}$. 


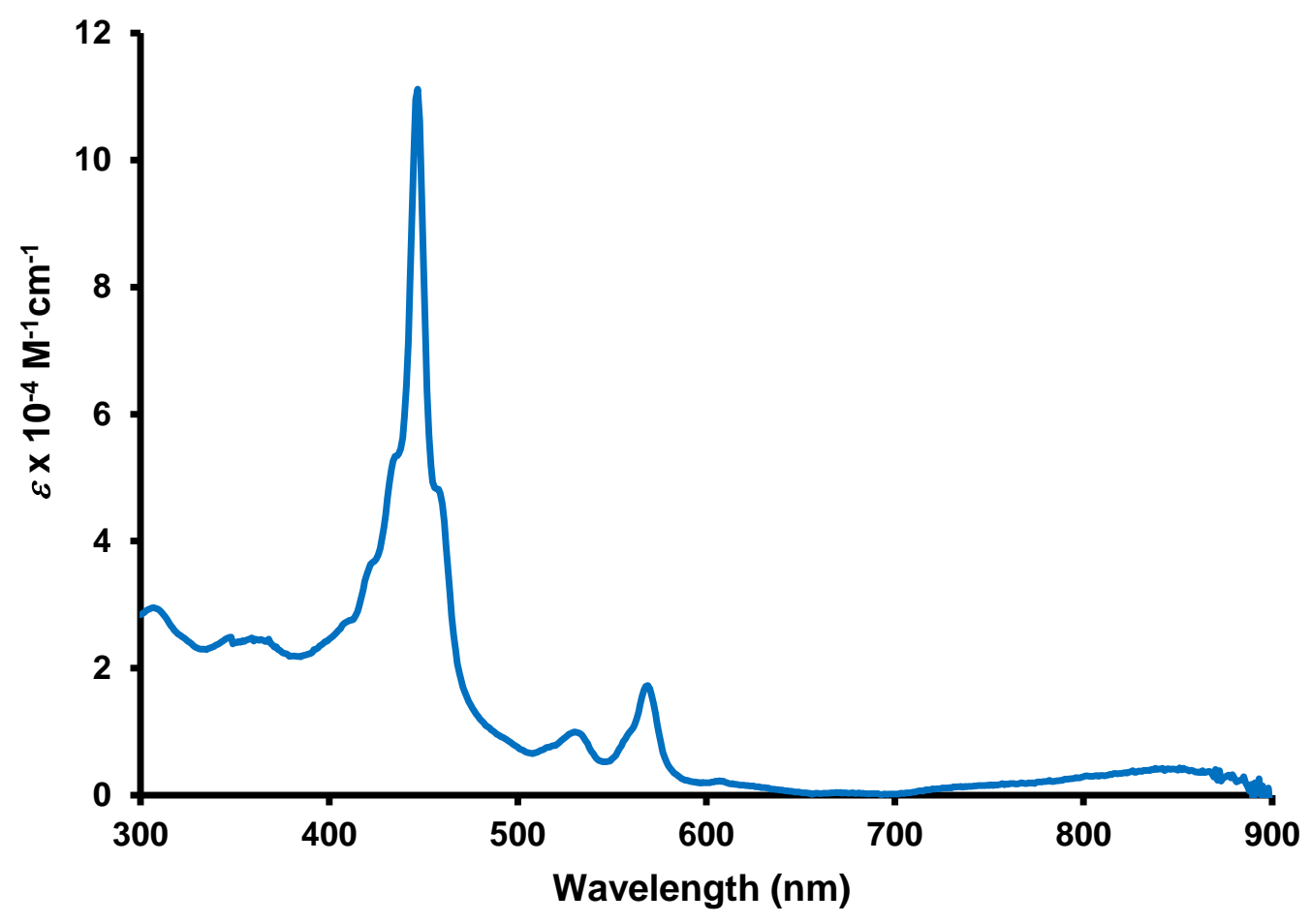

Figure C-12. UV-vis spectrum of rhodium(III) naphthocarbaporphyrin 92 in $\mathrm{CH}_{2} \mathrm{Cl}_{2}$.

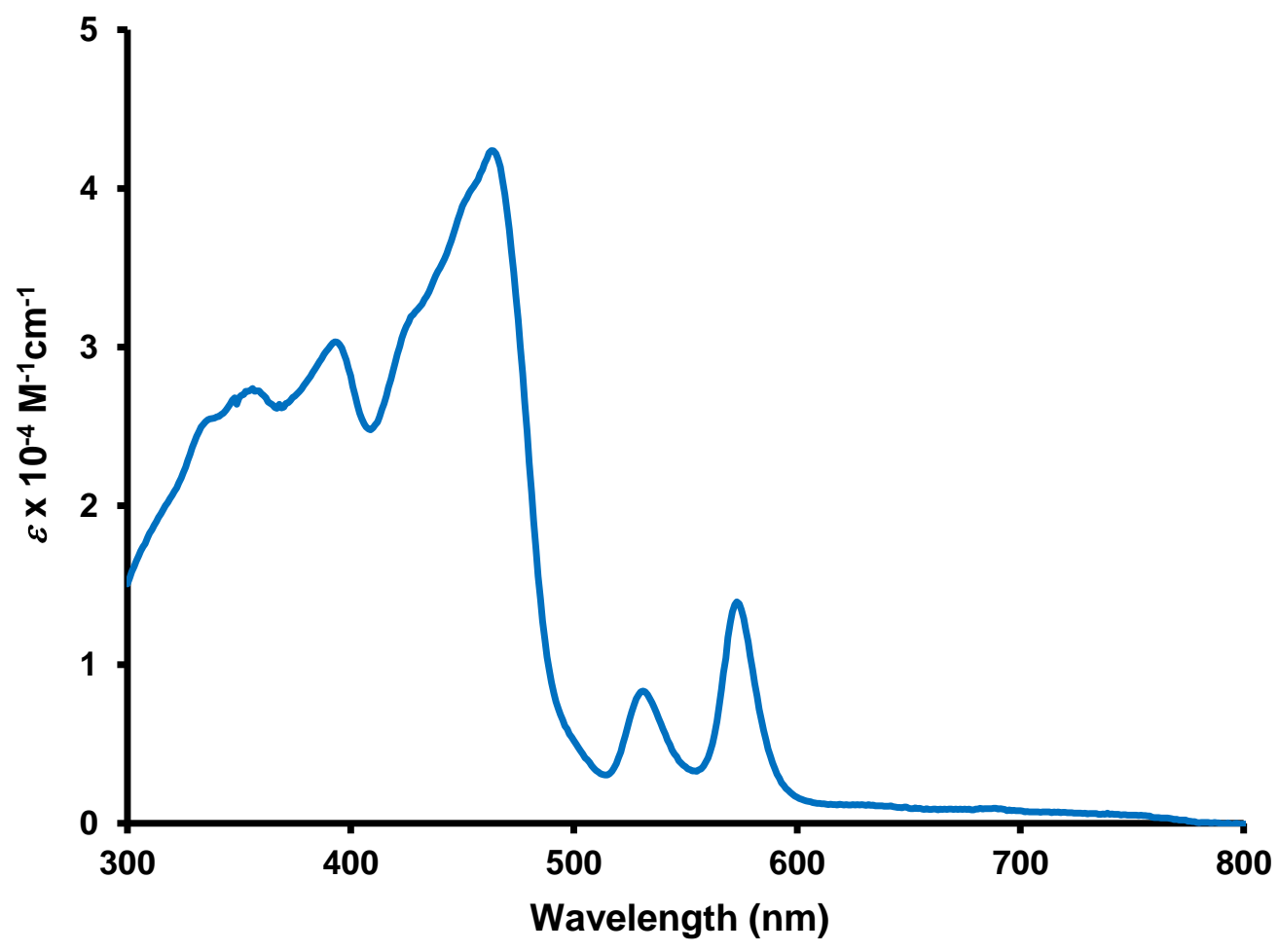

Figure C-13. UV-vis spectrum of rhodium(III) carbaporphyrin 90 in $\mathrm{CH}_{2} \mathrm{Cl}_{2}$. 


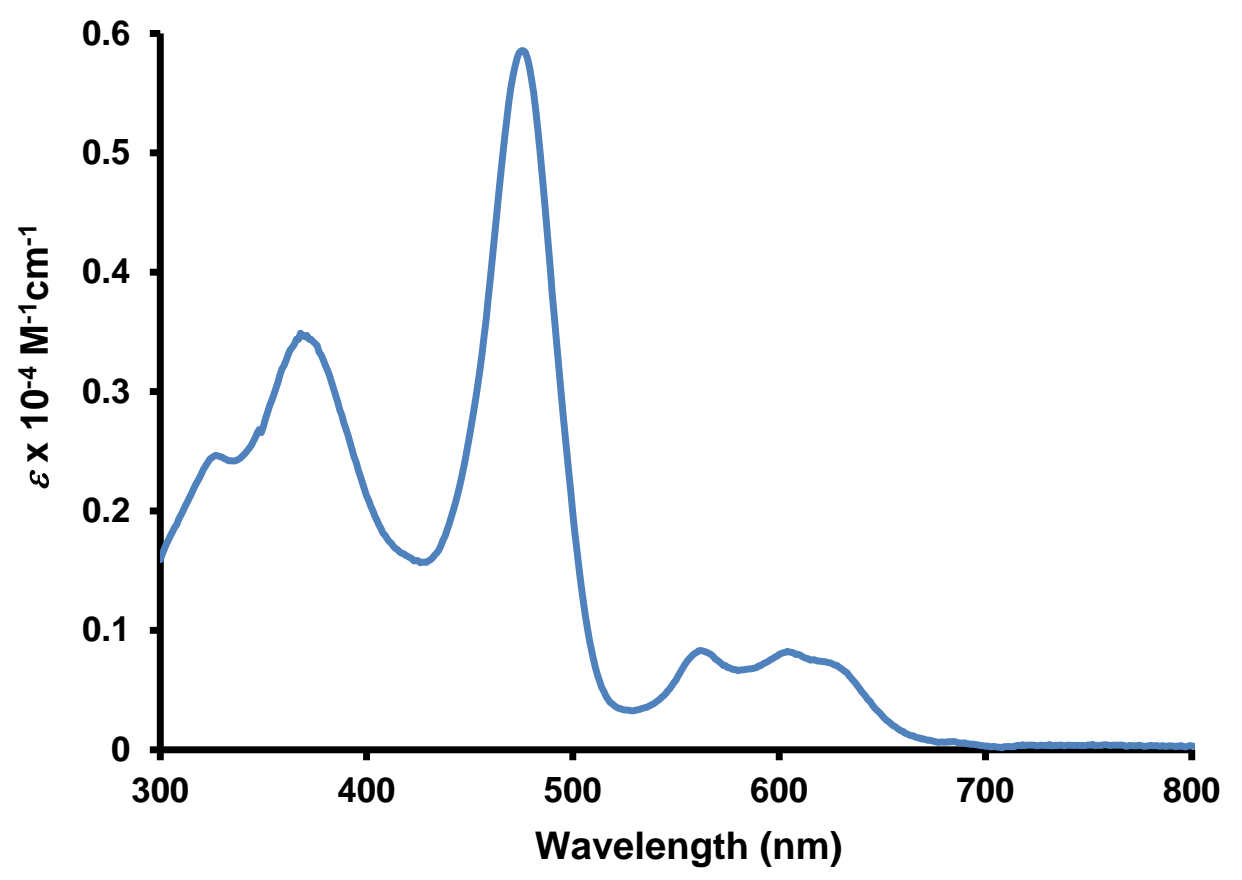

Figure C-14. UV-vis spectrum of rhodium(I) 21-methylcarbaporphyrin complex 94 in $\mathrm{CH}_{2} \mathrm{Cl}_{2}$.

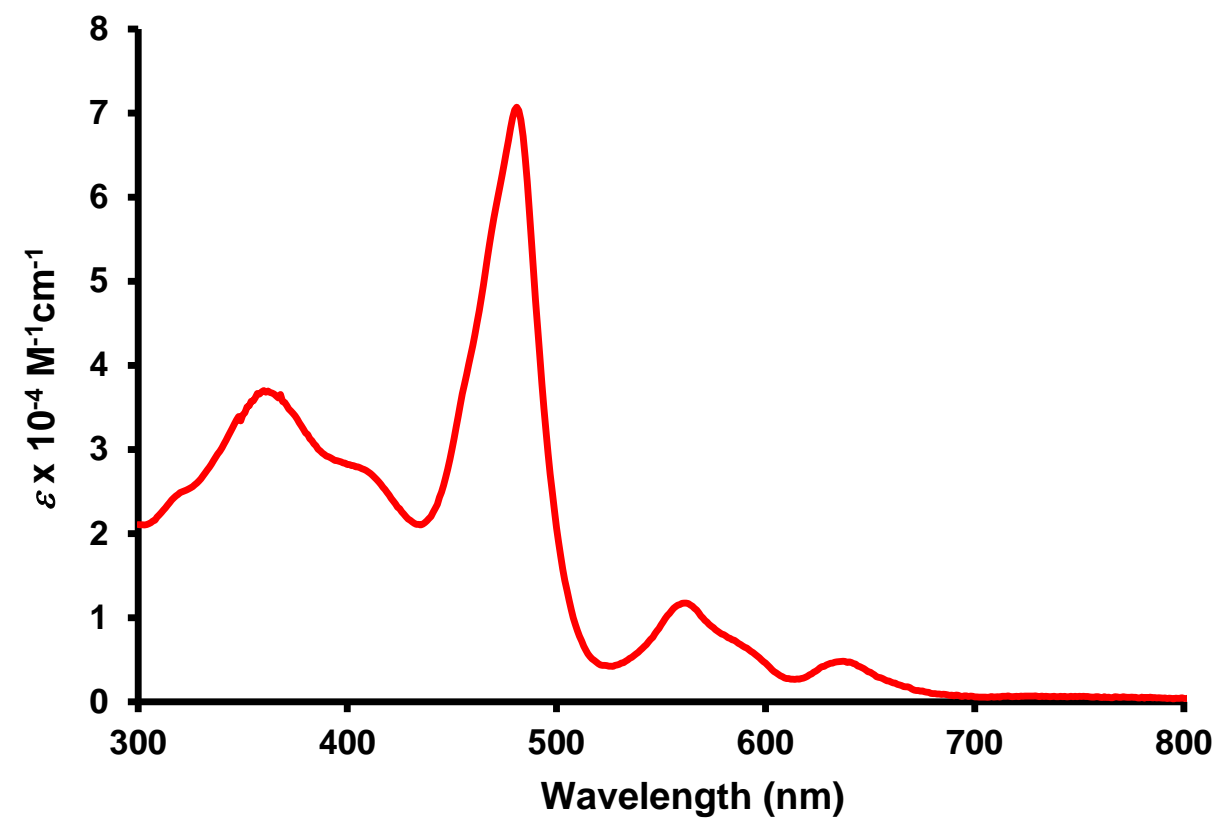

Figure C-15. UV-vis spectrum of rhodium(I) 22-methylcarbaporphyrin complex 93 in $\mathrm{CH}_{2} \mathrm{Cl}_{2}$. 


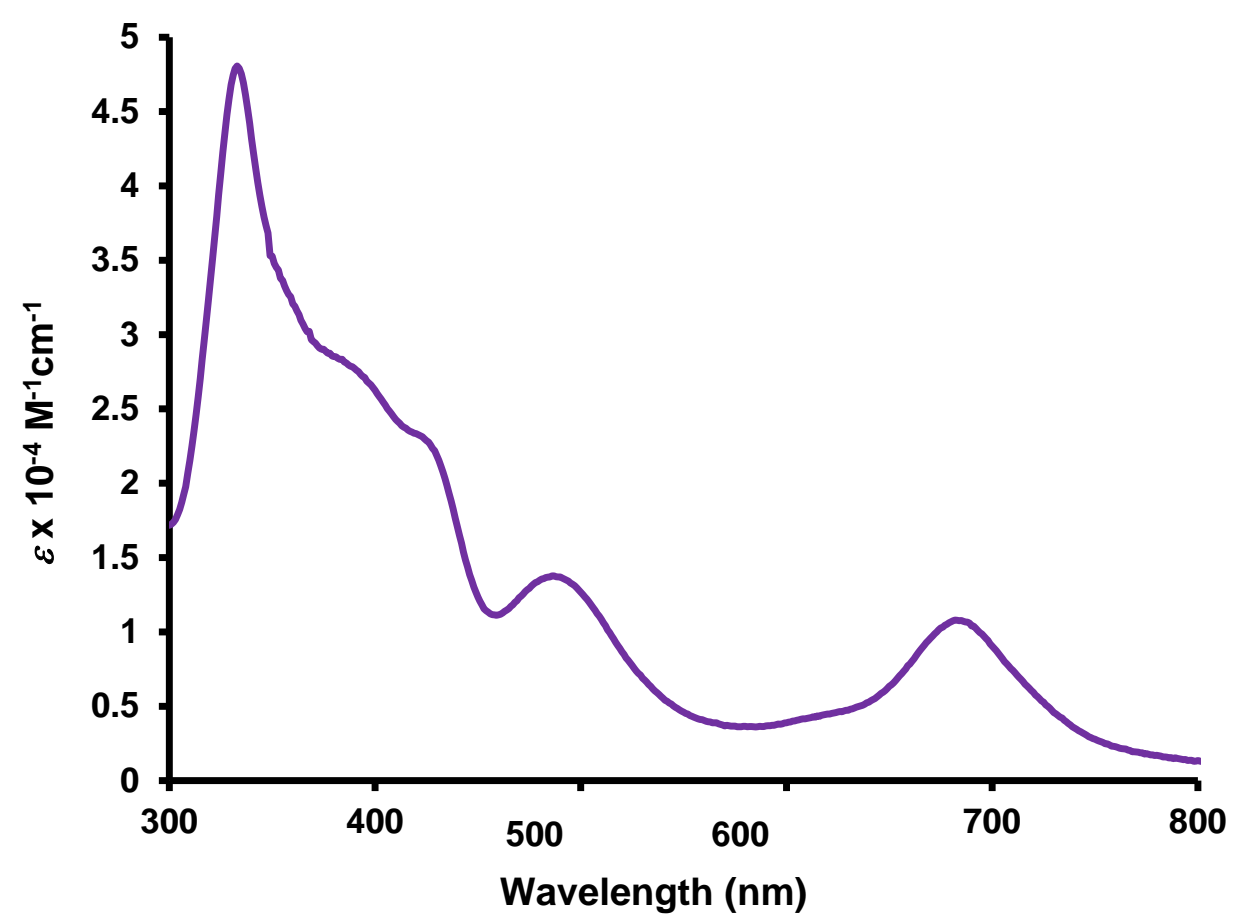

Figure C-16. UV-vis spectrum of rhodium(III) carbaporphyrin complex 95 in $\mathrm{CH}_{2} \mathrm{Cl}_{2}$. 


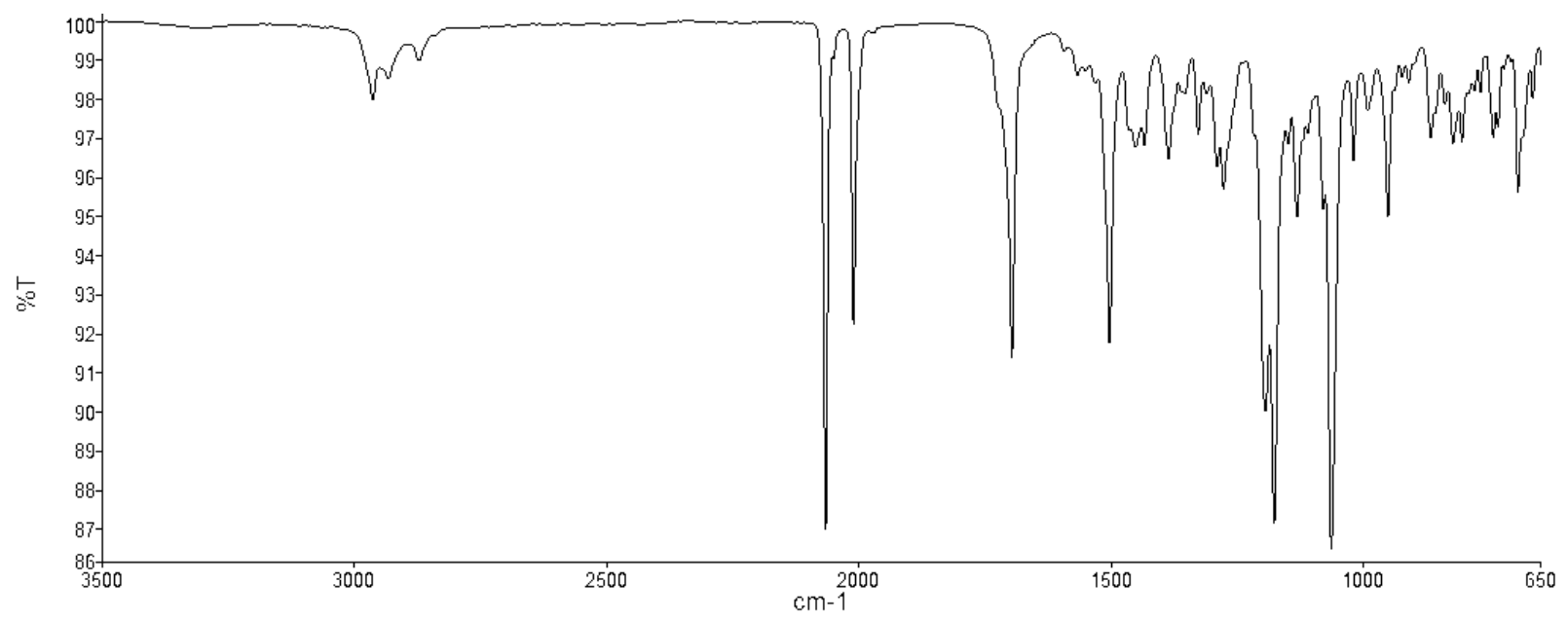

Figure C-17. IR spectrum of rhodium(I) carbaporphyrin 89.

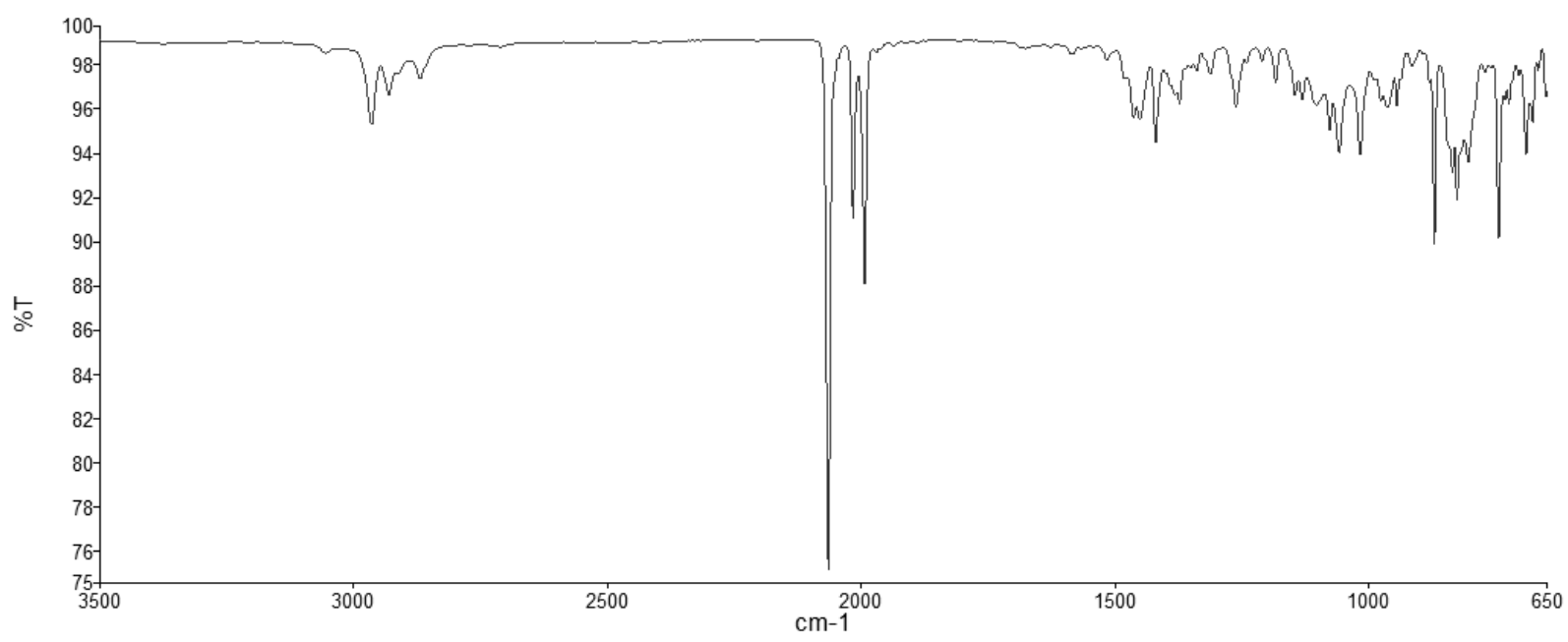

Figure C-18. IR spectrum of rhodium(I) naphthocarbaporphyrin 91. 


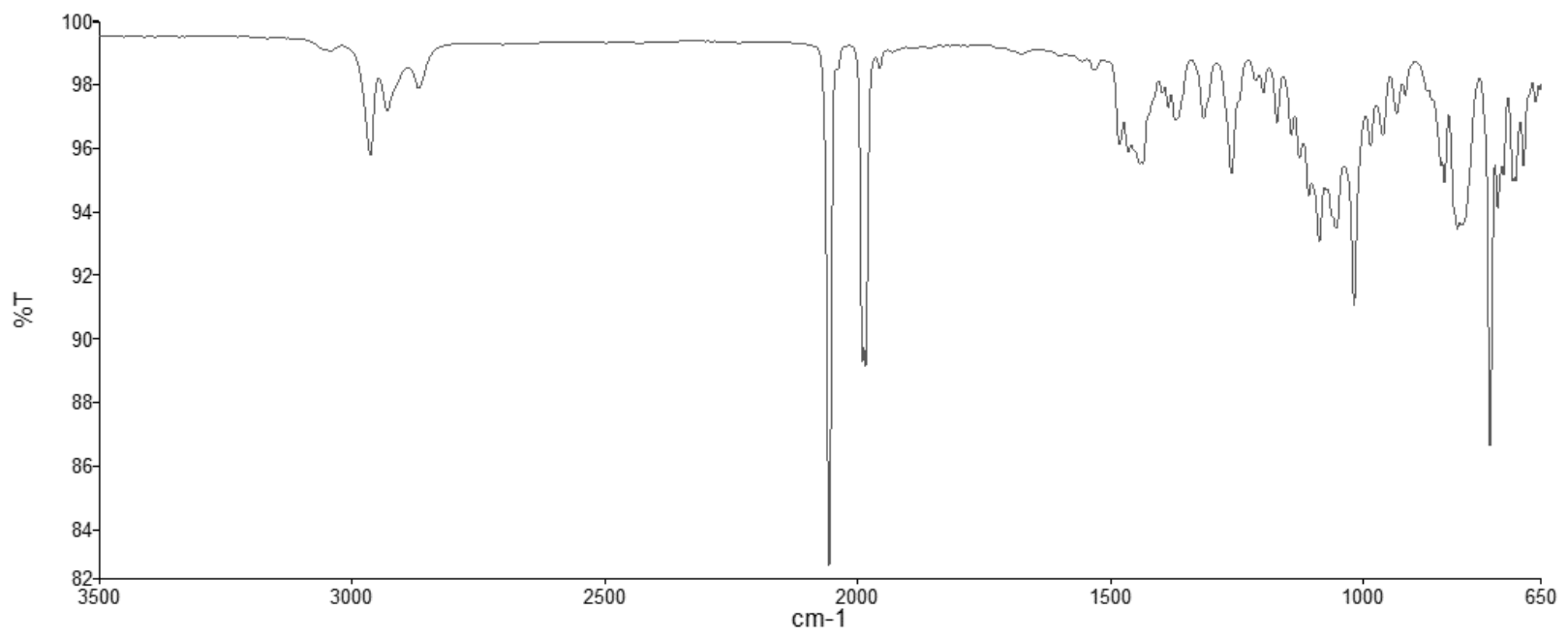

Figure C-19. IR spectrum of rhodium(I) 22-methylcarbaporphyrin 93.

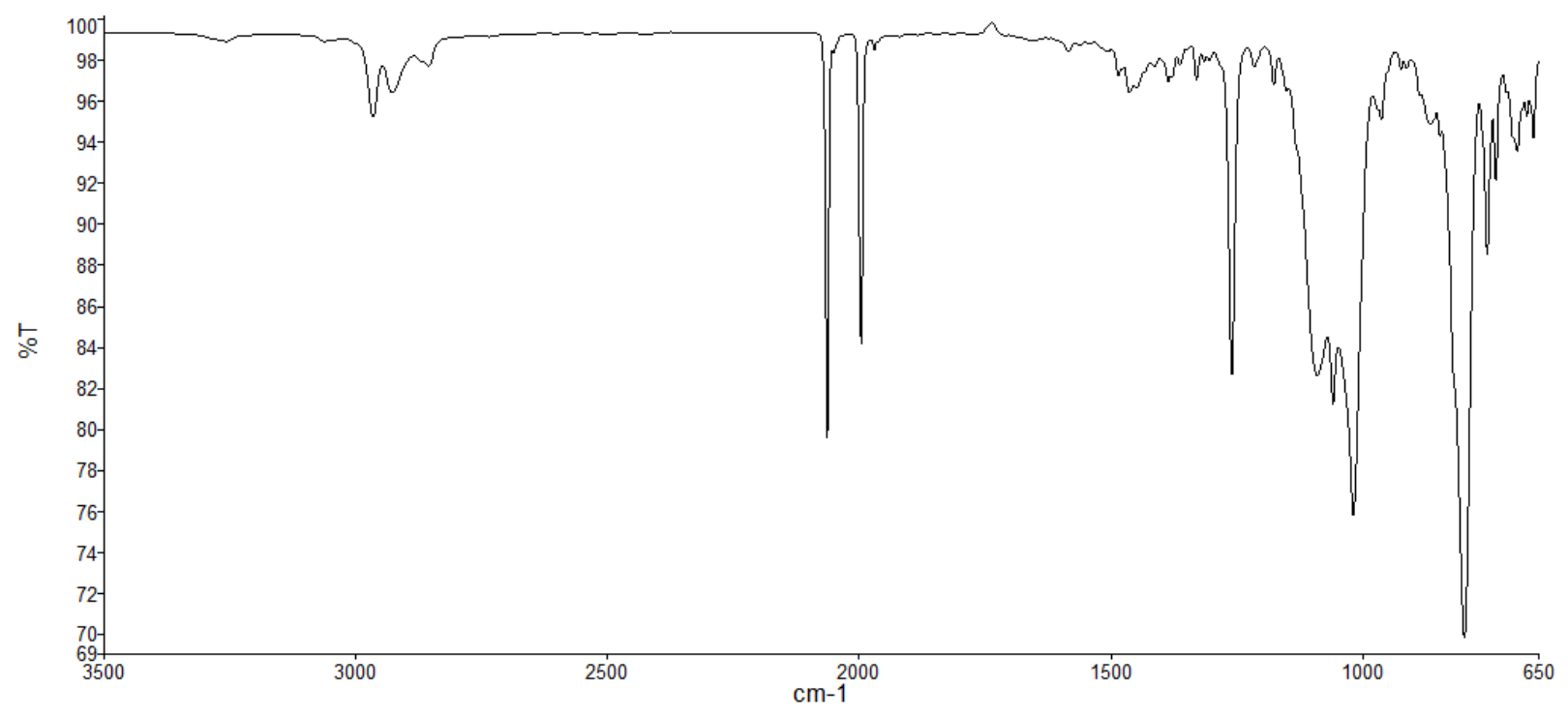

Figure C-20. IR spectrum of rhodium(I) 21-methylcarbaporphyrin 94. 

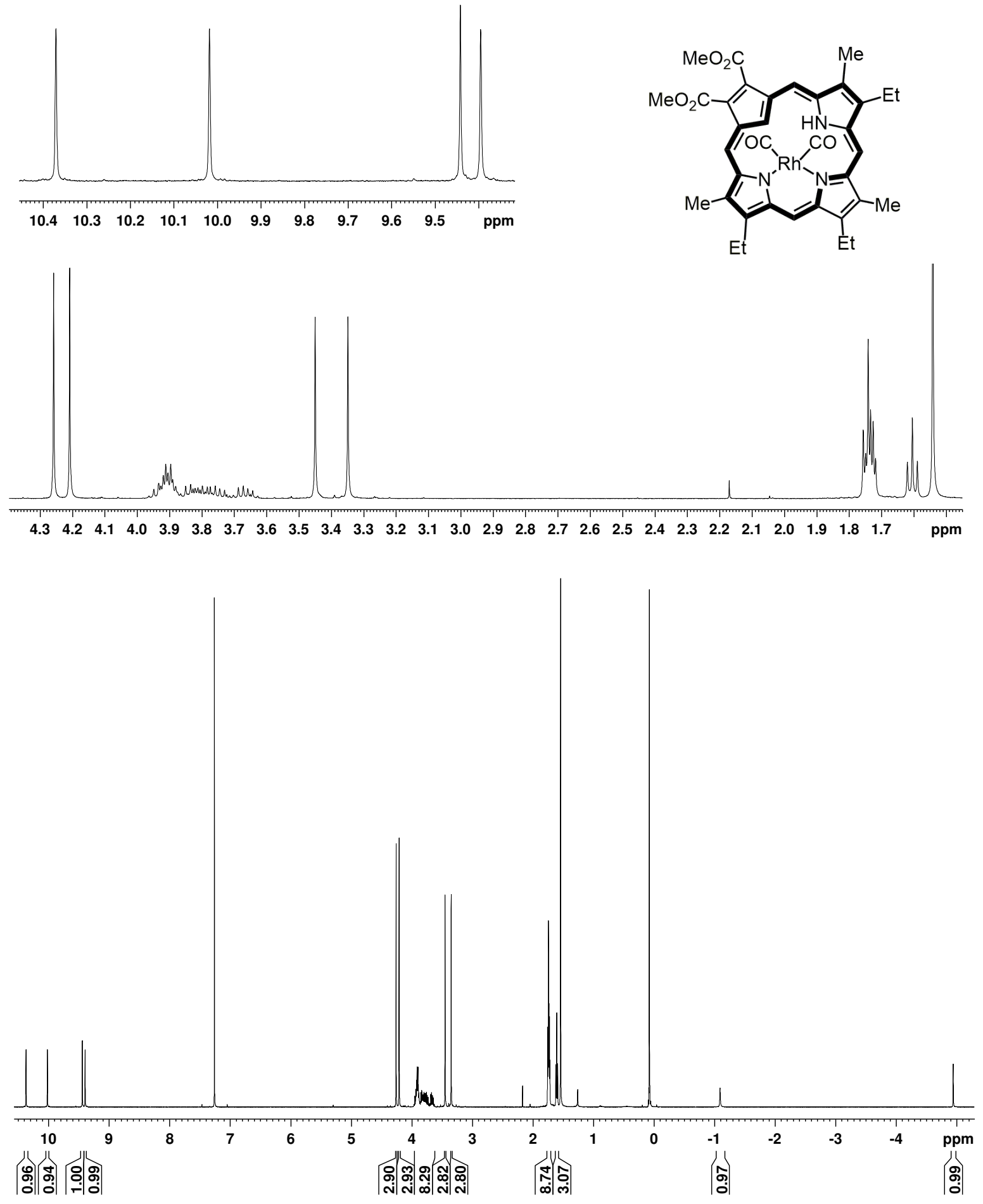

Figure C-21. $500 \mathrm{MHz}{ }^{1} \mathrm{H}$ NMR spectrum of rhodium(I) carbaporphyrin 89 in $\mathrm{CDCl}_{3}$. 


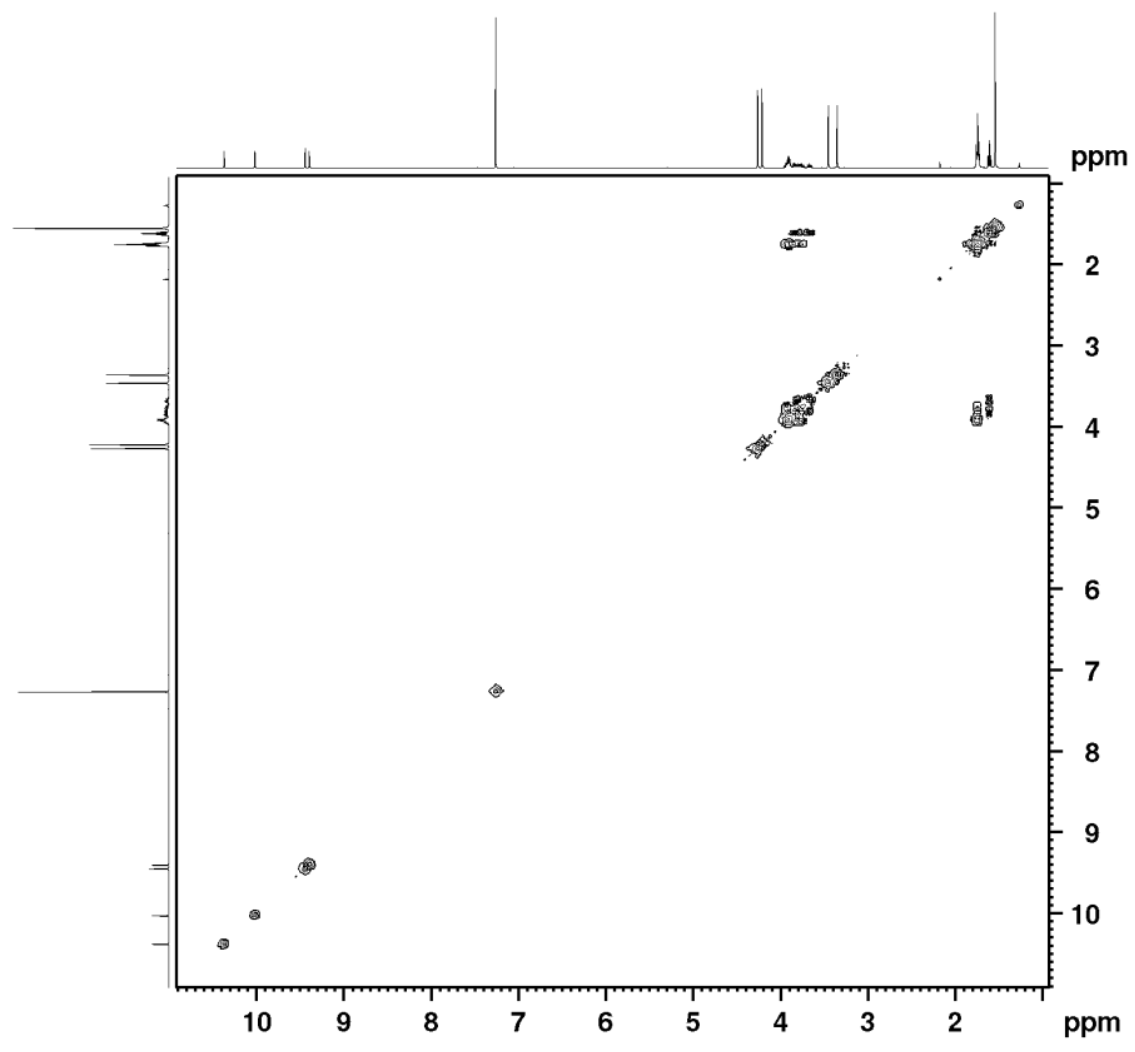

Figure C-22. ${ }^{1} \mathrm{H}-{ }^{1} \mathrm{H}$ COSY NMR spectrum of rhodium(I) carbaporphyrin 89 in $\mathrm{CDCl}_{3}$.

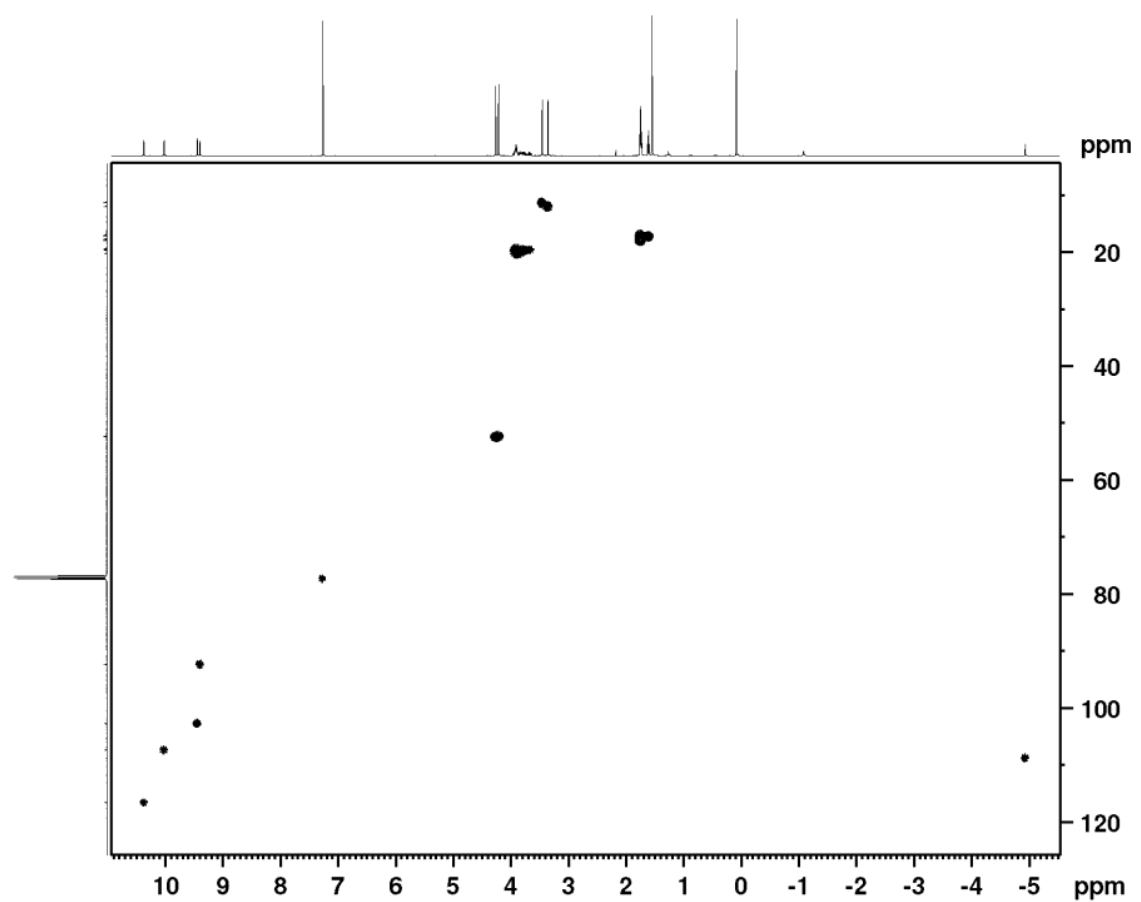

Figure C-23. HSQC NMR spectrum of rhodium(I) carbaporphyrin 89 in $\mathrm{CDCl}_{3}$. 


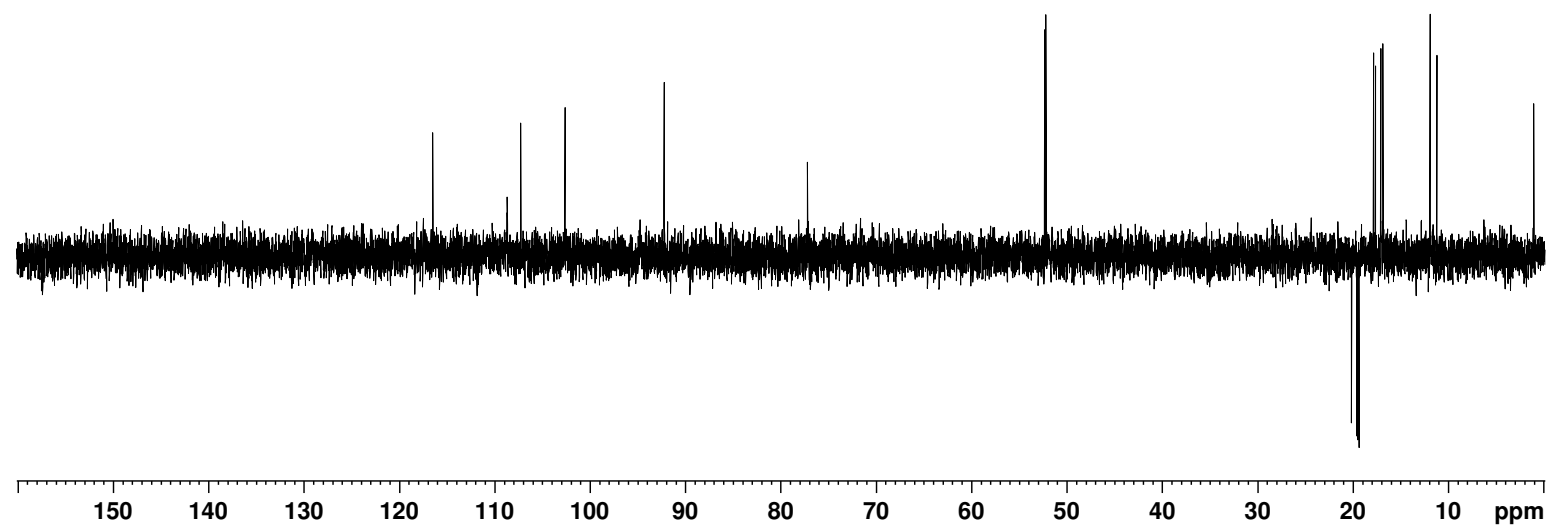

Figure C-24. DEPT-135 NMR spectrum of rhodium(I) carbaporphyrin 89 in $\mathrm{CDCl}_{3}$.
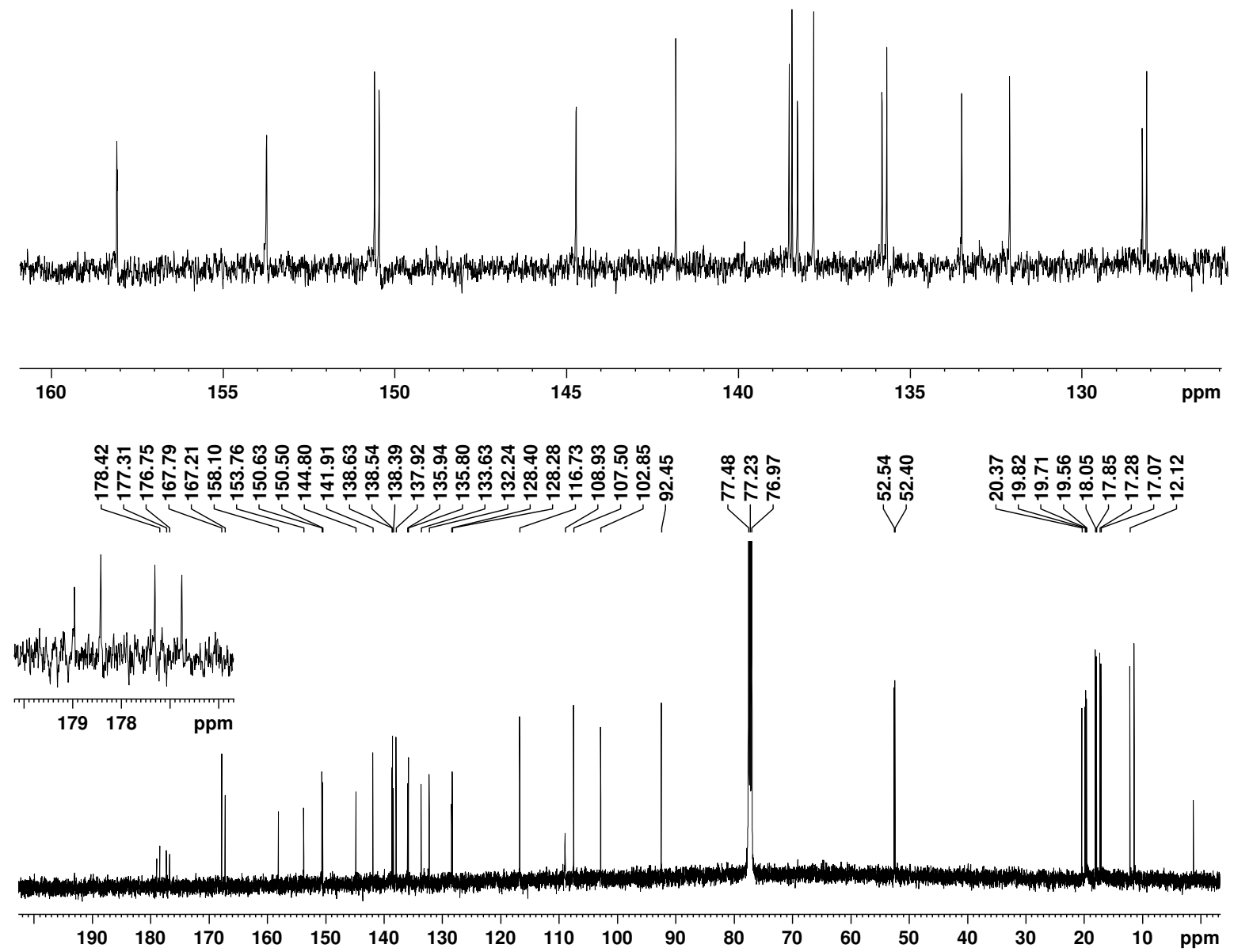

Figure C-25. $500 \mathrm{MHz}{ }^{1} \mathrm{H}$ NMR spectrum of rhodium(I) carbaporphyrin 89 in $\mathrm{CDCl}_{3}$. 

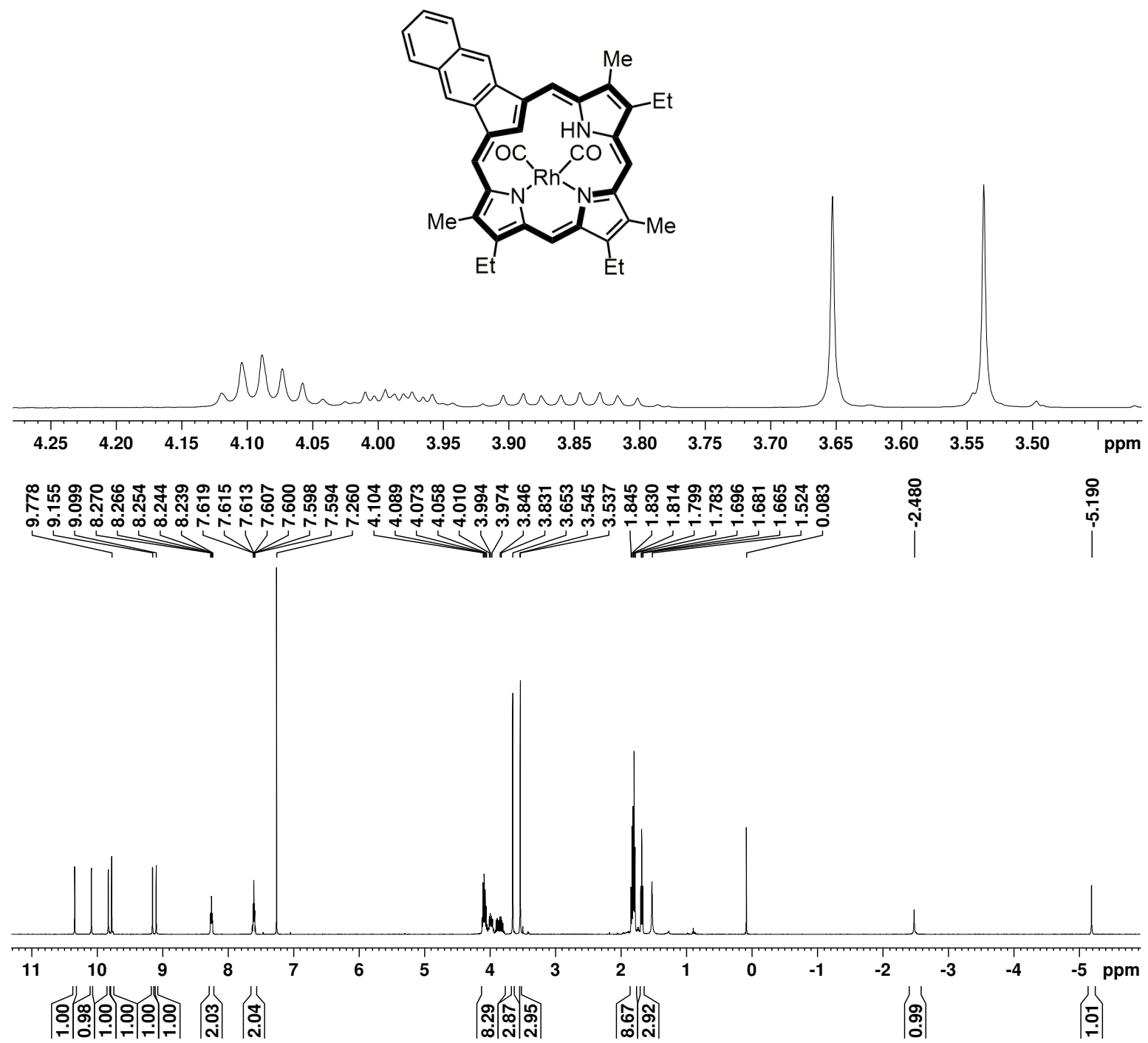

Figure C-26. $500 \mathrm{MHz}{ }^{1} \mathrm{H}$ NMR spectrum of rhodium(I) naphthocarbaporphyrin 91 in $\mathrm{CDCl}^{3}$. 


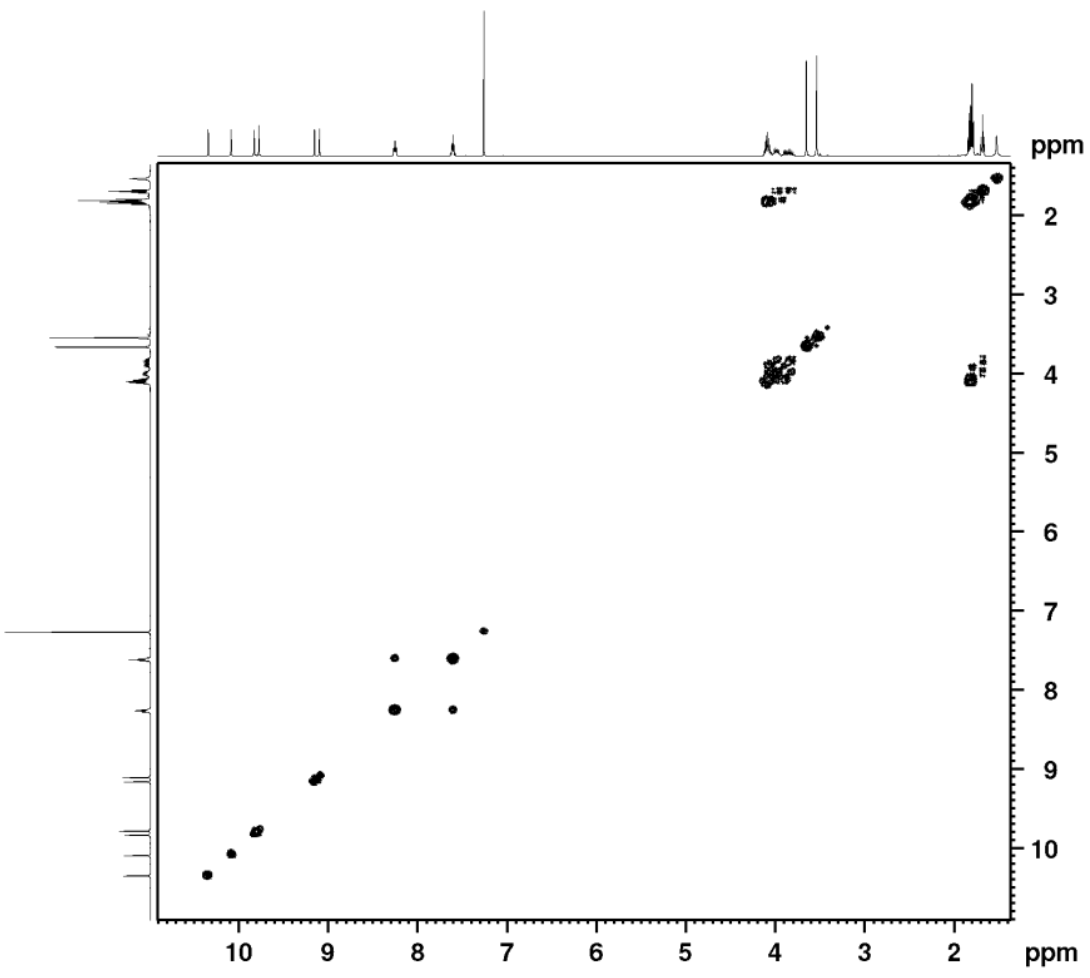

Figure C-27. ${ }^{1} \mathrm{H}-{ }^{1} \mathrm{H}$ COSY NMR spectrum of rhodium(I) naphthocarbaporphyrin 91 in $\mathrm{CDCl}_{3}$.

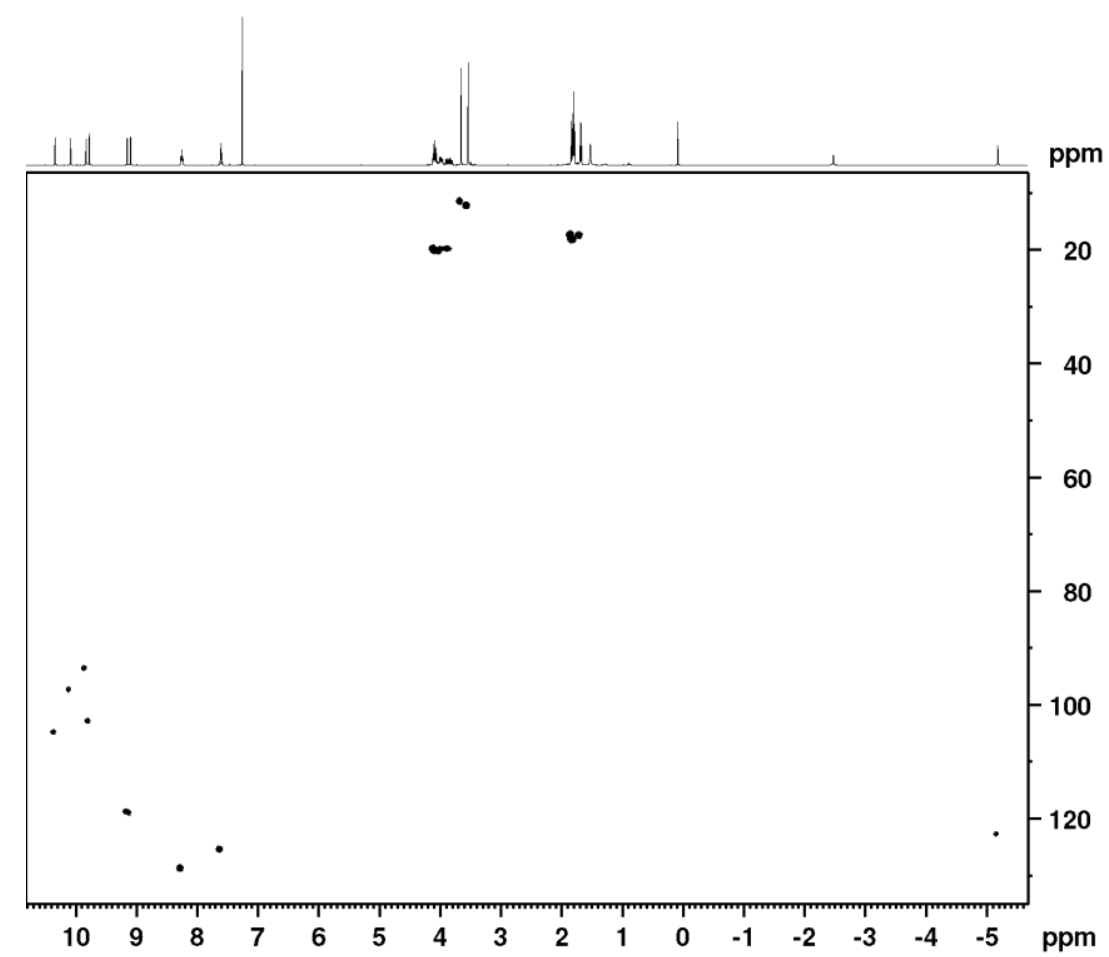

Figure C-28. HSQC NMR spectrum of rhodium(I) naphthocarbaporphyrin 91 in $\mathrm{CDCl}_{3}$. 


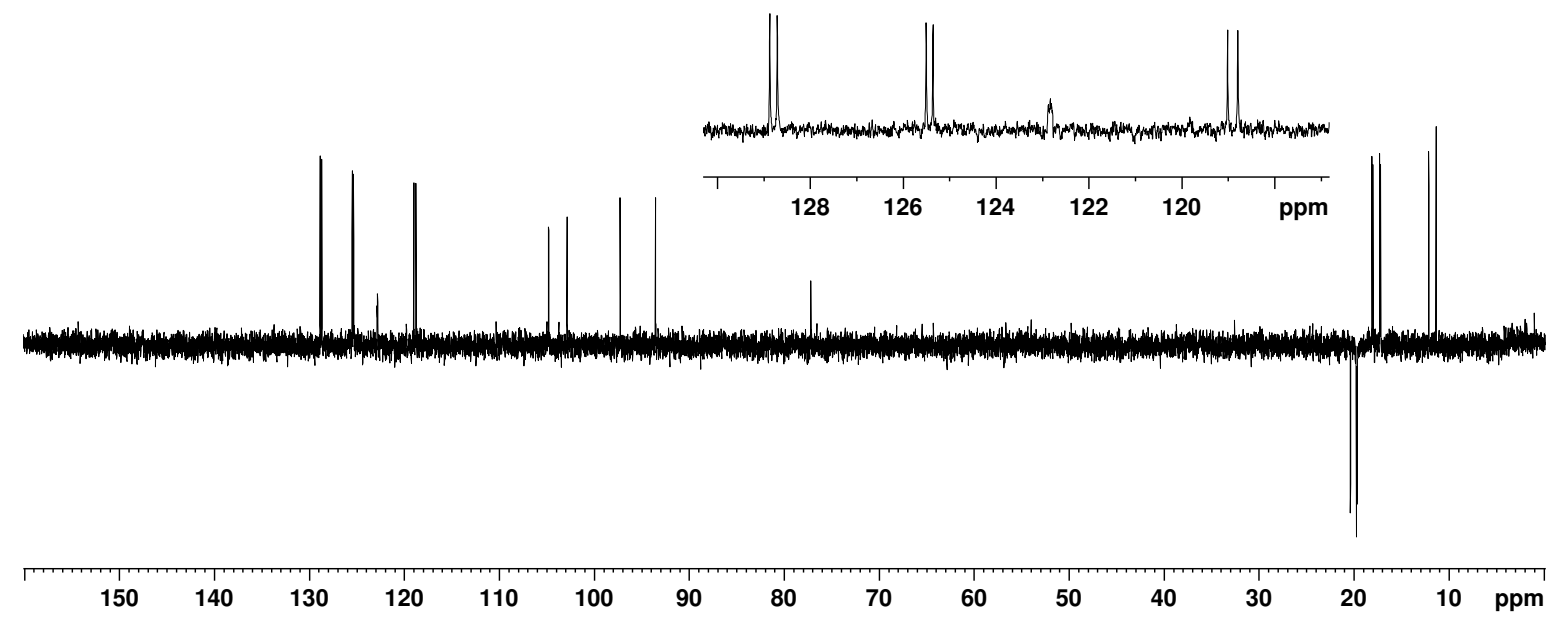

Figure C-29. DEPT-135 NMR spectrum of rhodium(I) naphthocarbaporphyrin 91 in $\mathrm{CDCl}_{3}$.

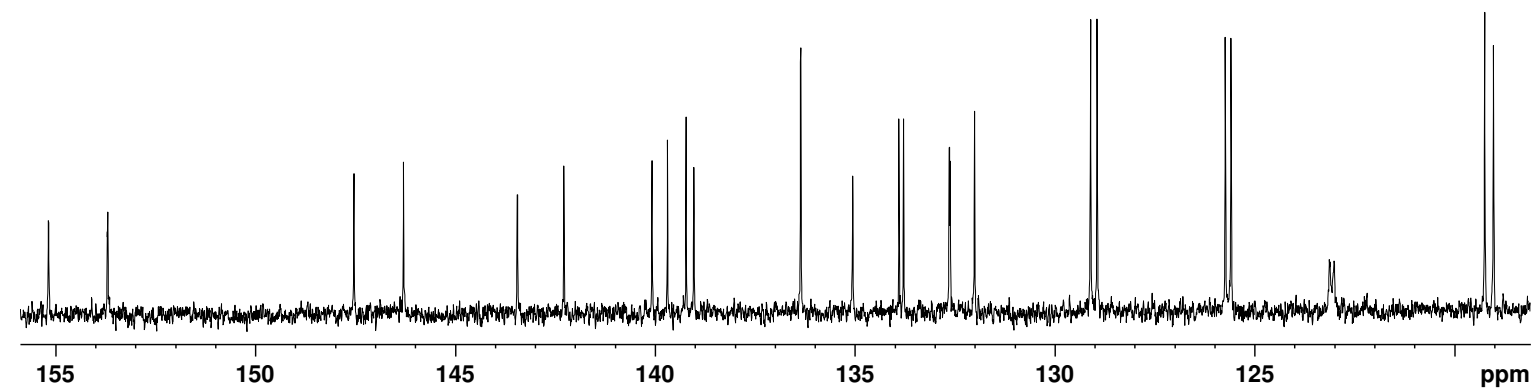

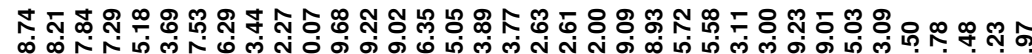

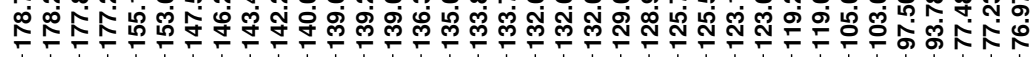

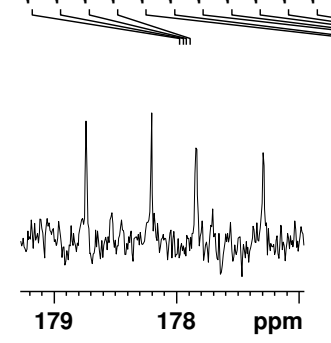

웅ำ

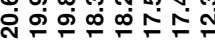
at

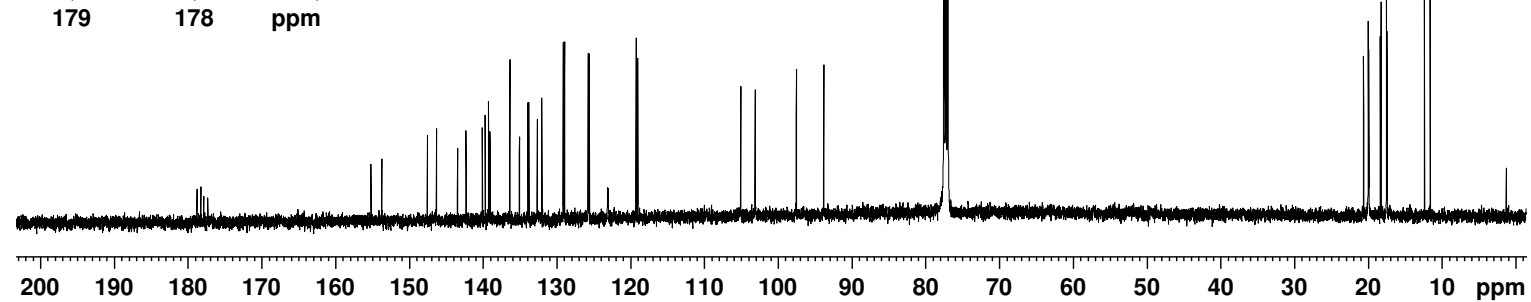

Figure C-30. $125 \mathrm{MHz}{ }^{13} \mathrm{C}$ NMR spectrum of rhodium(I) naphthocarbaporphyrin 91 in $\mathrm{CDCl}_{3}$. 

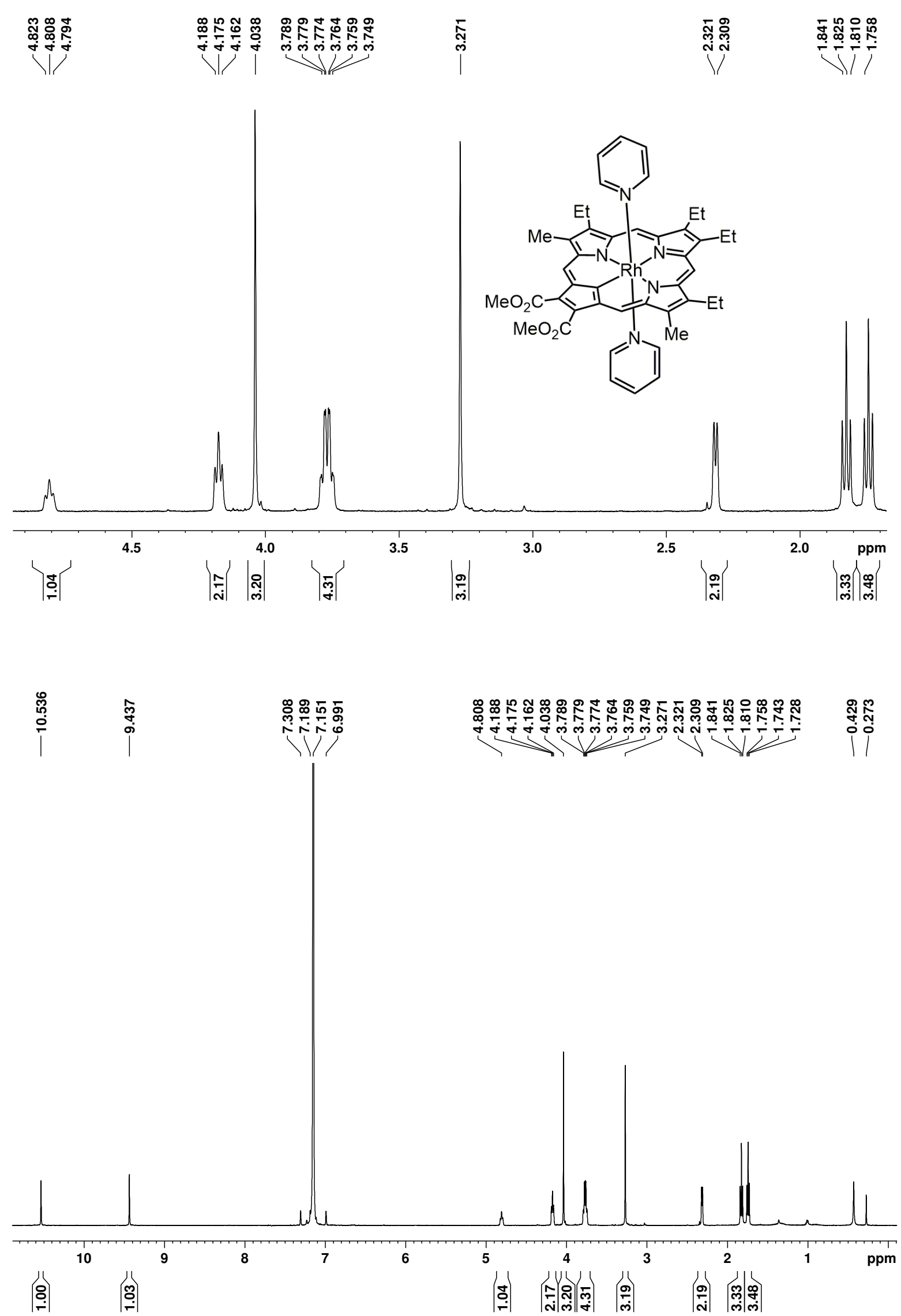

Figure C-31. $500 \mathrm{MHz}{ }^{1} \mathrm{H}$ NMR spectrum of rhodium(III) carbaporphyrin 90 in $\mathrm{C}_{6} \mathrm{D}_{6}$. 


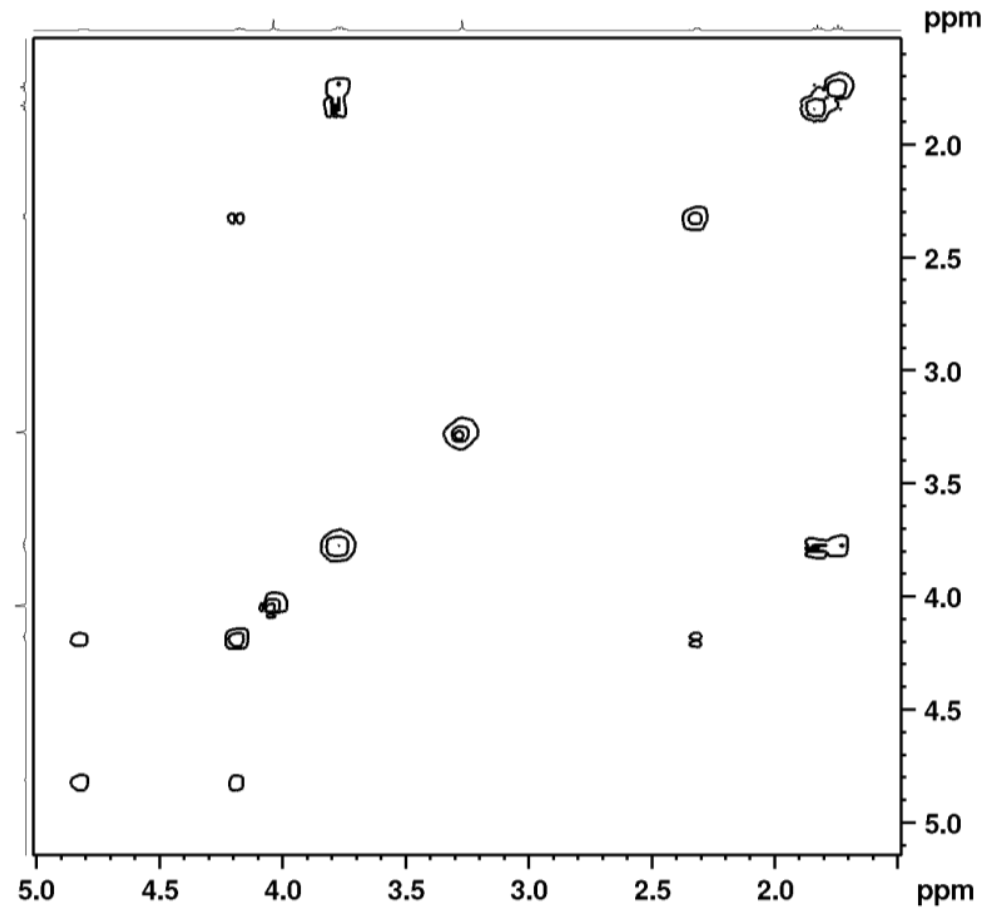

Figure C-32. ${ }^{1} \mathrm{H}-{ }^{1} \mathrm{H}$ COSY NMR spectrum of rhodium(III) carbaporphyrin 90 in $\mathrm{C}_{6} \mathrm{D}_{6}$ at 60 ${ }^{\circ} \mathrm{C}$.

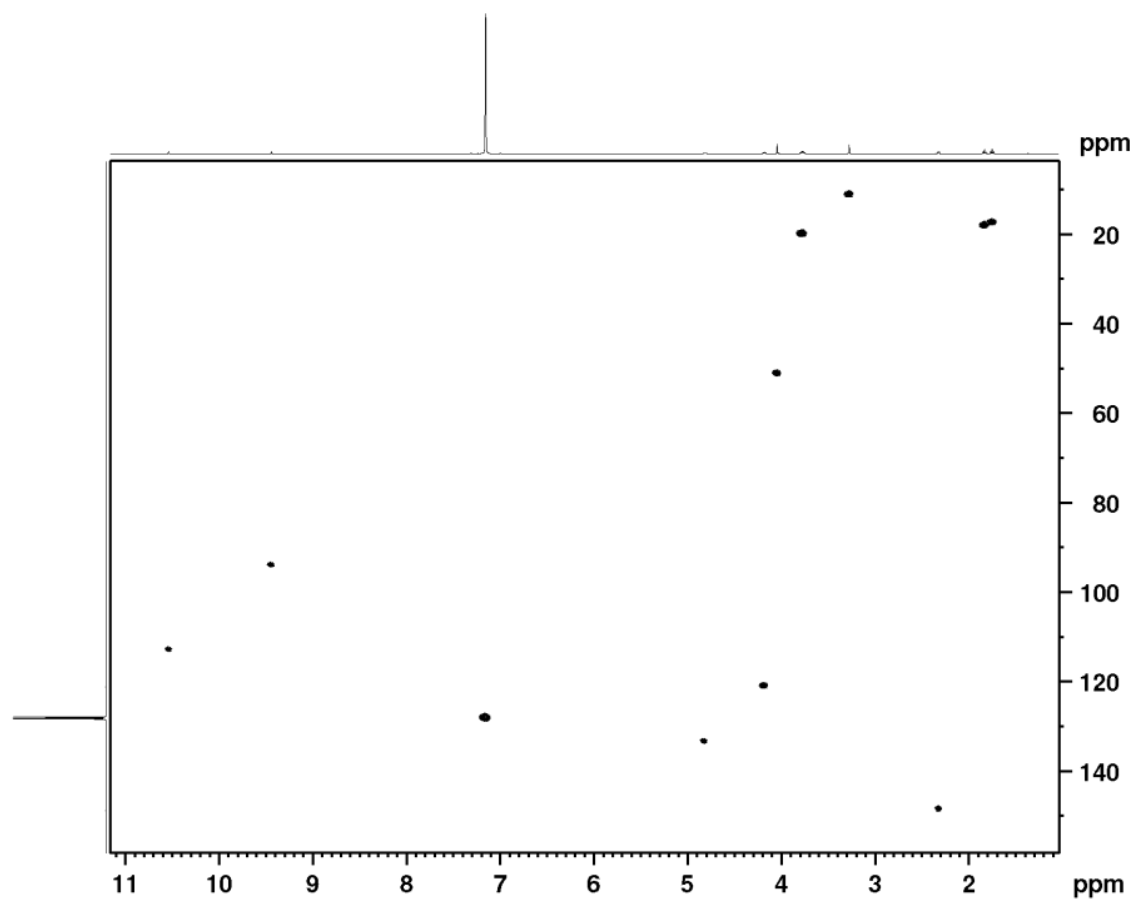

Figure C-33 HSQC NMR spectrum of rhodium(III) carbaporphyrin 90 in $\mathrm{C}_{6} \mathrm{D}_{6}$ at $60{ }^{\circ} \mathrm{C}$. 


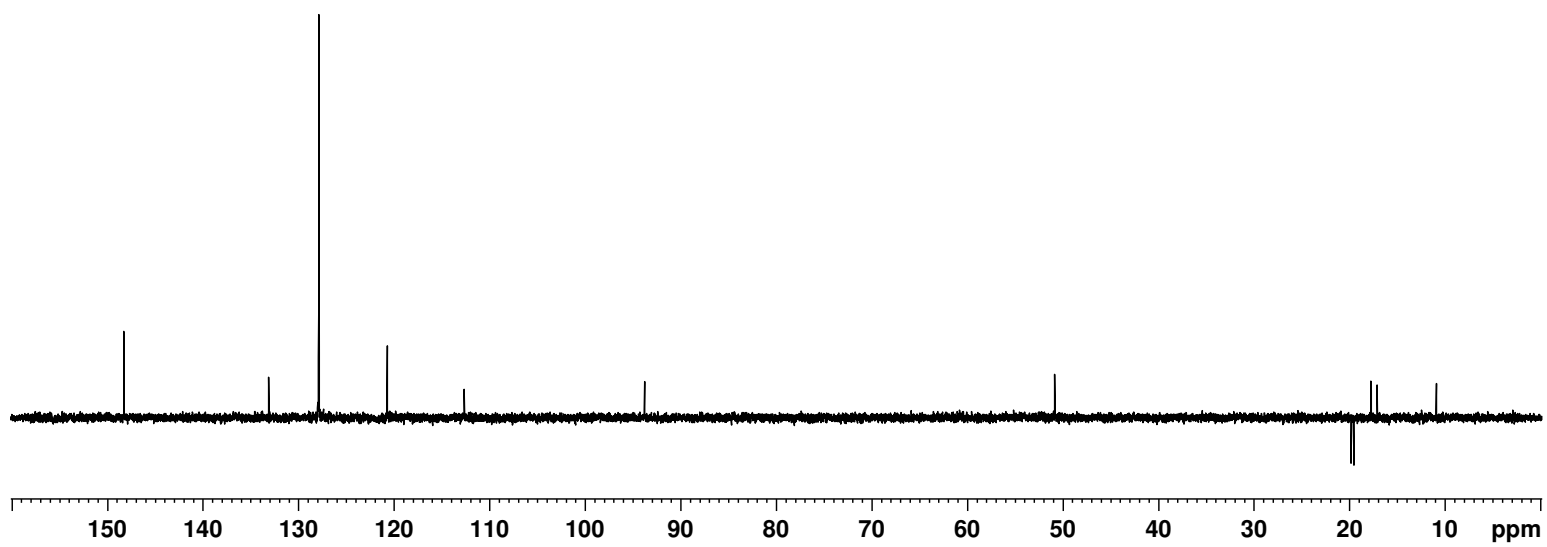

Figure C-34. DEPT-135 NMR spectrum of rhodium(III) carbaporphyrin 90 in $\mathrm{C}_{6} \mathrm{D}_{6}$.
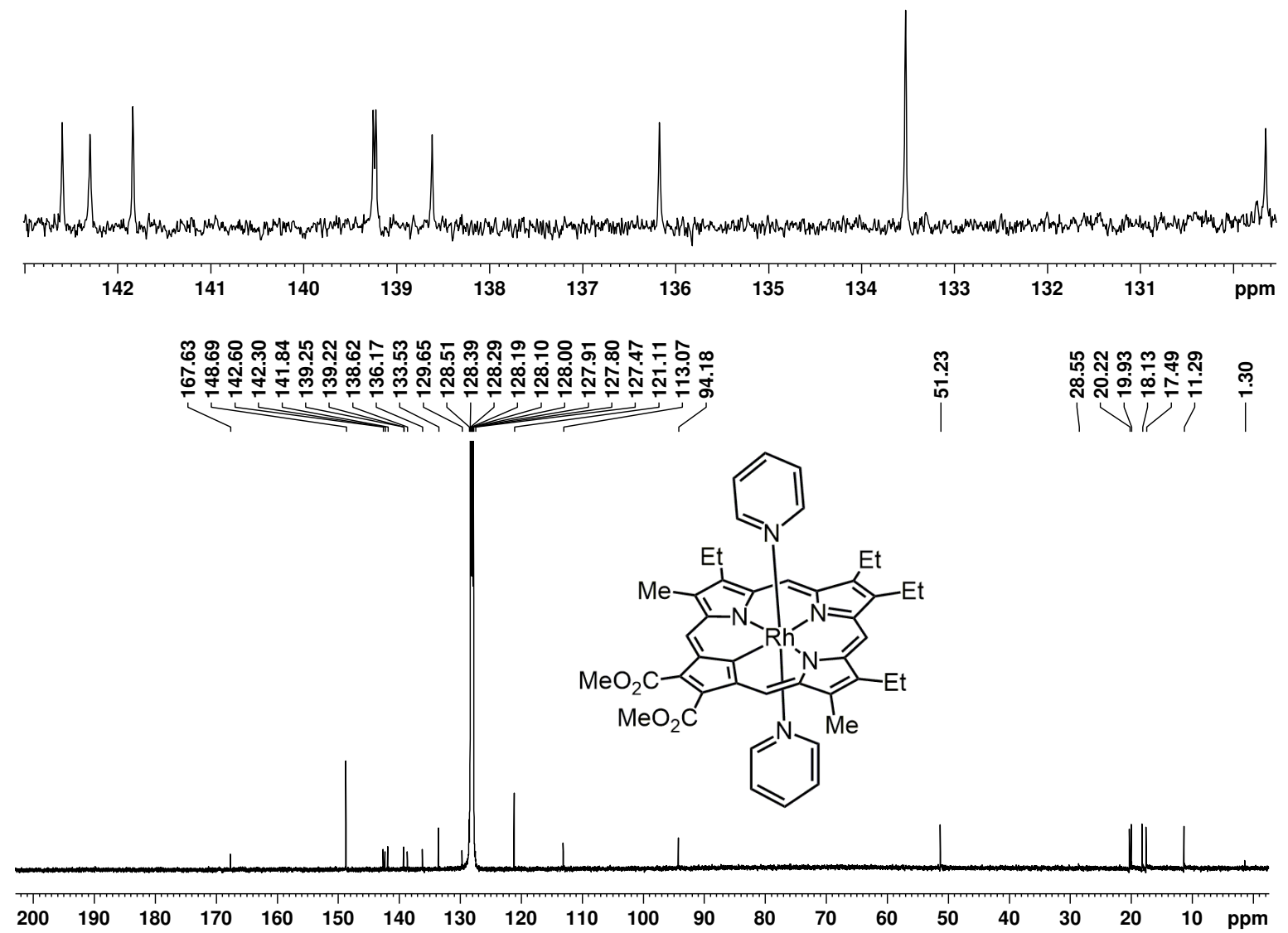

Figure C-35. $125 \mathrm{MHz}{ }^{13} \mathrm{C}$ NMR spectrum of rhodium(III) carbaporphyrin 90 in $\mathrm{C}_{6} \mathrm{D}_{6}$. 


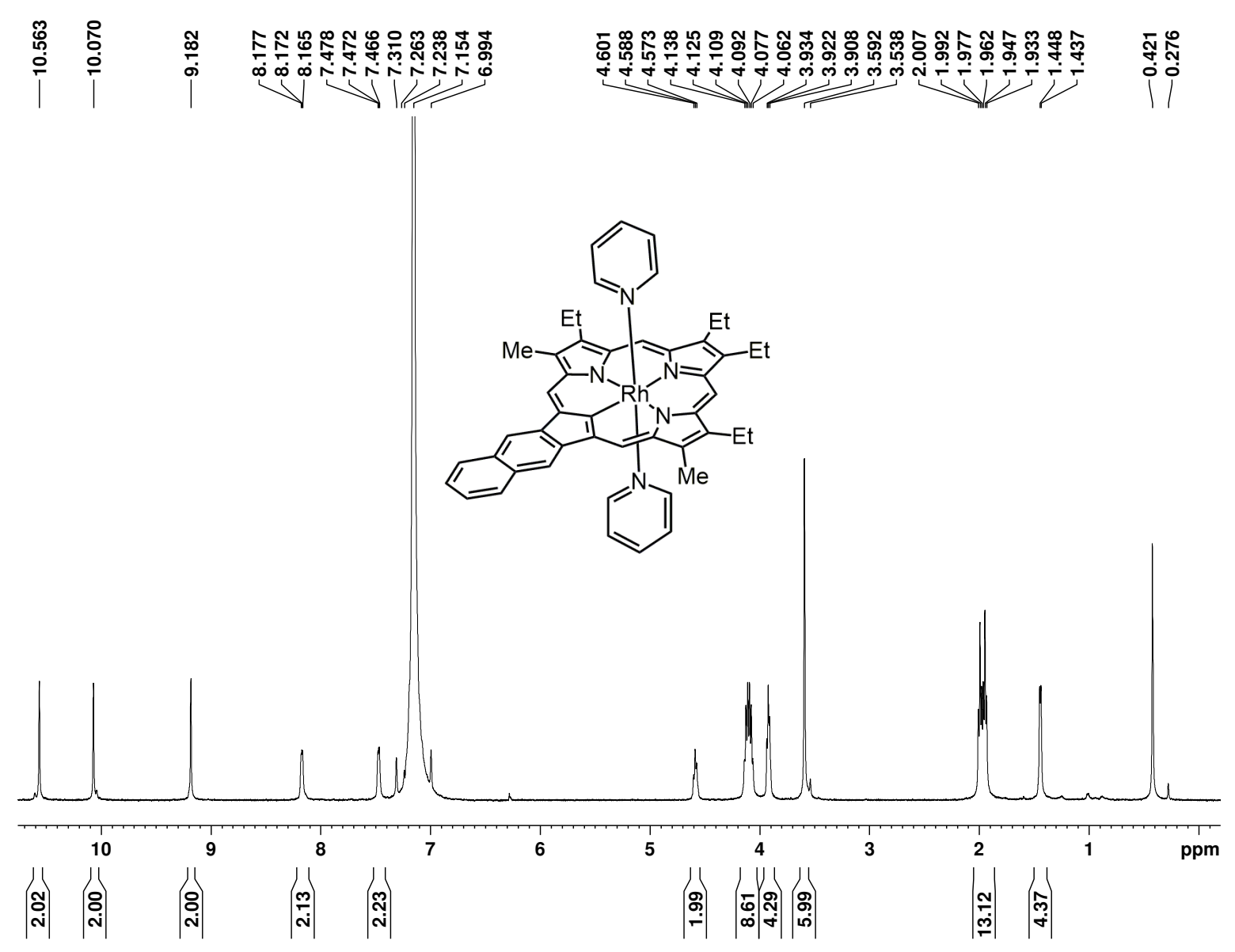

Figure C-36. $500 \mathrm{MHz}{ }^{1} \mathrm{H}$ NMR spectrum of rhodium(III) naphthocarbaporphyrin 92 in $d_{6}$-benzene at $60{ }^{\circ} \mathrm{C}$. 


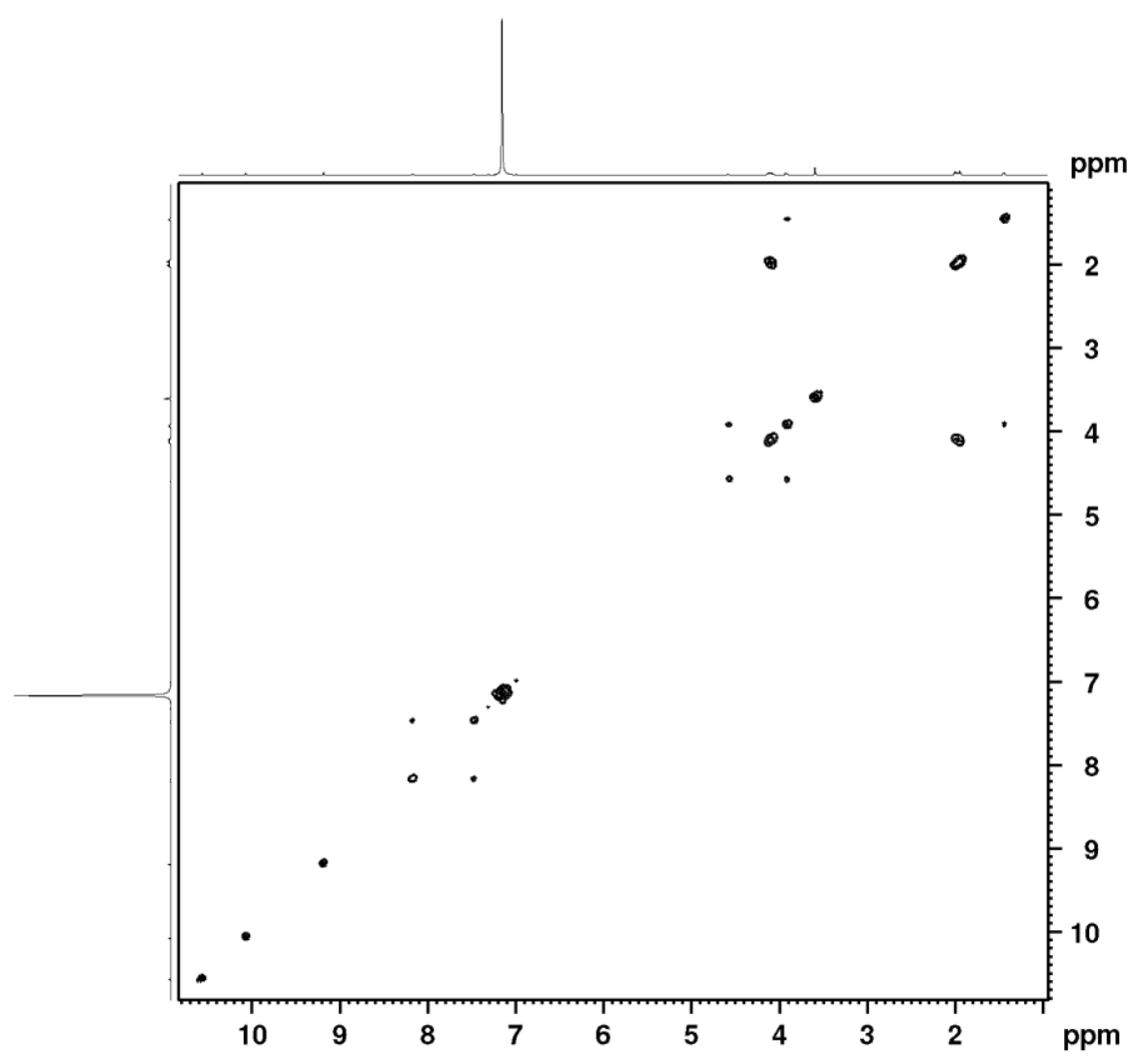

Figure C-37. ${ }^{1} \mathrm{H}-{ }^{1} \mathrm{H}$ COSY NMR spectrum of rhodium(III) naphthocarbaporphyrin 92 in $\mathrm{C}_{6} \mathrm{D}_{6}$ at $60{ }^{\circ} \mathrm{C}$.

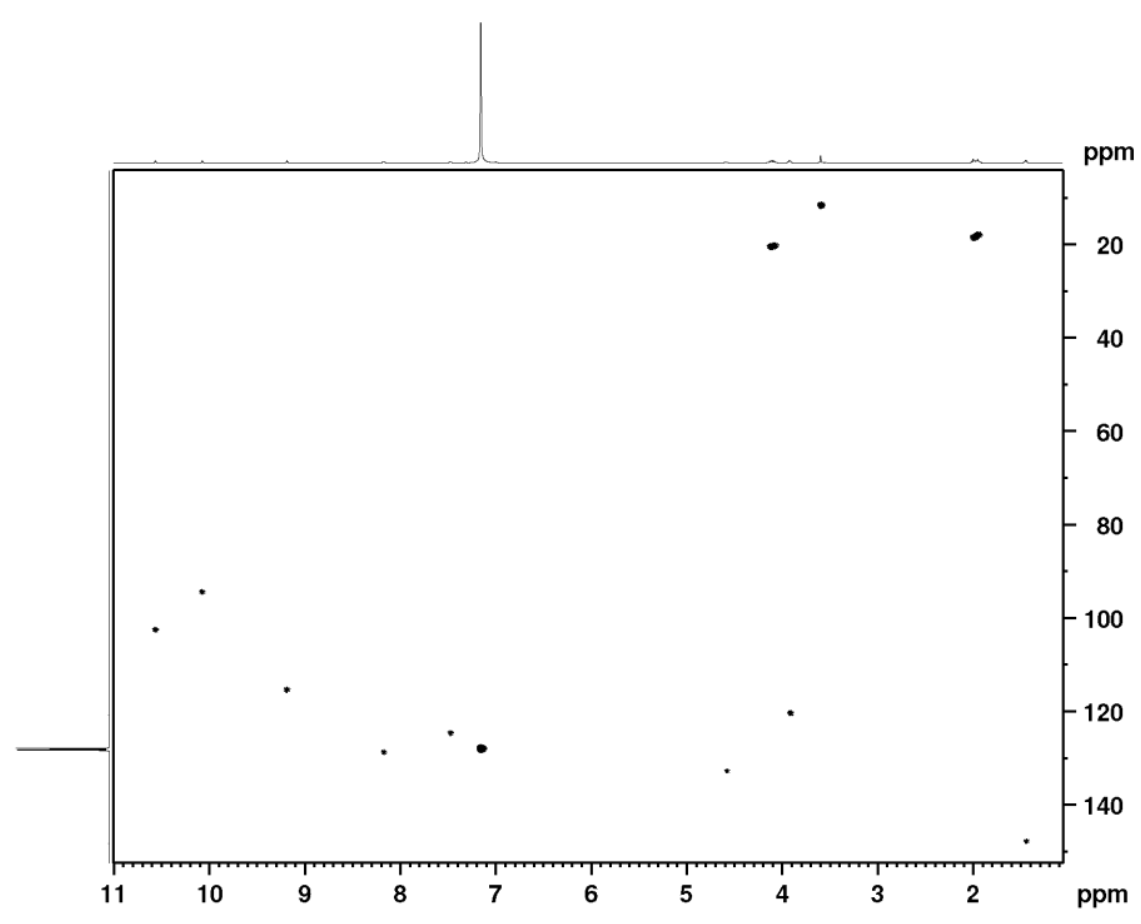

Figure C-38. HSQC NMR spectrum of rhodium(III) naphthocarbaporphyrin 92 in $\mathrm{C}_{6} \mathrm{D}_{6}$ at 60 ${ }^{\circ} \mathrm{C}$. 


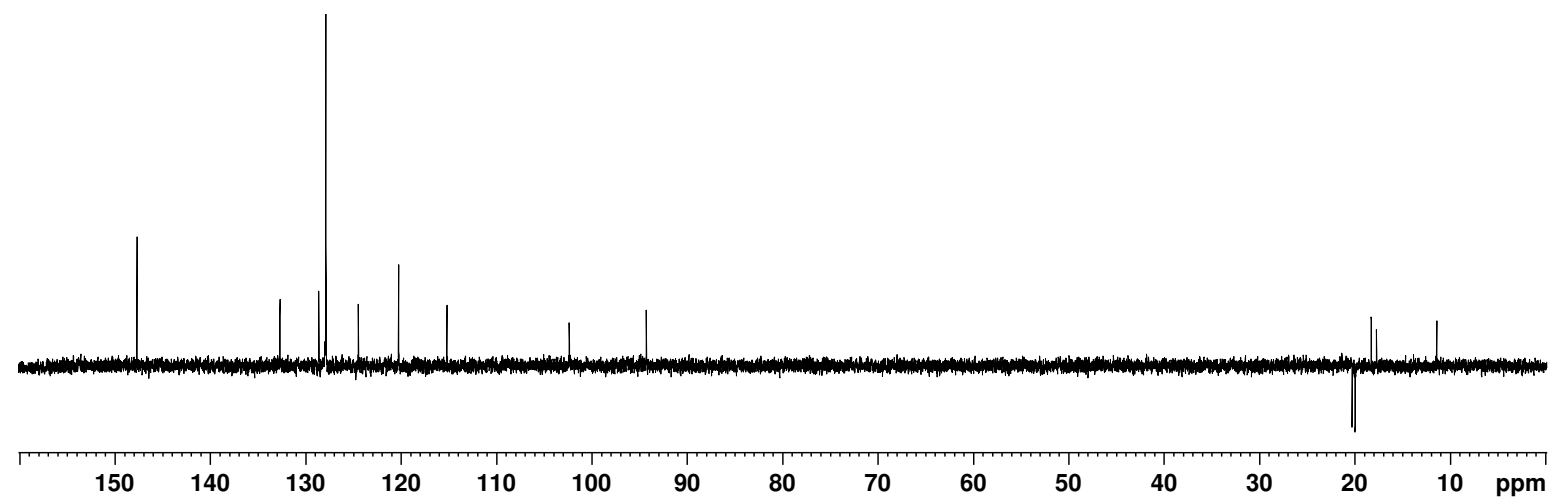

Figure C-39. DEPT-135 NMR spectrum of rhodium(III) naphthocarbaporphyrin 92 in $d_{\sigma^{-}}$ benzene at $60^{\circ} \mathrm{C}$.
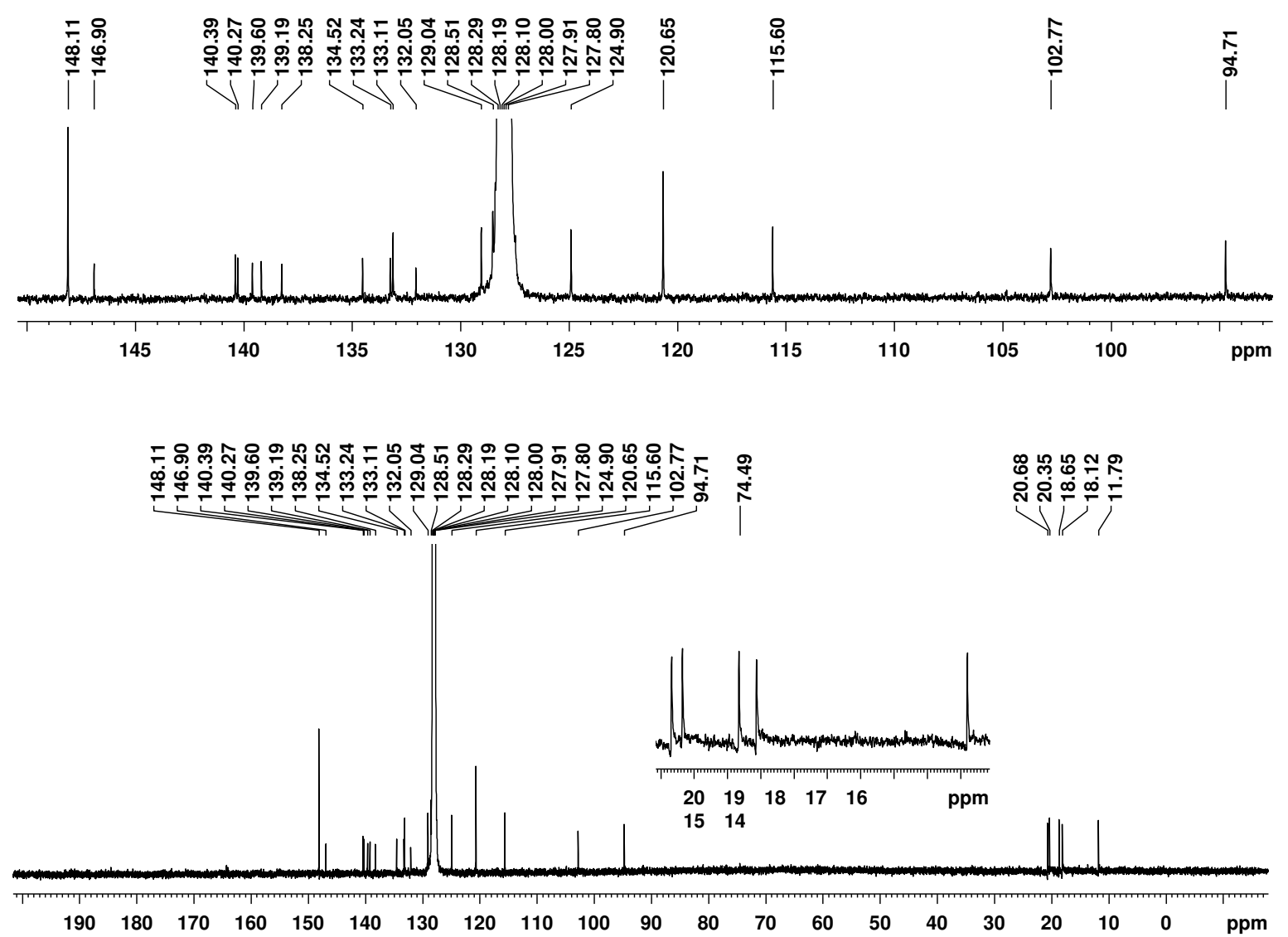

Figure C-40. $125 \mathrm{MHz}{ }^{13} \mathrm{C}$ NMR spectrum of rhodium(III) naphthocarbaporphyrin 92 in $d_{6}$-benzene at ${ }^{\circ} 60 \mathrm{C}$. 


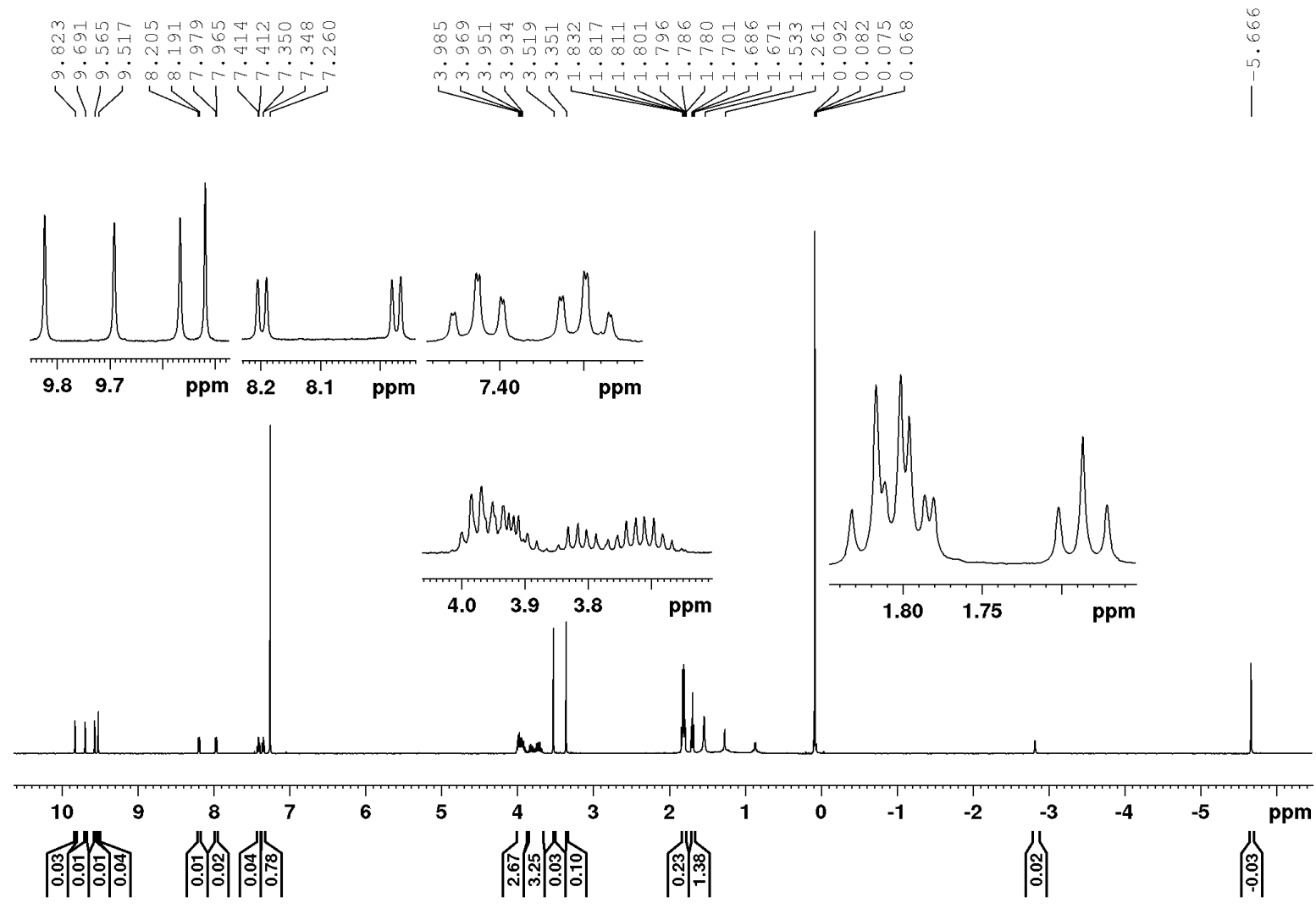

Figure C-41. $500 \mathrm{MHz}{ }^{1} \mathrm{H}$ NMR spectrum of rhodium(I) 21-methylcarbaporphyrin 94 in $\mathrm{CDCl}_{3}$. 


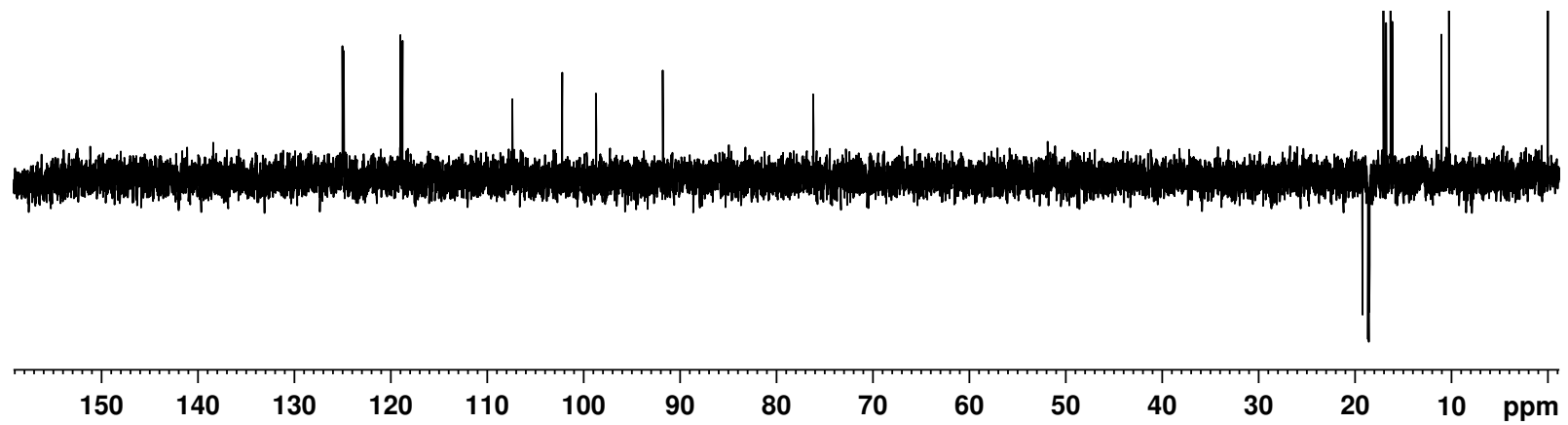

Figure C-42. DEPT-135 NMR spectrum of rhodium(I) 21-methylcarbaporphyrin 94 in $\mathrm{CDCl}_{3}$.

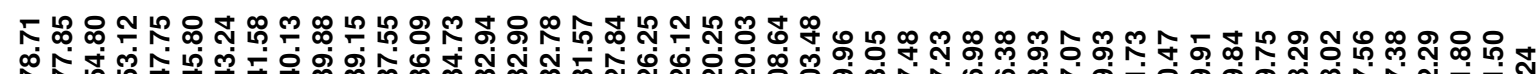

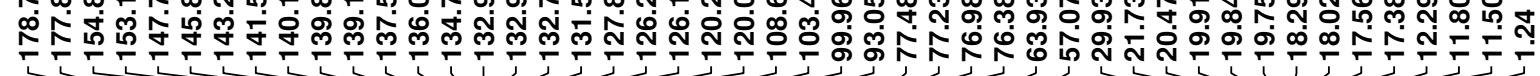

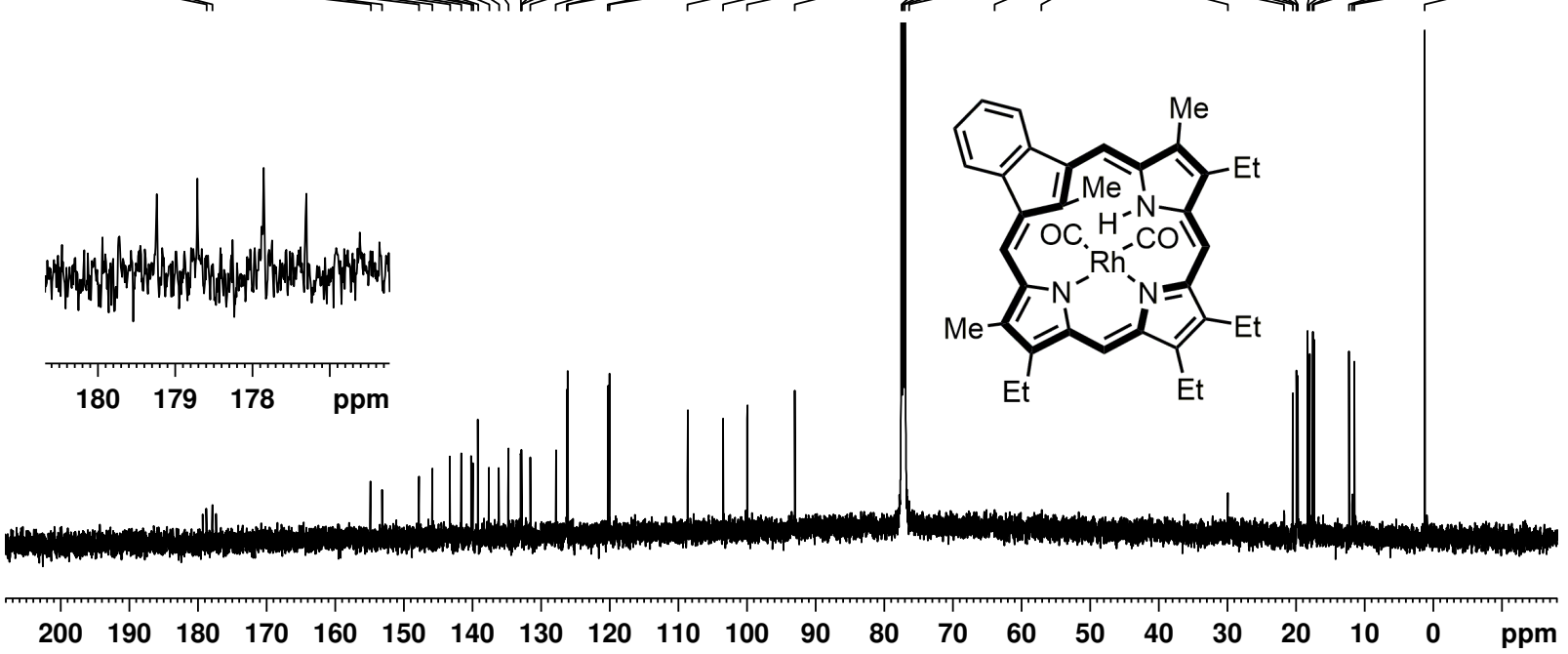

Figure C-43. $125 \mathrm{MHz}$ carbon-13 NMR spectrum of rhodium(I) 21-methylcarbaporphyrin 94 in $\mathrm{CDCl}_{3}$. 


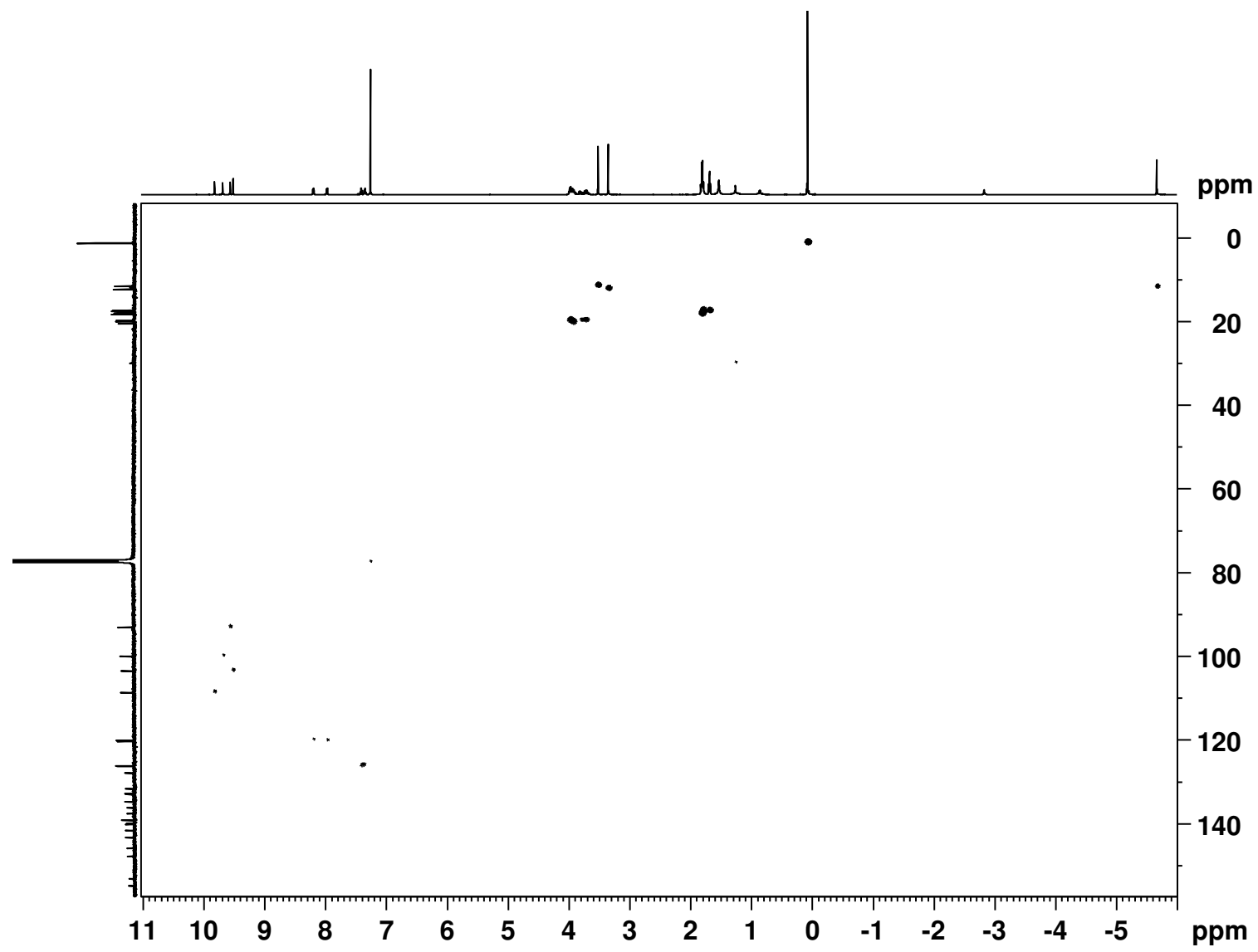

Figure C-44. HSQC NMR spectrum of rhodium(I) 21-methylcarbaporphyrin 94 in $\mathrm{CDCl}_{3}$. 


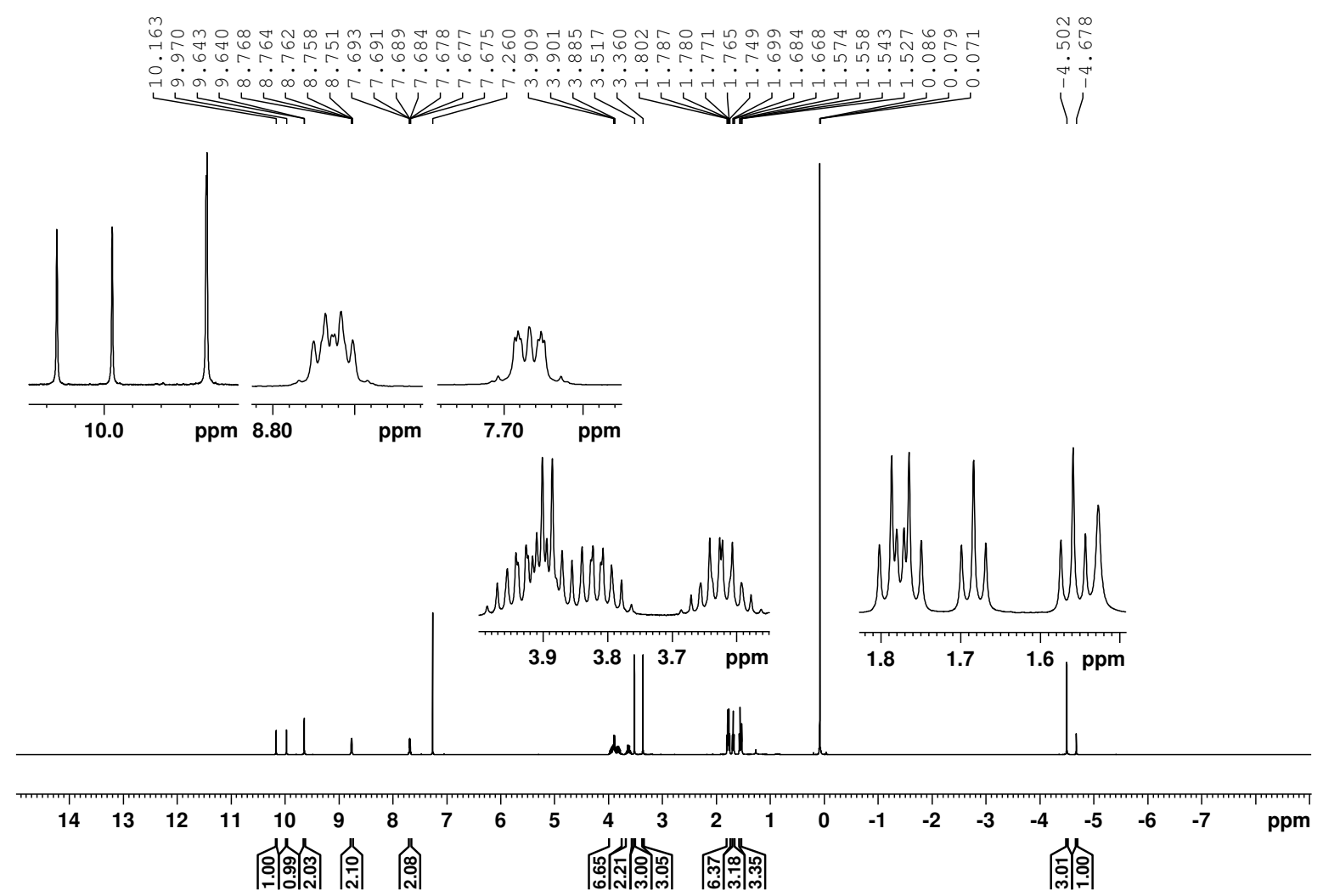

Figure C-45. $500 \mathrm{MHz}{ }^{1} \mathrm{H}$ NMR spectrum of rhodium(I) 22-methylcarbaporphyrin 93 in $\mathrm{CDCl}_{3}$. 


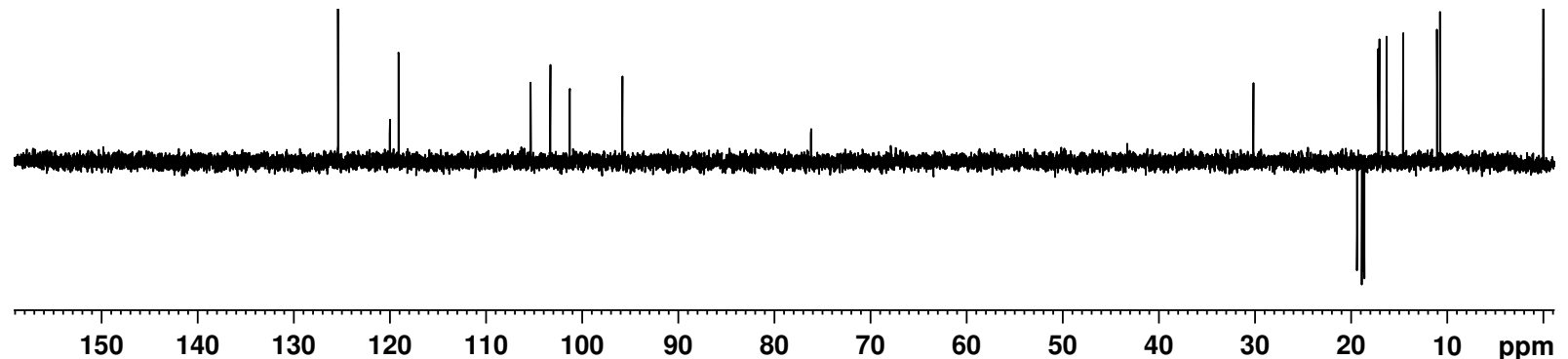

Figure C-46. DEPT-135 NMR spectrum of rhodium(I) 22-methylcarbaporphyrin 93 in $\mathrm{CDCl}_{3}$.

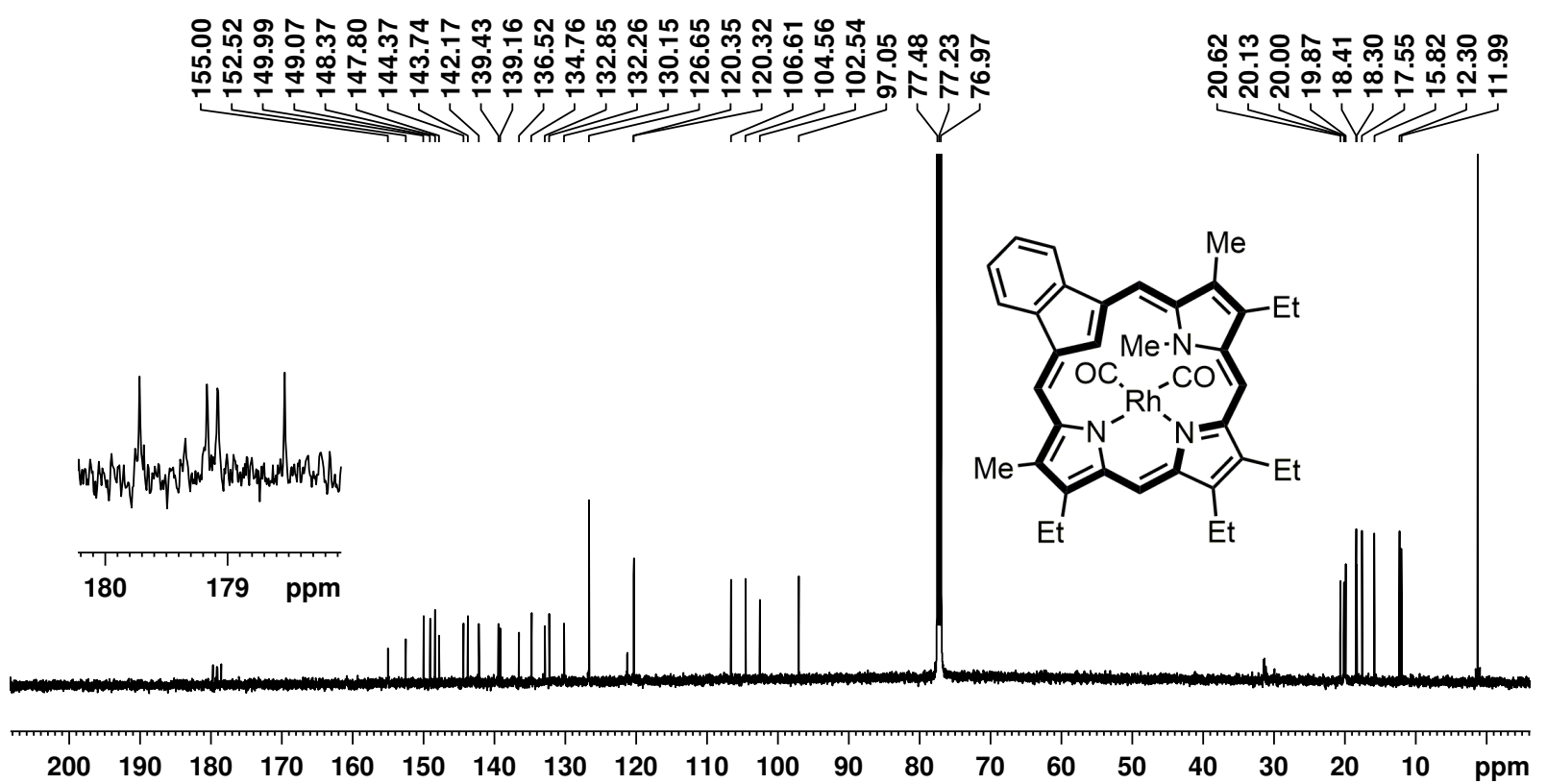

Figure C-47. $125 \mathrm{MHz}{ }^{13} \mathrm{C}$ NMR spectrum of rhodium(I) 22-methylcarbaporphyrin 93 in $\mathrm{CDCl}_{3}$. 


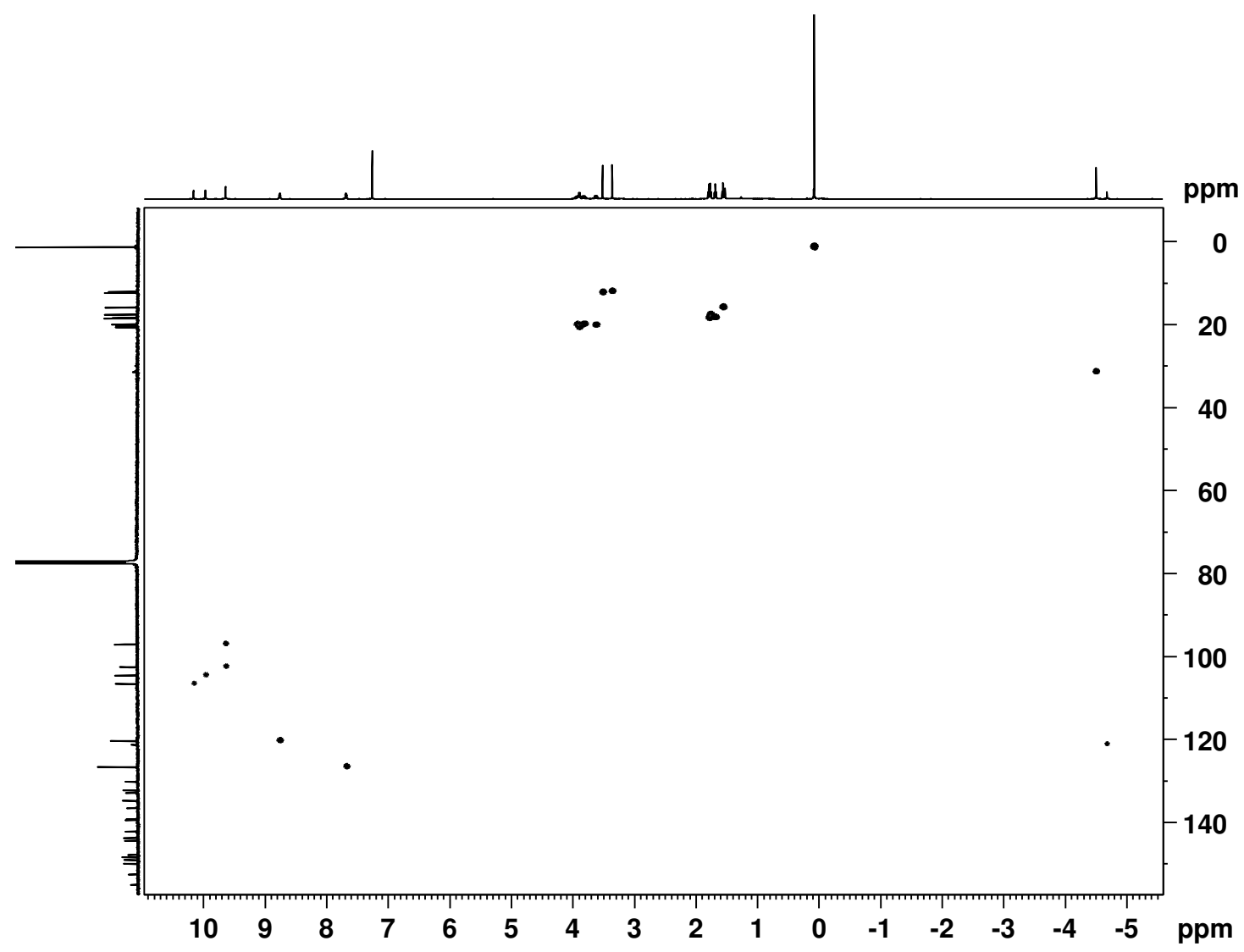

Figure C-48. HSQC NMR spectrum of rhodium(I) 22-methylcarbaporphyrin 93 in $\mathrm{CDCl}_{3}$. 


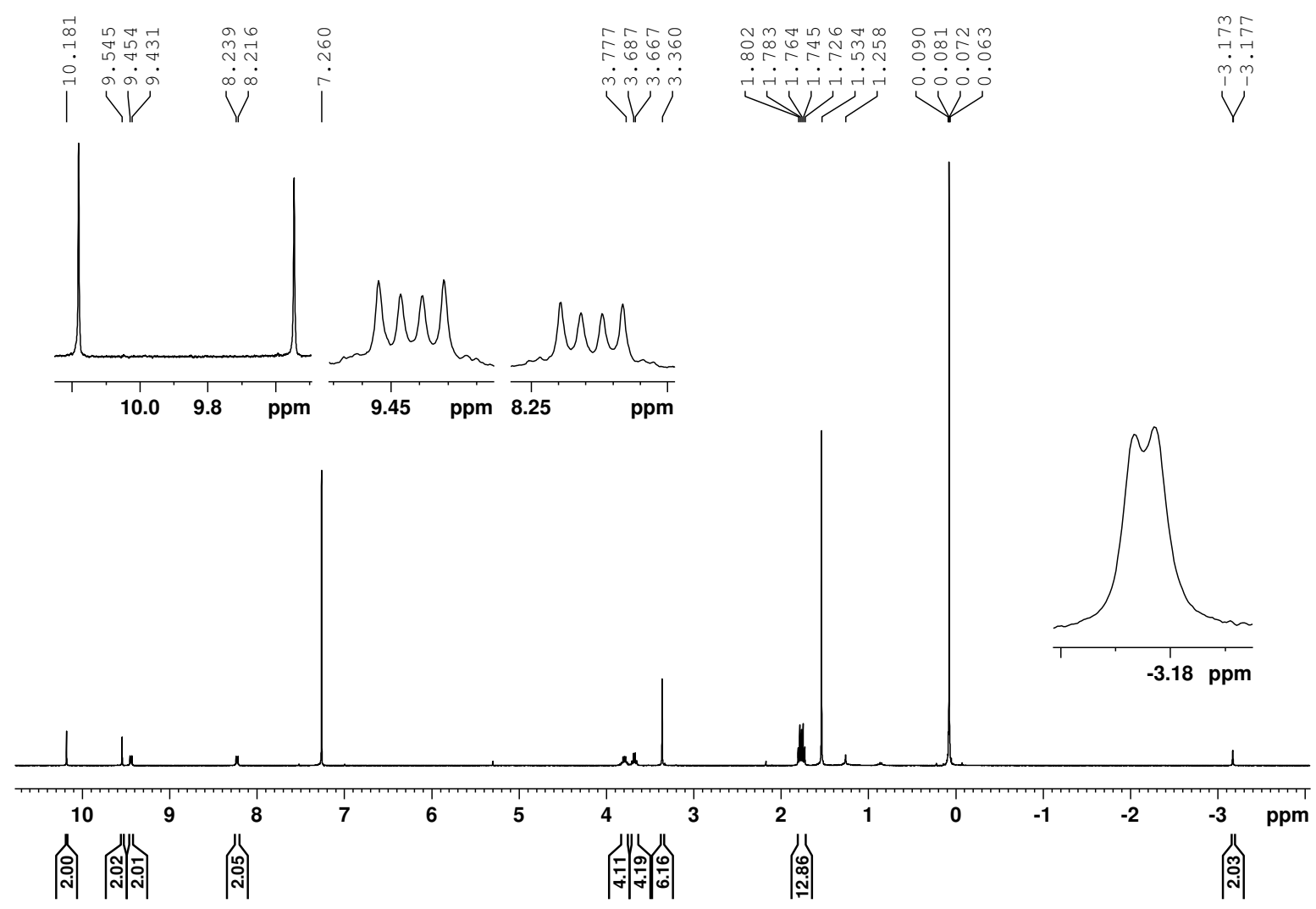

Figure C-49. $400 \mathrm{MHz}{ }^{1} \mathrm{H}$ NMR spectrum of rhodium(III) methylene-bridged complex 95 in $\mathrm{CDCl}_{3}$. 


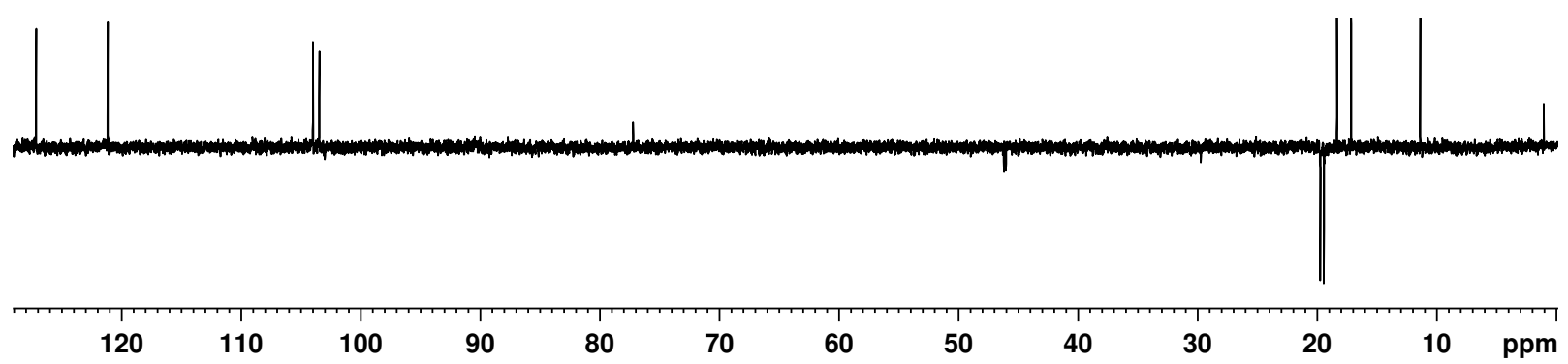

Figure C-50. DEPT-135 NMR spectrum of rhodium(III) methylene-bridged complex 95 in $\mathrm{CDCl}_{3}$.

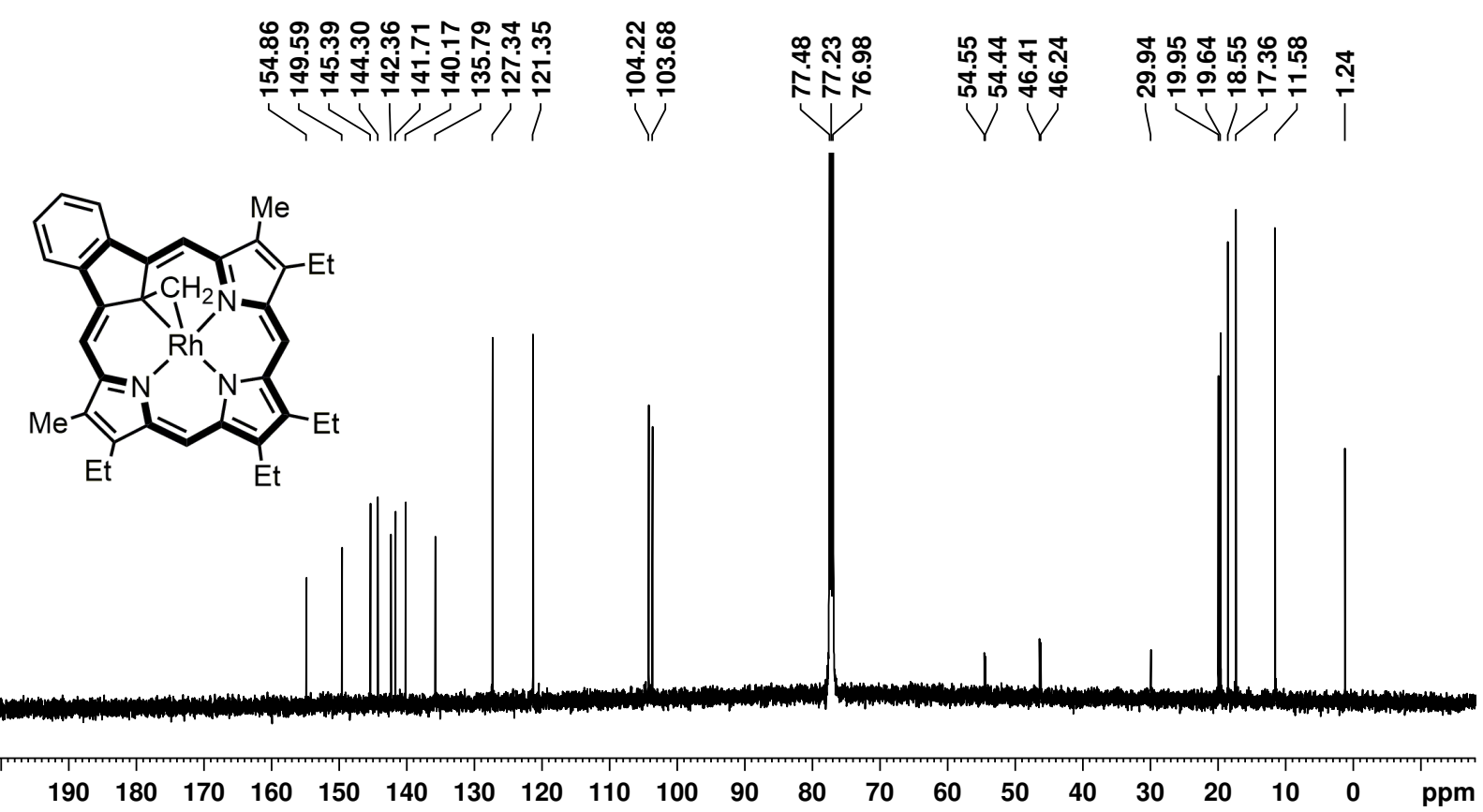

Figure C-51. $125 \mathrm{MHz}{ }^{13} \mathrm{C}$ NMR spectrum of rhodium(III) methylene-bridged complex 95 in $\mathrm{CDCl}_{3}$. 


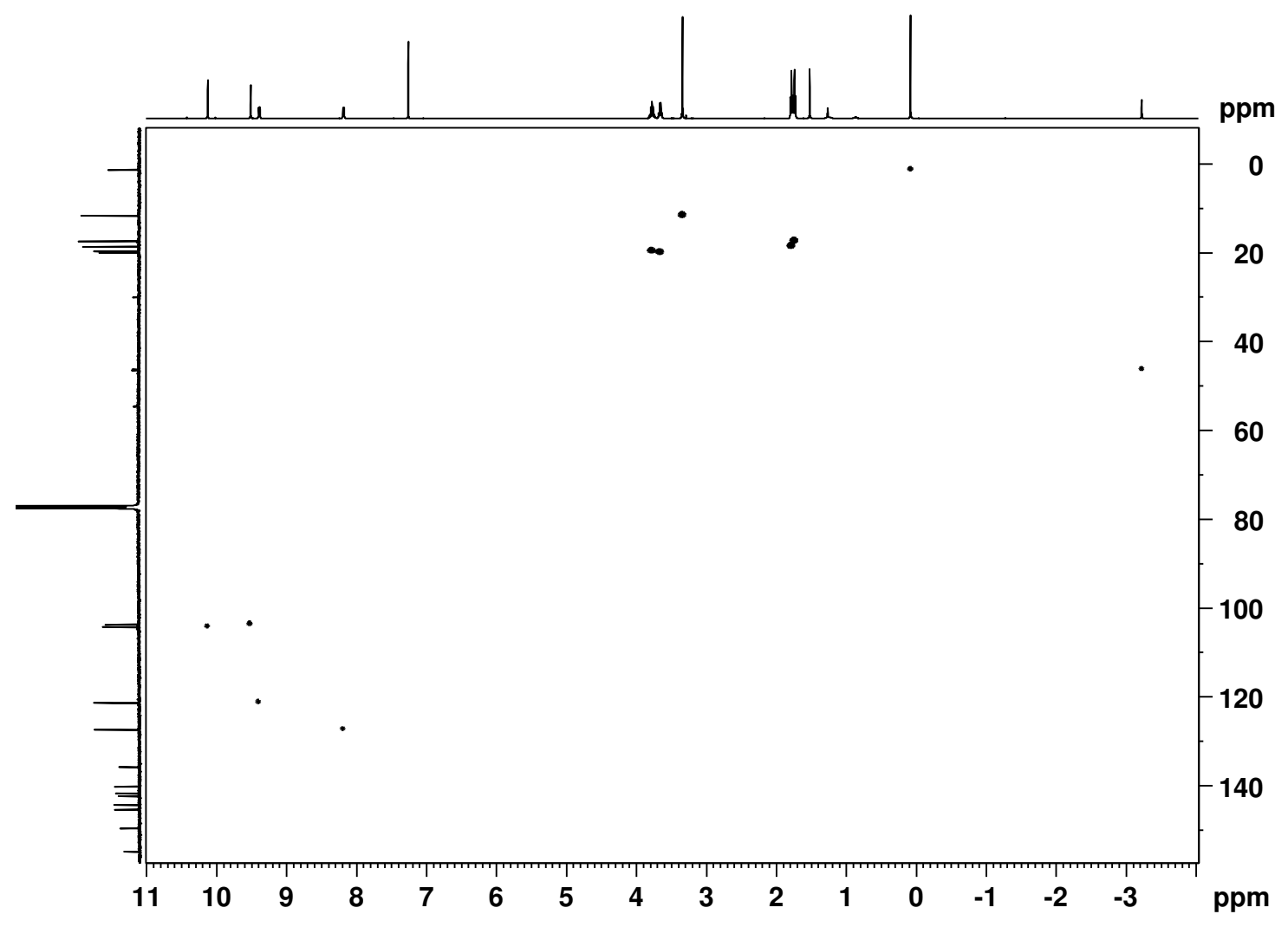

Figure C-52. HSQC NMR spectrum of rhodium(III) methylene-bridged complex 95 in $\mathrm{CDCl}_{3}$. 


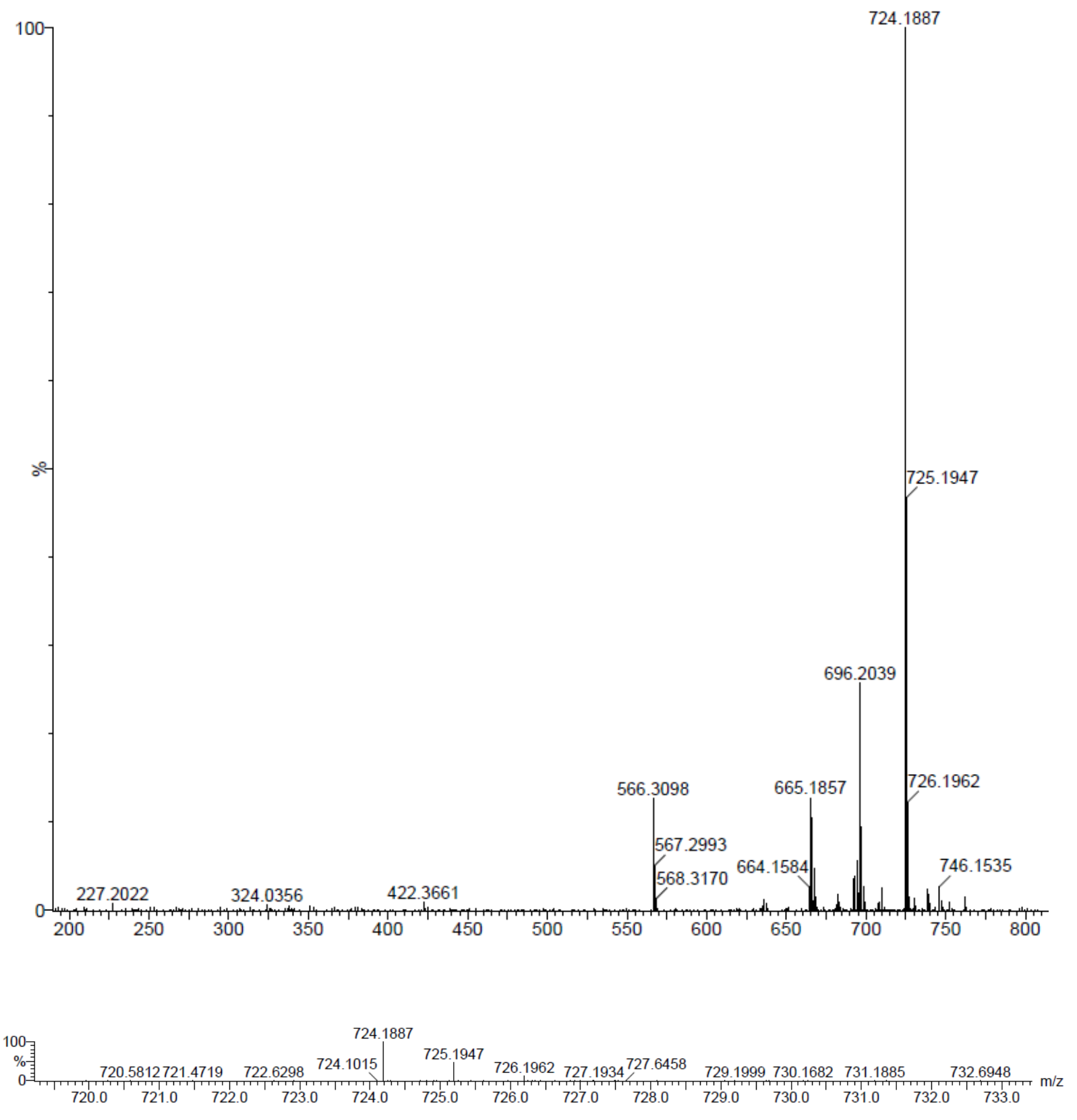

Figure C-53. ESI MS of rhodium(I) carbaporphyrin 89. 

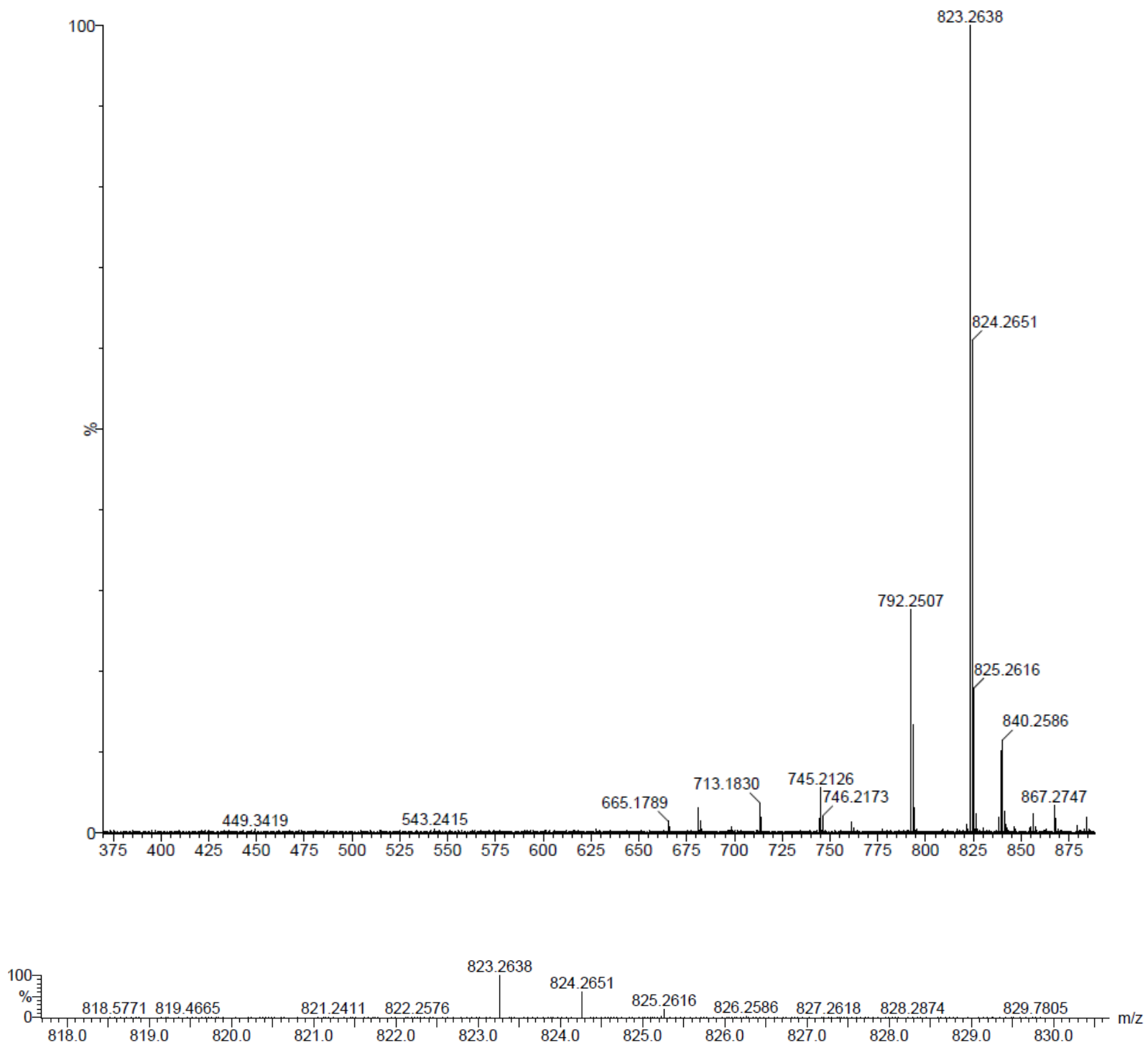

Figure C-54. ESI MS of rhodium(I) carbaporphyrin 91. 

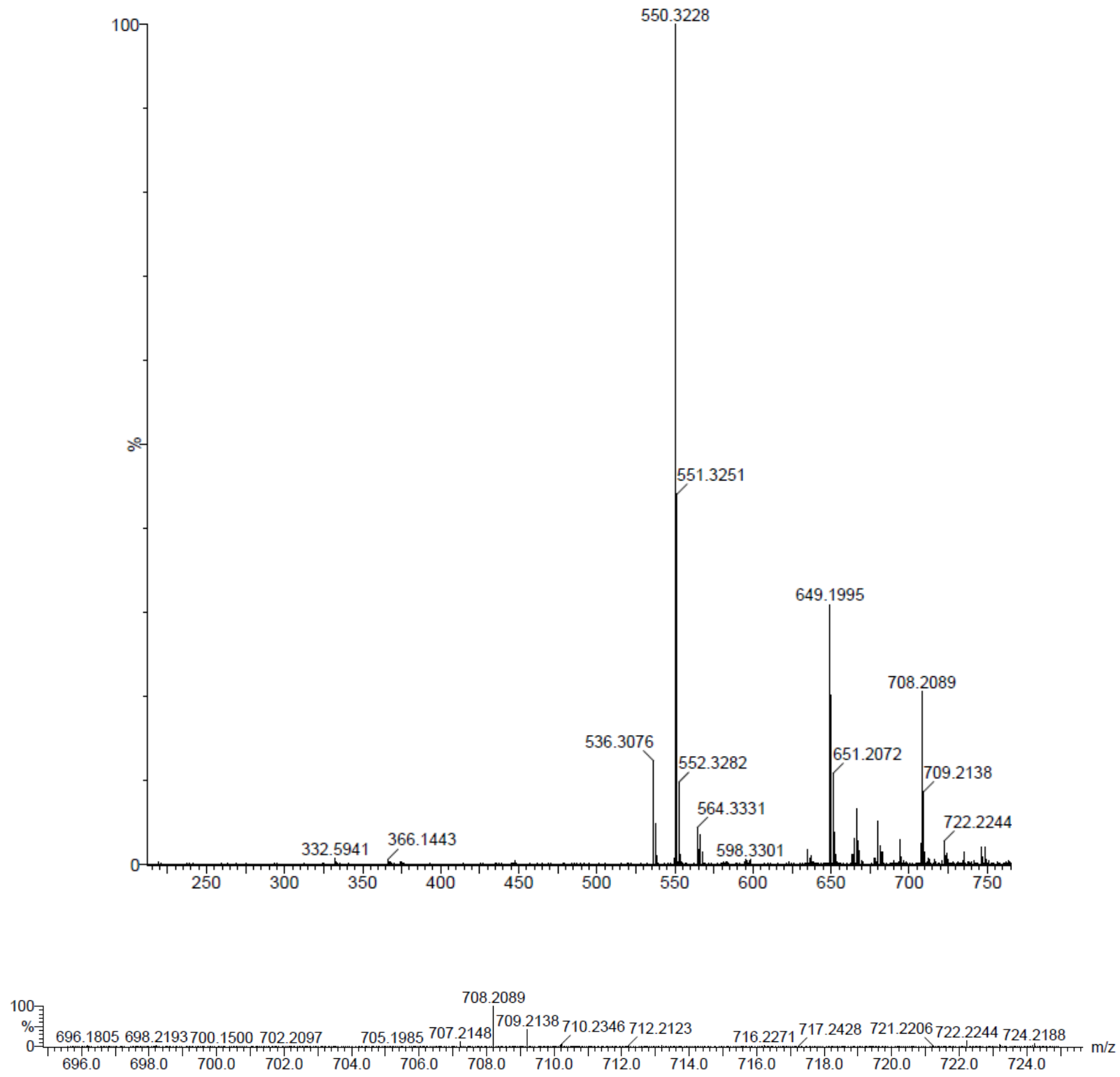

Figure C-55. ESI MS of rhodium(III) carbaporphyrin diester 91. 


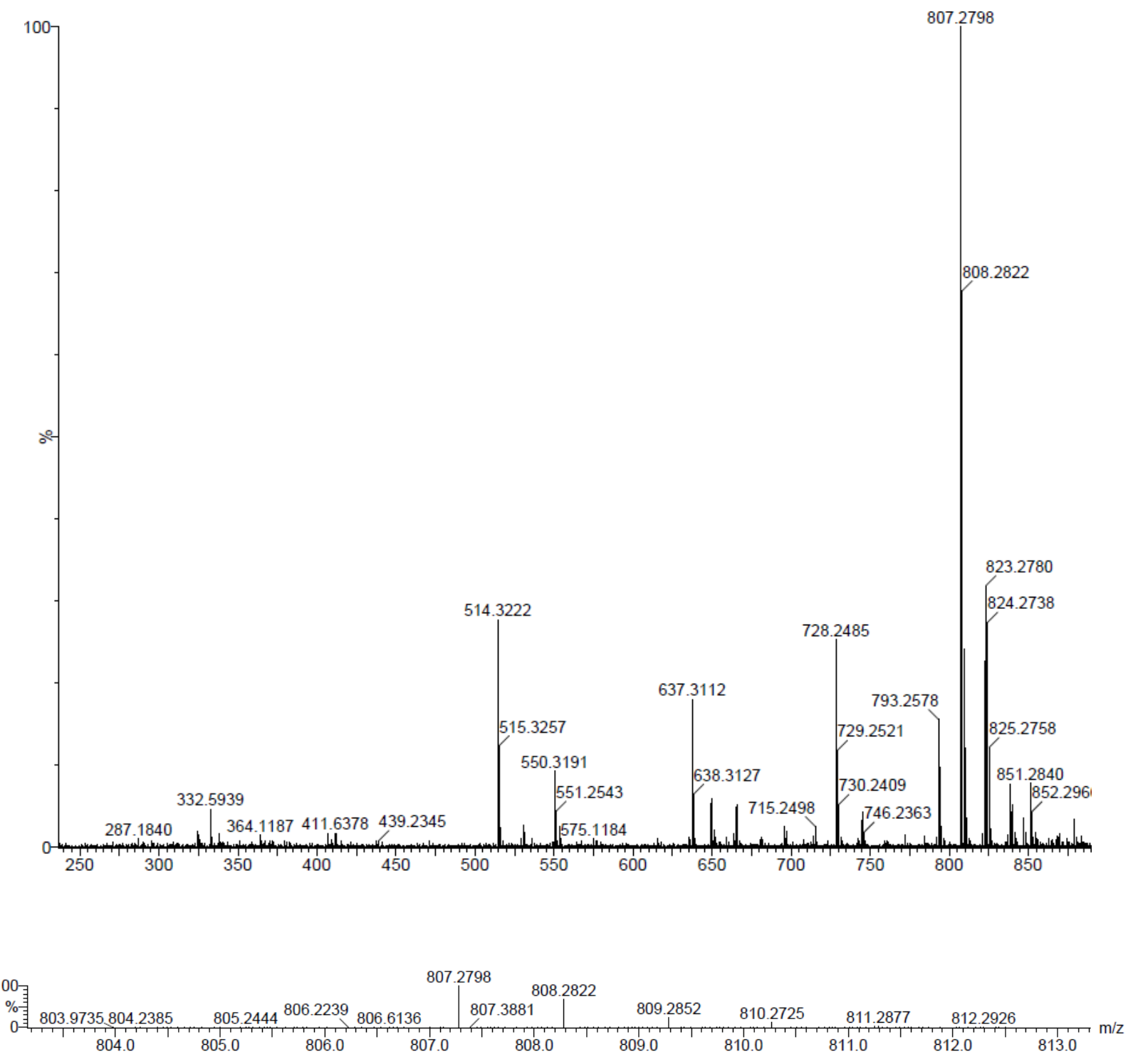

Figure C-56. ESI MS of rhodium(III) naphthocarbaporphyrin 92. 

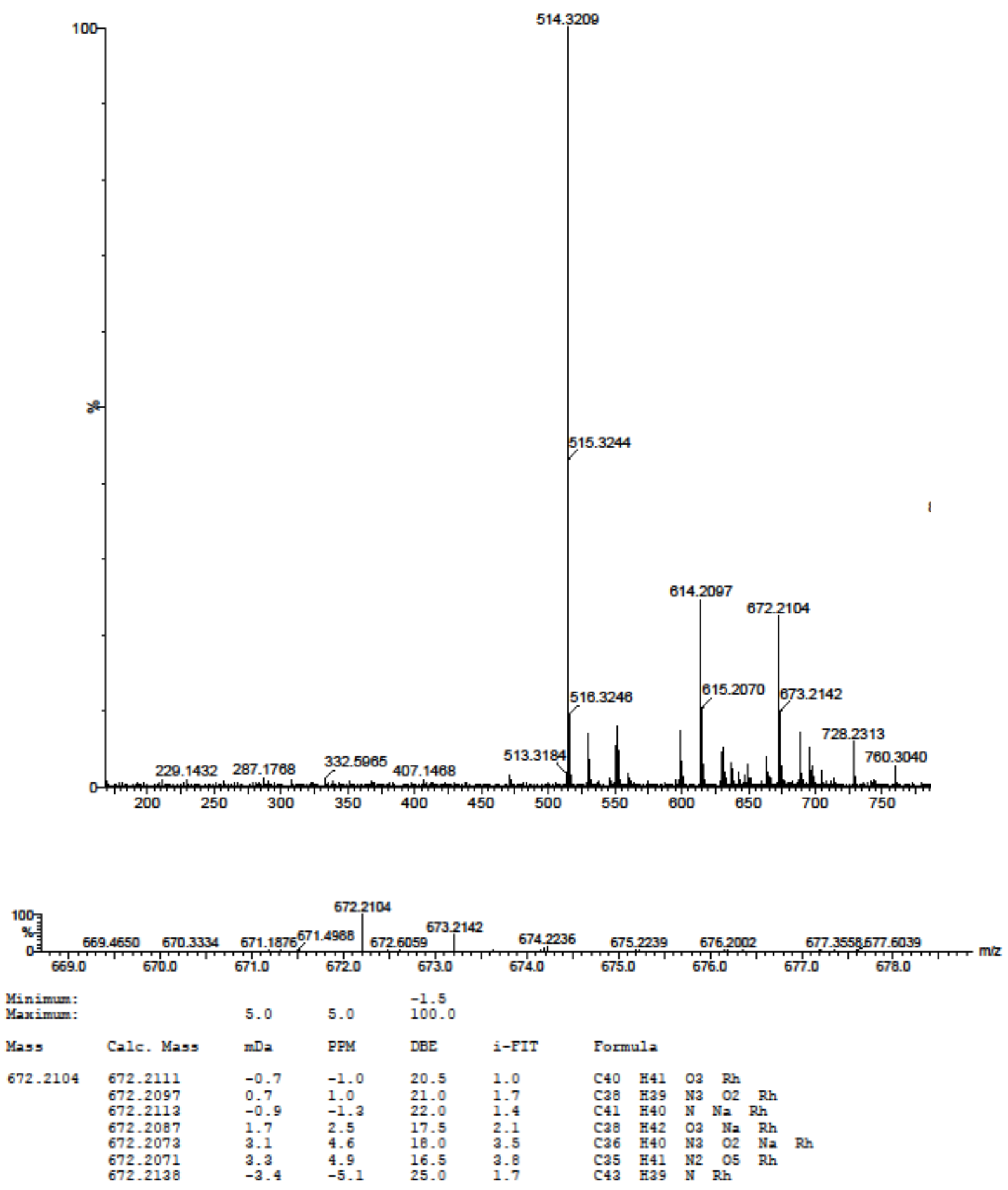

Figure C-57. ESI MS of rhodium(I) complex 94. 

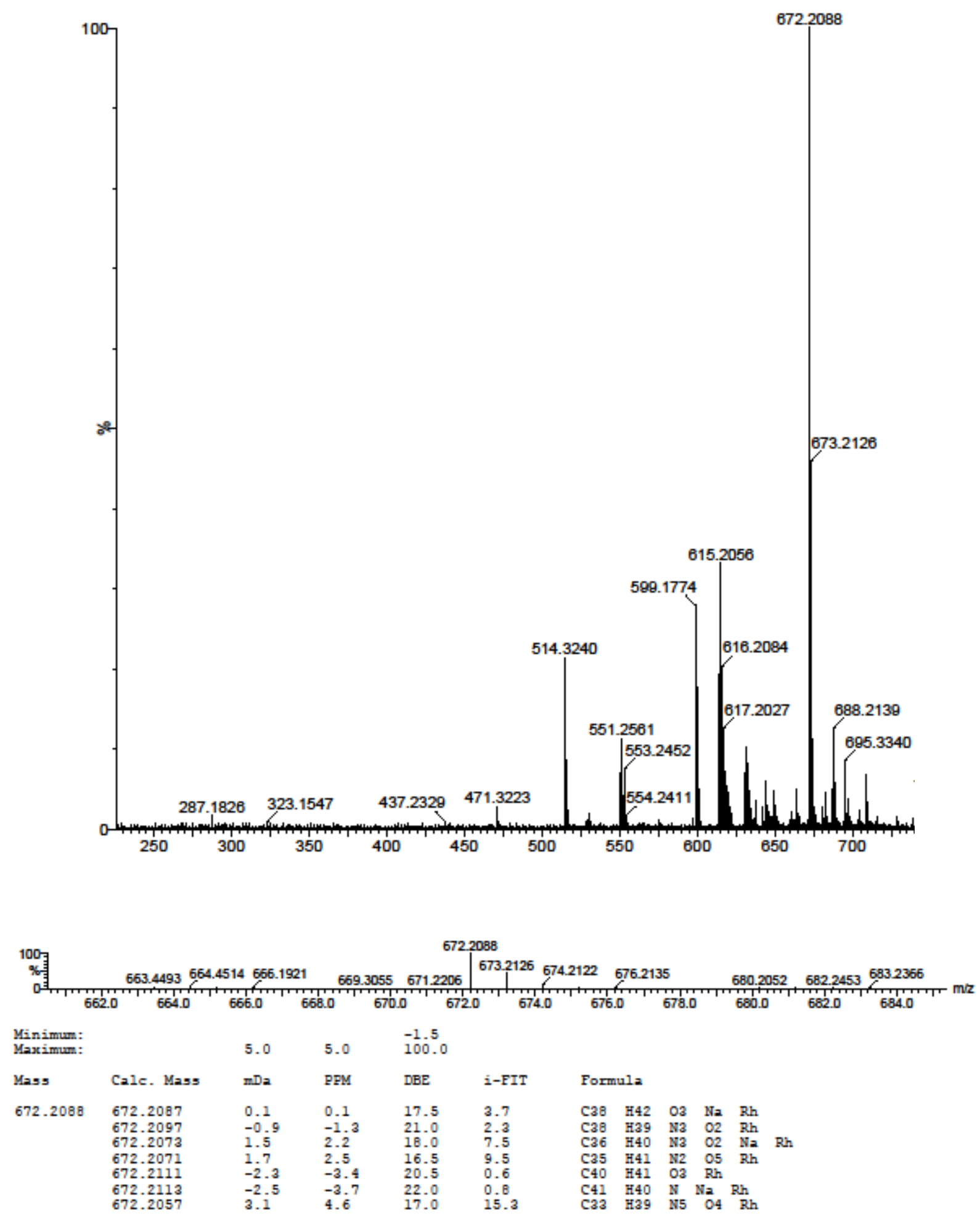

Figure C-58. ESI MS of rhodium(I) complex 93. 


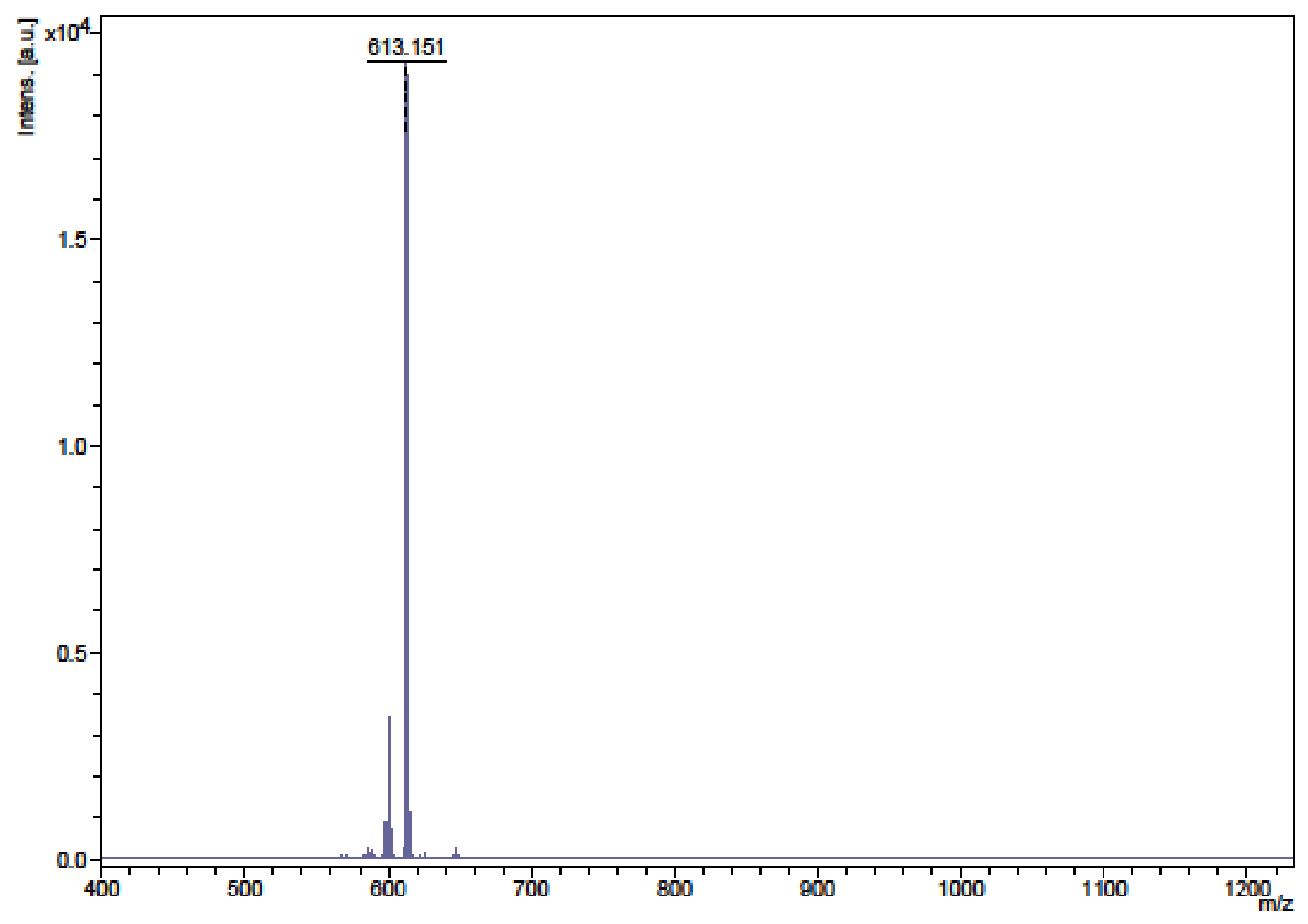

Figure C-59. Electron impact mass spectrum of rhodium(III) complex 95. 

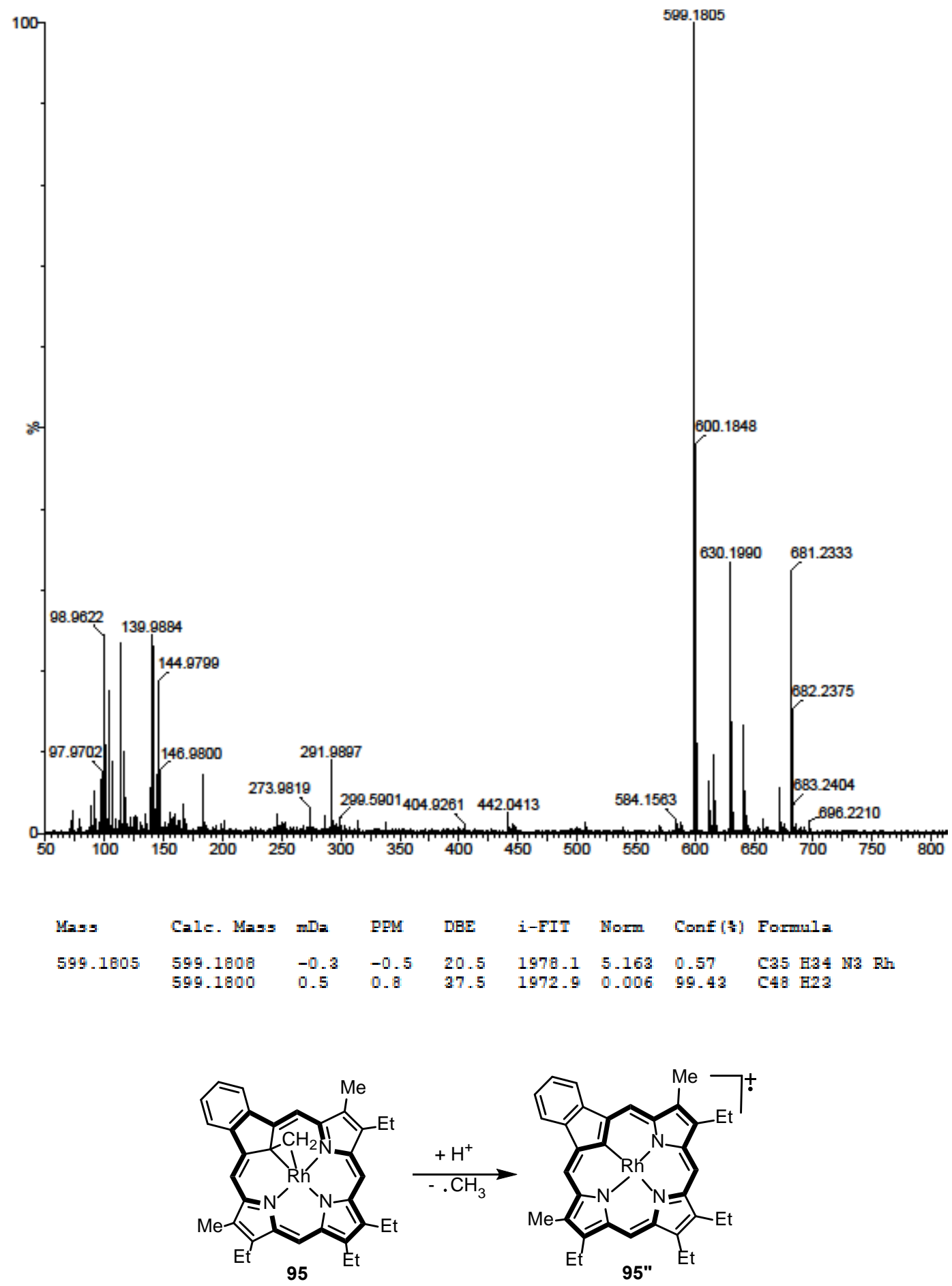

Figure C-60. Electrospray ionization mass spectrum of rhodium(III) complex 95 showing that complete degradation had occurred. The molecular ion corresponds to rhodium complex 95". 


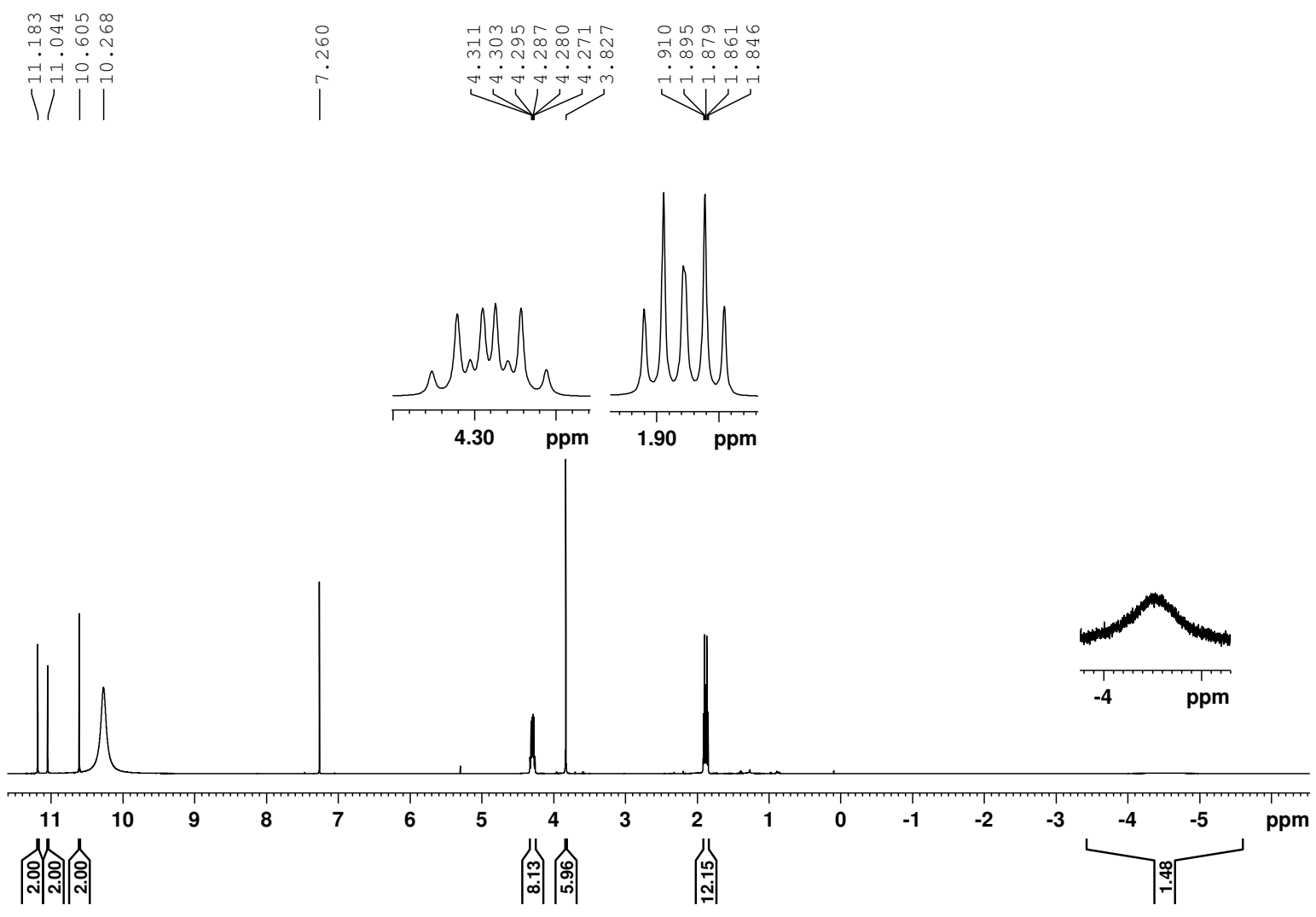

Figure D-1. $500 \mathrm{MHz}{ }^{1} \mathrm{H}$ NMR spectrum of 21-oxaporphyrin $96 \mathrm{aH}_{2}{ }^{2+}$ in TFA-CDCl 3

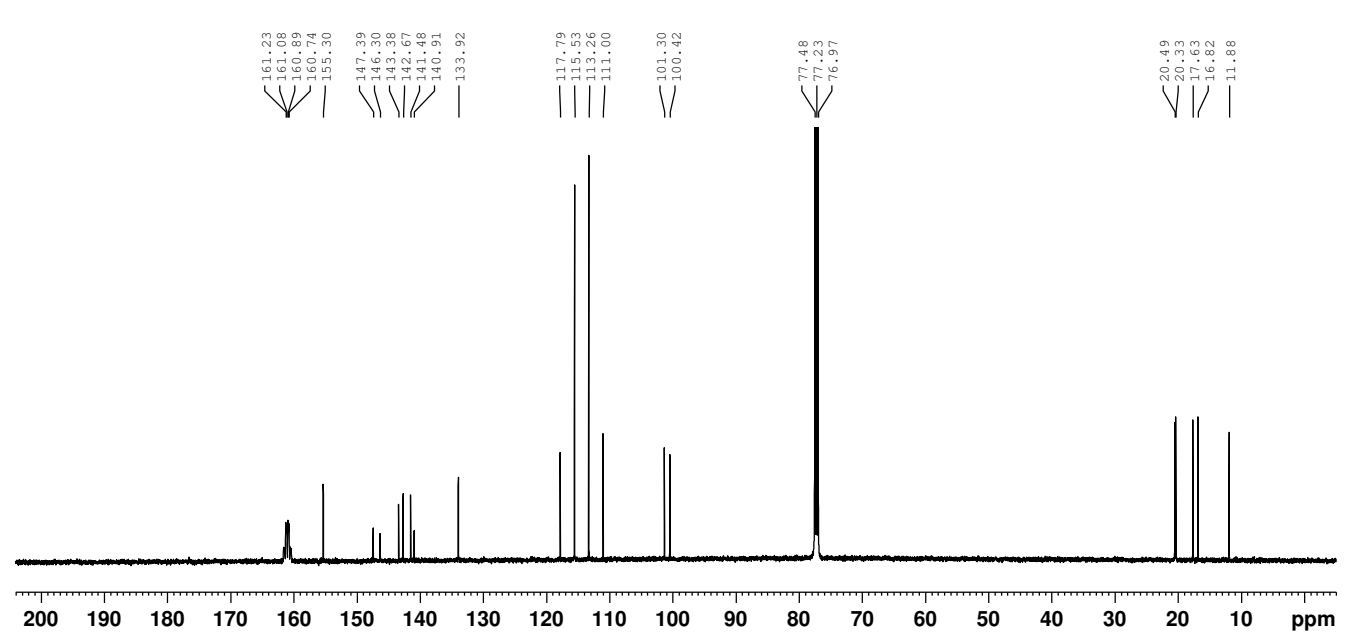

Figure D-2. $125 \mathrm{MHz}{ }^{13} \mathrm{C}$ NMR spectrum of 21-oxaporphyrin $96 \mathrm{aH}_{2}{ }^{2+}$ in TFA-CDCl 3 


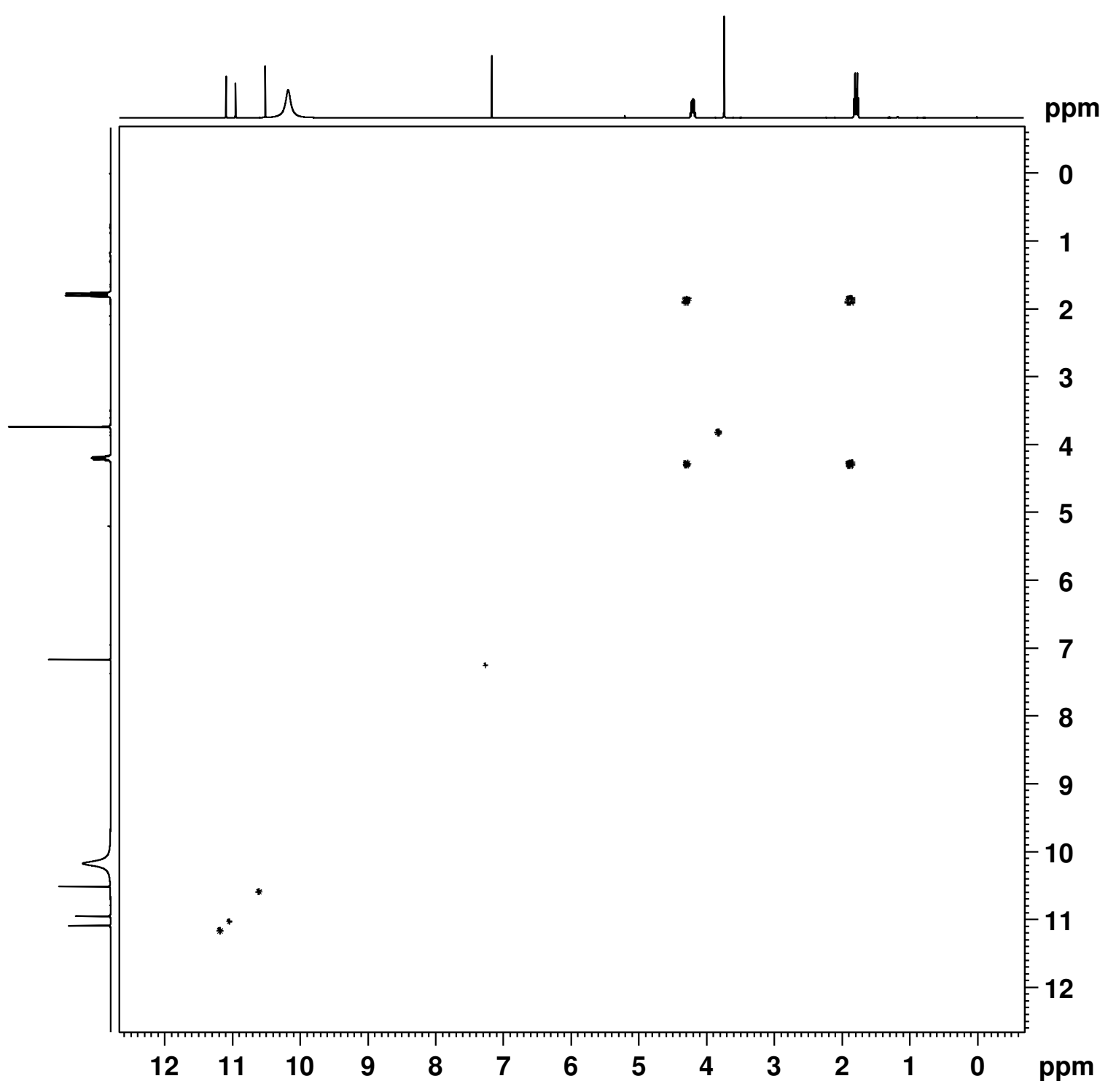

Figure D-3. ${ }^{1} \mathrm{H}-{ }^{1} \mathrm{H}$ COSY NMR spectrum of 21-oxaporphyrin $96 \mathrm{aH}_{2}{ }^{2+}$ in TFA-CDCl 3

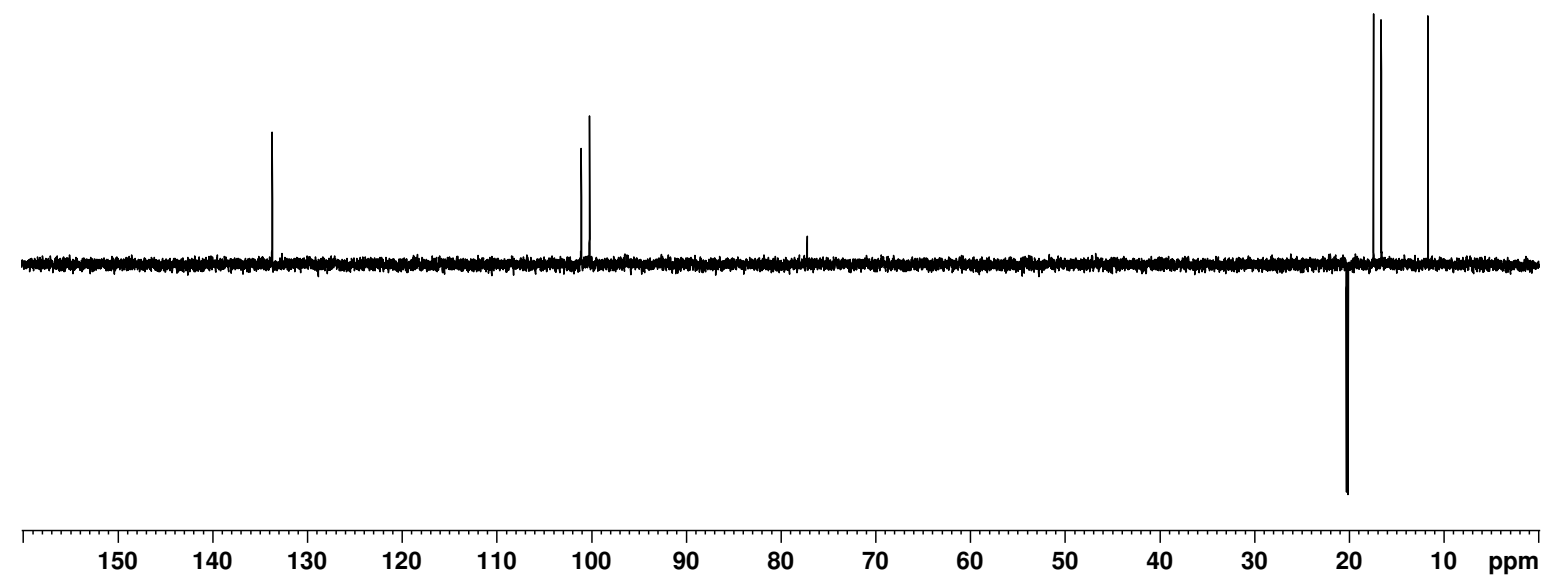

Figure D-4. DEPT-135 NMR spectrum of 21-oxaporphyrin $96 \mathbf{a H}_{2}{ }^{2+}$ in TFA-CDCl 3 


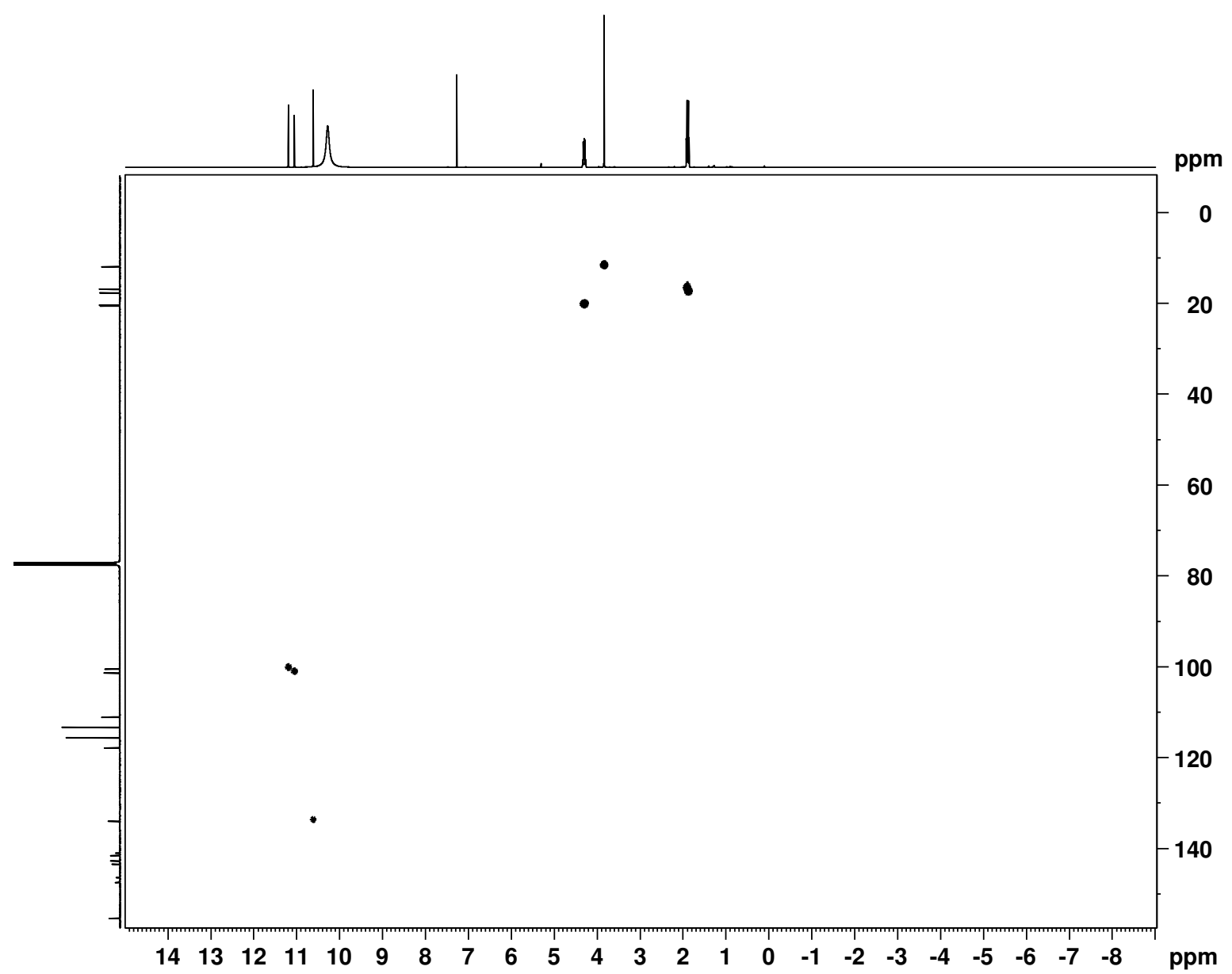

Figure D-5. HSQC NMR spectrum of 21-oxaporphyrin $96 \mathrm{aH}_{2}{ }^{2+}$ in TFA-CDCl $\mathrm{Cl}_{3}$ 


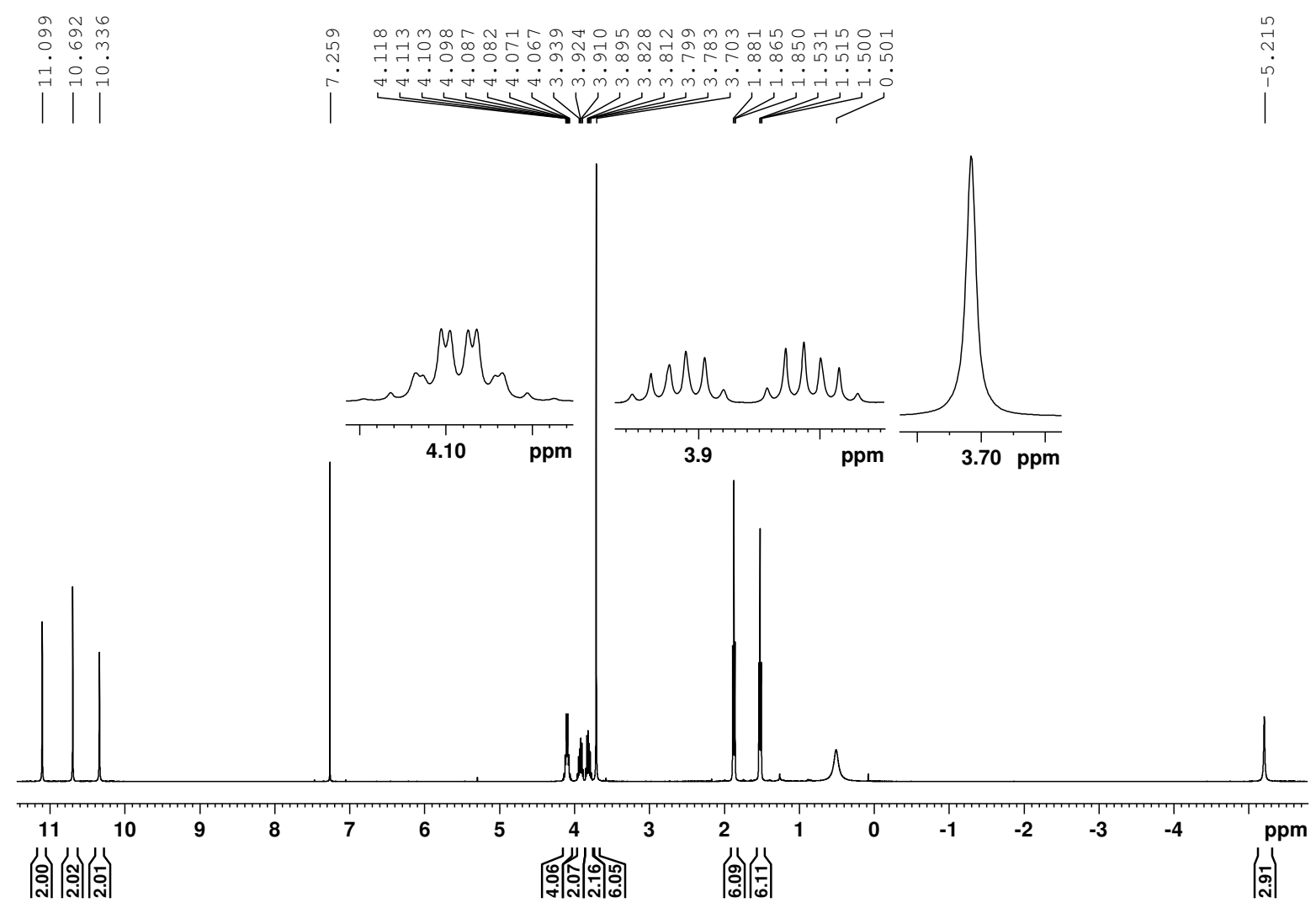

Figure D-6. $500 \mathrm{MHZ}{ }^{1} \mathrm{H}$ NMR spectrum of $N$-methyl oxaporphyrin $96 \mathbf{b}$ in $\mathrm{CDCl}_{3}$

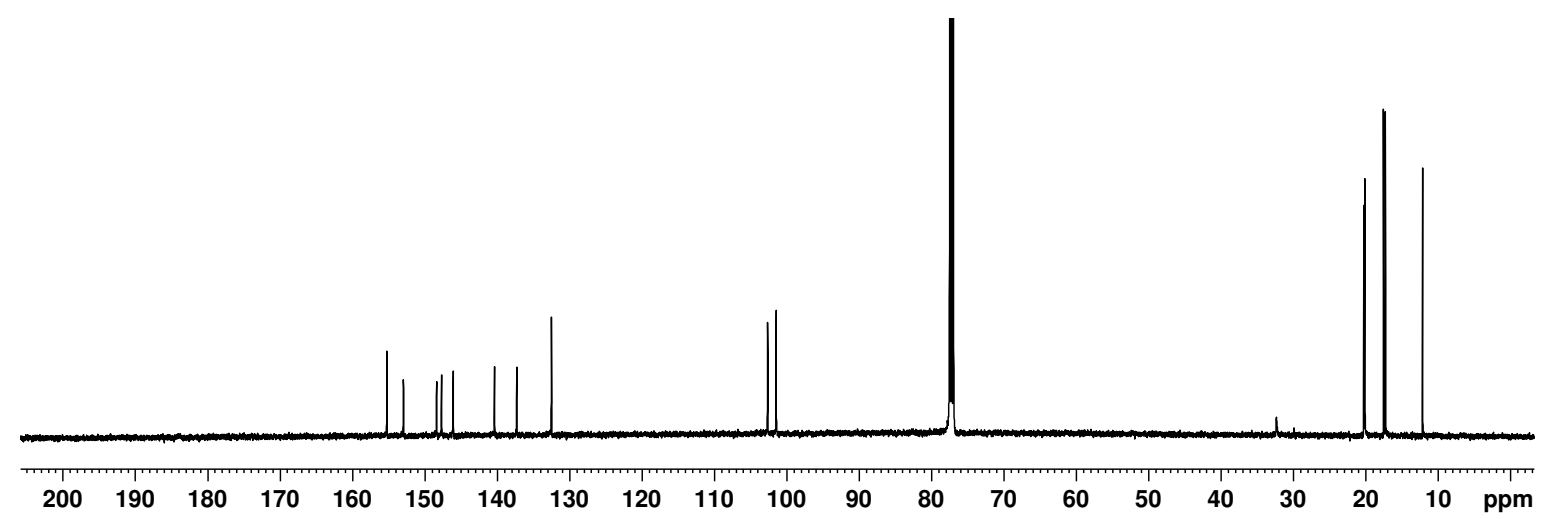

Figure D-7. $125 \mathrm{MHz}{ }^{13} \mathrm{C}$ NMR spectrumof $N$-methyl oxaporphyrin $96 \mathbf{b}$ in $\mathrm{CDCl}_{3}$ 


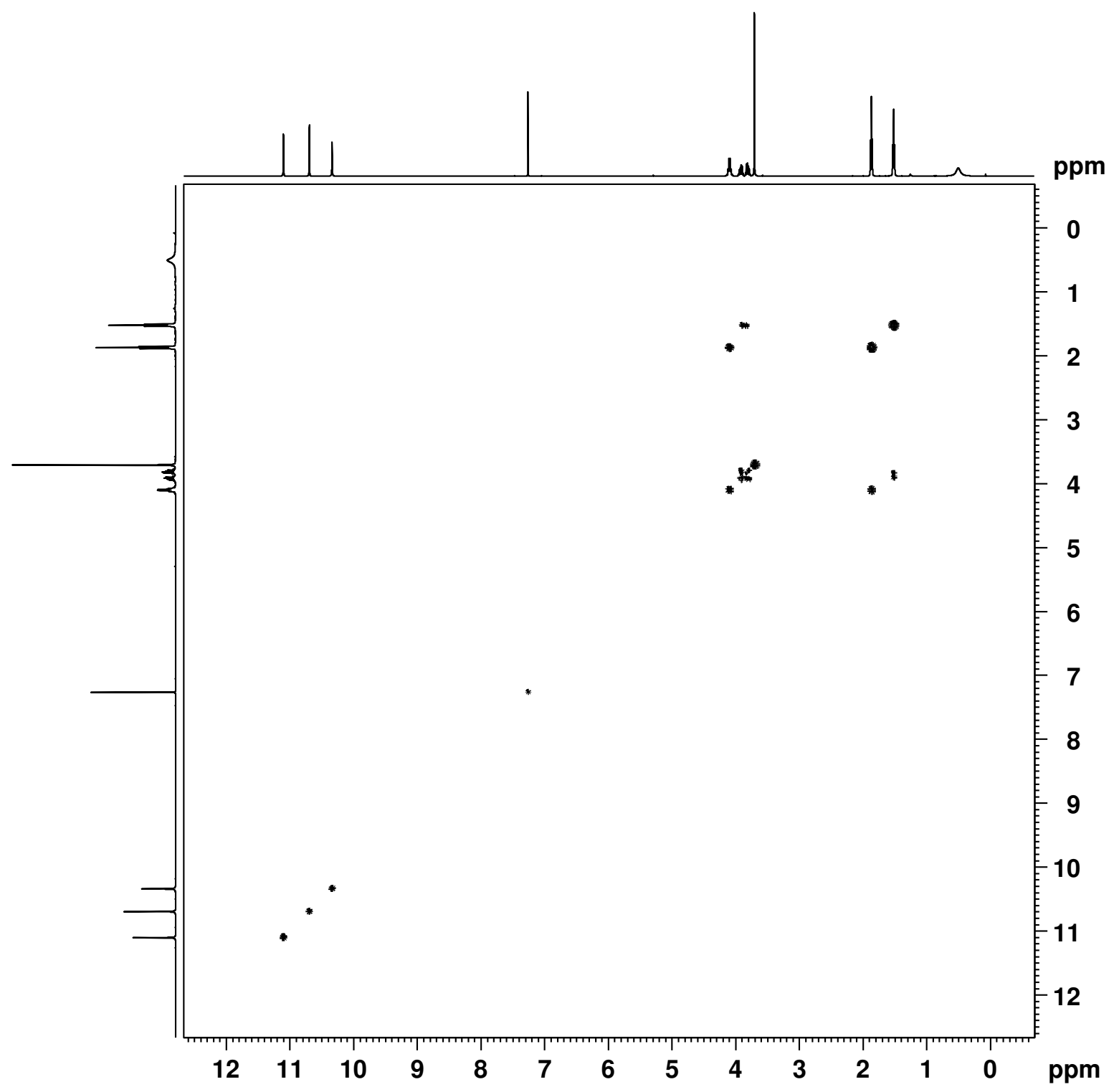

Figure D-8. ${ }^{1} \mathrm{H}-{ }^{1} \mathrm{H}$ COSY NMR spectrum of $\mathrm{N}$-methyl oxaporphyrin $96 \mathbf{b}$ in $\mathrm{CDCl}_{3}$

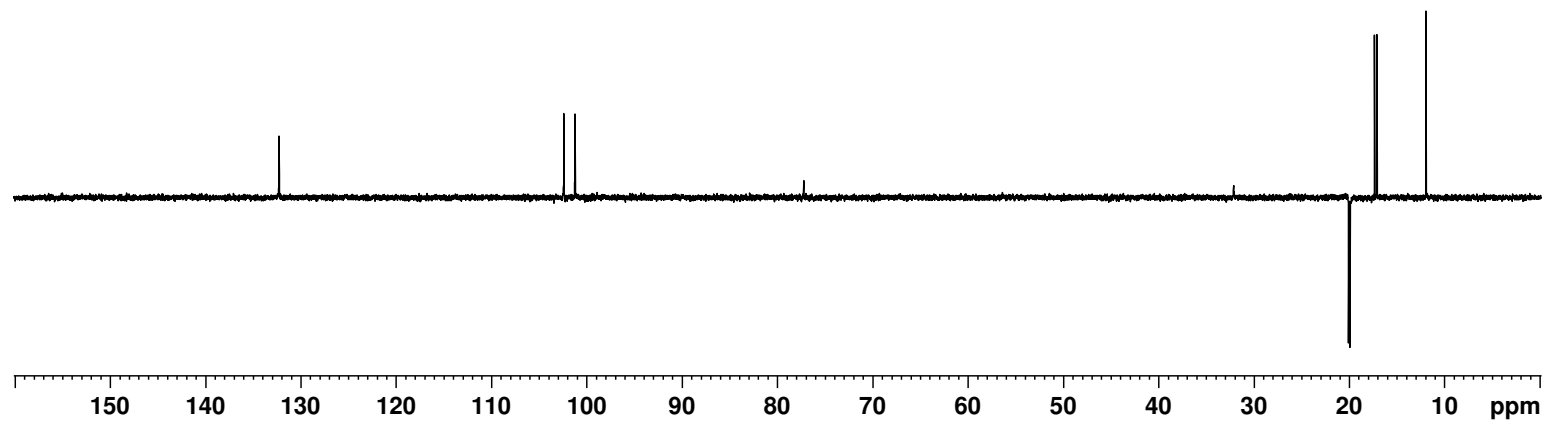

Figure D-9. DEPT-135 NMR spectrum of $N$-methyl oxaporphyrin 96b in $\mathrm{CDCl}_{3}$ 


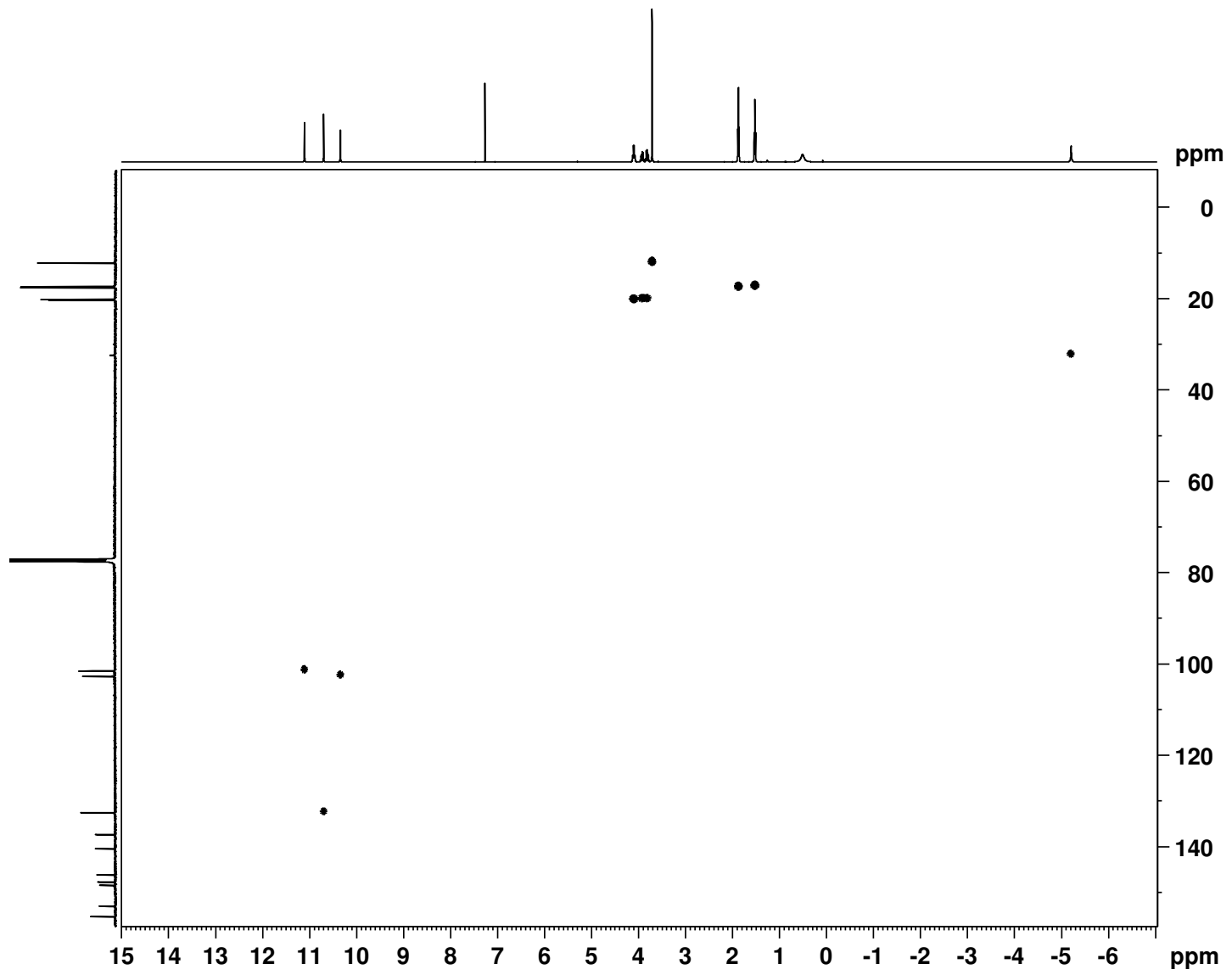

Figure D-10. HSQC NMR spectrum of $N$-methyl oxaporphyrin $96 \mathbf{b}$ in $\mathrm{CDCl}_{3}$ 


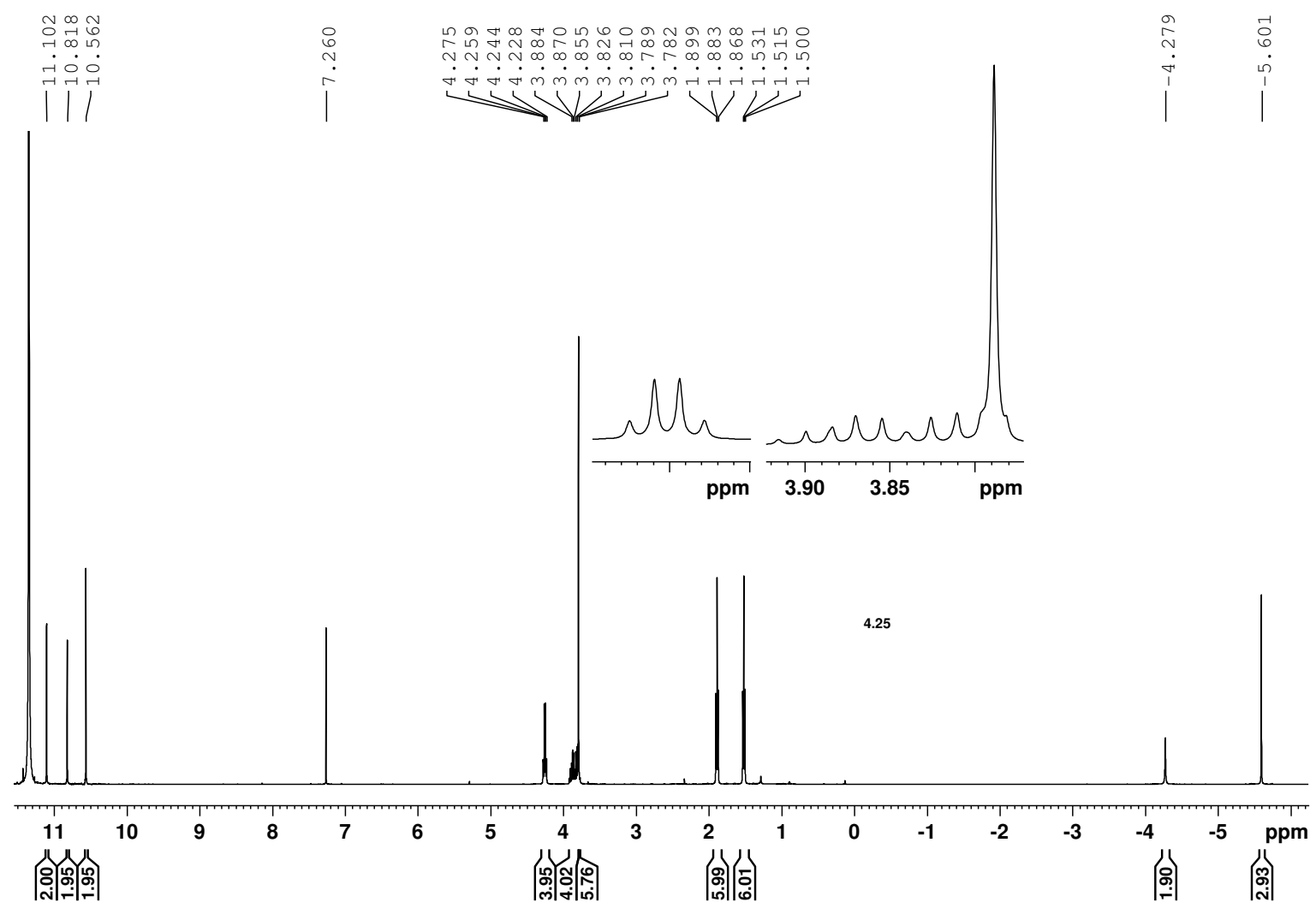

Figure D-11. $500 \mathrm{MHz}{ }^{1} \mathrm{H}$ NMR spectrum of $N$-methyl oxaporphyrin 96b in TFA-CDCl 3

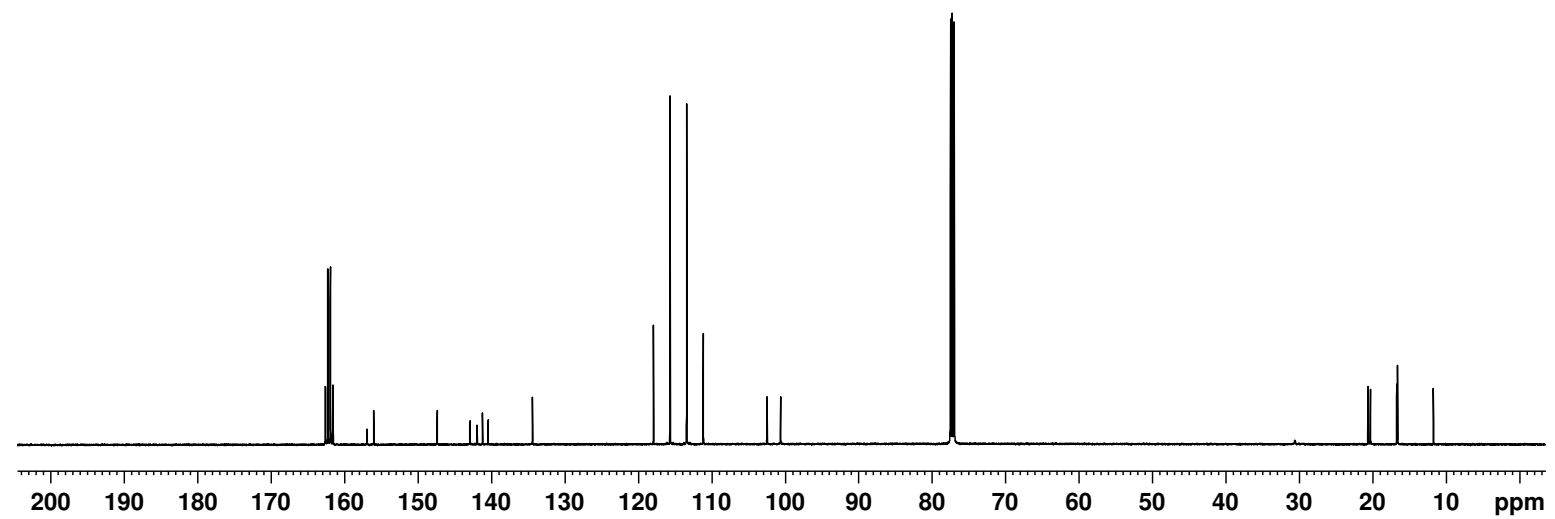

Figure D-12. $125 \mathrm{MHz}{ }^{13} \mathrm{C}$ NMR spectrum of $\mathrm{N}$-methyl oxaporphyrin $96 \mathbf{b}$ in TFA-CDCl ${ }_{3}$ 


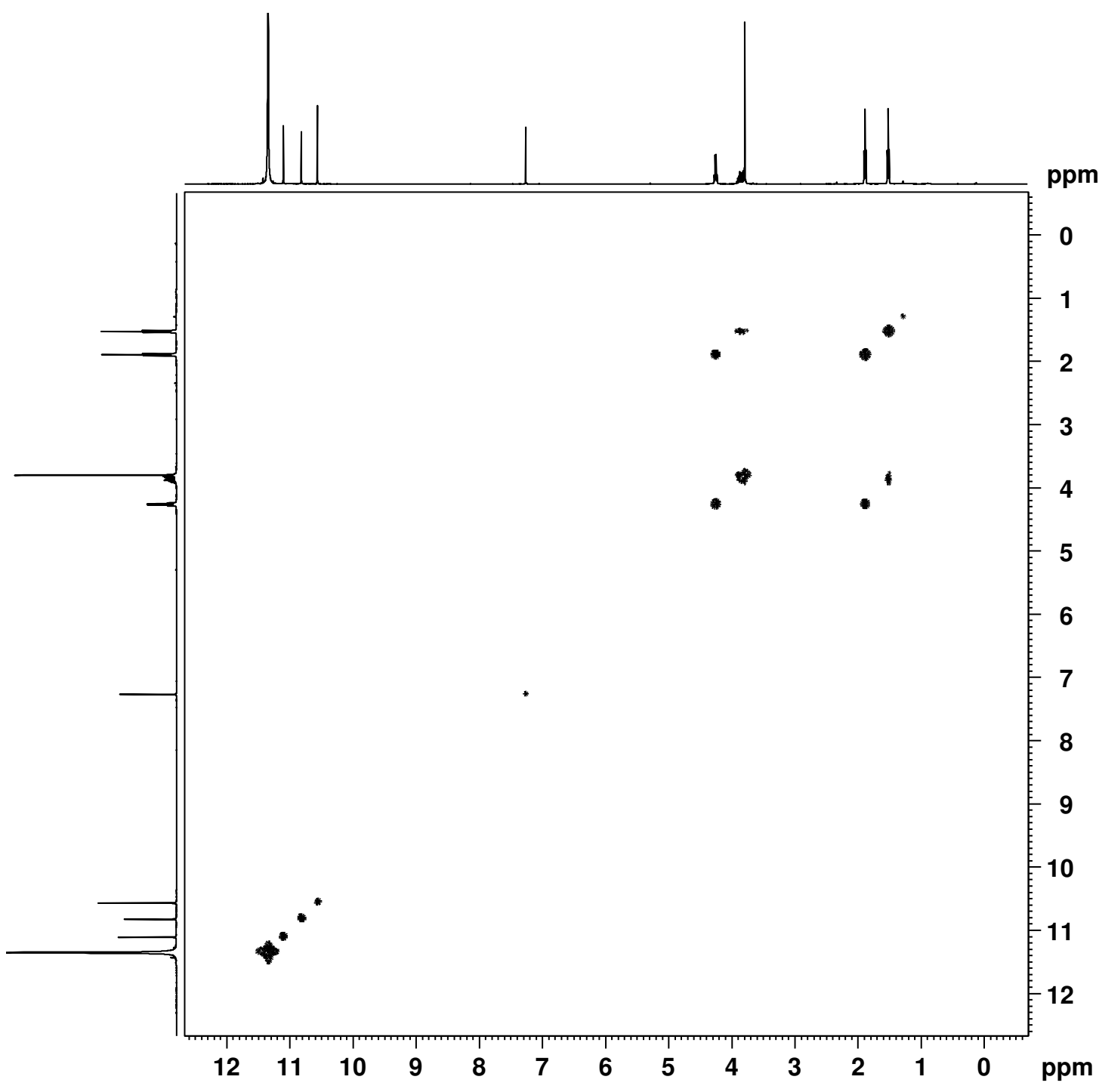

Figure D-13. ${ }^{1} \mathrm{H}-{ }^{1} \mathrm{H}$ COSY NMR spectrum of $\mathrm{N}$-methyl oxaporphyrin $96 \mathbf{b}$ in TFA-CDCl 3

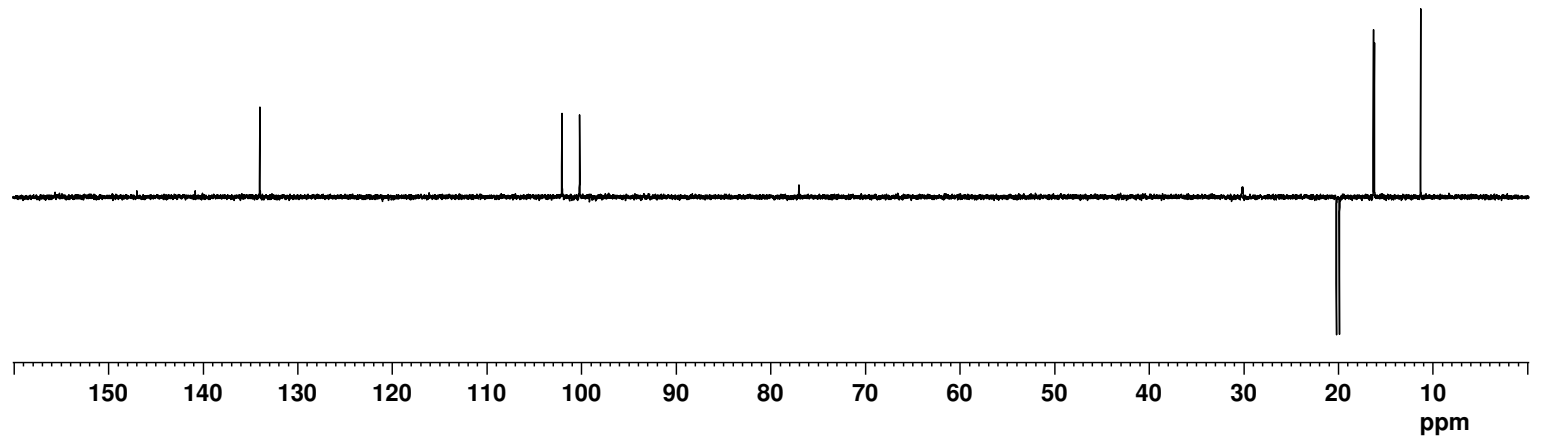

Figure D-14. DEPT-135 NMR spectrum of $N$-methyl oxaporphyrin 96b in TFA-CDCl 3 


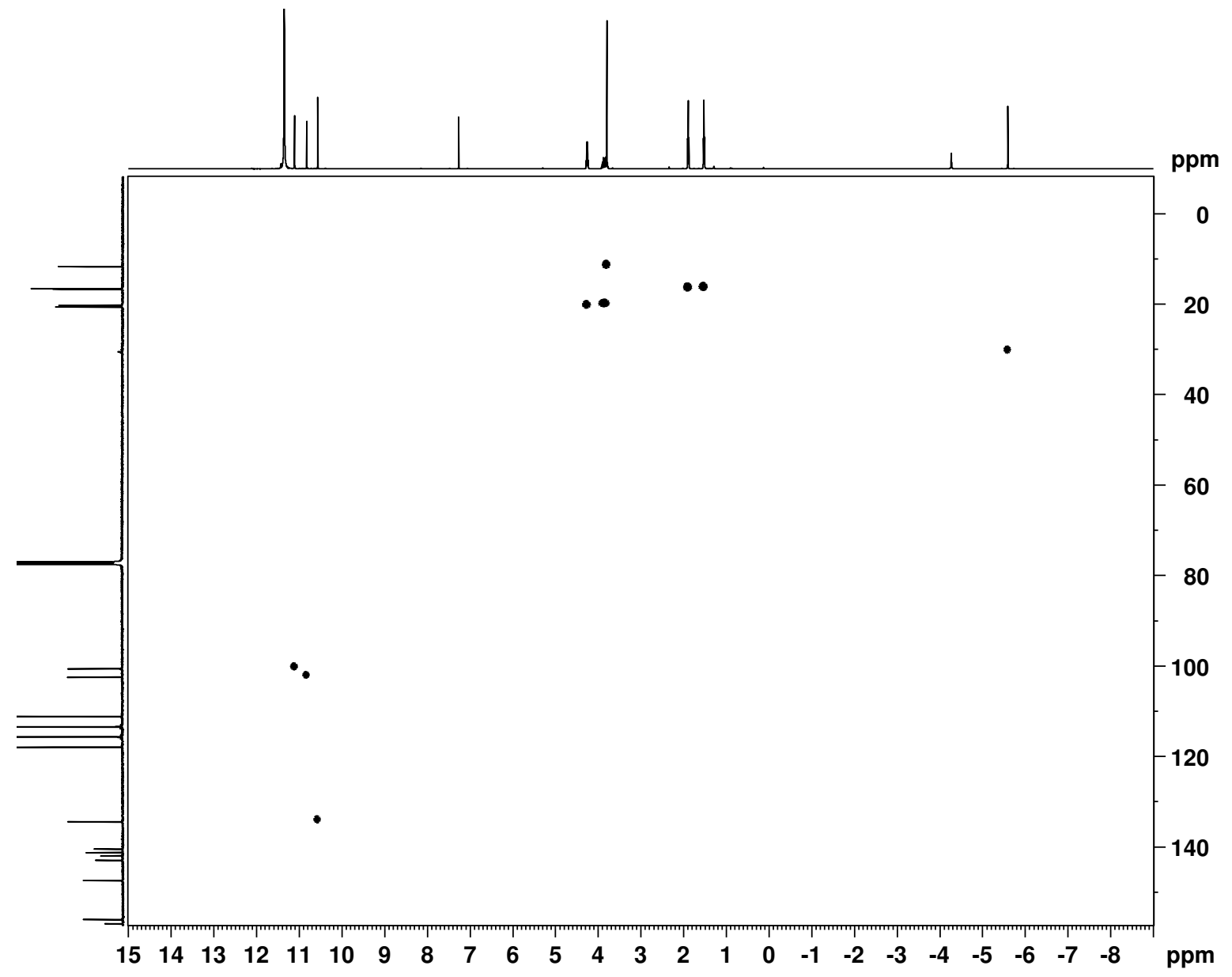

Figure D-15. HSQC NMR spectrum of $N$-methyl oxaporphyrin 96b in TFA-CDCl 


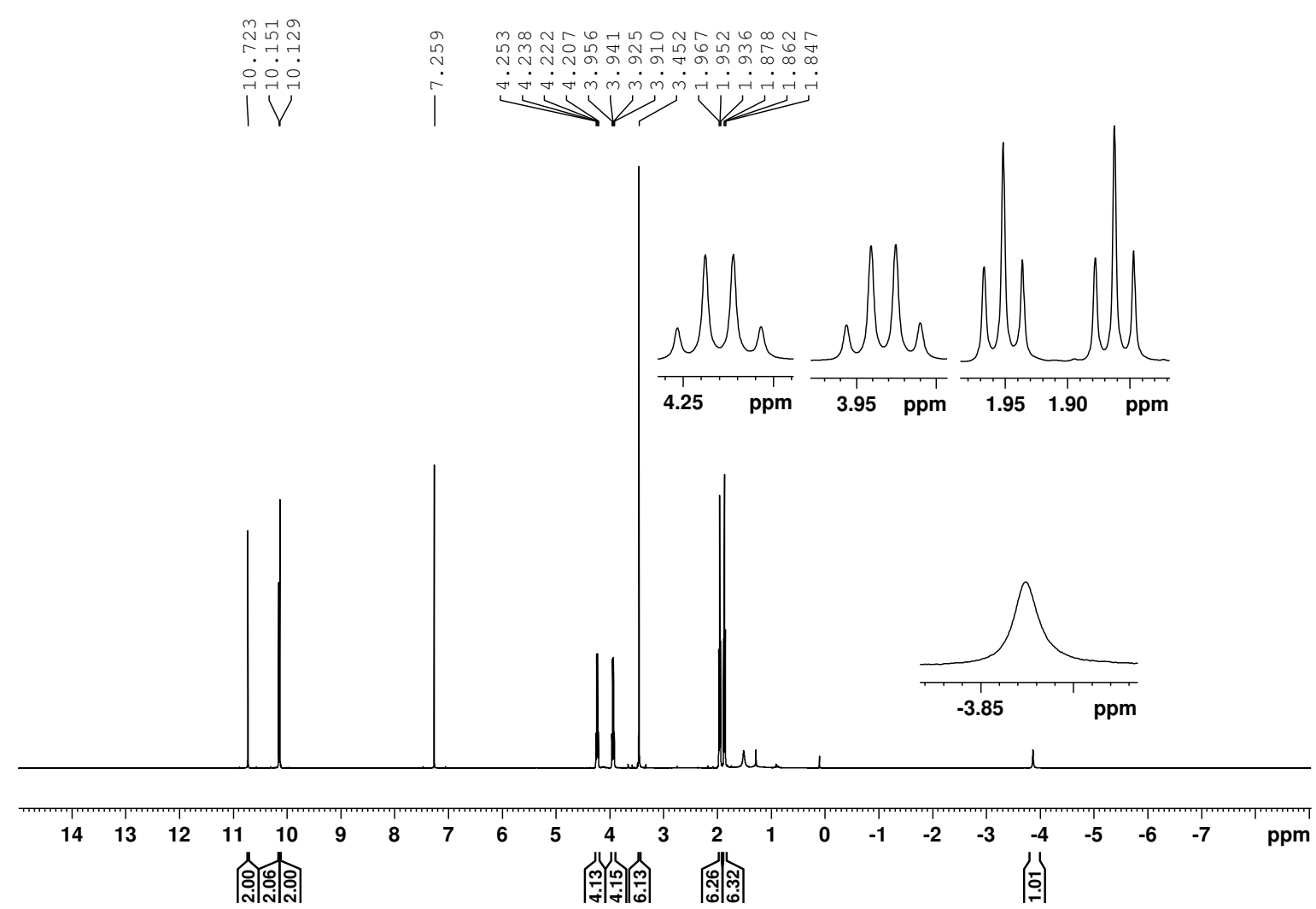

Figure D-16. $500 \mathrm{MHz}{ }^{1} \mathrm{H}$ NMR spectrum of 21-thiaporphyrin 97a in $\mathrm{CDCl}_{3}$

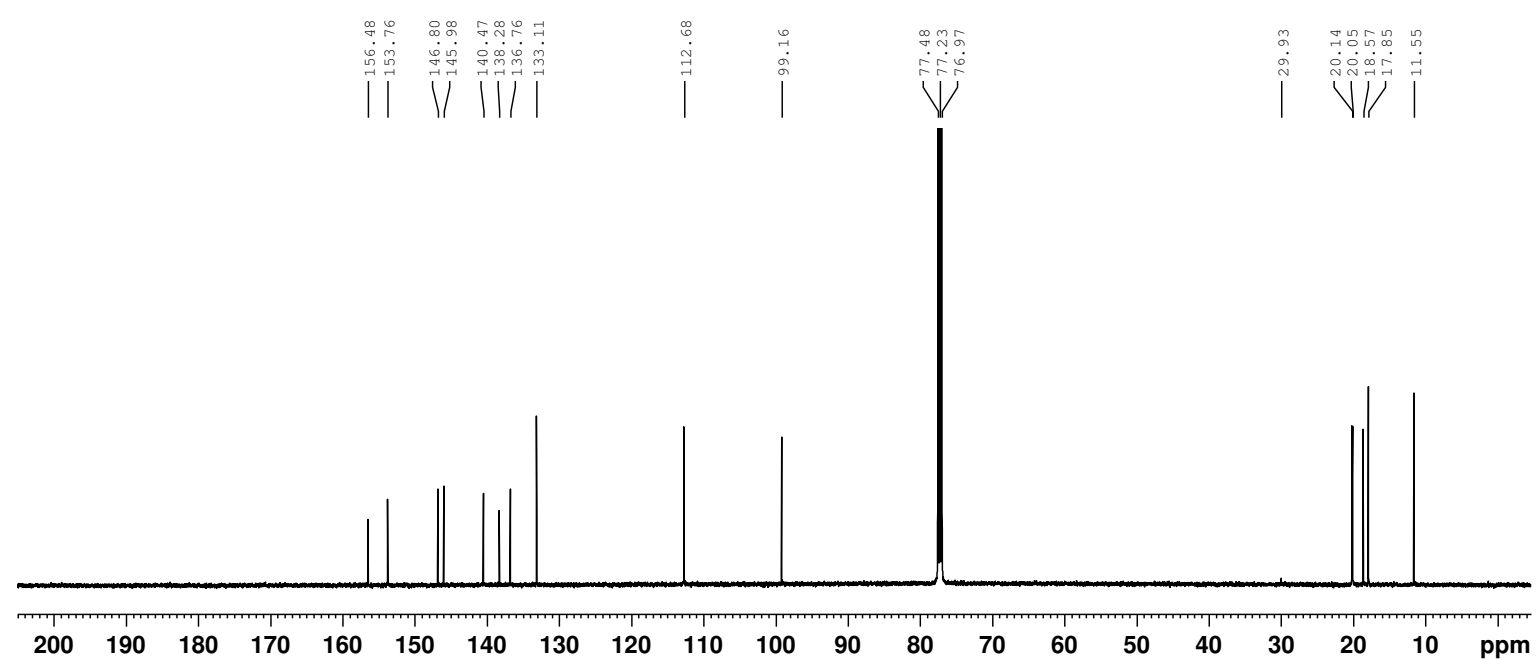

Figure D-17. $125 \mathrm{MHz}{ }^{13} \mathrm{C}$ NMR spectrum of 21-thiaporphyrin 97a in $\mathrm{CDCl}_{3}$ 


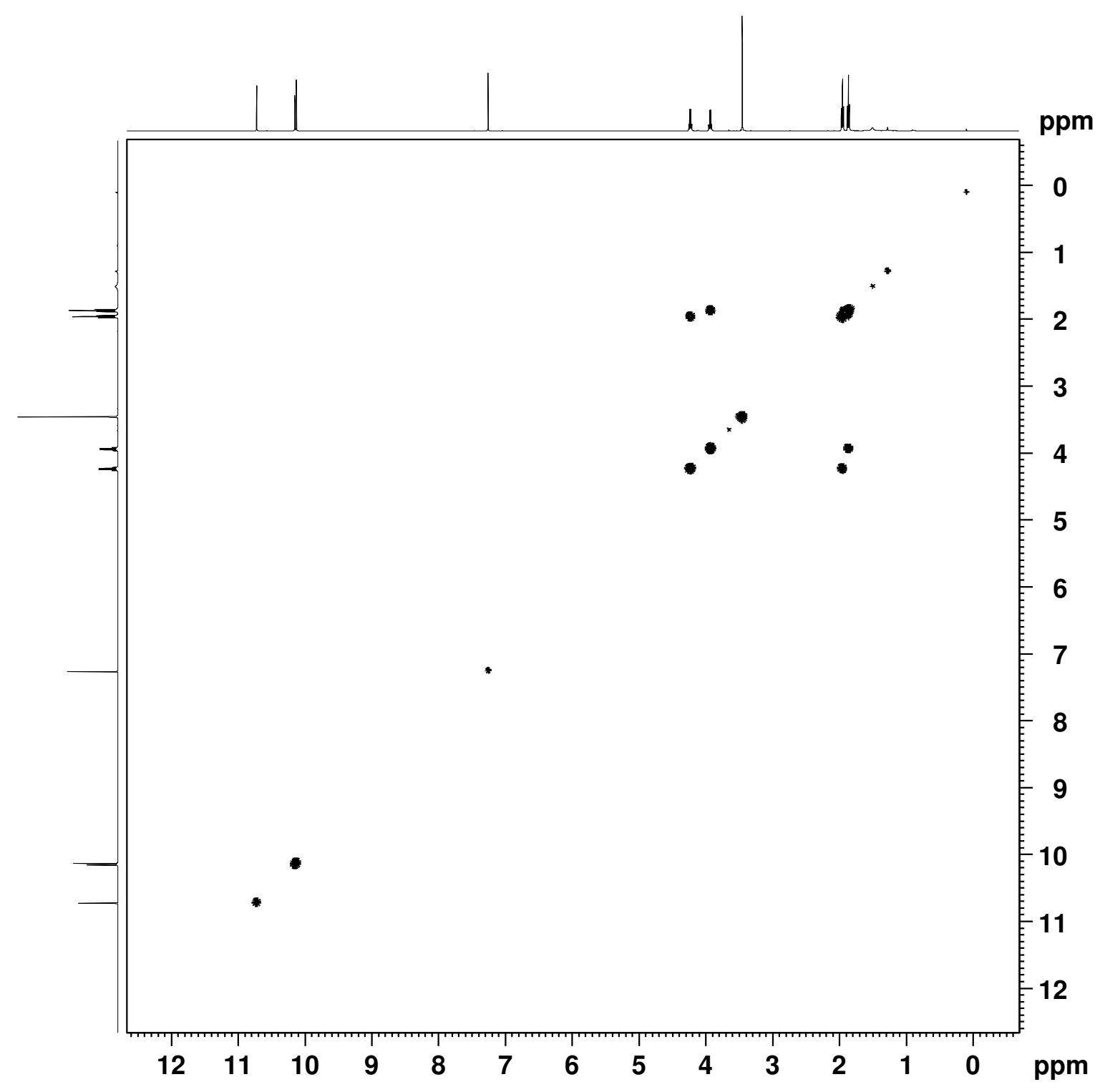

Figure D-18. ${ }^{1} \mathrm{H}-{ }^{1} \mathrm{H}$ COSY NMR spectrum of 21-thiaporphyrin 97a in $\mathrm{CDCl}_{3}$

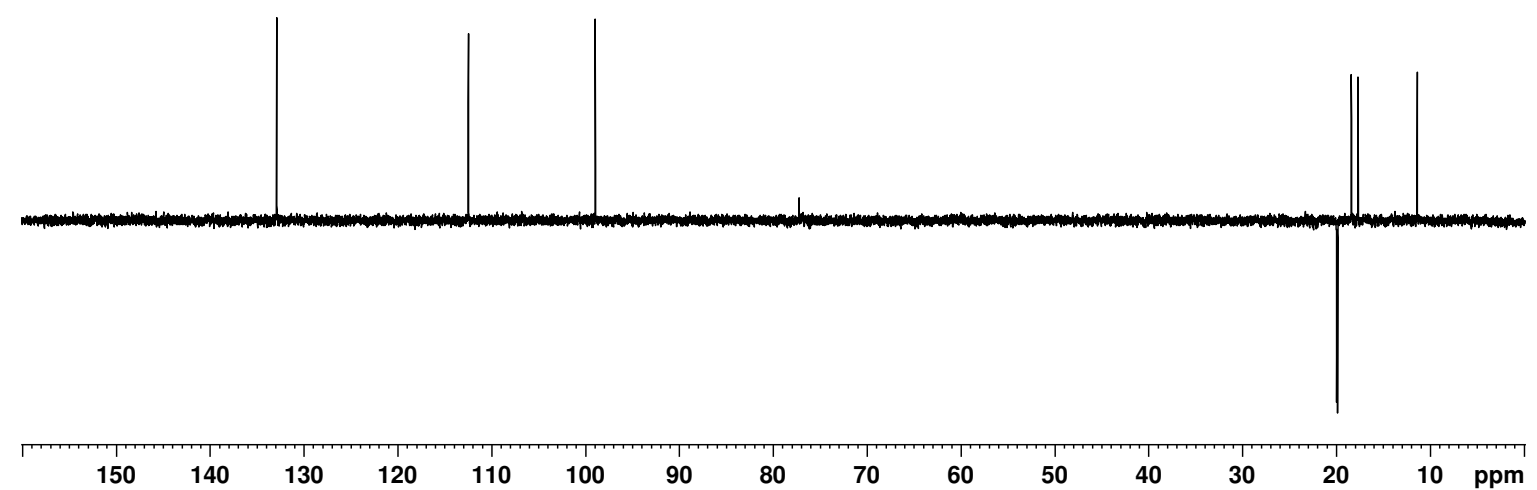

Figure D-19. DEPT-135 NMR spectrum of 21-thiaporphyrin 97a in $\mathrm{CDCl}_{3}$ 


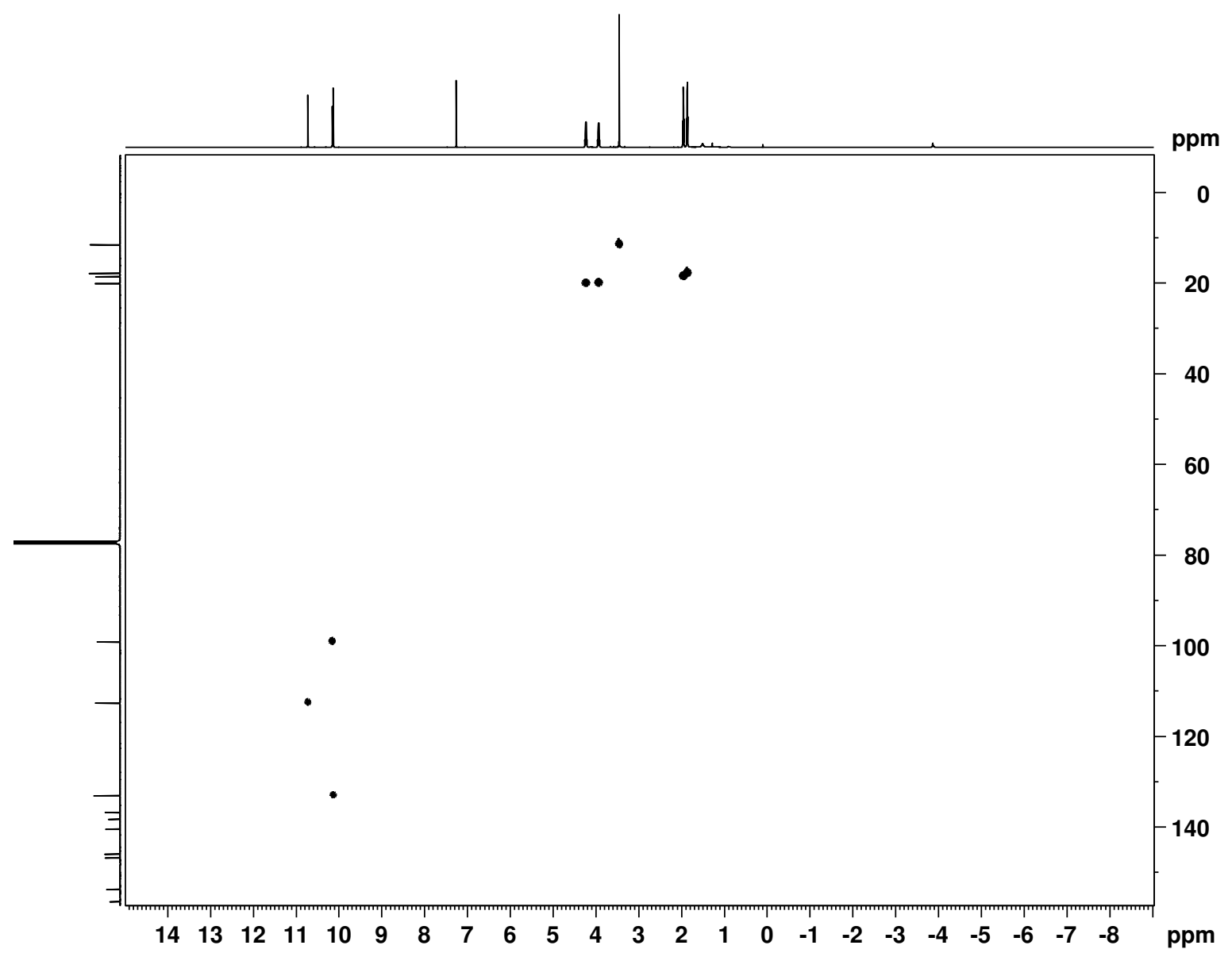

Figure D-20. HSQC NMR spectrum of 21-thiaporphyrin 97a in $\mathrm{CDCl}_{3}$ 


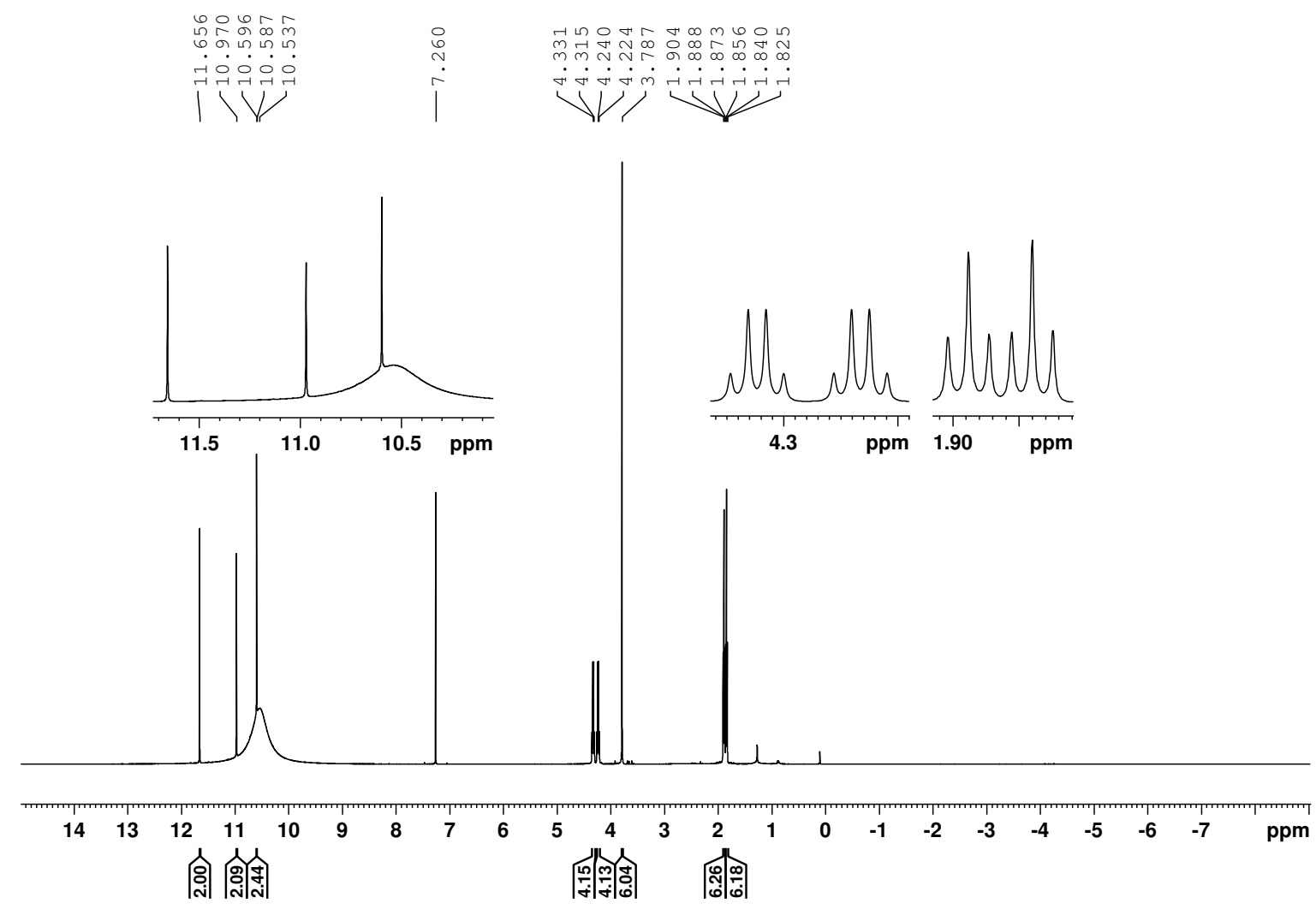

Figure D-21. $500 \mathrm{MHz}{ }^{1} \mathrm{H}$ NMR spectrum of 21-thiaporphyrin $97 \mathrm{aH}_{2}{ }^{2+}$ in TFA-CDCl ${ }_{3}$

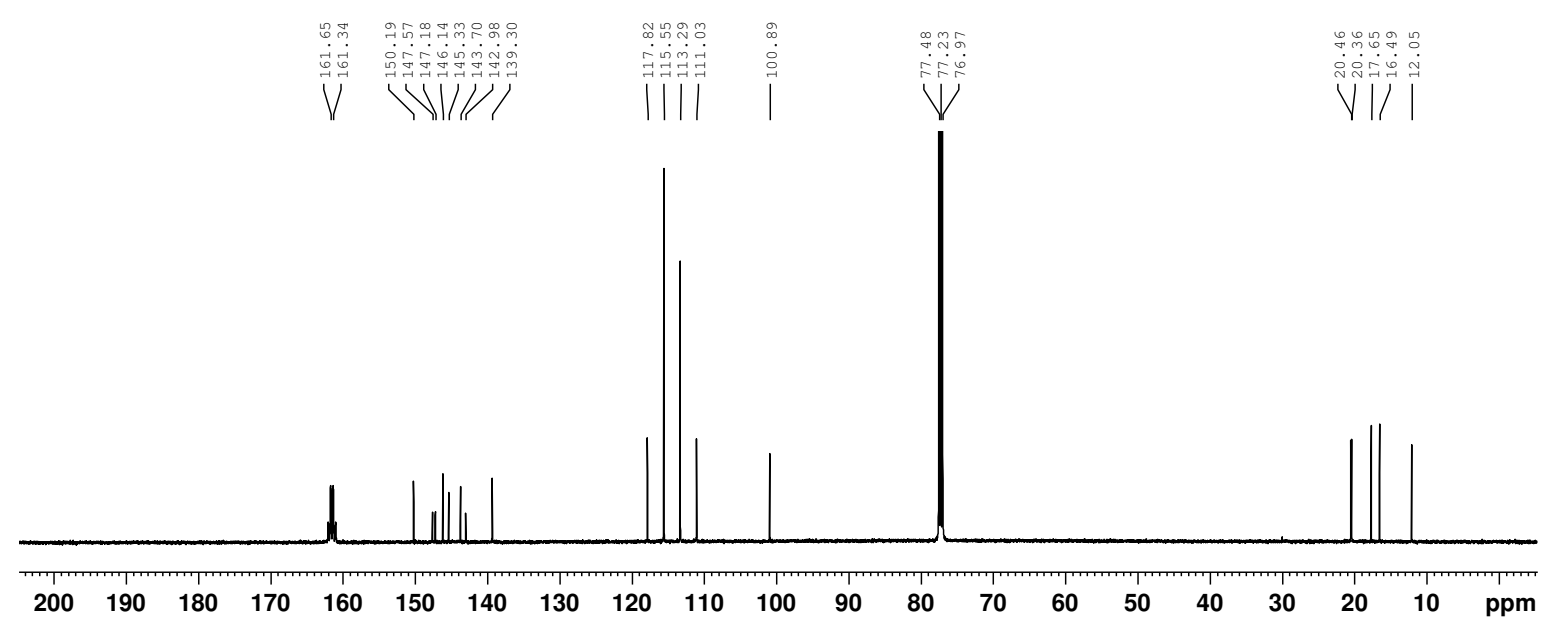

Figure D-22. $125 \mathrm{MHz}{ }^{13} \mathrm{C}$ NMR spectrum of 21-thiaporphyrin $97 \mathrm{aH}_{2}{ }^{2+}$ in TFA-CDCl 3 


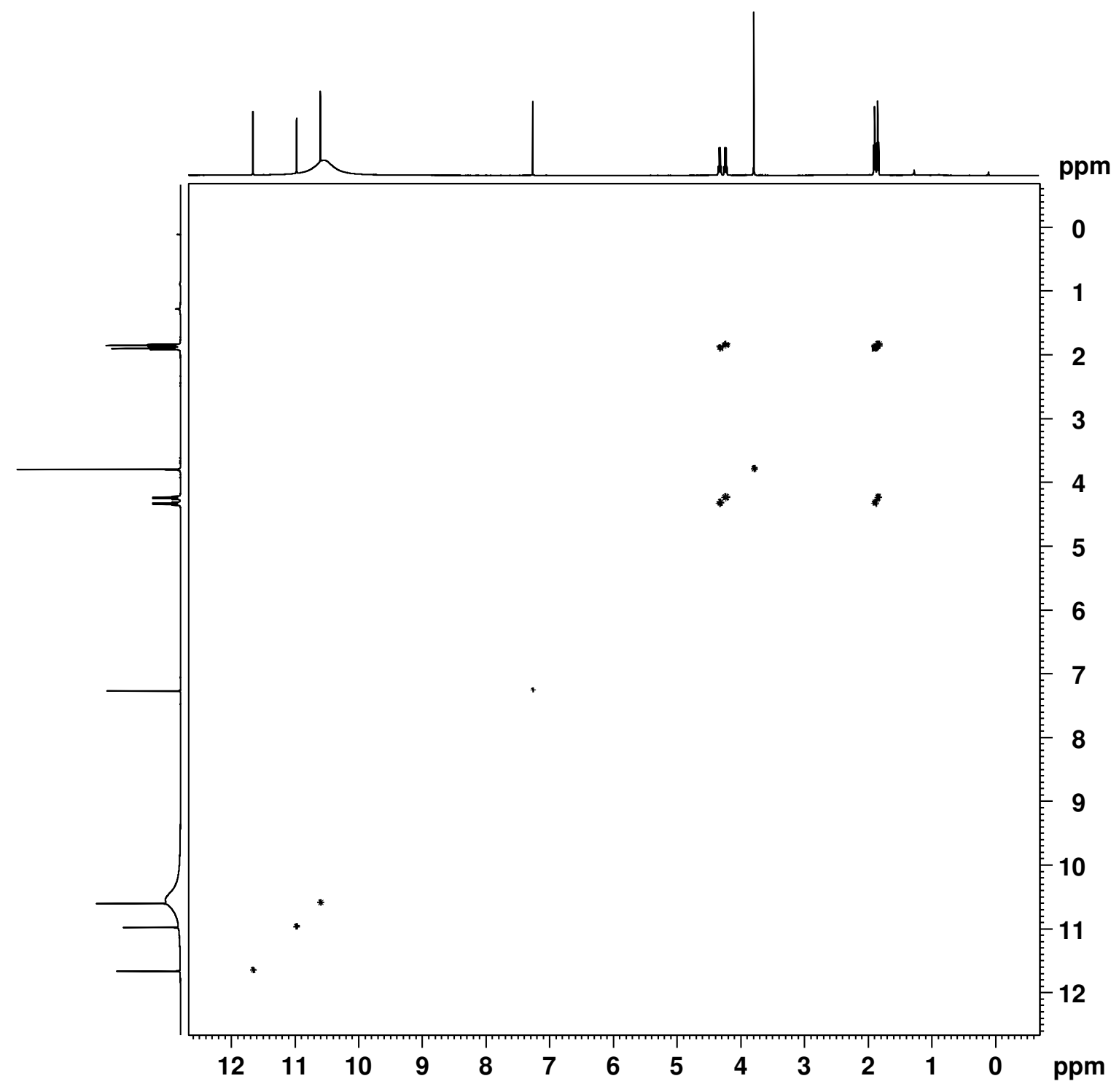

Figure D-23. ${ }^{1} \mathrm{H}-{ }^{1} \mathrm{H}$ COSY NMR spectrum of 21-thiaporphyrin $97 \mathbf{a H}_{2}{ }^{2+}$ in $\mathrm{TFA}-\mathrm{CDCl}_{3}$

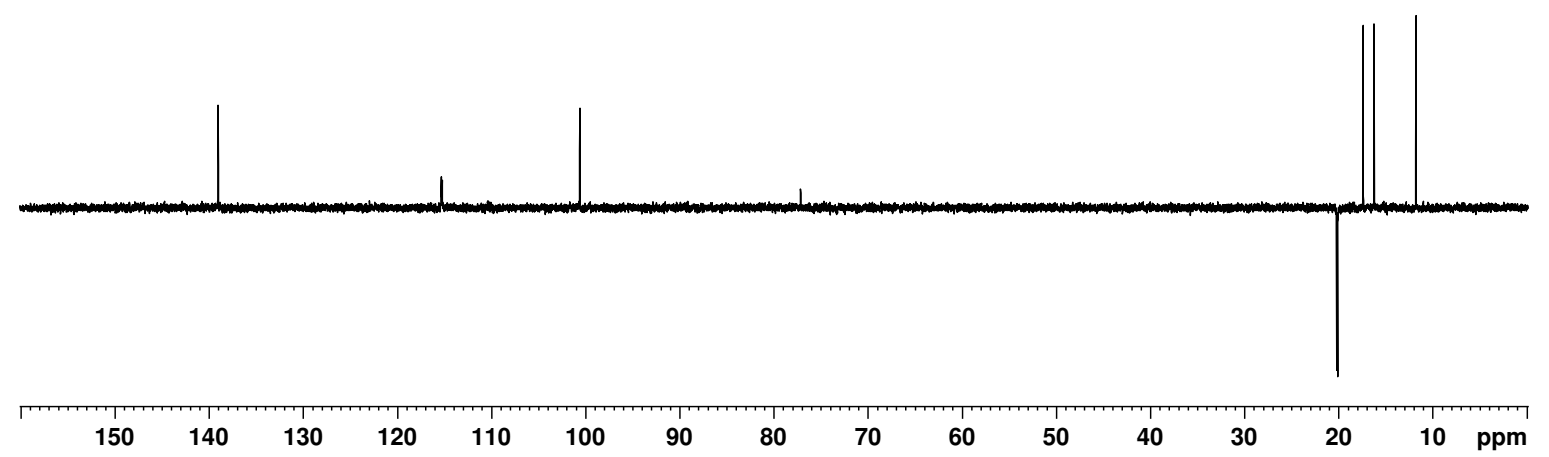

Figure D-24. DEPT-135 NMR spectrum of 21-thiaporphyrin $\mathbf{9 7} \mathbf{a H}_{2}{ }^{2+}$ in TFA-CDCl 3 


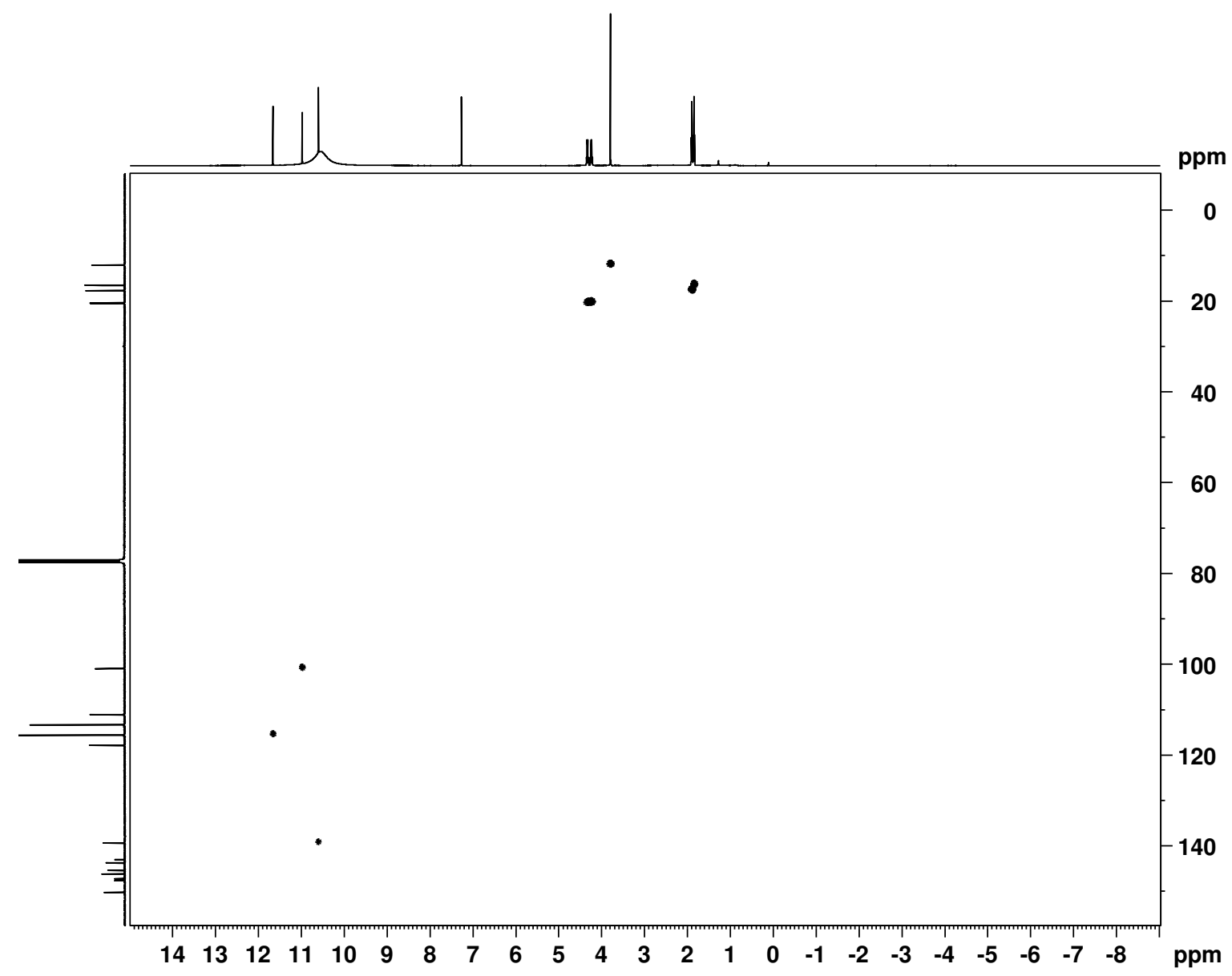

Figure D-25. HQSC NMR spectrum of 21-thiaporphyrin $\mathbf{9 7 a H _ { 2 }}{ }^{2+}$ in TFA-CDCl $\mathrm{Cl}_{3}$ 

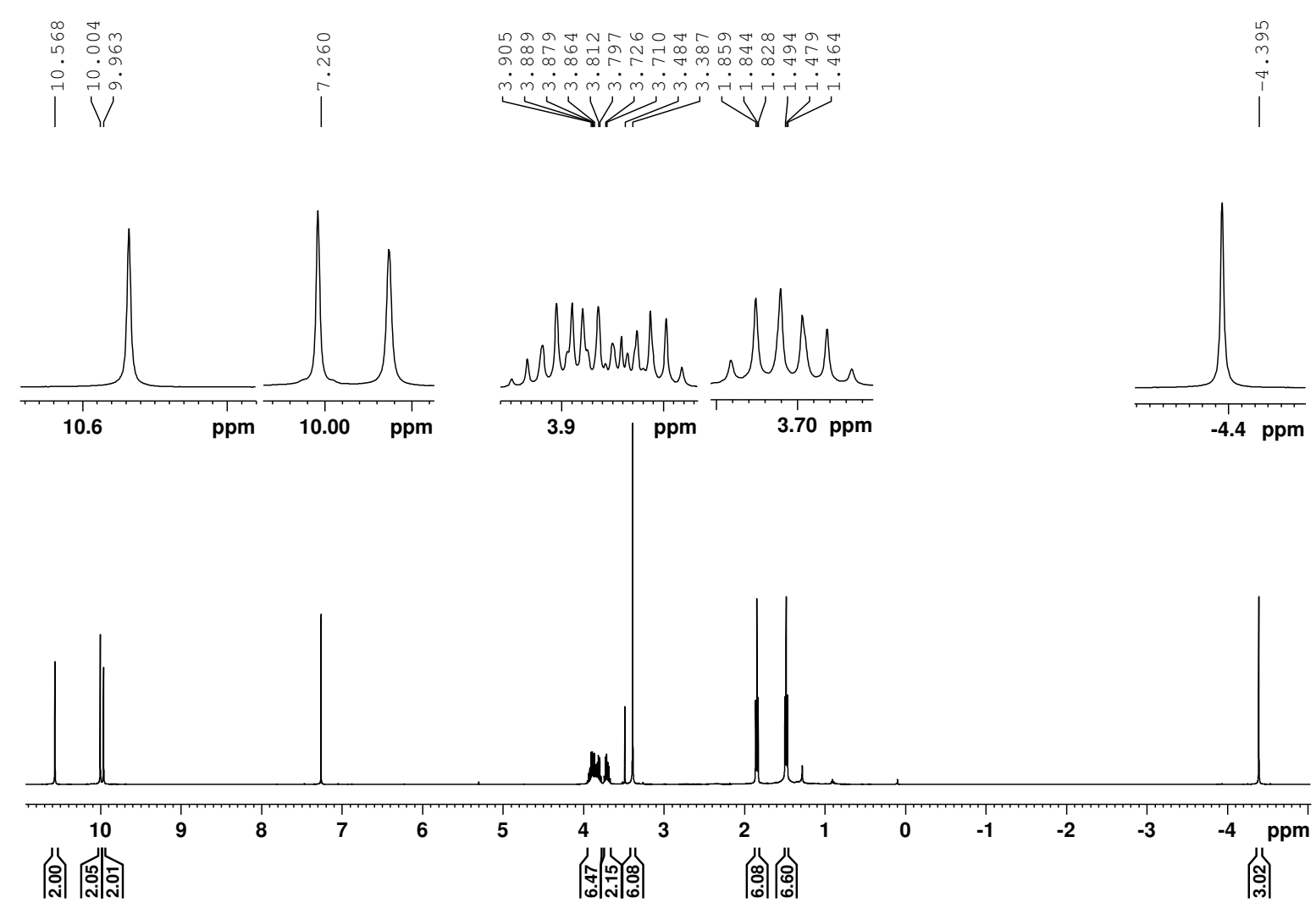

Figure D-26. $500 \mathrm{MHz}{ }^{1} \mathrm{H}$ NMR spectrum of $N$-methyl thiaporphyrin 97b in $\mathrm{CDCl}_{3}$

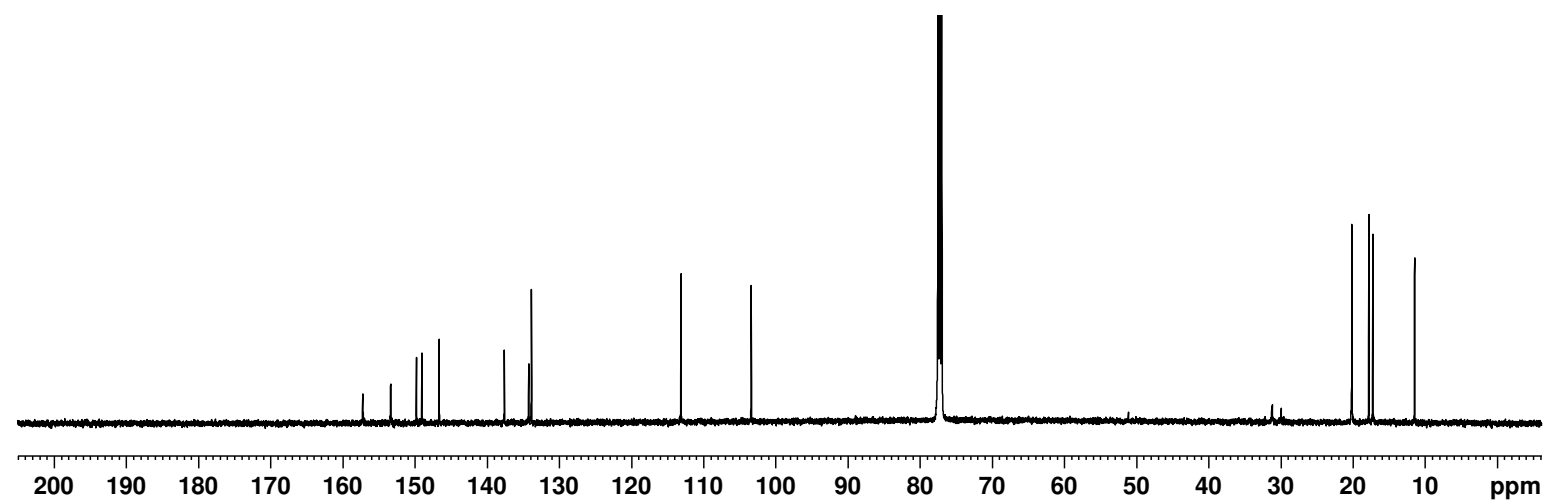

Figure D-27. $125 \mathrm{MHz}{ }^{13} \mathrm{C} \mathrm{NMR}$ spectrum of $N$-methyl thiaporphyrin 97b in $\mathrm{CDCl}_{3}$ 


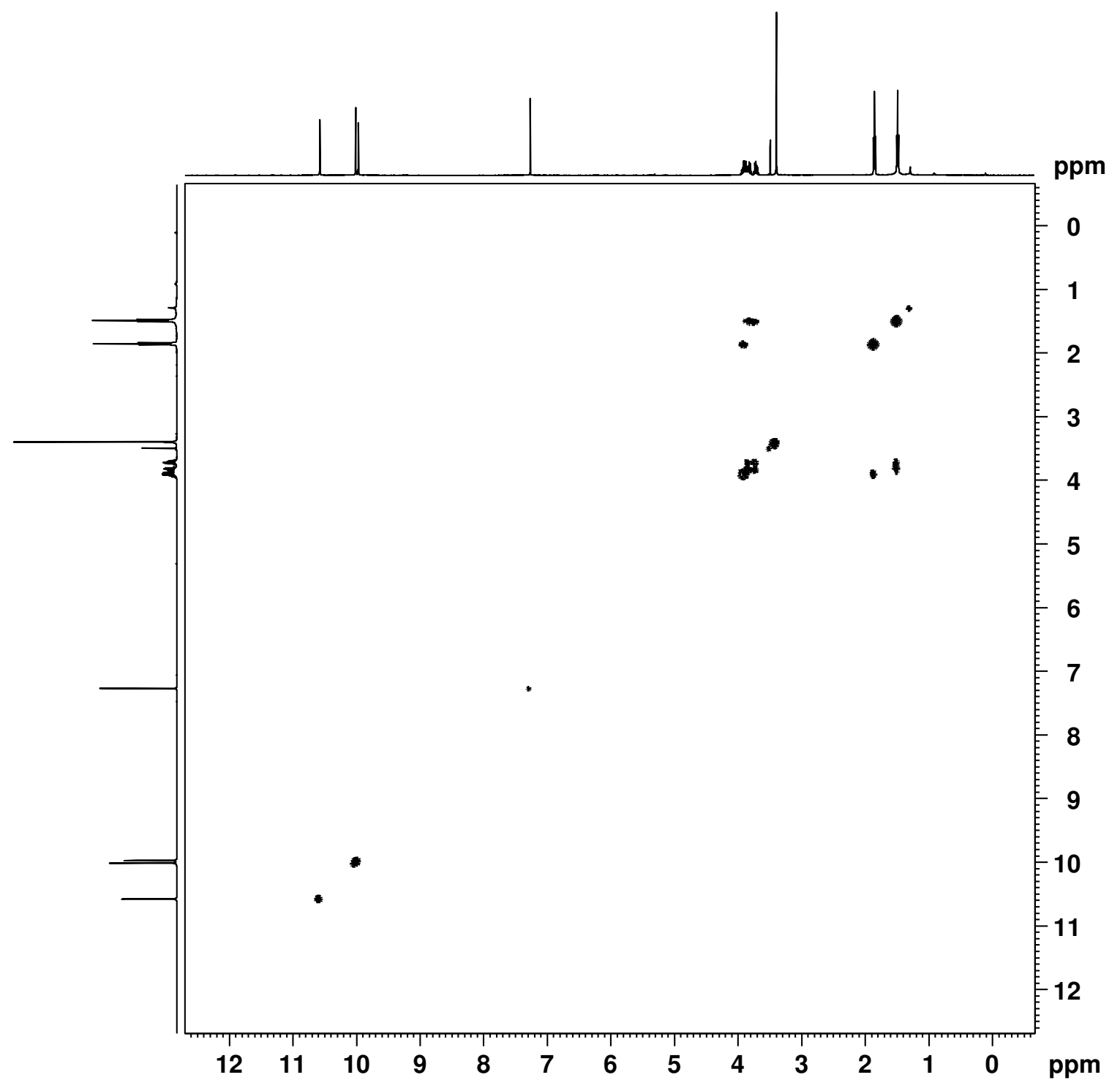

Figure D-28. ${ }^{1} \mathrm{H}-{ }^{1} \mathrm{H}$ COSY NMR spectrum of $N$-methyl thiaporphyrin 97b in $\mathrm{CDCl}_{3}$

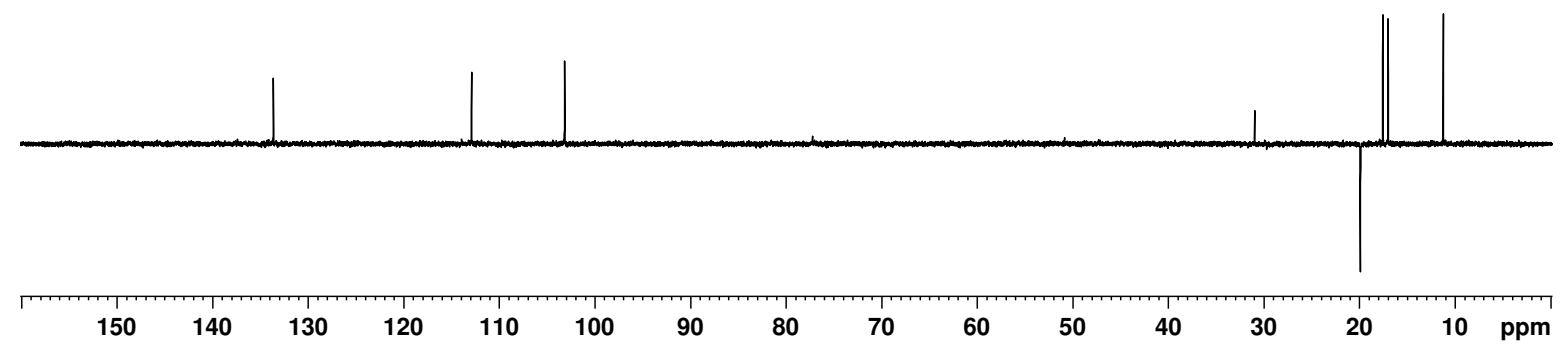

Figure D-29. DEPT-135 NMR spectrum of $N$-methyl thiaporphyrin 97b in $\mathrm{CDCl}_{3}$ 


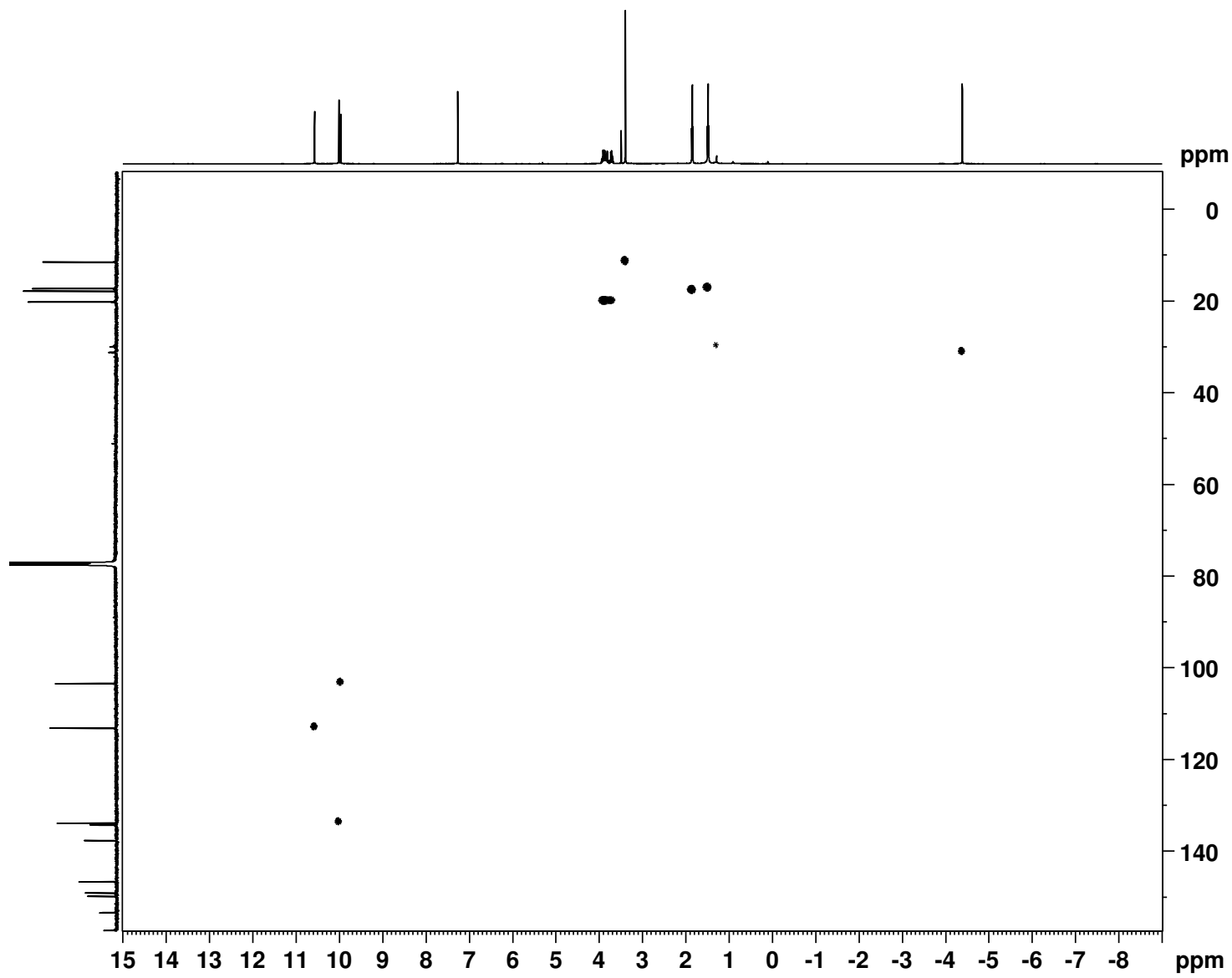

Figure D-30. HSQC NMR spectrum of $N$-methyl thiaporphyrin $97 \mathbf{b}$ in $\mathrm{CDCl}_{3}$ 


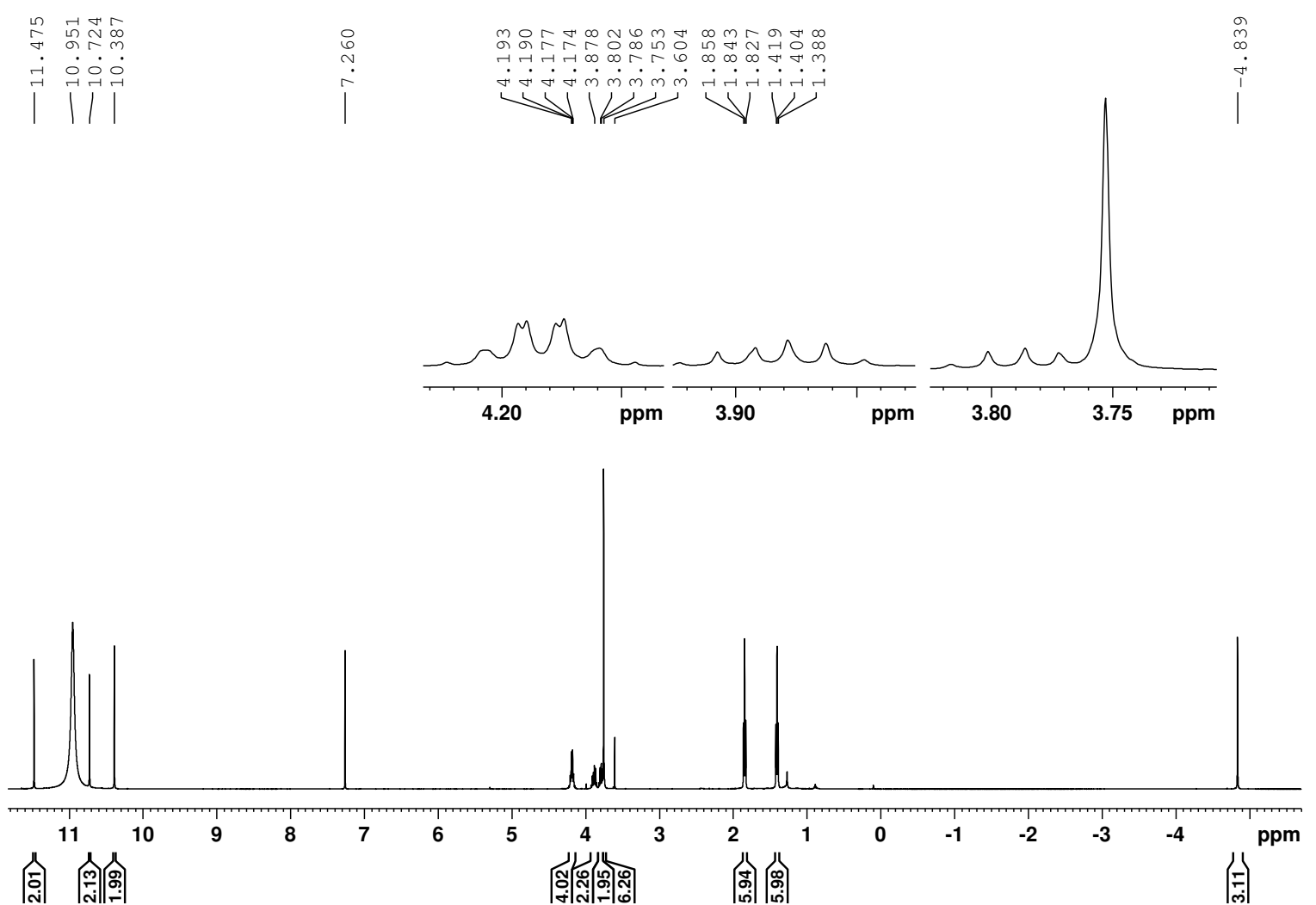

Figure D-31. $500 \mathrm{MHz}{ }^{1} \mathrm{H}$ NMR spectrum of $N$-methyl thiaporphyrin $97 \mathbf{b}$ in TFA-CDCl ${ }_{3}$

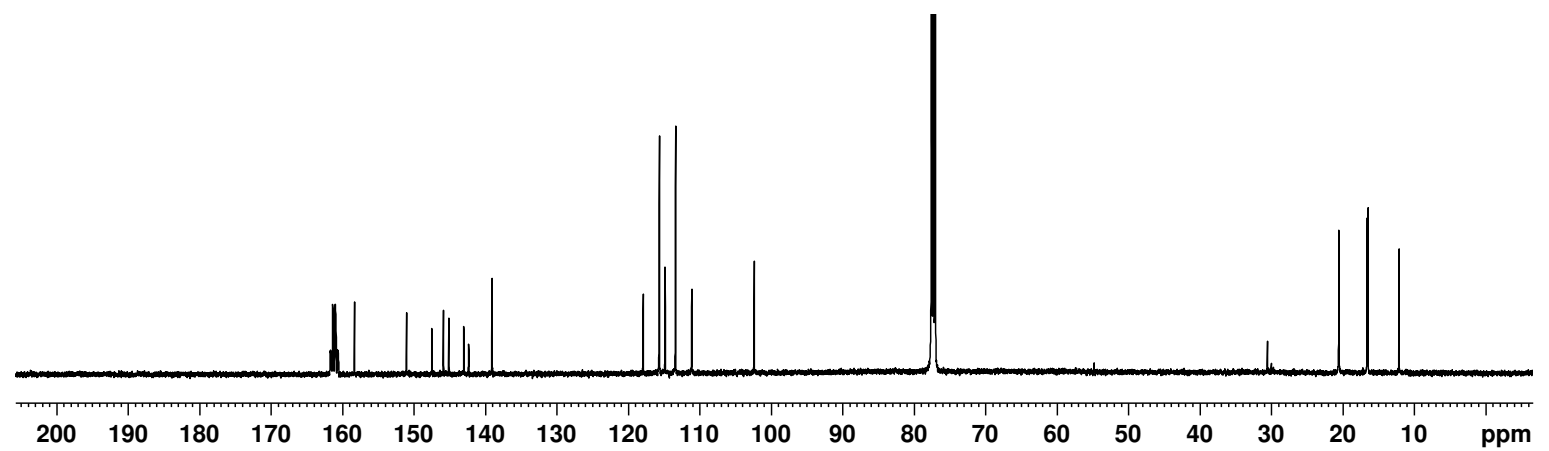

Figure D-32. $125 \mathrm{MHz}{ }^{13} \mathrm{C}$ NMR spectrum of $\mathrm{N}$-methyl thiaporphyrin $97 \mathbf{b}$ in $\mathrm{TFA}-\mathrm{CDCl}_{3}$ 


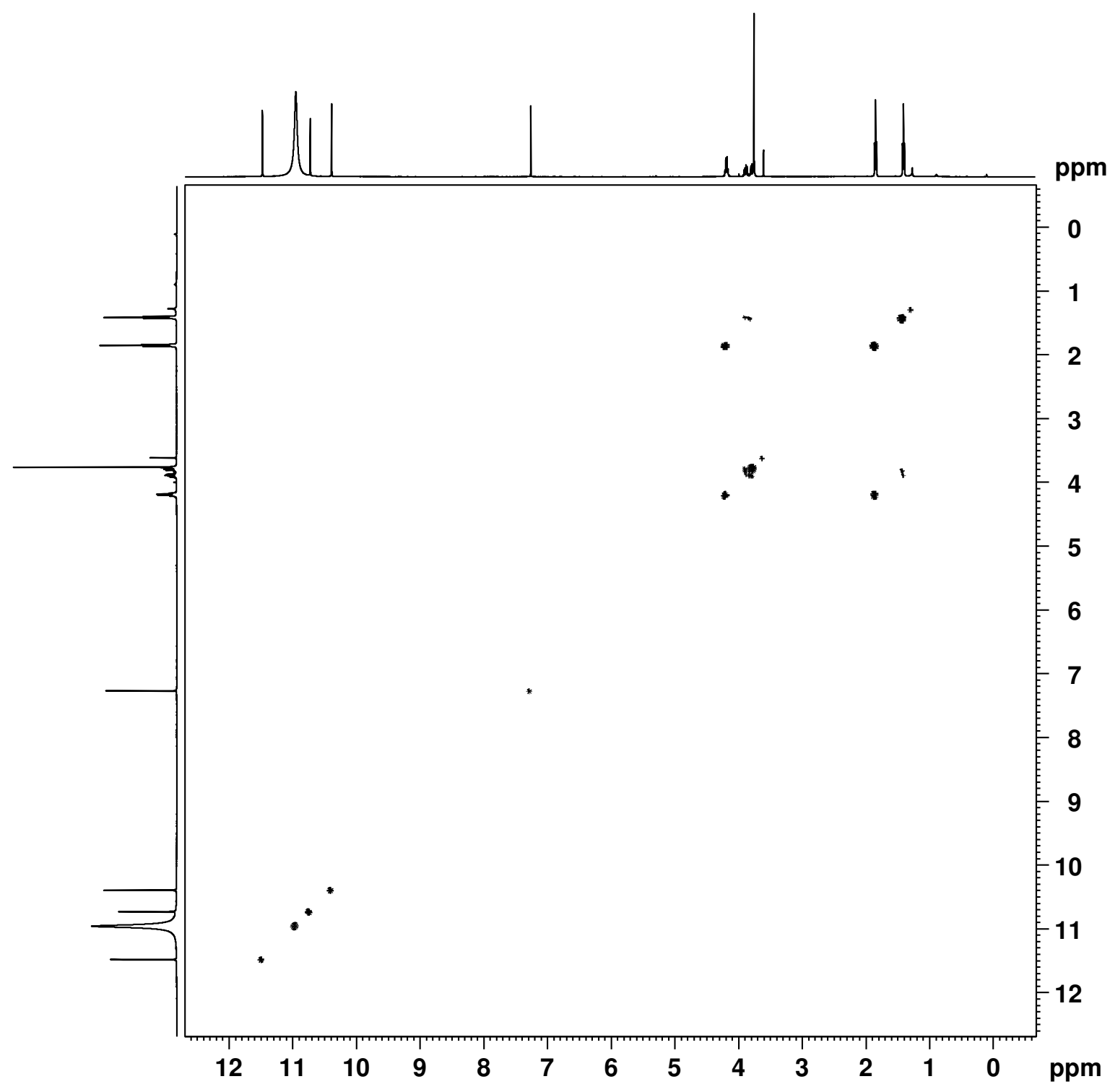

Figure D-33. ${ }^{1} \mathrm{H}-{ }^{1} \mathrm{H}$ COSY NMR spectrum of $N$-methyl thiaporphyrin $97 \mathbf{b}$ in TFA-CDCl ${ }_{3}$

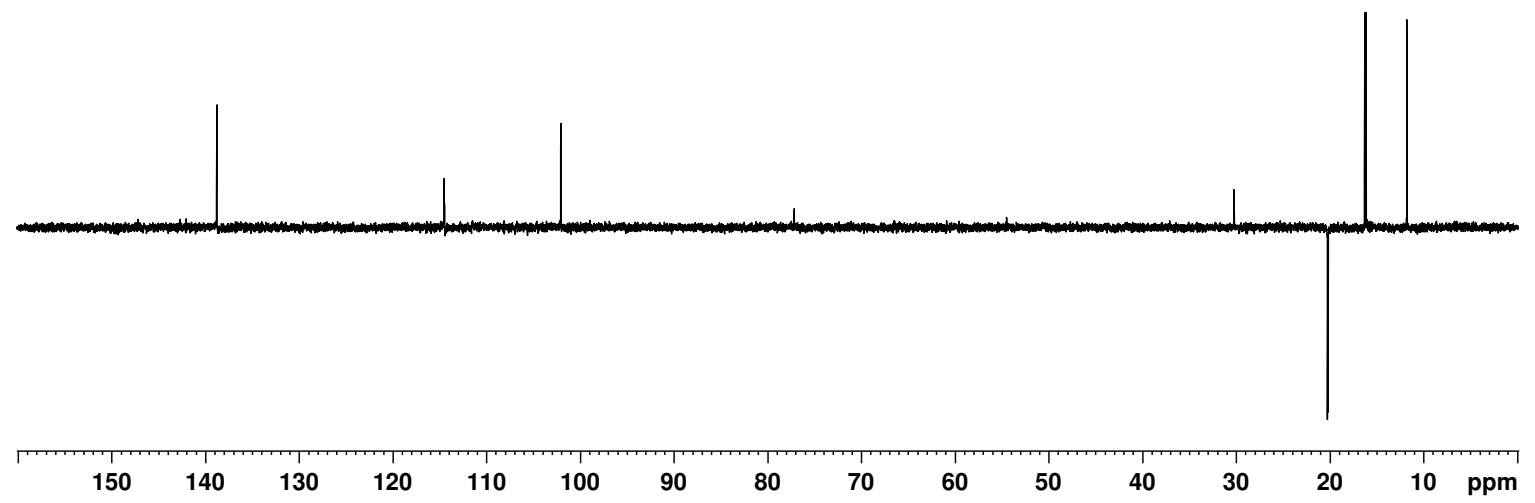

Figure D-34. DEPT-135 NMR spectrum of $N$-methyl thiaporphyrin 97b in TFA-CDCl 3 


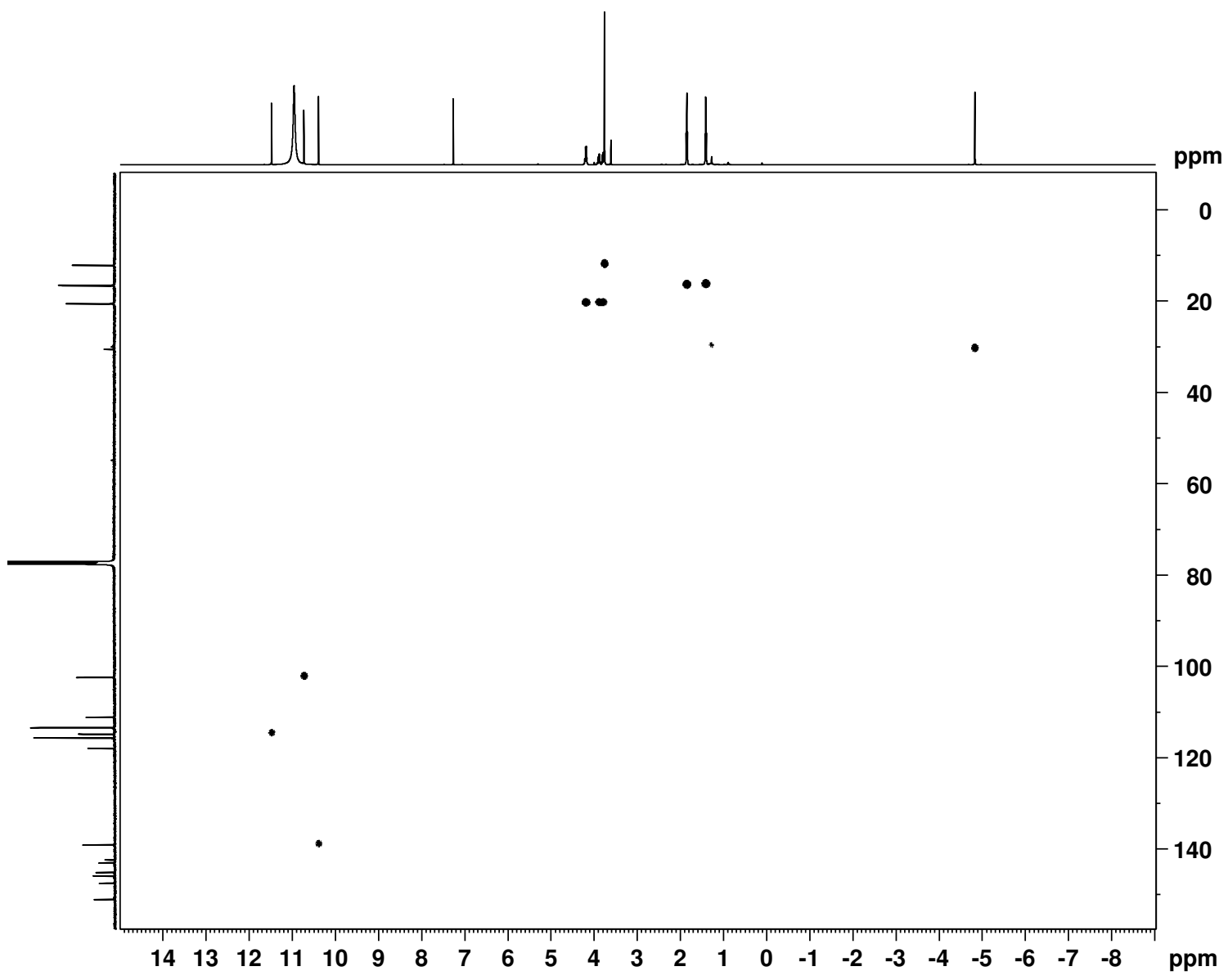

Figure D-35. HSQC NMR spectrum of $N$-methyl thiaporphyrin 97b in TFA-CDCl 3 

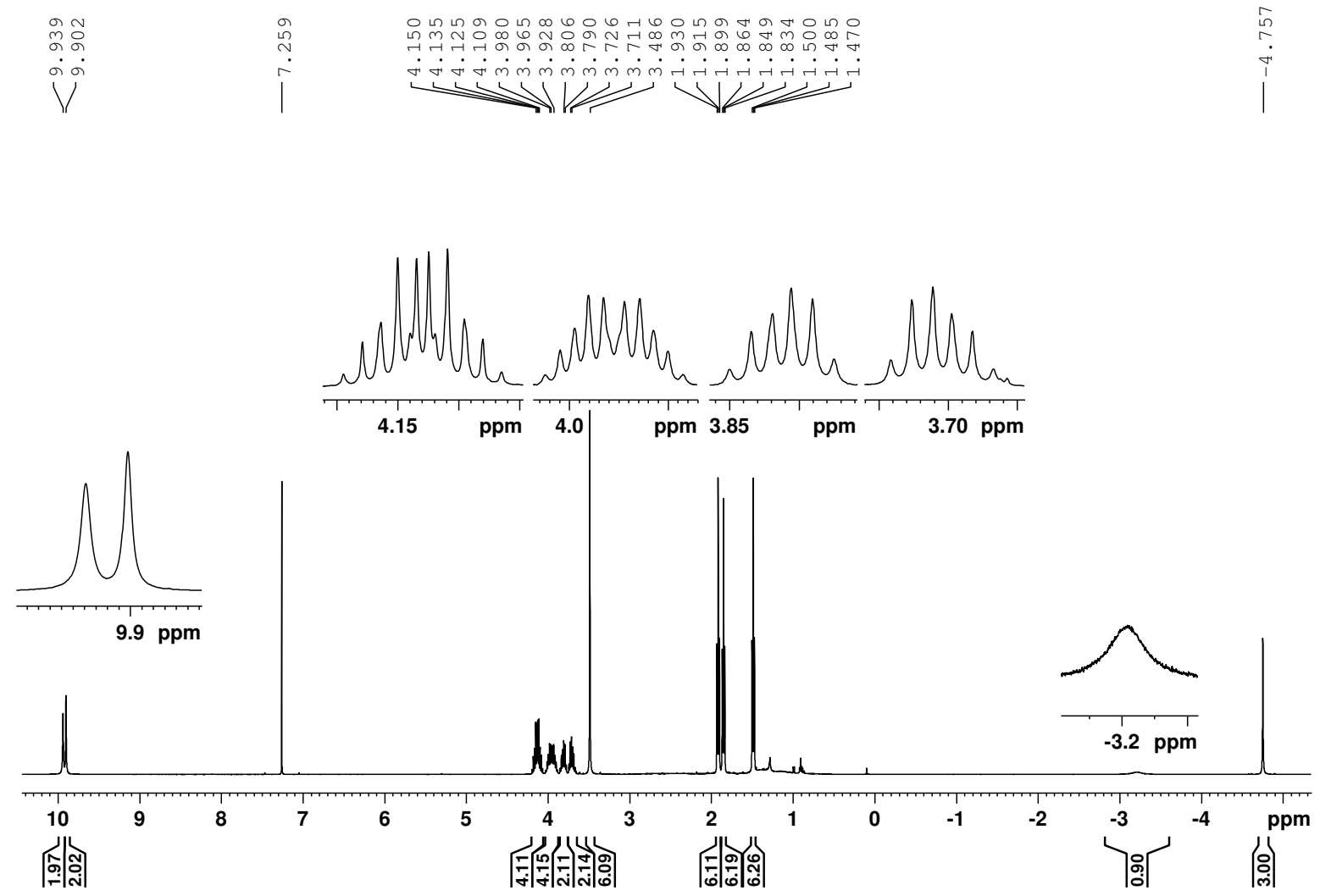

Figure D-36. 500MHz ${ }^{1} \mathrm{H}$ NMR spectrum of $N$-methyl porphyrin 99b in $\mathrm{CDCl}_{3}$

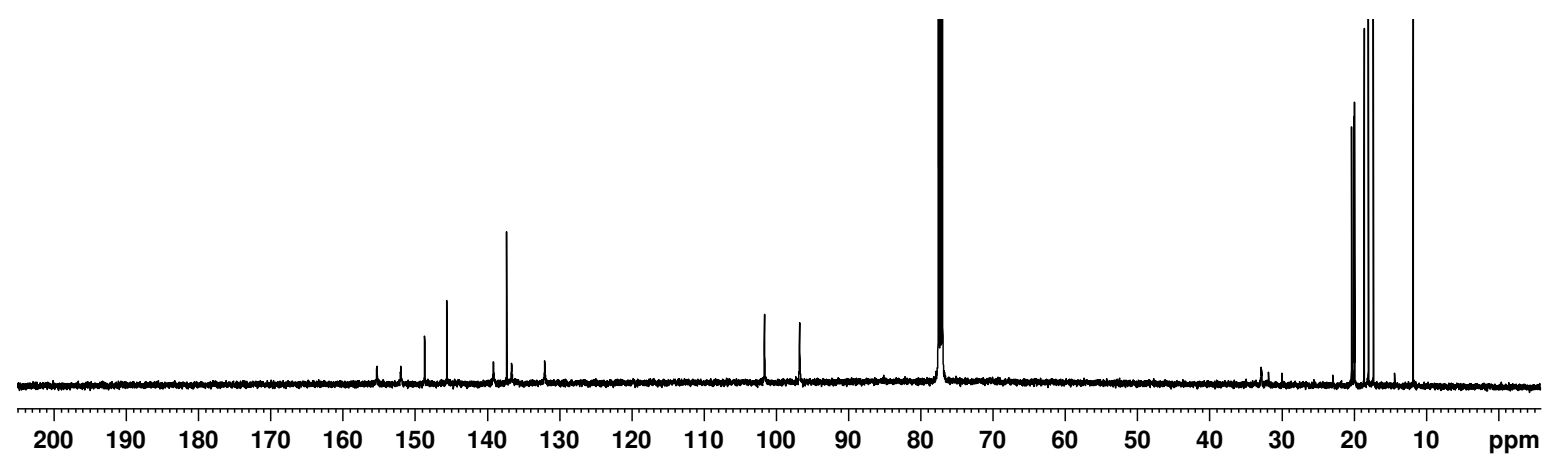

Figure D-37. $125 \mathrm{MHz}{ }^{13} \mathrm{C}$ NMR spectrum of $N$-methyl porphyrin $99 \mathrm{~b}$ in $\mathrm{CDCl}_{3}$ 


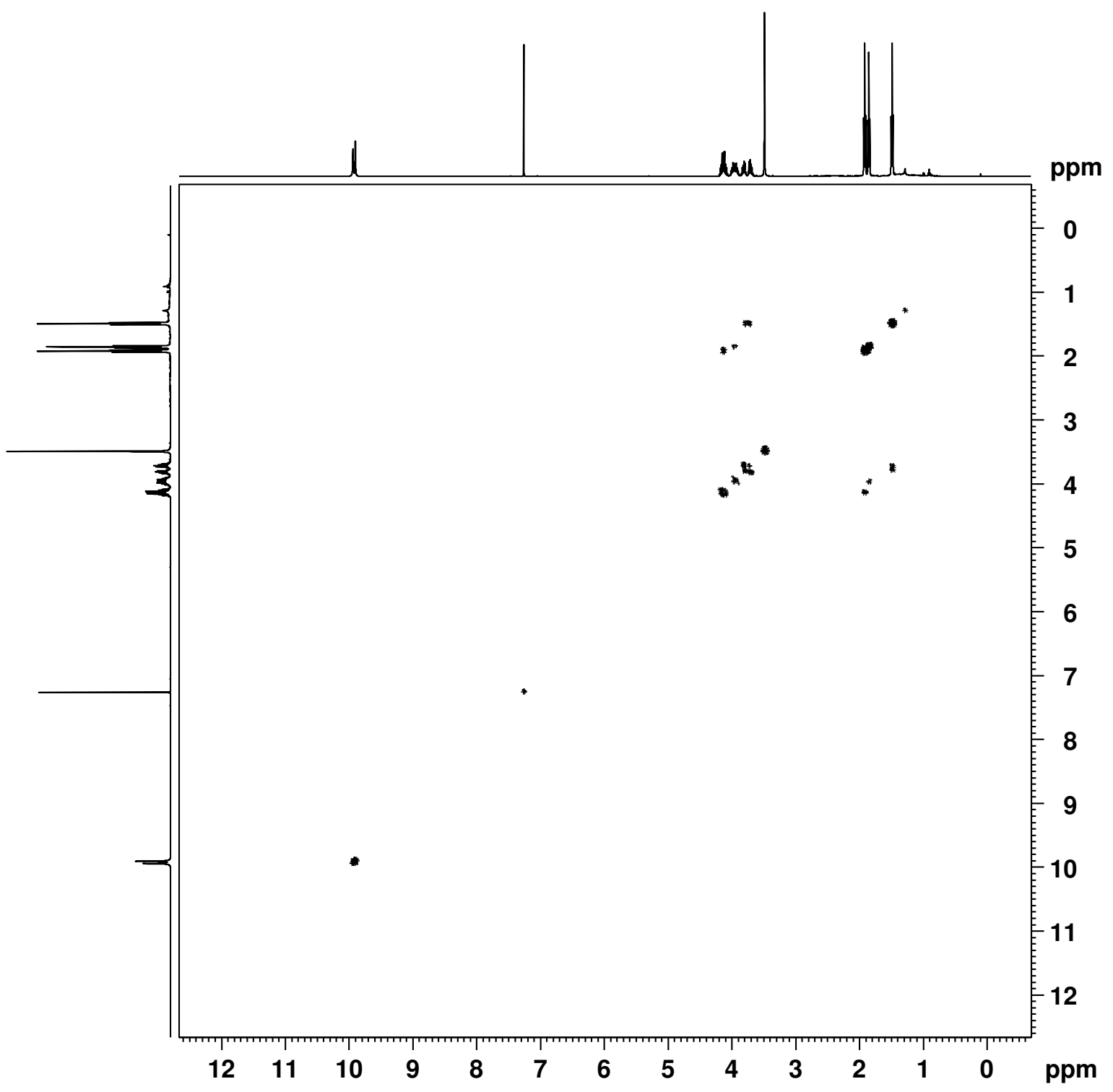

Figure D-38. ${ }^{1} \mathrm{H}-{ }^{1} \mathrm{H}$ COSY NMR spectrum of $N$-methyl porphyrin $99 \mathbf{b}$ in $\mathrm{CDCl}_{3}$

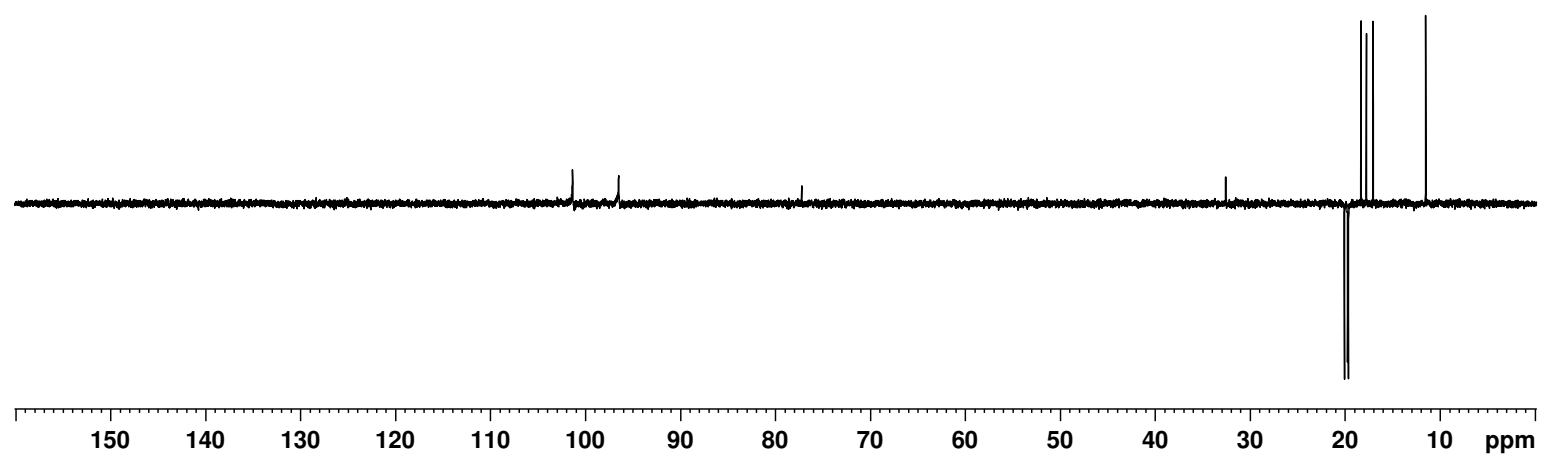

Figure D-39. DEPT-135 NMR spectrum of $N$-methyl porphyrin 99b in $\mathrm{CDCl}_{3}$ 


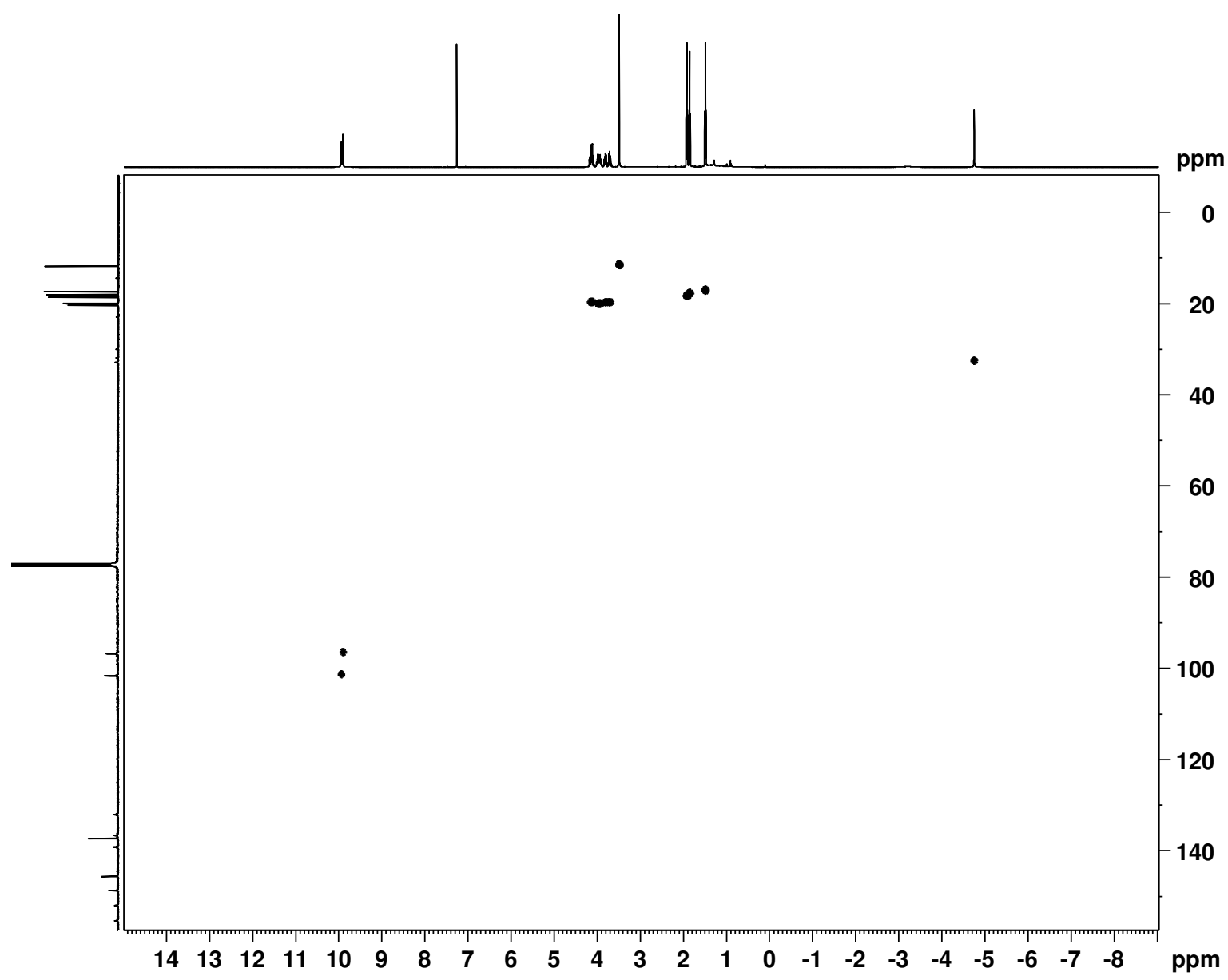

Figure D-40. HSQC NMR spectrum of $N$-methyl porphyrin 99 b in $\mathrm{CDCl}_{3}$ 


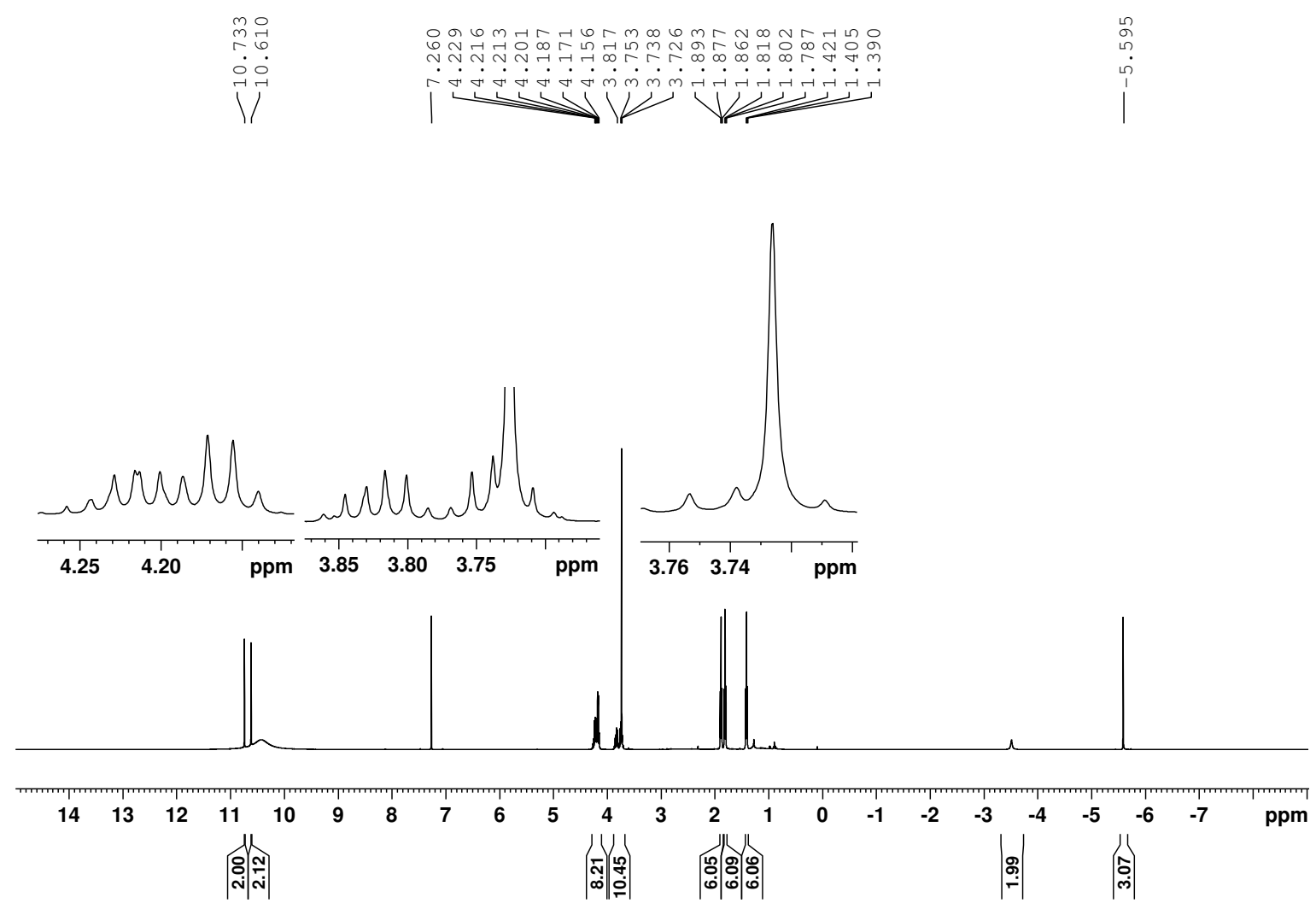

Figure D-41. $500 \mathrm{MHz}{ }^{1} \mathrm{H}$ NMR spectrum of $N$-methyl porphyrin 99b in TFA-CDCl 3

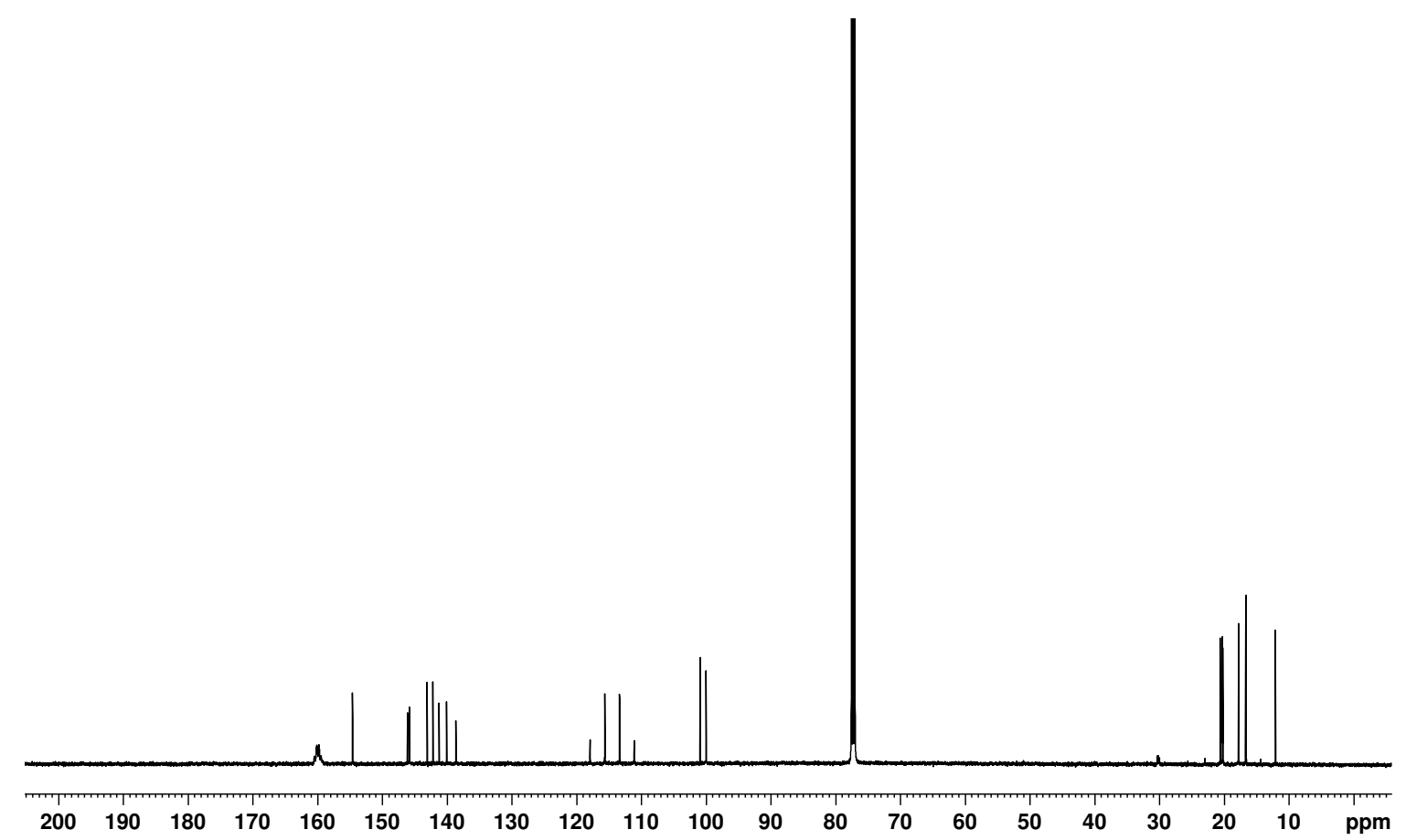

Figure D-42. $125 \mathrm{MHz}{ }^{13} \mathrm{C}$ NMR spectrum of $N$-methyl porphyrin 99b in TFA-CDCl ${ }_{3}$ 


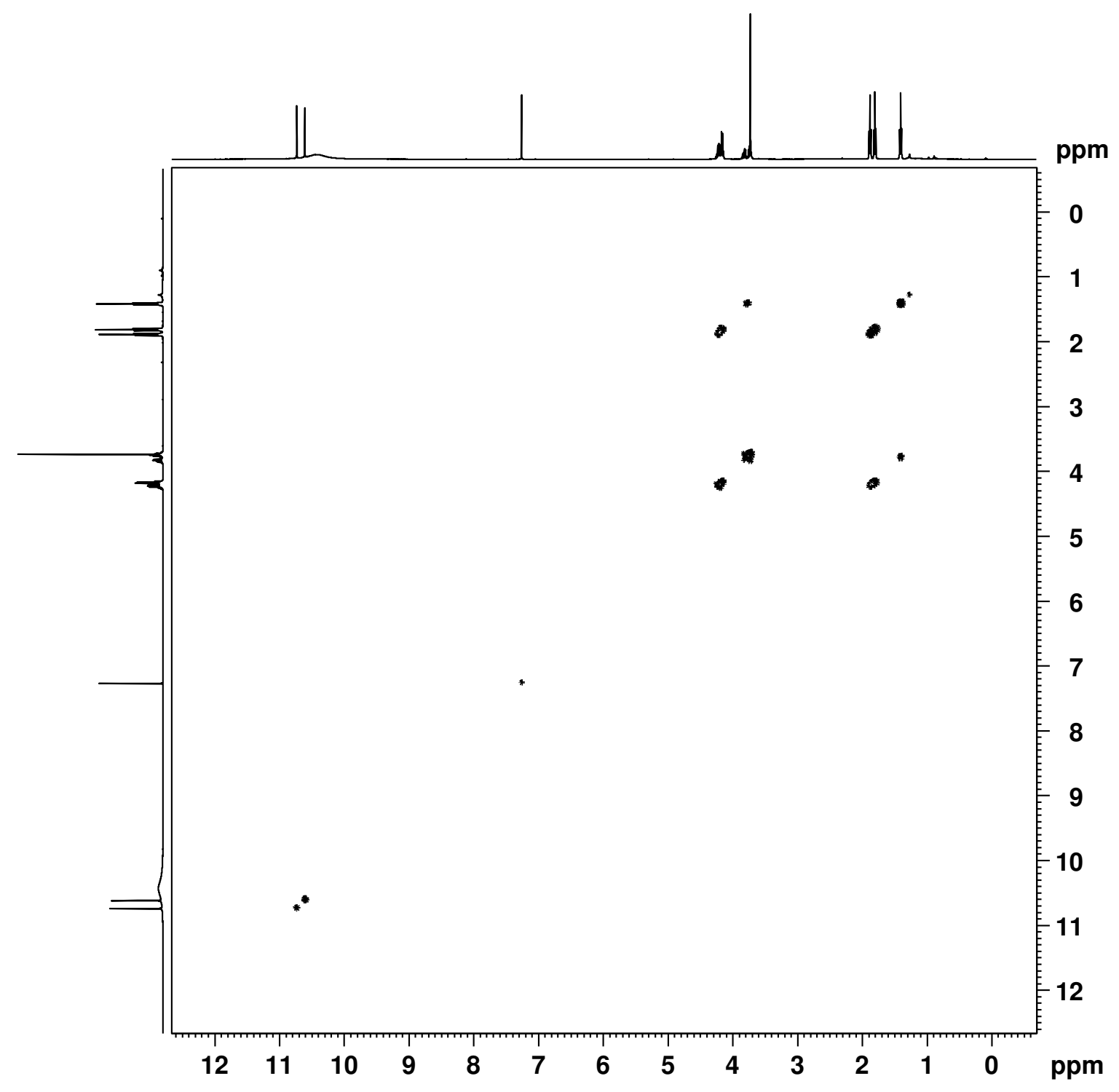

Figure D-43. ${ }^{1} \mathrm{H}-{ }^{1} \mathrm{H}$ COSY NMR spectrum of $\mathrm{N}$-methyl porphyrin 99b in TFA-CDCl $\mathrm{Cl}_{3}$

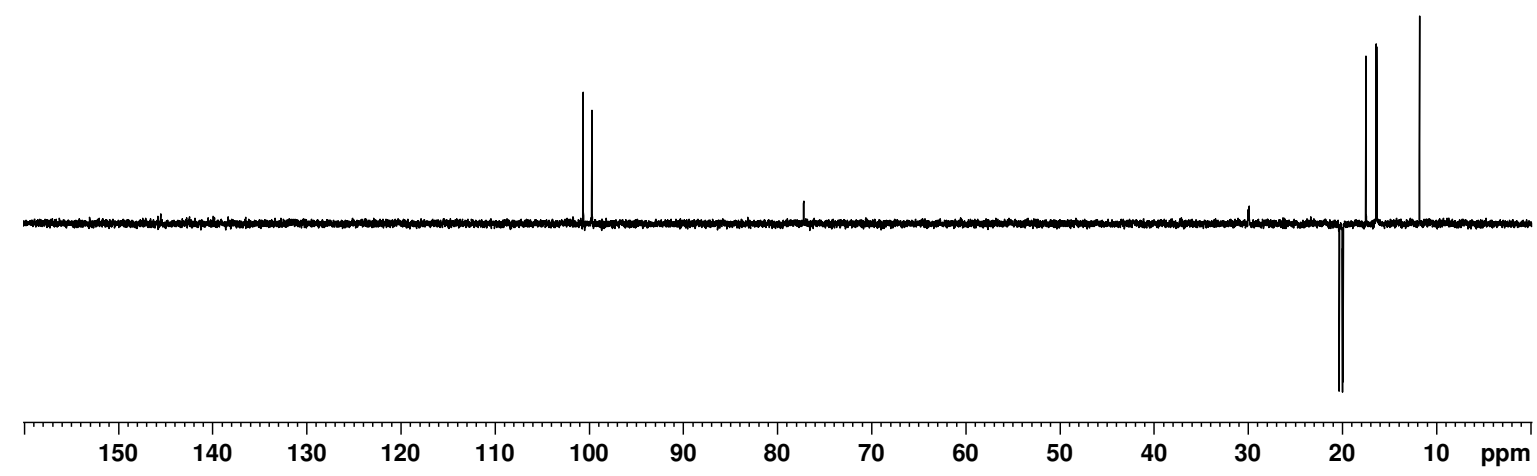

Figure D-44. DEPT-135 NMR spectrum of $N$-methyl porphyrin 99b in TFA-CDCl 3 


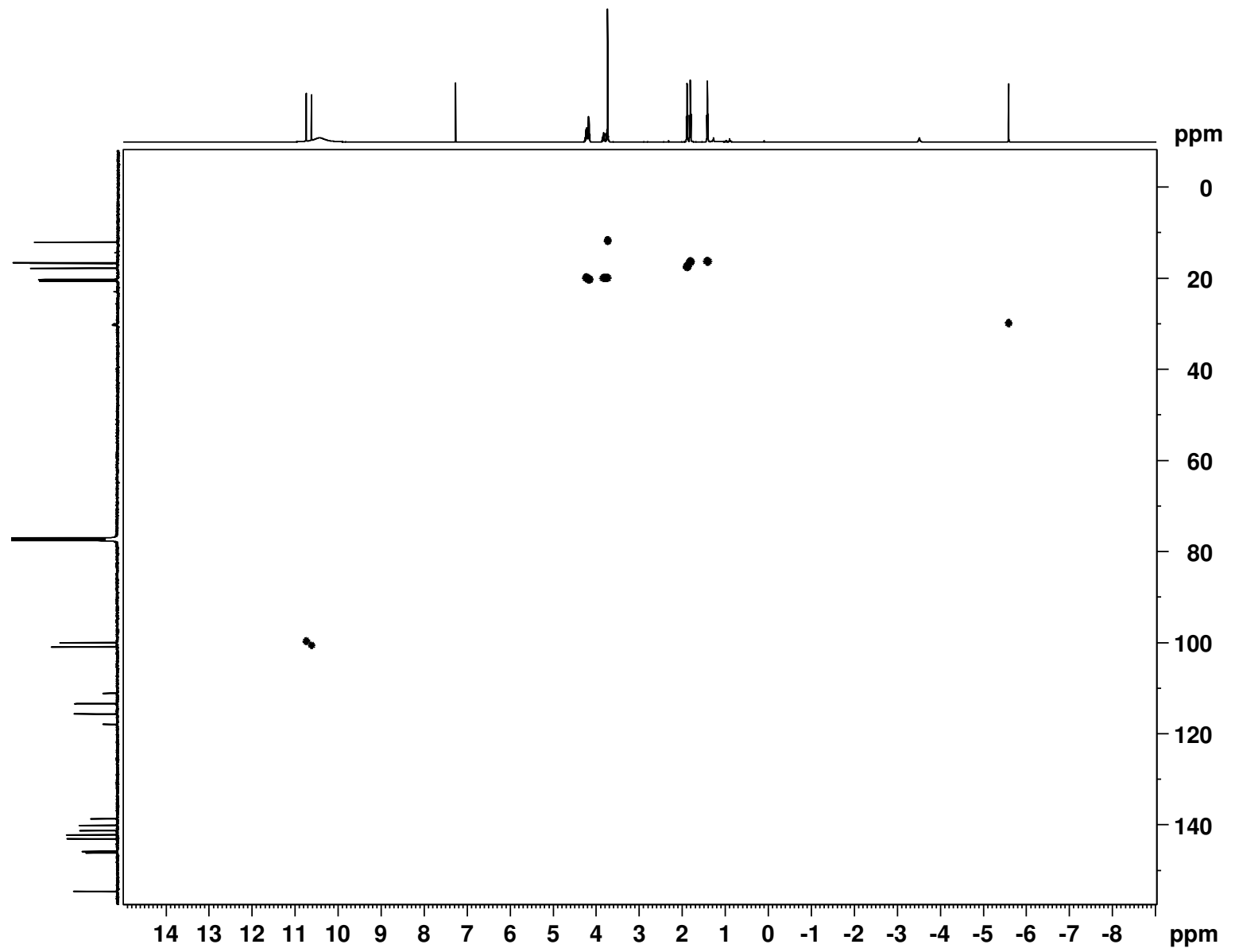

Figure D-45. HSQC NMR spectrum of $\mathrm{N}$-methyl porphyrin 99b in TFA-CDCl $\mathrm{Cl}_{3}$ 


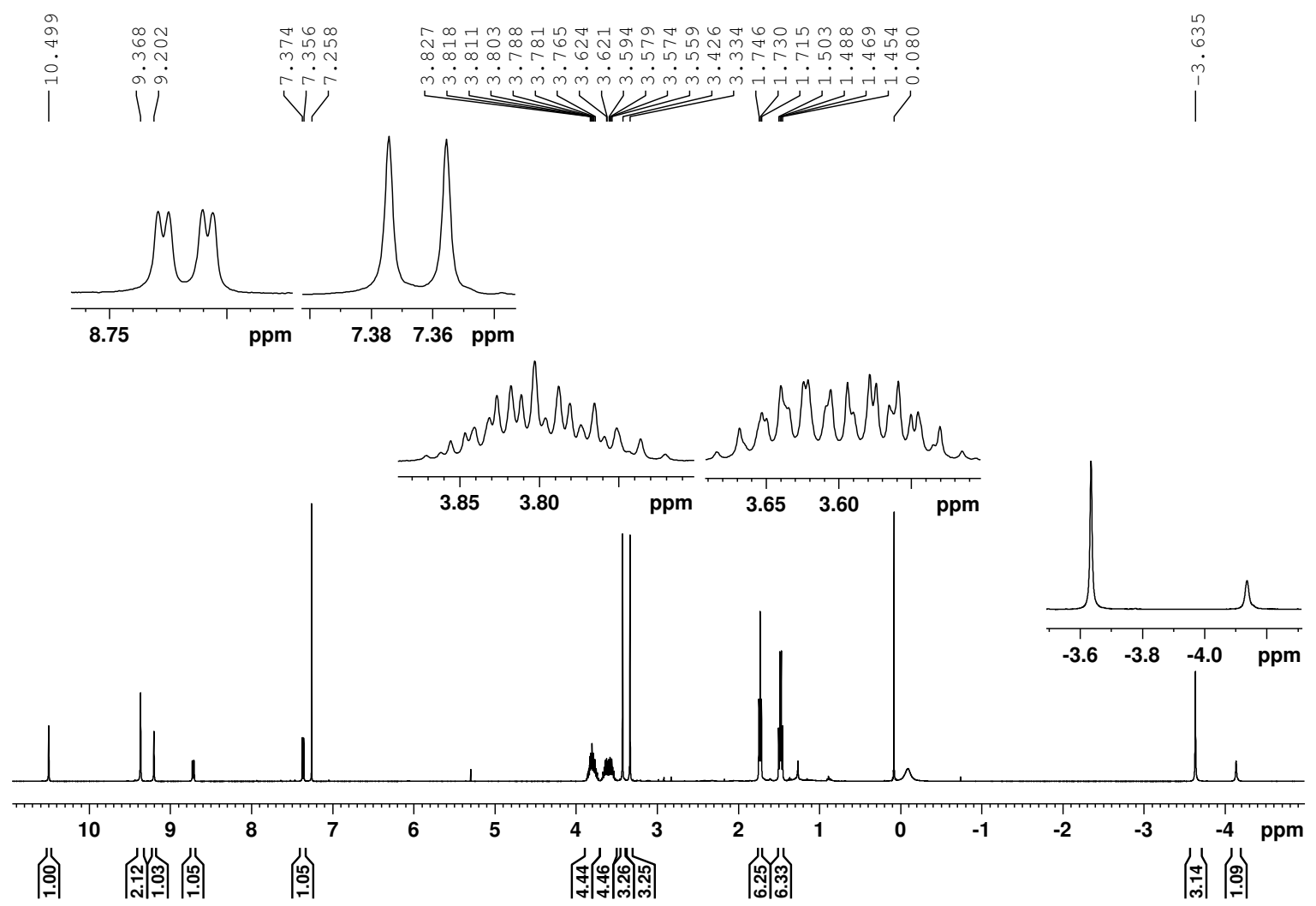

Figure D-46. $500 \mathrm{MHz}{ }^{1} \mathrm{H}$ NMR spectrum of $N$-methyl oxybenziporphyrin 100a in $\mathrm{CDCl}_{3}$

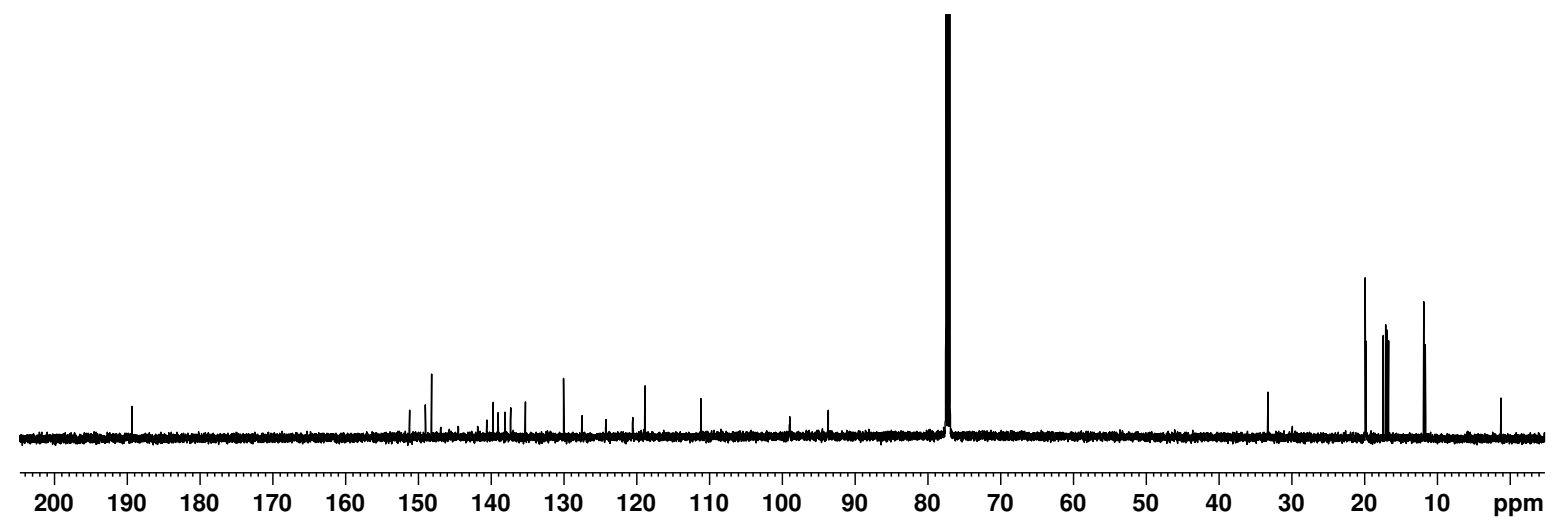

Figure D-47. $125 \mathrm{MHz}{ }^{13} \mathrm{C}$ NMR spectrum of $N$-methyl oxybenziporphyrin 100a in $\mathrm{CDCl}_{3}$ 


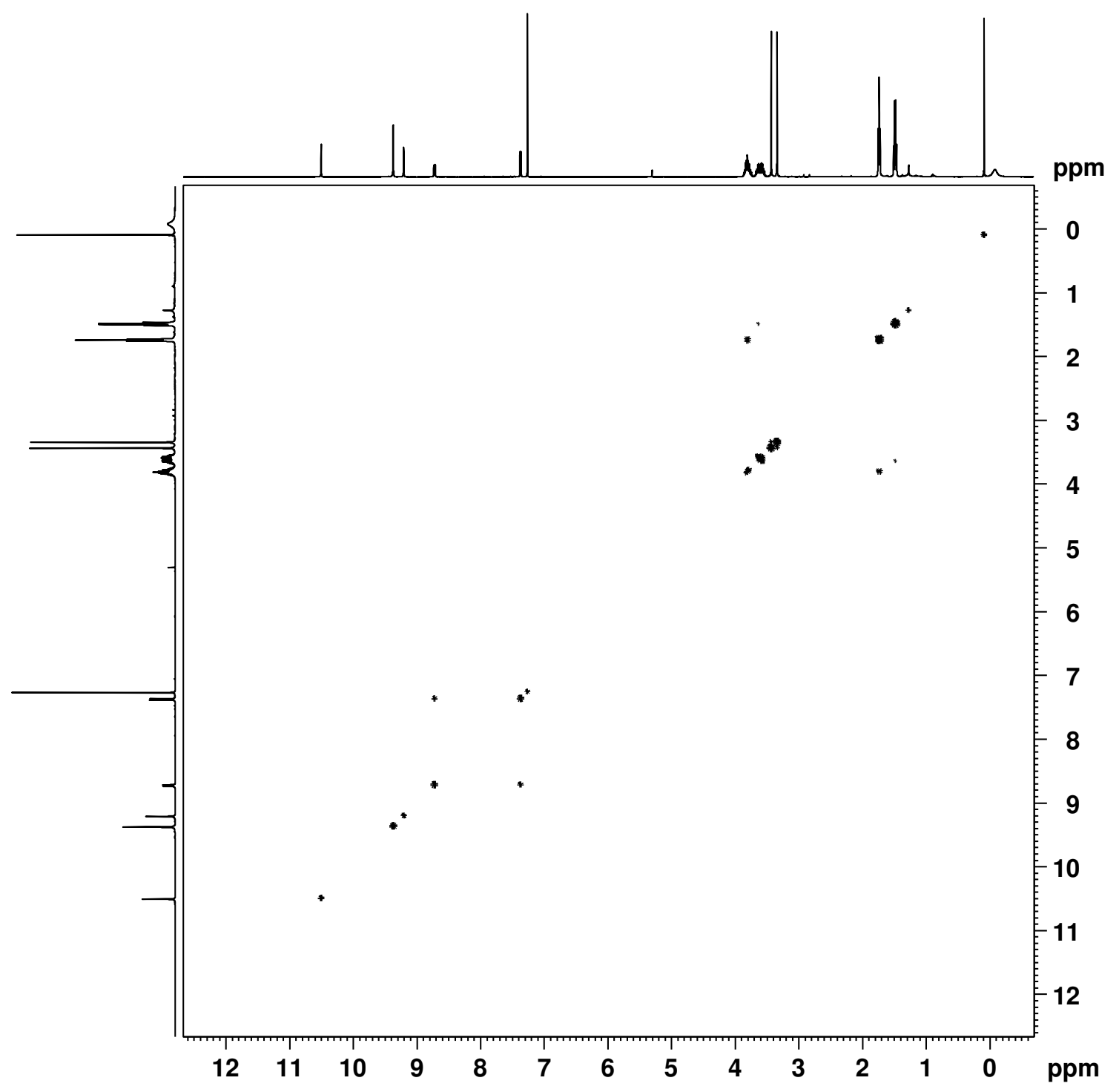

Figure D-48. ${ }^{1} \mathrm{H}-{ }^{1} \mathrm{H}$ COSY NMR spectrum of $N$-methyl oxybenziporphyrin 100a in $\mathrm{CDCl}_{3}$

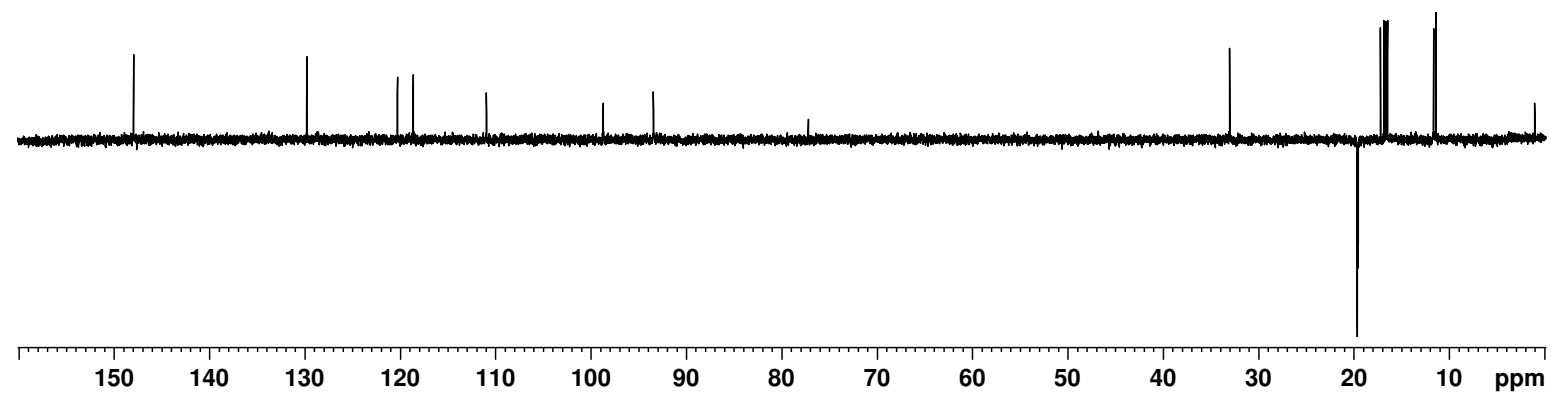

Figure D-49. DEPT-135 NMR spectrum of $N$-methyl oxybenziporphyrin 100a in $\mathrm{CDCl}_{3}$ 


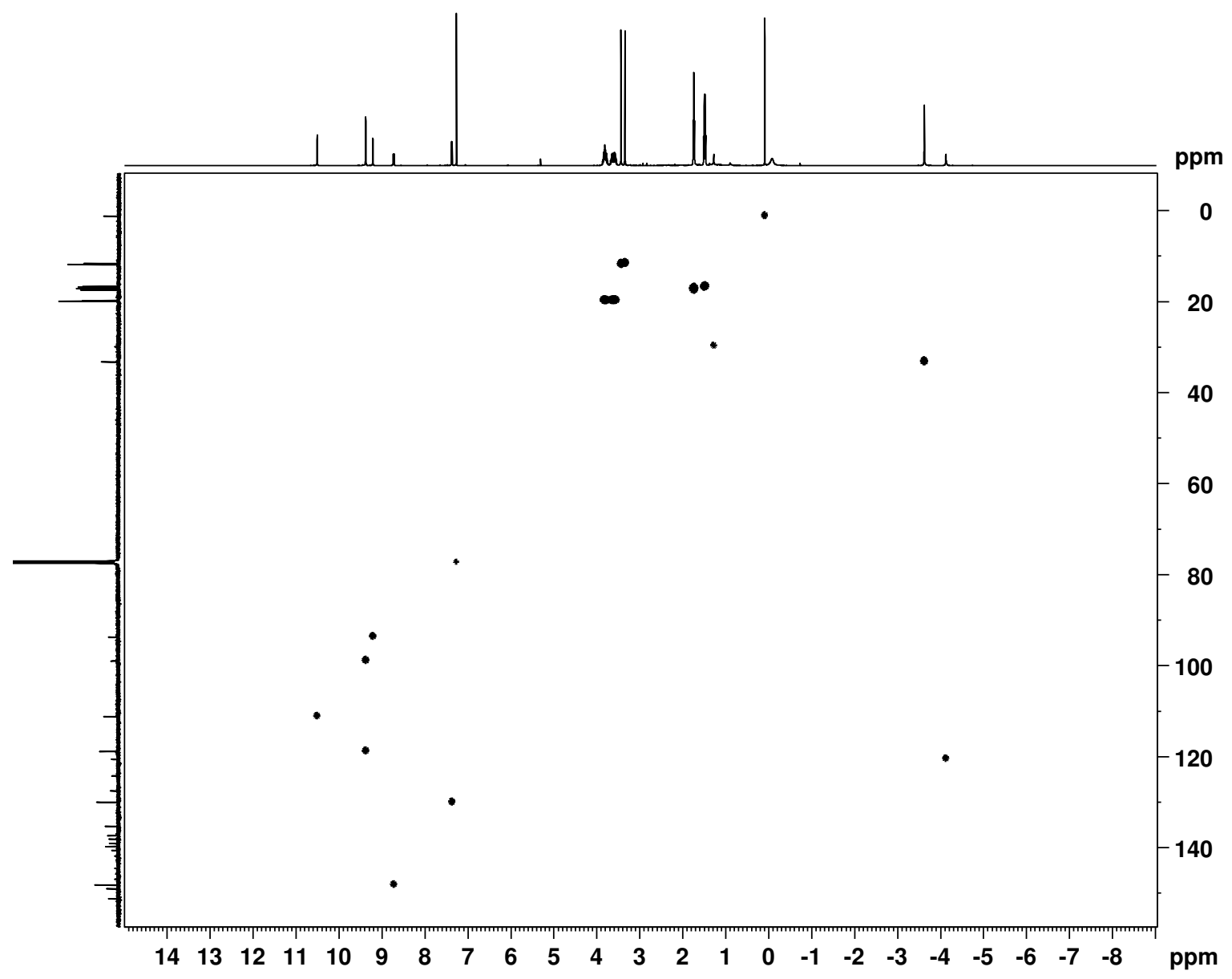

Figure D-50. HSQC NMR spectrum of $N$-methyl oxybenziporphyrin 100a in $\mathrm{CDCl}_{3}$ 


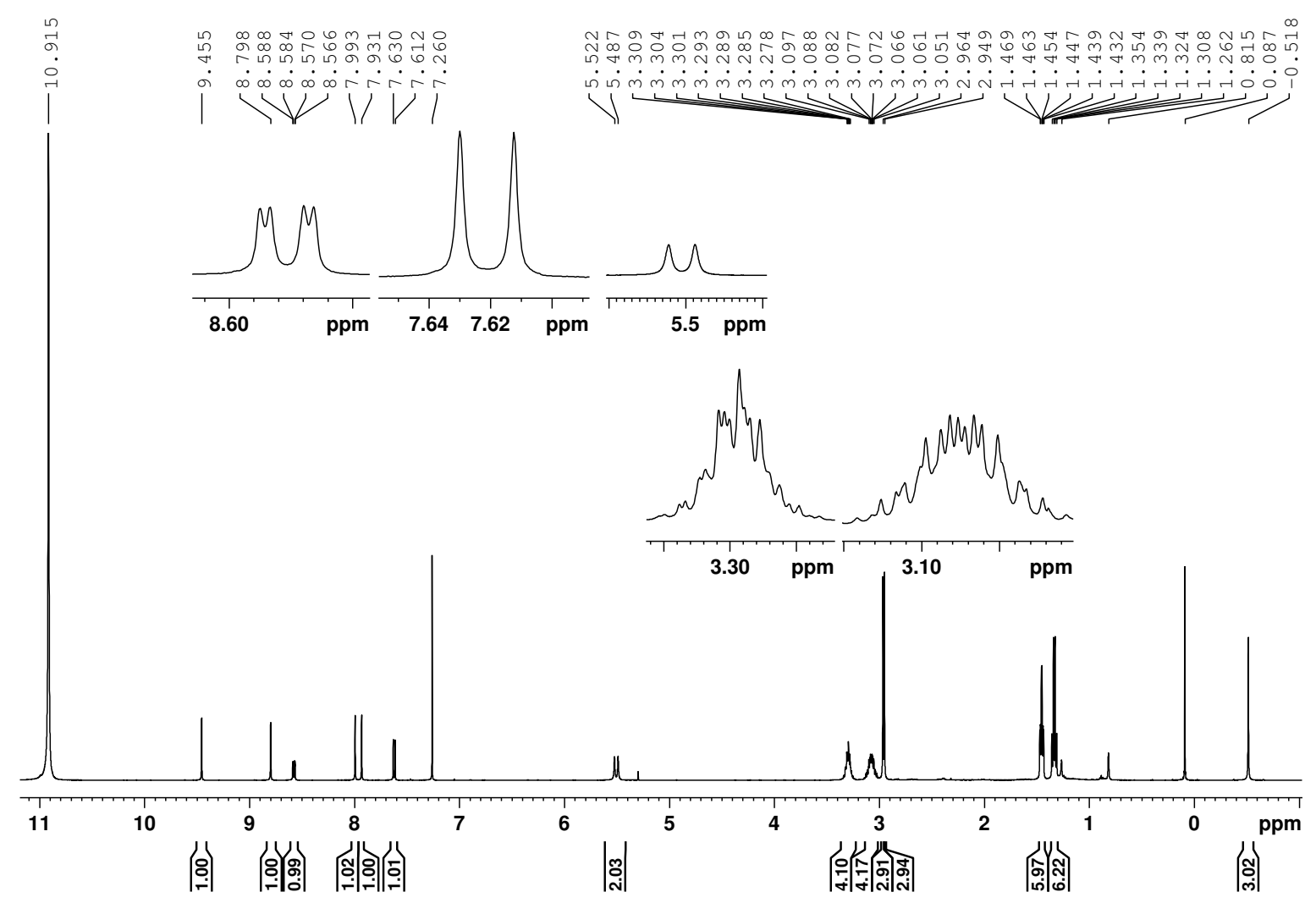

Figure D-51. $500 \mathrm{MHz}{ }^{1} \mathrm{H}$ NMR spectrum of $N$-methyl oxybenziporphyrin 100a in TFA$\mathrm{CDCl}^{3}$

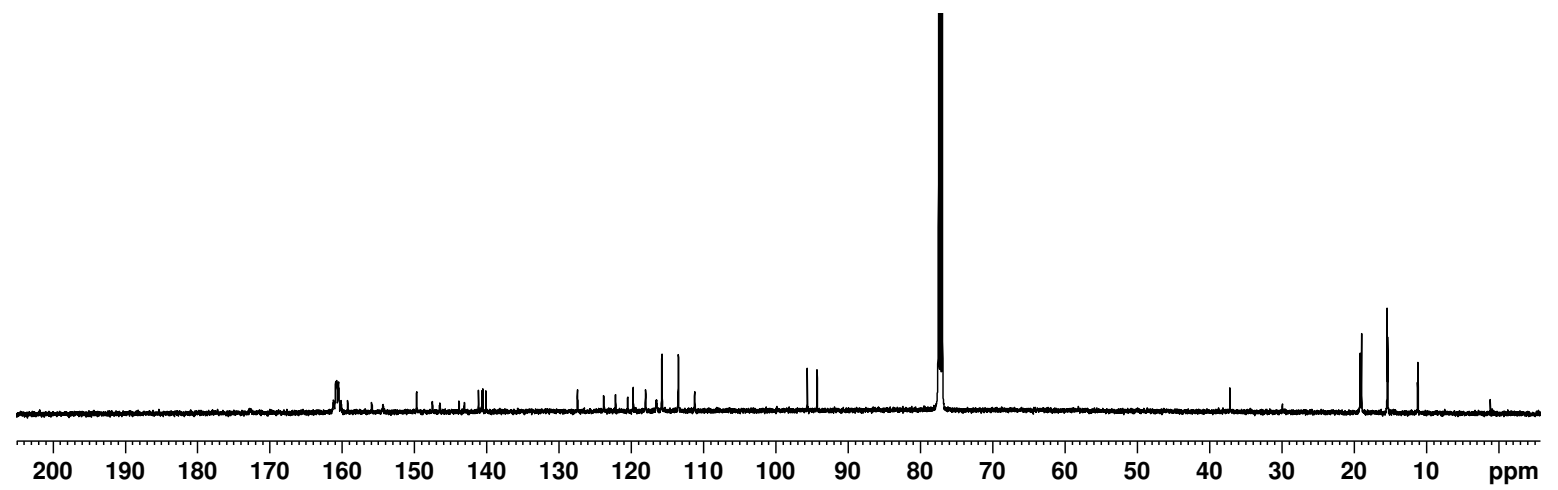

Figure D-52. $125 \mathrm{MHz}{ }^{13} \mathrm{C}$ NMR spectrum of $N$-methyl oxybenziporphyrin 100a in TFA$\mathrm{CDCl}^{3}$ 


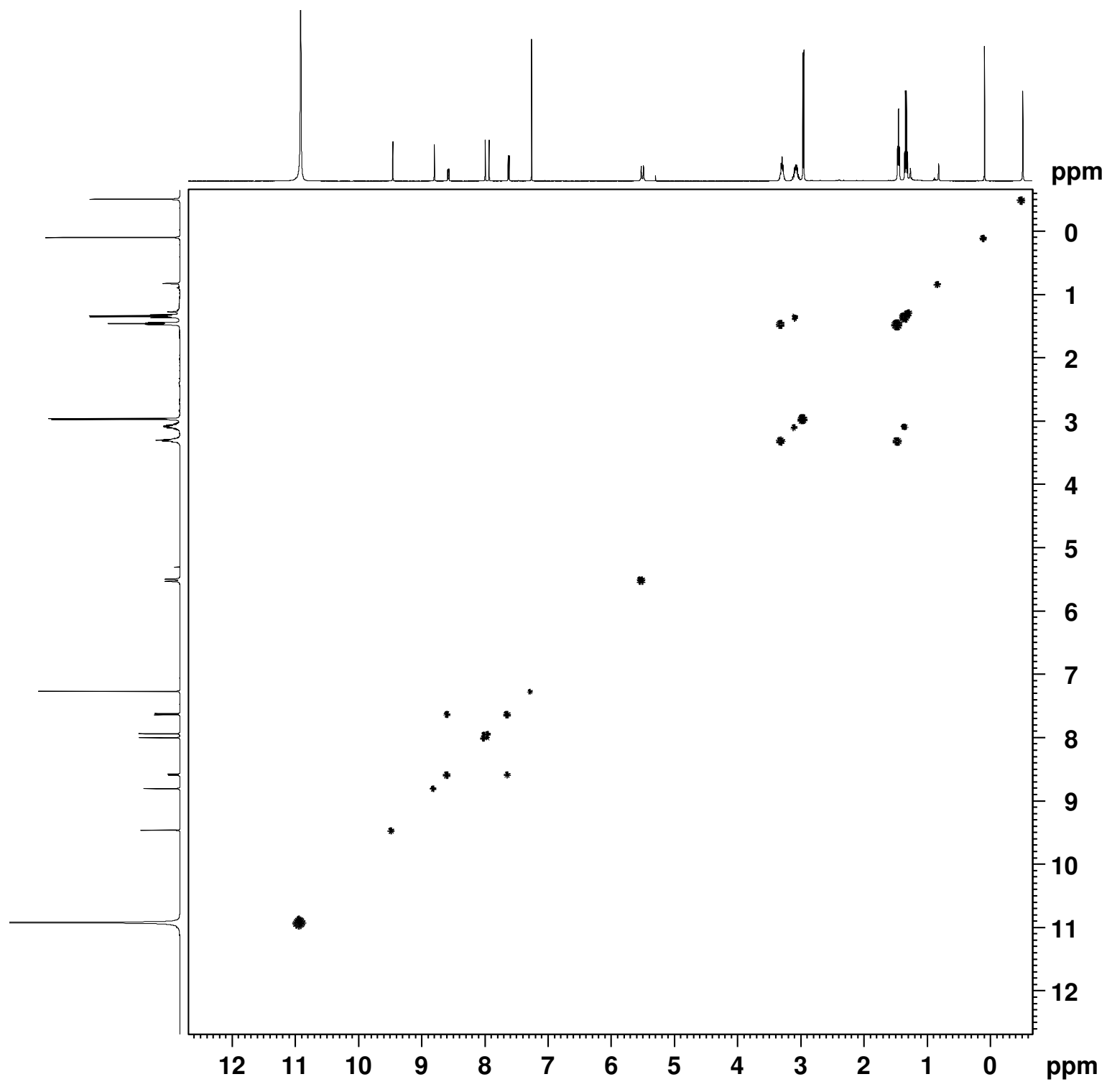

Figure D-53. ${ }^{1} \mathrm{H}-{ }^{1} \mathrm{H}$ COSY NMR spectrum of $N$-methyl oxybenziporphyrin 100a in TFA-CDCl ${ }_{3}$

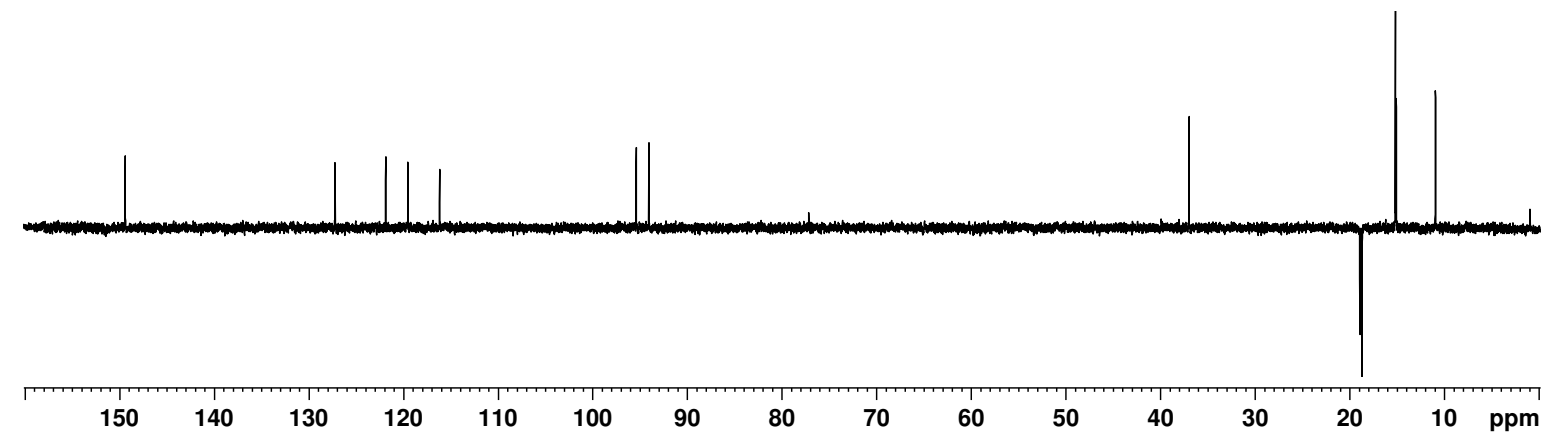

Figure D-54. DEPT-135 NMR spectrum of $N$-methyl oxybenziporphyrin 100a in TFA$\mathrm{CDCl}^{3}$ 


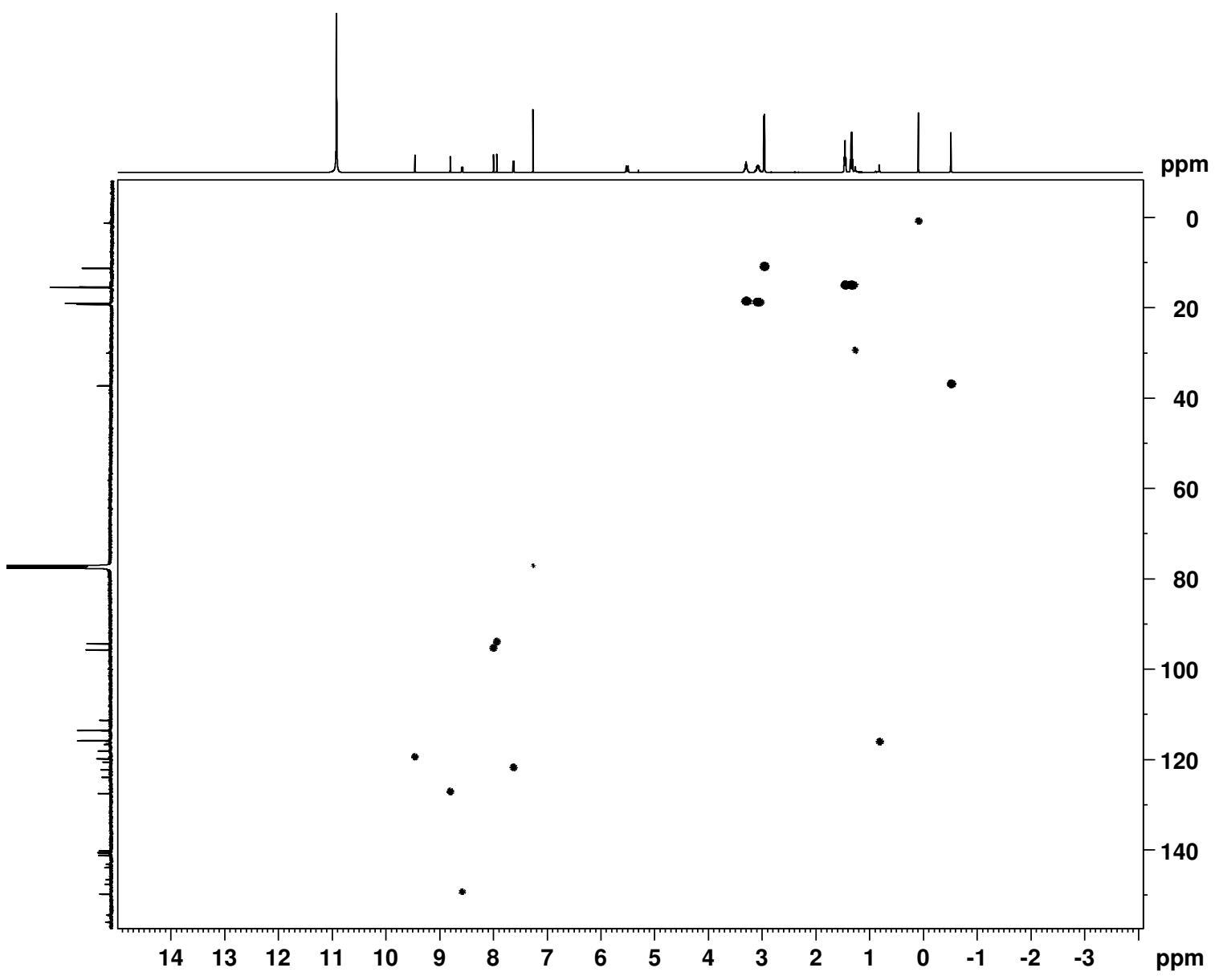

Figure D-55. HSQC NMR spectrum of $N$-methyl oxybenziporphyrin 100a in TFA$\mathrm{CDCl}^{3}$ 

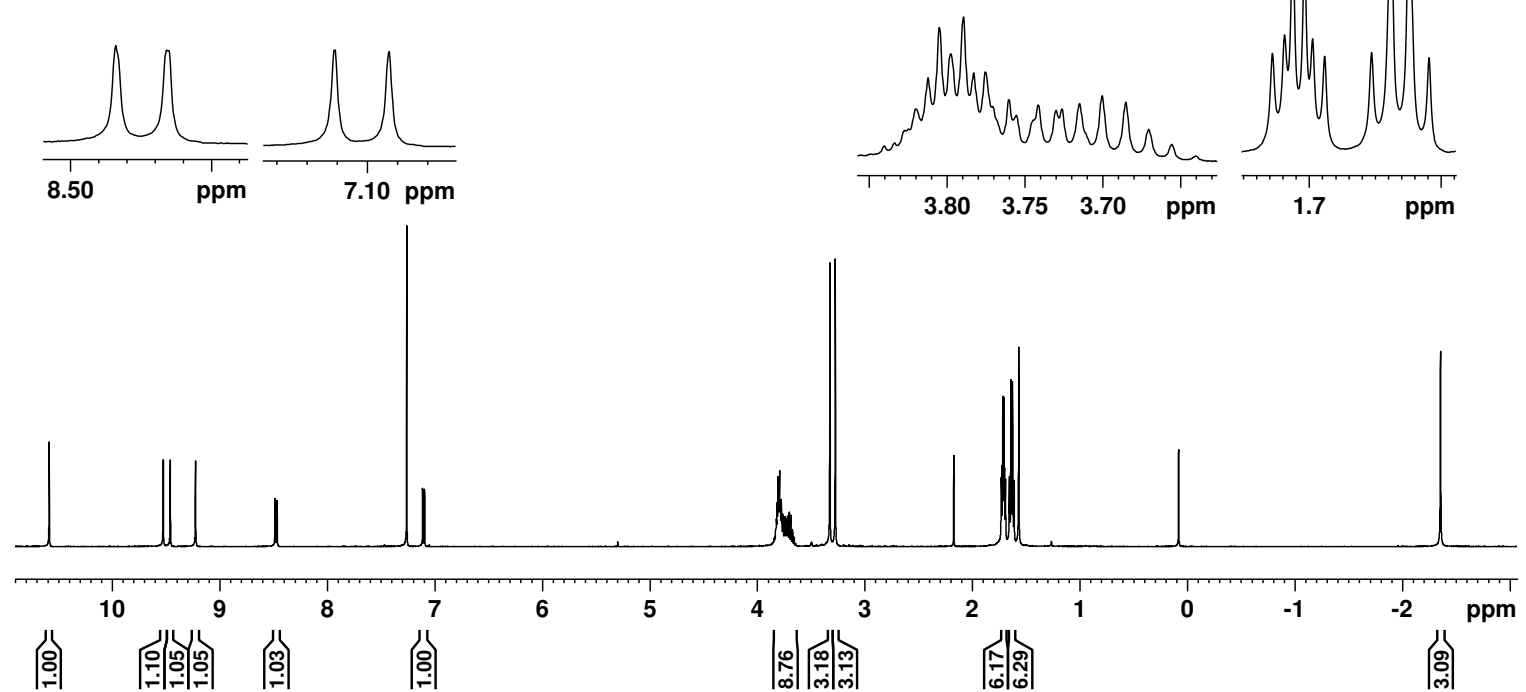

Figure D-56. $500 \mathrm{MHz}{ }^{1} \mathrm{H}$ NMR spectrum of $N$-methyl palladium(II) oxybenziporphyrin 102 in $\mathrm{CDCl}^{3}$

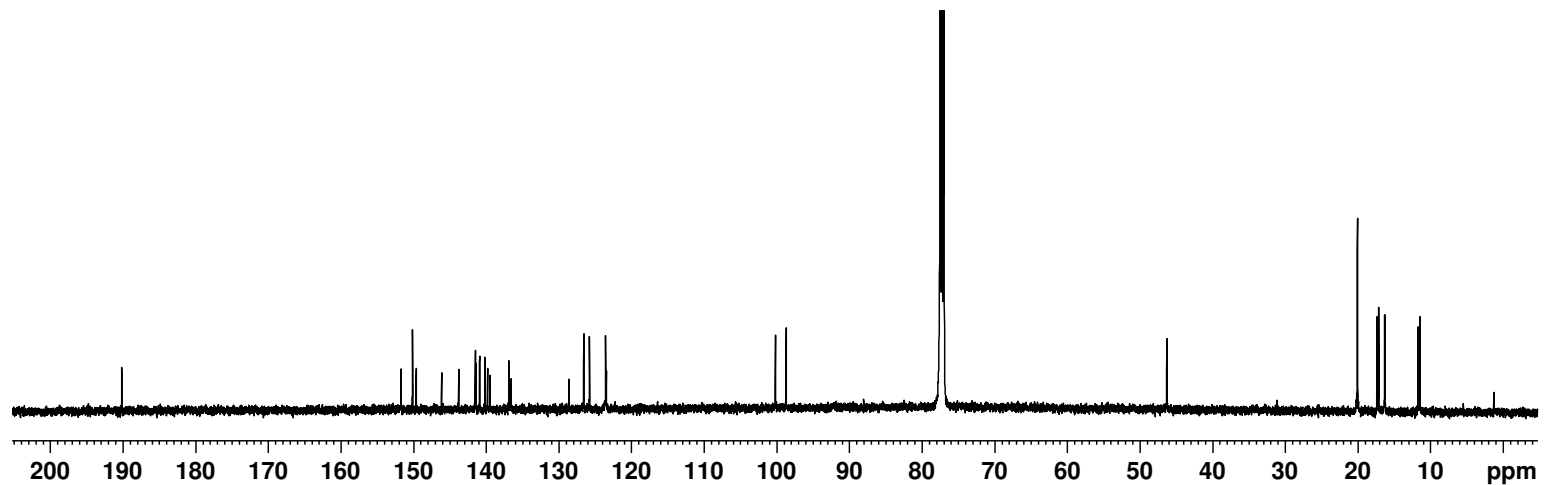

Figure D-57. $125 \mathrm{MHz}{ }^{13} \mathrm{C}$ NMR spectrum of $N$-methyl palladium(II) oxybenziporphyrin 102 in $\mathrm{CDCl}^{3}$ 


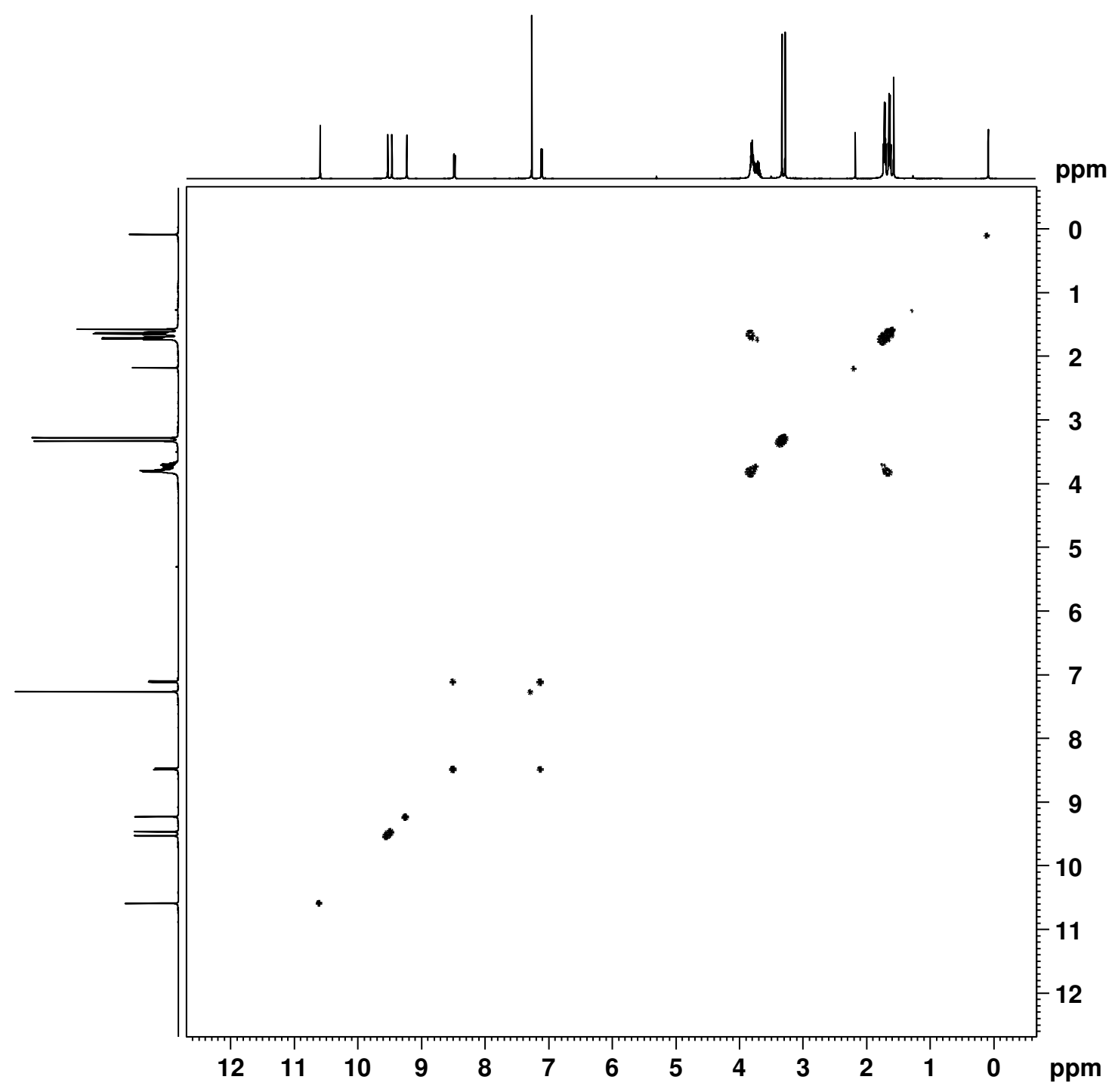

Figure D-58. ${ }^{1} \mathrm{H}-{ }^{1} \mathrm{H}$ COSY spectrum of $N$-methyl palladium(II) oxybenziporphyrin 102 in $\mathrm{CDCl}_{3}$

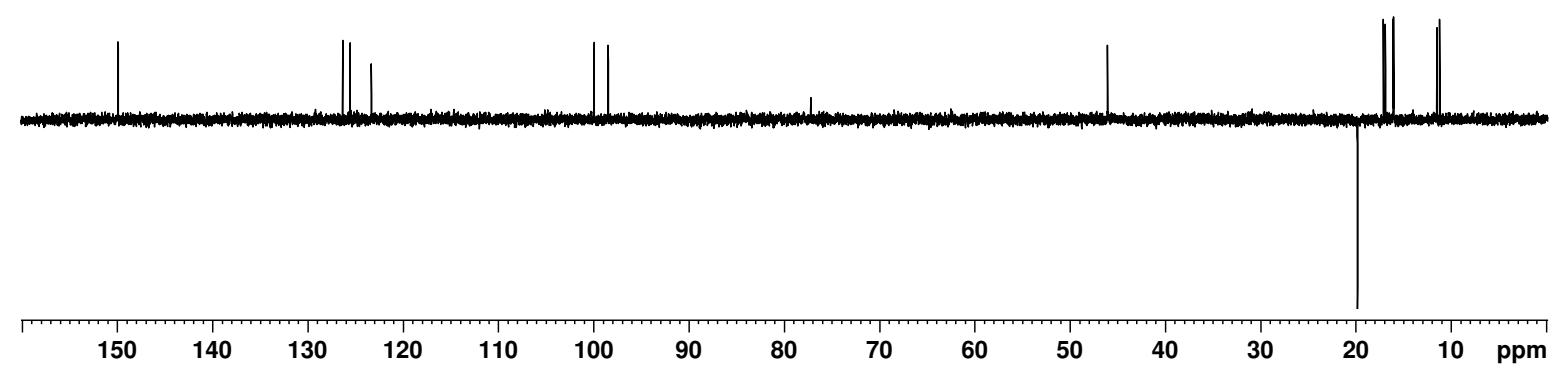

Figure D-59. DEPT-135 NMR spectrum of $N$-methyl palladium(II) oxybenziporphyrin 102 in $\mathrm{CDCl}^{3}$ 


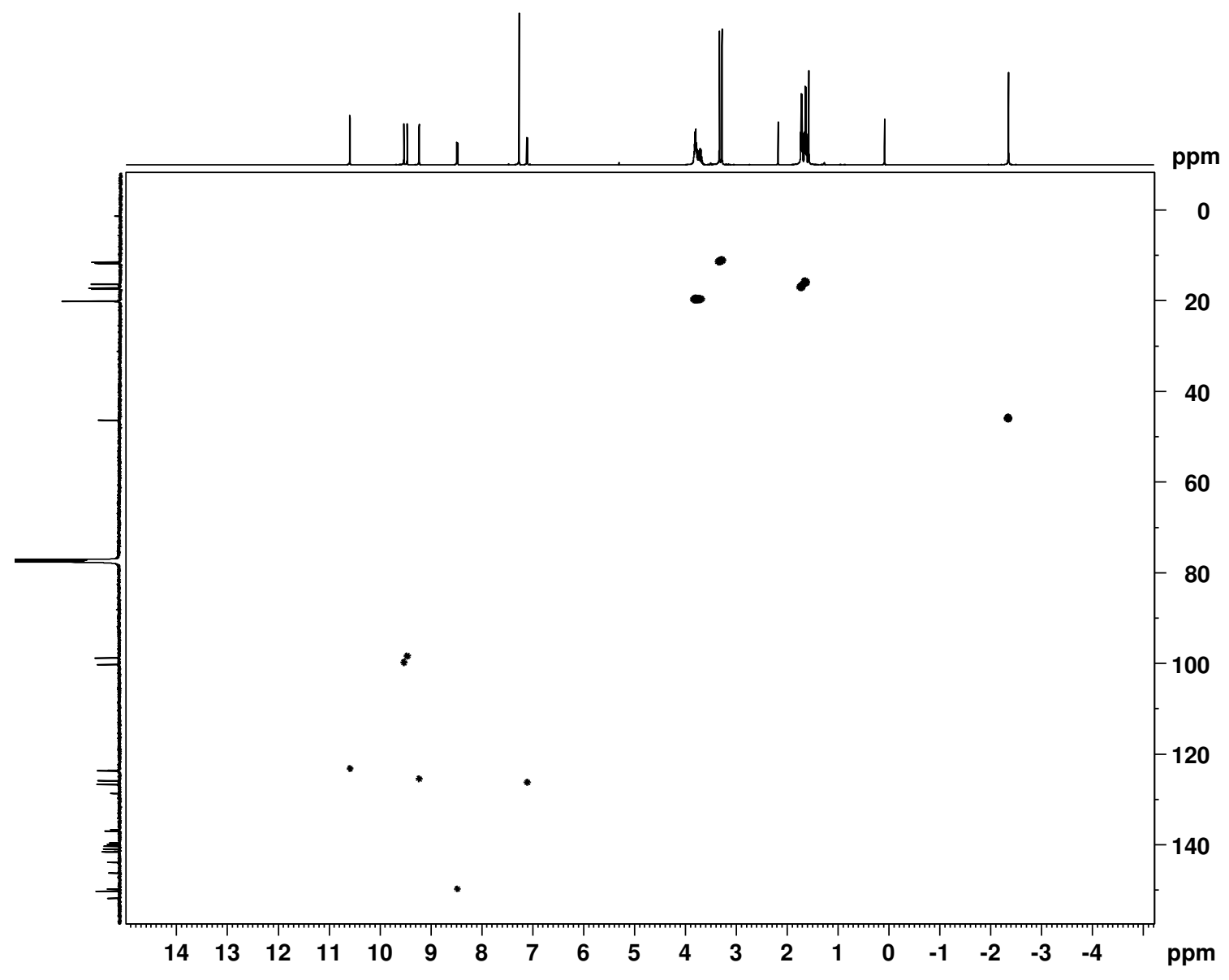

Figure D-60. HSQC NMR spectrum of $N$-methyl palladium(II) oxybenziporphyrin 102 in $\mathrm{CDCl}^{3}$ 

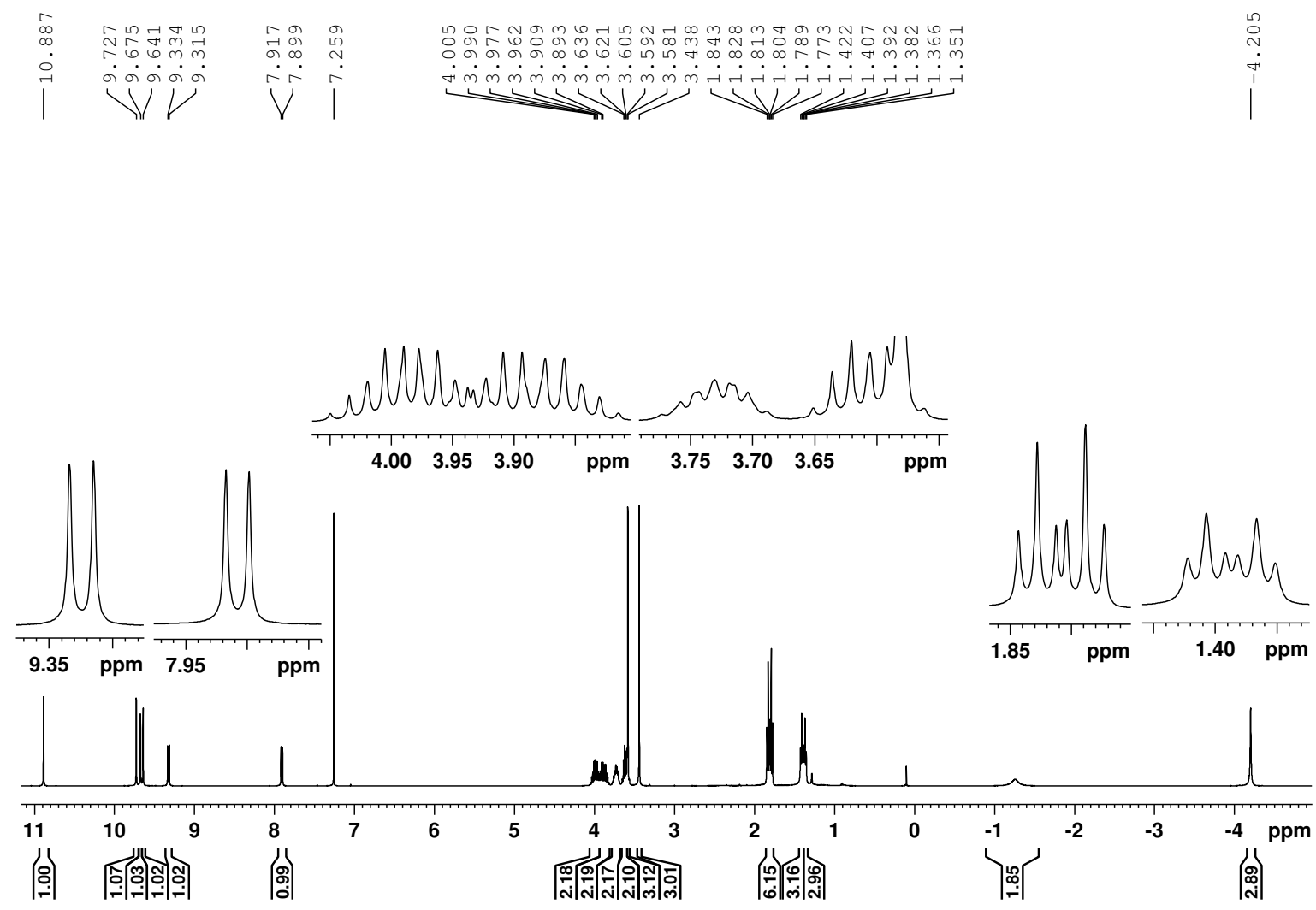

Figure D-61. $500 \mathrm{MHz}{ }^{1} \mathrm{H}$ NMR spectrum of $\mathrm{N}$-methyl oxypyriporphyrin $100 \mathbf{b}$ in $\mathrm{CDCl}_{3}$

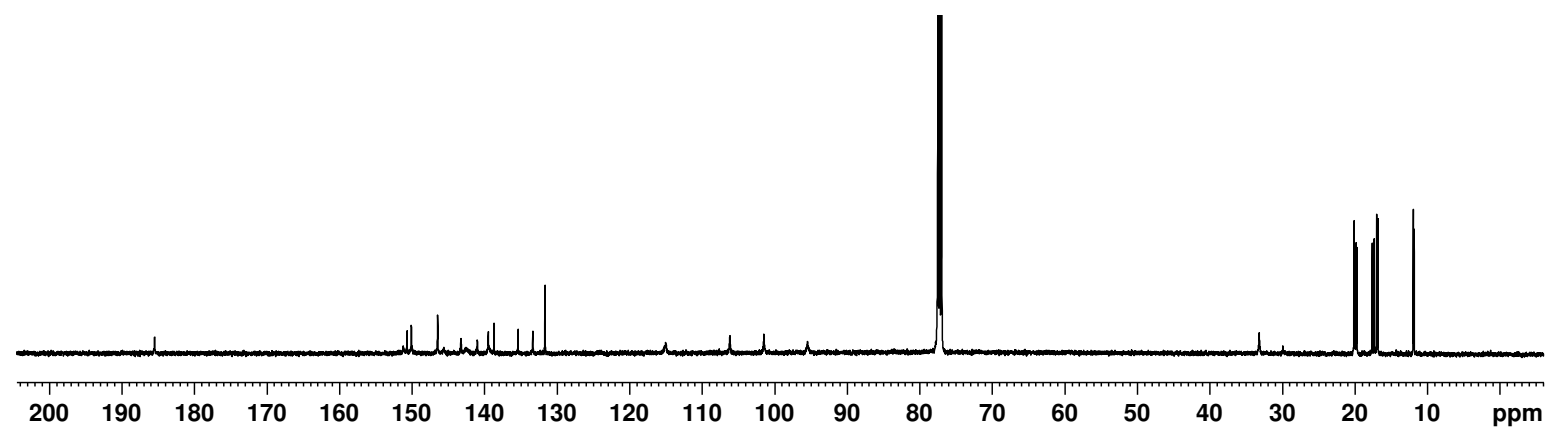

Figure D-62. $125 \mathrm{MHz}{ }^{13} \mathrm{C}$ NMR spectrum of $\mathrm{N}$-methyl oxypyriporphyrin $\mathbf{1 0 0 b}$ in $\mathrm{CDCl}_{3}$ 


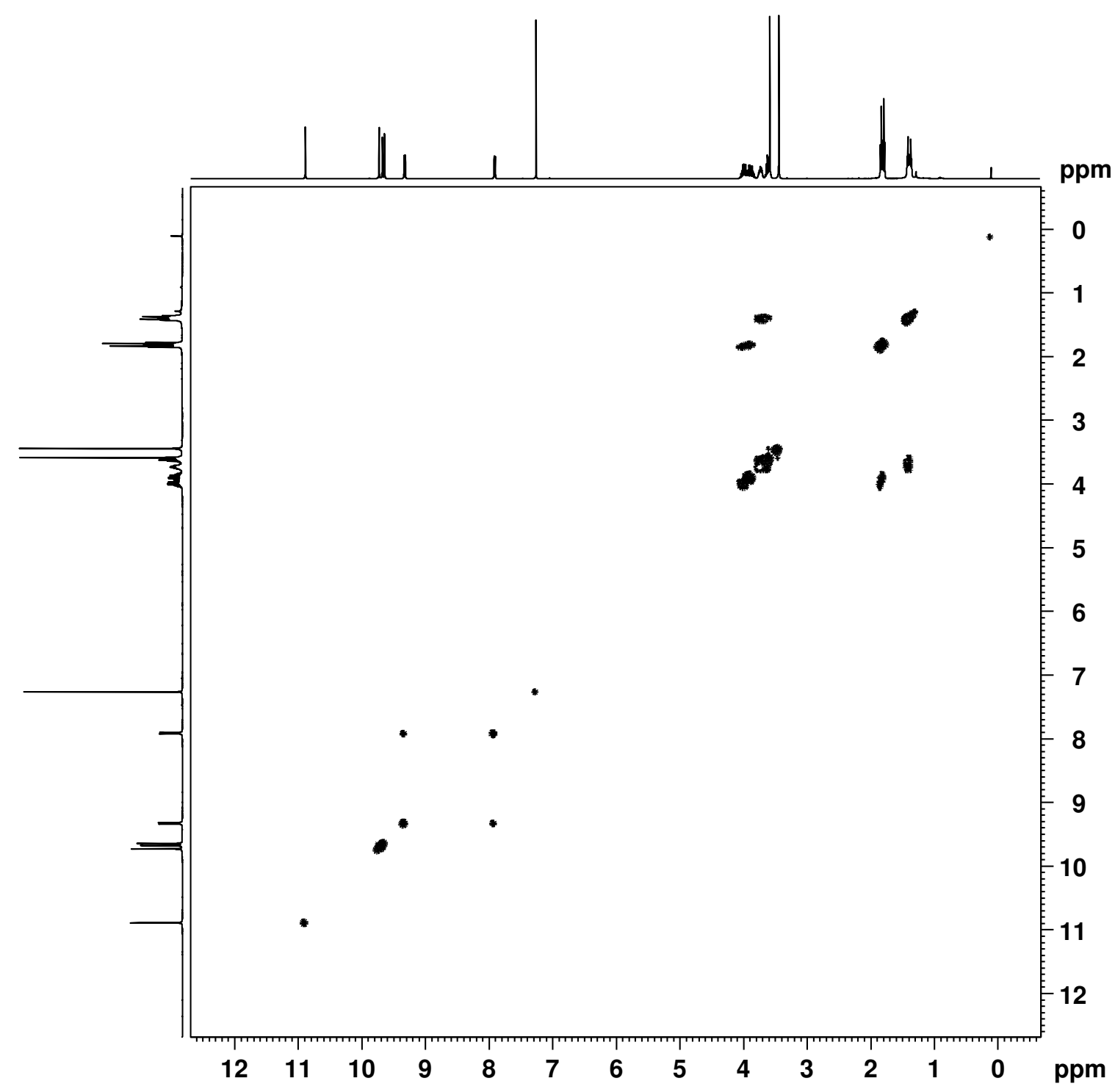

Figure D-63. ${ }^{1} \mathrm{H}-{ }^{1} \mathrm{H}$ COSY NMR spectrum of $N$-methyl oxypyriporphyrin $\mathbf{1 0 0 b}$ in $\mathrm{CDCl}_{3}$

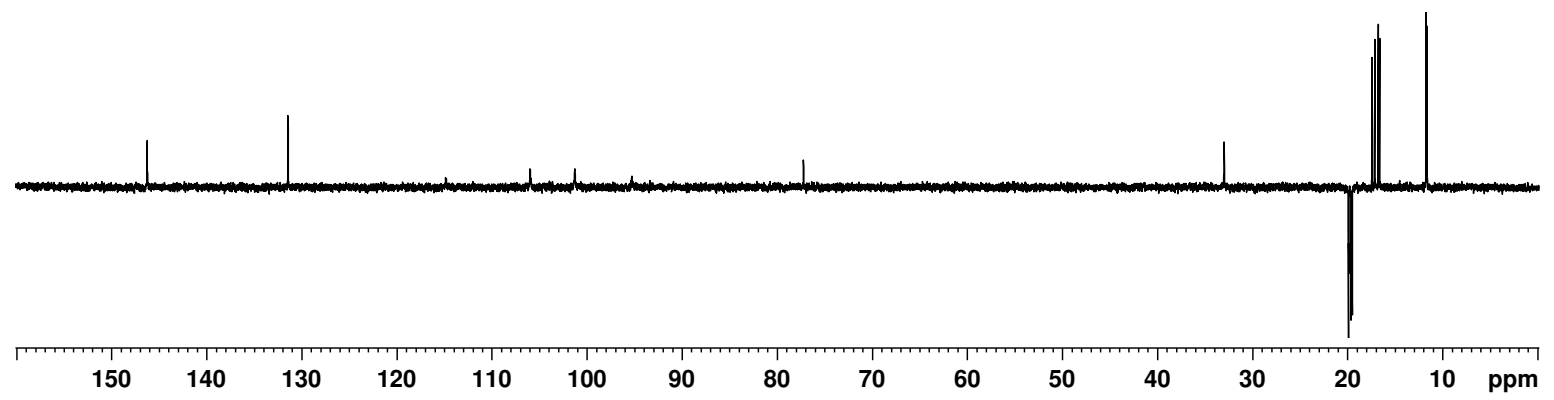

Figure D-64. DEPT-135 NMR spectrum of $N$-methyl oxypyriporphyrin 100b in $\mathrm{CDCl}_{3}$ 


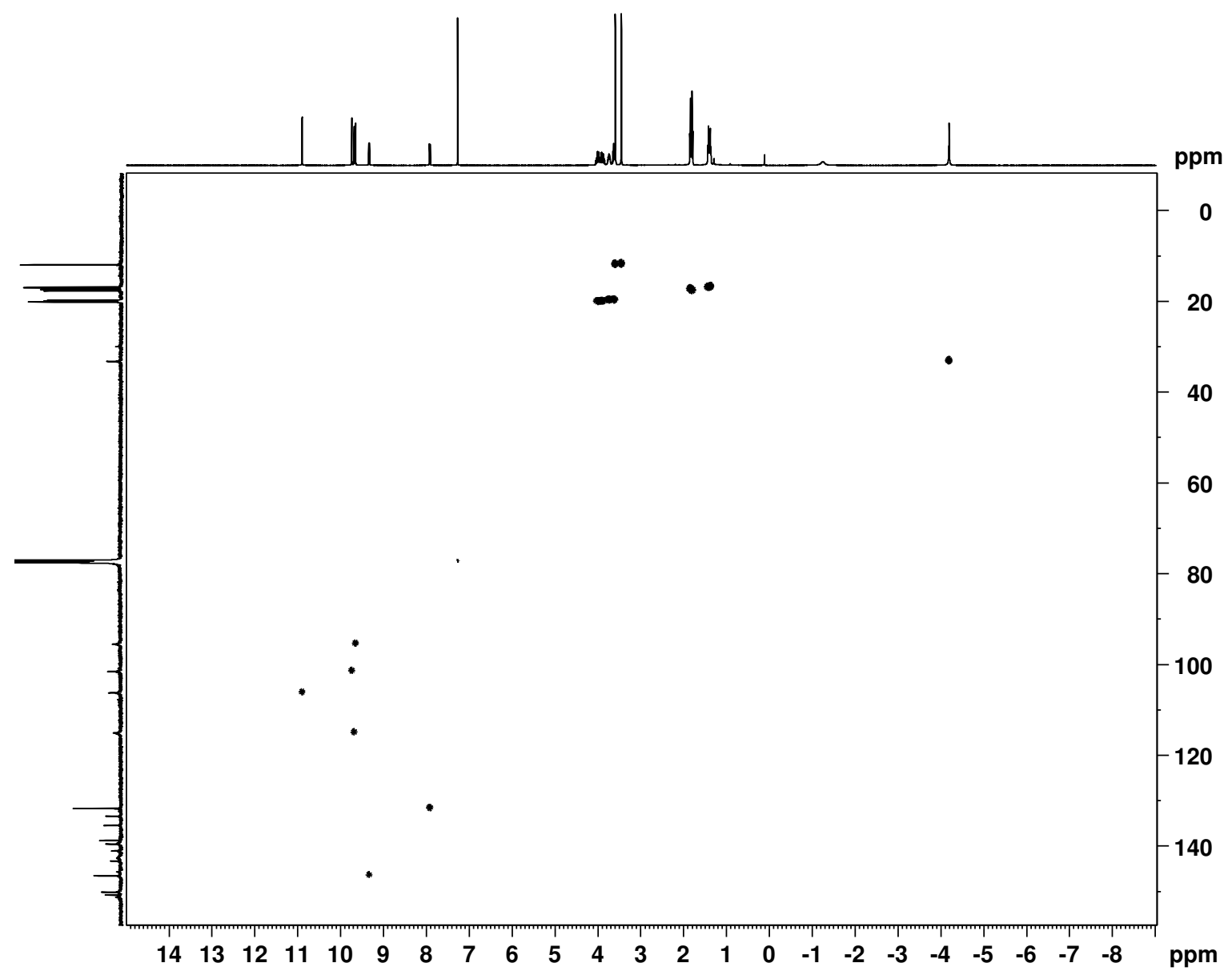

Figure D-65. HSQC NMR spectrum of $N$-methyl oxypyriporphyrin $\mathbf{1 0 0 b}$ in $\mathrm{CDCl}_{3}$ 


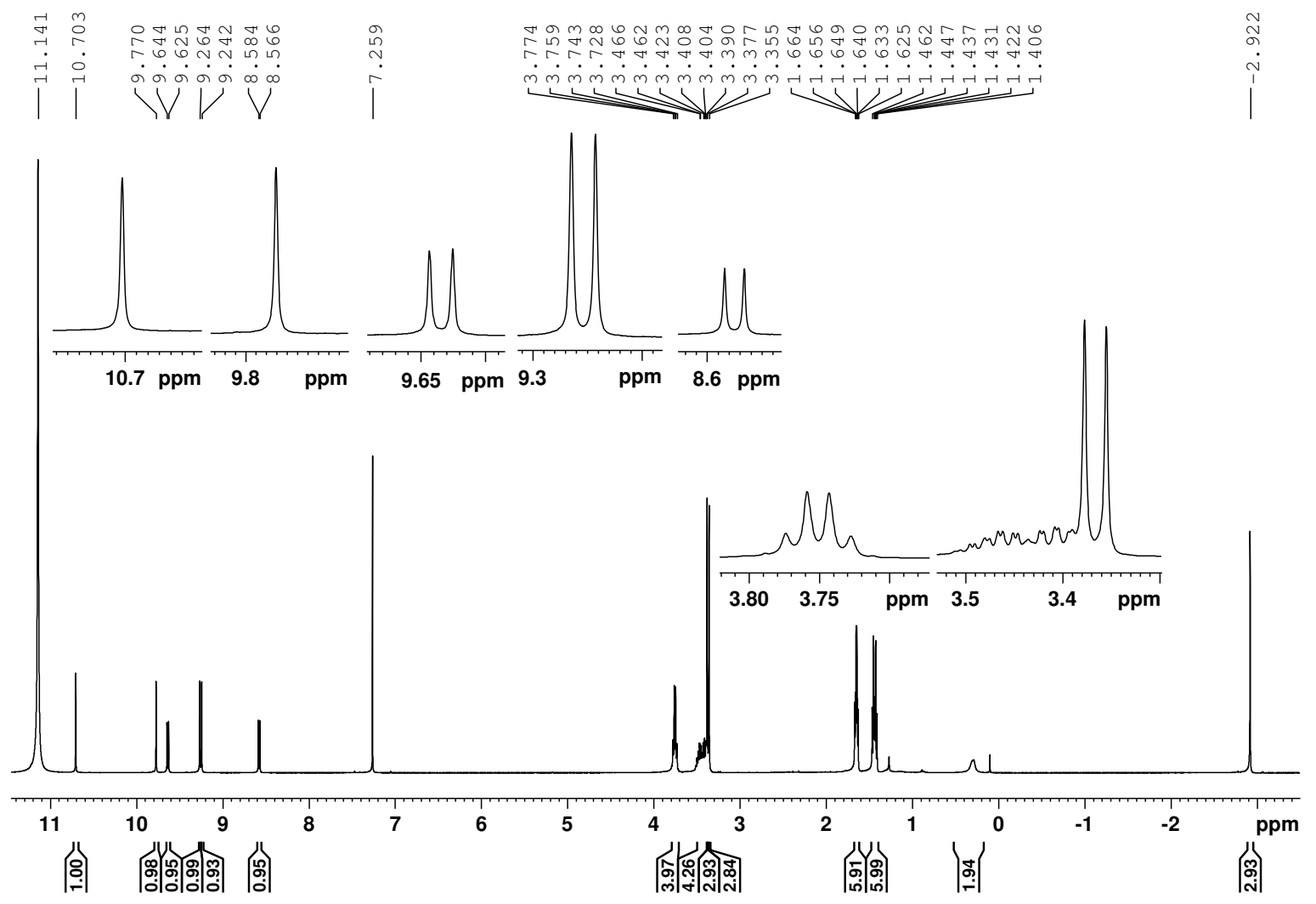

Figure D-66. $500 \mathrm{MHz}{ }^{1} \mathrm{H}$ NMR spectrum of $N$-methyl oxypyriporphyrin 100b in TFA$\mathrm{CDCl}^{3}$

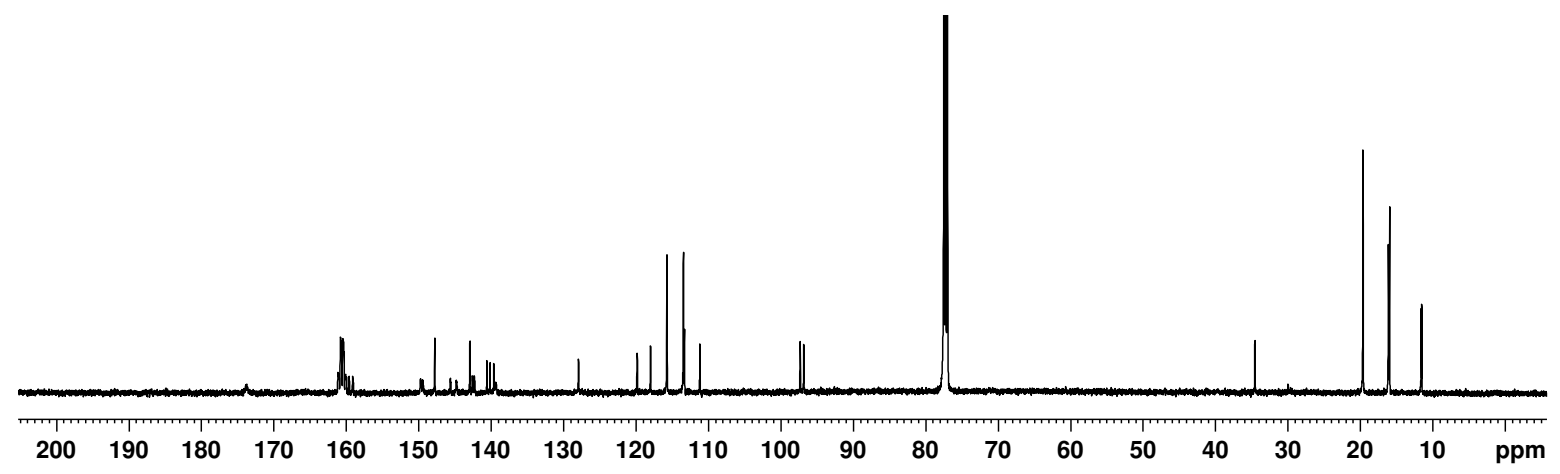

Figure D-67. $125 \mathrm{MHz}{ }^{13} \mathrm{C}$ NMR spectrum of $N$-methyl oxypyriporphyrin 100b in TFA$\mathrm{CDCl} 3$ 


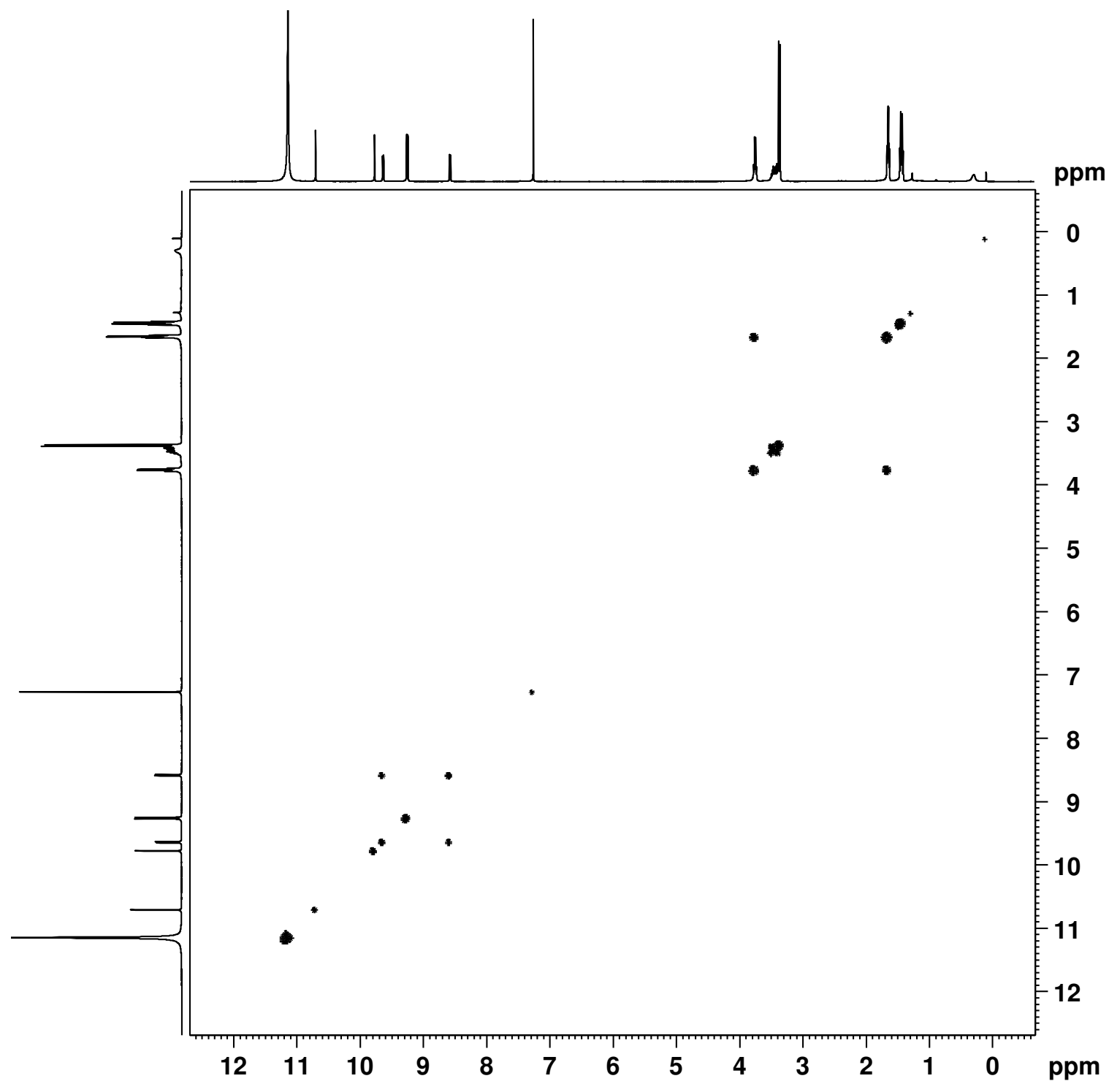

Figure D-68. ${ }^{1} \mathrm{H}-{ }^{1} \mathrm{H}$ COSY NMR spectrum of $N$-methyl oxypyriporphyrin 100b in TFA-CDCl ${ }_{3}$

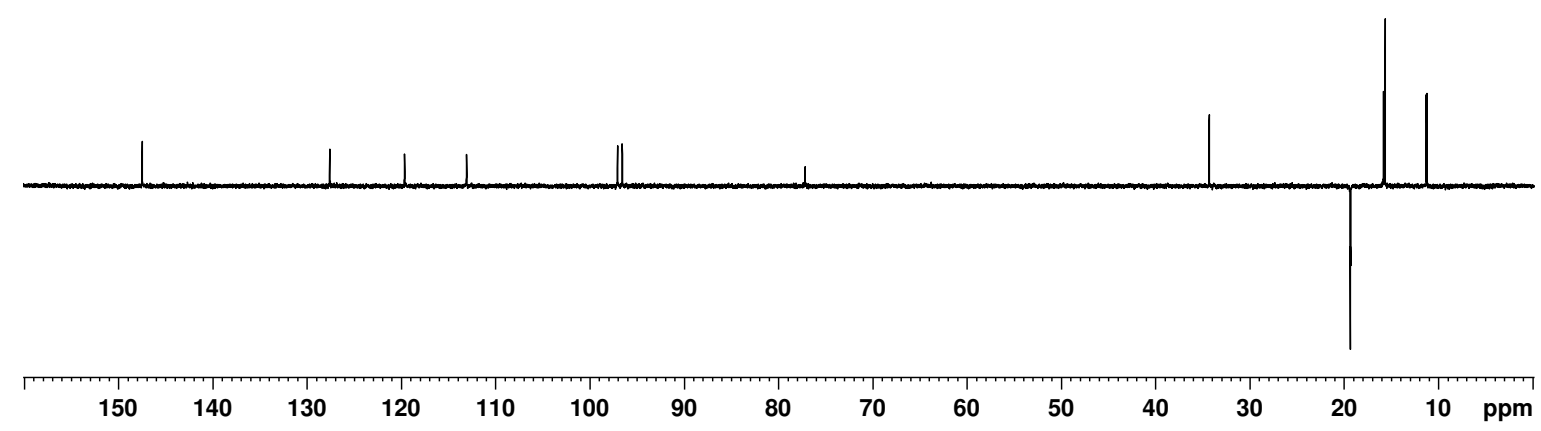

Figure D-69. DEPT-135 NMR spectrum of $N$-methyl oxypyriporphyrin 100b in TFA$\mathrm{CDCl}^{3}$ 


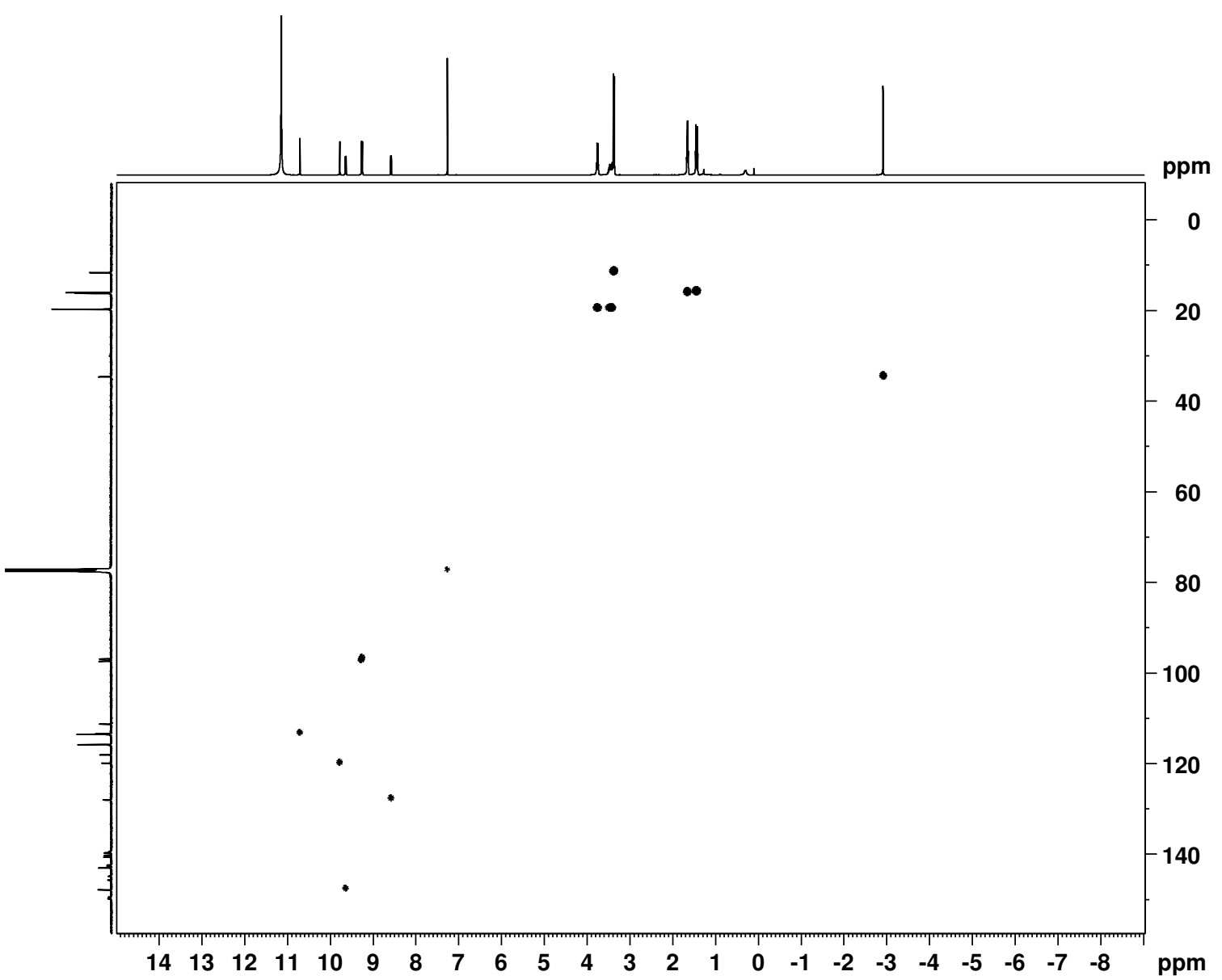

Figure D-70. HSQC NMR spectrum of $N$-methyl oxypyriporphyrin $100 \mathbf{b}$ in TFA-CDCl 3 


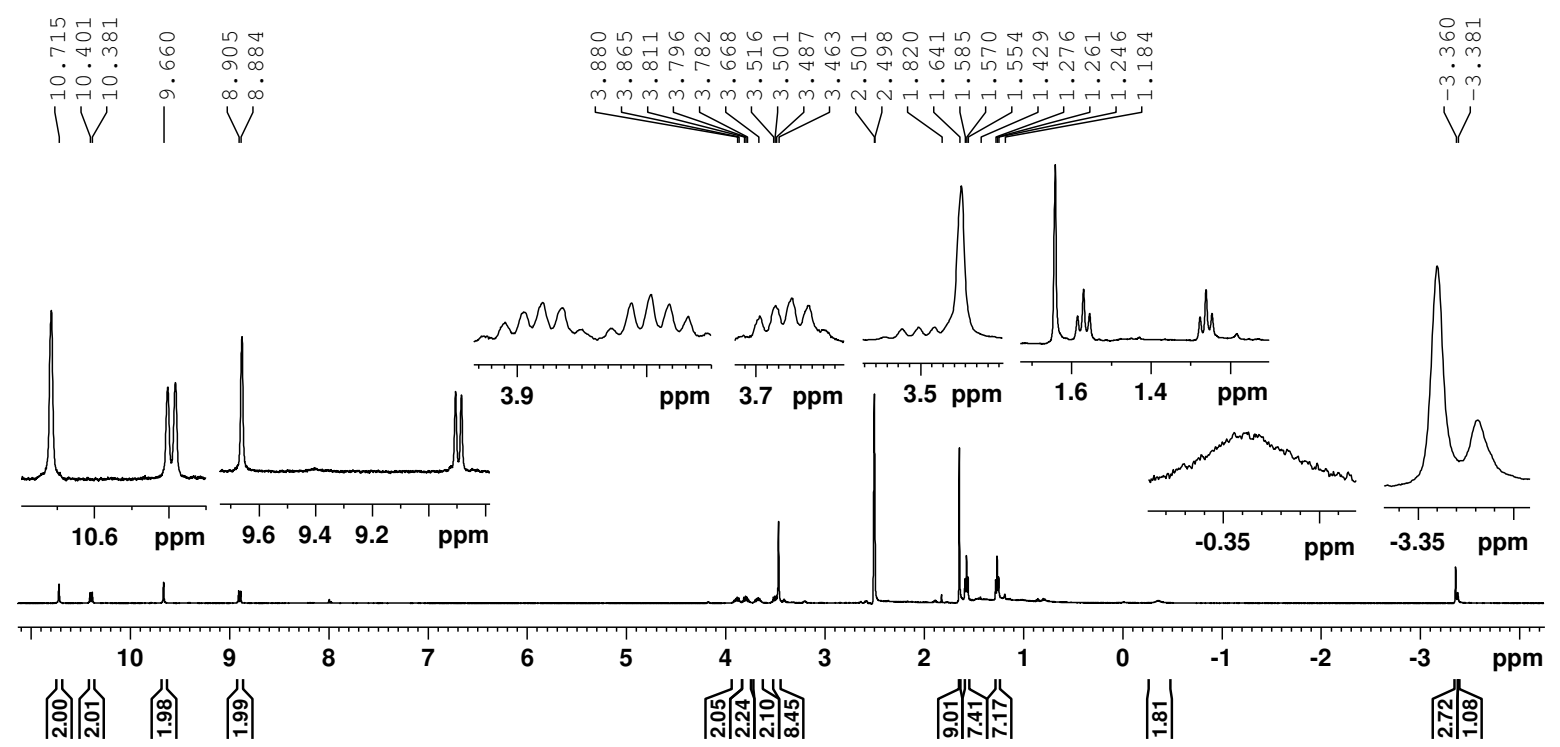

Figure D-71. $500 \mathrm{MHz}{ }^{1} \mathrm{H}$ NMR spectrum of $\mathrm{N}$-methyl tert-butyl azuliporphyrin $103 \mathrm{H}_{2}{ }^{2+}$ in TFA-d6-DMSO

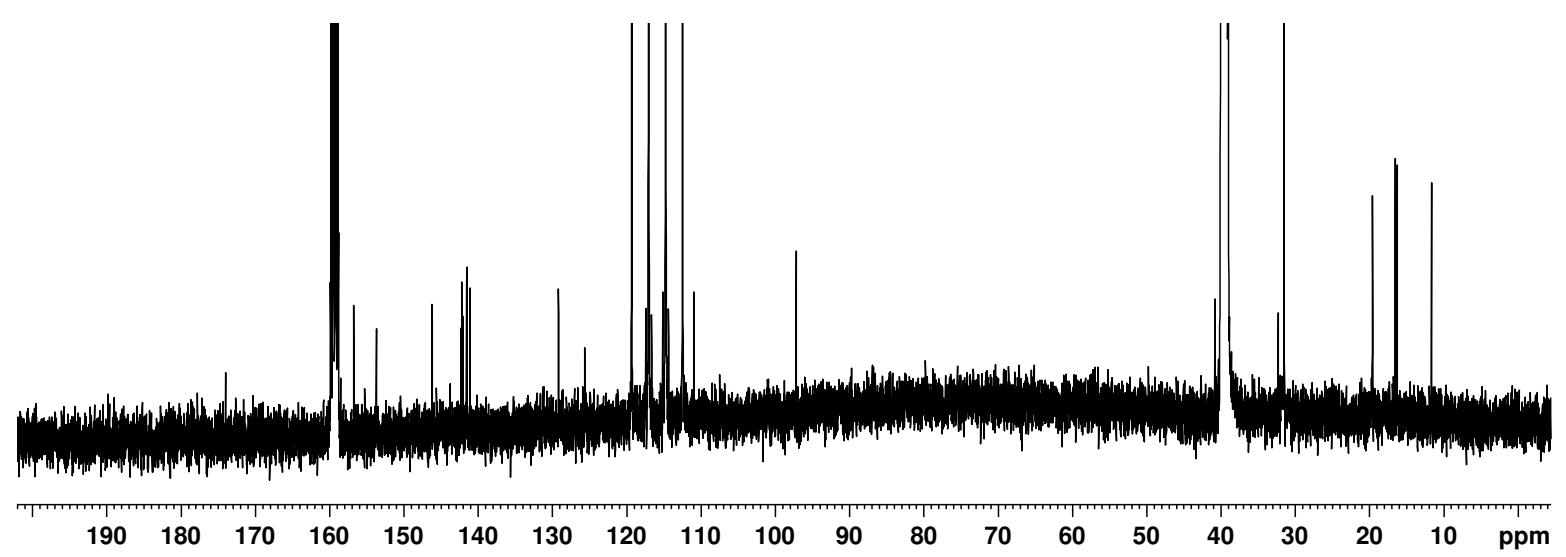

Figure D-72. $125 \mathrm{MHz}{ }^{13} \mathrm{C}$ NMR spectrum of $\mathrm{N}$-methyl tert-butyl azuliporphyrin $\mathbf{1 0 3}_{2}{ }^{2+}$ in TFA- $d 6$-DMSO 


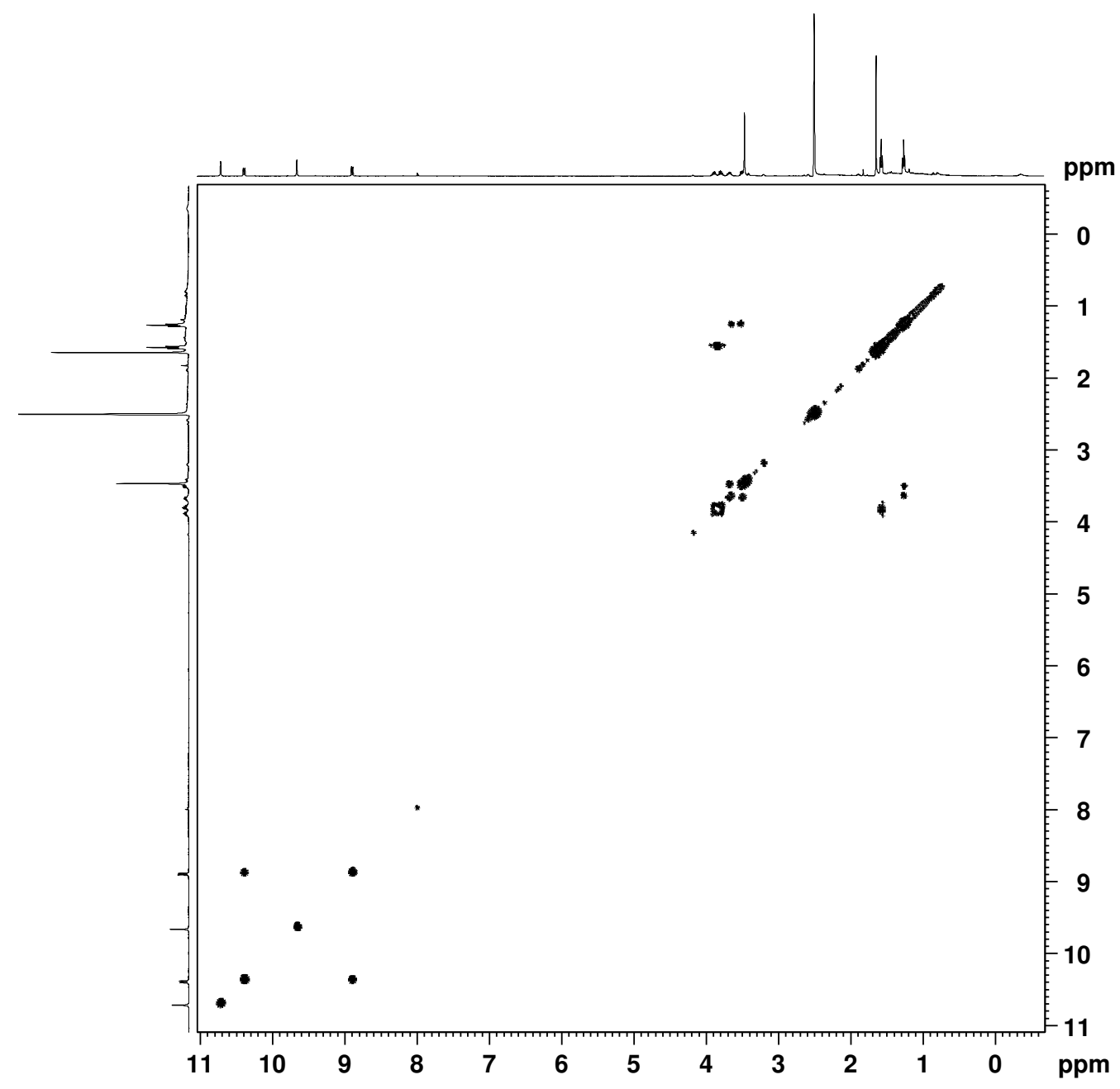

Figure D-73. ${ }^{1} \mathrm{H}-{ }^{1} \mathrm{H}$ COSY NMR spectrum of $N$-methyl tert-butyl azuliporphyrin $103 \mathrm{H}_{2}{ }^{2+}$ in TFA- $d 6$-DMSO

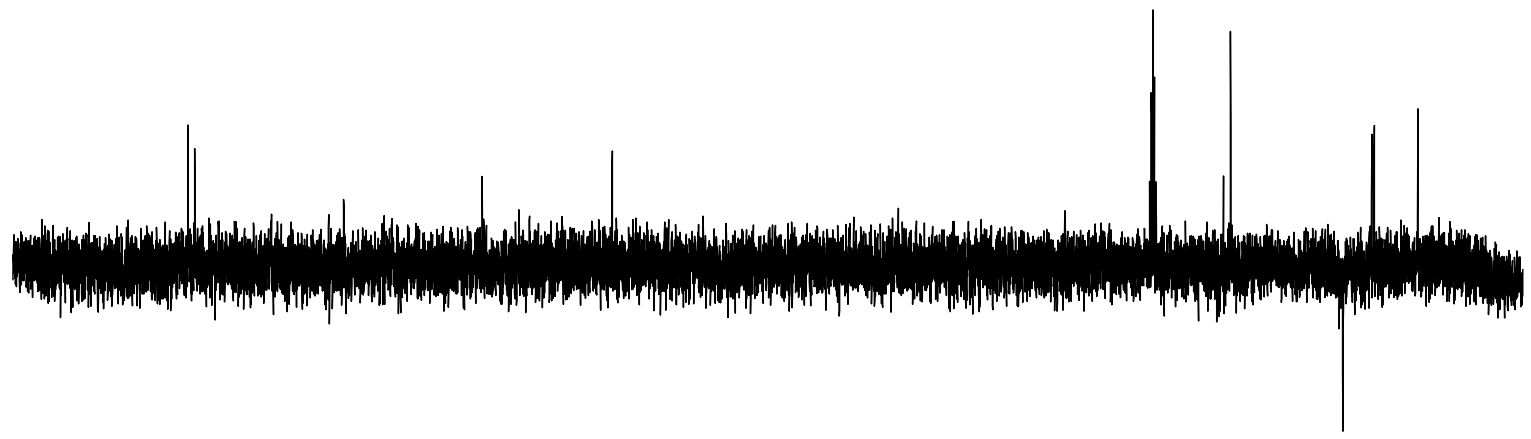

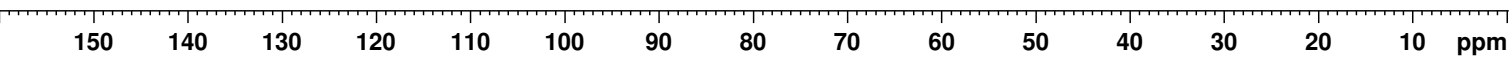

Figure D-74. DEPT $135 \mathrm{NMR}$ spectrum of $\mathrm{N}$-methyl tert-butyl azuliporphyrin $\mathbf{1 0 3 H}_{2}{ }^{2+}$ in TFA-d6-DMSO 


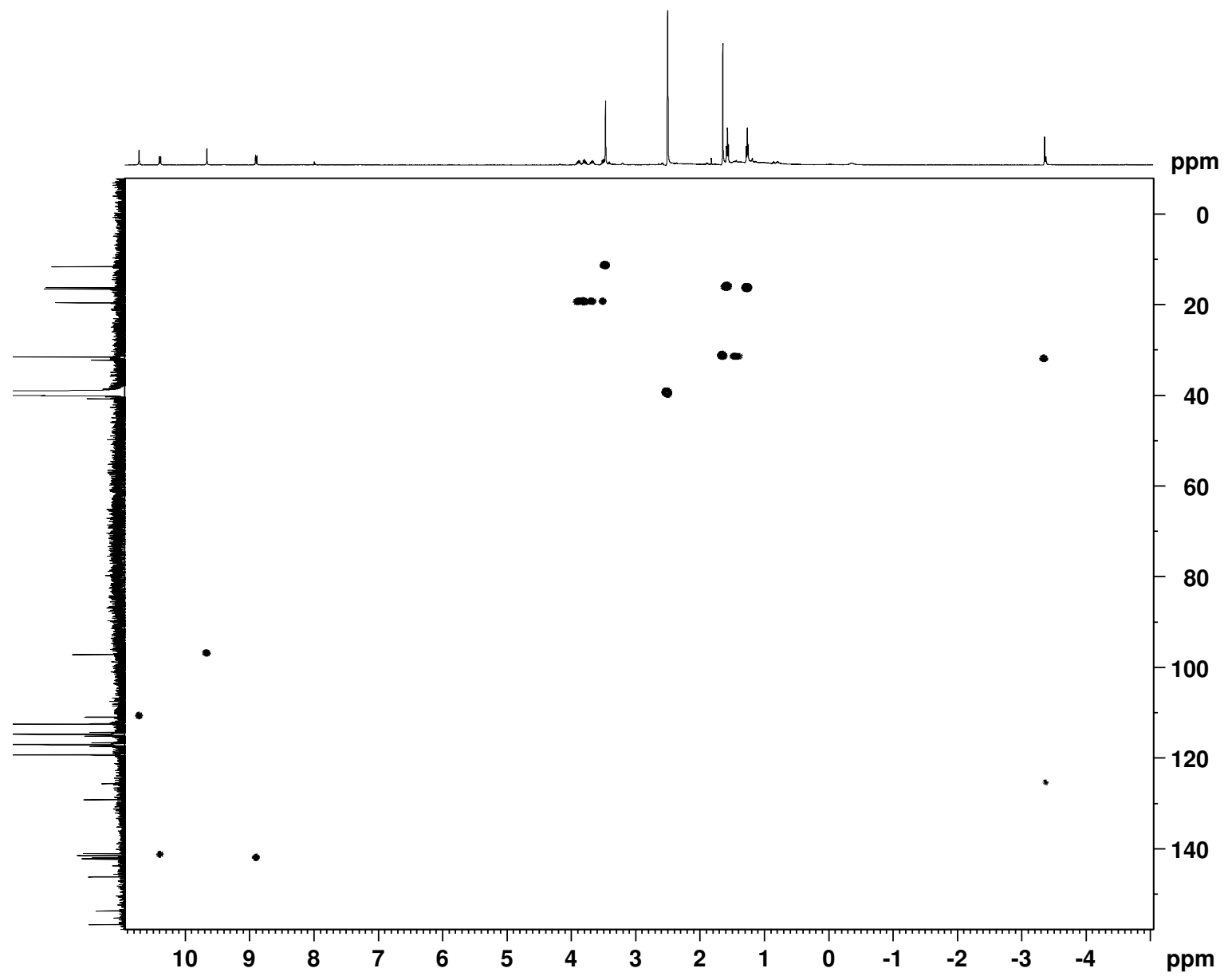

Figure D-75. HSQC NMR spectrum of $N$-methyl tert-butyl azuliporphyrin $103 \mathrm{H}_{2}{ }^{2+}$ in TFA- $d 6$-DMSO 


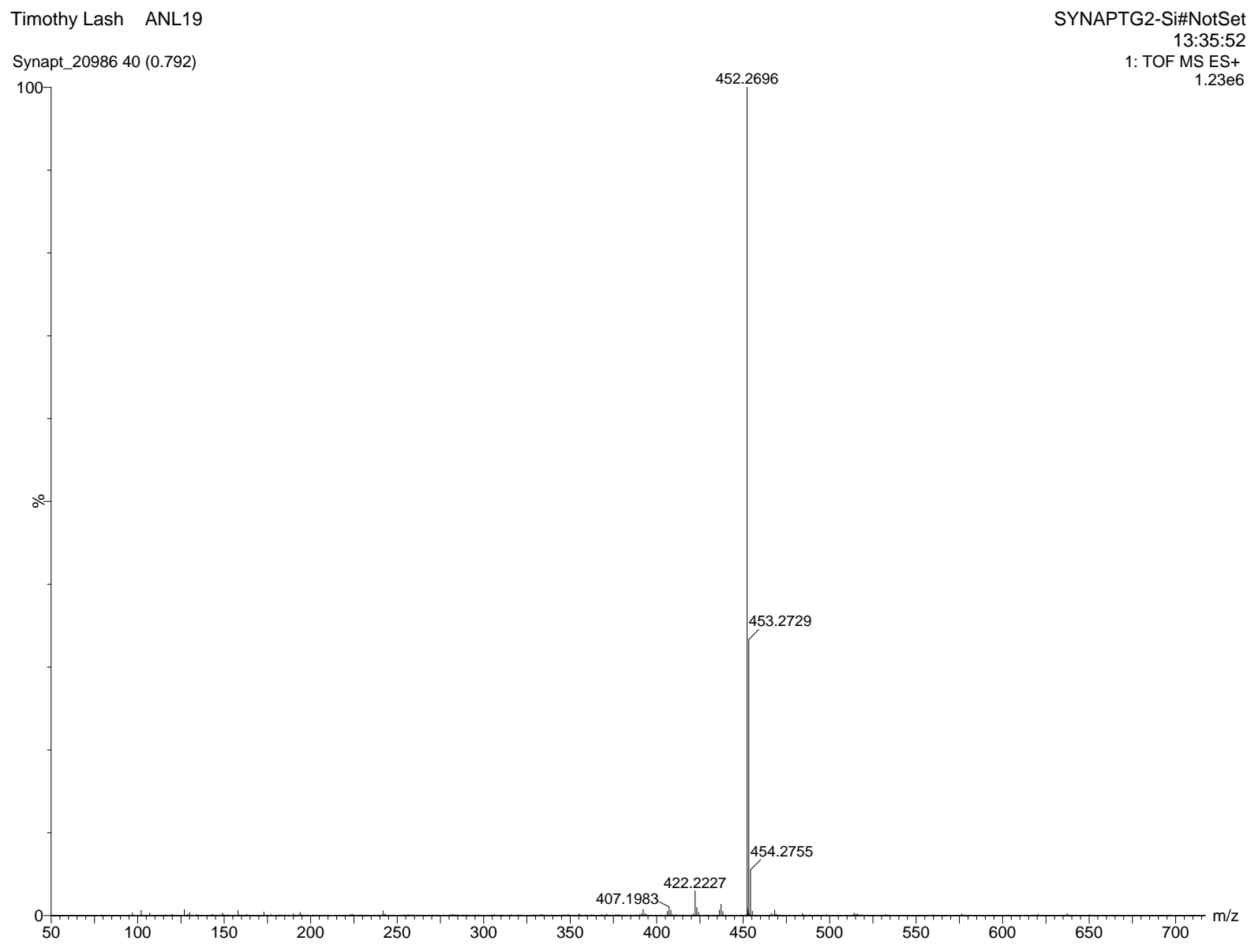

Figure D-76. ESI MS of 21-oxaporphyrin 96a 


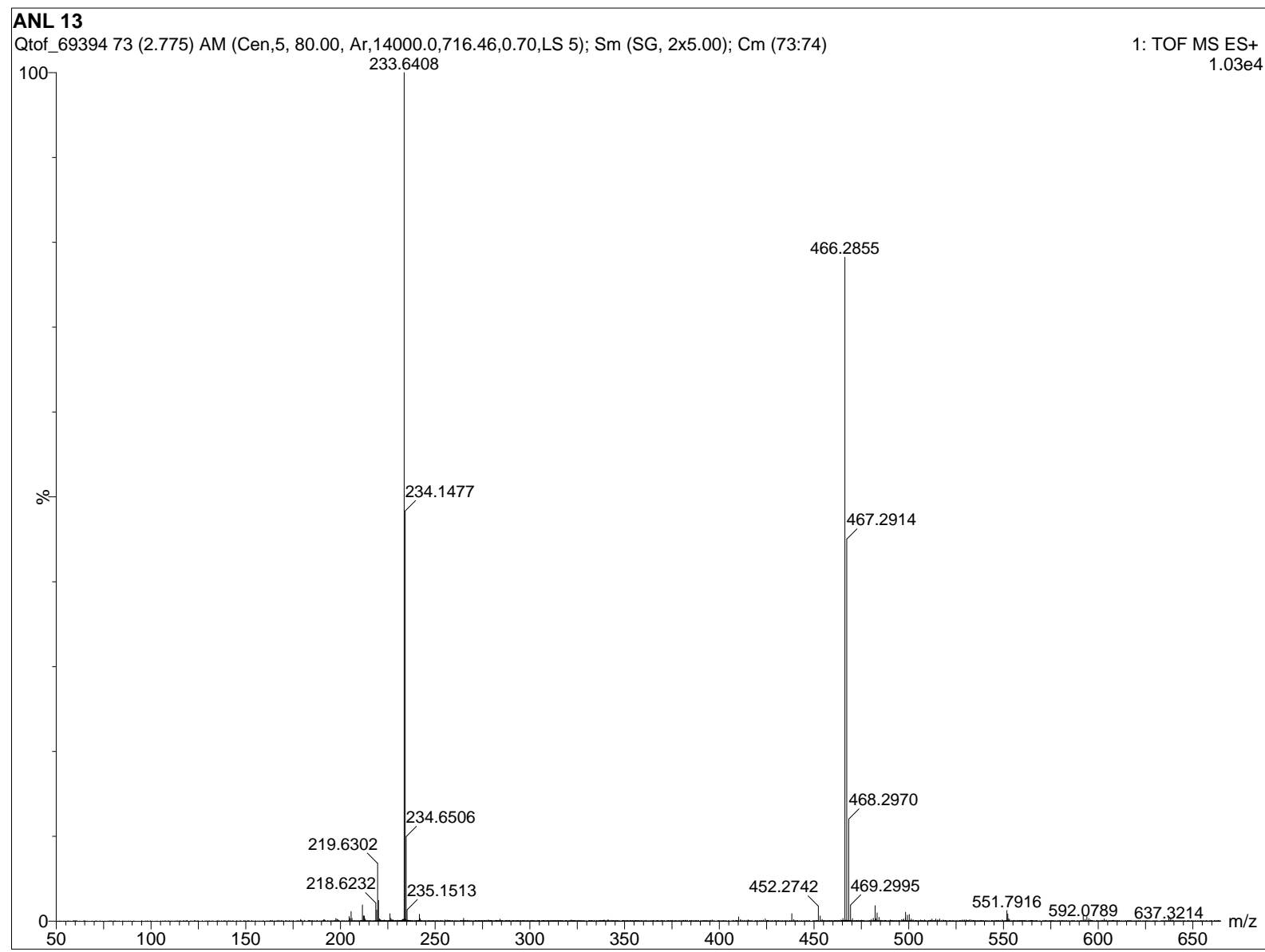

Figure D-77. ESI MS of $N$-methyl oxaporphyrin 96b 


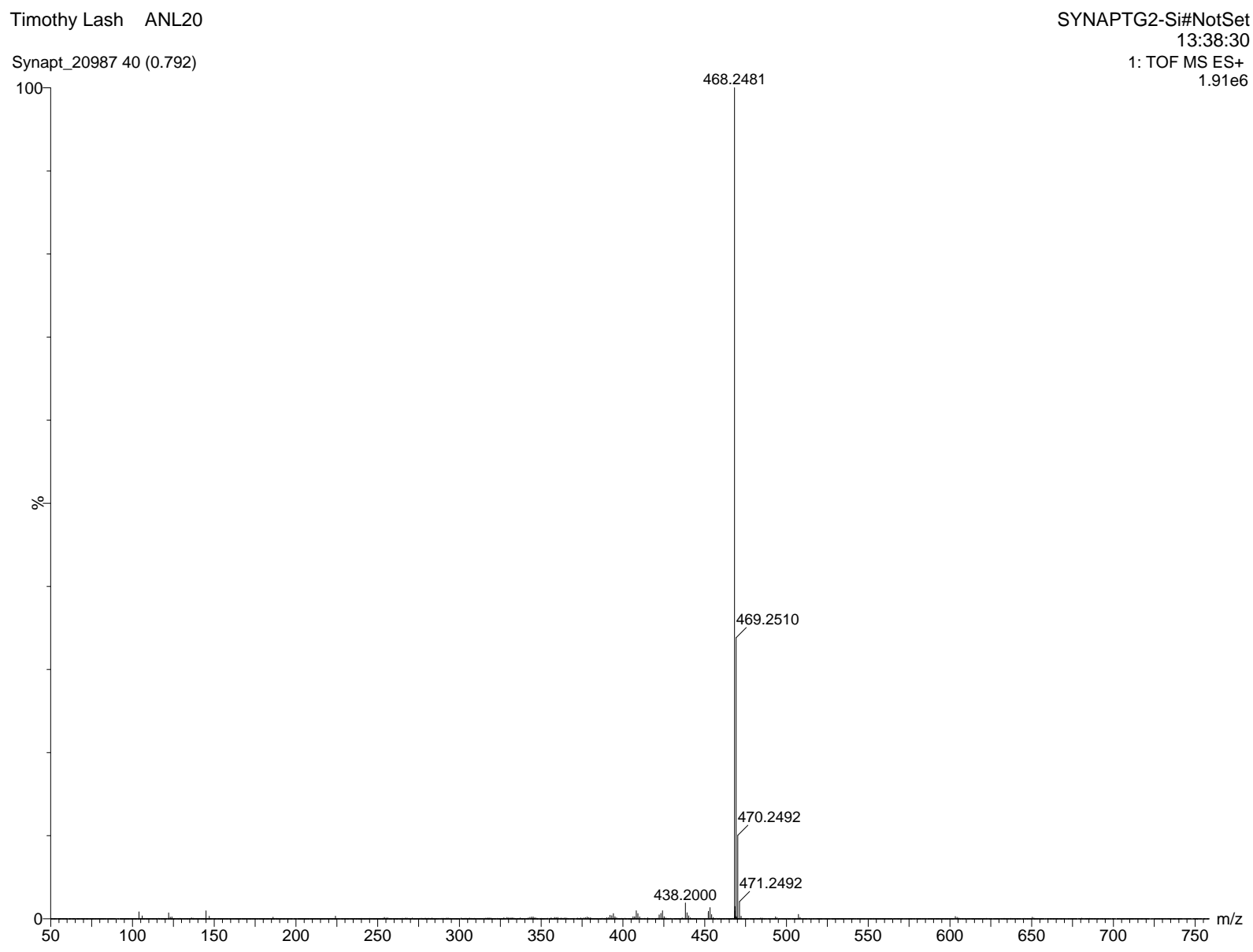

Figure D-78. ESI MS of 21-thiaporphyrin 97a 


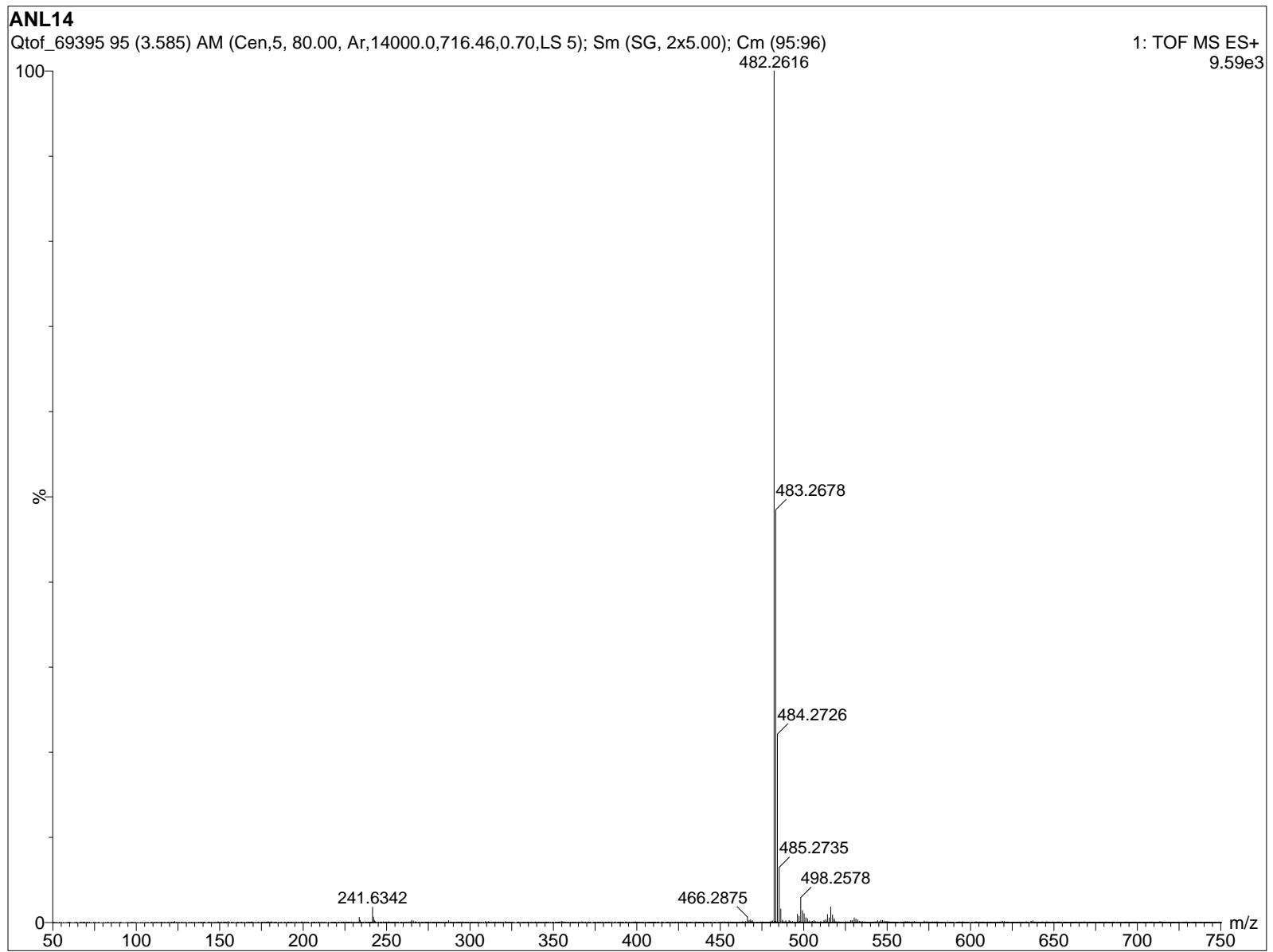

Figure D-79. ESI MS of $N$-methyl thiaporphyrin 97b 


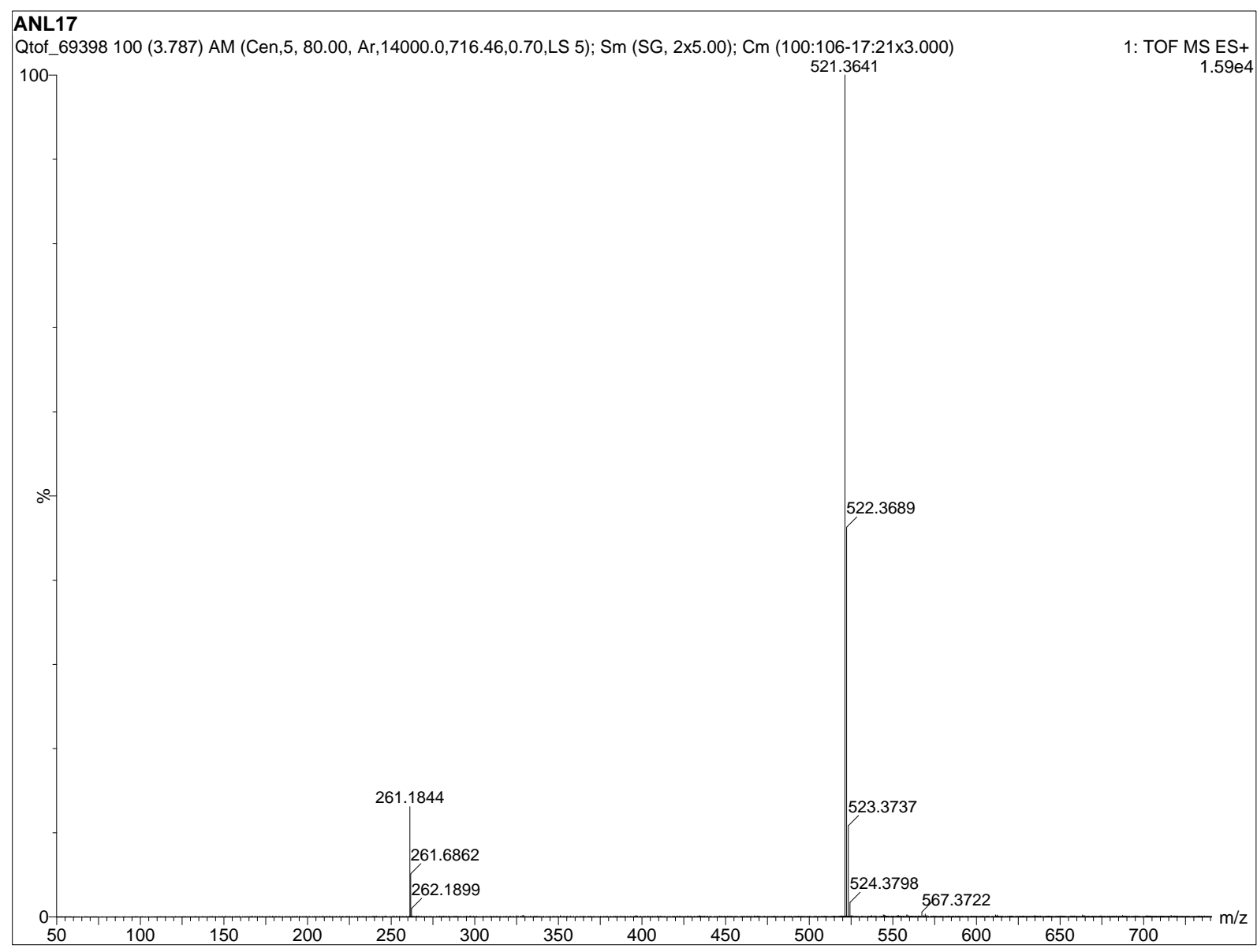

Figure D-80. ESI MS of $N$-methyl porphyrin 99b 


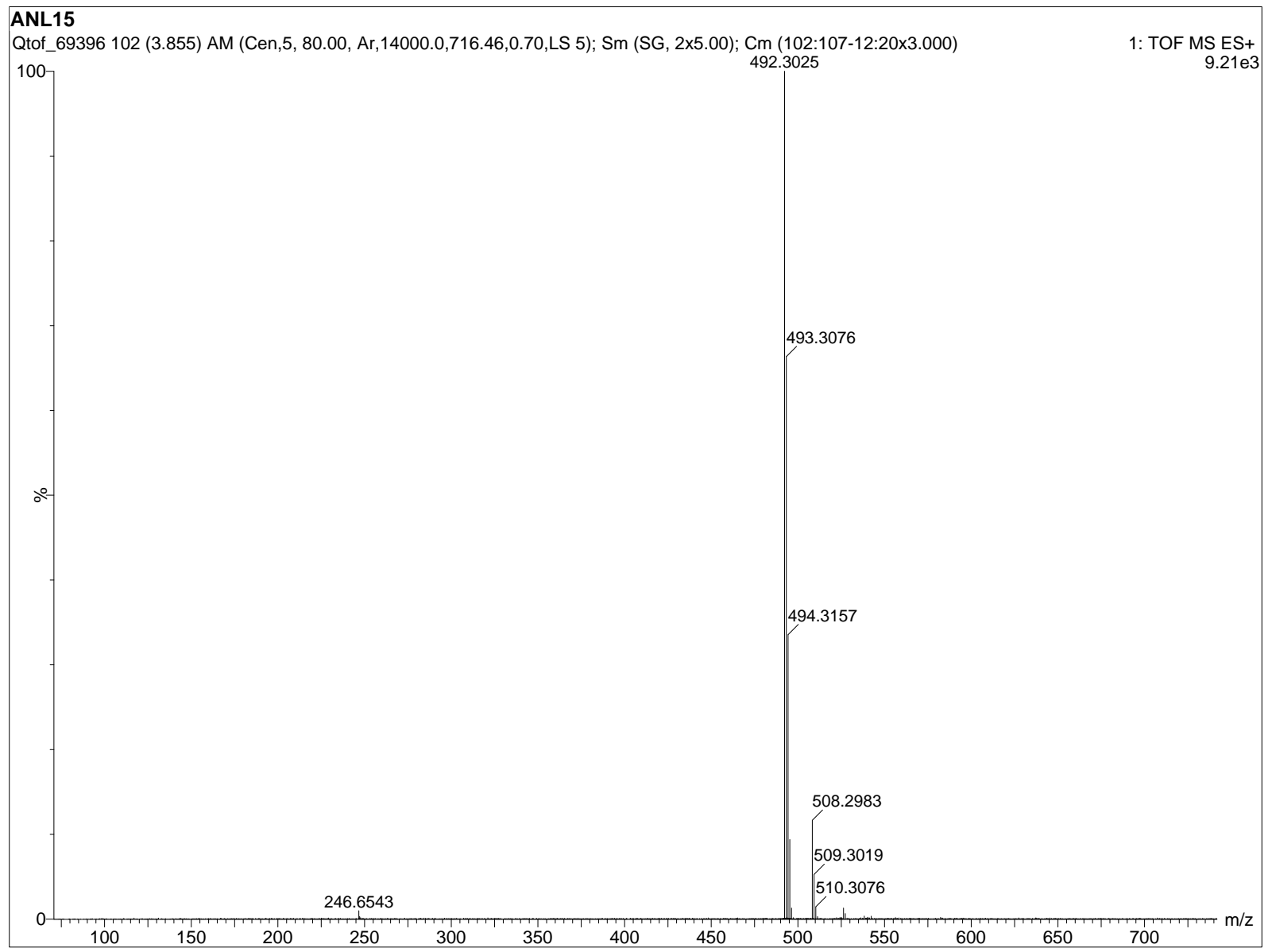

Figure D-81. ESI MS of $N$-methyl oxybenziporphyrin 100a 


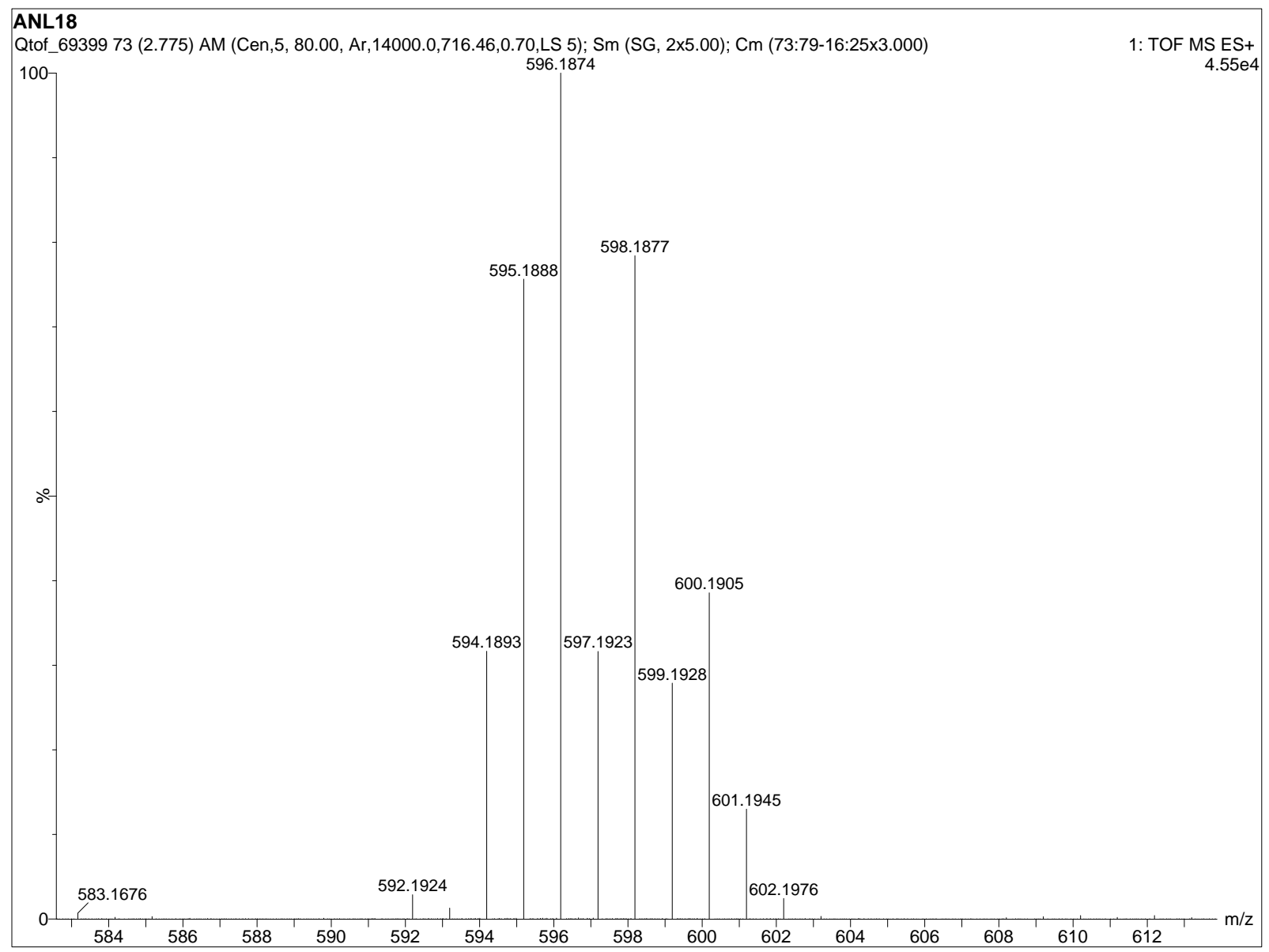

Figure D-82. ESI MS of $N$-methyl palladium(II) oxybenziporphyrin 102 


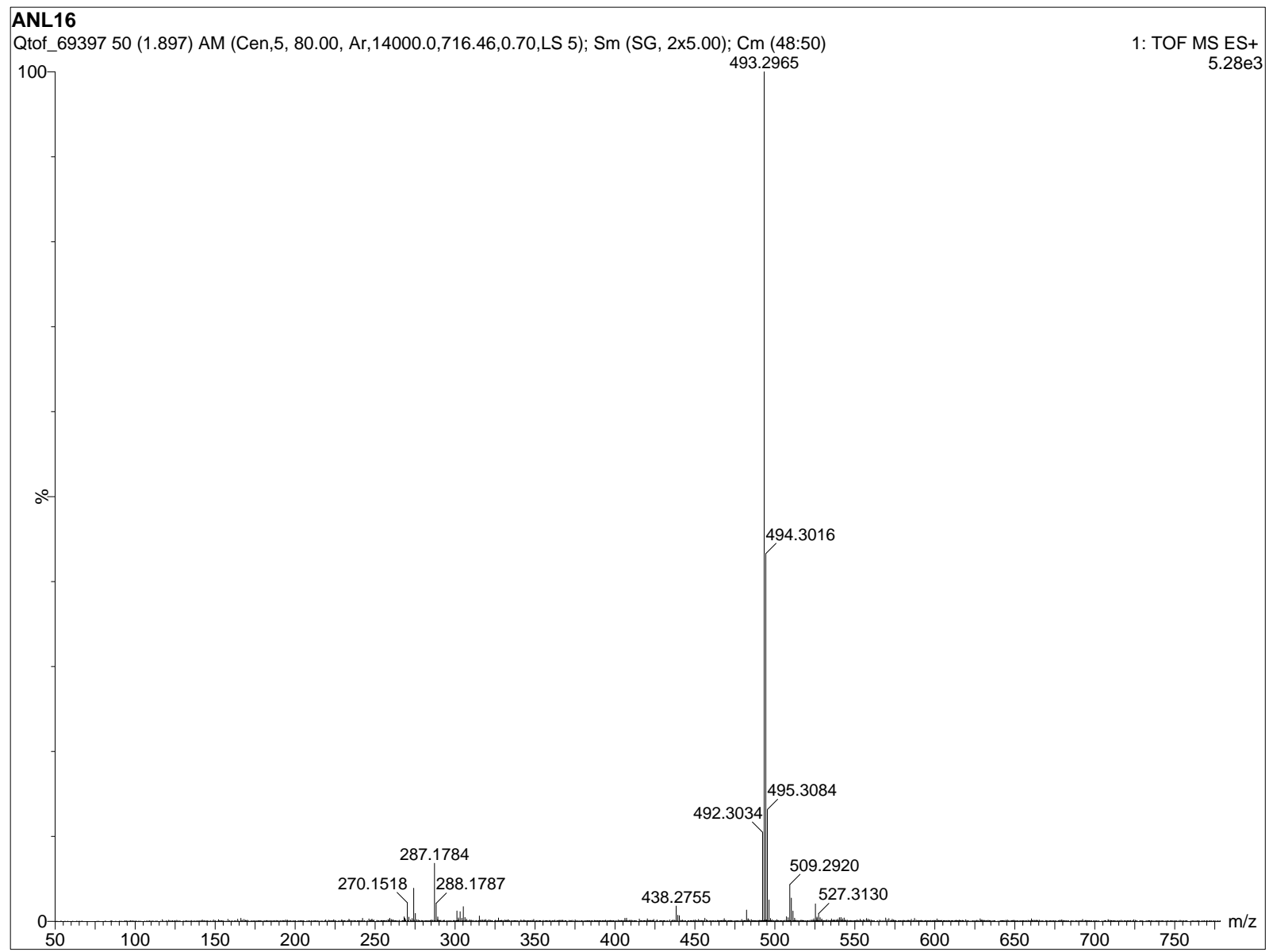

Figure D-83. ESI MS of $N$-methyl oxypyriporphyrin 100b 


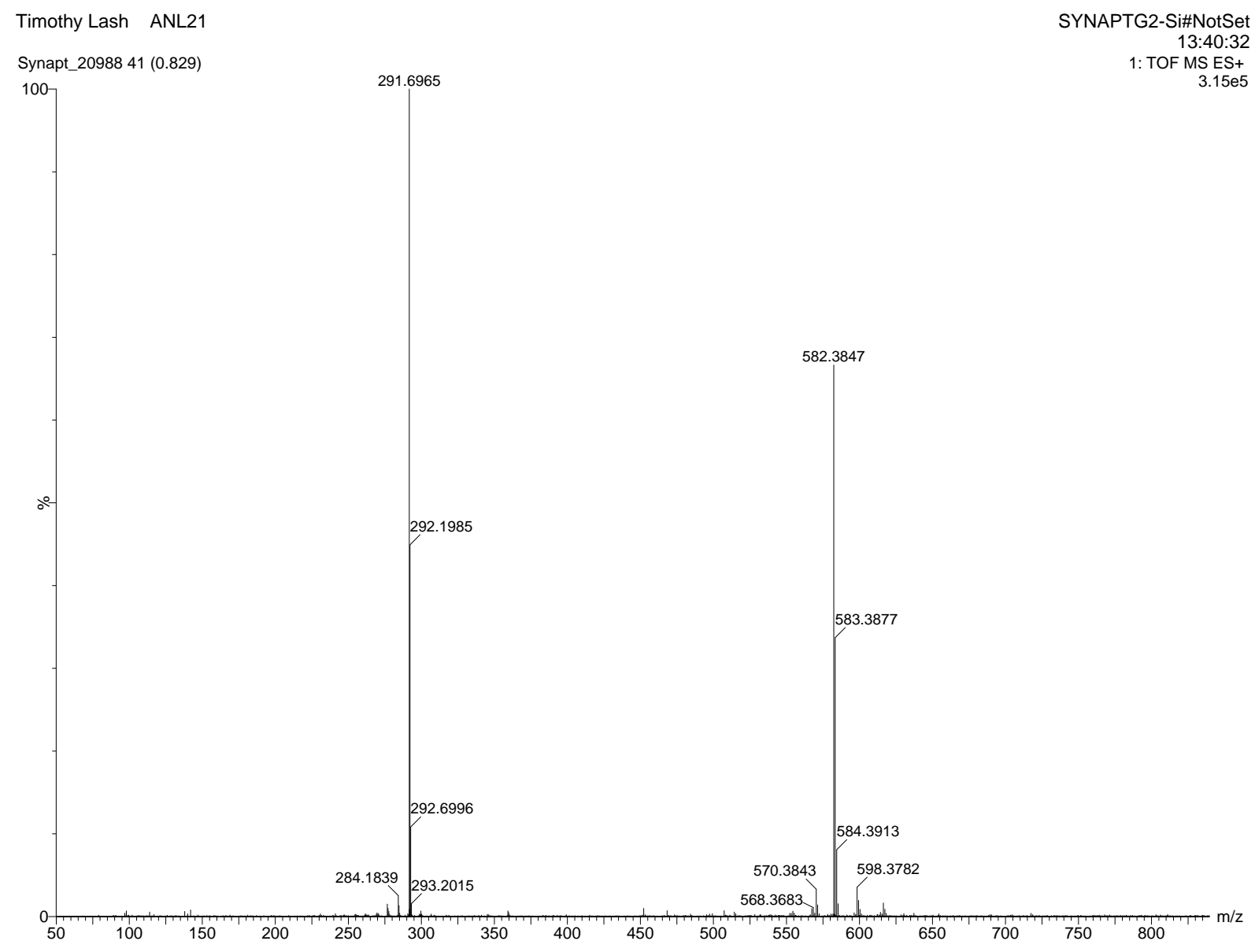

Figure D-84. ESI MS of $N$-methyl tert-Butyl azuliporphyrin $103 \mathrm{H}_{2}{ }^{2+}$ 


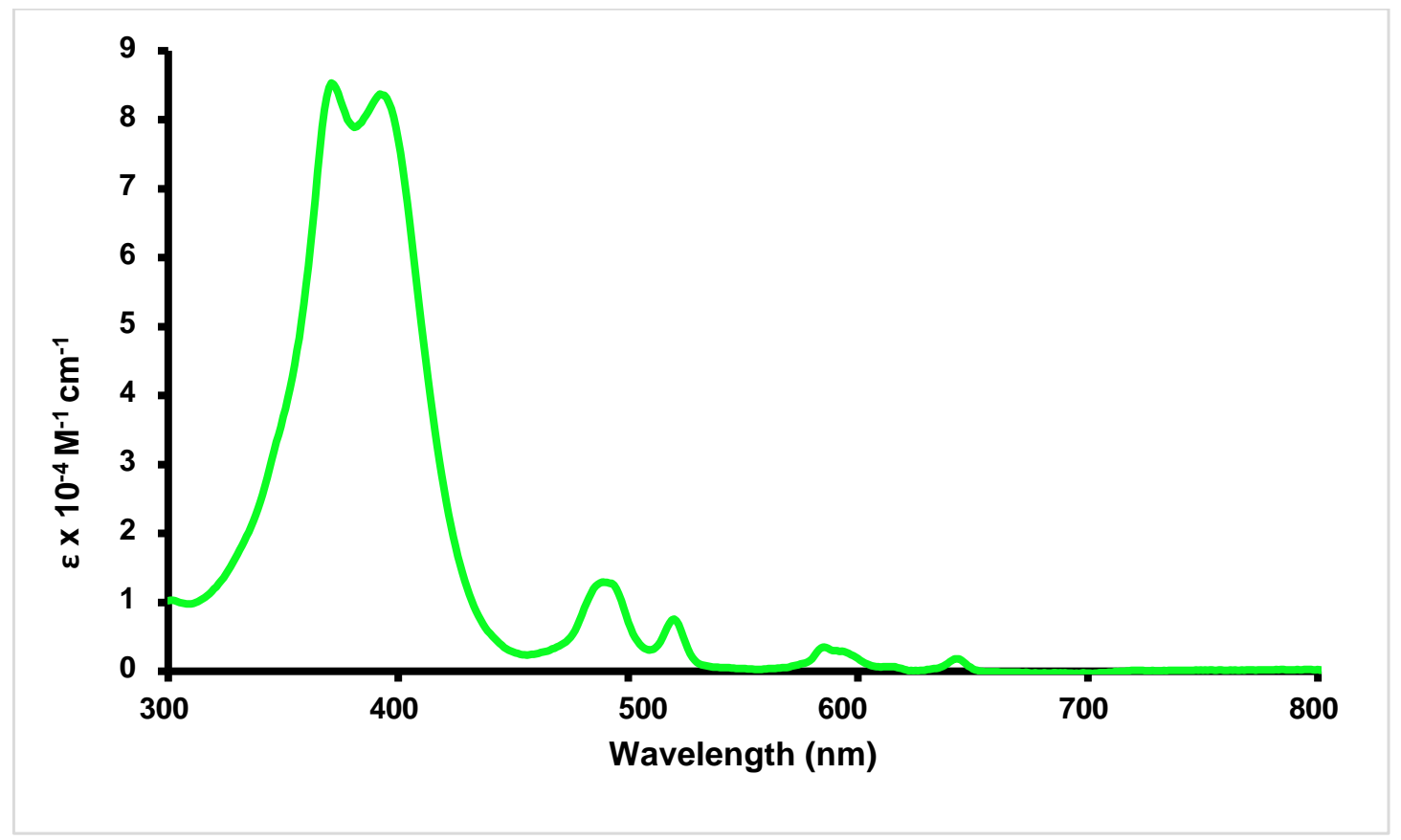

Figure D-85. UV-Vis spectrum of 21-oxaporphyrin 96a in 1\% triethylamine-dichloromethane

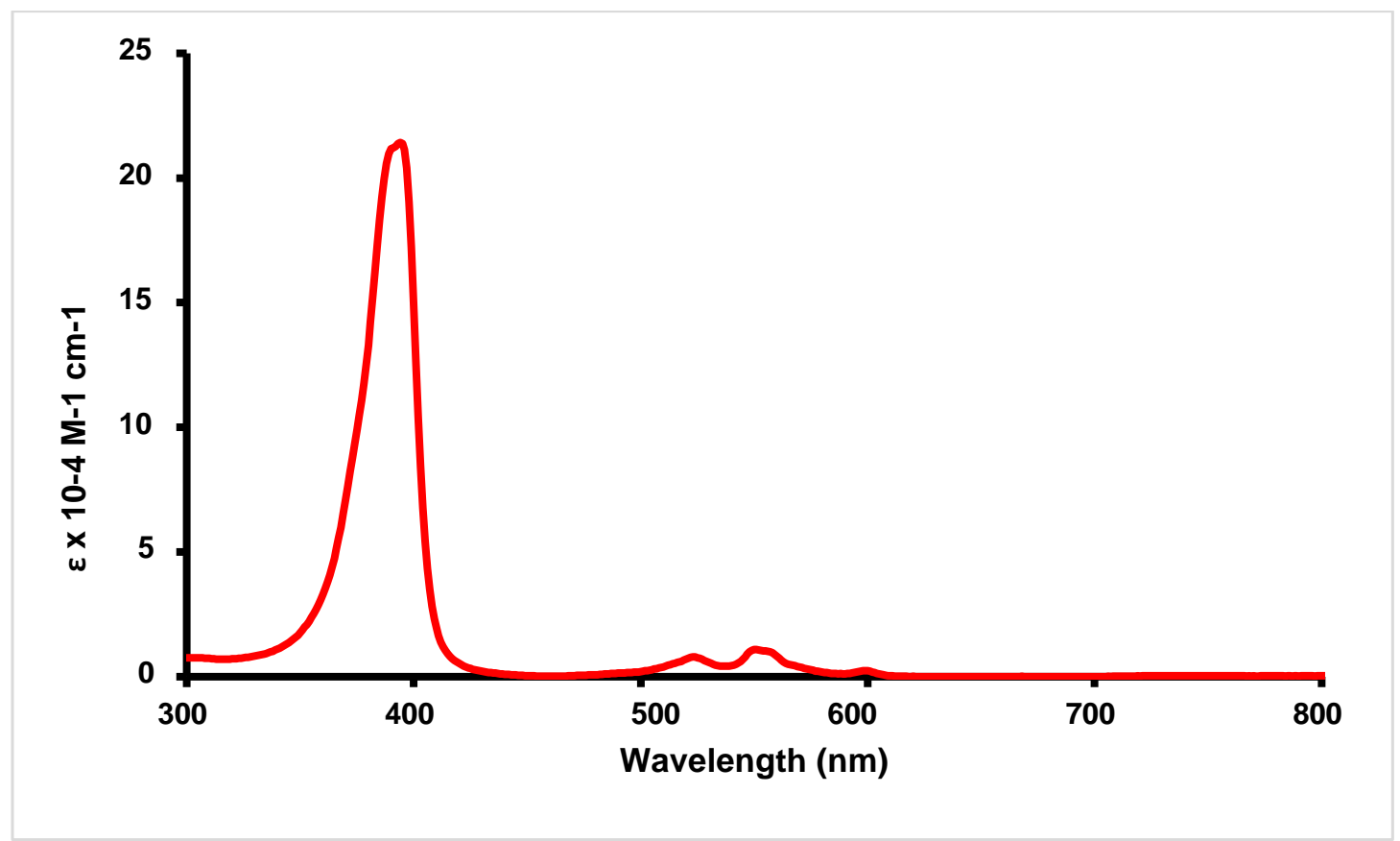

Figure D-86. UV-Vis spectrum of 21-oxaporphyrin 96a in 1\% TFA-dichloromethane 


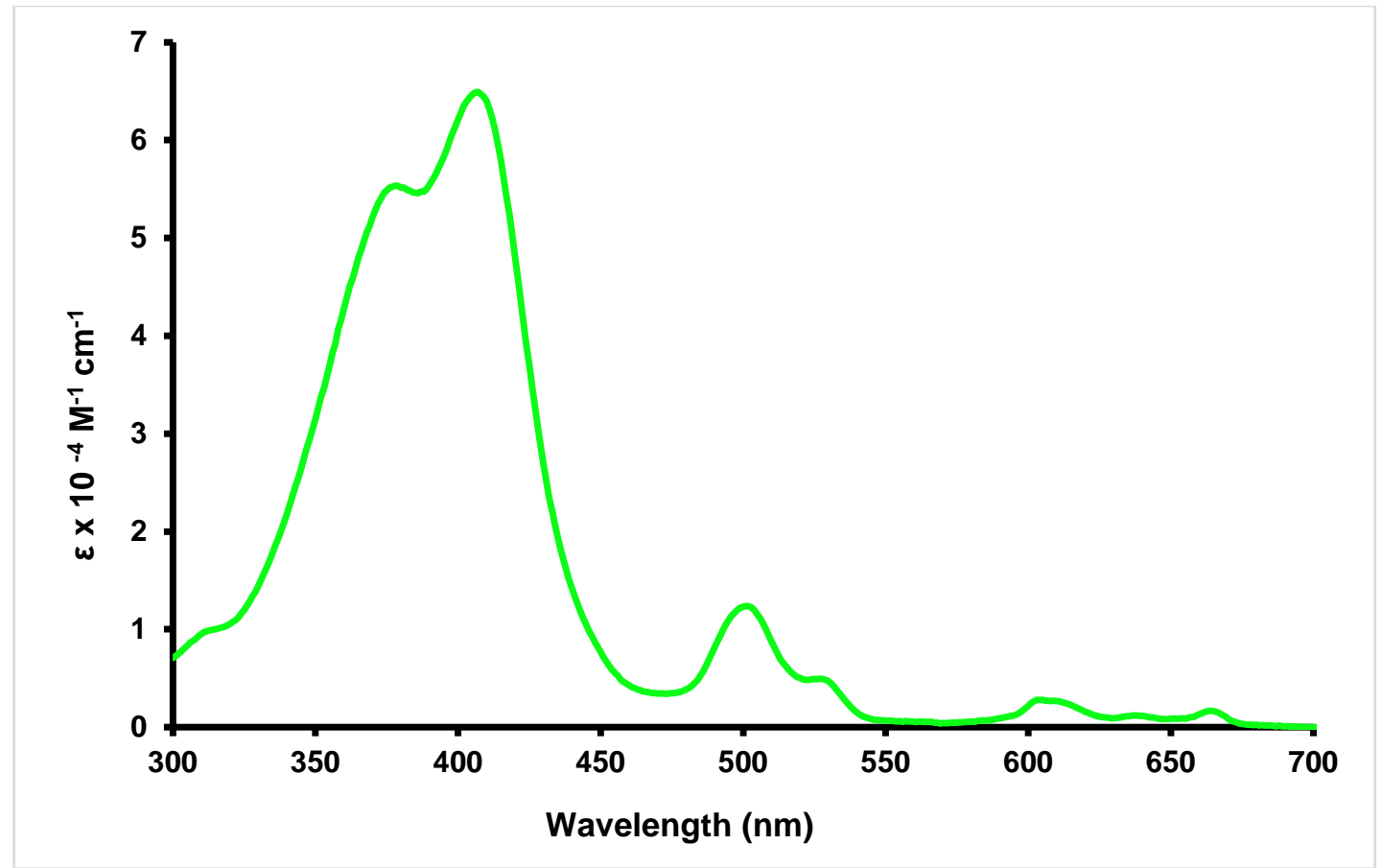

Figure D-87. UV-Vis spectrum of $N$-methyl oxaporphyrin $96 \mathbf{b}$ in $1 \%$ triethylaminedichloromethane.

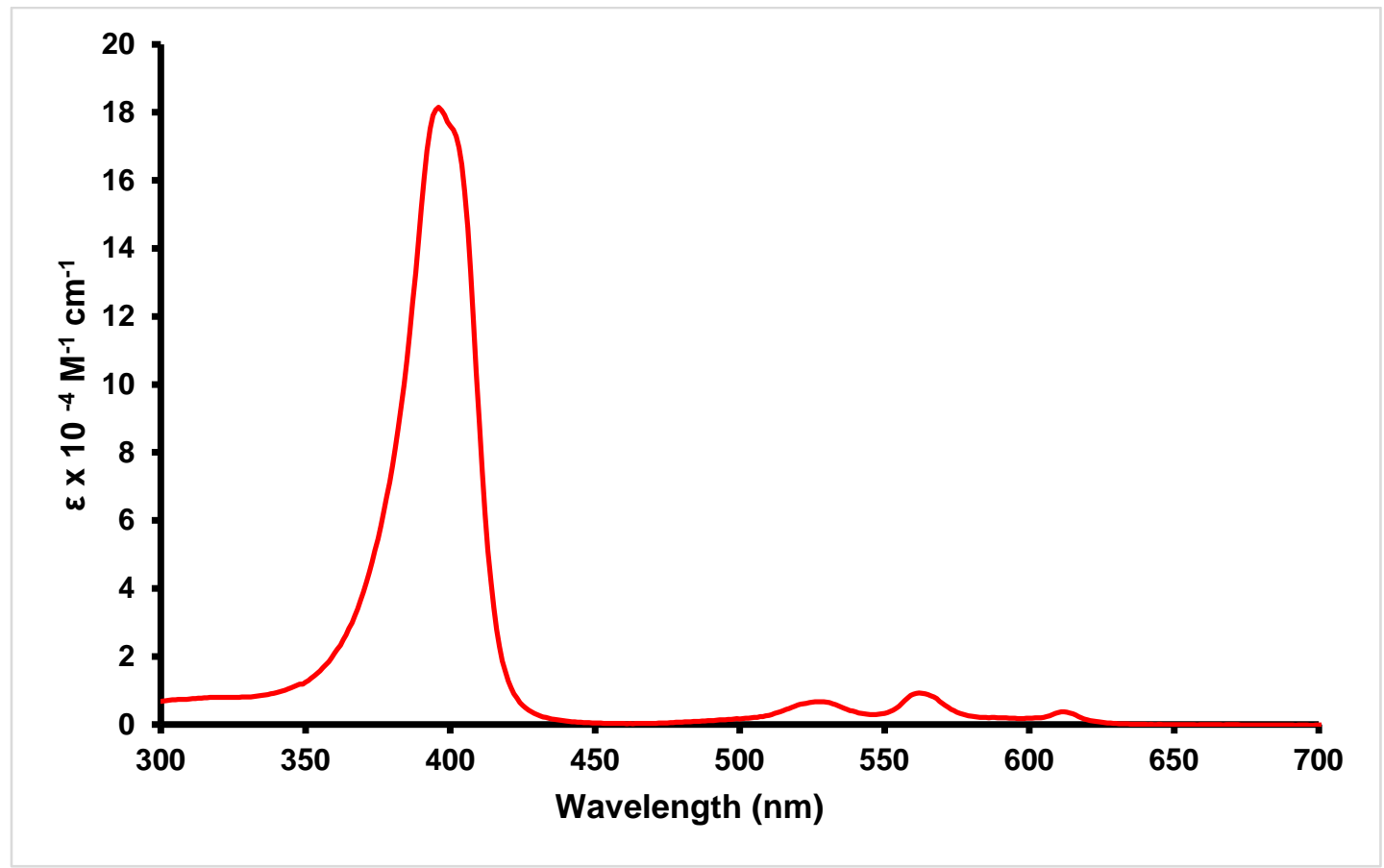

Figure D-88. UV-Vis spectrum of $N$-methyl oxaporphyrin $96 \mathbf{b}$ in 1\% TFA-dichloromethane. 


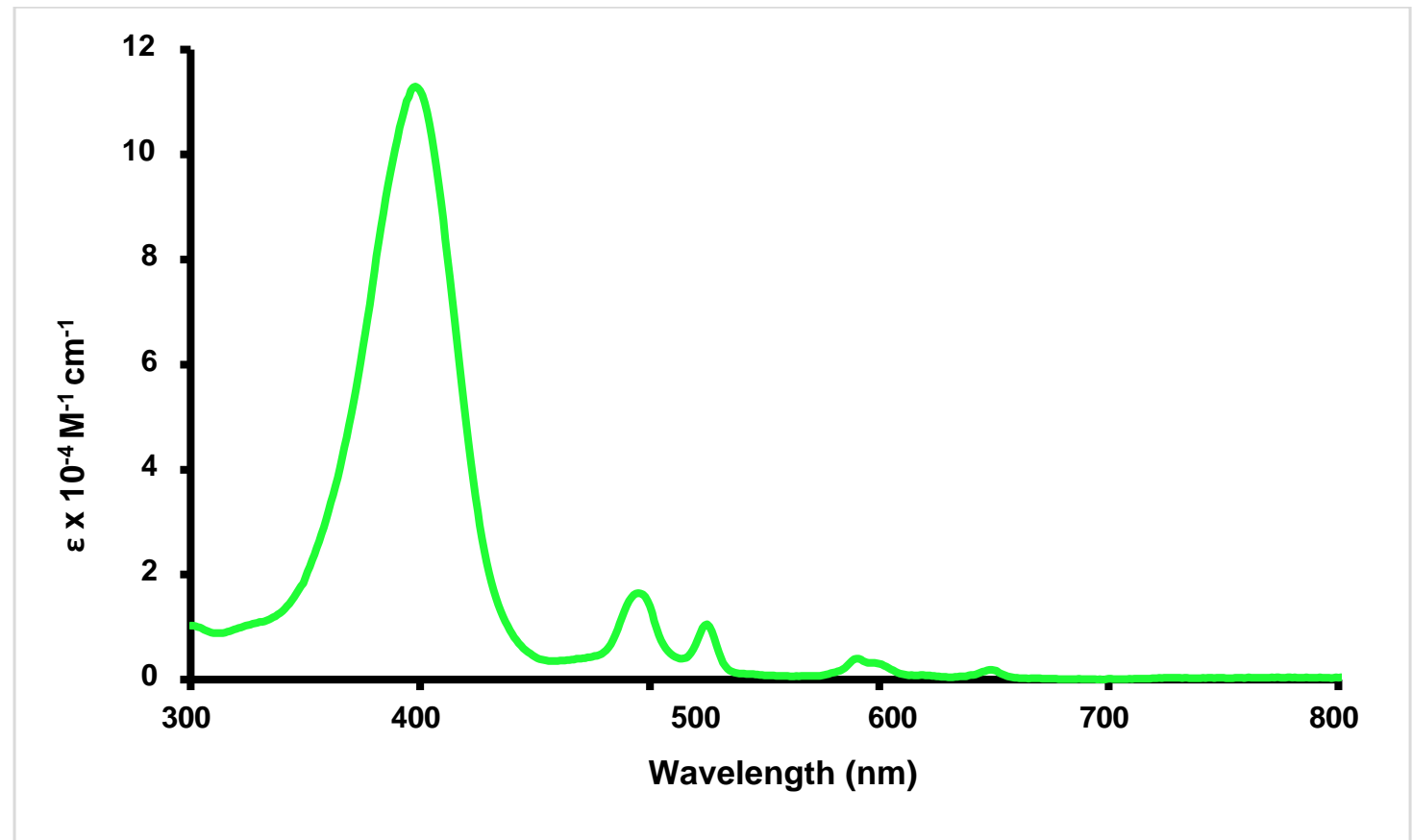

Figure D-89. UV-Vis spectrum of 21-thiaporphyrin 97a in 1\% triethylamine-dichloromethane

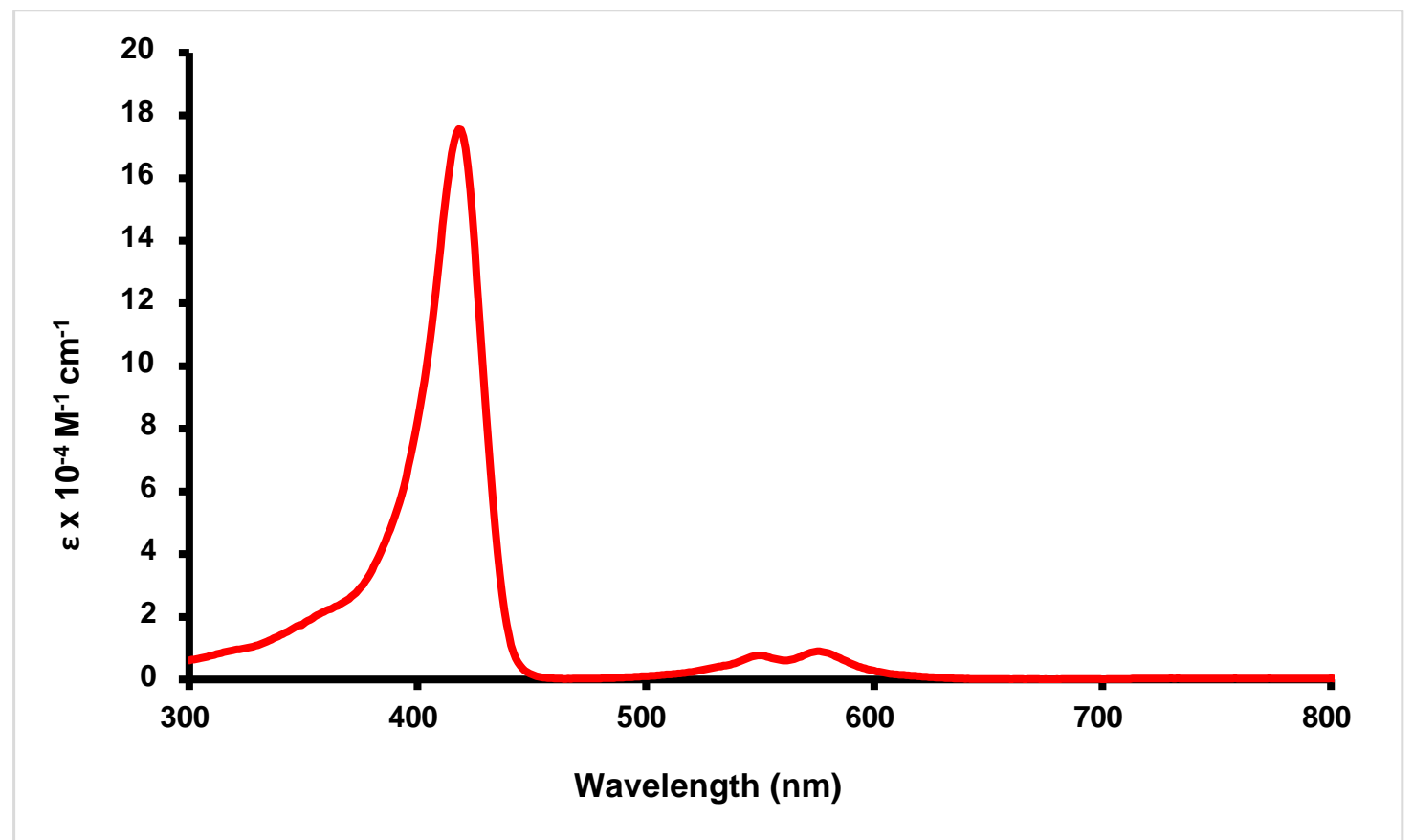

Figure D-90. UV-Vis spectrum of 21-thiaporphyrin 97a in 1\% TFA-dichloromethane 


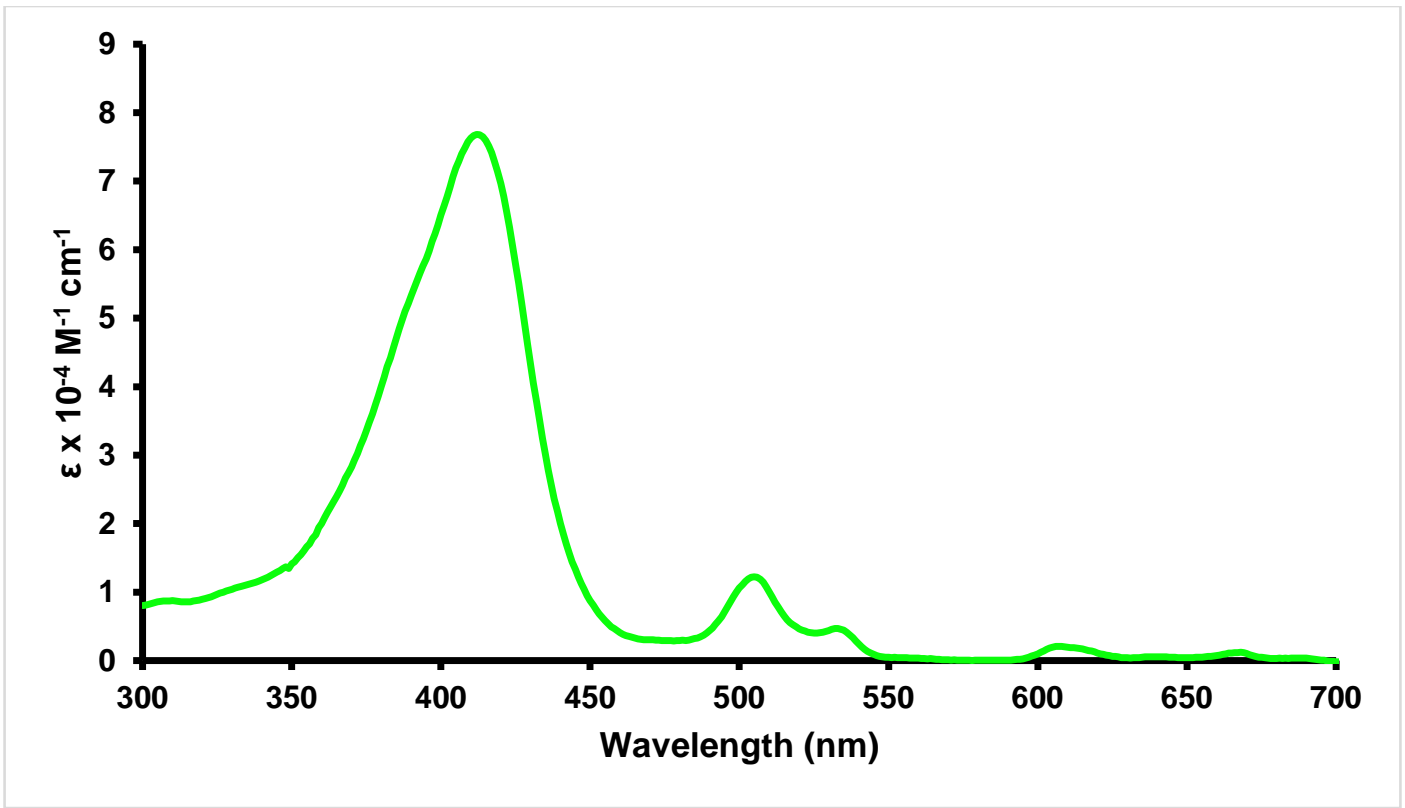

Figure D-91. UV-Vis spectrum of $N$-methyl thiaporphyrin 97b in 1\% triethylaminedichloromethane.

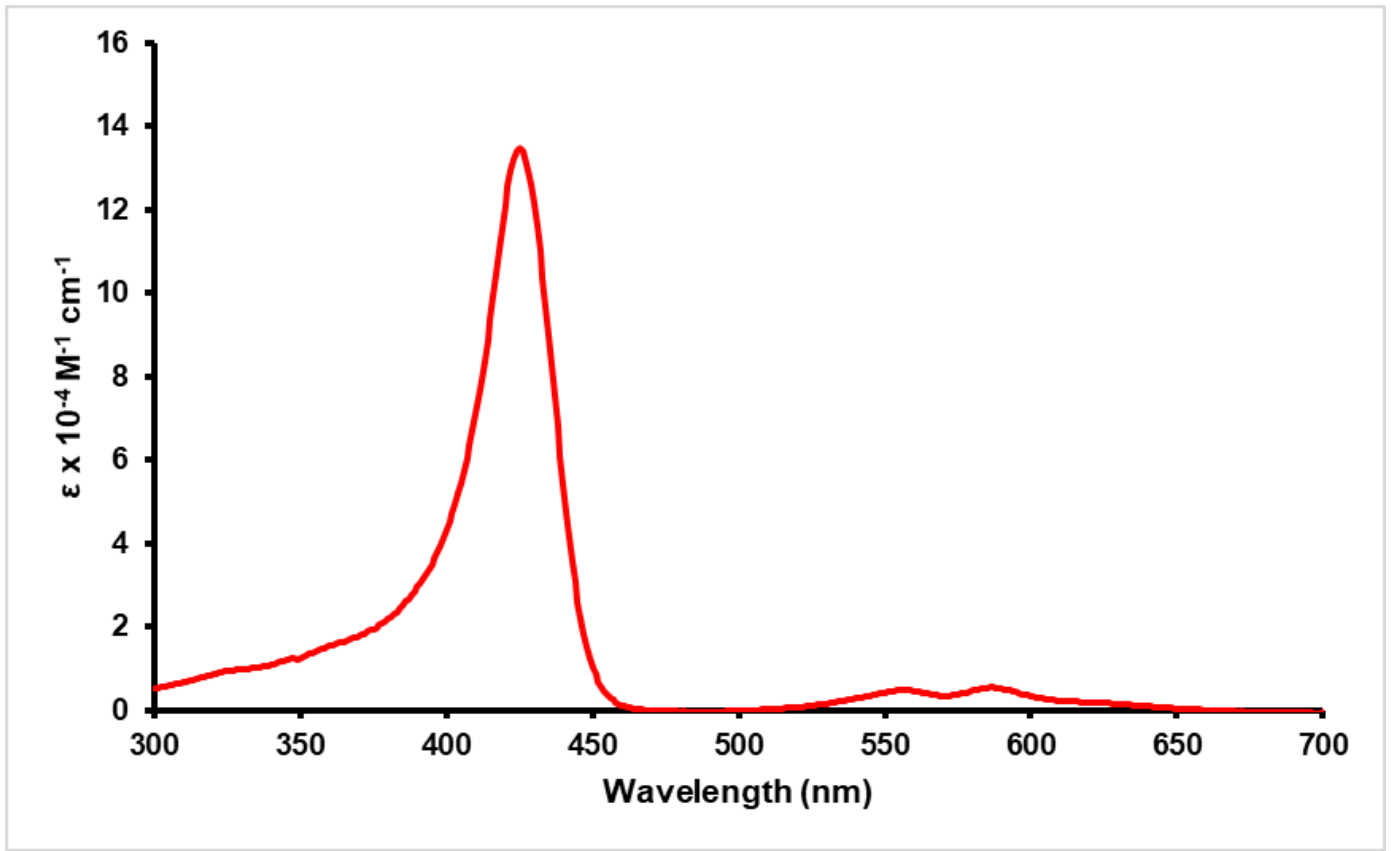

Figure D-92. UV-Vis spectrum of $N$-methyl thiaporphyrin 96b in 1\% TFA-dichloromethane. 


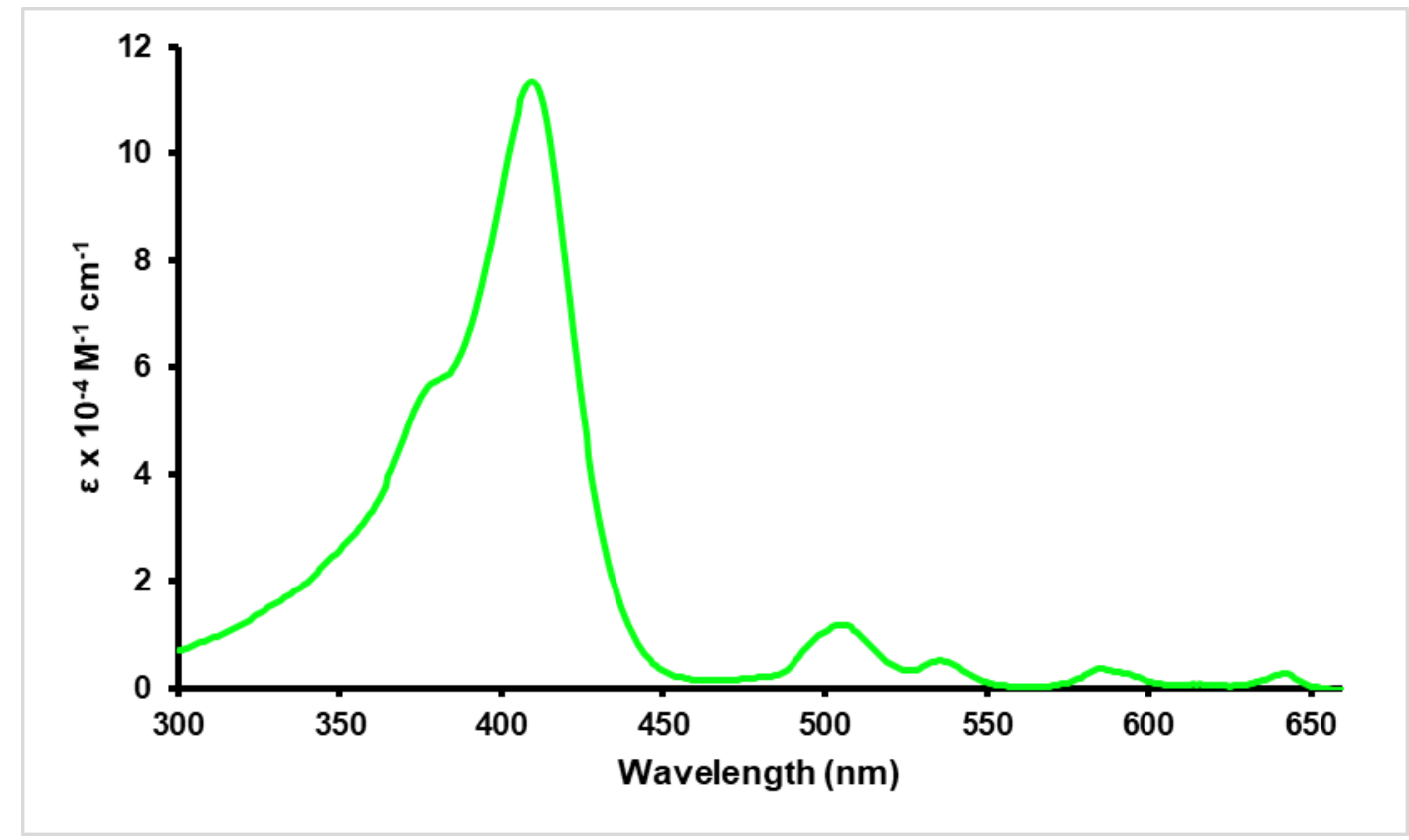

Figure D-93. UV-Vis spectrum of $N$-methyl porphyrin 99b in 1\% triethylaminedichloromethane.

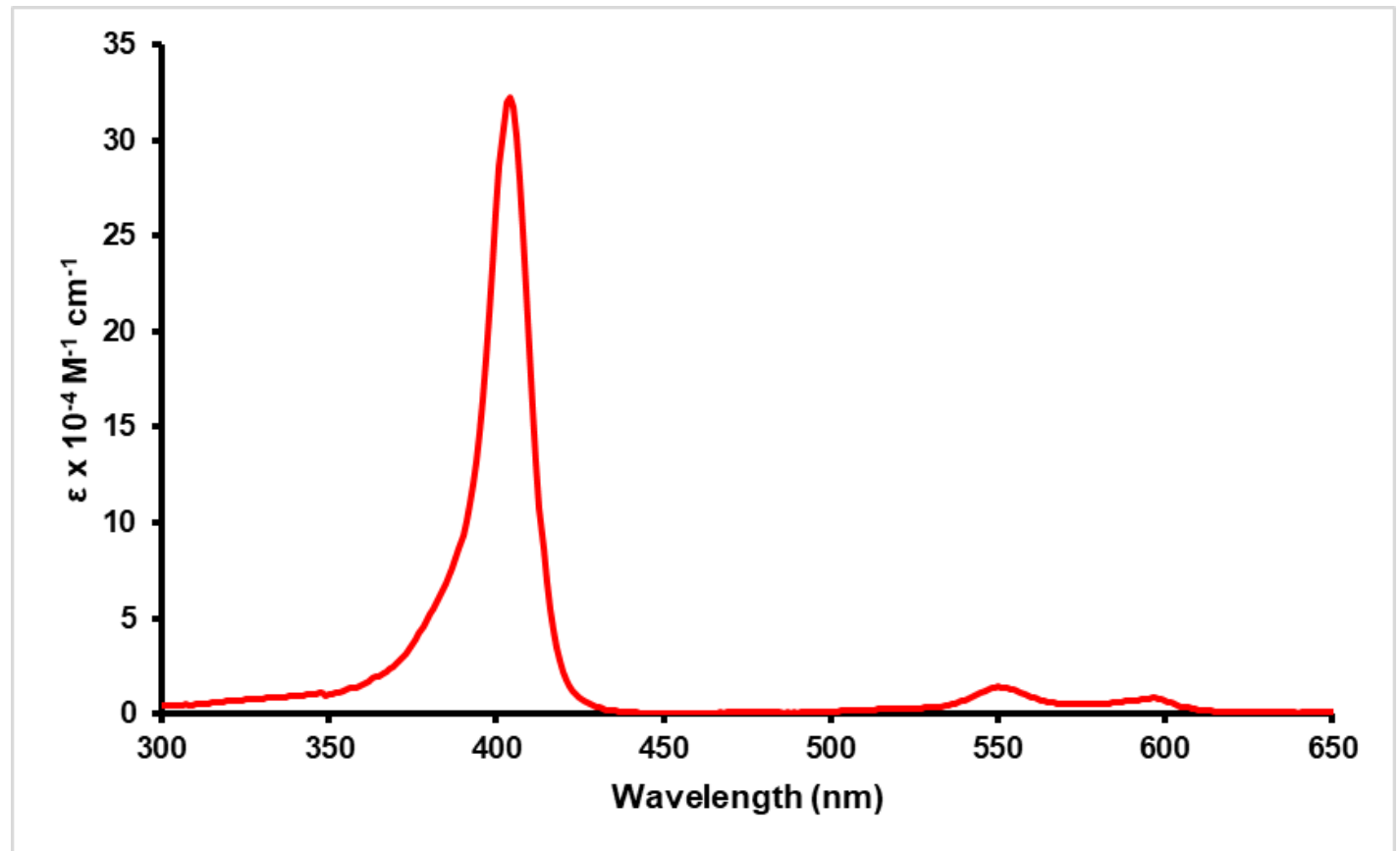

Figure D-94. UV-Vis spectrum of $N$-methyl porphyrin 99b in 1\% TFA-dichloromethane. 


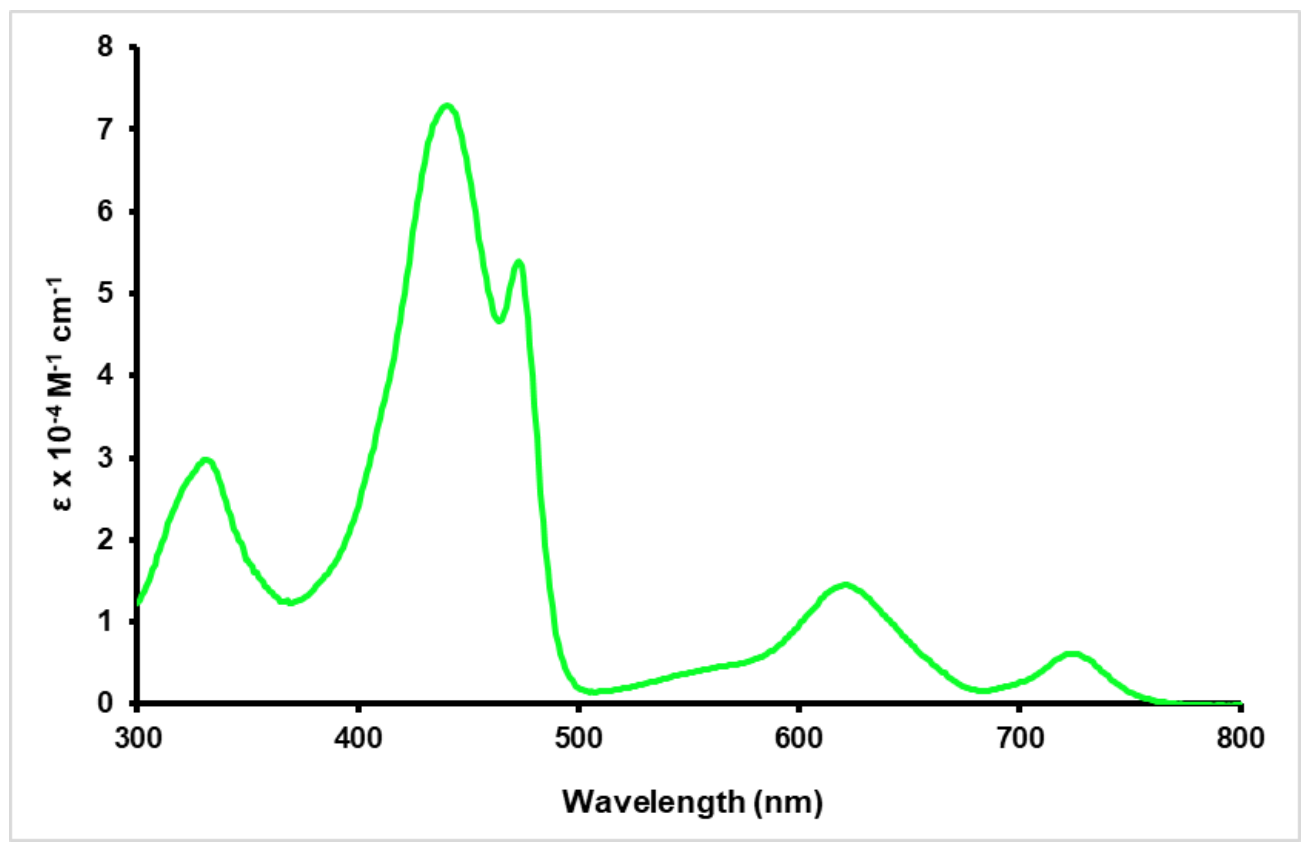

Figure D-95. UV-Vis spectrum of $N$-methyl oxybenziporphyrin 100a in 1\% triethylamine-dichloromethane.

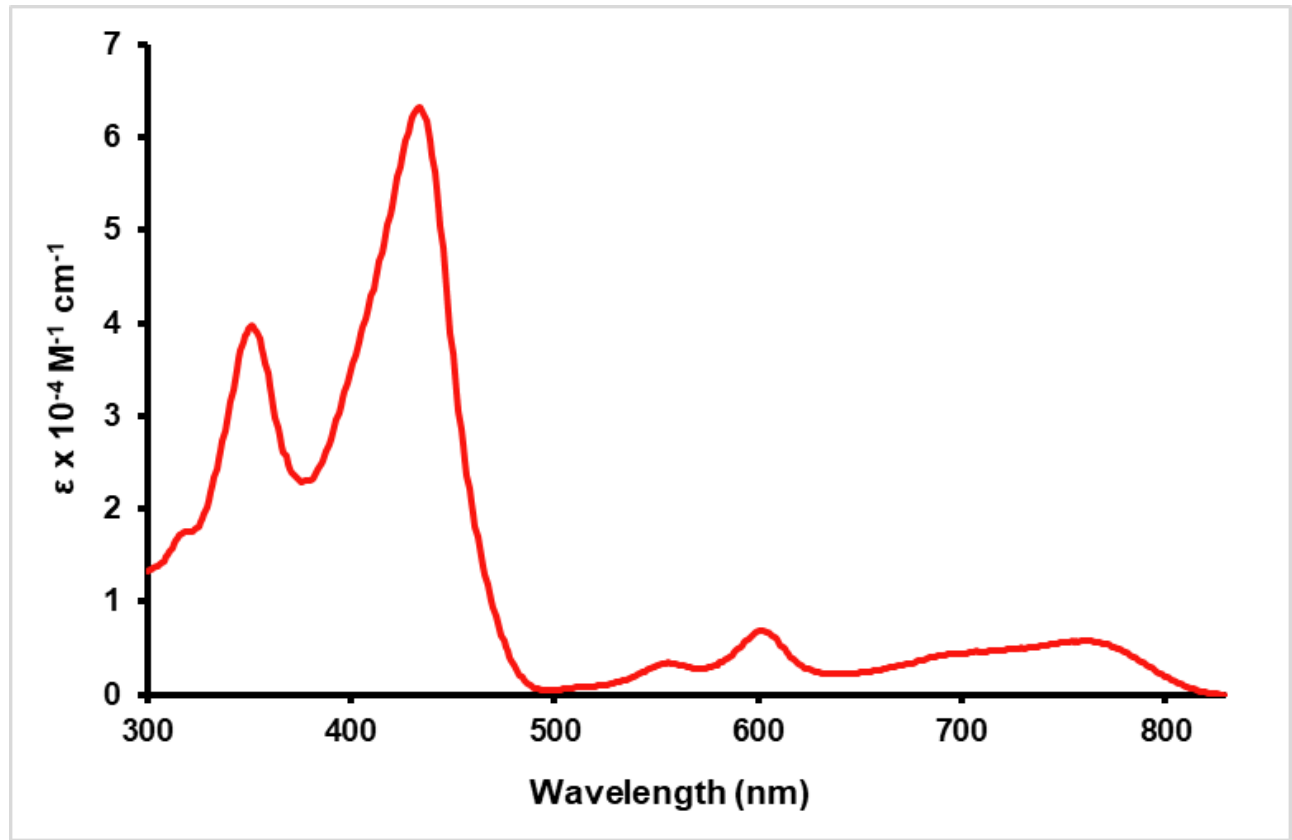

Figure D-96. UV-Vis spectrum of $N$-methyl oxybenziporphyrin 100a in 1\% TFAdichloromethane. 


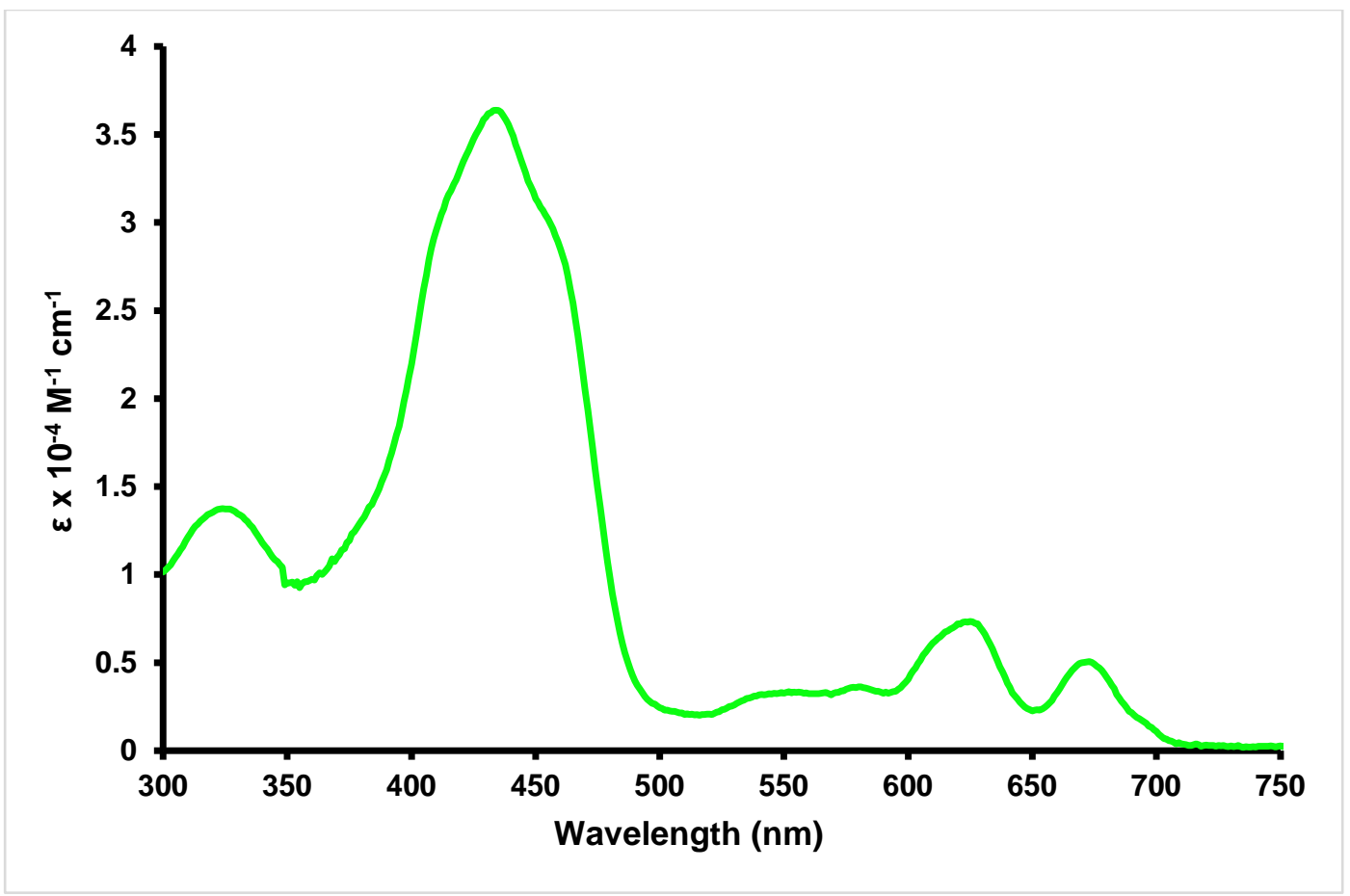

Figure D-97. UV-Vis spectrum of $N$-methyl oxypyriporphyrin 100b in 1\% triethylamine-dichloromethane.

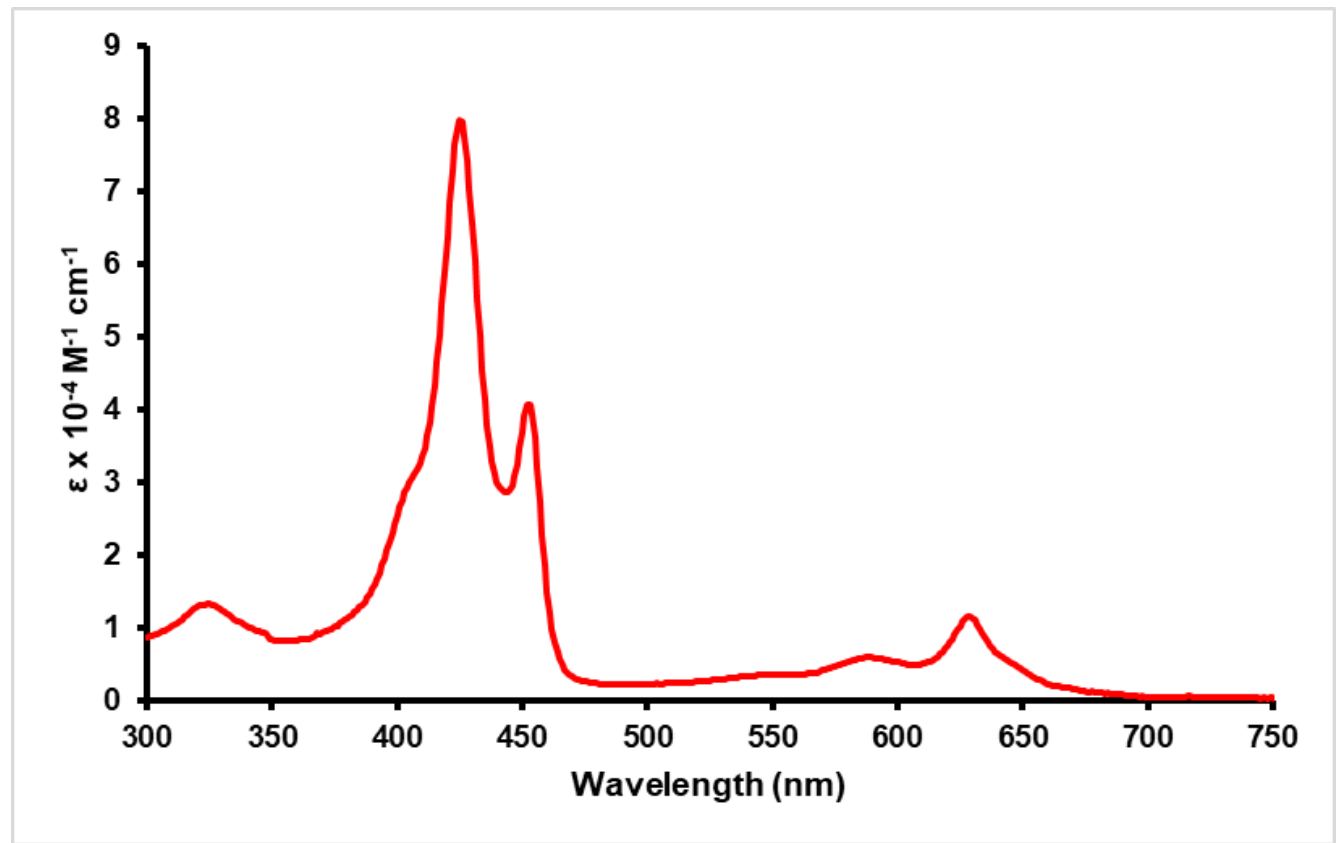

Figure D-98. UV-Vis spectrum of $N$-methyl oxypyriporphyrin 100b in 1\% TFA-dichloromethane. 


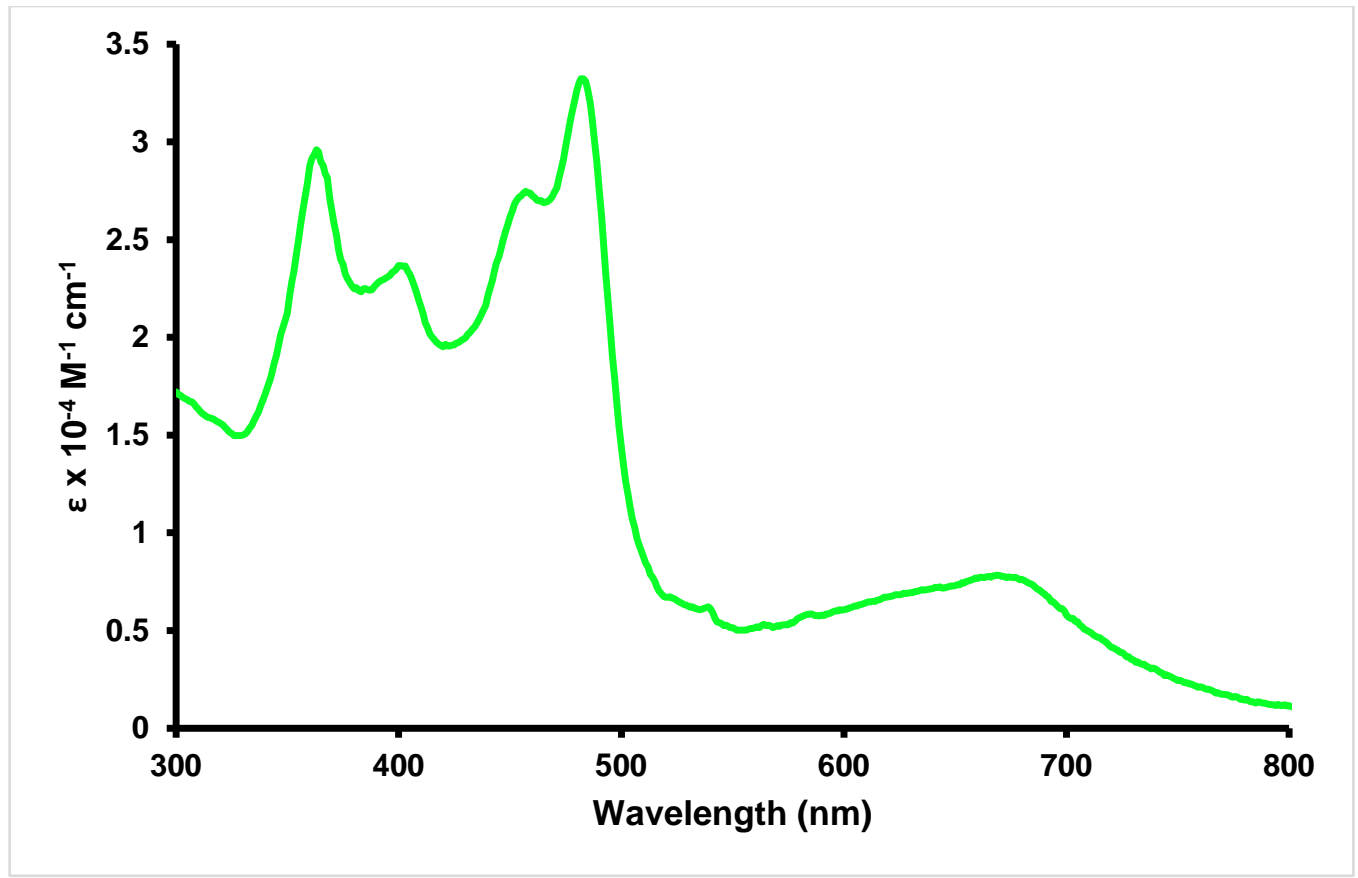

Figure D-99. UV-Vis spectrum of $N$-methyl tert-butyl azuliporphyrin $103 \mathrm{H}_{2}{ }^{2+}$ in $1 \%$ triethylamine-dichloromethane.

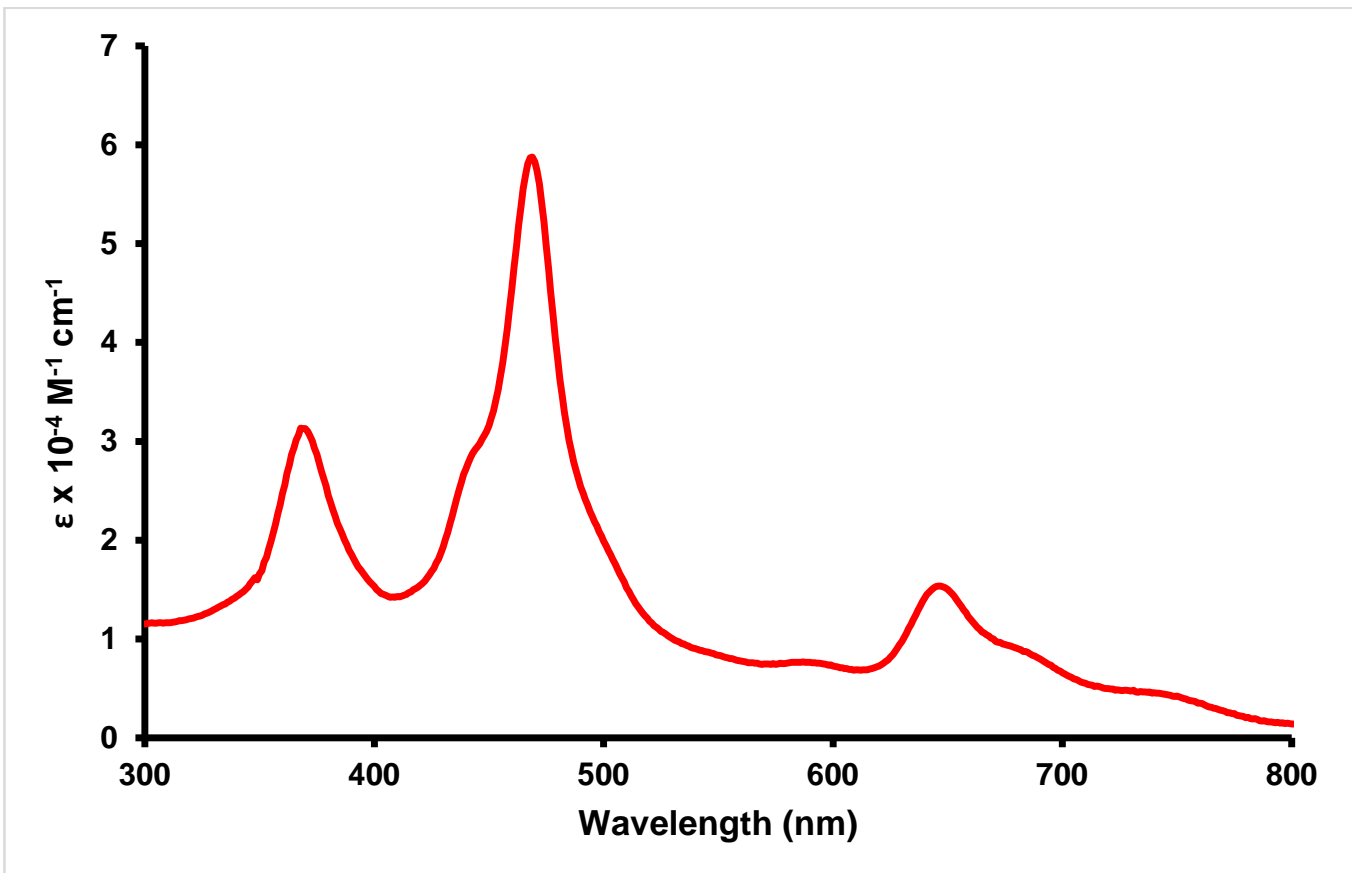

Figure D-100. UV-Vis spectrum of $N$-methyl tert-butyl azuliporphyrin $103 \mathrm{H}_{2}{ }^{2+}$ in 1\% TFA-dichloromethane. 